\title{
Supporting Information
}

Multicomponent Coupling Reaction of Perfluoroarenes with 1,3-Butadiene and Aryl Grignard

\section{Reagents Promoted by an Anionic Ni(II) Complex}

Takanori Iwasaki,* Asuka Fukuoka, Xin Min, Wataru Yokoyama, Hitoshi Kuniyasu, and Nobuaki

Kambe*

Department of Applied Chemistry, Graduate School of Engineering, Osaka University, Suita, Osaka 565-0871, Japan

Table of Contents

Additional Results

Materials and Methods

Synthesis of Substrates

General Procedures

Characterization Data of the Isolated Compounds

References

${ }^{1} \mathrm{H}$ and ${ }^{13} \mathrm{C}$ NMR Spectra of Products
S2-S16

S17

S18-S22

S21

S22-S39

S40

S41-S181 


\section{Additional Results}

\section{Synthesis of $\operatorname{NiPh}\left(\eta^{1}, \eta^{3}-\mathrm{Cr}_{8} \mathrm{H}_{12}\right) \cdot \operatorname{Li}\left(12-\right.$ crown-4) ${ }_{2} 1^{\prime}$ (Scheme 2)}

In to a suspension of $\mathrm{Ni}(\mathrm{cod})_{2}(266 \mathrm{mg}, 0.969 \mathrm{mmol})$ in $\mathrm{Et}_{2} \mathrm{O}(5 \mathrm{~mL})$ in a Schlenk tube was added $\mathrm{PhLi}$ (in cyclohexane/Et $2 \mathrm{O}, 1.09 \mathrm{M}, 1.2 \mathrm{~mL}, 1.31 \mathrm{mmol}$ ) and 1,3-butadiene (135 mL as gas, $6.03 \mathrm{mmol})$ at $-78^{\circ} \mathrm{C}$, then the mixture was allowed to warm to $\mathrm{rt}$ and stirred for $20 \mathrm{~min}$. The resulting orange solution was again cooled with a dry-ice ethanol bath and slightly depressurized. The Schlenk tube was brought into a glove box. The solution was diluted with $10 \mathrm{~mL}$ of toluene and filtrated through a glass wool. Into the filtrate was added 12-crown-4 $(0.60 \mathrm{~mL}, 3.71 \mathrm{~mL})$ to form orange precipitates. The orange solid was quickly collected by filtration, washed with toluene $(3 \mathrm{~mL})$ and pentane $(3 \mathrm{~mL} \times 3)$, and dried in vacuo to give a thermally unstable orange solid (467 $\mathrm{mg}, 80 \%):{ }^{1} \mathrm{H}$ NMR $\left(400 \mathrm{MHz}, \mathrm{THF}-d_{8},-20{ }^{\circ} \mathrm{C}\right): \delta 7.61(\mathrm{dd}, J=$ $\left.7.2,1.2 \mathrm{~Hz}, 2 \mathrm{H}, \mathrm{H}_{\mathrm{j}}\right), 6.68\left(\mathrm{t}, J=7.2 \mathrm{~Hz}, 2 \mathrm{H}, \mathrm{H}_{\mathrm{k}}\right), 6.49(\mathrm{dt}, J=7.2$, $\left.1.2 \mathrm{~Hz}, 1 \mathrm{H}, \mathrm{H}_{\mathrm{l}}\right), 5.98\left(\mathrm{td}, J=11.2,7.2 \mathrm{~Hz}, 1 \mathrm{H}, \mathrm{H}_{\mathrm{g}}\right), 4.10(\mathrm{br} \mathrm{q}, J=$

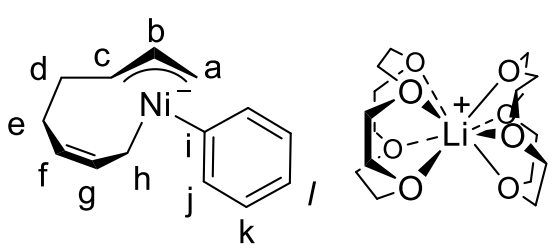
$\left.8.0 \mathrm{~Hz}, 1 \mathrm{H}, \mathrm{H}_{\mathrm{f}}\right), 3.79\left(\mathrm{td}, J=12.4,7.2 \mathrm{~Hz}, 1 \mathrm{H}, \mathrm{H}_{\mathrm{b}}\right), 3.64(\mathrm{~s}, 32 \mathrm{H}$, 12-crown-4), 2.15 (dd, $\left.J=11.2,4.0 \mathrm{~Hz}, 1 \mathrm{H}, \mathrm{H}_{\mathrm{h}}\right), 2.05-1.92\left(\mathrm{~m}, 2 \mathrm{H}, \mathrm{H}_{\mathrm{d}}, \mathrm{H}_{\mathrm{e}}\right), 1.98\left(\mathrm{~d}, J=7.6 \mathrm{~Hz}, 1 \mathrm{H}, \mathrm{H}_{\mathrm{a}}\right)$, $1.70\left(\mathrm{td}, J=10.8,5.2 \mathrm{~Hz}, 1 \mathrm{H}, \mathrm{H}_{\mathrm{c}}\right), 1.62\left(\mathrm{~d}, J=12.8 \mathrm{~Hz}, 1 \mathrm{H}, \mathrm{H}_{\mathrm{a}}\right), 1.60-1.52\left(\mathrm{~m}, 1 \mathrm{H}, \mathrm{H}_{\mathrm{e}}\right), 1.15(\mathrm{dd}, J=$ 7.2, $4.4 \mathrm{~Hz}, 1 \mathrm{H}, \mathrm{H}_{\mathrm{h}}$ ), 0.68 (q like, $\left.J=10.4 \mathrm{~Hz}, 1 \mathrm{H}, \mathrm{H}_{\mathrm{d}}\right):{ }^{13} \mathrm{C}$ NMR $\left(100 \mathrm{MHz}, \mathrm{THF}-d_{8},-20{ }^{\circ} \mathrm{C}\right): \delta 177.8$ $\left(\mathrm{C}_{\mathrm{i}}\right), 140.8\left(\mathrm{C}_{\mathrm{j}}\right), 135.6\left(\mathrm{C}_{\mathrm{g}}\right), 123.5\left(\mathrm{C}_{\mathrm{k}}\right), 117.6\left(\mathrm{C}_{1}\right), 104.4\left(\mathrm{C}_{\mathrm{b}}\right), 100.2\left(\mathrm{C}_{\mathrm{f}}\right), 68.3$ (12-crown-4), $57.6\left(\mathrm{C}_{\mathrm{c}}\right)$, $49.3\left(C_{a}\right), 28.9\left(C_{d}\right), 25.4\left(C_{e}\right), 19.1\left(C_{h}\right)$. These data are in accord with those of a similar compounds reported by us. ${ }^{\mathrm{S} 1}$ Although the complex 1 ' could be stocked at $-25{ }^{\circ} \mathrm{C}$ for several days, it turned to black sticky oil within several minutes at room temperature in a solid state probably due to the thermal decomposition. Thereby, the complex 1' does not have melting point and the elemental analysis resulted in failure. 


\section{X-ray crystallographic analysis}

Single crystals of $\mathrm{Ni}\left(\mathrm{C}_{6} \mathrm{H}_{5}\right)\left(\mathrm{C}_{8} \mathrm{H}_{12}\right) \cdot \mathrm{Li}(12 \text {-crown-4) })_{2}$ 1' suitable for X-ray crystallography were obtained by recrystallization of $\mathrm{Ni}\left(\mathrm{C}_{6} \mathrm{H}_{5}\right)\left(\mathrm{C}_{8} \mathrm{H}_{12}\right) \cdot \mathrm{Li}(12 \text {-crown-4) })_{2} \mathbf{1}$ ' from THF and pentane at $-25{ }^{\circ} \mathrm{C}$ under $\mathrm{N}_{2}$ atmosphere. The crystal was mounted on the CryoLoop (Hampton Research Corp.) with a layer of light mineral oil (dried over metallic $\mathrm{Na}$ at $100{ }^{\circ} \mathrm{C}$ for overnight) and placed in a nitrogen stream at $123(2) \mathrm{K}$. Measurements were made on a Rigaku RAXIS-RAPID Imaging Plate diffractometer $(\mathrm{Cu}-\mathrm{K} \alpha)$. The structure of the complex 1' was solved by direct methods (SIR2008 $\left.{ }^{\mathrm{S} 2}\right)$. The structure was refined on $F^{2}$ by full-matrix least-squares method using SHELXL97. ${ }^{\text {S3 }}$ Non-hydrogen atoms were anisotropically refined. Hydrogen atoms were refined using the riding model. The function minimized was $\left[\sum w\left(F_{\mathrm{o}}{ }^{2}-{F_{\mathrm{c}}}^{2}\right)^{2}\right]\left(w=1 /\left[\sigma^{2}\left(F_{\mathrm{o}}^{2}\right)+(0.0690 \mathrm{P})^{2}+0.3525 \mathrm{P}\right]\right)$, where $\mathrm{P}=\left(F_{\mathrm{o}}{ }^{2}+2 F_{\mathrm{c}}{ }^{2}\right) / 3$ with $\sigma^{2}\left(F_{\mathrm{o}}{ }^{2}\right)$ for counting statistics. The function $R 1$ and $w R 2$ were $\left(\Sigma|| F_{\mathrm{o}}|-| F_{\mathrm{c}} \| / \Sigma\left|F_{\mathrm{o}}\right|\right)$ and $\left[\Sigma\left(w\left(F_{\mathrm{o}}{ }^{2}-F_{\mathrm{c}}{ }^{2}\right)^{2}\right) /\right.$ $\left.\Sigma \mathrm{w}\left(F_{\mathrm{o}}\right)^{2}\right]^{1 / 2}$, respectively. The ORTEP-3 program was used to draw the molecule. ${ }^{\mathrm{S} 4}$

The 12-crown-4 ligands on $\mathrm{Li}^{+}$were disordered locating in two different orientations with $0.831(2): 0.169$ (2) ratio, probably by the rotation of the whole cationic moiety $\mathrm{Li}(12$-crown-4) 2 along its central axis by about 30 degrees. As the result, one of the methylene carbon in each $\mathrm{CH}_{2} \mathrm{CH}_{2}$ unit of the 12-crown-4 ligand in the minor orientation overlaps with that of the 12-crown-4 ligand in the major orientation. Due to the difficulties in refining the hydrogen atoms of the 12-crown-4 in the minor orientation, hydrogen atoms were attached to the 12-crown-4 only in the major orientation and the whole crystal structure was refined. 
Table S1. Crystal Data and Data Collection Parameters of $\mathrm{NiPh}\left(\eta^{1}, \eta^{3}-\mathrm{C}_{8} \mathrm{H}_{12}\right) \cdot \mathrm{Li}(12-\mathrm{crown}-4)_{2} 1^{\prime}$.

\begin{tabular}{|c|c|}
\hline empirical formula & $\mathrm{C}_{30} \mathrm{H}_{49} \mathrm{LiNiO}_{8}$ \\
\hline formula weight & 603.35 \\
\hline color, description & yellow, block \\
\hline temperature, $\mathrm{K}$ & $123(2)$ \\
\hline crystal system & Triclinic \\
\hline space group & $\mathrm{P}-1(\# 2)$ \\
\hline$a, \AA$ & $8.67174(16)$ \\
\hline$b, \AA$ & $12.0843(2)$ \\
\hline$c, \AA$ & $15.4857(3)$ \\
\hline$\alpha,{ }^{\circ}$ & $86.3290(8)$ \\
\hline$\beta, \circ$ & $78.9210(8)$ \\
\hline$\gamma,{ }^{\circ}$ & $74.0440(8)$ \\
\hline$V, \AA^{3}$ & $1531.07(5)$ \\
\hline$Z$ & 2 \\
\hline Dcalcd, $\mathrm{g} / \mathrm{cm}^{-3}$ & 1.309 \\
\hline $2 \theta_{\max }$, deg & 143.4 \\
\hline limiting indices & $-10 \leq h \leq 10,-14 \leq k \leq 14,-19 \leq l \leq 18$ \\
\hline absorption coefficient, & 1.298 \\
\hline $\max$. and $\min$. & $0.878(\max ) / 0.715(\min )$ \\
\hline $\mathrm{F}(000)$ & 648.00 \\
\hline crystal size, mm & $0.35 \times 0.20 \times 0.10$ \\
\hline goodness-of-fit on $F^{2}$ & 1.075 \\
\hline no. of reflections measured & 31816 \\
\hline unique data $\left(R_{\text {int }}\right)$ & $5807(0.0421)$ \\
\hline$R 1, w R 2[I>2 \sigma(I)]$ & $0.0415,0.1162$ \\
\hline$R 1, w R 2$ (all data) & $0.0465,0.1193$ \\
\hline residual electron density, e $\AA^{-3}$ & $0.88(\max ),-0.41(\min )$ \\
\hline
\end{tabular}




\section{NMR studies of the reaction of $\mathrm{Ni}(0)$ with 1,3-butadiene and $\mathrm{PhMgBr}$}

In order to confirm the formation of the similar anionic $\mathrm{Ni}$ complexes carrying a magnesium counter cation, $\mathrm{Ni}(\operatorname{cod})_{2}$ was treated with $\mathrm{PhMgBr}$ in the presence of 1,3-butadiene (eq S1) and examined by NMR. A THF solution of $\mathrm{PhMgBr}(40 \mu \mathrm{mol})$ in a NMR tube was evacuated to remove the solvent and into the residue were added $\mathrm{Ni}(\operatorname{cod})_{2}(5.6 \mathrm{mg}, 20 \mu \mathrm{mol})$ and a THF- $d_{8}$ solution of 1,3-butadiene $(0.6 \mathrm{~mL}$, $0.17 \mathrm{M}, 102 \mu \mathrm{mol})$ in this order at $\mathrm{rt}$ under $\mathrm{N}_{2}$ atmosphere. ${ }^{1} \mathrm{H}$ NMR of the mixture at $-20{ }^{\circ} \mathrm{C}$ showed new sharp signals of $\mathbf{1 b}$ along with free COD ligand (Figure S1a). The observed new signals well match with the isolated complex 1' (Figure S1b). In addition, ${ }^{13} \mathrm{C}$ NMR also indicated good similarity between the both complexes as shown in Figure S2. These results suggest that a similar anionic Ni complex was formed by aryl Grignard reagents and the complex may exist as solvent separated ion par in THF.

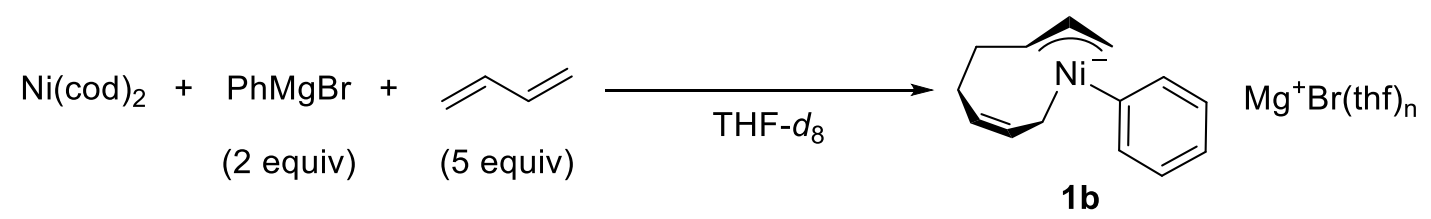

(a) ${ }^{1} \mathrm{H}$ NMR of the reaction in eq $\mathrm{S} 1$ in THF- $d_{8}$ at $-20^{\circ} \mathrm{C}$

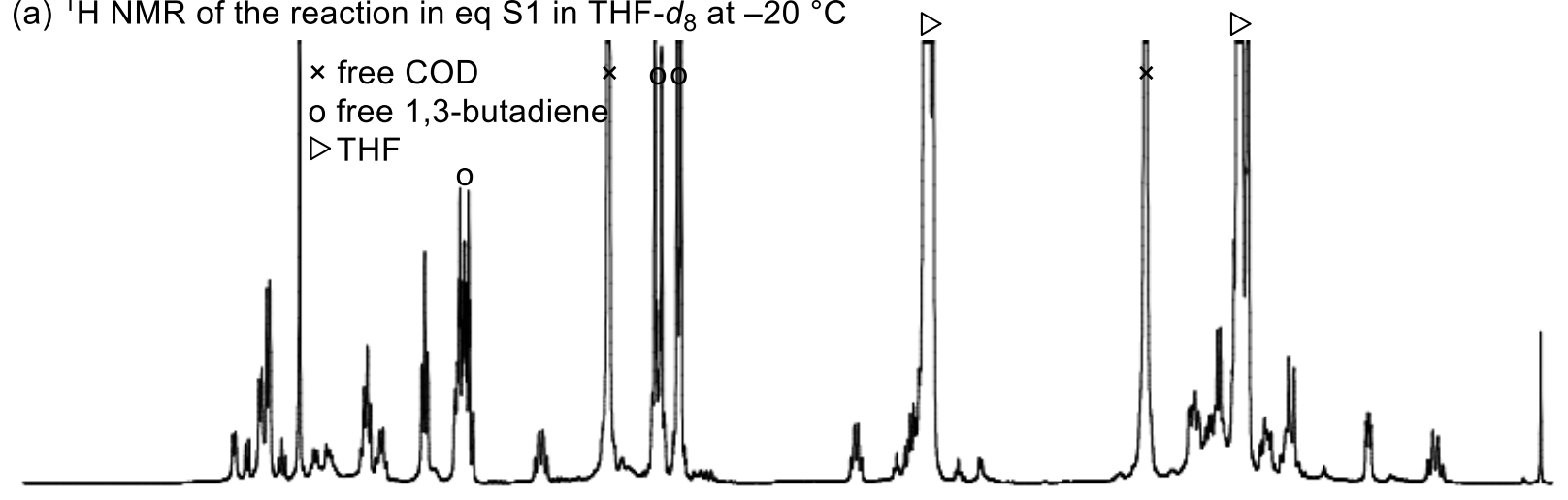

(b) ${ }^{1} \mathrm{H}$ NMR of complex 1 ' in THF- $d_{8}$ at $-20{ }^{\circ} \mathrm{C}$

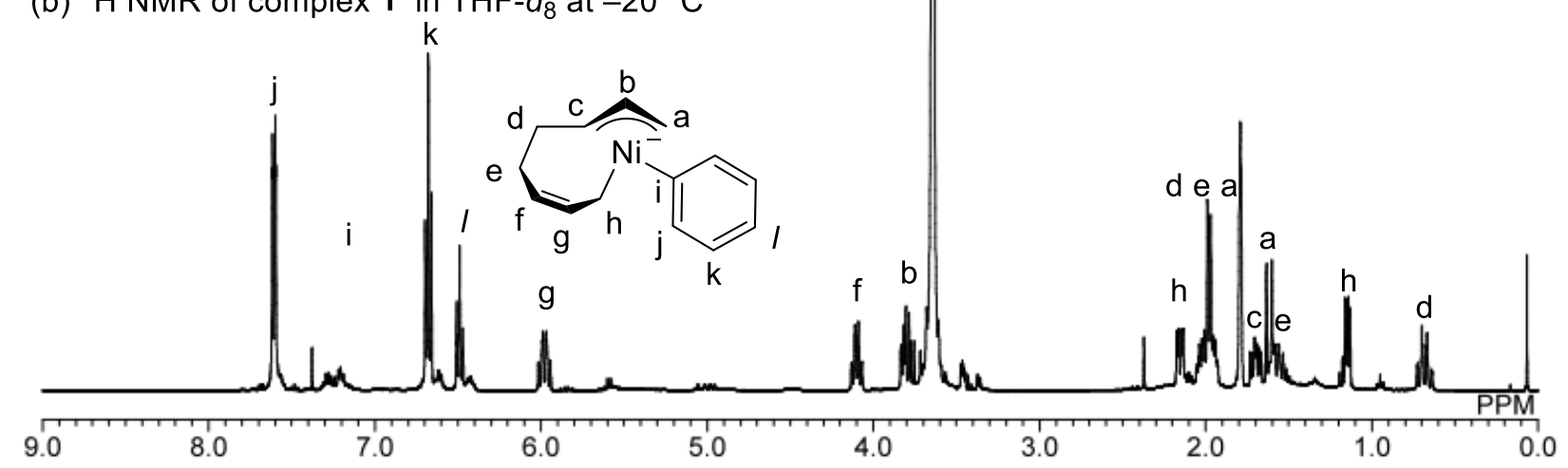

Figure S1. ${ }^{1} \mathrm{H}$ NMR spectra at $-20^{\circ} \mathbf{C}$ of $\mathbf{1 b}$ prepared by the reaction in eq $\mathrm{S} 1$ (a) and the complex $\mathbf{1}$ ' in THF- $d_{8}(\mathrm{~b})$. 
(a) ${ }^{13} \mathrm{C}$ NMR of the reaction in eq $\mathrm{S} 1 \mathrm{in} \mathrm{THF}-d_{8}$ at $-20{ }^{\circ} \mathrm{C}$

$x$ free COD

o free 1,3-butadiene

$\triangle \mathrm{THF}$

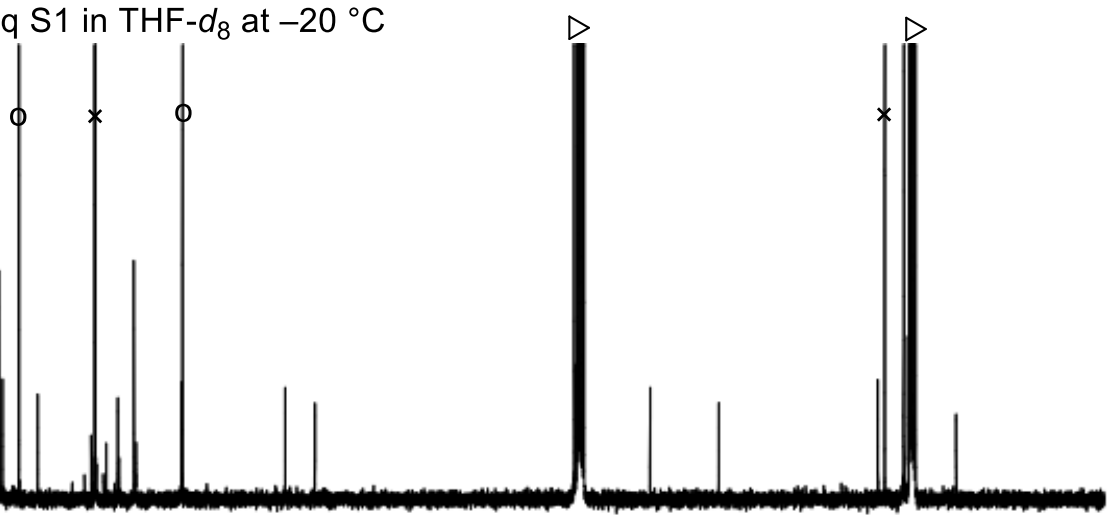

(b) ${ }^{13} \mathrm{C}$ NMR of complex $11^{\prime}$ in THF- $d_{8}$ at $-20^{\circ} \mathrm{C}$

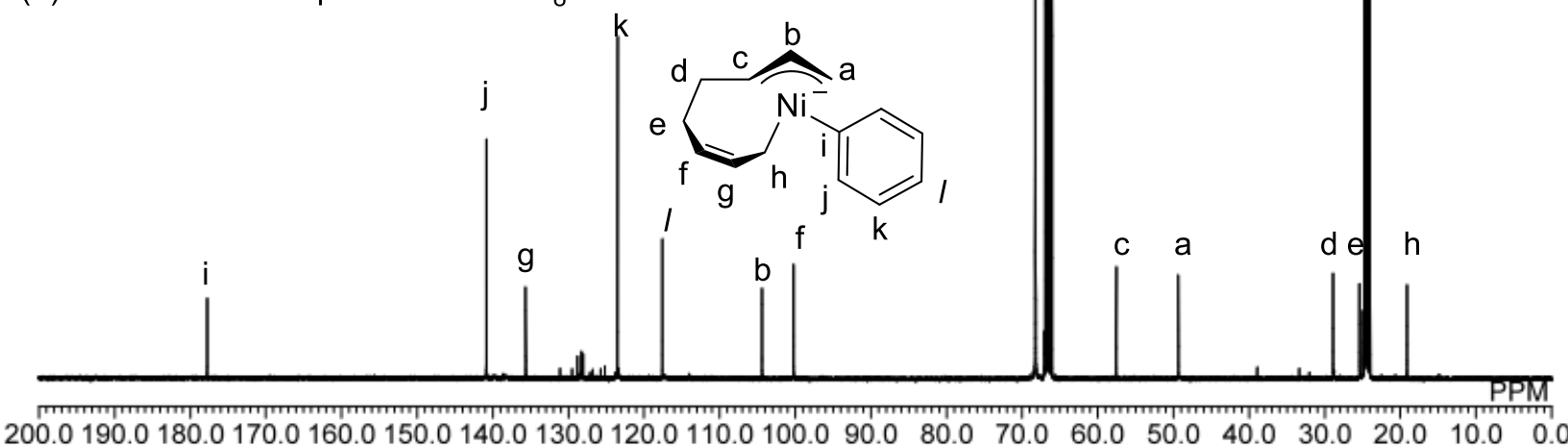

Figure S2. ${ }^{13} \mathrm{C}$ NMR spectra at $-20^{\circ} \mathrm{C}$ of $\mathbf{1 b}$ prepared by the reaction in eq $\mathrm{S} 1$ (a) and the complex $\mathbf{1}$ ' in $\mathrm{THF}-d_{8}(\mathrm{~b})$. 


\section{Four-component reaction using nickelate complex 1' as catalyst}

When the nickelate complex 1' was employed as the catalyst for the reaction of hexafluorobenzene (2a) with $p$-TolMgBr 3c and 1,3-butadiene (eq S2), the four-component coupling reaction smoothly proceeded to give $\mathbf{4 a c}$ in $41 \%$ yield along with the formation of $\mathbf{4 a b}$ containing a phenyl group derived from the Ni complex $\mathbf{1}$ ' in $7 \%$ yield.

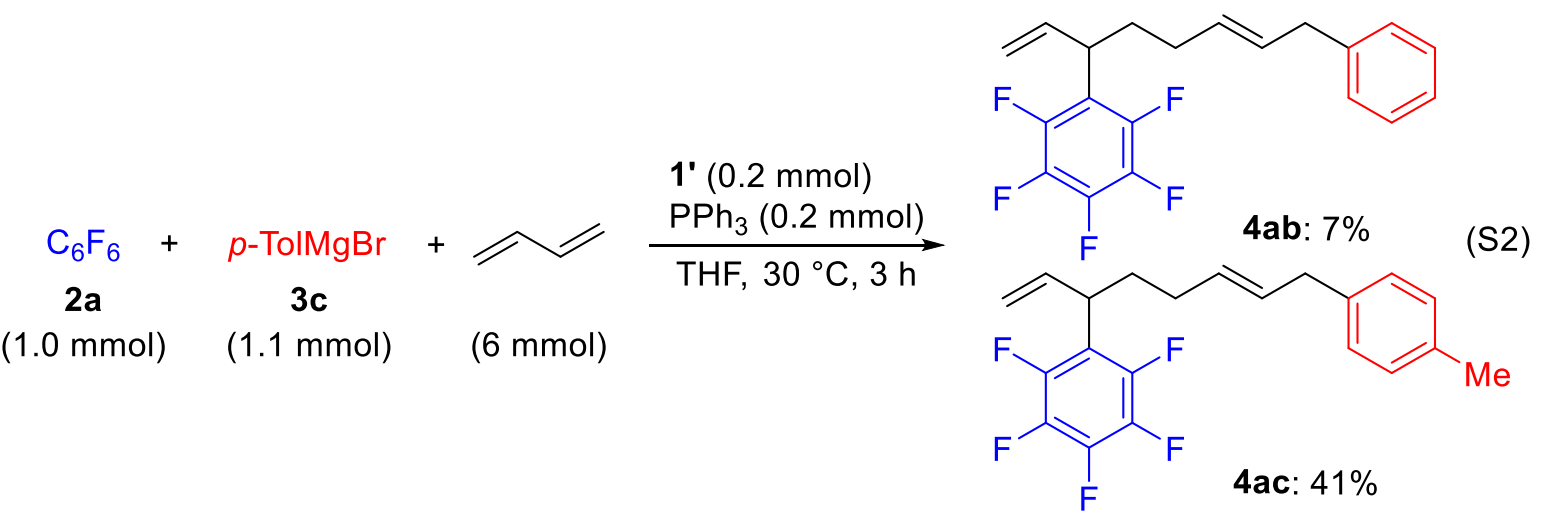

\section{Procedure for the reaction using Ni complex 1' as a catalyst (eq S2)}

In a glove box, Ni complex 1' (127.1 $\mathrm{mg}, 0.211 \mathrm{mmol})$ was added to a pre-cooled test tube containing $\mathrm{PPh}_{3}(52.9 \mathrm{mg}, 0.202 \mathrm{mmol})$. The test tube was sealed with a septum and brought out of the glove box. $p$-Tolylmagnesium bromide (in THF, $0.93 \mathrm{M}, 1.2 \mathrm{~mL}, 1.12 \mathrm{mmol}$ ), 1,3-butadiene (135 mL as gas, $6.0 \mathrm{mmol})$, and $\mathrm{C}_{6} \mathrm{~F}_{6}(180.4 \mathrm{mg}, 0.970 \mathrm{mmol})$ were added at $-78{ }^{\circ} \mathrm{C}$ followed by stirring at $30^{\circ} \mathrm{C}$ for $3 \mathrm{~h}$. The reaction was quenched by $1 \mathrm{~N} \mathrm{HCl}$, and undecane $(20 \mu \mathrm{L})$ was added as an internal standard. The organic compounds were extracted with $\mathrm{Et}_{2} \mathrm{O}$, and analyzed by GC. 


\section{Screening of catalysts and ligands}

Results of the reactions using various catalysts and ligands are summarized in Table $\mathrm{S} 2$. When $\mathrm{C}_{6} \mathrm{~F}_{6}$ 2a and $p$-fluorophenylmagnesium bromide (3a, 2 equiv), and 1,3-butadiene (3 equiv) were treated with $10 \mathrm{~mol} \%$ of $\mathrm{NiBr}_{2}(\mathrm{dme})$ in $\mathrm{THF}$ at $30{ }^{\circ} \mathrm{C}$ for 3 hours, the corresponding four-component coupling product 4 aa was yielded in $33 \%$ yield (entry 1). Then we tested various phosphine ligands (entries 2-10). The use of $20 \mathrm{~mol} \%$ of $\mathrm{PPh}_{3}$ and $\mathrm{P}(p \text {-Tol })_{3}$ as the ligand improved the yield of 4 aa to $75 \%$ yields (entries 2 and 3). On the other hand, almost no positive effects were observed when sterically congested $\mathrm{P}(o \text {-Tol })_{3}$ and electron deficient $\mathrm{P}\left(p-\mathrm{FC}_{6} \mathrm{H}_{4}\right)_{3}$ were used (entries 4 and 5). Among trialkylphosphines employed, $\mathrm{PCy}_{3}$ improved the yield of 4 aa to $72 \%$ (entry 6), whereas $\mathrm{P}(n \text {-Bu })_{3}$ did not work well and more sterically hindered $\mathrm{P}(t-\mathrm{Bu})_{3}$ completely suppressed the reaction (entries 7 and 8). Tris(diethylamino)phosphine was ineffective (entry 9). Chelating ligands, 1,2-bis(diphenylphosphino)ethane (dppe) and $N, N, N^{\prime}, N^{\prime}$-tetramethylethylenediamine (TMEDA), largely affected the reaction (entries 10 and 11). The addition of 12-crown-4 did not show positive effect (entry 12).

In the reaction of entry 2 , a significant amount of disubstituted product 4'aa arising from the further reaction of 4aa was observed. Actually, when the amount of the Grignard reagent 3a was reduced to 1.3 equiv, the further reaction was suppressed to $<5 \%$ yield and the

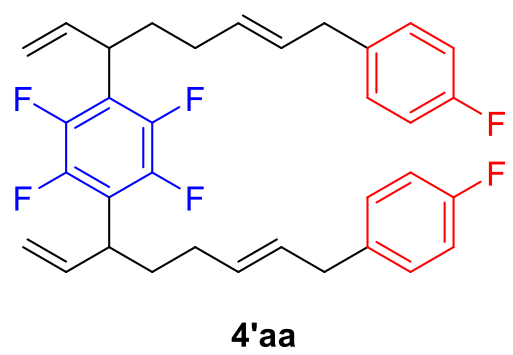
yield of 4aa improved to $82 \%$ (entry 13). Among the Ni salts tested, $\mathrm{NiCl}_{2}$ gave 4aa in a comparable yield as in the case of $\mathrm{NiBr}_{2}$ (dme) (entry 14). We thus decided to use $\mathrm{NiCl}_{2}$ as the precatalyst. Next, we examined the effects of the amounts of the $\mathrm{PPh}_{3}$ ligand and found that the reducing the amount of $\mathrm{PPh}_{3}$ to $10 \mathrm{~mol} \%$ (one equivalent to $\mathrm{Ni}$ ) improved the yield of 4 aa to $92 \%$ (entry 15). When the amount of $\mathrm{NiCl}_{2}$ and $\mathrm{PPh}_{3}$ was reduced to $5 \mathrm{~mol} \%$, the reaction did not complete within 3 hours (entry 16). Other group 10 metals $\mathrm{PdCl}_{2}$ and $\mathrm{PtCl}_{2}$ did not show the catalytic activity for the present four-component coupling reaction (entries 17 and 18). 
Table S2. Ni-Catalyzed Four-Component Coupling Reaction of Hexafluorobenzene, p-Fluorophenyl Grignard Reagent, and 1,3-Butadiene ${ }^{a}$
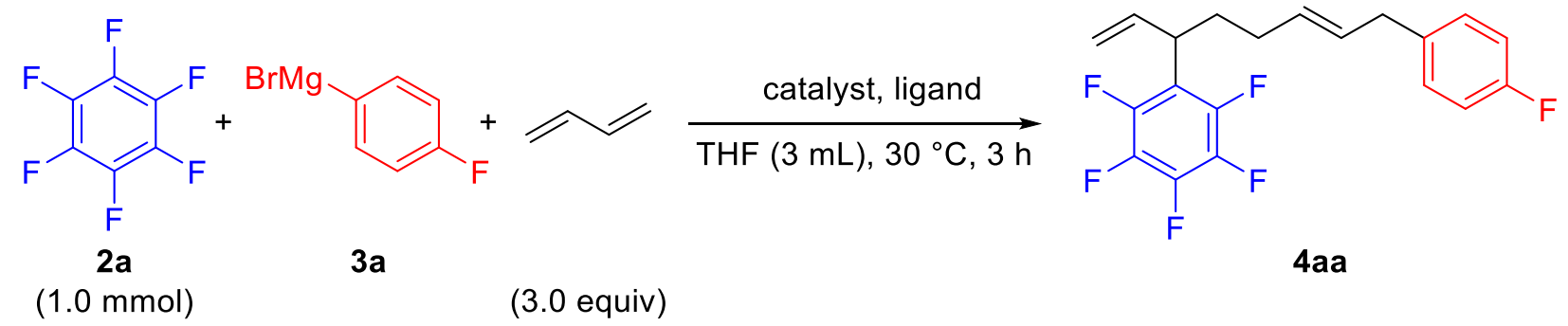

4aa

\begin{tabular}{|c|c|c|c|c|}
\hline entry & catalyst (mol \%) & ligand $(\mathrm{mol} \%)$ & $\operatorname{ArMgBr} 3 \mathbf{a}$ & yield $(\%)^{b}$ \\
\hline 1 & $\mathrm{NiBr}_{2}(\mathrm{dme})(10)$ & none & 2.0 equiv & 33 \\
\hline 2 & $\mathrm{NiBr}_{2}(\mathrm{dme})(10)$ & $\mathrm{PPh}_{3}(20)$ & 2.0 equiv & 75 \\
\hline 3 & $\mathrm{NiBr}_{2}(\mathrm{dme})(10)$ & $\mathrm{P}(p-\mathrm{Tol})_{3}(20)$ & 2.0 equiv & 75 \\
\hline 4 & $\mathrm{NiBr}_{2}(\mathrm{dme})(10)$ & $\mathrm{P}(o-\mathrm{Tol})_{3}(20)$ & 2.0 equiv & 32 \\
\hline 5 & $\mathrm{NiBr}_{2}(\mathrm{dme})(10)$ & $\mathrm{P}\left(p-\mathrm{FC}_{6} \mathrm{H}_{4}\right)_{3}(20)$ & 2.0 equiv & 44 \\
\hline 6 & $\mathrm{NiBr}_{2}(\mathrm{dme})(10)$ & $\mathrm{PCy}_{3}(20)$ & 2.0 equiv & 72 \\
\hline 7 & $\mathrm{NiBr}_{2}(\mathrm{dme})(10)$ & $\mathrm{P}(n-\mathrm{Bu})_{3}(20)$ & 2.0 equiv & 34 \\
\hline 8 & $\mathrm{NiBr}_{2}(\mathrm{dme})(10)$ & $\mathrm{P}(t-\mathrm{Bu})_{3}(20)$ & 2.0 equiv & n.d. \\
\hline 9 & $\mathrm{NiBr}_{2}(\mathrm{dme})(10)$ & $\mathrm{P}\left(\mathrm{NEt}_{2}\right)_{3}$ & 2.0 equiv & 40 \\
\hline 10 & $\mathrm{NiBr}_{2}(\mathrm{dme})(10)$ & dppe (10) & 2.0 equiv & 2 \\
\hline 11 & $\mathrm{NiBr}_{2}(\mathrm{dme})(10)$ & TMEDA (20) & 2.0 equiv & 12 \\
\hline 12 & $\mathrm{NiBr}_{2}(\mathrm{dme})(10)$ & 12-crown-4 (20) & 2.0 equiv & 30 \\
\hline 13 & $\mathrm{NiBr}_{2}(\mathrm{dme})(10)$ & $\mathrm{PPh}_{3}(20)$ & 1.3 equiv & 82 \\
\hline 14 & $\mathrm{NiCl}_{2}(10)$ & $\mathrm{PPh}_{3}(20)$ & 1.3 equiv & 88 \\
\hline 15 & $\mathrm{NiCl}_{2}(10)$ & $\mathrm{PPh}_{3}(10)$ & 1.3 equiv & $92(81)$ \\
\hline 16 & $\mathrm{NiCl}_{2}(5)$ & $\mathrm{PPh}_{3}(5)$ & 1.3 equiv & 53 \\
\hline 17 & $\mathrm{PdCl}_{2}(10)$ & $\mathrm{PPh}_{3}(10)$ & 2.0 equiv & n.d. \\
\hline 18 & $\mathrm{PtCl}_{2}(10)$ & $\mathrm{PPh}_{3}(10)$ & 2.0 equiv & n.d. \\
\hline
\end{tabular}

${ }^{a}$ A mixture of hexafluorobenzene 2a $(1 \mathrm{mmol}), p$-fluorophenylmagnesium bromide (3a) (in THF), 1,3-butadiene ( $3 \mathrm{mmol}$ ), catalyst, and ligand in THF (total $3 \mathrm{~mL}$ ) was stirred at $30{ }^{\circ} \mathrm{C}$ for $3 \mathrm{~h}$. ${ }^{b}$ Determined by GC. Isolation yield is in parenthesis. 


\section{Stoichiometric reactions}

In order to prove the active catalytic intermediate for $\mathrm{C}-\mathrm{C}$ bond forming step with perfluoroarenes, we conducted stoichiometric reactions of $\mathrm{Ni}(\operatorname{cod})_{2}, \mathrm{C}_{6} \mathrm{~F}_{6} \mathbf{2 a}$ ( 1 equiv), and 0 to 1 equiv of an aryl Grignard reagent 3a in the presence of 3 equiv of 1,3-butadiene and 1 equiv of $\mathrm{PPh}_{3}$ in $\mathrm{THF}$ at $30{ }^{\circ} \mathrm{C}$ for 3 hours (Scheme S1). Firstly, Ni(cod) 2 was treated with 2a (1 equiv), 1,3-butadiene (3 equiv) and $\mathrm{PPh}_{3}$ (1 equiv) at $30{ }^{\circ} \mathrm{C}$ for 3 hours in the absence of Grignard reagent 3a and the reaction mixture was quenched with $1 \mathrm{~N} \mathrm{HCl}$ aq. GC analysis of the resulting mixture showed that $96 \%$ of $\mathbf{2 a}$ was recovered unreacted. When the same reaction was conducted using 0.5 equiv of $\mathbf{3 a}, 68 \%$ of $\mathbf{2 a}$ was consumed and the corresponding four-component coupling product 4aa was formed in $43 \%$ yield. The conversion of $\mathbf{2 a}$ reached to $>99 \%$ giving $4 \mathbf{a a}$ in $82 \%$ yield when 1 equiv of 3a was employed. These results clearly indicate that a Grignard reagent 3a does promote the $\mathrm{C}-\mathrm{C}$ bond formation with hexafluorobenzene (2a) probably by forming an anionic Ni complex as an active catalytic intermediate toward perfluoroarenes.

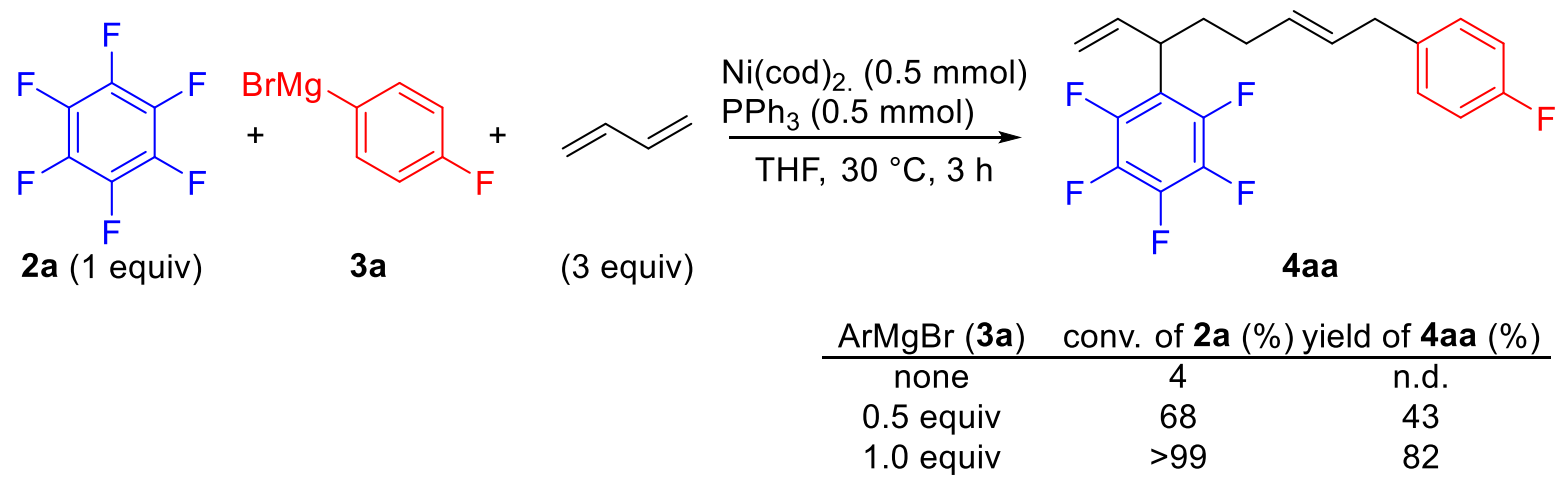

Scheme S1. Control experiments for the reactions of $\mathrm{Ni}(0)$ with $2 \mathrm{a}$, 3a and 1,3-butadiene in the presence of $\mathrm{PPh}_{3}$. 


\section{Mechanistic studies using 9,10-dihydroanthracene}

In order to check the possibility of a radical pathway, we conducted the reaction using 9,10-dihydroanthracene as a radical scavenger (Scheme S2). When 1 equiv of 9,10-dihydroanthracene was added to the reaction of $\mathbf{2 a}, \mathbf{3 a}$, and 1,3-butadiene in the presence of $10 \mathrm{~mol} \%$ of $\mathrm{NiCl}_{2}$ and $\mathrm{PPh}_{3}$, the corresponding four-component coupling product 4aa was obtained in 59\% yield. Although the yield of $4 \mathbf{a a}$ became somewhat lower than the reaction without 9,10-dihydroanthracene, the reaction did proceed. In addition, when $\mathrm{Ni}(\operatorname{cod})_{2}$ was used instead of $\mathrm{NiCl}_{2}$, the similar yield of $\mathbf{4 a a}$ was observed, indicating that the present catalytic reaction including the initiation step, i. e. reduction of $\mathrm{Ni}$ (II) into $\mathrm{Ni}(0)$ by aryl Grignard reagents, does not involve the formation of radical intermediates.
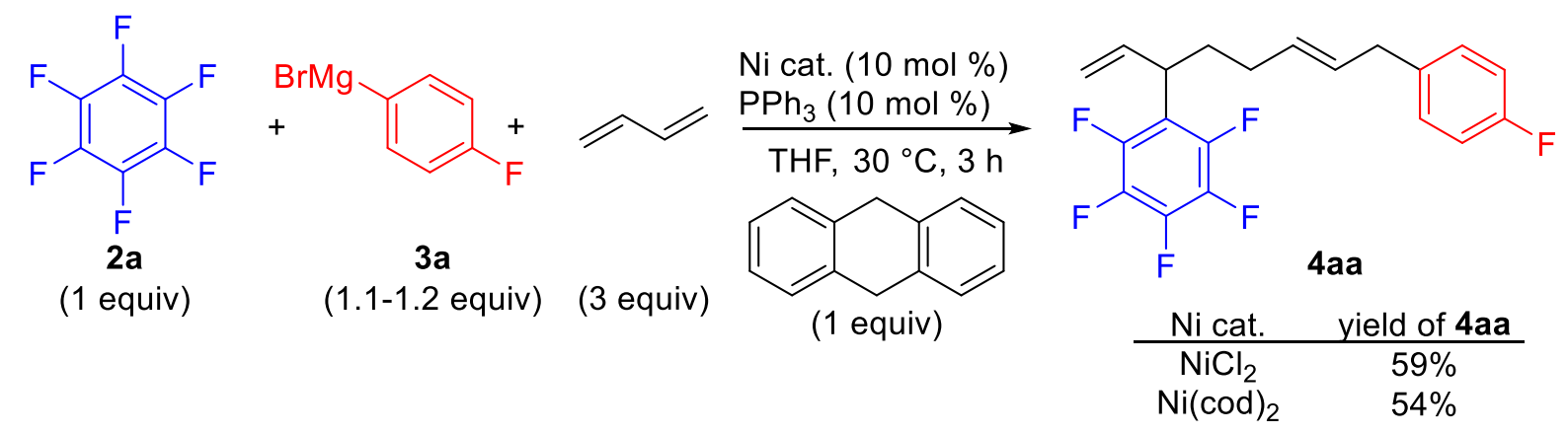

Scheme S2. The four-component coupling reaction in the presence of 9,10-dihydroathracene. 


\section{Kinetic studies with $\mathrm{Ni} / \mathrm{PPh}_{3}=1$}

Using hexafluorobenzene (2a), p-fluorophenylmagnesium bromide (3a), and 1,3-butadiene as the substrates, we conducted kinetic studies in the presence of $\mathrm{NiBr}_{2}(\mathrm{dme})$ as the catalyst precursor and $\mathrm{PPh}_{3}$ keeping the molar ratio of $\mathrm{Ni} / \mathrm{PPh}_{3}=1$. The reaction was conducted employing various concentrations of hexafluorobenzene (2a) under the standard conditions as depicted in eq S3 and quenched after $10 \mathrm{~min}$ by adding $1 \mathrm{~N} \mathrm{HCl}$ aq. The reaction rates $v(=\mathrm{d}[\mathbf{4 a a}] / \mathrm{d} t)$, were calculated based on the GC analysis and the results as the average of three runs were shown below (Table S3). The $\log -\log$ plots of the formed products in $\mathrm{mol} / \mathrm{L} \cdot \mathrm{s}$ against the initial concentration of $\mathbf{2 a}\left[\mathrm{C}_{6} \mathrm{~F}_{6}\right]_{0}$ was represented in Figures S3, suggesting that the reaction obeys zeroth-order kinetics respect to $2 \mathbf{a}$.

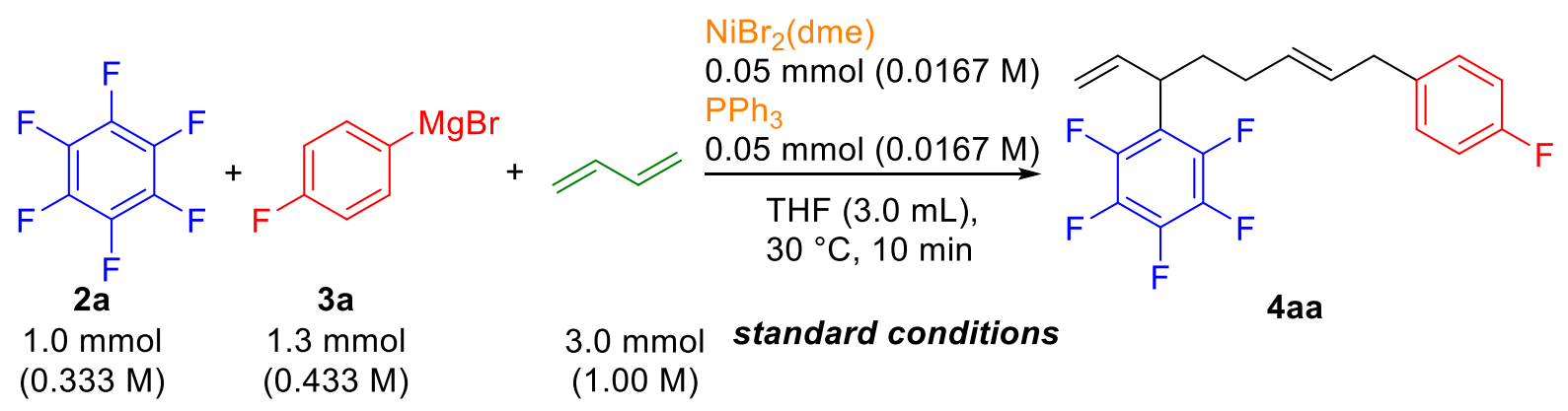

\section{A Typical Procedure under the Standard Conditions}

An oven-dried test tube was charged with a stirring bar, $\mathrm{NiBr}_{2}(\mathrm{dme})(16 \mathrm{mg}, 0.05 \mathrm{mmol})$, and $\mathrm{PPh}_{3}$ $(13 \mathrm{mg}, 0.05 \mathrm{mmol})$ and sealed with a septum cap. The test tube was evacuated and refiled with dry nitrogen. THF $(1.7 \mathrm{~mL})$ and undecane $(16 \mathrm{mg})$ as an internal standard were added via syringe at ambient temperature. 1,3-Butadiene (67 mL as gas, $3.0 \mathrm{mmol}$ ) and $p$-fluorophenylmagnesium bromide (3a) (in THF, $1.3 \mathrm{~mL}, 1.3 \mathrm{mmol}$ ) were added to the mixture cooled at $-78^{\circ} \mathrm{C}$ in a dry-ice bath. Then the solution was warmed in a water bath and stirred at $30{ }^{\circ} \mathrm{C}$ for 10 minutes. The reaction mixture was carefully quenched by $1 \mathrm{~N} \mathrm{HCl}$ aq. and the product was extracted with $\mathrm{Et}_{2} \mathrm{O}$. The yield of 4 aa was determined by GC analysis. 
Reactions using different concentrations of perfluorobenzene (2a)

Table S3. Concentrations of $\mathrm{C}_{6} \mathrm{~F}_{6} 2 \mathrm{a}$, the reaction rates and yields of $4 \mathrm{aa}$.

\begin{tabular}{llll}
\hline entry & {$\left[\mathrm{C}_{6} \mathrm{~F}_{6}\right]_{0} \times 10^{1}[\mathrm{M}]$} & ${\text { reaction rate } \mathrm{v} \times 10^{5}[\mathrm{M} / \mathrm{s}]^{a}}^{a}$ & yield of 4aa $(\%)^{b}$ \\
\hline 1 & 1.33 & $9.70 \pm 0.43$ & 44 \\
2 & 2.00 & $8.10 \pm 0.36$ & 24 \\
3 & 2.67 & $8.67 \pm 0.48$ & 19 \\
4 & 3.33 & $8.74 \pm 0.43$ & 16 \\
5 & 4.00 & $8.10 \pm 0.62$ & 12 \\
6 & 4.67 & $8.74 \pm 0.43$ & 11 \\
\hline
\end{tabular}

${ }^{a}$ Average of three runs. ${ }^{b}$ Based on $2 \mathbf{a}$.

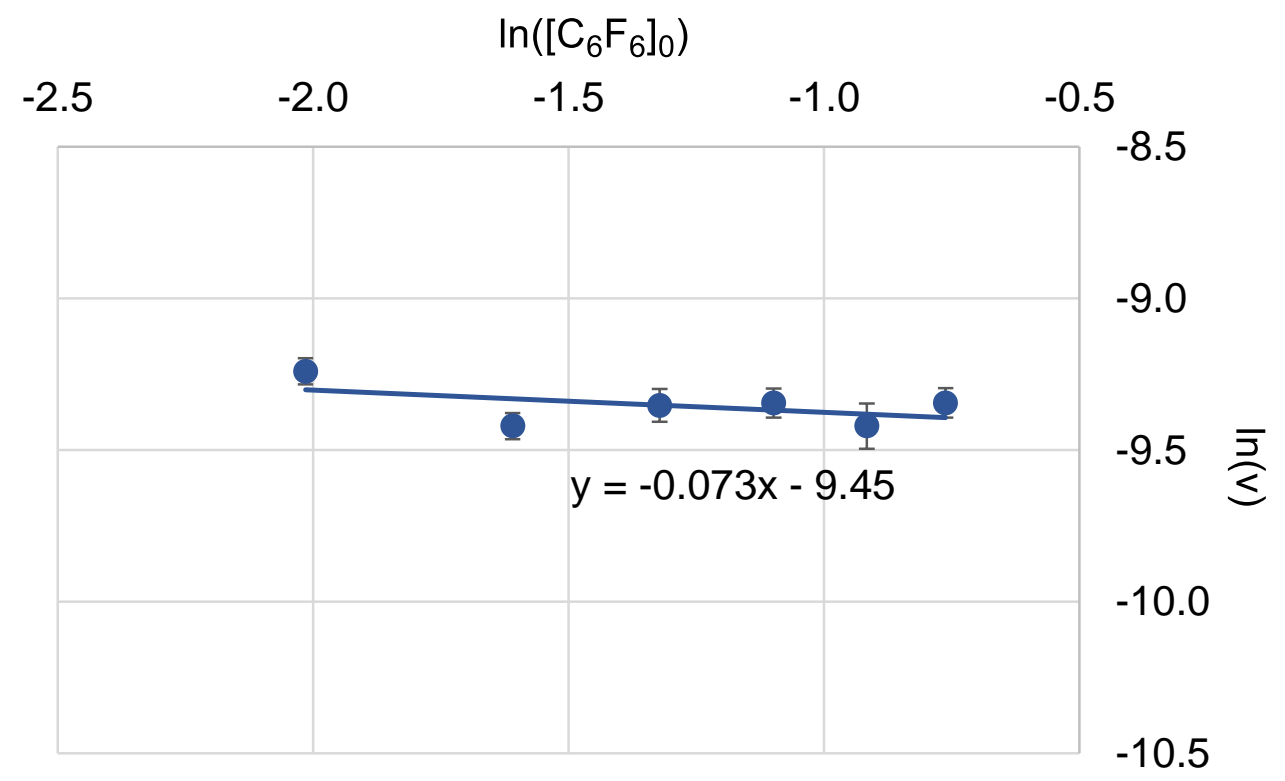

Figure S3. Log-log plot of the reaction rate versus $\mathrm{C}_{6} \mathrm{~F}_{6} \mathbf{2} \mathbf{a}$ concentration 


\section{Reactions using different concentrations of $\mathbf{P P h}_{3}$}

\subsection{Concentrations of $\mathrm{PPh}_{3}$ and reaction rates.}

We conducted further kinetic studies with respect to $\mathrm{PPh}_{3}$ in the presence of $0.0167 \mathrm{M}$ of $\mathrm{NiBr}_{2}(\mathrm{dme})$ fixed. The molar ratio of $\mathrm{PPh}_{3}$ against $\mathrm{NiBr}_{2}(\mathrm{dme})$ was varied from 0 to 7 (Table $\mathrm{S} 4$ ). Figure $\mathrm{S} 4$ shows a plot of reaction rate $v$ against the mole ratio of $\mathrm{PPh}_{3} / \mathrm{Ni}$. The initial rate of the reaction increased with increasing the mole ration of $\mathrm{PPh}_{3} / \mathrm{Ni}$ up to ca. 3. Further addition of $\mathrm{PPh}_{3}$ slightly retarded the reaction. Thus we conducted kinetic studies on the reaction at 1:3 ratio of $\mathrm{Ni}$ and $\mathrm{PPh}_{3}$.

Table S4. Mole ratio of $\mathrm{PPh}_{3}$ to $\mathrm{Ni}$ catalyst ${ }^{a}$ and the reaction rates.

\begin{tabular}{lll}
\hline entry & $\mathrm{PPh}_{3} / \mathrm{NiBr}_{2}(\mathrm{dme})$ & reaction rate $\mathrm{v} \times 10^{5}[\mathrm{M} / \mathrm{s}]^{b}$ \\
\hline 1 & 0 & $2.12 \pm 0.14$ \\
2 & 0.5 & $5.68 \pm 0.15$ \\
3 & 1.0 & $8.59 \pm 0.73$ \\
4 & 1.5 & $11.28 \pm 0.41$ \\
5 & 2.0 & $11.70 \pm 0.27$ \\
6 & 3.0 & $12.91 \pm 0.50$ \\
7 & 5.0 & $11.80 \pm 0.50$ \\
8 & 7.0 & $10.02 \pm 0.42$ \\
\hline
\end{tabular}

${ }^{a} \mathrm{Amount}$ of $\mathrm{PPh}_{3}$ was changed from 0 to $0.117 \mathrm{M}$ and the concentration of $\mathrm{NiBr}_{2}(\mathrm{dme})$ was fixed as 0.0167 M. ${ }^{b}$ Average of three runs.

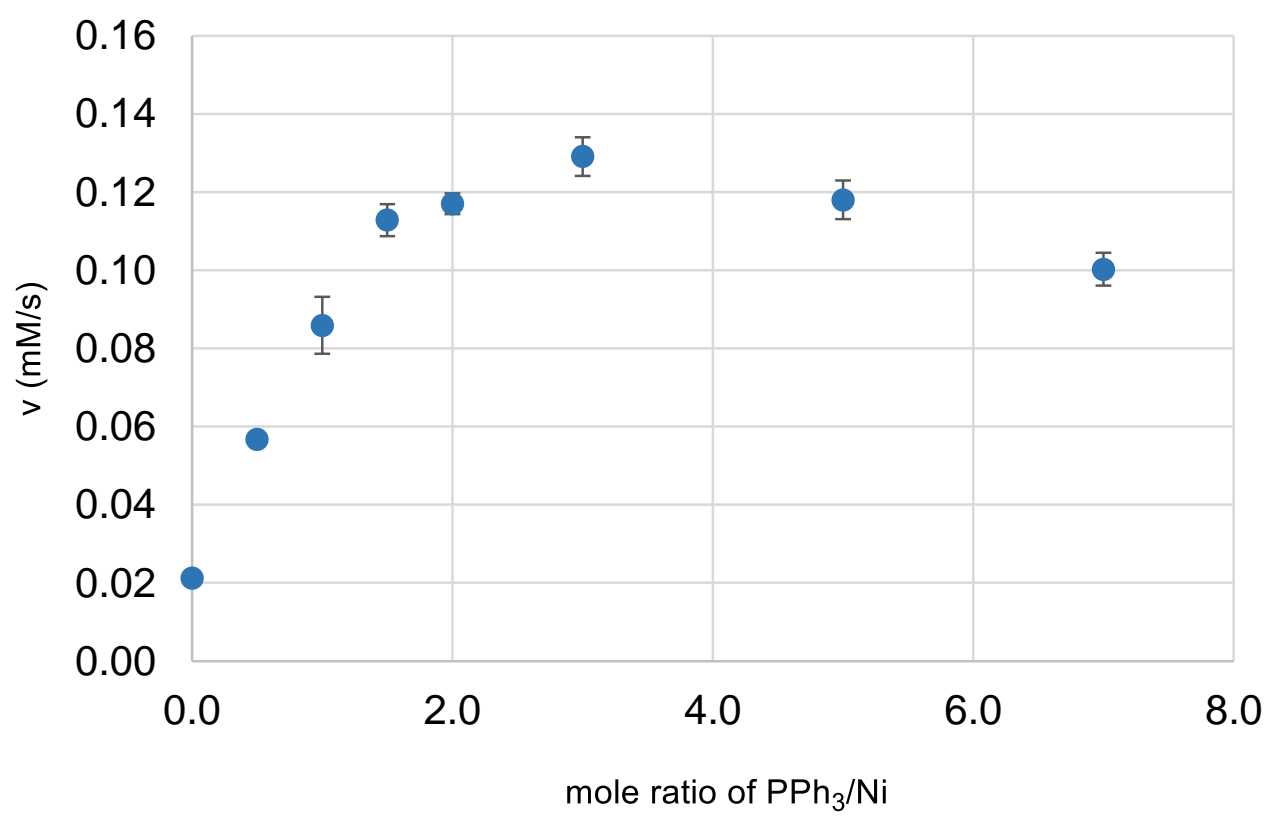

Figure S4. Plot of reaction rate versus mole ratio of $\mathrm{PPh}_{3}$ to Ni catalyst 


\subsection{Kinetic studies with $\mathrm{PPh}_{3} / \mathrm{Ni}=3$}

We conducted kinetic studies by changing the concentrations of $\mathbf{2 a}$ in a similar way to section 8 using $\mathrm{NiBr}_{2}(\mathrm{dme})$ and $\mathrm{PPh}_{3}$ in 1:3 ratio (eq $\left.\mathrm{S} 4\right)$. Under these conditions, the reaction rate was independent on the wide range of concentrations of $\mathrm{C}_{6} \mathrm{~F}_{6}$ (Figure S5).

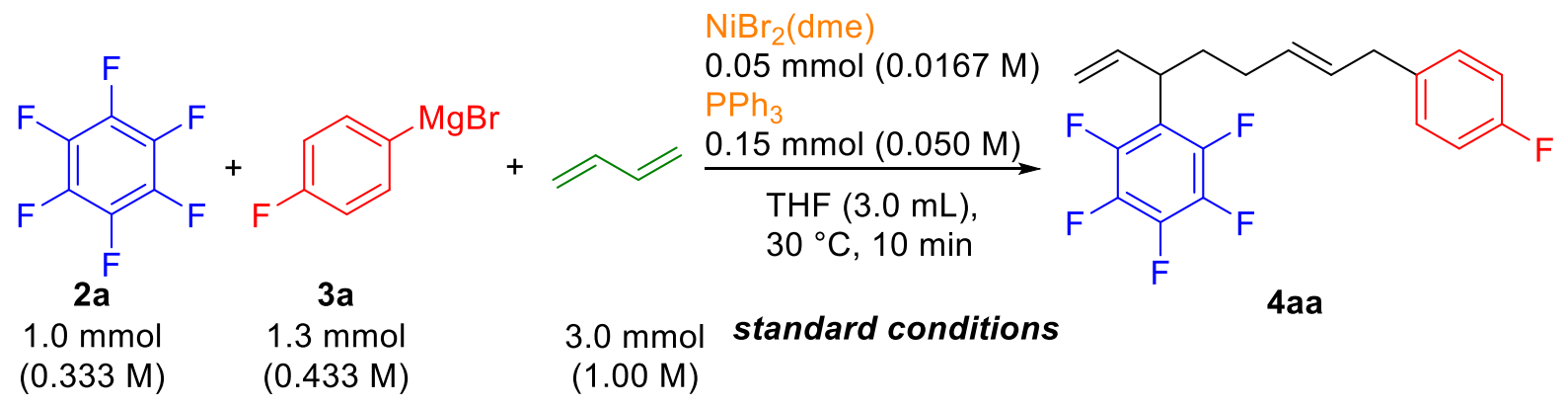

Reactions using different concentrations of $\mathrm{C}_{6} \mathrm{~F}_{6} 2 \mathrm{2a}$ with the ratio of $\mathrm{PPh}_{3} / \mathrm{Ni}$ catalyst $=3$

Table S5. Concentrations of $\mathrm{C}_{6} \mathrm{~F}_{6} 2 \mathrm{a}$, the reaction rates and yields of $4 \mathrm{aa}$

\begin{tabular}{llll}
\hline entry & {$\left[\mathrm{C}_{6} \mathrm{~F}_{6}\right]_{0} \times 10^{1}[\mathrm{M}]$} & ${\text { reaction rate } \mathrm{v} \times 10^{4}[\mathrm{M} / \mathrm{s}]^{a}}^{a}$ & ${\text { yield of 4aa }(\%)^{b}}^{b}$ \\
\hline 1 & 1.33 & $0.83 \pm 0.12$ & 37 \\
2 & 2.00 & $1.11 \pm 0.07$ & 33 \\
3 & 2.67 & $1.13 \pm 0.05$ & 26 \\
4 & 3.33 & $1.13 \pm 0.08$ & 20 \\
5 & 4.00 & $1.23 \pm 0.02$ & 18 \\
6 & 4.67 & $1.14 \pm 0.10$ & 15 \\
\hline
\end{tabular}

${ }^{a}$ Average of three runs. ${ }^{b}$ Based on $\mathbf{2 a}$. 


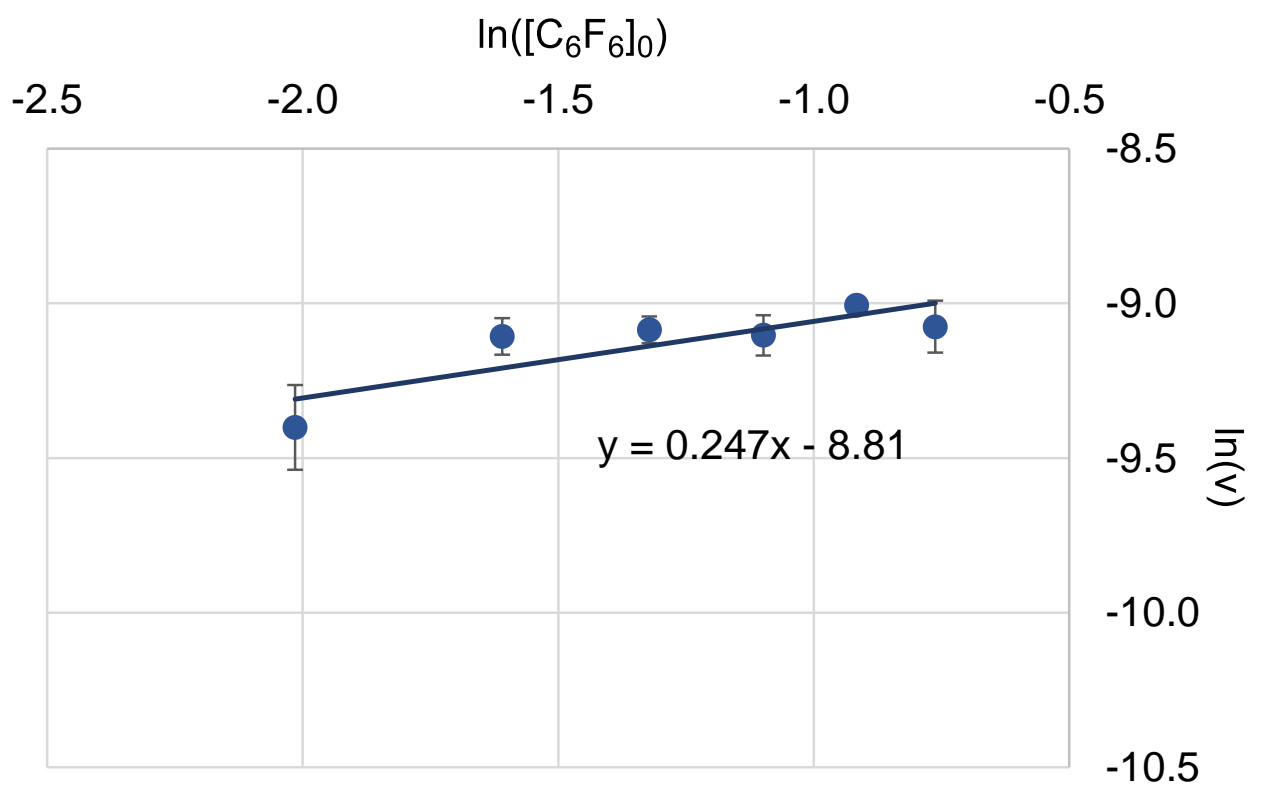

Figure S5. Log-log plot of the reaction rates versus $\mathrm{C}_{6} \mathrm{~F}_{6}(\mathbf{2 a})$ concentrations 


\section{Materials and Methods}

Nuclear magnetic resonance $\left({ }^{1} \mathrm{H}\right.$ NMR, ${ }^{13} \mathrm{C}$ NMR, ${ }^{19} \mathrm{~F}$ NMR, and ${ }^{31} \mathrm{P}$ NMR $)$ spectra were recorded on a JEOL JNM-Alice 400 spectrometer operating at $400 \mathrm{MHz}\left({ }^{1} \mathrm{H} \mathrm{NMR}\right), 100 \mathrm{MHz}\left({ }^{13} \mathrm{C}\right.$ NMR), 376 $\mathrm{MHz}\left({ }^{19} \mathrm{~F} \mathrm{NMR}\right)$, and $162 \mathrm{MHz}\left({ }^{31} \mathrm{P} \mathrm{NMR}\right)$ in $5 \mathrm{~mm}$ NMR tubes. All ${ }^{1} \mathrm{H}$ NMR chemical shifts were reported in ppm relative to an internal reference tetramethylsilane at $\delta 0.00$ or residual protiated solvent (THF- $d_{7}$ at $\left.\delta 3.53\right)$. All ${ }^{13} \mathrm{C}$ NMR chemical shifts were reported in ppm relative to the carbon of chloroform- $d_{1}$ as $\delta 77.00$ or THF- $d_{8}$ as $\delta 66.50$. The ${ }^{19} \mathrm{~F}$ NMR chemical shifts were reported in ppm relative to external references of $\alpha, \alpha, \alpha$-trifluorotoluene as $\delta-63.90$. Infrared spectra were recorded with a JASCO Corporation FT/IR-4200 instrument equipped ATR PRO450-S. HPLC separations were performed on a recycling preparative HPLC (Shimadzu Prominence) equipped with Shodex K2001 and 2002 columns (GPC) using $\mathrm{CHCl}_{3}$ as an eluent unless otherwise noted. GC analyses were performed on a Shimadzu GC-2014 instrument equipped with a GL Sciences InertCap 5 capillary column (I.D. 0.25 $\mathrm{mm}$, Length $30 \mathrm{~m}$, df $0.25 \mu \mathrm{m}$ ) using an internal standard. Conventional and high resolution mass spectra were recorded with a JEOL JMS-DX303HF spectrometer (EI and CI) with a double-focusing magnetic sector mass instrument or JEOL JMS-T100TD (DART) with a time-of-flight mass instrument. Melting points were measured using Stanford Research Systems OptiMelt MPA 100 with a glass capillary.

All manipulations involving air- and moisture-sensitive compounds were carried out by the standard Schlenk techniques under a nitrogen atmosphere. Dehydrated THF and $\mathrm{Et}_{2} \mathrm{O}$ were purchased from Kanto Chemical Company and purified by SPS ${ }^{\mathrm{S} 5}$ prior to use. Dehydrated toluene and pentane were purchased from Wako Pure Chemical Industries and used as received. THF- $d_{8}$ and 12-crown-4 were dried over sodium benzophenone ketyl and metallic $\mathrm{Na}$, respectively, and distilled prior to use. $o$-Isopropylphenylmagnesium bromide (3g) and 4-dimethylaminophenylmagnesium bromide (3l) was prepared by a standard procedure from the corresponding bromide and $\mathrm{Mg}$. Other THF solutions of Grignard reagents were purchased from Aldrich or TCI. A cyclohexane/ether solution of phenyllithium was purchased from Kanto. These organomagnesium and lithium reagents were used after titration using $\mathrm{I}_{2}$. $\mathrm{NiBr}_{2}$ (dme) (Aldrich), $\mathrm{Ni}$ (cod) $)_{2}$ (Kanto), 1,3-butadiene (TCI), $\mathrm{NiCl}_{2}$ (Wako), and all other commercially available reagents were used as received. 


\section{Synthesis of Substrates}

\section{Perfluorophenyl methyl sulfane $(2 \mathrm{e})^{\mathrm{S} 6}$}

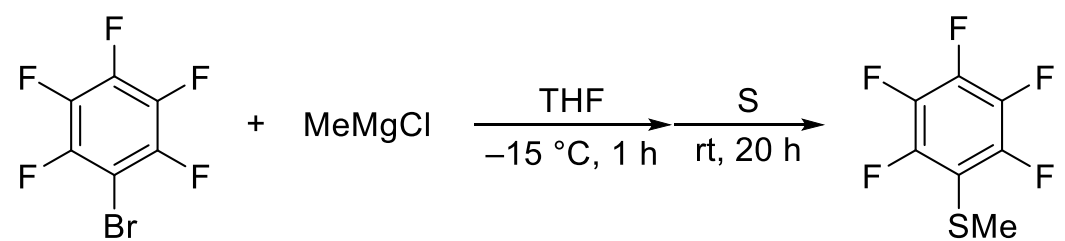

Methylmagnesium chloride $(8.8 \mathrm{~mL}, 2.52 \mathrm{M}$ in THF, $22.2 \mathrm{mmol}$ ) was slowly added to a stirring solution of bromopentafluorobenzene $(4.94 \mathrm{~g}, 20.2 \mathrm{mmol})$ in THF $(17 \mathrm{~mL})$ at $-15{ }^{\circ} \mathrm{C}$. After $1 \mathrm{~h}, \mathrm{GC}$ analysis showed the absence of bromopentafluorobenzene, then sulfur $(0.72 \mathrm{~g}, 22.4 \mathrm{mmol})$ was added and stirred at room temperature for $20 \mathrm{~h}$. The reaction was quenched by $1 \mathrm{~N} \mathrm{HCl}$ aq. and extracted with $\mathrm{Et}_{2} \mathrm{O}$. The ether layer was separated, dried over $\mathrm{MgSO}_{4}$, and filtered. The filtrate was evaporated and the crude product was purified by flash chromatography (hexane) to yield the product as a yellow oil ( $0.80 \mathrm{~g}$, $3.7 \mathrm{mmol}, 19 \%) .{ }^{1} \mathrm{H}$ NMR (400 MHz, $\left.\mathrm{CDCl}_{3}\right): \delta 2.48(\mathrm{~s}, 3 \mathrm{H}) ;{ }^{19} \mathrm{~F}$ NMR $\left(376 \mathrm{MHz}, \mathrm{CDCl}_{3}\right): \delta-133.4$ (dd, $J=23.5,7.0 \mathrm{~Hz}, 2 \mathrm{~F}),-153.6(\mathrm{t}, J=20.9 \mathrm{~Hz}, 1 \mathrm{~F}),-161.1$ (td, $J=22.4,7.4 \mathrm{~Hz}, 2 \mathrm{~F})$.

\section{1-Trimethylsilyl-2,3,4,5,6-perfluorobenzene $(2 \mathrm{~g})^{\mathrm{S} 7}$}<smiles>Fc1c(F)c(F)c(Br)c(F)c1F</smiles><smiles>CS(=O)(=O)c1c(F)c(F)c(F)c(F)c1F</smiles>

Bromopentafluorobenzene (4.41 g, $17.9 \mathrm{mmol})$, chlorotrimethylsilane $(2.18 \mathrm{~g}, 20.0 \mathrm{mmol})$, and hexane $(50 \mathrm{~mL})$ were placed into a flask. $\mathrm{P}\left(\mathrm{NEt}_{2}\right)_{3}(4.95 \mathrm{~g}, 20.0 \mathrm{mmol})$ was added and the solution stirred at room temperature for $1 \mathrm{~h}$. The reaction mixture was filtered, concentrated, and purified by a short pad of silica gel column chromatography (hexane) to yield the product as a colorless oil ( $2.79 \mathrm{~g}$, $11.6 \mathrm{mmol}, 65 \%) .{ }^{1} \mathrm{H}$ NMR $\left(400 \mathrm{MHz}, \mathrm{CDCl}_{3}\right): \delta 0.40(\mathrm{t}, J=1.6 \mathrm{~Hz}, 9 \mathrm{H}) ;{ }^{19} \mathrm{~F}$ NMR $\left(376 \mathrm{MHz}, \mathrm{CDCl}_{3}\right)$ : $\delta-127.2-127.3(\mathrm{~m}, 2 \mathrm{~F}),-152.0-152.1$ (m, 1F), -161.4--161.6 (m, 2F).

\section{Perfluorophenyl 4-methylbenzenesulfonate $(2 \mathrm{~h})^{\mathrm{S8}}$}

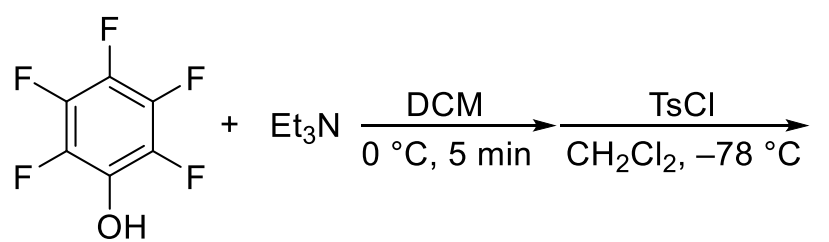<smiles>Fc1c(F)c(F)c(O[Sb])c(F)c1F</smiles>

To a solution of pentafluorophenol $(3.41 \mathrm{~g}, 18.5 \mathrm{mmol})$ in $\mathrm{CH}_{2} \mathrm{Cl}_{2}(30 \mathrm{~mL})$ was added triethylamine $(2.7 \mathrm{~mL}, 19.4 \mathrm{mmol})$ at $0{ }^{\circ} \mathrm{C}$. After $5 \mathrm{~min}$, the solution was transferred by cannula to a stirring solution 
of tosyl chloride $(3.54 \mathrm{~g}, 18.6 \mathrm{mmol})$ in $\mathrm{CH}_{2} \mathrm{Cl}_{2}(50 \mathrm{~mL})$ at $-78{ }^{\circ} \mathrm{C}$. The mixture was then allowed to warm to room temperature and stirred for $3 \mathrm{~h} .1 \mathrm{~N} \mathrm{HCl}$ aq. was then added and the products were extracted with $\mathrm{CH}_{2} \mathrm{Cl}_{2}$. The organic layer was separated, dried over $\mathrm{MgSO}_{4}$, and filtered. The filtrate was evaporated and the crude product was purified by a short pad of silica gel column chromatography $\left(\mathrm{Et}_{2} \mathrm{O} /\right.$ hexane $\left.=20 / 80\right)$ to yield the product as a white powder $(5.77 \mathrm{~g}, 17.1 \mathrm{mmol}, 92 \%) .{ }^{1} \mathrm{H}$ NMR $(400$ $\left.\mathrm{MHz} \mathrm{CDCl}_{3}\right): \delta 7.86(\mathrm{~d}, J=8.8 \mathrm{~Hz}, 2 \mathrm{H}), 7.41(\mathrm{~d}, J=8.4 \mathrm{~Hz}, 2 \mathrm{H}), 2.51(\mathrm{~s}, 3 \mathrm{H}) ;{ }^{19} \mathrm{~F} \mathrm{NMR}(376 \mathrm{MHz}$, $\left.\mathrm{CDCl}_{3}\right): \delta-150.4-150.5(\mathrm{~m}, 2 \mathrm{~F}),-155.3(\mathrm{t}, J=21.6 \mathrm{~Hz}, 1 \mathrm{~F}),-160.9-161.1(\mathrm{~m}, 2 \mathrm{~F})$.

\section{1,2,3,4,5-Pentafluoro-6-(methoxymethoxy)benzene (2j) $)^{\mathrm{S9}}$}

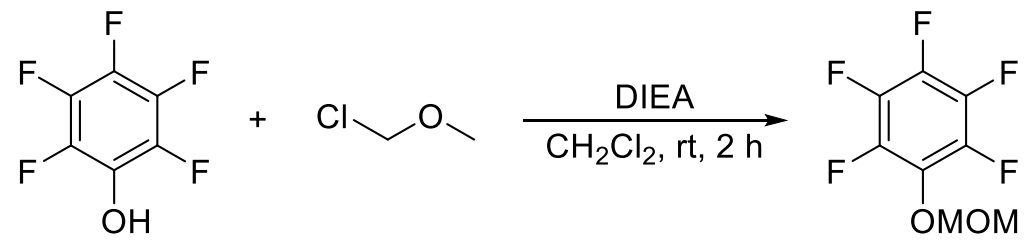

Chloromethyl methyl ether $(1.9 \mathrm{~mL}, 25.0 \mathrm{mmol})$ was added to a solution of pentafluorophenol (3.78 g, $20.6 \mathrm{mmol})$ and $N$-ethyldiisopropylamine $(4.3 \mathrm{~mL}, 24.7 \mathrm{mmol})$ in $\mathrm{CH}_{2} \mathrm{Cl}_{2}(20 \mathrm{~mL})$ at $0{ }^{\circ} \mathrm{C}$. The mixture was kept standing for $2 \mathrm{~h}$ at room temperature, and poured into $1 \mathrm{~N} \mathrm{NaOH}$ aq. The product was extracted with $\mathrm{Et}_{2} \mathrm{O}$ and the concentration under vacuo gave the title compound as a colorless oil (3.26 $\mathrm{g}$, $14.3 \mathrm{mmol}, 70 \%) .{ }^{1} \mathrm{H}$ NMR $\left(400 \mathrm{MHz}, \mathrm{CDCl}_{3}\right): \delta 3.60(\mathrm{~s}, 3 \mathrm{H}), 5.16(\mathrm{~s}, 2 \mathrm{H}) ;{ }^{13} \mathrm{C}\left\{{ }^{1} \mathrm{H}\right\} \mathrm{NMR}(100 \mathrm{MHz}$, $\left.\mathrm{CDCl}_{3}\right): \delta 142.4\left(\mathrm{dm},{ }^{1} J_{\mathrm{C}-\mathrm{F}}=250.8 \mathrm{~Hz}\right), 137.9\left(\mathrm{dm},{ }^{1} J_{\mathrm{C}-\mathrm{F}}=248.9 \mathrm{~Hz}\right), 131.0(\mathrm{~m}), 99.4\left(\mathrm{t},{ }^{3} J_{\mathrm{C}-\mathrm{F}}=2.9 \mathrm{~Hz}\right)$, 57.4 (a carbon signal from the pentafluorophenyl ring was not observed); ${ }^{19} \mathrm{~F} \mathrm{NMR}\left(376 \mathrm{MHz}, \mathrm{CDCl}_{3}\right)$ : $\delta-155.9-156.0(\mathrm{~m}, 2 \mathrm{~F}),-161.8(\mathrm{tt}, J=20.0,2.3 \mathrm{~Hz}, 1 \mathrm{~F}),-163.1--163.2(\mathrm{~m}, 2 \mathrm{~F})$.

\section{$N, N$-Diethyl-2,3,4,5,6-pentafluorobenzamide $(2 \mathrm{k})^{\mathrm{S10}}$}

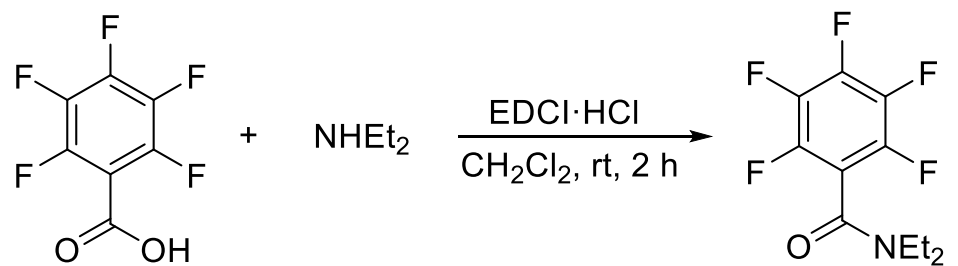

Pentafluorobenzoic acid (3.19 g, $15.0 \mathrm{mmol})$, 1-(3-dimethylaminopropyl)-3-ethylcarbodiimide hydrochloride (EDCI. $\mathrm{HCl})(3.84 \mathrm{~g}, 20.0 \mathrm{mmol})$ and $\mathrm{CH}_{2} \mathrm{Cl}_{2}(60 \mathrm{~mL})$ were placed into a flask in dry nitrogen atmosphere. Then diethylamine $(3.1 \mathrm{~mL}, 30.1 \mathrm{mmol})$ was added at $0{ }^{\circ} \mathrm{C}$ and then stirred at room temperature for $2 \mathrm{~h}$. The reaction mixture was washed with saturated aqueous solution of $\mathrm{NaHCO}_{3}$. The ether layer was separated, dried over $\mathrm{Na}_{2} \mathrm{SO}_{4}$ and filtered. The filtrate was evaporated and the crude product was purified by flash chromatography (hexane) to yield the title compound as a yellow oil (1.67 $\mathrm{g}, 8.7 \mathrm{mmol}, 58 \%)$. ${ }^{1} \mathrm{H} \mathrm{NMR}\left(400 \mathrm{MHz} \mathrm{CDCl}_{3}\right): \delta 3.59(\mathrm{q}, J=7.2 \mathrm{~Hz}, 2 \mathrm{H}), 3.22(\mathrm{q}, J=7.2 \mathrm{~Hz}, 2 \mathrm{H})$, $1.27(\mathrm{t}, J=7.2 \mathrm{~Hz}, 3 \mathrm{H}), 1.14(\mathrm{t}, J=7.0 \mathrm{~Hz}, 3 \mathrm{H}) ;{ }^{19} \mathrm{~F}$ NMR $\left(376 \mathrm{MHz}, \mathrm{CDCl}_{3}\right): \delta-141.3-141.4(\mathrm{~m}, 2 \mathrm{~F})$, 
$-152.5-152.6(\mathrm{~m}, 1 \mathrm{~F}),-159.7-159.9(\mathrm{~m}, 2 \mathrm{~F})$.

\section{2,3,4,5,6-Pentafluoro- $N, N$-dimethylaniline $(2 l)^{\mathrm{S} 11}$}

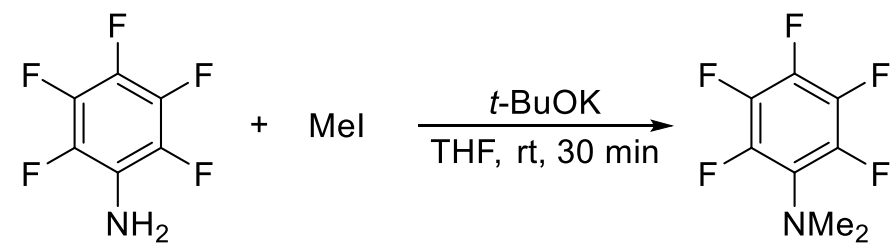

2,3,4,5,6-Pentafluoroaniline (2.42 g, $13.2 \mathrm{mmol})$, iodomethane ( $2.1 \mathrm{~mL}, 33.7 \mathrm{mmol})$, and THF ( 80 $\mathrm{mL})$ were placed into a flask under dry nitrogen atmosphere. Then ${ }^{t} \mathrm{BuOK}(4.52 \mathrm{~g}, 40.2 \mathrm{mmol})$ was added and the mixture was stirred at room temperature for $30 \mathrm{~min}$. The following filtration, concentrated, and purification by flash chromatography (hexane) afforded the product as a colorless oil ( $0.60 \mathrm{~g}, 2.8$ mmol, 21\%). ${ }^{1} \mathrm{H}$ NMR (400 MHz, $\left.\mathrm{CDCl}_{3}\right): \delta 2.90(\mathrm{t}, J=1.8 \mathrm{~Hz}, 6 \mathrm{H}) ;{ }^{19} \mathrm{~F}$ NMR $\left(376 \mathrm{MHz}, \mathrm{CDCl}_{3}\right): \delta$ -150.7 (d, $J=19.6 \mathrm{~Hz}, 2 \mathrm{~F}),-164.0-164.1$ (m, 2F), -164.6-164.8 (m, 1F).

\section{1,2,3,4-Tetrafluorodibenzo $[b, e][1,4]$ dioxine $(2 \mathrm{t})^{\mathrm{S} 12}$}<smiles>Oc1ccccc1F</smiles>

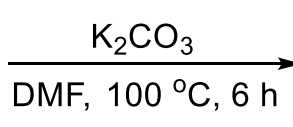<smiles>Fc1c(F)c(F)c2c(c1F)Oc1ccccc1O2</smiles>

Hexafluorobenzene $(5.8 \mathrm{~mL}, 50.5 \mathrm{mmol})$, potassium carbonate $(2.90 \mathrm{~g}, 21.0 \mathrm{mmol})$, catechol $(2.20 \mathrm{~g}$, $20.0 \mathrm{mmol})$, and DMF $(60 \mathrm{~mL})$ were placed into a flask under dry nitrogen atmosphere. The reaction mixture was stirred at $100{ }^{\circ} \mathrm{C}$ for $6 \mathrm{~h}$, and then cooled to room temperature. The mixture was acidified with $1 \mathrm{~N} \mathrm{HCl}$ aq. to $\mathrm{pH} 4$ and extracted with ethyl acetate. The organic layer was washed with water, dried over $\mathrm{MgSO}_{4}$, and filtered. The filtrate was evaporated and the crude product was purified by a short pad of silica gel column chromatography (EtOAc) to yield the product as a white powder $(4.92 \mathrm{~g}$, $19.2 \mathrm{mmol}, 96 \%) .{ }^{1} \mathrm{H} \mathrm{NMR}\left(400 \mathrm{MHz}, \mathrm{CDCl}_{3}\right): \delta 7.02-6.96(\mathrm{~m}, 4 \mathrm{H}) ;{ }^{19} \mathrm{~F} \mathrm{NMR}\left(376 \mathrm{MHz}, \mathrm{CDCl}_{3}\right): \delta$ $-162.4-162.5$ (m, 2F), -165.1--165.2 (m, 2F). 
General procedure for Ni-catalyzed four-component coupling of perfluoroarenes, aryl Grignard reagents, and 1,3-butadiene

An oven-dried test tube was charged with $\mathrm{NiCl}_{2}$ (13 mg, $\left.10 \mathrm{~mol} \%\right), \mathrm{PPh}_{3}(26 \mathrm{mg}, 10 \mathrm{~mol} \%$ ), and a stirring bar and closed with a septum cap in a glove box. Perfluoroarene $(1.0 \mathrm{mmol})$ and THF were added via syringe at ambient temperature. Grignard reagent (in THF, 1.2-1.3 mmol) and 1,3-butadiene (67 mL as gas, $3.0 \mathrm{mmol}$ ) were added at $-78{ }^{\circ} \mathrm{C}$ and then stirred in a preheated water bath at $30^{\circ} \mathrm{C}$. The reaction mixture was diluted with $\mathrm{Et}_{2} \mathrm{O}$ and carefully quenched by $1 \mathrm{~N} \mathrm{HCl}$ aq. The products were extracted by $\mathrm{Et}_{2} \mathrm{O}$ for three times, dried over $\mathrm{Na}_{2} \mathrm{SO}_{4}$, concentrated, filtered through a short pad of silica gel (hexane or $\mathrm{Et}_{2} \mathrm{O}$ as an eluent), and purified by $\mathrm{GPC}\left(\mathrm{CHCl}_{3}\right.$ as eluent) to give the desired product. 
Characterization Data for the Isolated Compounds

(E)-8-(4-Fluorophenyl)-3-perfluorophenyl-1,6-octadiene (4aa):

The general procedure was followed using hexafluorobenzene (193.8 $\mathrm{mg}, 1.042 \mathrm{mmol}$ ) and 4-fluorophenylmagnesium bromide (in THF, 0.80 M, $1.6 \mathrm{~mL}, 1.3 \mathrm{mmol})$. Purification by GPC gave the desired product 4aa as a colorless oil (311.7 $\mathrm{mg}, 81 \%)$ along with disubstituted byproduct 4'aa (28.3 mg, 5\%): IR (neat): 1521, 1500, 1223, 1158, 1117 , 976, 825, 667, $556 \mathrm{~cm}^{-1}:{ }^{1} \mathrm{H}$ NMR $\left(400 \mathrm{MHz}, \mathrm{CDCl}_{3}\right): \delta 7.11(\mathrm{t}, J=6.8$ $\mathrm{Hz}, 2 \mathrm{H}$ ), 6.96 (t, $J=8.4 \mathrm{~Hz}, 2 \mathrm{H}$ ), 6.01 (quin, $J=8.4 \mathrm{~Hz}, 1 \mathrm{H}$ ), 5.53 (dt, $J=15.2,6.4 \mathrm{~Hz}, 1 \mathrm{H}), 5.42(\mathrm{dt}, J=15.2,6.4 \mathrm{~Hz}, 1 \mathrm{H}), 5.11(\mathrm{br} \mathrm{s}, 1 \mathrm{H})$, $5.07(\mathrm{~d}, J=10.8 \mathrm{~Hz}, 1 \mathrm{H}), 3.74(\mathrm{q}, J=7.6 \mathrm{~Hz}, 1 \mathrm{H}), 3.28(\mathrm{~d}, J=6.4 \mathrm{~Hz}$,
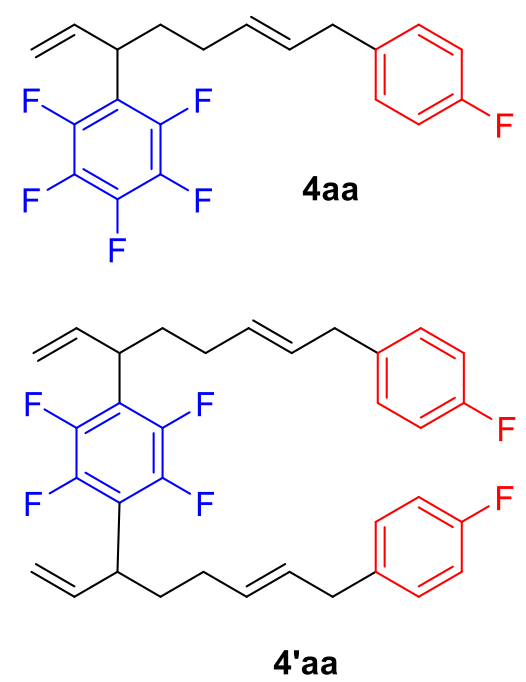
2H), 2.04-1.83 (m, 4H): ${ }^{13} \mathrm{C}\left\{{ }^{1} \mathrm{H}\right\}$ NMR (100 MHz, $\left.\mathrm{CDCl}_{3}\right): 161.3(\mathrm{~d}$, $\left.{ }^{1} J_{\mathrm{C}-\mathrm{F}}=242.2 \mathrm{~Hz}\right), 144.9\left(\mathrm{dm},{ }^{1} J_{\mathrm{C}-\mathrm{F}}=244.1 \mathrm{~Hz}\right), 139.6\left(\mathrm{dm},{ }^{1} J_{\mathrm{C}-\mathrm{F}}=250.8 \mathrm{~Hz}\right), 137.5\left(\mathrm{dm},{ }^{1} J_{\mathrm{C}-\mathrm{F}}=250.8\right.$ $\mathrm{Hz}), 137.4,136.2\left(\mathrm{~d},{ }^{4} J_{\mathrm{C}-\mathrm{F}}=2.9 \mathrm{~Hz}\right), 130.3,130.1,129.8\left(\mathrm{~d},{ }^{3} J_{\mathrm{C}-\mathrm{F}}=8.6 \mathrm{~Hz}\right), 117.0\left(\mathrm{t},{ }^{2} J_{\mathrm{C}-\mathrm{F}}=19.1 \mathrm{~Hz}\right)$, 116.8, $115.1\left(\mathrm{~d},{ }^{2} J_{\mathrm{C}-\mathrm{F}}=21.0 \mathrm{~Hz}\right), 39.4,38.1,32.8,30.4:{ }^{19} \mathrm{~F}$ NMR $\left(376 \mathrm{MHz}, \mathrm{CDCl}_{3}\right): \delta-117.5(\mathrm{~s}, 1 \mathrm{~F})$, -142.1 (m, 2F), $-157.2(\mathrm{~m}, 1 \mathrm{~F}),-162.3(\mathrm{~m}, 2 \mathrm{~F}):$ MS (DART) $\mathrm{m} / z$ (relative intensity, \%): 369 ([M-H] $]^{+}$ 18), 279 (100): HRMS (DART) $m / z$ [M-H] ${ }^{+}$Calcd for $\mathrm{C}_{20} \mathrm{H}_{15} \mathrm{~F}_{6}$ 369.1078; Found 369.1073.

\section{1,4-Bis((E)-8-(4-fluorophenyl)-1,6-octadien-3-yl)-2,3,5,6-tetrafluorobenzene (4'aa):}

${ }^{1} \mathrm{H}$ NMR (400 MHz, $\left.\mathrm{CDCl}_{3}\right): \delta 7.11(\mathrm{dd}, J=8.8,5.6 \mathrm{~Hz}, 4 \mathrm{H}), 6.96(\mathrm{t}, J=8.8 \mathrm{~Hz}, 4 \mathrm{H}), 6.03$ (quin, $J=$ $8.8 \mathrm{~Hz}, 1 \mathrm{H}), 5.52(\mathrm{dt}, J=15.2,6.4 \mathrm{~Hz}, 2 \mathrm{H}), 5.43(\mathrm{dt}, J=15.2,6.4 \mathrm{~Hz}, 2 \mathrm{H}), 5.10(\mathrm{~d}, J=4.4 \mathrm{~Hz}, 2 \mathrm{H})$, 5.06 (br s, 2H), 3.75 (q, $J=7.6 \mathrm{~Hz}, 2 \mathrm{H}), 3.28(\mathrm{~d}, J=6.4 \mathrm{~Hz}, 4 \mathrm{H}), 2.08-1.81(\mathrm{~m}, 8 \mathrm{H}):{ }^{19} \mathrm{~F}$ NMR $(376$ $\left.\mathrm{MHz}, \mathrm{CDCl}_{3}\right): \delta-117.6(\mathrm{~s}, 2 \mathrm{~F}),-143.3(\mathrm{~s}, 4 \mathrm{~F})$.

\section{(E)-3-Perfluorophenyl-8-phenyl-1,6-octadiene (4ab):}

The general procedure was followed using hexafluorobenzene $(192.7 \mathrm{mg}$, $1.036 \mathrm{mmol}$ ) and phenylmagnesium bromide (in THF, $0.95 \mathrm{M}, 1.4 \mathrm{~mL}, 1.3$ mmol). Purification by GPC gave the desired product $\mathbf{4 a b}$ as a colorless oil (280.7 mg, 77\%) along with disubstituted byproduct 4'ab (33.1 mg, 6\%): IR (neat): 1521, 1499, 1118, 975, 744, $700 \mathrm{~cm}^{-1}:{ }^{1} \mathrm{H}$ NMR $(400 \mathrm{MHz}$,

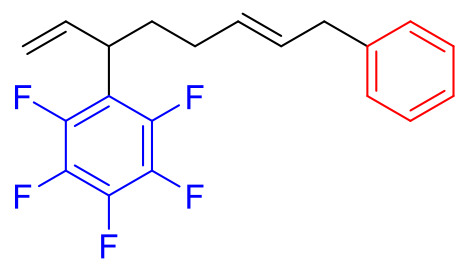
$\left.\mathrm{CDCl}_{3}\right): \delta 7.28(\mathrm{t}, J=7.6 \mathrm{~Hz}, 2 \mathrm{H}), 7.20-7.15(\mathrm{~m}, 3 \mathrm{H}), 6.05-5.96(\mathrm{~m}, 1 \mathrm{H}), 5.56(\mathrm{dt}, J=15.2,6.8 \mathrm{~Hz}$, $1 \mathrm{H}), 5.44$ (dt, $J=15.2,6.8 \mathrm{~Hz}, 1 \mathrm{H}), 5.10$ (br s, $1 \mathrm{H}), 5.07$ (d, $J=9.2 \mathrm{~Hz}, 1 \mathrm{H}), 3.75$ (q, $J=8.0 \mathrm{~Hz}, 1 \mathrm{H})$, $3.32(\mathrm{~d}, J=6.8 \mathrm{~Hz}, 2 \mathrm{H}), 2.03-1.84(\mathrm{~m}, 4 \mathrm{H}):{ }^{13} \mathrm{C}\left\{{ }^{1} \mathrm{H}\right\} \mathrm{NMR}\left(100 \mathrm{MHz}, \mathrm{CDCl}_{3}\right): 144.9\left(\mathrm{dm},{ }^{1} J_{\mathrm{C}-\mathrm{F}}=\right.$ $244.1 \mathrm{~Hz}), 140.6,139.6\left(\mathrm{dm},{ }^{1} J_{\mathrm{C}-\mathrm{F}}=250.8 \mathrm{~Hz}\right), 137.5,137.5\left(\mathrm{dm},{ }^{1} J_{\mathrm{C}-\mathrm{F}}=250.8 \mathrm{~Hz}\right), 130.3,130.1$, 128.44, 128.35, 125.9, $117.1\left(\mathrm{tt},{ }^{2} J_{\mathrm{C}-\mathrm{F}}=15.7,{ }^{3} J_{\mathrm{C}-\mathrm{F}}=1.9 \mathrm{~Hz}\right), 116.7,39.4,39.0,32.8,30.4:{ }^{19} \mathrm{~F}$ NMR $\left(376 \mathrm{MHz}, \mathrm{CDCl}_{3}\right): \delta-142.1(\mathrm{~d}, J=23.3 \mathrm{~Hz}, 2 \mathrm{~F}),-157.2(\mathrm{~m}, 1 \mathrm{~F}),-162.3(\mathrm{~m}, 2 \mathrm{~F}):$ MS (DART) $\mathrm{m} / z$ (relative intensity, \%): $351\left([\mathrm{M}-\mathrm{H}]^{+}, 30\right), 279$ (68), 239 (12), 195 (12), 133 (43): HRMS (DART) $\mathrm{m} / z$ 
$[\mathrm{M}-\mathrm{H}]^{+}$Calcd for $\mathrm{C}_{20} \mathrm{H}_{16} \mathrm{~F}_{5}$ 351.1172; Found 351.1187.

\section{(E)-8-(3-Methylphenyl)-3-perfluorophenyl-1,6-octadiene (4ac):}

The general procedure was followed using hexafluorobenzene (183.0 $\mathrm{mg}, 0.984 \mathrm{mmol}$ ) and 4-methylphenylmagnesium bromide (in THF, $0.80 \mathrm{M}, 1.6 \mathrm{~mL}, 1.3 \mathrm{mmol}$ ). Purification by GPC gave the desired product 4ac as a colorless oil (225.6 mg, 63\%) along with disubstituted

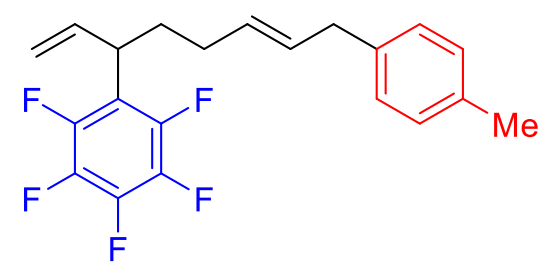
byproduct 4'ac (107.9 mg, 20\%): IR (neat): 1521, 1499, 1118, 975, $807 \mathrm{~cm}^{-1}$ : ${ }^{1} \mathrm{H}$ NMR (400 MHz, $\mathrm{CDCl}_{3}$ ): $\delta 7.09(\mathrm{~d}, J=8.0 \mathrm{~Hz}, 2 \mathrm{H}), 7.05(\mathrm{~d}, J=8.0 \mathrm{~Hz}, 2 \mathrm{H}), 6.00$ (quin, $J=8.4 \mathrm{~Hz}, 1 \mathrm{H}$ ), $5.54(\mathrm{dt}, J=$ $15.2,6.8 \mathrm{~Hz}, 1 \mathrm{H}), 5.42$ (dt, $J=15.2,6.4 \mathrm{~Hz}, 1 \mathrm{H}), 5.10$ (br s, 1H), 5.06 (d, $J=6.8 \mathrm{~Hz}, 1 \mathrm{H}), 3.75$ (q, $J=$ $7.6 \mathrm{~Hz}, 1 \mathrm{H}), 3.27(\mathrm{~d}, J=6.8 \mathrm{~Hz}, 2 \mathrm{H}), 2.31(\mathrm{~s}, 3 \mathrm{H}), 2.04-1.82(\mathrm{~m}, 4 \mathrm{H}):{ }^{13} \mathrm{C}\left\{{ }^{1} \mathrm{H}\right\} \mathrm{NMR}(100 \mathrm{MHz}$, $\left.\mathrm{CDCl}_{3}\right): 144.9\left(\mathrm{dm},{ }^{1} J_{\mathrm{C}-\mathrm{F}}=244.1 \mathrm{~Hz}\right), 139.6\left(\mathrm{dm},{ }^{1} J_{\mathrm{C}-\mathrm{F}}=249.8 \mathrm{~Hz}\right), 137.54,137.53\left(\mathrm{dm},{ }^{1} J_{\mathrm{C}-\mathrm{F}}=250.8\right.$ $\mathrm{Hz}), 137.49,135.4,130.5,129.8,129.0,128.3,117.1\left(\mathrm{t},{ }^{2} J_{\mathrm{C}-\mathrm{F}}=17.6 \mathrm{~Hz}\right), 116.7,39.4,38.5,32.8,30.4$, 20.9: ${ }^{19} \mathrm{~F}$ NMR $\left(376 \mathrm{MHz}, \mathrm{CDCl}_{3}\right): \delta-142.1(\mathrm{~d}, J=23.3 \mathrm{~Hz}, 2 \mathrm{~F}),-157.3(\mathrm{~m}, 1 \mathrm{~F}),-162.3(\mathrm{~m}, 2 \mathrm{~F}): \mathrm{MS}$ (DART) $m / z$ (relative intensity, \%): $365\left([\mathrm{M}-\mathrm{H}]^{+}, 70\right), 279$ (100): HRMS (DART) $\mathrm{m} / z$ [M-H] $]^{+}$Calcd for $\mathrm{C}_{21} \mathrm{H}_{18} \mathrm{~F}_{5}$ 365.1329; Found 365.1318.

\section{(E)-8-(3-Methylphenyl)-3-perfluorophenyl-1,6-octadiene (4ad):}

The general procedure was followed using hexafluorobenzene $(183.0 \mathrm{mg}$, $0.984 \mathrm{mmol}$ ) and 3-methylphenylmagnesium bromide (in THF, $0.52 \mathrm{M}$, $2.5 \mathrm{~mL}, 1.3 \mathrm{mmol})$. Purification by GPC gave the desired product $4 \mathbf{a d}$ as a colorless oil (286.6 $\mathrm{mg}, 80 \%$ ) along with disubstituted byproduct 4'ad

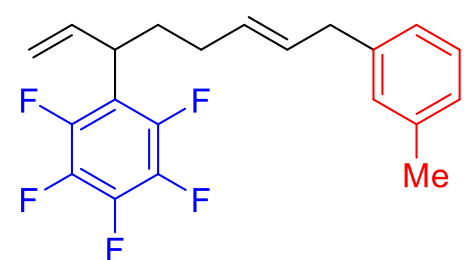
(24.6 mg, 5\%): IR (neat): $1521,1118,976,743 \mathrm{~cm}^{-1}:{ }^{1} \mathrm{H} \mathrm{NMR}\left(400 \mathrm{MHz}, \mathrm{CDCl}_{3}\right): \delta 7.17(\mathrm{t}, J=7.2 \mathrm{~Hz}$, $1 \mathrm{H}$ ), 7.01-6.95 (m, 3H), 6.01 (quin, $J=8.8 \mathrm{~Hz}, 1 \mathrm{H}$ ), 5.55 (dt, $J=15.2,6.4 \mathrm{~Hz}, 1 \mathrm{H}), 5.44(\mathrm{dt}, J=15.2$, $6.4 \mathrm{~Hz}, 1 \mathrm{H}), 5.10$ (br s, 1H), $5.07(\mathrm{~d}, J=7.2 \mathrm{~Hz}, 1 \mathrm{H}), 3.75$ (q, $J=7.6 \mathrm{~Hz}, 1 \mathrm{H}), 3.28(\mathrm{~d}, J=6.4 \mathrm{~Hz}, 2 \mathrm{H})$, $2.32(\mathrm{~s}, 3 \mathrm{H}), 2.04-1.83(\mathrm{~m}, 4 \mathrm{H}):{ }^{13} \mathrm{C}\left\{{ }^{1} \mathrm{H}\right\} \mathrm{NMR}\left(100 \mathrm{MHz}, \mathrm{CDCl}_{3}\right): 144.9\left(\mathrm{dm},{ }^{1} J_{\mathrm{C}-\mathrm{F}}=244.1 \mathrm{~Hz}\right), 140.6$, $139.6\left(\mathrm{dm},{ }^{1} J_{\mathrm{C}-\mathrm{F}}=251.8 \mathrm{~Hz}\right), 138.0,137.52\left(\mathrm{dm},{ }^{1} J_{\mathrm{C}-\mathrm{F}}=250.8 \mathrm{~Hz}\right), 137.49,130.4,129.9,129.2,128.3$, 126.7, 125.4, $117.1\left(\mathrm{tt},{ }^{2} J_{\mathrm{C}-\mathrm{F}}=17.1,{ }^{3} J_{\mathrm{C}-\mathrm{F}}=1.9 \mathrm{~Hz}\right), 116.7,39.4,38.9,32.8,30.4,21.3:{ }^{19} \mathrm{~F}$ NMR $(376$ $\mathrm{MHz}_{\mathrm{CDCl}}$ ): $\delta-142.0(\mathrm{~d}, J=22.9 \mathrm{~Hz}, 2 \mathrm{~F}),-157.2(\mathrm{~m}, 1 \mathrm{~F}),-162.3(\mathrm{~m}, 2 \mathrm{~F}):$ MS (DART) $\mathrm{m} / z$ (relative intensity, \%): 365 ([M-H $\left.]^{+}, 70\right), 279$ (100): HRMS (DART) $m / z[\mathrm{M}-\mathrm{H}]^{+}$Calcd for $\mathrm{C}_{21} \mathrm{H}_{18} \mathrm{~F}_{5} 365.1329$; Found 365.1319.

\section{(E)-8-(3-Methoxyphenyl)-3-perfluorophenyl-1,6-octadiene (4ae):}

The general procedure was followed using hexafluorobenzene $(195.1 \mathrm{mg}$, $1.049 \mathrm{mmol}$ ) and 3-methoxyphenylmagnesium bromide (in THF, $0.97 \mathrm{M}$, $1.4 \mathrm{~mL}, 1.3 \mathrm{mmol})$. Purification by GPC gave the desired product 4 ae as a

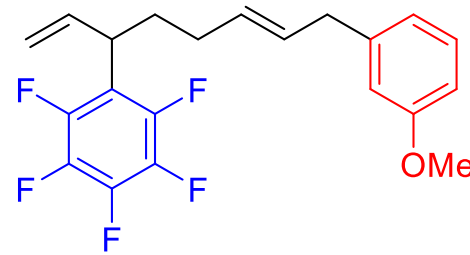


colorless oil (318.0 mg, 79\%) along with disubstituted byproduct 4'ae (35.8 mg, 6\%): ${ }^{1} \mathrm{H}$ NMR (400 $\left.\mathrm{MHz}_{2} \mathrm{CDCl}_{3}\right): \delta 7.20(\mathrm{t}, J=7.6 \mathrm{~Hz}, 1 \mathrm{H}), 6.77-6.72(\mathrm{~m}, 3 \mathrm{H}), 6.05-5.96(\mathrm{~m}, 1 \mathrm{H}), 5.55(\mathrm{dt}, J=14.8,6.8$ $\mathrm{Hz}, 1 \mathrm{H}), 5.45$ (dt, $J=15.2,6.4 \mathrm{~Hz}, 1 \mathrm{H}), 5.11$ (br s, $1 \mathrm{H}), 5.07$ (d, $J=6.8 \mathrm{~Hz}, 1 \mathrm{H}), 3.78$ (s, 3H), 3.75 (q, $J$ $=8.0 \mathrm{~Hz}, 1 \mathrm{H}), 3.29(\mathrm{~d}, J=6.4 \mathrm{~Hz}, 2 \mathrm{H}), 2.04-1.83(\mathrm{~m}, 4 \mathrm{H}):{ }^{13} \mathrm{C}\left\{{ }^{1} \mathrm{H}\right\}$ NMR $\left(100 \mathrm{MHz}, \mathrm{CDCl}_{3}\right): 145.0$ $\left(\mathrm{dm},{ }^{1} J_{\mathrm{C}-\mathrm{F}}=245.0 \mathrm{~Hz}\right), 142.4,139.7\left(\mathrm{dm},{ }^{1} J_{\mathrm{C}-\mathrm{F}}=250.8 \mathrm{~Hz}\right), 137.6\left(\mathrm{dm},{ }^{1} J_{\mathrm{C}-\mathrm{F}}=250.8 \mathrm{~Hz}\right), 137.5,130.2$, 130.1, 129.4, 120.9, $117.1\left(\mathrm{t},{ }^{2} J_{\mathrm{C}-\mathrm{F}}=17.2 \mathrm{~Hz}\right), 116.8,114.3,111.2,55.1,39.5,39.1,32.9,30.5:{ }^{19} \mathrm{~F}$ NMR $\left(376 \mathrm{MHz}, \mathrm{CDCl}_{3}\right): \delta-142.0(\mathrm{~m}, 2 \mathrm{~F}),-157.2(\mathrm{~m}, 1 \mathrm{~F}),-162.3(\mathrm{~m}, 2 \mathrm{~F}):$ MS (DART) $\mathrm{m} / z$ (relative intensity, \%): $383\left([\mathrm{M}+\mathrm{H}]^{+}, 20\right), 367$ (54), 239 (15), 195 (16), 147 (52), 133 (100): HRMS (DART) m/z $[\mathrm{M}+\mathrm{H}]^{+}$Calcd for $\mathrm{C}_{21} \mathrm{H}_{20} \mathrm{~F}_{5} \mathrm{O} 383.1434$; Found 383.1415.

\section{(E)-8-(2-Methylphenyl)-3-perfluorophenyl-1,6-octadiene (4af):}

The general procedure was followed using hexafluorobenzene $(181.2 \mathrm{mg}$, $0.974 \mathrm{mmol}$ ) and 2-methylphenylmagnesium bromide (in THF, $0.82 \mathrm{M}$, $1.6 \mathrm{~mL}, 1.3 \mathrm{mmol})$. Purification by GPC gave the desired product 4 af as a

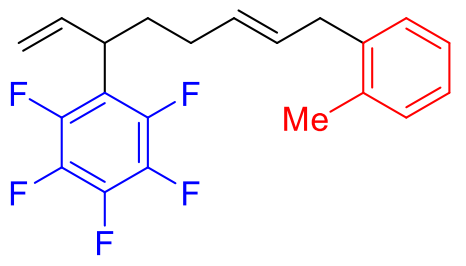
colorless oil (248.4 mg, 70\%) along with disubstituted byproduct 4'af (113.3 mg, 21\%): IR (neat): 1521, 1499, 1118, 975, 779, $699 \mathrm{~cm}^{-1}:{ }^{1} \mathrm{H}$ NMR (400 MHz, $\mathrm{CDCl}_{3}$ ): $\delta 7.18-7.04(\mathrm{~m}, 4 \mathrm{H}$ ), 6.00 (quin, $J=8.8$ $\mathrm{Hz}, 1 \mathrm{H}), 5.53$ (dt, $J=15.2,7.6 \mathrm{~Hz}, 1 \mathrm{H}), 5.35$ (dt, $J=15.2,7.6 \mathrm{~Hz}, 1 \mathrm{H}), 5.10$ (br s, $1 \mathrm{H}), 5.06(\mathrm{~d}, J=8.8$ $\mathrm{Hz}, 1 \mathrm{H}), 3.73(\mathrm{q}, J=7.6 \mathrm{~Hz}, 1 \mathrm{H}), 3.30(\mathrm{~d}, J=6.4 \mathrm{~Hz}, 2 \mathrm{H}), 2.27(\mathrm{~s}, 3 \mathrm{H}), 2.05-1.79(\mathrm{~m}, 4 \mathrm{H}):{ }^{13} \mathrm{C}\left\{{ }^{1} \mathrm{H}\right\}$ NMR (100 MHz, $\left.\mathrm{CDCl}_{3}\right): 144.9\left(\mathrm{dm},{ }^{1} J_{\mathrm{C}-\mathrm{F}}=245.1 \mathrm{~Hz}\right), 139.6\left(\mathrm{dm},{ }^{1} J_{\mathrm{C}-\mathrm{F}}=250.8 \mathrm{~Hz}\right), 138.6,137.54$ $\left(\mathrm{dm},{ }^{1} J_{\mathrm{C}-\mathrm{F}}=250.8 \mathrm{~Hz}\right), 137.48,136.2,130.1,129.9,129.4,128.9,126.2,126.0,117.1\left(\mathrm{tt},{ }^{2} J_{\mathrm{C}-\mathrm{F}}=16.7\right.$, $\left.{ }^{3} J_{\mathrm{C}-\mathrm{F}}=1.9 \mathrm{~Hz}\right), 116.7,39.5,36.4,32.9,30.5,19.2:{ }^{19} \mathrm{~F}$ NMR $\left(376 \mathrm{MHz}, \mathrm{CDCl}_{3}\right): \delta-142.0(\mathrm{~d}, J=23.3$ $\mathrm{Hz}, 2 \mathrm{~F}),-157.3(\mathrm{~m}, 1 \mathrm{~F}),-162.3(\mathrm{~m}, 2 \mathrm{~F}): \mathrm{MS}$ (DART) $\mathrm{m} / z$ (relative intensity, \%): $365\left([\mathrm{M}-\mathrm{H}]^{+}, 15\right), 279$ (100): HRMS (DART) $m / z$ [M-H] $]^{+}$Calcd for $\mathrm{C}_{21} \mathrm{H}_{18} \mathrm{~F}_{5}$ 365.1329; Found 365.1334.

\section{(E)-8-(2-Isopropylphenyl)-3-perfluorophenyl-1,6-octadiene (4ag):}

The general procedure was followed using hexafluorobenzene $(187.6 \mathrm{mg}$, $1.01 \mathrm{mmol}$ ) and 2-isopropylphenylmagnesium bromide (in THF, $0.97 \mathrm{M}$, $1.3 \mathrm{~mL}, 1.3 \mathrm{mmol})$. Purification by GPC gave the desired product $4 \mathbf{a g}$ as a colorless oil (214.5 mg, 54\%): ${ }^{1} \mathrm{H}$ NMR (400 MHz, $\left.\mathrm{CDCl}_{3}\right): \delta 7.27$ (d, $J=$

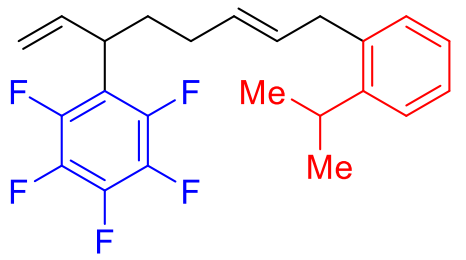
$7.2 \mathrm{~Hz}, 1 \mathrm{H}), 7.21-7.18(\mathrm{~m}, 1 \mathrm{H}), 7.11-7.10(\mathrm{~m}, 2 \mathrm{H}), 6.04-5.95(\mathrm{~m}, 1 \mathrm{H}), 5.56(\mathrm{dt}, J=15.2,6.4 \mathrm{~Hz}, 1 \mathrm{H})$, $5.32(\mathrm{dt}, J=15.2,6.4 \mathrm{~Hz}, 1 \mathrm{H}), 5.09(\mathrm{br} \mathrm{s}, 1 \mathrm{H}), 5.06(\mathrm{~d}, J=8.8 \mathrm{~Hz}, 1 \mathrm{H}), 3.73(\mathrm{q}, J=7.6 \mathrm{~Hz}, 1 \mathrm{H}), 3.36(\mathrm{~d}$, $J=6.4 \mathrm{~Hz}, 2 \mathrm{H}), 3.15(\mathrm{sept}, J=6.8 \mathrm{~Hz}, 1 \mathrm{H}), 2.05-1.77(\mathrm{~m}, 4 \mathrm{H}), 1.21(\mathrm{~d}, J=6.8 \mathrm{~Hz}, 6 \mathrm{H}):{ }^{13} \mathrm{C}\left\{{ }^{1} \mathrm{H}\right\} \mathrm{NMR}$ $\left(100 \mathrm{MHz}, \mathrm{CDCl}_{3}\right): 146.7,144.9\left(\mathrm{dm},{ }^{1} J_{\mathrm{C}-\mathrm{F}}=246.0 \mathrm{~Hz}\right), 139.6\left(\mathrm{dm},{ }^{1} J_{\mathrm{C}-\mathrm{F}}=250.8 \mathrm{~Hz}\right), 137.5\left(\mathrm{dm},{ }^{1} J_{\mathrm{C}-\mathrm{F}}\right.$ $=250.8 \mathrm{~Hz}), 137.5,137.1,130.5,129.6,129.5,126.6,125.7,125.2,117.0\left(\mathrm{tt},{ }^{2} J_{\mathrm{C}-\mathrm{F}}=16.7,{ }^{3} J_{\mathrm{C}-\mathrm{F}}=1.9\right.$ $\mathrm{Hz}), 116.7,39.4,35.8,32.8,30.5,28.7,23.8:{ }^{19} \mathrm{~F} \mathrm{NMR}\left(376 \mathrm{MHz}, \mathrm{CDCl}_{3}\right): \delta-142.0(\mathrm{~m}, 2 \mathrm{~F}),-157.2(\mathrm{t}$, $J=21.4 \mathrm{~Hz}, 1 \mathrm{~F}),-162.3(\mathrm{~m}, 2 \mathrm{~F}):$ MS (DART) $m / z$ (relative intensity, \%): $393\left([\mathrm{M}-\mathrm{H}]^{+}, 4\right), 391(20)$, 
239 (7), 195 (15), 177 (28), 163 (100): HRMS (DART) $m / z[\mathrm{M}-\mathrm{H}]^{+}$Calcd for $\mathrm{C}_{23} \mathrm{H}_{22} \mathrm{~F}_{5}$ 393.1642; Found 393.1671.

\section{(E)-4-(6-(Perfluorophenyl)octa-2,7-dien-1-yl)-1,1'-biphenyl}

(4ah):

The general procedure was followed using hexafluorobenzene (183.9 $\mathrm{mg}, 0.99 \mathrm{mmol}$ ) and biphenylmagnesium bromide (in THF,

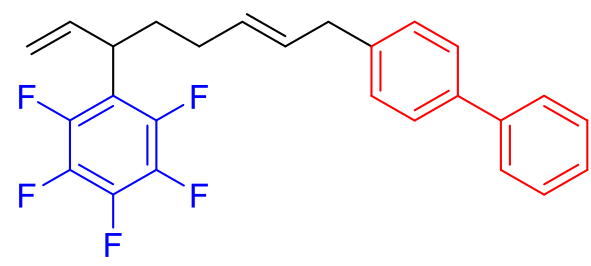

1.21 M, $1.1 \mathrm{~mL}, 1.3 \mathrm{mmol})$. Purification by GPC gave the desired product 4 ah as a colorless oil (228.6 mg, 54\%): IR (neat): 1521, 1498, 1117, 974, 761, $698 \mathrm{~cm}^{-1}$ : IR (neat) : 1521, 1498, 1117, 974, 761, 698 $\mathrm{cm}^{-1} ;{ }^{1} \mathrm{H} \mathrm{NMR}\left(400 \mathrm{MHz}, \mathrm{CDCl}_{3}\right): \delta 7.58(\mathrm{~d}, J=8.0 \mathrm{~Hz}, 2 \mathrm{H}), 7.52(\mathrm{~d}, J=8.0 \mathrm{~Hz}, 2 \mathrm{H}), 7.43(\mathrm{t}, J=7.6$ $\mathrm{Hz}, 2 \mathrm{H}), 7.33(\mathrm{t}, J=7.6 \mathrm{~Hz}, 1 \mathrm{H}), 7.24(\mathrm{~d}, J=8.4 \mathrm{~Hz}, 2 \mathrm{H}), 6.06-5.97(\mathrm{~m}, 1 \mathrm{H}), 5.63-5.45(\mathrm{~m}, 2 \mathrm{H})$, 5.00-5.07 (m, 2H), $3.76(\mathrm{dt}, J=7.6,7.6 \mathrm{~Hz}, 1 \mathrm{H}), 3.36(\mathrm{~d}, J=6.4 \mathrm{~Hz}, 2 \mathrm{H}), 2.08-1.83(\mathrm{~m}, 4 \mathrm{H}):{ }^{13} \mathrm{C}$ NMR $\left(100 \mathrm{MHz} \mathrm{CDCl}_{3}\right): \delta 141.1,139.8,139.0,137.5,130.3,130.1,128.9,128.7,127.1,127.03,127.0,116.7$, 39.4, 38.6, 32.9, 30.4 (carbon signals from the pentafluorophenyl ring were not observed): ${ }^{19} \mathrm{~F}$ NMR $\left(376 \mathrm{MHz}, \mathrm{CDCl}_{3}\right): \delta-142.02(\mathrm{dd}, J=21.8,7.9 \mathrm{~Hz}, 2 \mathrm{~F}),-157.22(\mathrm{t}, J=21.1 \mathrm{~Hz}, 1 \mathrm{~F}),-162.28(\mathrm{ddd}, J=$ 21.8, 21.1, 7.9 Hz, 2F): MS (EI): $m / z$ (relative intensity, \%) 428 ([M] $\left.{ }^{+}, 87\right), 219(14), 180$ (75), 167 (100): HRMS (EI) $m / z[M]^{+}$Calcd for $\mathrm{C}_{26} \mathrm{H}_{21} \mathrm{~F}_{5}$ 428.1563; Found 428.1565.

\section{(E)-8-(4-Fluoro-2-methylphenyl)-3-perfluorophenyl-1,6-octadiene}

\section{(4ai):}

The general procedure was followed using hexafluorobenzene (185.7 $\mathrm{mg}, 0.998 \mathrm{mmol}$ ) and 4-fluoro-2-methylphenylmagnesium bromide (in

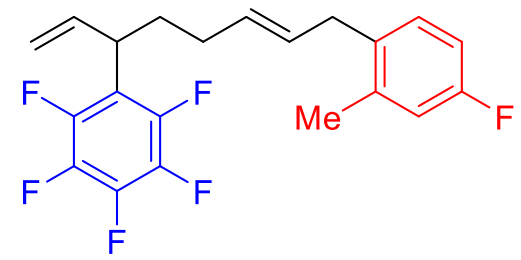
THF, 0.48 M, $2.7 \mathrm{~mL}, 1.3 \mathrm{mmol}$ ). Purification by GPC gave the desired product 4ai as a colorless oil (261.4 mg, 69\%) along with disubstituted byproduct 4'ai (14.7 mg, 3\%): ${ }^{1} \mathrm{H}$ NMR (400 MHz, $\left.\mathrm{CDCl}_{3}\right): \delta$ $7.04(\mathrm{dd}, J=8.0,5.6 \mathrm{~Hz}, 1 \mathrm{H}), 6.86-6.79(\mathrm{~m}, 2 \mathrm{H}), 6.04-5.95(\mathrm{~m}, 1 \mathrm{H}), 5.50(\mathrm{dt}, J=15.2,6.4 \mathrm{~Hz}, 1 \mathrm{H})$, $5.32(\mathrm{dt}, J=15.2,6.4 \mathrm{~Hz}, 1 \mathrm{H}), 5.10(\mathrm{~d}, J=4.4 \mathrm{~Hz}, 1 \mathrm{H}), 5.06(\mathrm{~d}, J=11.2 \mathrm{~Hz}, 1 \mathrm{H}), 3.72(\mathrm{q}, J=7.6 \mathrm{~Hz}$, $1 \mathrm{H}), 3.25(\mathrm{~d}, J=6.4 \mathrm{~Hz}, 2 \mathrm{H}), 2.26(\mathrm{~s}, 3 \mathrm{H}), 2.05-1.77(\mathrm{~m}, 4 \mathrm{H}):{ }^{13} \mathrm{C}\left\{{ }^{1} \mathrm{H}\right\} \mathrm{NMR}\left(100 \mathrm{MHz}, \mathrm{CDCl}_{3}\right): 161.2$ $\left(\mathrm{d},{ }^{1} J_{\mathrm{C}-\mathrm{F}}=242.1 \mathrm{~Hz}\right), 144.9\left(\mathrm{dm},{ }^{1} J_{\mathrm{C}-\mathrm{F}}=245.1 \mathrm{~Hz}\right), 139.8\left(\mathrm{dm},{ }^{1} J_{\mathrm{C}-\mathrm{F}}=205.9 \mathrm{~Hz}\right), 138.4,138.3,137.5$ $\left(\mathrm{dm},{ }^{1} J_{\mathrm{C}-\mathrm{F}}=250.8 \mathrm{~Hz}\right), 137.4,134.2\left(\mathrm{~d},{ }^{3} J_{\mathrm{C}-\mathrm{F}}=2.9 \mathrm{~Hz}\right), 130.24,130.16,130.0,129.2,117.0\left(\mathrm{tt},{ }^{2} J_{\mathrm{C}-\mathrm{F}}=\right.$ $\left.17.1,{ }^{3} J_{\mathrm{C}-\mathrm{F}}=1.9 \mathrm{~Hz}\right), 116.6\left(\mathrm{~d},{ }^{2} J_{\mathrm{C}-\mathrm{F}}=20.0 \mathrm{~Hz}\right), 112.4\left(\mathrm{~d},{ }^{2} J_{\mathrm{C}-\mathrm{F}}=20.0 \mathrm{~Hz}\right), 39.4,35.7,32.8,30.4,19.4$ $\left(\mathrm{d},{ }^{3} J_{\mathrm{C}-\mathrm{F}}=1.9 \mathrm{~Hz}\right):{ }^{19} \mathrm{~F} \mathrm{NMR}\left(376 \mathrm{MHz}, \mathrm{CDCl}_{3}\right): \delta-117.9(\mathrm{t}, J=14.3 \mathrm{~Hz}, 1 \mathrm{~F}),-142.1(\mathrm{~d}, J=23.3 \mathrm{~Hz}$, 2F), -157.2 (t, $J=23.3 \mathrm{~Hz}, 1 \mathrm{~F}),-162.3$ (t, $J=23.3 \mathrm{~Hz}, 2 \mathrm{~F}):$ MS (DART) $\mathrm{m} / z$ (relative intensity, \%): 383 ([M-H] $\left.]^{+}, 9\right), 279$ (11), 195 (11), 123 (15), 102 (100): HRMS (DART) $m / z$ [M-H] ${ }^{+}$Calcd for $\mathrm{C}_{21} \mathrm{H}_{18} \mathrm{~F}_{6}$ 383.1234; Found 383.1262. 


\section{(E)-8-(4-Chloro-2-methylphenyl)-3-perfluorophenyl-1,6-octadiene (4aj):}

The general procedure was followed using hexafluorobenzene (182.2 $\mathrm{mg}, 0.979 \mathrm{mmol}$ ) and 4-chloro-2-methylphenylmagnesium bromide (in THF, 0.51 M, $2.6 \mathrm{~mL}, 1.3 \mathrm{mmol}$ ). Purification by GPC gave the desired product 4aj as a colorless oil (309.2 $\mathrm{mg}, 79 \%)$ along with disubstituted byproduct 4'aj (23.2 mg, 4\%): $\left.{ }^{1} \mathrm{H} \mathrm{NMR} \mathrm{(400} \mathrm{MHz}, \mathrm{CDCl}_{3}\right): \delta$

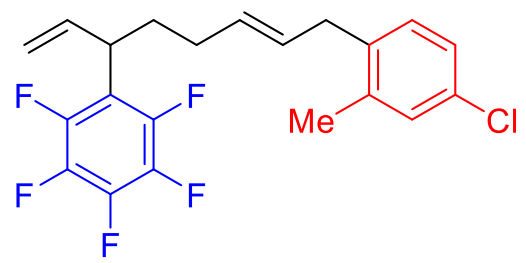
7.11-7.07 (m, 2H), $7.01(\mathrm{~d}, J=8.0 \mathrm{~Hz}, 1 \mathrm{H}), 6.04-5.95(\mathrm{~m}, 1 \mathrm{H}), 5.49(\mathrm{dt}, J=15.2,6.4 \mathrm{~Hz}, 1 \mathrm{H}), 5.33(\mathrm{dt}$, $J=15.2,6.4 \mathrm{~Hz}, 1 \mathrm{H}), 5.10(\mathrm{br} \mathrm{s}, 1 \mathrm{H}), 5.07(\mathrm{~d}, J=10.4 \mathrm{~Hz}, 1 \mathrm{H}), 3.72(\mathrm{q}, J=7.6 \mathrm{~Hz}, 1 \mathrm{H}), 3.24(\mathrm{~d}, J=$ $6.4 \mathrm{~Hz}, 2 \mathrm{H}), 2.24(\mathrm{~s}, 3 \mathrm{H}), 2.05-1.77(\mathrm{~m}, 4 \mathrm{H}):{ }^{13} \mathrm{C}\left\{{ }^{1} \mathrm{H}\right\} \mathrm{NMR}\left(100 \mathrm{MHz}, \mathrm{CDCl}_{3}\right): 144.9\left(\mathrm{dm},{ }^{1} J_{\mathrm{C}-\mathrm{F}}=\right.$ $245.1 \mathrm{~Hz}), 139.6\left(\mathrm{dm},{ }^{1} J_{\mathrm{C}-\mathrm{F}}=249.8 \mathrm{~Hz}\right), 138.1,137.5\left(\mathrm{dm},{ }^{1} J_{\mathrm{C}-\mathrm{F}}=251.7 \mathrm{~Hz}\right), 137.4,137.1,131.5,130.3$, 130.2, 129.8, 128.8, 125.9, $117.0\left(\mathrm{tt},{ }^{2} J_{\mathrm{C}-\mathrm{F}}=17.1,{ }^{3} J_{\mathrm{C}-\mathrm{F}}=1.9 \mathrm{~Hz}\right), 116.7,39.4,35.8,32.8,30.4,19.1:{ }^{19} \mathrm{~F}$ NMR (376 MHz, $\left.\mathrm{CDCl}_{3}\right): \delta-142.1(\mathrm{~d}, J=16.9 \mathrm{~Hz}, 2 \mathrm{~F}),-157.2(\mathrm{~m}, 1 \mathrm{~F}),-162.3(\mathrm{~m}, 2 \mathrm{~F})$ : MS (DART) $\mathrm{m} / \mathrm{z}$ (relative intensity, \%): 399 ([M-H] $\left.]^{+}, 12\right), 380$ (11), 279 (10), 195 (11), 123 (17), 102 (100): HRMS (DART) $m / z$ [M-H] $]^{+}$Calcd for $\mathrm{C}_{21} \mathrm{H}_{18} \mathrm{ClF}_{5}$ 399.0939; Found 399.0949.

\section{(E)-8-(4-Methoxy-2-methylphenyl)-3-perfluorophenyl-1,6-octadiene (4ak):}

The general procedure was followed using hexafluorobenzene (188.2 $\mathrm{mg}, 1.01 \mathrm{mmol}$ ) and 4-methoxyl-2-methylphenylmagnesium bromide (in THF, $0.48 \mathrm{M}, 2.7 \mathrm{~mL}, 1.3 \mathrm{mmol}$ ). Purification by GPC gave the desired product 4ak as a colorless oil (309.9 $\mathrm{mg}, 78 \%$ ) along with disubstituted byproduct 4'ak (42.9 mg, 7\%): ${ }^{1} \mathrm{H}$ NMR (400 MHz,

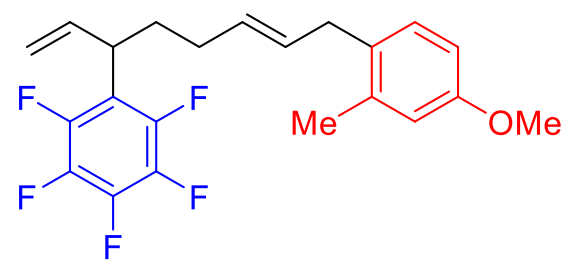
$\left.\mathrm{CDCl}_{3}\right): \delta 7.01(\mathrm{~d}, J=8.0 \mathrm{~Hz}, 1 \mathrm{H}), 6.70(\mathrm{~s}, 1 \mathrm{H}), 6.68(\mathrm{~d}, J=8.0 \mathrm{~Hz}, 1 \mathrm{H}), 6.00$ (quin, $J=8.4 \mathrm{~Hz}, 1 \mathrm{H}$ ), $5.51(\mathrm{dt}, J=15.2,6.4 \mathrm{~Hz}, 1 \mathrm{H}), 5.32(\mathrm{dt}, J=15.2,6.4 \mathrm{~Hz}, 1 \mathrm{H}), 5.10(\mathrm{~s}, 1 \mathrm{H}), 5.06(\mathrm{~d}, J=8.4 \mathrm{~Hz}, 1 \mathrm{H})$, $3.77(\mathrm{~s}, 3 \mathrm{H}), 3.73(\mathrm{q}, J=8.0 \mathrm{~Hz}, 1 \mathrm{H}), 3.23(\mathrm{~d}, J=6.4 \mathrm{~Hz}, 2 \mathrm{H}), 2.25(\mathrm{~s}, 3 \mathrm{H}), 2.05-1.77(\mathrm{~m}, 4 \mathrm{H}):{ }^{13} \mathrm{C}\left\{{ }^{1} \mathrm{H}\right\}$ NMR (100 MHz, $\left.\mathrm{CDCl}_{3}\right): 157.9,144.9\left(\mathrm{dm},{ }^{1} J_{\mathrm{C}-\mathrm{F}}=244.1 \mathrm{~Hz}\right), 139.6\left(\mathrm{dm},{ }^{1} J_{\mathrm{C}-\mathrm{F}}=250.8 \mathrm{~Hz}\right), 137.52$ $\left(\mathrm{dm},{ }^{1} J_{\mathrm{C}-\mathrm{F}}=251.7 \mathrm{~Hz}\right), 137.47,130.8,129.9,129.8,129.5,117.1\left(\mathrm{tt},{ }^{2} J_{\mathrm{C}-\mathrm{F}}=16.2,{ }^{3} J_{\mathrm{C}-\mathrm{F}}=1.9 \mathrm{~Hz}\right), 116.7$, 115.8, 110.9, 55.1, 39.4, 35.6, 32.9, 30.4, 19.5: ${ }^{19} \mathrm{~F}$ NMR (376 MHz, $\left.\mathrm{CDCl}_{3}\right): \delta-142.1(\mathrm{~d}, J=22.9 \mathrm{~Hz}$, $2 \mathrm{~F}),-157.3(\mathrm{~m}, 1 \mathrm{~F}),-162.3(\mathrm{~m}, 2 \mathrm{~F}):$ MS (DART) $\mathrm{m} / \mathrm{z}$ (relative intensity, \%): $395\left([\mathrm{M}-\mathrm{H}]^{+}, 12\right), 151(25)$, 135 (100): HRMS (DART) $m / z$ [M-H] ${ }^{+}$Calcd for $\mathrm{C}_{22} \mathrm{H}_{20} \mathrm{~F}_{5} \mathrm{O}$ 395.1434; Found 395.1442.

\section{(E)-8-(4-Dimethylaminophenyl)-3-perfluorophenyl-1,6-octadien e $(4 \mathrm{a} l)$ :}

The general procedure was followed using hexafluorobenzene (185.2 $\mathrm{mg}, 0.995 \mathrm{mmol})$ and 4-dimethylaminophenylmagnesium bromide (in THF, $0.75 \mathrm{M}, 1.7 \mathrm{~mL}, 1.3 \mathrm{mmol}$ ). The reaction was

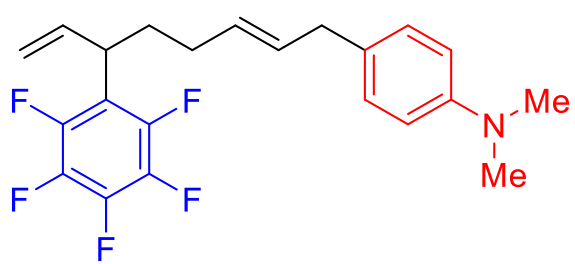
quenched by adding water instead of $1 \mathrm{~N} \mathrm{HCl}$ aq. Purification by $\mathrm{GPC}\left(\mathrm{CHCl}_{3}\right.$ containing $0.1 \% \mathrm{Et}_{3} \mathrm{~N}$ as 
an eluent) gave the desired product $\mathbf{4 a} l$ as a pale yellow oil (286.4 $\mathrm{mg}, 73 \%)$ along with disubstituted byproduct 4'al (27.9 mg, 5\%): ${ }^{1} \mathrm{H}$ NMR (400 MHz, $\left.\mathrm{CDCl}_{3}\right): \delta 7.03(\mathrm{~d}, J=8.4 \mathrm{~Hz}, 2 \mathrm{H}), 6.69(\mathrm{~d}, J=8.4$ $\mathrm{Hz}, 2 \mathrm{H}$ ), 6.00 (quin, $J=8.4 \mathrm{~Hz}, 1 \mathrm{H}), 5.54$ (dt, $J=15.2,6.8 \mathrm{~Hz}, 1 \mathrm{H}), 5.41(\mathrm{dt}, J=15.2,6.4 \mathrm{~Hz}, 1 \mathrm{H}), 5.10$ (br s, 1H), 5.07 (d, $J=6.4 \mathrm{~Hz}, 1 \mathrm{H}), 3.75$ (q, $J=7.6 \mathrm{~Hz}, 1 \mathrm{H}), 3.22$ (d, $J=6.8 \mathrm{~Hz}, 2 \mathrm{H}), 2.90$ (s, 6H), 2.07-1.78 (m, 4H): ${ }^{13} \mathrm{C}\left\{{ }^{1} \mathrm{H}\right\} \mathrm{NMR}\left(100 \mathrm{MHz}, \mathrm{CDCl}_{3}\right): 149.2,144.9\left(\mathrm{dm},{ }^{1} J_{\mathrm{C}-\mathrm{F}}=251.2 \mathrm{~Hz}\right), 140.0(\mathrm{dm}$, $\left.{ }^{1} J_{\mathrm{C}-\mathrm{F}}=249.9 \mathrm{~Hz}\right), 137.5,137.4\left(\mathrm{dm},{ }^{1} J_{\mathrm{C}-\mathrm{F}}=218.9 \mathrm{~Hz}\right), 131.1,129.3,129.0,128.8,117.1\left(\mathrm{t},{ }^{2} J_{\mathrm{C}-\mathrm{F}}=14.7\right.$ $\mathrm{Hz}), 116.6,113.0,40.9,39.4,38.0,32.9,30.4:{ }^{19} \mathrm{~F} \mathrm{NMR}\left(376 \mathrm{MHz}, \mathrm{CDCl}_{3}\right): \delta-142.0(\mathrm{dd}, J=22.6,7.9$ $\mathrm{Hz}, 2 \mathrm{~F}),-157.4(\mathrm{t}, J=21.1 \mathrm{~Hz}, 1 \mathrm{~F}),-162.4(\mathrm{td}, J=22.6,8.3 \mathrm{~Hz}, 2 \mathrm{~F}): \mathrm{MS}$ (DART) $\mathrm{m} / z$ (relative intensity, \%): 396 ([M+H] $]^{+}, 100$ ), 295 (10): HRMS (DART) $m / z[\mathrm{M}-\mathrm{H}]^{+}$Calcd for $\mathrm{C}_{22} \mathrm{H}_{23} \mathrm{~F}_{5} \mathrm{~N} 396.1751$; Found 396.1749.

\section{(E)-8-(3-Methyl-2-thienyl)-3-perfluorophenyl-1,6-octadiene (4am):}

The general procedure was followed using hexafluorobenzene $(185.5 \mathrm{mg}$, $0.997 \mathrm{mmol}$ ) and 3-methyl-2-thienylmagnesium bromide (in THF, $0.53 \mathrm{M}$, $2.5 \mathrm{~mL}, 1.3 \mathrm{mmol}$ ). Purification by GPC gave the desired product $\mathbf{4 a m}$ as a

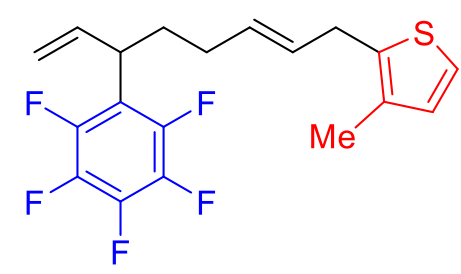
pale yellow oil (179.2 mg, 49\%) along with disubstituted byproduct 4'am (4.6 mg, 1\%): ${ }^{1} \mathrm{H}$ NMR (400 $\left.\left.\mathrm{MHz}_{\mathrm{CDCl}}\right)\right): \delta .02(\mathrm{~d}, J=5.2 \mathrm{~Hz}, 1 \mathrm{H}), 6.79(\mathrm{~d}, J=5.2 \mathrm{~Hz}, 1 \mathrm{H}), 6.05-5.96(\mathrm{~m}, 1 \mathrm{H}), 5.54(\mathrm{dt}, J=15.2$, $6.4 \mathrm{~Hz}, 1 \mathrm{H}), 5.44(\mathrm{dt}, J=15.2,6.4 \mathrm{~Hz}, 1 \mathrm{H}), 5.11(\mathrm{~s}, 1 \mathrm{H}), 5.08(\mathrm{~d}, J=4.4 \mathrm{~Hz}, 1 \mathrm{H}), 3.75(\mathrm{q}, J=8.0 \mathrm{~Hz}$, $1 \mathrm{H}), 3.40(\mathrm{~d}, J=6.4 \mathrm{~Hz}, 2 \mathrm{H}), 2.14(\mathrm{~s}, 3 \mathrm{H}), 2.05-1.78(\mathrm{~m}, 4 \mathrm{H}):{ }^{13} \mathrm{C}\left\{{ }^{1} \mathrm{H}\right\} \mathrm{NMR}\left(100 \mathrm{MHz}, \mathrm{CDCl}_{3}\right): 144.9$ $\left(\mathrm{dm},{ }^{1} J_{\mathrm{C}-\mathrm{F}}=245.0 \mathrm{~Hz}\right), 139.6\left(\mathrm{dm},{ }^{1} J_{\mathrm{C}-\mathrm{F}}=250.8 \mathrm{~Hz}\right), 137.5\left(\mathrm{dm},{ }^{1} J_{\mathrm{C}-\mathrm{F}}=247.9 \mathrm{~Hz}\right), 137.4,136.3,132.8$, 130.2, 130.1, 129.0, 121.4, $117.0\left(\mathrm{tt},{ }^{2} J_{\mathrm{C}-\mathrm{F}}=17.1,{ }^{3} J_{\mathrm{C}-\mathrm{F}}=1.9 \mathrm{~Hz}\right), 116.8,39.4,32.7,30.9,30.3,13.5:{ }^{19} \mathrm{~F}$ NMR (376 MHz, $\left.\mathrm{CDCl}_{3}\right): \delta-142.0(\mathrm{~d}, J=22.9 \mathrm{~Hz}, 2 \mathrm{~F}),-157.2(\mathrm{~m}, 1 \mathrm{~F}),-162.3(\mathrm{~m}, 2 \mathrm{~F}):$ MS (DART) $\mathrm{m} / \mathrm{z}$ (relative intensity, \%): $373\left([\mathrm{M}+\mathrm{H}]^{+}, 40\right), 279$ (30), 239 (18), 195 (42), 151 (100): HRMS (DART) $m / z,[\mathrm{M}+\mathrm{H}]^{+}$Calcd for $\mathrm{C}_{19} \mathrm{H}_{18} \mathrm{~F}_{5} \mathrm{~S}$ 373.1049; Found 373.1057.

\section{(E)-8-(2-Methyl-1-naphthyl)-3-perfluorophenyl-1,6-octadiene (4an):}

The general procedure was followed using hexafluorobenzene (187.3 $\mathrm{mg}, 1.01 \mathrm{mmol}$ ) and 2-methyl-1-naphthylmagnesium bromide (in THF, $0.15 \mathrm{M}, 8.7 \mathrm{~mL}, 1.3 \mathrm{mmol})$ for $18 \mathrm{~h}$. Purification by GPC gave the desired product 4an as an orange oil (127.1 mg, 30\%): ${ }^{1} \mathrm{H}$ NMR (400

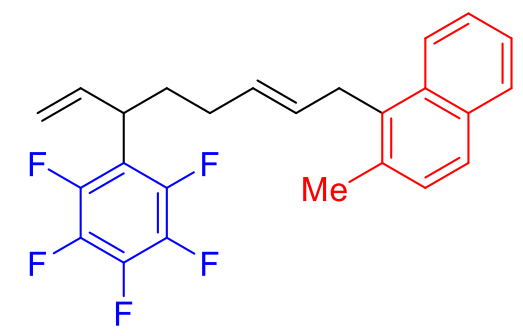
$\left.\left.\mathrm{MHz}_{\mathrm{CDCl}}\right)\right): \delta 7.97(\mathrm{~d}, J=8.8 \mathrm{~Hz}, 1 \mathrm{H}), 7.78(\mathrm{~d}, J=8.0 \mathrm{~Hz}, 1 \mathrm{H}), 7.63(\mathrm{~d}, J=8.8 \mathrm{~Hz}, 1 \mathrm{H}), 7.46(\mathrm{t}, J=$ $8.0 \mathrm{~Hz}, 1 \mathrm{H}), 7.39(\mathrm{t}, J=8.0 \mathrm{~Hz}, 1 \mathrm{H}), 7.30(\mathrm{~d}, J=8.0 \mathrm{~Hz}, 1 \mathrm{H}), 5.94$ (quin, $J=8.0 \mathrm{~Hz}, 1 \mathrm{H}), 5.59(\mathrm{dt}, J=$ $15.2,6.4 \mathrm{~Hz}, 1 \mathrm{H}), 5.25(\mathrm{dt}, J=15.2,6.4 \mathrm{~Hz}, 1 \mathrm{H}), 5.05-4.98(\mathrm{~m}, 2 \mathrm{H}), 3.76(\mathrm{~d}, J=5.6 \mathrm{~Hz}, 2 \mathrm{H}), 3.66(\mathrm{q}, J$ $=7.6 \mathrm{~Hz}, 1 \mathrm{H}), 2.46(\mathrm{~s}, 3 \mathrm{H}), 1.98-1.68(\mathrm{~m}, 4 \mathrm{H}):{ }^{13} \mathrm{C}\left\{{ }^{1} \mathrm{H}\right\} \mathrm{NMR}\left(100 \mathrm{MHz}, \mathrm{CDCl}_{3}\right): 144.8\left(\mathrm{dm},{ }^{1} J_{\mathrm{C}-\mathrm{F}}=\right.$ $245.0 \mathrm{~Hz}), 139.5\left(\mathrm{dm},{ }^{1} J_{\mathrm{C}-\mathrm{F}}=250.7 \mathrm{~Hz}\right), 137.5\left(\mathrm{dm},{ }^{1} J_{\mathrm{C}-\mathrm{F}}=251.7 \mathrm{~Hz}\right), 137.4,133.5,133.1,132.5,132.3$, $132.1,132.0,131.91,131.88,129.5,129.1,128.7,128.5,128.43,128.41,126.3,125.8,124.5,123.8$, 
$117.0\left(\mathrm{tt},{ }^{2} J_{\mathrm{C}-\mathrm{F}}=17.7,{ }^{3} J_{\mathrm{C}-\mathrm{F}}=1.9 \mathrm{~Hz}\right), 116.6,39.3,32.7,31.4,30.4,20.0:{ }^{19} \mathrm{~F}$ NMR $\left(376 \mathrm{MHz}, \mathrm{CDCl}_{3}\right)$ : $\delta-142.0(\mathrm{~d}, J=11.7 \mathrm{~Hz}, 2 \mathrm{~F}),-157.2(\mathrm{t}, J=22.9 \mathrm{~Hz}, 1 \mathrm{~F}),-162.3$ (quin, $J=11.7 \mathrm{~Hz}, 2 \mathrm{~F}$ ): MS (DART) m/z (relative intensity, \%): 417 ([M+H]+, 11), 380 (18), 373 (12), 279 (96), 239 (14), 195 (39), 151 (38), 102 (100): HRMS (DART) $m / z$ [M+H] $]^{+}$Calcd for $\mathrm{C}_{25} \mathrm{H}_{22} \mathrm{~F}_{5}$ 417.1642; Found 417.1653.

\section{(E)-2-(6-(Perfluorophenyl)octa-2,7-dien-1-yl)naphthalene (4ao):}

The general procedure was followed using hexafluorobenzene (183.4 $\mathrm{mg}, 0.99 \mathrm{mmol}$ ) and 2-naphthylmagnesium bromide (in THF, 1.43 M, $0.9 \mathrm{~mL}, 1.3 \mathrm{mmol})$. Purification by GPC gave the desired product $4 \mathbf{a o}$ as an orange oil (187.2 $\mathrm{mg}, 47 \%)$ : IR (neat): 1521, 1499, 1117, 975,

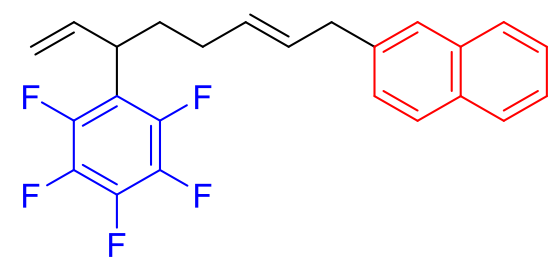
818, $745 \mathrm{~cm}^{-1}:{ }^{1} \mathrm{H}$ NMR (400 MHz, $\left.\mathrm{CDCl}_{3}\right): \delta 7.81-7.76(\mathrm{~m}, 3 \mathrm{H}), 7.60(\mathrm{~s}, 1 \mathrm{H}), 7.40-7.47(\mathrm{~m}, 2 \mathrm{H}), 7.31$ (dd, $J=8.4,1.6 \mathrm{~Hz}, 1 \mathrm{H}), 6.06-5.96(\mathrm{~m}, 1 \mathrm{H}), 5.67-5.46(\mathrm{~m}, 2 \mathrm{H}), 5.04-5.10(\mathrm{~m}, 2 \mathrm{H}), 3.76(\mathrm{dt}, J=7.6,7.6$ $\mathrm{Hz}, 1 \mathrm{H}), 3.48(\mathrm{~d}, J=6.4 \mathrm{~Hz}, 2 \mathrm{H}), 2.09-1.83(\mathrm{~m}, 4 \mathrm{H}):{ }^{13} \mathrm{C} \mathrm{NMR}\left(100 \mathrm{MHz}, \mathrm{CDCl}_{3}, 20{ }^{\circ} \mathrm{C}\right): \delta 138.1$, 137.5, 133.6, 132.0, 130.4, 130.1, 127.9, 127.6, 127.4, 127.3, 126.5, 125.9, 125.2, 116.8, 39.4, 39.1, 32.8, 30.4 (carbon signals of the pentafluorophenyl ring were not observed): ${ }^{19} \mathrm{~F} N \mathrm{NM}\left(376 \mathrm{MHz}, \mathrm{CDCl}_{3}, 20\right.$ $\left.{ }^{\circ} \mathrm{C}\right): \delta-142.00(\mathrm{dd}, J=22.0,7.9 \mathrm{~Hz}, 2 \mathrm{~F}),-157.20(\mathrm{t}, J=20.9 \mathrm{~Hz}, 1 \mathrm{~F}),-162.25$ (ddd, $J=22.0,20.9,7.9$ $\mathrm{Hz}, 2 \mathrm{~F}$ ): MS (EI) $\mathrm{m} / \mathrm{z}$ (relative intensity, \%): 402 ([M] $\left.{ }^{+}, 99\right), 193$ (15), 179 (25), 167 (84), 154 (62), 141 (100), 128 (26): HRMS (EI) $m / z$ [M] ${ }^{+}$Calcd for $\mathrm{C}_{24} \mathrm{H}_{19} \mathrm{~F}_{5}$ 402.1407; Found 402.1404.

\section{(E)-1,2,4,5-Tetrafluoro-3-(8-(4-fluorophenyl)octa-1,6-dien-3-yl)-6-m ethylbenzene (4ba) and its regioisomer ( $p: m=94: 6)$ :}

The general procedure was followed using 2,3,4,5,6-pentafluorotoluene (180.7 $\mathrm{mg}, 0.992 \mathrm{mmol}$ ) and 4-fluorophenylmagnesium bromide (in THF, $0.776 \mathrm{M}, 1.55 \mathrm{~mL}, 1.2 \mathrm{mmol}$ ). Purification by GPC gave $\mathbf{4 b a}$ as a

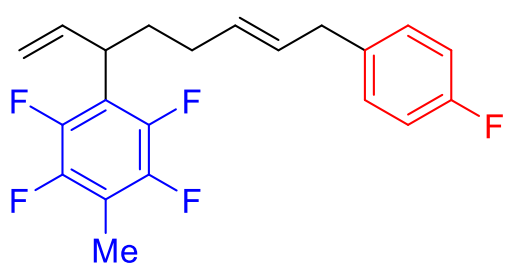
mixture of two regioisomers in $p: m=94: 6$ ratio (determined by ${ }^{19} \mathrm{~F}$ NMR ) as a colorless oil (294.1 mg, 81\%): ${ }^{1} \mathrm{H}$ NMR $\left(400 \mathrm{MHz}, \mathrm{CDCl}_{3}\right): \delta 7.13-7.09(\mathrm{~m}, 2 \mathrm{H}), 6.98-6.94(\mathrm{~m}, 2 \mathrm{H}), 6.08-5.99(\mathrm{~m}, 1 \mathrm{H})$, 5.56-5.49 (m, 1H), 5.47-5.40 (m, 1H), 5.09-5.05 (m, 2H), $3.76(\mathrm{q}, J=7.6 \mathrm{~Hz}, 1 \mathrm{H}), 3.28(\mathrm{~d}, J=6.4 \mathrm{~Hz}$, $2 \mathrm{H}), 2.23(\mathrm{~s}, 2.8 \mathrm{H}), 2.16(\mathrm{~s}, 0.22 \mathrm{H}), 2.08-1.84(\mathrm{~m}, 4 \mathrm{H}) ;{ }^{13} \mathrm{C}\left\{{ }^{1} \mathrm{H}\right\} \mathrm{NMR}\left(100 \mathrm{MHz}, \mathrm{CDCl}_{3}\right): \delta 161.3(\mathrm{~d}$, $\left.{ }^{1} J_{\mathrm{C}-\mathrm{F}}=242.2 \mathrm{~Hz}\right), 145.1\left(\mathrm{dm},{ }^{1} J_{\mathrm{C}-\mathrm{F}}=242.2 \mathrm{~Hz}\right), 144.4\left(\mathrm{dm},{ }^{1} J_{\mathrm{C}-\mathrm{F}}=231.7 \mathrm{~Hz}\right), 138.1,136.3\left(\mathrm{~d},{ }^{4} J_{\mathrm{C}-\mathrm{F}}=\right.$ $2.8 \mathrm{~Hz}), 130.6,129.9,129.8\left(\mathrm{~d},{ }^{3} J_{\mathrm{C}-\mathrm{F}}=7.6 \mathrm{~Hz}\right), 116.2,115.0\left(\mathrm{~d},{ }^{2} J_{\mathrm{C}-\mathrm{F}}=21.0 \mathrm{~Hz}\right), 39.7,38.1,32.9,30.4$, $7.4\left(\mathrm{t},{ }^{3} J_{\mathrm{C}-\mathrm{F}}=2.4 \mathrm{~Hz}\right) ;{ }^{19} \mathrm{~F} \mathrm{NMR}\left(376 \mathrm{MHz}, \mathrm{CDCl}_{3}\right): \delta-117.5-117.6(\mathrm{~m}, 1 \mathrm{~F}),-121.6(\mathrm{~d}, J=9.4 \mathrm{~Hz}$, $0.06 \mathrm{~F}),-139.5(\mathrm{~d}, J=20.7 \mathrm{~Hz}, 0.06 \mathrm{~F}),-139.8(\mathrm{~d}, J=21.4 \mathrm{~Hz}, 0.06 \mathrm{~F}),-144.3(\mathrm{~s}, 3.8 \mathrm{~F}),-166.1(\mathrm{td}, J=$ 21.4, 11.4 Hz, 0.06F); HRMS (DART) $m / z[\mathrm{M}+\mathrm{H}]^{+}$Calcd for $\mathrm{C}_{21} \mathrm{H}_{20} \mathrm{~F}_{5}$ 367.1485; Found 367.1486. 
(E)-1,2,4,5-Tetrafluoro-6-(trifluoromethyl)-3-(8-(4-fluorophenyl)oct a-1,6-dien-3-yl)benzene (4ca):

The general procedure was followed using octafluorotoluene $(235.8 \mathrm{mg}$, $0.999 \mathrm{mmol}$ ) and 4-fluorophenylmagnesium bromide (in THF, 0.776 M,

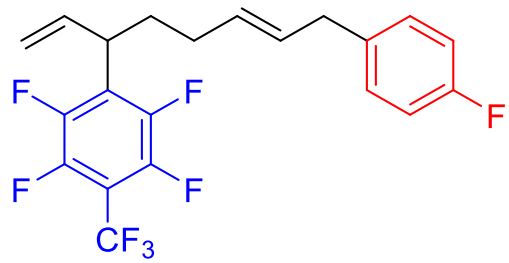
$1.55 \mathrm{~mL}, 1.2 \mathrm{mmol})$. Purification by GPC gave the desired product 4ca as a yellow oil (245.0 $\mathrm{mg}, 58 \%$, single isomer): ${ }^{1} \mathrm{H}$ NMR (400 $\left.\mathrm{MHz}, \mathrm{CDCl}_{3}\right): \delta$ 7.13-7.09 (m, 2H), 6.99-6.94 (m, 2H), 6.04-5.97 (m, $1 \mathrm{H}), 5.58-5.51(\mathrm{~m}, 1 \mathrm{H}), 5.46-5.39(\mathrm{~m}, 1 \mathrm{H}), 5.15-5.10(\mathrm{~m}, 2 \mathrm{H}), 3.83(\mathrm{q}, J=7.6 \mathrm{~Hz}, 1 \mathrm{H}), 3.28(\mathrm{~d}, J=6.4$ $\mathrm{Hz}, 2 \mathrm{H}), 2.05-1.85(\mathrm{~m}, 4 \mathrm{H}) ;{ }^{13} \mathrm{C}\left\{{ }^{1} \mathrm{H}\right\} \mathrm{NMR}\left(100 \mathrm{MHz}, \mathrm{CDCl}_{3}\right): \delta 161.4\left(\mathrm{~d},{ }^{1} J_{\mathrm{C}-\mathrm{F}}=242.1 \mathrm{~Hz}\right), 145.0$ $\left(\mathrm{dm},{ }^{1} J_{\mathrm{C}-\mathrm{F}}=240.3 \mathrm{~Hz}\right), 144.1\left(\mathrm{dm},{ }^{1} J_{\mathrm{C}-\mathrm{F}}=238.4 \mathrm{~Hz}\right), 136.5,136.1\left(\mathrm{~d},{ }^{4} J_{\mathrm{C}-\mathrm{F}}=3.8 \mathrm{~Hz}\right), 130.4,130.0$, $129.8\left(\mathrm{~d},{ }^{3} J_{\mathrm{C}-\mathrm{F}}=7.6 \mathrm{~Hz}\right), 127.1\left(\mathrm{t},{ }^{2} J_{\mathrm{C}-\mathrm{F}}=16.2 \mathrm{~Hz}\right), 117.6,115.0\left(\mathrm{~d},{ }^{2} J_{\mathrm{C}-\mathrm{F}}=21.0 \mathrm{~Hz}\right), 40.1,38.1,32.6(\mathrm{~d}$, ${ }^{3} J_{\mathrm{C}-\mathrm{F}}=1.9 \mathrm{~Hz}$ ), 30.3 (carbon signals from the $\mathrm{CF}_{3}$ and its ipso-carbon were not observed); ${ }^{19} \mathrm{~F}$ NMR $\left(376 \mathrm{MHz}, \mathrm{CDCl}_{3}\right): \delta-56.2(\mathrm{t}, J=21.4 \mathrm{~Hz}, 3 \mathrm{~F}),-117.4-117.5(\mathrm{~m}, 1 \mathrm{~F}),-140.2-140.3(\mathrm{~m}, 2 \mathrm{~F})$, -140.8-140.9 (m, 2F); HRMS (DART) $m / z$ [M+H] $]^{+}$Calcd for $\mathrm{C}_{21} \mathrm{H}_{17} \mathrm{~F}_{8}$ 421.1203; Found 420.1204.

\section{(E)-1,2,4,5-Tetrafluoro-3-(8-(4-fluorophenyl)octa-1,6-dien-3-yl)-6-m ethoxybenzene (4da) and its regioisomers $(p: m: o=61: 35: 4)$ :}

The general procedure was followed using 2,3,4,5,6-pentafluoroanisole (197.5 mg, $0.997 \mathrm{mmol}$ ) and 4-fluorophenylmagnesium bromide (in

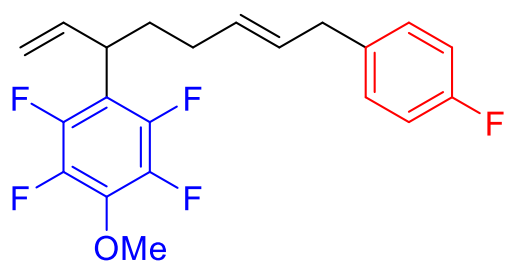
THF, $0.776 \mathrm{M}, 1.55 \mathrm{~mL}, 1.2 \mathrm{mmol})$. Purification by GPC gave 4da as a mixture of three regioisomers in $p: m: o=61: 35: 4$ ratio (determined by $\left.{ }^{19} \mathrm{~F} \mathrm{NMR}\right)$ as a colorless oil $(281.1 \mathrm{mg}, 74 \%):{ }^{1} \mathrm{H} \mathrm{NMR}(400 \mathrm{MHz}$, $\left.\mathrm{CDCl}_{3}\right): \delta 7.13-7.09(\mathrm{~m}, 2 \mathrm{H}), 7.00-6.94(\mathrm{~m}, 2 \mathrm{H}), 6.06-5.98(\mathrm{~m}, 1 \mathrm{H})$, 5.56-5.50 (m, 1H), 5.47-5.40 (m, 1H), 5.09-5.05 (m, 2H), 4.05-4.04 (m, 1.87H), 3.97-3.96 (m, 1.08H), 3.87-3.86 (m, 0.12H), $3.72(\mathrm{q}, J$ $=7.6 \mathrm{~Hz}, 1 \mathrm{H}), 3.28(\mathrm{~d}, J=6.4 \mathrm{~Hz}, 2 \mathrm{H}), 2.04-1.85(\mathrm{~m}, 4 \mathrm{H}) ;{ }^{13} \mathrm{C}\left\{{ }^{1} \mathrm{H}\right\} \mathrm{NMR}\left(100 \mathrm{MHz}, \mathrm{CDCl}_{3}\right): \delta 161.3$ $\left(\mathrm{d},{ }^{1} J_{\mathrm{C}-\mathrm{F}}=242.2 \mathrm{~Hz}\right), 145.0\left(\mathrm{dm},{ }^{1} J_{\mathrm{C}-\mathrm{F}}=243.1 \mathrm{~Hz}\right), 141.0\left(\mathrm{dm},{ }^{1} J_{\mathrm{C}-\mathrm{F}}=245.0 \mathrm{~Hz}\right), 138.0,136.3\left(\mathrm{~d},{ }^{4} J_{\mathrm{C}-\mathrm{F}}=\right.$ $2.9 \mathrm{~Hz}), 130.5,129.9,129.8\left(\mathrm{~d},{ }^{3} J_{\mathrm{C}-\mathrm{F}}=7.7 \mathrm{~Hz}\right), 116.2,115.0\left(\mathrm{~d},{ }^{2} J_{\mathrm{C}-\mathrm{F}}=20.9 \mathrm{~Hz}\right), 62.3\left(\mathrm{t},{ }^{3} J_{\mathrm{C}-\mathrm{F}}=2.9 \mathrm{~Hz}\right)$, $62.1\left(\mathrm{t},{ }^{3} J_{\mathrm{C}-\mathrm{F}}=3.4 \mathrm{~Hz}\right), 39.4,38.1,32.9,30.4\left(\mathrm{t},{ }^{3} J_{\mathrm{C}-\mathrm{F}}=1.9 \mathrm{~Hz}\right)$ (carbon signals from two ipso position in tetrafluoroanisyl ring were not observed); ${ }^{19} \mathrm{~F} \mathrm{NMR}\left(376 \mathrm{MHz}, \mathrm{CDCl}_{3}\right): \delta-117.5-117.6(\mathrm{~m}, 1 \mathrm{~F})$, $-136.5(\mathrm{~d}, J=5.6 \mathrm{~Hz}, 0.34 \mathrm{~F}),-142.2(\mathrm{~d}, J=22.6 \mathrm{~Hz}, 0.05 \mathrm{~F}),-143.4(\mathrm{~d}, J=22.6 \mathrm{~Hz}, 0.34 \mathrm{~F}),-143.8$ (dd, $J=21.6,8.1 \mathrm{~Hz}, 1.2 \mathrm{~F}),-152.7(\mathrm{~d}, J=20.7 \mathrm{~Hz}, 0.33 \mathrm{~F}),-156.2(\mathrm{dd}, J=20.1,8.1 \mathrm{~Hz}, 0.05 \mathrm{~F}),-158.2(\mathrm{dd}$, $J=21.8,7.9 \mathrm{~Hz}, 1.3 \mathrm{~F}),-158.9(\mathrm{t}, J=20.5 \mathrm{~Hz}, 0.05 \mathrm{~F}),-163.6(\mathrm{t}, J=21.6 \mathrm{~Hz}, 0.05 \mathrm{~F}),-163.9(\mathrm{td}, J=$ 21.6, 8.4 Hz, 0.32F); HRMS (DART) $m / z[\mathrm{M}-\mathrm{H}]^{+}$Calcd for $\mathrm{C}_{21} \mathrm{H}_{18} \mathrm{~F}_{5} \mathrm{O}$ 381.1278; Found 381.1280.

(E)-2,3,5,6-Tetrafluoro-4-(8-(4-fluorophenyl)octa-1,6-dien-3-yl)-1-m ethylthiobenzene (4ea):

The general procedure was followed using methyl(perfluorophenyl)sulfane $\quad(217.8 \mathrm{mg}, \quad 1.017 \mathrm{mmol})$ and

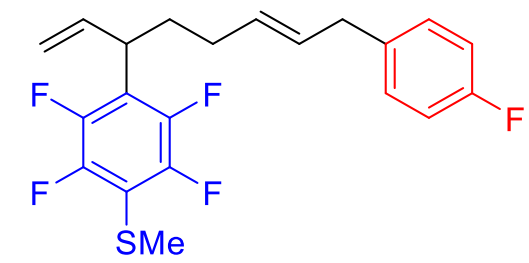


4-fluorophenylmagnesium bromide (in THF, $0.776 \mathrm{M}, 1.55 \mathrm{~mL}, 1.2 \mathrm{mmol}$ ). Purification by GPC gave the desired product 4ea as a yellow oil (305.7 $\mathrm{mg}, 75 \%$, single isomer); ${ }^{1} \mathrm{H} \mathrm{NMR}\left(400 \mathrm{MHz}, \mathrm{CDCl}_{3}\right)$ : 6 7.13-7.09 (m, 2H), 6.99-6.93 (m, 2H), 6.07-5.98 (m, 1H), 5.57-5.50 (m, 1H), 5.46-5.40 (m, 1H), 5.11-5.07 (m, 2H), $3.78(\mathrm{q}, J=7.6 \mathrm{~Hz}, 1 \mathrm{H}), 3.28(\mathrm{~d}, J=6.4 \mathrm{~Hz}, 2 \mathrm{H}), 2.49(\mathrm{~s}, 3 \mathrm{H}), 2.09-1.81(\mathrm{~m}, 4 \mathrm{H})$; ${ }^{13} \mathrm{C}\left\{{ }^{1} \mathrm{H}\right\}$ NMR $\left(100 \mathrm{MHz}, \mathrm{CDCl}_{3}\right): \delta 161.3\left(\mathrm{~d},{ }^{1} J_{\mathrm{C}-\mathrm{F}}=242.2 \mathrm{~Hz}\right), 146.6\left(\mathrm{dm},{ }^{1} J_{\mathrm{C}-\mathrm{F}}=244.1 \mathrm{~Hz}\right), 144.7$ $\left(\mathrm{dm},{ }^{1} J_{\mathrm{C}-\mathrm{F}}=243.2 \mathrm{~Hz}\right), 137.5,136.2\left(\mathrm{~d},{ }^{4} J_{\mathrm{C}-\mathrm{F}}=3.8 \mathrm{~Hz}\right), 130.4,130.0,129.7\left(\mathrm{~d},{ }^{3} J_{\mathrm{C}-\mathrm{F}}=7.6 \mathrm{~Hz}\right), 121.9(\mathrm{t}$, $\left.{ }^{2} J_{\mathrm{C}-\mathrm{F}}=17.2 \mathrm{~Hz}\right), 116.7,115.0\left(\mathrm{~d},{ }^{2} J_{\mathrm{C}-\mathrm{F}}=20.9 \mathrm{~Hz}\right), 113.6\left(\mathrm{t},{ }^{2} J_{\mathrm{C}-\mathrm{F}}=20.1 \mathrm{~Hz}\right), 39.9\left(\mathrm{~d},{ }^{4} J_{\mathrm{C}-\mathrm{F}}=1.9 \mathrm{~Hz}\right), 38.1$, $32.8\left(\mathrm{~d},{ }^{3} J_{\mathrm{C}-\mathrm{F}}=2.0 \mathrm{~Hz}\right), 30.4,17.7\left(\mathrm{t},{ }^{3} J_{\mathrm{C}-\mathrm{F}}=3.4 \mathrm{~Hz}\right) ;{ }^{19} \mathrm{~F} \mathrm{NMR}\left(376 \mathrm{MHz}, \mathrm{CDCl}_{3}\right): \delta-117.5-117.6(\mathrm{~m}$, $1 \mathrm{~F}),-135.4(\mathrm{dd}, J=22.9,11.7 \mathrm{~Hz}, 2 \mathrm{~F}),-142.2(\mathrm{dd}, J=22.9,11.7 \mathrm{~Hz}, 2 \mathrm{~F})$; HRMS (DART) $\mathrm{m} / z[\mathrm{M}]^{+}$ Calcd for $\mathrm{C}_{21} \mathrm{H}_{19} \mathrm{~F}_{5} \mathrm{~S} 398.1128$; Found 398.1134.

(E)-1-Chloro-2,3,5,6-tetrafluoro-4-(8-(4-fluorophenyl)octa-1,6-dien3-yl)benzene (4fa) and its regioisomers $(p: m: o=86: 6: 8)$ :

The general procedure was followed using chloropentafluorobenzene (203.8 $\mathrm{mg}, 1.006 \mathrm{mmol}$ ) and 4-fluorophenylmagnesium bromide (in THF, $0.776 \mathrm{M}, 1.55 \mathrm{~mL}, 1.2 \mathrm{mmol})$. Purification by GPC gave $\mathbf{4 f a}$ as a

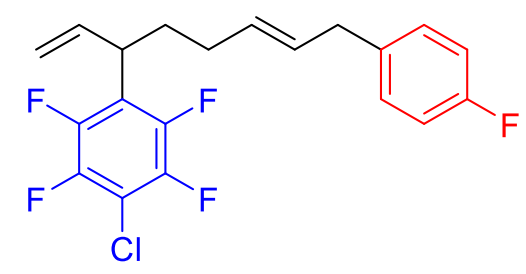
mixture of three regioisomers in $p: m: o=86: 6: 8$ ratio (determined by ${ }^{19} \mathrm{~F} \mathrm{NMR}$ ) as a colorless oil (279.5 $\mathrm{mg}, 72 \%$ ): major isomer: ${ }^{1} \mathrm{H} \mathrm{NMR}\left(400 \mathrm{MHz}, \mathrm{CDCl}_{3}\right)$ : $\delta$ 7.12-7.09 (m, 2H), 6.98-6.93 (m, 2H), 6.06-5.97 (m, 1H), 5.57-5.50 (m, 1H), 5.46-5.39 (m, 1H), 5.11-5.07 (m, 2H), $3.78(\mathrm{q}, J=8.0 \mathrm{~Hz}, 1 \mathrm{H})$, $3.28(\mathrm{~d}, J=6.4 \mathrm{~Hz}, 2 \mathrm{H}), 2.05-1.84(\mathrm{~m}, 4 \mathrm{H}) ;{ }^{13} \mathrm{C}\left\{{ }^{1} \mathrm{H}\right\} \mathrm{NMR}\left(100 \mathrm{MHz}, \mathrm{CDCl}_{3}\right): \delta 161.3\left(\mathrm{~d},{ }^{1} J_{\mathrm{C}-\mathrm{F}}=242.2\right.$ $\mathrm{Hz}), 144.9\left(\mathrm{dm},{ }^{1} J_{\mathrm{C}-\mathrm{F}}=247.0 \mathrm{~Hz}\right), 144.1\left(\mathrm{dm},{ }^{1} J_{\mathrm{C}-\mathrm{F}}=252.7 \mathrm{~Hz}\right), 137.3,136.2\left(\mathrm{~d},{ }^{4} J_{\mathrm{C}-\mathrm{F}}=2.9 \mathrm{~Hz}\right), 130.2$, $130.1,129.8\left(\mathrm{~d},{ }^{3} J_{\mathrm{C}-\mathrm{F}}=7.7 \mathrm{~Hz}\right), 121.1\left(\mathrm{t},{ }^{2} J_{\mathrm{C}-\mathrm{F}}=15.8 \mathrm{~Hz}\right), 116.9,115.1\left(\mathrm{~d},{ }^{2} J_{\mathrm{C}-\mathrm{F}}=20.9 \mathrm{~Hz}\right), 39.8,38.1$, $32.7\left(\mathrm{~d},{ }^{3} J_{\mathrm{C}-\mathrm{F}}=1.9 \mathrm{~Hz}\right.$ ), 30.4 (a carbon signal from the ipso carbon of the perfluoroaryl ring was not observed); ${ }^{19} \mathrm{~F}$ NMR $\left(376 \mathrm{MHz}, \mathrm{CDCl}_{3}\right): \delta-117.5-117.6(\mathrm{~m}, 1.17 \mathrm{~F}),-119.5(\mathrm{~d}, J=8.6 \mathrm{~Hz}, 0.07 \mathrm{~F})$, $-135.8(\mathrm{dd}, J=21.6,3.6 \mathrm{~Hz}, 0.07 \mathrm{~F}),-136.3(\mathrm{dd}, J=21.1,8.3 \mathrm{~Hz}, 0.10 \mathrm{~F}),-136.4(\mathrm{~d}, J=21.4 \mathrm{~Hz}, 0.07 \mathrm{~F}$ ), -139.1 (m, 0.10F), -141.2-141.3 (m, 2F), -141.4-141.5 (m, 2F), -156.5 (t, $J=20.9 \mathrm{~Hz}, 0.10 \mathrm{~F})$, -156.9 (t, $J=20.9 \mathrm{~Hz}, 0.10 \mathrm{~F}),-136.4(\mathrm{td}, J=21.6,9.4 \mathrm{~Hz}, 0.07 \mathrm{~F})$; HRMS (EI+) $\mathrm{m} / z[\mathrm{M}]^{+}$Calcd for $\mathrm{C}_{20} \mathrm{H}_{16} \mathrm{ClF}_{5}$ 386.0861; Found 386.0861.

\section{(E)-2,3,5,6-Tetrafluoro-4-(8-(4-fluorophenyl)octa-1,6-dien-3-yl)-1-tr} imetylsilylbenzene (4ga):

The general procedure was followed using trimethyl(perfluorophenyl)silane $(238.8 \quad \mathrm{mg}, \quad 0.994 \quad \mathrm{mmol}) \quad$ and 4-fluorophenylmagnesium bromide (in THF, $0.776 \mathrm{M}, 1.55 \mathrm{~mL}, 1.2$

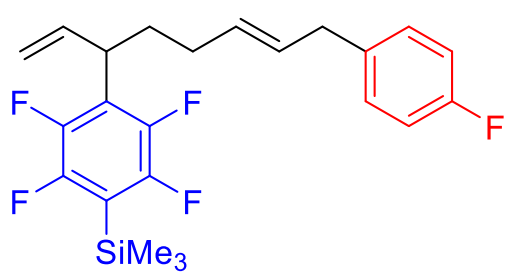
mmol). Purification by GPC gave the desired product 4ga as a colorless oil (308.5 mg, 73\%, single isomer); ${ }^{1} \mathrm{H}$ NMR (400 MHz, $\left.\mathrm{CDCl}_{3}\right): \delta 7.13-7.10(\mathrm{~m}, 2 \mathrm{H}), 7.00-6.95(\mathrm{~m}, 2 \mathrm{H}), 6.10-6.01(\mathrm{~m}, 1 \mathrm{H})$, 
5.58-5.51 (m, 1H), 5.48-5.41 (m, 1H), 5.12-5.07 (m, 2H), $3.78(\mathrm{q}, J=7.6 \mathrm{~Hz}, 1 \mathrm{H}), 3.28(\mathrm{~d}, J=6.4 \mathrm{~Hz}$, $2 \mathrm{H}), 2.09-1.84(\mathrm{~m}, 4 \mathrm{H}), 0.39(\mathrm{t}, J=1.4 \mathrm{~Hz}, 9 \mathrm{H}) ;{ }^{13} \mathrm{C}\left\{{ }^{1} \mathrm{H}\right\} \mathrm{NMR}\left(100 \mathrm{MHz}, \mathrm{CDCl}_{3}\right): \delta 161.4\left(\mathrm{~d},{ }^{1} J_{\mathrm{C}-\mathrm{F}}=\right.$ $242.2 \mathrm{~Hz}), 149.5\left(\mathrm{dm},{ }^{1} J_{\mathrm{C}-\mathrm{F}}=239.3 \mathrm{~Hz}\right), 145.0\left(\mathrm{dm},{ }^{1} J_{\mathrm{C}-\mathrm{F}}=251.7 \mathrm{~Hz}\right), 137.9,136.4\left(\mathrm{~d},{ }^{4} J_{\mathrm{C}-\mathrm{F}}=2.9 \mathrm{~Hz}\right)$, 130.6, 129.9, $129.8\left(\mathrm{~d},{ }^{3} J_{\mathrm{C}-\mathrm{F}}=7.6 \mathrm{~Hz}\right), 123.6\left(\mathrm{t},{ }^{2} J_{\mathrm{C}-\mathrm{F}}=16.2 \mathrm{~Hz}\right), 116.5,115.1\left(\mathrm{~d},{ }^{2} J_{\mathrm{C}-\mathrm{F}}=20.9 \mathrm{~Hz}\right), 40.1$, $38.2,32.9\left(\mathrm{~d},{ }^{3} J_{\mathrm{C}-\mathrm{F}}=1.9 \mathrm{~Hz}\right), 30.5,0.0\left(\mathrm{t},{ }^{3} J_{\mathrm{C}-\mathrm{F}}=2.9 \mathrm{~Hz}\right)$ (a carbon signal from the ipso carbon of the perfluoroaryl ring was not observed); $\left.{ }^{19} \mathrm{~F} \mathrm{NMR} \mathrm{(376} \mathrm{MHz,} \mathrm{CDCl}_{3}\right)$ : $\delta-117.5-117.6(\mathrm{~m}, 1 \mathrm{~F}),-128.7$ (dd, $J=23.5,14.1 \mathrm{~Hz}, 2 \mathrm{~F}),-143.0(\mathrm{dd}, J=23.5,14.9 \mathrm{~Hz}, 2 \mathrm{~F})$; HRMS (DART) $\mathrm{m} / z[\mathrm{M}-\mathrm{H}]^{+}$Calcd for $\mathrm{C}_{23} \mathrm{H}_{24} \mathrm{~F}_{5} \mathrm{Si} 423.1567$; Found 423.1577.

\section{(E)-2,3,5,6-tetrafluoro-4-(8-(4-fluorophenyl)octa-1,6-dien-3-yl)phen} yl-4-methylbenzenesulfonate (4ha) and its regioisomers $(p: m: o=$

96:2:2):

The general procedure was followed using perfluorophenyl 4-methylbenzenesulfonate $\quad(338.8 \quad \mathrm{mg}, \quad 1.002 \quad \mathrm{mmol}) \quad$ and

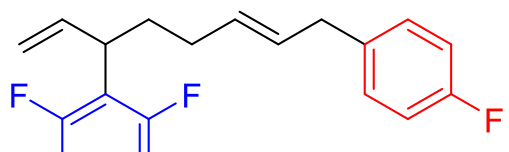
4-fluorophenylmagnesium bromide (in THF, $0.776 \mathrm{M}, 1.55 \mathrm{~mL}, 1.2 \mathrm{mmol}$ ). Purification by GPC gave 4ha as a mixture of three regioisomers in $p: m: o=96: 2: 2$ (determined by ${ }^{19} \mathrm{~F} \mathrm{NMR}$ ) as a colorless oil (273.3 mg, 52\%): ${ }^{1} \mathrm{H}$ NMR (400 MHz, $\left.\mathrm{CDCl}_{3}\right): \delta 7.86(\mathrm{~d}, J=8.4 \mathrm{~Hz}, 2 \mathrm{H}), 7.39(\mathrm{~d}, J=7.6 \mathrm{~Hz}$, 2H), 7.13-7.09 (m, 2H), 6.99-6.93 (m, 2H), 6.04-5.96 (m, 1H), 5.57-5.50 (m, 1H), 5.46-5.39 (m, 1H), 5.12-5.07 (m, 2H), $3.76(\mathrm{q}, J=7.6 \mathrm{~Hz}, 1 \mathrm{H}), 3.28(\mathrm{~d}, J=6.4 \mathrm{~Hz}, 2 \mathrm{H}), 2.49(\mathrm{~s}, 3 \mathrm{H}), 2.07-1.80(\mathrm{~m}, 4 \mathrm{H})$; ${ }^{13} \mathrm{C}\left\{{ }^{1} \mathrm{H}\right\}$ NMR $\left(100 \mathrm{MHz}, \mathrm{CDCl}_{3}\right): \delta 161.3\left(\mathrm{~d},{ }^{1} J_{\mathrm{C}-\mathrm{F}}=242.2 \mathrm{~Hz}\right), 146.4,144.7\left(\mathrm{dm},{ }^{1} J_{\mathrm{C}-\mathrm{F}}=257.4 \mathrm{~Hz}\right)$, $141.6\left(\mathrm{dm},{ }^{1} J_{\mathrm{C}-\mathrm{F}}=249.8 \mathrm{~Hz}\right), 137.1,136.2\left(\mathrm{~d},{ }^{4} J_{\mathrm{C}-\mathrm{F}}=2.9 \mathrm{~Hz}\right), 132.1,130.2,130.16,130.0,129.8\left(\mathrm{~d},{ }^{3} J_{\mathrm{C}-\mathrm{F}}\right.$ $=7.6 \mathrm{~Hz}), 128.5,121.1\left(\mathrm{t},{ }^{2} J_{\mathrm{C}-\mathrm{F}}=16.7 \mathrm{~Hz}\right), 117.1,115.0\left(\mathrm{~d},{ }^{2} J_{\mathrm{C}-\mathrm{F}}=20.9 \mathrm{~Hz}\right), 40.8,38.1,32.7,30.3,21.8$ (a carbon signal from the ipso carbon of the perfluoroaryl ring was not observed); ${ }^{19} \mathrm{~F} \mathrm{NMR}(376 \mathrm{MHz}$, $\left.\mathrm{CDCl}_{3}\right): \delta-117.5-117.6(\mathrm{~m}, 1 \mathrm{~F}),-130.3(\mathrm{~d}, J=8.6 \mathrm{~Hz}, 0.05 \mathrm{~F}),-135.9(\mathrm{~d}, J=22.2 \mathrm{~Hz}, 0.05 \mathrm{~F}),-139.2$ (dd, $J=21.4,8.3 \mathrm{~Hz}, 0.05 \mathrm{~F}),-141.6(\mathrm{dd}, J=21.8,7.9 \mathrm{~Hz}, 1.92 \mathrm{~F}),-146.0--146.1$ (m, 0.05F), -148.5 (dd, $J=20.9,8.1 \mathrm{~Hz}, 0.05 \mathrm{~F}),-151.6-151.7(\mathrm{~m}, 1.92 \mathrm{~F}),-156.8(\mathrm{t}, J=21.2 \mathrm{~Hz}, 0.05 \mathrm{~F}),-157.2(\mathrm{t}, J=21.4$ $\mathrm{Hz}, 0.05 \mathrm{~F}$ ), $-161.8\left(\mathrm{td}, J=21.8,8.9 \mathrm{~Hz}, 0.05 \mathrm{~F}\right.$ ); HRMS (DART) $\mathrm{m} / z[\mathrm{M}+\mathrm{H}]^{+}$Calcd for $\mathrm{C}_{27} \mathrm{H}_{24} \mathrm{~F}_{5} \mathrm{O}_{3} \mathrm{~S}$ 523.1366; Found 523.1378.

\section{(E)-2-(2,3,5,6-Tetrafluoro-4-(8-(4-fluorophenyl)octa-1,6-dien-3-yl)p} henyl)-1,3-dioxolane (4ia):

The general procedure was followed using 2-(perfluorophenyl)-1,3-dioxolane (240.4 $\mathrm{mg}, 1.001 \mathrm{mmol})$ and 4-fluorophenylmagnesium bromide (in THF, $0.776 \mathrm{M}, 1.55 \mathrm{~mL}, 1.2$ mmol). Purification by GPC gave the desired product 4 ia as a colorless

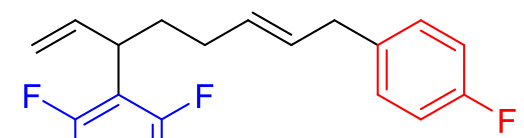
oil (343.6 mg, 81\%, single isomer); ${ }^{1} \mathrm{H}$ NMR (400 $\left.\mathrm{MHz}, \mathrm{CDCl}_{3}\right): \delta 7.12-7.08(\mathrm{~m}, 2 \mathrm{H}), 6.99-6.94(\mathrm{~m}$, 
$2 \mathrm{H}), 6.22(\mathrm{~s}, 1 \mathrm{H}), 6.06-5.97(\mathrm{~m}, 1 \mathrm{H}), 5.56-5.49(\mathrm{~m}, 1 \mathrm{H}), 5.45-5.38(\mathrm{~m}, 1 \mathrm{H}), 5.10-5.05(\mathrm{~m}, 2 \mathrm{H})$, 4.23-4.19 (m, 2H), 4.05-4.02 (m, 2H), $3.78(\mathrm{q}, J=8.0 \mathrm{~Hz}, 1 \mathrm{H}), 3.28(\mathrm{~d}, J=6.4 \mathrm{~Hz}, 2 \mathrm{H}), 2.02-1.86$ (m, $4 \mathrm{H}) ;{ }^{13} \mathrm{C}\left\{{ }^{1} \mathrm{H}\right\} \mathrm{NMR}\left(100 \mathrm{MHz}, \mathrm{CDCl}_{3}\right): \delta 161.3\left(\mathrm{~d},{ }^{1} J_{\mathrm{C}-\mathrm{F}}=242.2 \mathrm{~Hz}\right), 145.2\left(\mathrm{dm},{ }^{1} J_{\mathrm{C}-\mathrm{F}}=250.8 \mathrm{~Hz}\right)$, $144.6\left(\mathrm{dm},{ }^{1} J_{\mathrm{C}-\mathrm{F}}=244.1 \mathrm{~Hz}\right), 137.4,136.2\left(\mathrm{~d},{ }^{4} J_{\mathrm{C}-\mathrm{F}}=2.8 \mathrm{~Hz}\right), 130.4,130.0,129.8\left(\mathrm{~d},{ }^{3} J_{\mathrm{C}-\mathrm{F}}=7.7 \mathrm{~Hz}\right)$, 116.7, $115.0\left(\mathrm{~d},{ }^{2} J_{\mathrm{C}-\mathrm{F}}=21.9 \mathrm{~Hz}\right), 96.7(\mathrm{~m}), 66.0,39.8\left(\mathrm{~d},{ }^{4} J_{\mathrm{C}-\mathrm{F}}=1.9 \mathrm{~Hz}\right), 38.1,32.7\left(\mathrm{~d},{ }^{3} J_{\mathrm{C}-\mathrm{F}}=2.0 \mathrm{~Hz}\right)$, 30.3 (carbon signals from two ipso carbons of the perfluoroaryl ring were not observed); ${ }^{19} \mathrm{~F}$ NMR (376 $\left.\mathrm{MHz}, \mathrm{CDCl}_{3}\right): \delta-117.6-117.7(\mathrm{~m}, 1 \mathrm{~F}),-142.8(\mathrm{dd}, J=21.2,12.6 \mathrm{~Hz}, 2 \mathrm{~F}),-145.0(\mathrm{dd}, J=21.6,12.2$ $\mathrm{Hz}, 2 \mathrm{~F}$ ); HRMS (DART) $m / z$ [M] $]^{+}$Calcd for $\mathrm{C}_{23} \mathrm{H}_{21} \mathrm{~F}_{5} \mathrm{O}_{2} 424.1462$; Found 424.1466.

\section{(E)-1,2,4,5-tetrafluoro-3-(8-(4-fluorophenyl)octa-1,6-dien-3-yl)-6-(m} ethoxymethoxy)benzene $(4 \mathrm{ja})$ and its regioisomers $(p: m: o=$ 64:32:4):

The general procedure was followed using 1,2,3,4,5-pentafluoro-6-(methoxymethoxy) benzene (227.1 mg, 0.996 mmol) and 4-fluorophenylmagnesium bromide (in THF, 0.776 M, 1.55

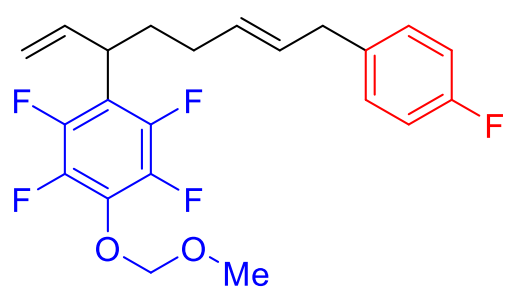
$\mathrm{mL}, 1.2 \mathrm{mmol}$ ). Purification by GPC gave $\mathbf{4 j a}$ as a mixture of three regioisomers in $p: m: o=64: 32: 4$ (determined by ${ }^{19} \mathrm{~F}$ NMR.) as a colorless oil (269.0 mg, 66\%): ${ }^{1} \mathrm{H}$ NMR (400 MHz, $\left.\mathrm{CDCl}_{3}\right): \delta 7.13-7.09$ $(\mathrm{m}, 2 \mathrm{H}), 6.99-6.94(\mathrm{~m}, 2 \mathrm{H}), 6.04-5.98(\mathrm{~m}, 1 \mathrm{H}), 5.55-5.49(\mathrm{~m}, 1 \mathrm{H}), 5.47-5.41(\mathrm{~m}, 1 \mathrm{H}), 5.17-5.03(\mathrm{~m}$, 4H), $3.73(\mathrm{q}, J=7.6 \mathrm{~Hz}, 1 \mathrm{H}), 3.60(\mathrm{~s}, 1.9 \mathrm{H}), 3.58(\mathrm{~s}, 1.0 \mathrm{H}), 3.56(\mathrm{~s}, 0.1 \mathrm{H}), 3.28(\mathrm{~d}, J=6.4 \mathrm{~Hz}, 2 \mathrm{H})$, 2.03-1.85 (m, 4H); ${ }^{13} \mathrm{C}\left\{{ }^{1} \mathrm{H}\right\} \mathrm{NMR}\left(100 \mathrm{MHz}, \mathrm{CDCl}_{3}\right): \delta 161.3\left(\mathrm{~d},{ }^{1} J_{\mathrm{C}-\mathrm{F}}=242.2 \mathrm{~Hz}\right), 145.0\left(\mathrm{dm},{ }^{1} J_{\mathrm{C}-\mathrm{F}}=\right.$ $243.2 \mathrm{~Hz}), 141.8\left(\mathrm{dm},{ }^{1} J_{\mathrm{C}-\mathrm{F}}=246.0 \mathrm{~Hz}\right), 138.0,137.9,136.3\left(\mathrm{~d},{ }^{4} J_{\mathrm{C}-\mathrm{F}}=2.9 \mathrm{~Hz}\right), 130.5\left(\mathrm{~d},{ }^{4} J_{\mathrm{C}-\mathrm{F}}=1.9 \mathrm{~Hz}\right)$, 129.9, $129.8\left(\mathrm{~d},{ }^{3} J_{\mathrm{C}-\mathrm{F}}=7.7 \mathrm{~Hz}\right), 116.4,116.3,115.0\left(\mathrm{~d},{ }^{2} J_{\mathrm{C}-\mathrm{F}}=21.0 \mathrm{~Hz}\right), 99.2\left(\mathrm{t},{ }^{4} J_{\mathrm{C}-\mathrm{F}}=1.9 \mathrm{~Hz}\right), 99.1(\mathrm{t}$, $\left.{ }^{3} J_{\mathrm{C}-\mathrm{F}}=3.4 \mathrm{~Hz}\right), 57.29,57.27,39.5,39.4,38.1,32.9\left(\mathrm{~d},{ }^{3} J_{\mathrm{C}-\mathrm{F}}=1.9 \mathrm{~Hz}\right), 30.41,30.40 ;{ }^{19} \mathrm{~F} \mathrm{NMR}(376 \mathrm{MHz}$, $\left.\mathrm{CDCl}_{3}\right): \delta-117.5-117.6(\mathrm{~m}, 1 \mathrm{~F}),-135.5(\mathrm{~d}, J=8.3 \mathrm{~Hz}, 0.30 \mathrm{~F}),-141.2(\mathrm{dd}, J=22.2,7.5 \mathrm{~Hz}, 0.04 \mathrm{~F})$, $-142.0(\mathrm{~d}, J=22.6 \mathrm{~Hz}, 0.30 \mathrm{~F}),-143.6(\mathrm{dd}, J=22.4,8.8 \mathrm{~Hz}, 1.25 \mathrm{~F}),-151.3(\mathrm{~d}, J=21.4 \mathrm{~Hz}, 0.29 \mathrm{~F})$, $-154.7(\mathrm{dd}, J=21.1,8.6 \mathrm{~Hz}, 0.04 \mathrm{~F}),-156.8(\mathrm{dd}, J=22.2,9.0 \mathrm{~Hz}, 1.18 \mathrm{~F}),-158.6$ (t, $J=20.9 \mathrm{~Hz}, 0.04 \mathrm{~F})$, $-162.6(\mathrm{t}, J=21.6 \mathrm{~Hz}, 0.04 \mathrm{~F}),-163.8(\mathrm{td}, J=22.1,8.8 \mathrm{~Hz}, 0.29 \mathrm{~F})$; HRMS (DART) $\mathrm{m} / z$ [M+H] ${ }^{+}$Calcd for $\mathrm{C}_{22} \mathrm{H}_{22} \mathrm{~F}_{5} \mathrm{O}_{2}$ 413.1540; Found 413.1552.

\section{(E)-N,N-Diethyl-2,3,5,6-tetrafluoro-4-(8-(4-fluorophenyl)octa-1,6-di} en-3-yl)benzamide (4ka) and its regioisomer ( $p: m$ or $o=96: 4)$ :

The general procedure was followed using $N, N$-diethyl-2,3,4,5,6-pentafluorobenzamide (269.6 mg, $1.009 \mathrm{mmol}$ ) and 4-fluorophenylmagnesium bromide (in THF, 0.776 M, $1.55 \mathrm{~mL}, 1.2$

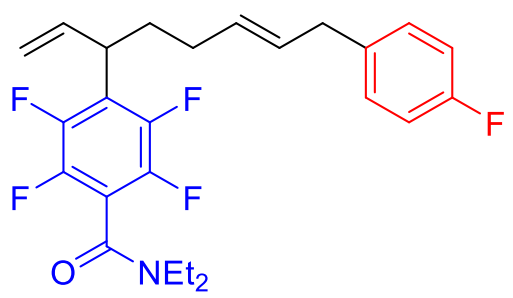
mmol). Purification by GPC gave $4 \mathbf{k a}$ as a mixture of two isomers in $p: m$ or $o=96: 4$ ratio (determined by GC, substitution pattern of the minor isomer could not be identified) as a colorless oil (222.2 mg, 
$49 \%$ ) along with a cross-coupling product $5 \mathbf{k a}$ as a colorless oil (47.2 $\mathrm{mg}, 14 \%$, single isomer): ${ }^{1} \mathrm{H}$ NMR (400 MHz, $\left.\mathrm{CDCl}_{3}\right): \delta$ 7.14-7.09 (m, 2H), 6.99-6.94 (m, 2H), 6.08-5.99 (m, 1H), 5.58-5.51 (m, 1H), $5.47-5.40(\mathrm{~m}, 1 \mathrm{H}), 5.13-5.09(\mathrm{~m}, 2 \mathrm{H}), 3.79(\mathrm{q}, J=7.6 \mathrm{~Hz}, 1 \mathrm{H}), 3.59(\mathrm{q}, J=7.2 \mathrm{~Hz}, 2 \mathrm{H}), 3.29(\mathrm{~d}, J=$ $6.4 \mathrm{~Hz}, 2 \mathrm{H}), 3.23$ (q, $J=7.2 \mathrm{~Hz}, 2 \mathrm{H}), 2.05-1.86(\mathrm{~m}, 4 \mathrm{H}), 1.26(\mathrm{t}, J=7.2 \mathrm{~Hz}, 3 \mathrm{H}), 1.13$ (t, $J=7.0 \mathrm{~Hz}$, $3 \mathrm{H}) ;{ }^{13} \mathrm{C}\left\{{ }^{1} \mathrm{H}\right\}$ NMR $\left(100 \mathrm{MHz}, \mathrm{CDCl}_{3}\right): \delta 161.3\left(\mathrm{~d},{ }^{1} J_{\mathrm{C}-\mathrm{F}}=242.2 \mathrm{~Hz}\right), 158.8,144.6\left(\mathrm{dm},{ }^{1} J_{\mathrm{C}-\mathrm{F}}=222.1\right.$ $\mathrm{Hz}), 142.3\left(\mathrm{dm},{ }^{1} J_{\mathrm{C}-\mathrm{F}}=230.7 \mathrm{~Hz}\right), 137.3,136.2\left(\mathrm{~d},{ }^{4} J_{\mathrm{C}-\mathrm{F}}=2.9 \mathrm{~Hz}\right), 130.3,130.1,129.8\left(\mathrm{~d},{ }^{3} J_{\mathrm{C}-\mathrm{F}}=7.6 \mathrm{~Hz}\right)$, $123.2\left(\mathrm{t},{ }^{2} J_{\mathrm{C}-\mathrm{F}}=16.7 \mathrm{~Hz}\right), 117.0,115.1\left(\mathrm{~d},{ }^{2} J_{\mathrm{C}-\mathrm{F}}=21.0 \mathrm{~Hz}\right), 114.2\left(\mathrm{t},{ }^{2} J_{\mathrm{C}-\mathrm{F}}=17.2 \mathrm{~Hz}\right), 43.2,39.9,39.6$, $38.1,32.8,30.3,14.0,12.7 ;{ }^{19} \mathrm{~F}$ NMR (376 MHz, $\mathrm{CDCl}_{3}$ ): $\delta-117.5-117.6(\mathrm{~m}, 1 \mathrm{~F}),-140.9(\mathrm{dd}, J=22.7$, $12.6 \mathrm{~Hz}, 1 \mathrm{~F}),-141.2(\mathrm{dd}, J=23.1,13.0 \mathrm{~Hz}, 1 \mathrm{~F}),-142.4-142.6(\mathrm{~m}, 2 \mathrm{~F})$; HRMS (DART) $\mathrm{m} / z . \mathrm{M}+\mathrm{H}]^{+}$ Calcd for $\mathrm{C}_{25} \mathrm{H}_{27} \mathrm{~F}_{5} \mathrm{NO}$ 452.2013; Found 452.2002.

\section{$N, N$-Diethyl-3,4,4',5,6-pentafluoro-[1,1'-biphenyl]-2-carboxamide (5ka):}

${ }^{1} \mathrm{H}$ NMR (400 MHz, $\left.\mathrm{CDCl}_{3}\right): \delta$ 7.41-7.38 (m, 2H), 7.15-7.09 (m, 2H), 3.72-3.63 (m, 1H), 3.07-2.97 (m, 2H), 2.85-2.76 (m, 1H), $0.92(\mathrm{t}, J=7.0 \mathrm{~Hz}, 3 \mathrm{H}), 0.84(\mathrm{t}, J$ $=7.2 \mathrm{~Hz}, 3 \mathrm{H}) ;{ }^{13} \mathrm{C}\left\{{ }^{1} \mathrm{H}\right\} \operatorname{NMR}\left(100 \mathrm{MHz}, \mathrm{CDCl}_{3}\right): \delta 163.1\left(\mathrm{~d},{ }^{1} J_{\mathrm{C}-\mathrm{F}}=247.9 \mathrm{~Hz}\right)$,

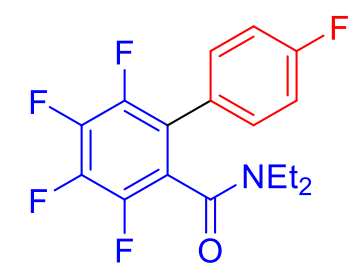
$131.7\left(\mathrm{~d},{ }^{3} J_{\mathrm{C}-\mathrm{F}}=7.6 \mathrm{~Hz}\right), 115.6\left(\mathrm{~d},{ }^{2} J_{\mathrm{C}-\mathrm{F}}=21.9 \mathrm{~Hz}\right), 42.6,38.8,13.6,11.9$ (carbon signals from the perfluoroaryl ring, carbonyl group, and ipso carbon of fluorophenyl group were not observed); ${ }^{19} \mathrm{~F}$ NMR (376 MHz, $\mathrm{CDCl}_{3}$ ): $\delta-111.6-111.7(\mathrm{~m}, 1 \mathrm{~F}),-140.2(\mathrm{ddd}, J=21.6,12.6,3.2 \mathrm{~Hz}, 1 \mathrm{~F}),-141.4$ (ddd, $J=$ $22.3,12.5,3.1 \mathrm{~Hz}, 1 \mathrm{~F}),-154.3(\mathrm{td}, J=20.7,3.4 \mathrm{~Hz}, 1 \mathrm{~F}),-154.6(\mathrm{td}, J=21.2,3.8 \mathrm{~Hz}, 1 \mathrm{~F})$; HRMS (DART) $m / z[\mathrm{M}+\mathrm{H}]^{+}$Calcd for $\mathrm{C}_{17} \mathrm{H}_{15} \mathrm{~F}_{5} \mathrm{NO} 344.1074$; Found 344.1073.

(E)-N,N-Diethyl-2,3,5,6-tetrafluoro-4-(8-(2,6-dimethylphenyl)octa-1,6dien-3-yl)benzamide (4kp):

The general procedure was followed using $N, N$-diethyl-2,3,4,5,6-pentafluorobenzamide $(267.4 \mathrm{mg}, 1.001 \mathrm{mmol})$ and (2,6-dimethylphenyl)magnesium bromide (in THF, $0.83 \mathrm{M}, 1.45 \mathrm{~mL}, 1.2$

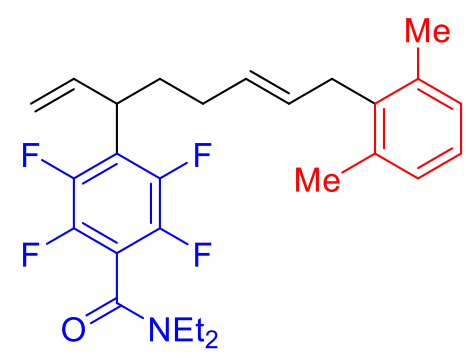
mmol). Purification by GPC gave the desired product $4 \mathbf{k p}$ as a colorless oil $(212.2 \mathrm{mg}$, 46\%, single isomer): ${ }^{1} \mathrm{H}$ NMR (400 MHz, $\left.\mathrm{CDCl}_{3}\right): \delta 7.02-7.01(\mathrm{~m}, 3 \mathrm{H}), 6.03-5.97(\mathrm{~m}, 1 \mathrm{H}), 5.52-5.45(\mathrm{~m}, 1 \mathrm{H})$, $5.26-5.21(\mathrm{~m}, 1 \mathrm{H}), 5.11-5.06(\mathrm{~m}, 2 \mathrm{H}), 3.74(\mathrm{q}, J=7.6 \mathrm{~Hz}, 1 \mathrm{H}), 3.59(\mathrm{q}, J=7.2 \mathrm{~Hz}, 2 \mathrm{H}), 3.34-3.32(\mathrm{~m}$, 2H), 3.23 (q, $J=7.2 \mathrm{~Hz}, 2 \mathrm{H}), 2.29$ (s, 6H), 2.02-1.73 (m, 4H), 1.26 (t, $J=7.2 \mathrm{~Hz}, 3 \mathrm{H}), 1.13$ (t, $J=7.2$ $\mathrm{Hz}, 3 \mathrm{H}) ;{ }^{13} \mathrm{C}\left\{{ }^{1} \mathrm{H}\right\}$ NMR $\left(100 \mathrm{MHz}, \mathrm{CDCl}_{3}\right): \delta 158.8,144.6\left(\mathrm{dm},{ }^{1} J_{\mathrm{C}-\mathrm{F}}=216.4 \mathrm{~Hz}\right), 142.3\left(\mathrm{dm},{ }^{1} J_{\mathrm{C}-\mathrm{F}}=\right.$ $222.1 \mathrm{~Hz}), 137.3,136.7,136.5,129.0,128.01,127.95,125.9,123.2\left(\mathrm{t},{ }^{2} J_{\mathrm{C}-\mathrm{F}}=16.7 \mathrm{~Hz}\right), 116.9,114.7(\mathrm{t}$, $\left.{ }^{2} J_{\mathrm{C}-\mathrm{F}}=22.0 \mathrm{~Hz}\right), 43.2,39.9\left(\mathrm{~d},{ }^{4} J_{\mathrm{C}-\mathrm{F}}=1.9 \mathrm{~Hz}\right), 39.6,32.8,32.4,30.4,19.8,14.0,12.7 ;{ }^{19} \mathrm{~F}$ NMR $(376$ $\left.\mathrm{MHz}, \mathrm{CDCl}_{3}\right): \delta-140.9(\mathrm{dd}, J=23.1,13.0 \mathrm{~Hz}, 1 \mathrm{~F}),-141.1(\mathrm{dd}, J=23.5,12.6 \mathrm{~Hz}, 1 \mathrm{~F}),-142.5-142.7$ (m, 2F); HRMS (DART) m/z [M+H] ${ }^{+}$Calcd for $\mathrm{C}_{27} \mathrm{H}_{32} \mathrm{~F}_{4} \mathrm{NO}$ 462.2420; Found 462.2394. 
(E)-2,3,5,6-tetrafluoro-4-(8-(4-fluorophenyl)octa-1,6-dien-3-yl)- $N, N$ dimethylaniline (4la) and its regioisomer $(p: m=90: 10)$ :

The general procedure was followed 2,3,4,5,6-pentafluoro- $N, N$-dimethylaniline $(212.5 \mathrm{mg}, 1.006 \mathrm{mmol})$ and

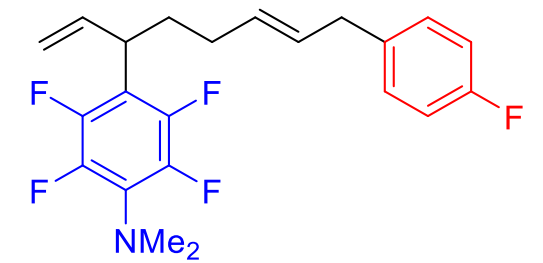
4-fluorophenylmagnesium bromide (in THF, $0.776 \mathrm{M}, 1.55 \mathrm{~mL}, 1.2 \mathrm{mmol}$ ). Purification by GPC gave 4la as a mixture of two isomers in $p: m=90: 10$ ratio (determined by ${ }^{19} \mathrm{~F}$ NMR.) as a colorless oil (224.3 $\mathrm{mg}, 56 \%):{ }^{1} \mathrm{H}$ NMR $\left(400 \mathrm{MHz}, \mathrm{CDCl}_{3}\right): \delta 7.13-7.09(\mathrm{~m}, 2 \mathrm{H}), 6.99-6.94(\mathrm{~m}, 2 \mathrm{H}), 6.07-5.98(\mathrm{~m}, 1 \mathrm{H})$, 5.55-5.49 (m, 1H), 5.47-5.42 (m, 1H), 5.08-5.04 (m, 2H), $3.70(\mathrm{q}, J=7.6 \mathrm{~Hz}, 1 \mathrm{H}), 3.28(\mathrm{~d}, J=6.4 \mathrm{~Hz}$, 2H), $2.92(\mathrm{t}, J=1.9 \mathrm{~Hz}, 5.3 \mathrm{H}), 2.85(\mathrm{t}, J=1.4 \mathrm{~Hz}, 0.7 \mathrm{H}), 2.02-1.84(\mathrm{~m}, 4 \mathrm{H}) ;{ }^{13} \mathrm{C}\left\{{ }^{1} \mathrm{H}\right\} \mathrm{NMR}(100 \mathrm{MHz}$, $\left.\mathrm{CDCl}_{3}\right): \delta 161.3\left(\mathrm{~d},{ }^{1} J_{\mathrm{C}-\mathrm{F}}=242.2 \mathrm{~Hz}\right), 145.2\left(\mathrm{dm},{ }^{1} J_{\mathrm{C}-\mathrm{F}}=231.7 \mathrm{~Hz}\right), 142.6\left(\mathrm{dm},{ }^{1} J_{\mathrm{C}-\mathrm{F}}=250.8 \mathrm{~Hz}\right), 138.4$, $136.3\left(\mathrm{~d},{ }^{4} J_{\mathrm{C}-\mathrm{F}}=2.9 \mathrm{~Hz}\right), 130.7,129.8,129.7\left(\mathrm{~d},{ }^{3} J_{\mathrm{C}-\mathrm{F}}=1.9 \mathrm{~Hz}\right), 115.9,115.1\left(\mathrm{~d},{ }^{2} J_{\mathrm{C}-\mathrm{F}}=20.9 \mathrm{~Hz}\right), 114.2$ $\left(\mathrm{t},{ }^{2} J_{\mathrm{C}-\mathrm{F}}=16.6 \mathrm{~Hz}\right), 43.7\left(\mathrm{t},{ }^{3} J_{\mathrm{C}-\mathrm{F}}=3.9 \mathrm{~Hz}\right), 43.4\left(\mathrm{t},{ }^{3} J_{\mathrm{C}-\mathrm{F}}=3.8 \mathrm{~Hz}\right), 39.4,38.1,33.0,30.4 ;{ }^{19} \mathrm{~F}$ NMR $(376$ $\left.\mathrm{MHz}, \mathrm{CDCl}_{3}\right): \delta-117.6-117.7(\mathrm{~m}, 1 \mathrm{~F}),-127.8(\mathrm{~d}, J=6.8 \mathrm{~Hz}, 0.10 \mathrm{~F}),-143.4(\mathrm{~d}, J=22.2 \mathrm{~Hz}, 0.10 \mathrm{~F})$, $-144.7(\mathrm{dd}, J=20.1,8.8 \mathrm{~Hz}, 1.77 \mathrm{~F}),-145.4(\mathrm{~d}, J=19.6 \mathrm{~Hz}, 0.09 \mathrm{~F}),-151.5(\mathrm{dd}, J=20.3,8.6 \mathrm{~Hz}, 1.76 \mathrm{~F})$, $-164.9(\mathrm{td}, J=21.4,8.9 \mathrm{~Hz}, 0.10 \mathrm{~F})$; HRMS (DART) $\mathrm{m} / z[\mathrm{M}]^{+}$Calcd for $\mathrm{C}_{22} \mathrm{H}_{22} \mathrm{~F}_{5} \mathrm{~N} 395.1672$; Found 395.1671 .

(E)-2,3,5,6-Tetrafluoro-4-(8-(4-fluorophenyl)octa-1,6-dien-3-yl)-1,1' -biphenyl (4ma):

The general procedure was followed using 2,3,4,5,6-pentafluoro-1,1'-biphenyl (244.9 $\mathrm{mg}, 1.003 \mathrm{mmol})$ and

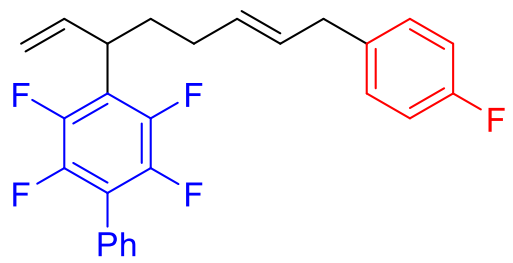
4-fluorophenylmagnesium bromide (in THF, $0.776 \mathrm{M}, 1.55 \mathrm{~mL}, 1.2 \mathrm{mmol}$ ). Purification by GPC gave the desired product $4 \mathrm{ma}$ as a colorless oil $\left(410.2 \mathrm{mg}\right.$, 95\%, single isomer); ${ }^{1} \mathrm{H} \mathrm{NMR}$ (400 $\left.\mathrm{MHz}, \mathrm{CDCl}_{3}\right)$ : א 7.50-7.41 (m, 5H), 7.14-7.10 (m, 2H), 6.99-6.94 (m, 2H), 6.13-6.05 (m, 1H), 5.60-5.53 (m, 1H), 5.50-5.43 (m, 1H), 5.16-5.11 (m, 2H), $3.84(\mathrm{q}, J=7.6 \mathrm{~Hz}, 1 \mathrm{H}), 3.29(\mathrm{~d}, J=6.4 \mathrm{~Hz}, 2 \mathrm{H}), 2.10-1.92(\mathrm{~m}$, $4 \mathrm{H}) ;{ }^{13} \mathrm{C}\left\{{ }^{1} \mathrm{H}\right\} \mathrm{NMR}\left(100 \mathrm{MHz}, \mathrm{CDCl}_{3}\right): \delta 161.4\left(\mathrm{~d},{ }^{1} J_{\mathrm{C}-\mathrm{F}}=242.1 \mathrm{~Hz}\right), 145.0\left(\mathrm{dm},{ }^{1} J_{\mathrm{C}-\mathrm{F}}=244.1 \mathrm{~Hz}\right)$, $143.8\left(\mathrm{dm},{ }^{1} J_{\mathrm{C}-\mathrm{F}}=235.5 \mathrm{~Hz}\right), 137.8,136.3\left(\mathrm{~d},{ }^{4} J_{\mathrm{C}-\mathrm{F}}=2.9 \mathrm{~Hz}\right), 130.5,130.1\left(\mathrm{t},{ }^{4} J_{\mathrm{C}-\mathrm{F}}=1.9 \mathrm{~Hz}\right), 130.0$, $129.8\left(\mathrm{~d},{ }^{3} J_{\mathrm{C}-\mathrm{F}}=7.7 \mathrm{~Hz}\right), 129.0,128.5,127.6,121.2\left(\mathrm{t},{ }^{2} J_{\mathrm{C}-\mathrm{F}}=16.7 \mathrm{~Hz}\right), 118.6\left(\mathrm{t},{ }^{3} J_{\mathrm{C}-\mathrm{F}}=16.7 \mathrm{~Hz}\right), 116.6$, $115.1\left(\mathrm{~d},{ }^{2} J_{\mathrm{C}-\mathrm{F}}=21.0 \mathrm{~Hz}\right), 39.9,38.1,32.9\left(\mathrm{t},{ }^{3} J_{\mathrm{C}-\mathrm{F}}=1.9 \mathrm{~Hz}\right), 30.5 ;{ }^{19} \mathrm{~F} \mathrm{NMR}\left(376 \mathrm{MHz}, \mathrm{CDCl}_{3}\right): \delta$ -117.5-117.6 (m, 1F), -143.0 (dd, $J=22.2,12.4 \mathrm{~Hz}, 2 \mathrm{~F}),-144.5$ (dd, $J=22.4,12.2 \mathrm{~Hz}, 2 \mathrm{~F})$; HRMS (DART) $\mathrm{m} / \mathrm{z}[\mathrm{M}]^{+}$Calcd for $\mathrm{C}_{26} \mathrm{H}_{21} \mathrm{~F}_{5}$ 428.1563; Found 428.1557.

(E)-2,2',3,3',4,5,5',6,6'-Nonafluoro-4' -(8-(4-fluorophenyl)octa-1,6-di en-3-yl)-1,1'-biphenyl (4na):

The general procedure was followed using decafluorobiphenyl (334.8 $\mathrm{mg}, 1.002 \mathrm{mmol}$ ) and 4-fluorophenylmagnesium bromide (in THF,

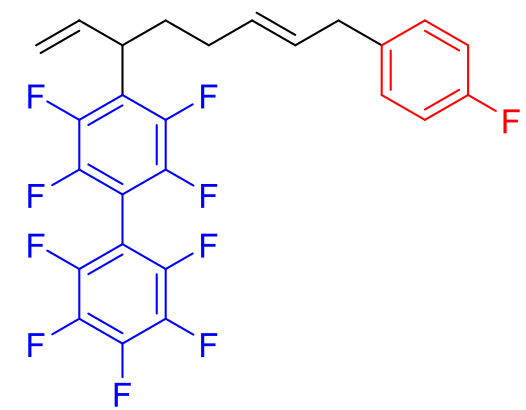


$0.776 \mathrm{M}, 1.55 \mathrm{~mL}, 1.2 \mathrm{mmol})$. Purification by GPC gave the desired product 4na as a colorless oil (244.9 mg, 47\%, single isomer); ${ }^{1} \mathrm{H} \mathrm{NMR}\left(400 \mathrm{MHz}, \mathrm{CDCl}_{3}\right): \delta 7.15-7.10(\mathrm{~m}, 2 \mathrm{H}), 7.00-6.94(\mathrm{~m}, 2 \mathrm{H})$, 6.12-6.03 (m, 1H), 5.61-5.54 (m, 1H), 5.49-5.42 (m, 1H), 5.18-5.14 (m, 2H), $3.87(\mathrm{q}, J=7.6 \mathrm{~Hz}, 1 \mathrm{H})$, $3.30(\mathrm{~d}, J=6.4 \mathrm{~Hz}, 2 \mathrm{H}), 2.10-1.92(\mathrm{~m}, 4 \mathrm{H}) ;{ }^{13} \mathrm{C}\left\{{ }^{1} \mathrm{H}\right\} \mathrm{NMR}\left(100 \mathrm{MHz}, \mathrm{CDCl}_{3}\right): \delta 161.4\left(\mathrm{~d},{ }^{1} J_{\mathrm{C}-\mathrm{F}}=242.2\right.$ $\mathrm{Hz}), 144.8\left(\mathrm{dm},{ }^{1} J_{\mathrm{C}-\mathrm{F}}=240.3 \mathrm{~Hz}\right), 144.6\left(\mathrm{dm},{ }^{1} J_{\mathrm{C}-\mathrm{F}}=252.7 \mathrm{~Hz}\right), 144.1\left(\mathrm{dm},{ }^{1} J_{\mathrm{C}-\mathrm{F}}=233.6 \mathrm{~Hz}\right), 142.3(\mathrm{dm}$, $\left.{ }^{1} J_{\mathrm{C}-\mathrm{F}}=215.5 \mathrm{~Hz}\right), 137.9\left(\mathrm{dm},{ }^{1} J_{\mathrm{C}-\mathrm{F}}=247.9 \mathrm{~Hz}\right), 137.0,136.2\left(\mathrm{~d},{ }^{4} J_{\mathrm{C}-\mathrm{F}}=3.8 \mathrm{~Hz}\right), 130.2(2 \mathrm{C}), 129.8(\mathrm{~d}$, $\left.{ }^{3} J_{\mathrm{C}-\mathrm{F}}=7.6 \mathrm{~Hz}\right), 124.9\left(\mathrm{t},{ }^{2} J_{\mathrm{C}-\mathrm{F}}=16.7 \mathrm{~Hz}\right), 117.3,115.1\left(\mathrm{~d},{ }^{2} J_{\mathrm{C}-\mathrm{F}}=21.0 \mathrm{~Hz}\right), 40.2\left(\mathrm{~d},{ }^{4} J_{\mathrm{C}-\mathrm{F}}=1.9 \mathrm{~Hz}\right), 38.1$, $32.8\left(\mathrm{~d},{ }^{3} J_{\mathrm{C}-\mathrm{F}}=1.9 \mathrm{~Hz}\right), 30.4$ (carbon signals from two ipso carbons of the perfluoroaryl ring were not observed); ${ }^{19} \mathrm{~F}$ NMR $\left(376 \mathrm{MHz}, \mathrm{CDCl}_{3}\right): \delta-117.5-117.6(\mathrm{~m}, 1 \mathrm{~F}),-137.3-137.4 \quad(\mathrm{~m}, 2 \mathrm{~F})$, -138.7-138.9 (m, 2F), -141.3 (dd, $J=21.6,11.5 \mathrm{~Hz}, 2 \mathrm{~F}),-150.5-150.6$ (m, 1F), -160.6--160.7 (m, $2 \mathrm{~F}$ ); HRMS (DART) $m / z[\mathrm{M}]^{+}$Calcd for $\mathrm{C}_{26} \mathrm{H}_{16} \mathrm{~F}_{10}$ 518.1092; Found 518.1099.

\section{(E)-2,3,5,6-Tetrafluoro-4-(8-(4-fluorophenyl)octa-1,6-dien-3-yl)-1-di} phenylphosphinobenzene (4oa):

The general procedure was followed using (perfluorophenyl)diphenylphosphane (352.2 $\mathrm{mg}, 1.000 \mathrm{mmol})$ and 4-fluorophenylmagnesium bromide (in THF, $0.776 \mathrm{M}, 1.55 \mathrm{~mL}, 1.2$

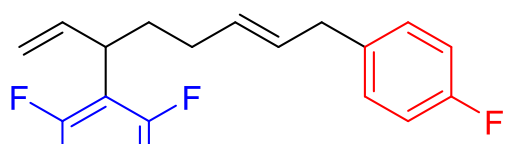
mmol). Purification by GPC gave the desired product $40 a$ as a yellow oil $(365.4 \mathrm{mg}$, 68\%, single isomer); ${ }^{1} \mathrm{H}$ NMR (400 MHz, $\left.\mathrm{CDCl}_{3}\right): \delta 7.45-7.41(\mathrm{~m}, 4 \mathrm{H}), 7.36-7.35(\mathrm{~m}, 6 \mathrm{H}), 7.12-7.08(\mathrm{~m}, 2 \mathrm{H})$, 6.97-6.93 (m, 2H), 6.08-5.99 (m, 1H), 5.57-5.50 (m, 1H), 5.46-5.39 (m, 1H), 5.13-5.09 (m, 2H), 3.79 $(\mathrm{q}, J=8.0 \mathrm{~Hz}, 1 \mathrm{H}), 3.26(\mathrm{~d}, J=6.4 \mathrm{~Hz}, 2 \mathrm{H}), 2.10-1.81(\mathrm{~m}, 4 \mathrm{H}) ;{ }^{13} \mathrm{C}\left\{{ }^{1} \mathrm{H}\right\} \mathrm{NMR}\left(100 \mathrm{MHz}, \mathrm{CDCl}_{3}\right)$ : $\delta 161.3\left(\mathrm{~d},{ }^{1} J_{\mathrm{C}-\mathrm{F}}=242.2 \mathrm{~Hz}\right), 148.0\left(\mathrm{dm},{ }^{1} J_{\mathrm{C}-\mathrm{F}}=242.2 \mathrm{~Hz}\right), 144.7\left(\mathrm{dm},{ }^{1} J_{\mathrm{C}-\mathrm{F}}=246.9 \mathrm{~Hz}\right), 137.4,136.2(\mathrm{~d}$, $\left.{ }^{4} J_{\mathrm{C}-\mathrm{F}}=3.8 \mathrm{~Hz}\right), 133.9\left(\mathrm{t},{ }^{3} J_{\mathrm{C}-\mathrm{F}}=1.9 \mathrm{~Hz}\right), 133.8\left(\mathrm{t},{ }^{3} J_{\mathrm{C}-\mathrm{F}}=1.9 \mathrm{~Hz}\right), 133.1,132.9,130.4,130.0,129.7(\mathrm{~d}$, $\left.{ }^{3} J_{\mathrm{C}-\mathrm{F}}=7.7 \mathrm{~Hz}\right), 129.2,124.8\left(\mathrm{t},{ }^{2} J_{\mathrm{C}-\mathrm{F}}=16.7 \mathrm{~Hz}\right), 117.0,115.1\left(\mathrm{~d},{ }^{2} J_{\mathrm{C}-\mathrm{F}}=21.0 \mathrm{~Hz}\right), 40.2,38.1,32.8,30.4$; ${ }^{19} \mathrm{~F}$ NMR $\left(376 \mathrm{MHz}, \mathrm{CDCl}_{3}\right): \delta-117.4-117.65(\mathrm{~m}, 1 \mathrm{~F}),-128.6-128.8(\mathrm{~m}, 2 \mathrm{~F}),-141.4(\mathrm{dd}, J=22.9$, $13.2 \mathrm{~Hz}, 2 \mathrm{~F}) ;{ }^{31} \mathrm{P}$ NMR $\left(162 \mathrm{MHz}, \mathrm{CDCl}_{3}\right): \delta-24.5(\mathrm{t}, J=37.8 \mathrm{~Hz}) ; \mathrm{HRMS}(\mathrm{DART}) \mathrm{m} / z[\mathrm{M}+\mathrm{H}]^{+} \mathrm{Calcd}$ for $\mathrm{C}_{32} \mathrm{H}_{27} \mathrm{~F}_{5} \mathrm{P}$ 537.1771; Found 537.1755.

\section{(E)-2-(2,3,5,6-Tetrafluoro-4-(8-(4-fluorophenyl)octa-1,6-dien-3-yl)p} henyl)pyridine (4pa):

The general procedure was followed using 2-(perfluorophenyl)pyridine (246.0 $\mathrm{mg}, 1.003 \mathrm{mmol}$ ) and 4-fluorophenylmagnesium bromide (in THF, $0.776 \mathrm{M}, 1.55 \mathrm{~mL}, 1.2 \mathrm{mmol}$ ). Purification by GPC gave the desired product 4 pa as a colorless oil (204.2 mg, 47\%, single isomer)

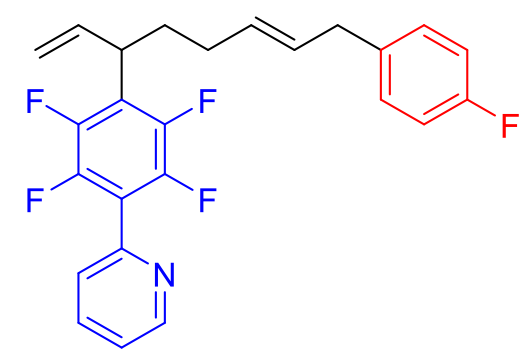
along with a cross-coupling product 2-(3,4,4',5,6-Pentafluoro-1,1'-biphenyl-2-yl)pyridine (5pa) as a byproduct (white powder, $81.8 \mathrm{mg}, 25 \%$, single isomer); ${ }^{1} \mathrm{H} \mathrm{NMR}\left(400 \mathrm{MHz}, \mathrm{CDCl}_{3}\right): \delta 8.78-8.76(\mathrm{~m}$, 
1H), 7.85-7.80 (m, 1H), 7.50-7.48 (m, 1H), 7.38-7.35 (m, 1H), 7.14-7.09 (m, 2H), 6.99-6.94 (m, 2H), 6.12-6.03 (m, 1H), 5.59-5.52 (m, 1H), 5.49-5.42 (m, 1H), 5.15-5.11 (m, 2H), $3.85(\mathrm{q}, J=8.0 \mathrm{~Hz}, 1 \mathrm{H})$, $3.29(\mathrm{~d}, J=6.4 \mathrm{~Hz}, 2 \mathrm{H}), 2.10-1.93(\mathrm{~m}, 4 \mathrm{H}) ;{ }^{13} \mathrm{C}\left\{{ }^{1} \mathrm{H}\right\} \mathrm{NMR}\left(100 \mathrm{MHz}, \mathrm{CDCl}_{3}\right): \delta 161.3\left(\mathrm{~d},{ }^{1} J_{\mathrm{C}-\mathrm{F}}=242.2\right.$ $\mathrm{Hz}), 150.0,144.9\left(\mathrm{dm},{ }^{1} J_{\mathrm{C}-\mathrm{F}}=244.2 \mathrm{~Hz}\right), 144.2\left(\mathrm{dm},{ }^{1} J_{\mathrm{C}-\mathrm{F}}=242.2 \mathrm{~Hz}\right), 147.9,137.6,136.6,136.3(\mathrm{~d}$, $\left.{ }^{4} J_{\mathrm{C}-\mathrm{F}}=3.9 \mathrm{~Hz}\right), 130.5,130.0,129.8\left(\mathrm{~d},{ }^{3} J_{\mathrm{C}-\mathrm{F}}=7.7 \mathrm{~Hz}\right), 125.9,123.5,122.5\left(\mathrm{t},{ }^{2} J_{\mathrm{C}-\mathrm{F}}=16.7 \mathrm{~Hz}\right), 118.0(\mathrm{t}$, $\left.{ }^{3} J_{\mathrm{C}-\mathrm{F}}=15.7 \mathrm{~Hz}\right), 116.7,115.1\left(\mathrm{~d},{ }^{2} J_{\mathrm{C}-\mathrm{F}}=20.9 \mathrm{~Hz}\right), 39.9,38.1,32.9\left(\mathrm{t},{ }^{3} J_{\mathrm{C}-\mathrm{F}}=1.9 \mathrm{~Hz}\right), 30.4 ;{ }^{19} \mathrm{~F}$ NMR $\left(376 \mathrm{MHz}, \mathrm{CDCl}_{3}\right): \delta-117.5-117.6(\mathrm{~m}, 1 \mathrm{~F}),-142.5(\mathrm{dd}, J=22.2,12.8 \mathrm{~Hz}, 2 \mathrm{~F}),-144.5(\mathrm{dd}, J=22.0$, $11.8 \mathrm{~Hz}, 2 \mathrm{~F}$ ); HRMS (DART) $\mathrm{m} / z$ [M+H] ${ }^{+}$Calcd for $\mathrm{C}_{25} \mathrm{H}_{21} \mathrm{~F}_{5} \mathrm{~N}$ 430.1594; Found 430.1597.

\section{2-(3,4,4',5,6-Pentafluoro-1,1'-biphenyl-2-yl)pyridine (5pa):}

m.p. $=80-85{ }^{\circ} \mathrm{C} ;{ }^{1} \mathrm{H}$ NMR $\left(400 \mathrm{MHz}, \mathrm{CDCl}_{3}\right): \delta 8.57-8.55(\mathrm{~m}, 1 \mathrm{H}), 7.59-7.55(\mathrm{~m}$, 1H), 7.20-7.17 (m, 1H), 7.07-7.01 (m, 3H), 6.95-6.90 (m, 2H); ${ }^{13} \mathrm{C}\left\{{ }^{1} \mathrm{H}\right\} \mathrm{NMR}$ $\left(100 \mathrm{MHz}, \mathrm{CDCl}_{3}\right): \delta 162.3\left(\mathrm{~d},{ }^{1} J_{\mathrm{C}-\mathrm{F}}=247.0 \mathrm{~Hz}\right), 149.6,145.4\left(\mathrm{dm},{ }^{1} J_{\mathrm{C}-\mathrm{F}}=251.4\right.$

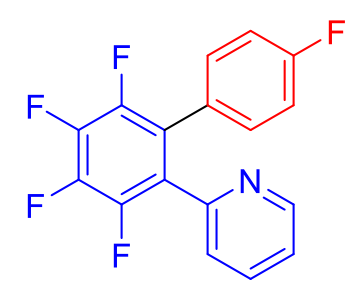
$\mathrm{Hz}), 144.9\left(\mathrm{dm},{ }^{1} J_{\mathrm{C}-\mathrm{F}}=244.2 \mathrm{~Hz}\right), 140.7\left(\mathrm{dm},{ }^{1} J_{\mathrm{C}-\mathrm{F}}=270.6 \mathrm{~Hz}\right), 140.0\left(\mathrm{dm},{ }^{1} J_{\mathrm{C}-\mathrm{F}}=259.0 \mathrm{~Hz}\right), 139.6$, 136.2, $132.2\left(\mathrm{~d},{ }^{3} J_{\mathrm{C}-\mathrm{F}}=3.9 \mathrm{~Hz}\right), 126.1,122.8,115.2\left(\mathrm{~d},{ }^{2} J_{\mathrm{C}-\mathrm{F}}=22.0 \mathrm{~Hz}\right)$ (carbon signals from the ipso carbons were not observed); ${ }^{19} \mathrm{~F}$ NMR $\left(376 \mathrm{MHz} \mathrm{CDCl}_{3}\right): \delta-113.0-113.1(\mathrm{~m}, 1 \mathrm{~F}),-141.0-141.1(\mathrm{~m}$, $1 \mathrm{~F}),-141.2-141.3(\mathrm{~m}, 1 \mathrm{~F}),-155.1-155.2(\mathrm{~m}, 1 \mathrm{~F}),-156.1-156.2(\mathrm{~m}, 1 \mathrm{~F}) ;$ HRMS (DART) $\mathrm{m} / z$ $[\mathrm{M}+\mathrm{H}]^{+}$Calcd for $\mathrm{C}_{17} \mathrm{H}_{9} \mathrm{~F}_{5} \mathrm{~N} 322.0655$; Found 322.0653 .

\section{(E)-2-(2,3,5,6-Tetrafluoro-4-(8-(4-fluorophenyl)octa-1,6-dien-3-yl)p henyl)-5-methyl-thiophene (4qa):}

The general procedure was followed using 5-methyl-2-(perfluorophenyl)thiophene $(264.1 \mathrm{mg}, 1.000 \mathrm{mmol})$ and 4-fluorophenylmagnesium bromide (in THF, $0.776 \mathrm{M}, 1.55 \mathrm{~mL}, 1.2$ mmol). Purification by GPC gave the desired product $\mathbf{4 q a}$ as a colorless

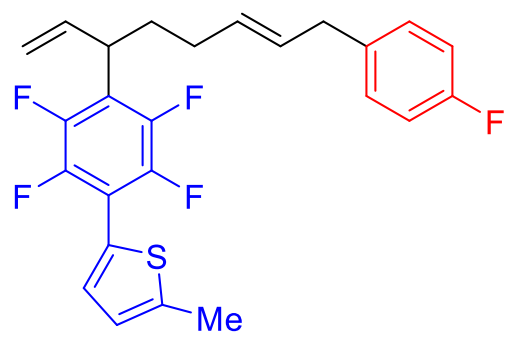
oil (431.5 mg, 96\%) with 98\% selectivity (substitution pattern of a minor isomer could not be identified); major isomer: ${ }^{1} \mathrm{H}$ NMR (400 MHz, $\left.\mathrm{CDCl}_{3}\right): \delta 7.38(\mathrm{~d}, J=3.6 \mathrm{~Hz}, 1 \mathrm{H}), 7.13-7.09(\mathrm{~m}, 2 \mathrm{H})$, 6.98-6.94 (m, 2H), 6.83-6.82 (m, 1H), 6.10-6.01 (m, 1H), 5.58-5.51 (m, 1H), 5.48-5.41 (m, 1H), 5.13-5.09 (m, 2H), $3.80(\mathrm{q}, J=8.0 \mathrm{~Hz}, 1 \mathrm{H}), 3.28(\mathrm{~d}, J=6.4 \mathrm{~Hz}, 2 \mathrm{H}), 2.54(\mathrm{~s}, 3 \mathrm{H}), 2.11-1.88(\mathrm{~m}, 4 \mathrm{H})$; ${ }^{13} \mathrm{C}\left\{{ }^{1} \mathrm{H}\right\}$ NMR $\left(100 \mathrm{MHz}, \mathrm{CDCl}_{3}\right): \delta 161.3\left(\mathrm{~d},{ }^{1} J_{\mathrm{C}-\mathrm{F}}=242.2 \mathrm{~Hz}\right), 145.1\left(\mathrm{dm},{ }^{1} J_{\mathrm{C}-\mathrm{F}}=247.9 \mathrm{~Hz}\right), 143.5$ $\left(\mathrm{dm},{ }^{1} J_{\mathrm{C}-\mathrm{F}}=247.9 \mathrm{~Hz}\right), 142.8\left(\mathrm{t},{ }^{3} J_{\mathrm{C}-\mathrm{F}}=3.8 \mathrm{~Hz}\right), 137.8,136.2\left(\mathrm{~d},{ }^{4} J_{\mathrm{C}-\mathrm{F}}=3.8 \mathrm{~Hz}\right), 130.5,130.1\left(\mathrm{t},{ }^{4} J_{\mathrm{C}-\mathrm{F}}=\right.$ $6.2 \mathrm{~Hz}), 130.0,129.8\left(\mathrm{~d},{ }^{3} J_{\mathrm{C}-\mathrm{F}}=8.6 \mathrm{~Hz}\right), 125.6,125.4\left(\mathrm{t},{ }^{2} J_{\mathrm{C}-\mathrm{F}}=13.4 \mathrm{~Hz}\right), 119.8\left(\mathrm{t},{ }^{2} J_{\mathrm{C}-\mathrm{F}}=17.2 \mathrm{~Hz}\right)$, $116.5,115.1\left(\mathrm{~d},{ }^{2} J_{\mathrm{C}-\mathrm{F}}=21.0 \mathrm{~Hz}\right), 112.7\left(\mathrm{t},{ }^{4} J_{\mathrm{C}-\mathrm{F}}=13.9 \mathrm{~Hz}\right), 40.8,38.1,32.9\left(\mathrm{~d},{ }^{3} J_{\mathrm{C}-\mathrm{F}}=2.0 \mathrm{~Hz}\right), 30.4$, 15.1; ${ }^{19} \mathrm{~F}$ NMR (376 MHz, $\left.\mathrm{CDCl}_{3}\right): \delta-117.5-117.6(\mathrm{~m}, 1 \mathrm{~F}),-141.3(\mathrm{dd}, J=21.4,11.3 \mathrm{~Hz}, 2 \mathrm{~F}),-143.3$ $\left(\mathrm{dd}, J=21.2,11.1 \mathrm{~Hz}, 2 \mathrm{~F}\right.$ ); HRMS (DART) $\mathrm{m} / z$ [M] ${ }^{+}$Calcd for $\mathrm{C}_{25} \mathrm{H}_{21} \mathrm{~F}_{5} \mathrm{~S}$ 448.1284; Found 448.1284. 
(E)-2,3,5,6-Tetrafluoro-4-(8-(4-fluorophenyl)octa-1,6-dien-3-yl)pyri dine (4ra):

The general procedure was followed using pentafluoropyridine (171.3 $\mathrm{mg}, 1.013 \mathrm{mmol}$ ) and 4-fluorophenylmagnesium bromide (in THF,

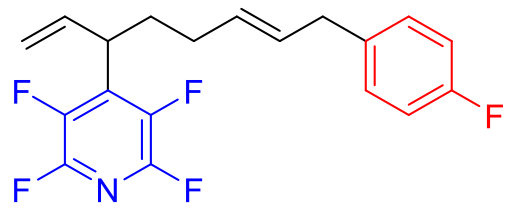
$0.776 \mathrm{M}, 1.55 \mathrm{~mL}, 1.2 \mathrm{mmol})$. Purification by GPC gave the desired product 4ra as a colorless oil (162.5 mg, 45\%, single isomer): ${ }^{1} \mathrm{H}$ NMR (400 MHz, $\left.\mathrm{CDCl}_{3}\right): \delta 7.12-7.08(\mathrm{~m}, 2 \mathrm{H}), 7.00-6.95(\mathrm{~m}, 2 \mathrm{H})$, 6.03-5.96 (m, 1H), 5.59-5.51 (m, 1H), 5.45-5.38 (m, 1H), 5.18-5.13 (m, 2H), $3.85(\mathrm{q}, J=7.6 \mathrm{~Hz}, 1 \mathrm{H})$, $3.28(\mathrm{~d}, J=6.8 \mathrm{~Hz}, 2 \mathrm{H}), 2.12-1.83(\mathrm{~m}, 4 \mathrm{H}) ;{ }^{13} \mathrm{C}\left\{{ }^{1} \mathrm{H}\right\} \mathrm{NMR}\left(100 \mathrm{MHz}, \mathrm{CDCl}_{3}\right): \delta 161.4\left(\mathrm{~d},{ }^{1} J_{\mathrm{C}-\mathrm{F}}=242.1\right.$ $\mathrm{Hz}), 143.6\left(\mathrm{dm},{ }^{1} J_{\mathrm{C}-\mathrm{F}}=245.0 \mathrm{~Hz}\right), 140.1\left(\mathrm{dm},{ }^{1} J_{\mathrm{C}-\mathrm{F}}=230.8 \mathrm{~Hz}\right), 136.6\left(\mathrm{t},{ }^{2} J_{\mathrm{C}-\mathrm{F}}=14.8 \mathrm{~Hz}\right), 136.1\left(\mathrm{~d},{ }^{4} J_{\mathrm{C}-\mathrm{F}}\right.$ $=3.8 \mathrm{~Hz}), 135.8,130.6,129.79,129.78\left(\mathrm{~d},{ }^{3} J_{\mathrm{C}-\mathrm{F}}=8.6 \mathrm{~Hz}\right), 118.2,115.1\left(\mathrm{~d},{ }^{2} J_{\mathrm{C}-\mathrm{F}}=21.0 \mathrm{~Hz}\right), 40.6,38.1$, $32.3\left(\mathrm{~d},{ }^{3} J_{\mathrm{C}-\mathrm{F}}=1.9 \mathrm{~Hz}\right), 30.2 ;{ }^{19} \mathrm{~F}$ NMR $\left(376 \mathrm{MHz}, \mathrm{CDCl}_{3}\right): \delta-91.2-91.3(\mathrm{~m}, 2 \mathrm{~F}),-117.4-117.5(\mathrm{~m}$, 1F), -143.5-143.6 (m, 2F); HRMS (DART) $\mathrm{m} / \mathrm{z}[\mathrm{M}+\mathrm{H}]^{+}$Calcd for $\mathrm{C}_{19} \mathrm{H}_{17} \mathrm{~F}_{5} \mathrm{~N} 354.1281$; Found 354.1273.

(E)-1,2,3,4,5,6,8-Heptafluoro-7-(8-(4-fluorophenyl)octa-1,6-dien-3-yl)naphthalene (4sa) and its regioisomer (7-isomer:8-isomer $=94: 6)$ :

The general procedure was followed using perfluoronaphthalene (271.3 mg, $0.997 \mathrm{mmol}$ ) and 4-fluorophenylmagnesium bromide (in THF, $0.776 \mathrm{M}, 1.55 \mathrm{~mL}, 1.2 \mathrm{mmol})$. Purification by GPC gave $4 \mathrm{sa}$ as a mixture of two regioisomers in 7-isomer:8-isomer $=94: 6$ (determined by GC) as a yellow oil (205.0 mg, 45\%): major isomer: ${ }^{1} \mathrm{H}$ NMR (400 MHz, $\left.\mathrm{CDCl}_{3}\right): \delta 7.12-7.08(\mathrm{~m}, 2 \mathrm{H}), 6.98-6.92(\mathrm{~m}, 2 \mathrm{H})$, 6.15-6.06 (m, 1H), 5.57-5.50 (m, 1H), 5.48-5.41 (m, 1H), 5.15-5.12 (m, 2H), 3.96 (q, $J=7.2 \mathrm{~Hz}, 1 \mathrm{H}), 3.28$ (d, $J=6.0 \mathrm{~Hz}, 2 \mathrm{H}), 2.09-1.92$ $(\mathrm{m}, 4 \mathrm{H}) ;{ }^{13} \mathrm{C}\left\{{ }^{1} \mathrm{H}\right\} \mathrm{NMR}\left(100 \mathrm{MHz}, \mathrm{CDCl}_{3}\right): \delta 161.3\left(\mathrm{~d},{ }^{1} J_{\mathrm{C}-\mathrm{F}}=243.1\right.$ $\mathrm{Hz}), 149.9\left(\mathrm{dm},{ }^{1} J_{\mathrm{C}-\mathrm{F}}=257.5 \mathrm{~Hz}\right), 146.8\left(\mathrm{dm},{ }^{1} J_{\mathrm{C}-\mathrm{F}}=247.9 \mathrm{~Hz}\right), 141.3$

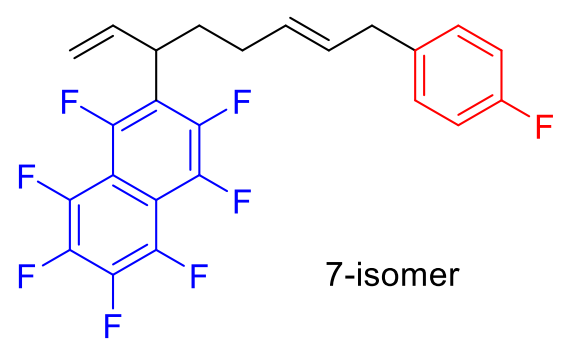
$\left(\mathrm{dm},{ }^{1} J_{\mathrm{C}-\mathrm{F}}=252.6 \mathrm{~Hz}\right), 141.0\left(\mathrm{dm},{ }^{1} J_{\mathrm{C}-\mathrm{F}}=247.0 \mathrm{~Hz}\right), 140.7\left(\mathrm{dm},{ }^{1} J_{\mathrm{C}-\mathrm{F}}\right.$

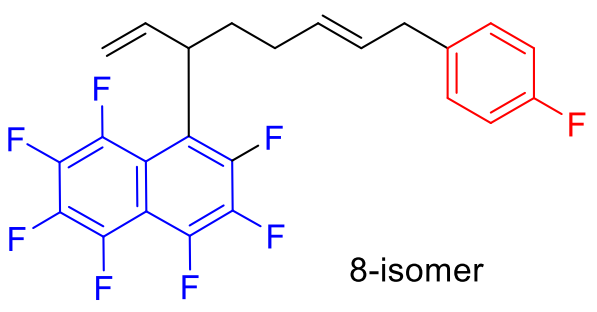
$=253.7 \mathrm{~Hz}), 139.3\left(\mathrm{dm},{ }^{1} J_{\mathrm{C}-\mathrm{F}}=236.4 \mathrm{~Hz}\right), 138.6\left(\mathrm{dm},{ }^{1} J_{\mathrm{C}-\mathrm{F}}=255.6 \mathrm{~Hz}\right), 137.5,136.2\left(\mathrm{~d},{ }^{4} J_{\mathrm{C}-\mathrm{F}}=2.9 \mathrm{~Hz}\right)$, 130.3, 130.2, $129.8\left(\mathrm{~d},{ }^{3} J_{\mathrm{C}-\mathrm{F}}=7.7 \mathrm{~Hz}\right), 117.1,115.0\left(\mathrm{~d},{ }^{2} J_{\mathrm{C}-\mathrm{F}}=21.0 \mathrm{~Hz}\right), 40.9,38.1,32.8\left(\mathrm{~d},{ }^{3} J_{\mathrm{C}-\mathrm{F}}=1.9\right.$ $\mathrm{Hz}$ ), 30.5 (carbon signals from the ipso, $4 \mathrm{a}$, and $8 \mathrm{a}$ carbons of the perfluoronaphthyl ring were not observed); ${ }^{19} \mathrm{~F}$ NMR $\left(376 \mathrm{MHz}, \mathrm{CDCl}_{3}\right): \delta-117.5-117.6(\mathrm{~m}, 1 \mathrm{~F}),-120.5(\mathrm{dd}, J=70.3,17.7 \mathrm{~Hz}, 1 \mathrm{~F})$, $-136.4(\mathrm{~d}, J=15.4 \mathrm{~Hz}, 1 \mathrm{~F}),-144.3-144.5$ (m, 1F), -146.3-146.6 (m, 1F), -148.8-149.1 (m, 1F), $-154.3(\mathrm{t}, J=18.8 \mathrm{~Hz}, 1 \mathrm{~F}),-156.0\left(\mathrm{t}, J=16.5 \mathrm{~Hz}, 1 \mathrm{~F}\right.$ ); HRMS (DART) $m / z[\mathrm{M}]^{+}$Calcd for $\mathrm{C}_{24} \mathrm{H}_{16} \mathrm{~F}_{8}$ 456.1124; Found 456.1124. 
(E)-1,2,3-Trifluoro-4-(8-(4-fluorophen yl)octa-1,6-dien-3-yl)dibenzo[b,e $][1,4] \mathrm{d}$ ioxine (4ta) (67:33):

The general procedure was followed using

1,2,3,4-tetrafluorodibenzo[b,e][1,4]dioxi ne $\quad(256.0 \mathrm{mg}, \quad 0.999 \mathrm{mmol})$ and

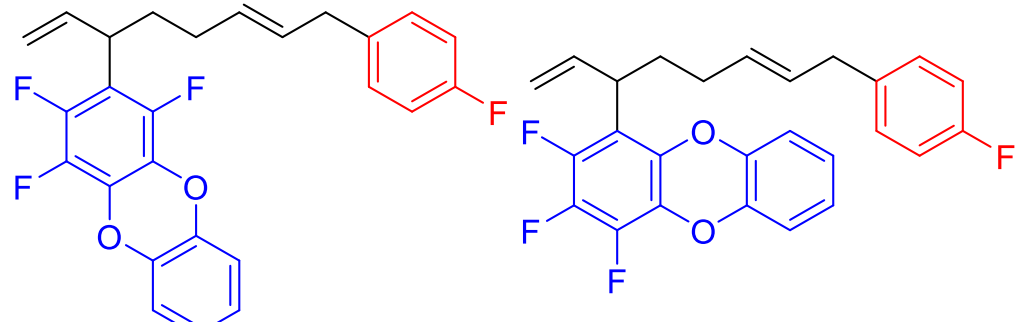

3-isomer

4-isomer

4-fluorophenylmagnesium bromide (in THF, 0.776 M, $1.55 \mathrm{~mL}, 1.2 \mathrm{mmol}$ ). Purification by GPC gave 4ta as a mixture of two regioisomers in 3-isomer:4-isomer $=67: 33$ (determined by ${ }^{19} \mathrm{~F} \mathrm{NMR}$ ) as a colorless oil $(301.9 \mathrm{mg}, 68 \%):{ }^{1} \mathrm{H}$ NMR $\left(400 \mathrm{MHz}, \mathrm{CDCl}_{3}\right): \delta 7.12-7.08(\mathrm{~m}, 2 \mathrm{H}), 6.97-6.93(\mathrm{~m}, 5.7 \mathrm{H})$, 6.84-6.81 (m, 0.3H), 6.06-6.00 (m, 1H), 5.53-5.42 (m, 2H), 511-5.05 (m, 2H), $3.80(\mathrm{q}, J=8.0 \mathrm{~Hz}$, $0.34 \mathrm{H}), 3.69(\mathrm{q}, J=8.0 \mathrm{~Hz}, 0.67 \mathrm{H}), 3.28(\mathrm{~d}, J=6.4 \mathrm{~Hz}, 2 \mathrm{H}), 2.05-1.81(\mathrm{~m}, 4 \mathrm{H}) ;{ }^{13} \mathrm{C}\left\{{ }^{1} \mathrm{H}\right\} \mathrm{NMR}(100$ $\left.\mathrm{MHz} \mathrm{CDCl}_{3}\right): \delta 161.3\left(\mathrm{~d},{ }^{1} J_{\mathrm{C}-\mathrm{F}}=242.2 \mathrm{~Hz}\right), 145.0\left(\mathrm{dm},{ }^{1} J_{\mathrm{C}-\mathrm{F}}=247.8 \mathrm{~Hz}\right), 144.6\left(\mathrm{dm},{ }^{1} J_{\mathrm{C}-\mathrm{F}}=240.2 \mathrm{~Hz}\right)$, $143.9\left(\mathrm{dm},{ }^{1} J_{\mathrm{C}-\mathrm{F}}=247.9 \mathrm{~Hz}\right), 138.8\left(\mathrm{dm},{ }^{1} J_{\mathrm{C}-\mathrm{F}}=238.2 \mathrm{~Hz}\right), 140.9,140.6\left(\mathrm{~d},{ }^{4} J_{\mathrm{C}-\mathrm{F}}=2.8 \mathrm{~Hz}\right), 140.2,138.4$ $\left(\mathrm{d},{ }^{4} J_{\mathrm{C}-\mathrm{F}}=1.9 \mathrm{~Hz}\right), 138.2,136.3\left(\mathrm{~d},{ }^{3} J_{\mathrm{C}-\mathrm{F}}=4.8 \mathrm{~Hz}\right), 131.2\left(\mathrm{dm},{ }^{1} J_{\mathrm{C}-\mathrm{F}}=268.6 \mathrm{~Hz}\right), 130.8,130.6,129.8$, $129.75,129.69,128.4\left(\mathrm{dm},{ }^{1} J_{\mathrm{C}-\mathrm{F}}=232.8 \mathrm{~Hz}\right), 125.0,124.8,124.71,124.66,116.83,116.80,116.6,116.5$, 116.0, 115.9, $115.0\left(\mathrm{~d},{ }^{2} J_{\mathrm{C}-\mathrm{F}}=20.9 \mathrm{~Hz}\right), 39.2\left(\mathrm{~d},{ }^{3} J_{\mathrm{C}-\mathrm{F}}=1.9 \mathrm{~Hz}\right), 39.1,38.2,38.1,32.9\left(\mathrm{~d},{ }^{3} J_{\mathrm{C}-\mathrm{F}}=1.9 \mathrm{~Hz}\right)$, $32.7\left(\mathrm{~d},{ }^{3} J_{\mathrm{C}-\mathrm{F}}=2.0 \mathrm{~Hz}\right), 30.4 ;{ }^{19} \mathrm{~F}$ NMR $\left(376 \mathrm{MHz}, \mathrm{CDCl}_{3}\right): \delta-117.5-117.6(\mathrm{~m}, 1 \mathrm{~F}),-142.2(\mathrm{~d}, J=9.4$ $\mathrm{Hz}, 0.64 \mathrm{~F}),-144.6(\mathrm{~d}, J=22.2 \mathrm{~Hz}, 0.31 \mathrm{~F}),-145.2(\mathrm{~d}, J=21.8 \mathrm{~Hz}, 0.64 \mathrm{~F}),-157.9(\mathrm{~d}, J=21.1 \mathrm{~Hz}$, $0.31 \mathrm{~F}),-163.3(\mathrm{dd}, J=22.2,9.8 \mathrm{~Hz}, 0.64 \mathrm{~F}),-165.7(\mathrm{t}, J=21.6 \mathrm{~Hz}, 0.31 \mathrm{~F})$; HRMS (DART) $\mathrm{m} / z$ [M] $^{+}$ Calcd for $\mathrm{C}_{26} \mathrm{H}_{20} \mathrm{~F}_{4} \mathrm{O}_{2} 440.1399$; Found 440.1410.

\section{(E)-1,2,4,5-Tetrafluoro-3-(8-(4-fluorophenyl)octa-1,6-dien-3-yl)benz ene (4ua) and its regioisomers $(p: m+o=87: 13)$ :}

The general procedure was followed using pentafluorobenzene (167.4 $\mathrm{mg}, 0.996 \mathrm{mmol}$ ) and 4-fluorophenylmagnesium bromide (in THF,

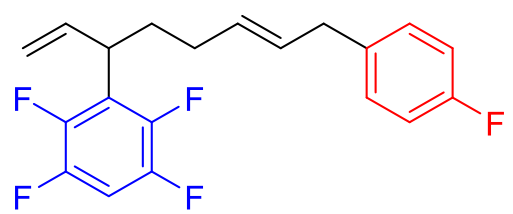
$0.776 \mathrm{M}, 1.55 \mathrm{~mL}, 1.2 \mathrm{mmol}$ ). Purification by GPC gave 4ua as a mixture of three regioisomers in $p: m+o=87: 13$ ratio (determined by GC and ${ }^{19} \mathrm{~F} \mathrm{NMR}$, the ratio of $m$ - and $o$ - isomers could not be determined) as a colorless oil $(265.5 \mathrm{mg}, 76 \%)$ along with a hydroarylation product (E)-1-fluoro-4-(octa-2,7-dien-1-yl)benzene (6a, colorless oil, $43.7 \mathrm{mg}, 21 \%$ ): major isomer: ${ }^{1} \mathrm{H}$ NMR (400 MHz, $\left.\mathrm{CDCl}_{3}\right): \delta 7.13-7.09(\mathrm{~m}, 2 \mathrm{H}), 6.98-6.87(\mathrm{~m}, 3 \mathrm{H}), 6.09-6.00(\mathrm{~m}, 1 \mathrm{H}), 5.57-5.50(\mathrm{~m}, 1 \mathrm{H})$, 5.47-5.40 (m, 1H), 5.10-5.07 (m, 2H), $3.80(\mathrm{q}, J=7.6 \mathrm{~Hz}, 1 \mathrm{H}), 3.28(\mathrm{~d}, J=5.6 \mathrm{~Hz}, 2 \mathrm{H}), 2.07-1.84(\mathrm{~m}$, $4 \mathrm{H}) ;{ }^{13} \mathrm{C}\left\{{ }^{1} \mathrm{H}\right\} \mathrm{NMR}\left(100 \mathrm{MHz}, \mathrm{CDCl}_{3}\right): \delta 161.3\left(\mathrm{~d},{ }^{1} J_{\mathrm{C}-\mathrm{F}}=242.2 \mathrm{~Hz}\right), 146.0\left(\mathrm{dm},{ }^{1} J_{\mathrm{C}-\mathrm{F}}=246.0 \mathrm{~Hz}\right)$, $144.6\left(\mathrm{dm},{ }^{1} J_{\mathrm{C}-\mathrm{F}}=241.2 \mathrm{~Hz}\right), 137.6,136.3\left(\mathrm{~d},{ }^{4} J_{\mathrm{C}-\mathrm{F}}=2.8 \mathrm{~Hz}\right), 130.4,130.0,129.8\left(\mathrm{~d},{ }^{3} J_{\mathrm{C}-\mathrm{F}}=7.6 \mathrm{~Hz}\right)$, $123.0\left(\mathrm{t},{ }^{2} J_{\mathrm{C}-\mathrm{F}}=16.2 \mathrm{~Hz}\right), 116.6,115.0\left(\mathrm{~d},{ }^{2} J_{\mathrm{C}-\mathrm{F}}=20.9 \mathrm{~Hz}\right), 103.8\left(\mathrm{t},{ }^{2} J_{\mathrm{C}-\mathrm{F}}=22.4 \mathrm{~Hz}\right), 39.9,38.1,32.8(\mathrm{~d}$, $\left.{ }^{3} J_{\mathrm{C}-\mathrm{F}}=1.9 \mathrm{~Hz}\right), 30.4 ;{ }^{19} \mathrm{~F}$ NMR $\left(376 \mathrm{MHz}, \mathrm{CDCl}_{3}\right): \delta-117.5-117.6(\mathrm{~m}, 1 \mathrm{~F}),-139.3-139.4(\mathrm{~m}, 2 \mathrm{~F})$, 
-142.6--142.8 (m, 2F); HRMS (EI+) $m / z[\mathrm{M}]^{+}$Calcd for $\mathrm{C}_{20} \mathrm{H}_{17} \mathrm{~F}_{5}$ 352.1250; Found 352.1246.

\section{(E)-1-Fluoro-4-(octa-2,7-dien-1-yl)benzene (6a):}

${ }^{1} \mathrm{H}$ NMR (400 MHz, $\left.\mathrm{CDCl}_{3}\right): \delta$ 7.14-7.10 (m, 2H), 6.98-6.94 (m, 2H),

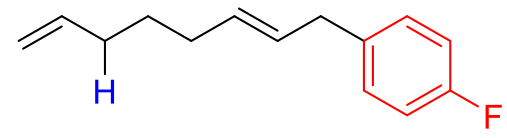
5.85-5.75 (m, 1H), 5.58-5.45 (m, 2H), 5.02-4.93 (m, 2H), $3.29(\mathrm{~d}, J=6.4 \mathrm{~Hz}, 2 \mathrm{H}), 2.08-2.02(\mathrm{~m}, 4 \mathrm{H})$, $1.51-1.43(\mathrm{~m}, 2 \mathrm{H}) ;{ }^{13} \mathrm{C}\left\{{ }^{1} \mathrm{H}\right\} \mathrm{NMR}\left(100 \mathrm{MHz}, \mathrm{CDCl}_{3}\right): \delta 161.3\left(\mathrm{~d},{ }^{1} J_{\mathrm{C}-\mathrm{F}}=242.2 \mathrm{~Hz}\right), 138.7,136.3(\mathrm{~d}$, $\left.{ }^{4} J_{\mathrm{C}-\mathrm{F}}=2.8 \mathrm{~Hz}\right), 131.8,129.8\left(\mathrm{~d},{ }^{3} J_{\mathrm{C}-\mathrm{F}}=7.7 \mathrm{~Hz}\right), 129.0,115.0\left(\mathrm{~d},{ }^{2} J_{\mathrm{C}-\mathrm{F}}=21.0 \mathrm{~Hz}\right), 114.5,38.2,33.2,32.9$, 28.6; ${ }^{19} \mathrm{~F}$ NMR (376 MHz, $\mathrm{CDCl}_{3}$ ): $\delta-117.6-117.7(\mathrm{~m}, 1 \mathrm{~F}) ;$ HRMS (DART) $\mathrm{m} / z[\mathrm{M}+\mathrm{H}]^{+}$Calcd for $\mathrm{C}_{14} \mathrm{H}_{18} \mathrm{~F}$ 205.1393; Found 205.1373. 


\section{References}

S1 Iwasaki, T.; Min, X.; Fukuoka, A.; Kuniyasu, H.; Kambe, N. Angew. Chem. Int. Ed. 2016, 55, 5550.

S2 Burla, M. C.; Caliandro, R.; Camalli, M.; Carrozzini, B.; Cascarano, G. L.; De Caro, L.; Giacovazzo, C.; Polidori, G.; Siliqi, D.; Spagna, R. J. Appl. Cryst. 2007, 40, 609.

S3 Sheldrick, G. M. Acta Cryst. A 2008, A64, 112.

S4 Farrugia, L. J. J. Appl. Cryst. 2012, 45, 849.

S5 Pangborn, A. M.; Giardello, M. A.; Grubbs, R. H.; Rosen, R. K.; Timmers, F. J. Organometallics 1996, $15,1518$.

S6 Ward, W. E.; Sicree, S.; Chen. B.; Tamborski, C. J. Fluorine. Chem. 1995, 73, 73.

S7 Bardin. V. V.; Pressman. L. S.; Rogoza. L. N.; Furin. G. G. J. Fluorine. Chem. 1991, 53, 213.

S8 Smith, C. A.; Cross, L. E.; Hughes, K.; Davis, R. E.; Judd, D. B.; Merritt, A. T. Tetrahedron Lett. 2009, 50, 4906.

S9 Marzi, E.; Gorecka, J.; Schlosser, M. Synthesis 2004, 10, 1609.

S10 Yu, D.; Lu, L.; Shen, Q. Org. Lett. 2013, 15, 940.

S11 Roberts. K. M.; Jones, J. P. Chem. Eur. J. 2010, 16, 8096.

S12 Senaweera, S. M.; Singh, A.; Weaver, J. D. J. Am. Chem. Soc. 2014, 136, 3002. 


\section{${ }^{1} \mathrm{H}$ NMR of $\mathbf{4 a a}$}

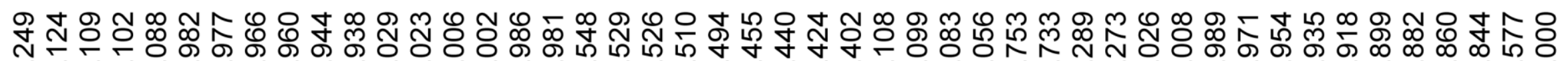

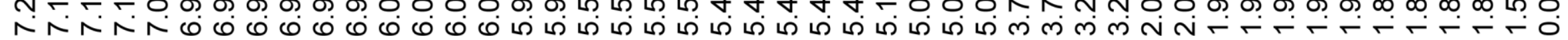
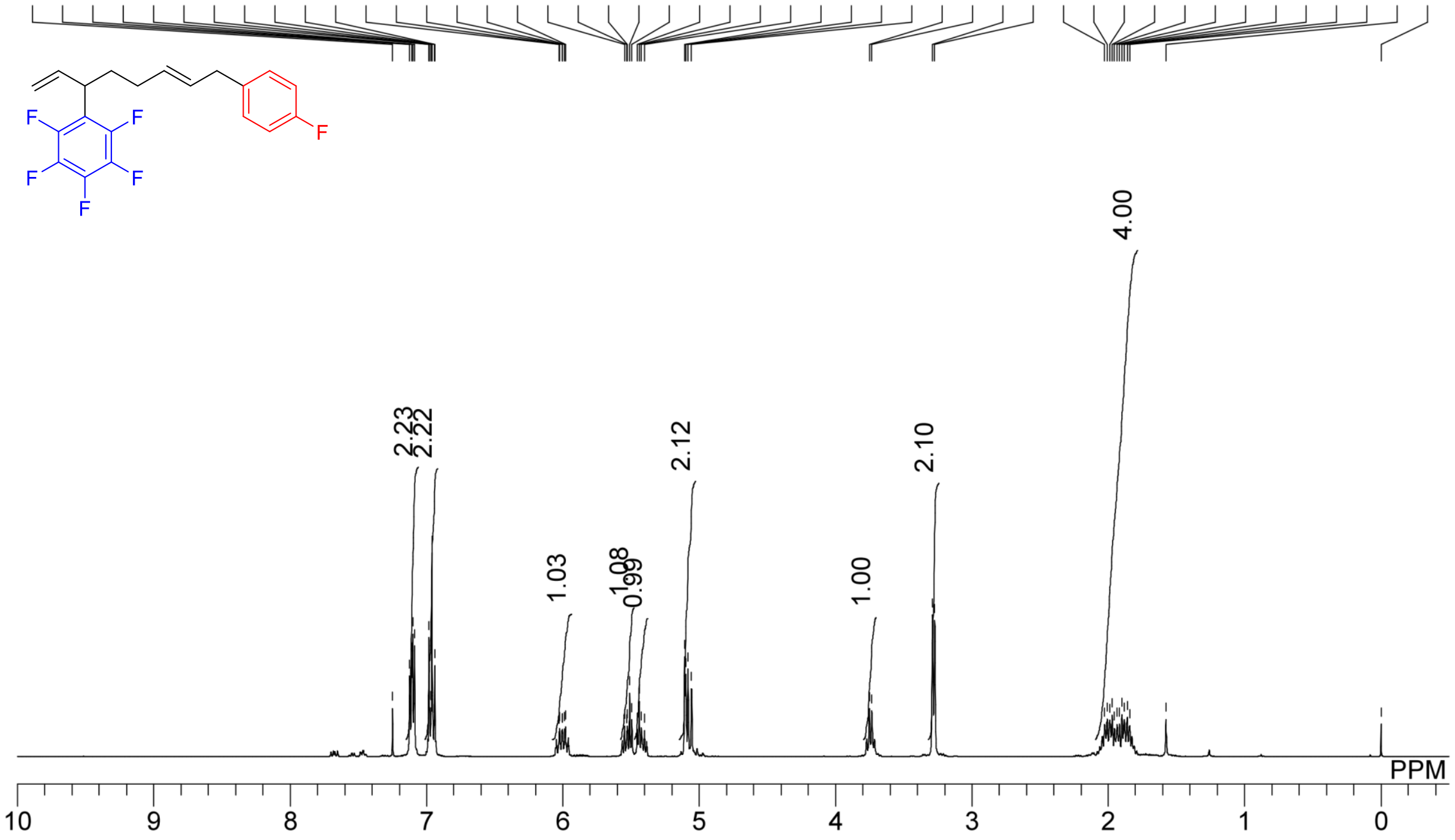
${ }^{13} \mathrm{C}\left\{{ }^{1} \mathrm{H}\right\}$ NMR of $\mathbf{4 a a}$

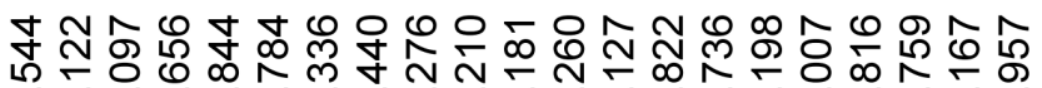
ஸ்
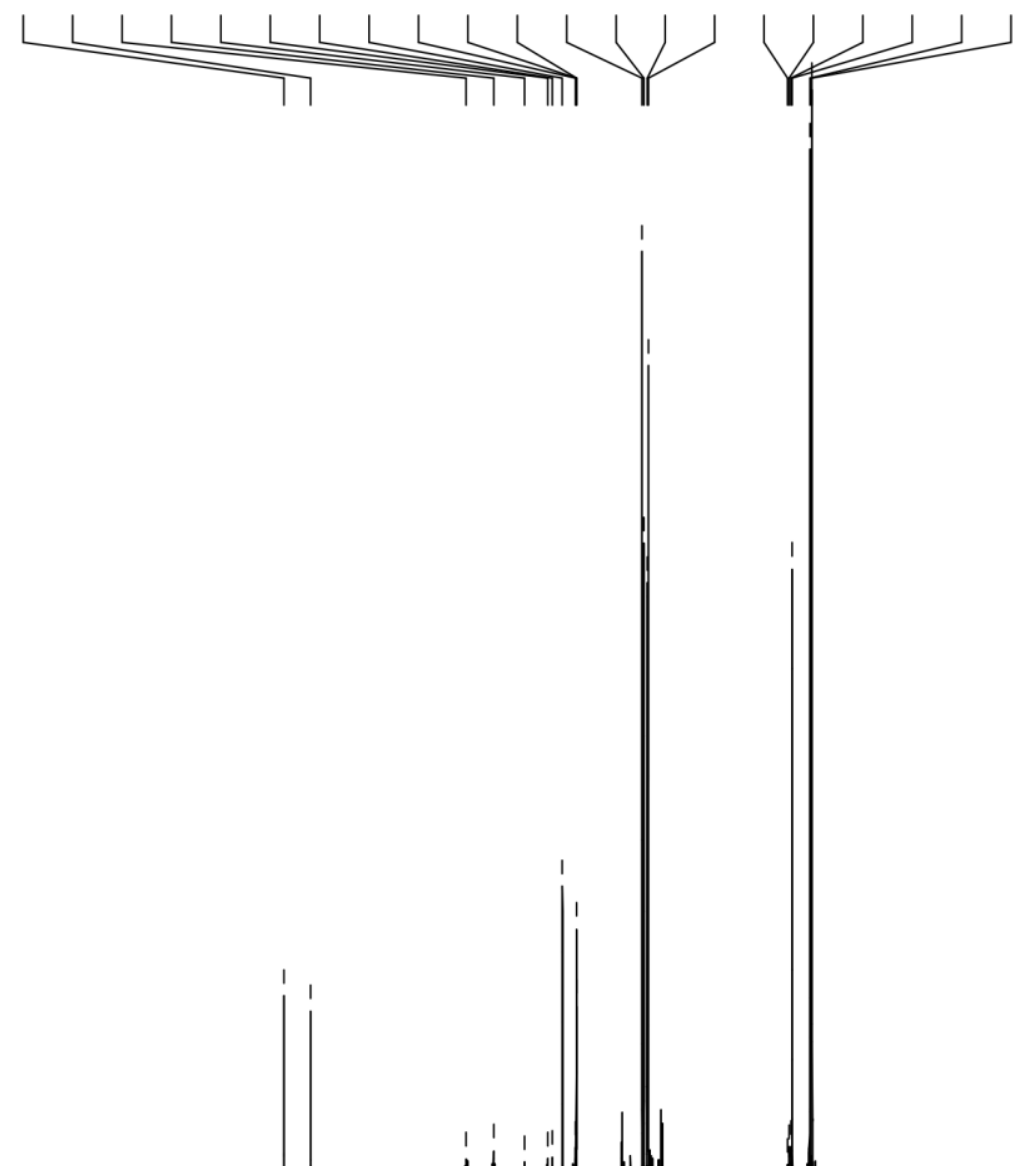
${ }^{13} \mathrm{C}\left\{{ }^{19} \mathrm{~F}\right\}$ NMR of $4 \mathbf{a a}$

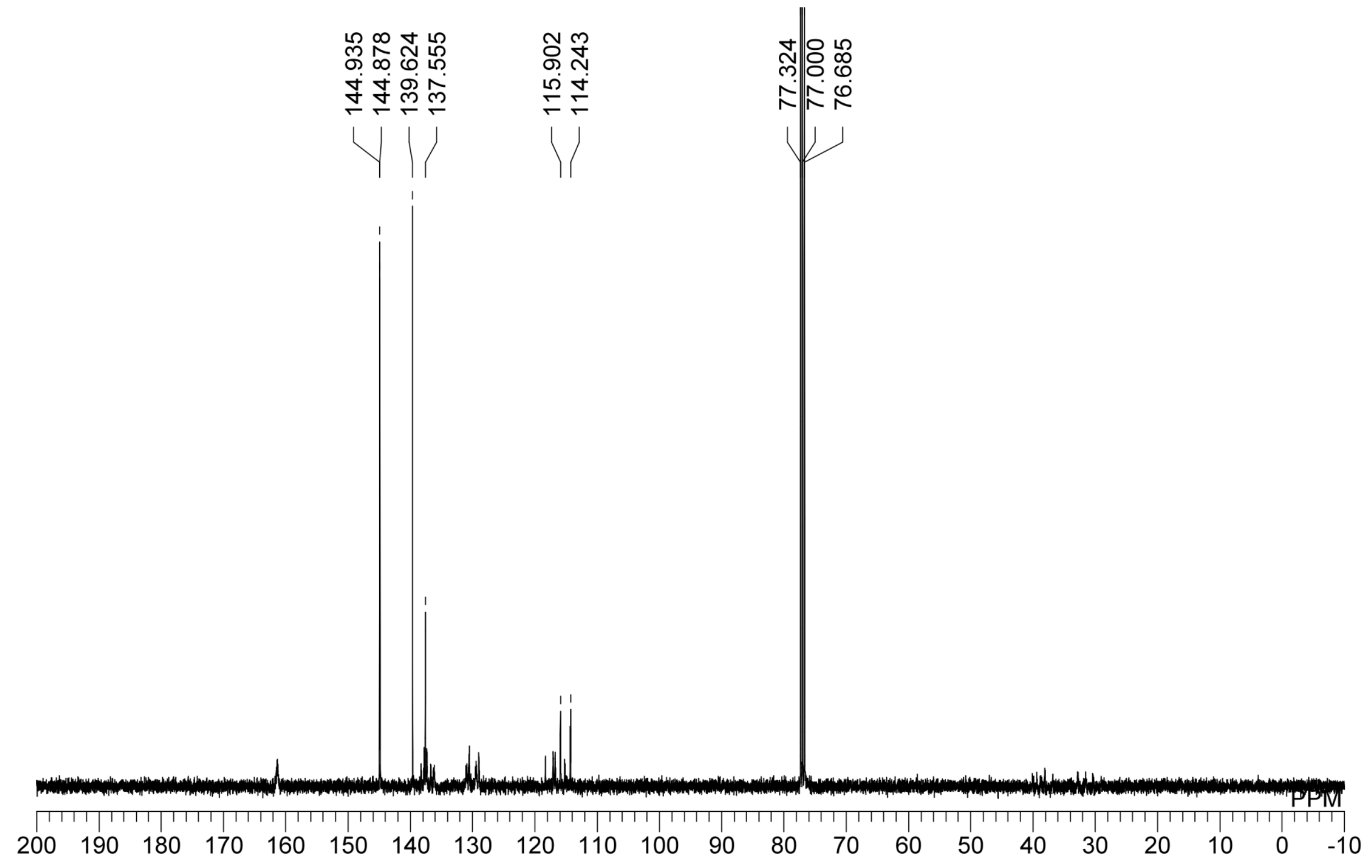


${ }^{19} \mathrm{~F}$ NMR of $4 \mathbf{a a}$
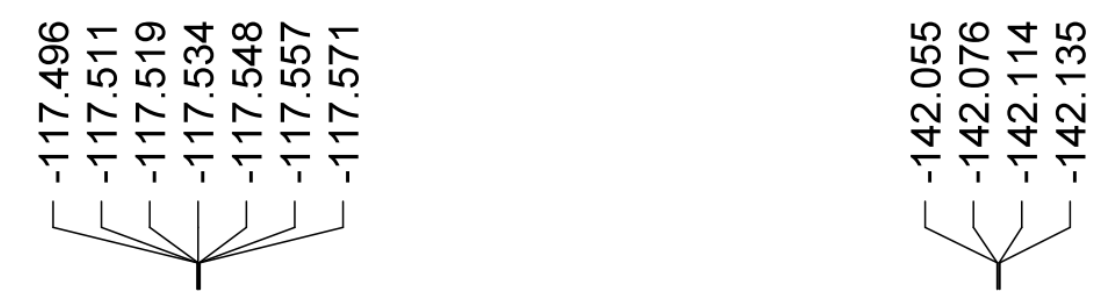

๒ Nิ

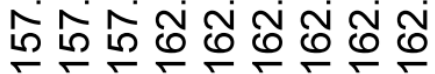
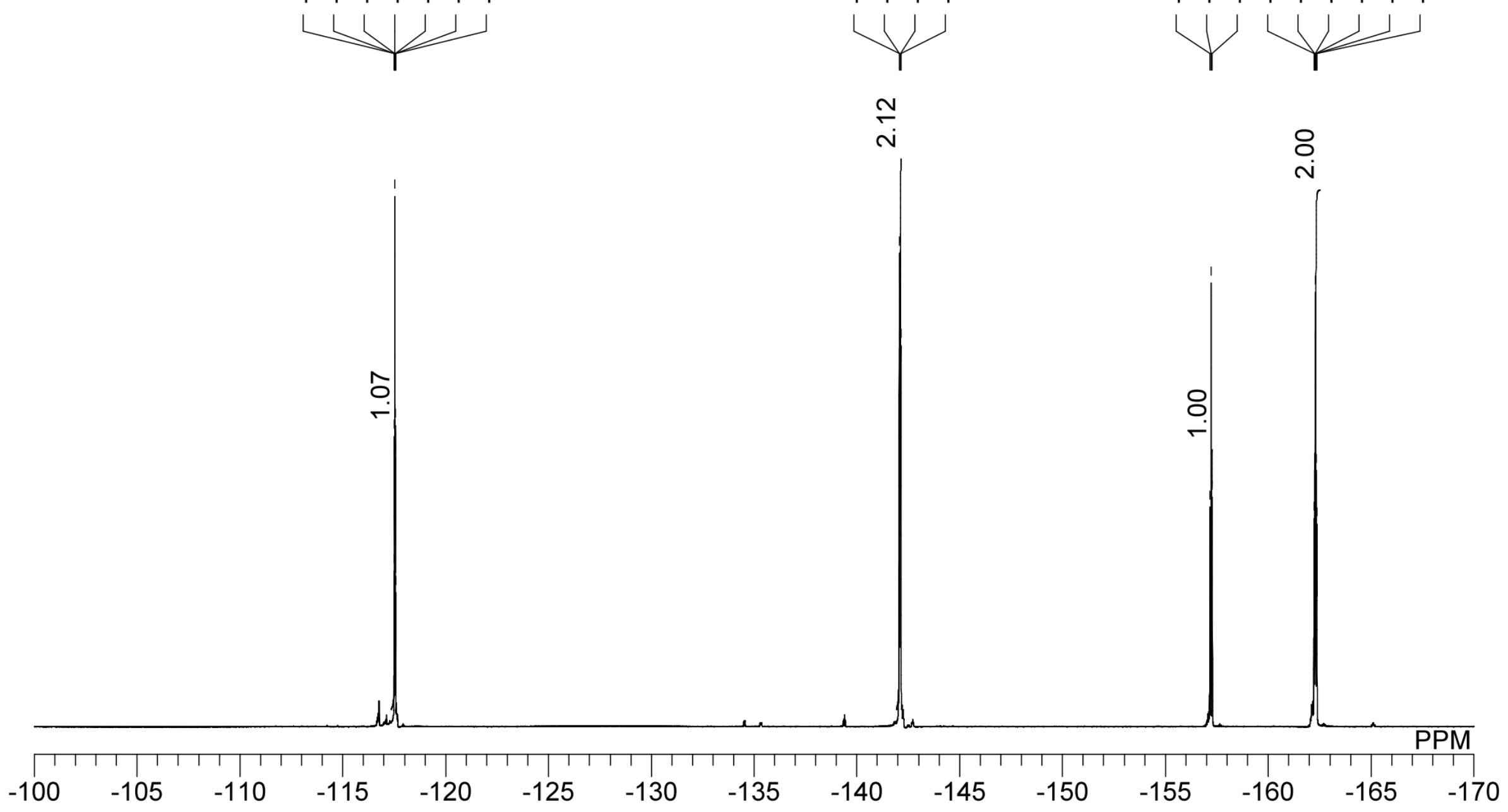


\section{${ }^{1} \mathrm{H}$ NMR of $4 \mathbf{a b}$}

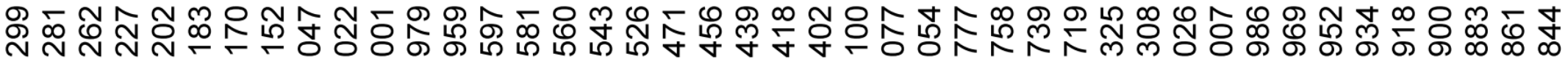

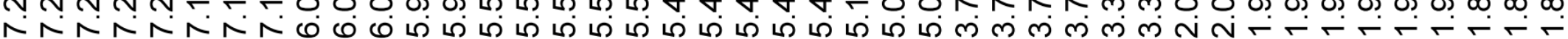

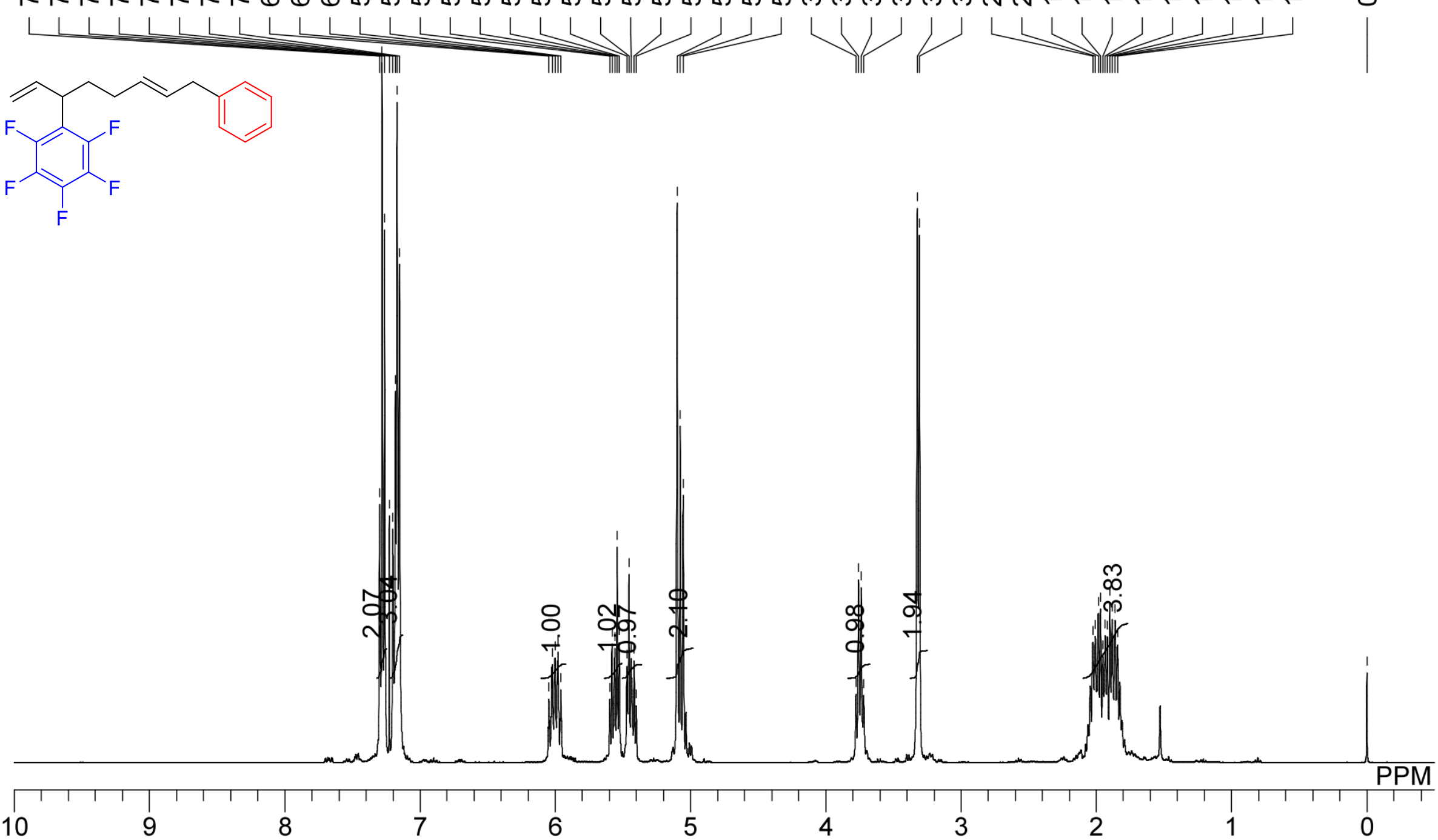


${ }^{13} \mathrm{C}\left\{{ }^{1} \mathrm{H}\right\}$ NMR of $\mathbf{4 a b}$

సิ 守守守览

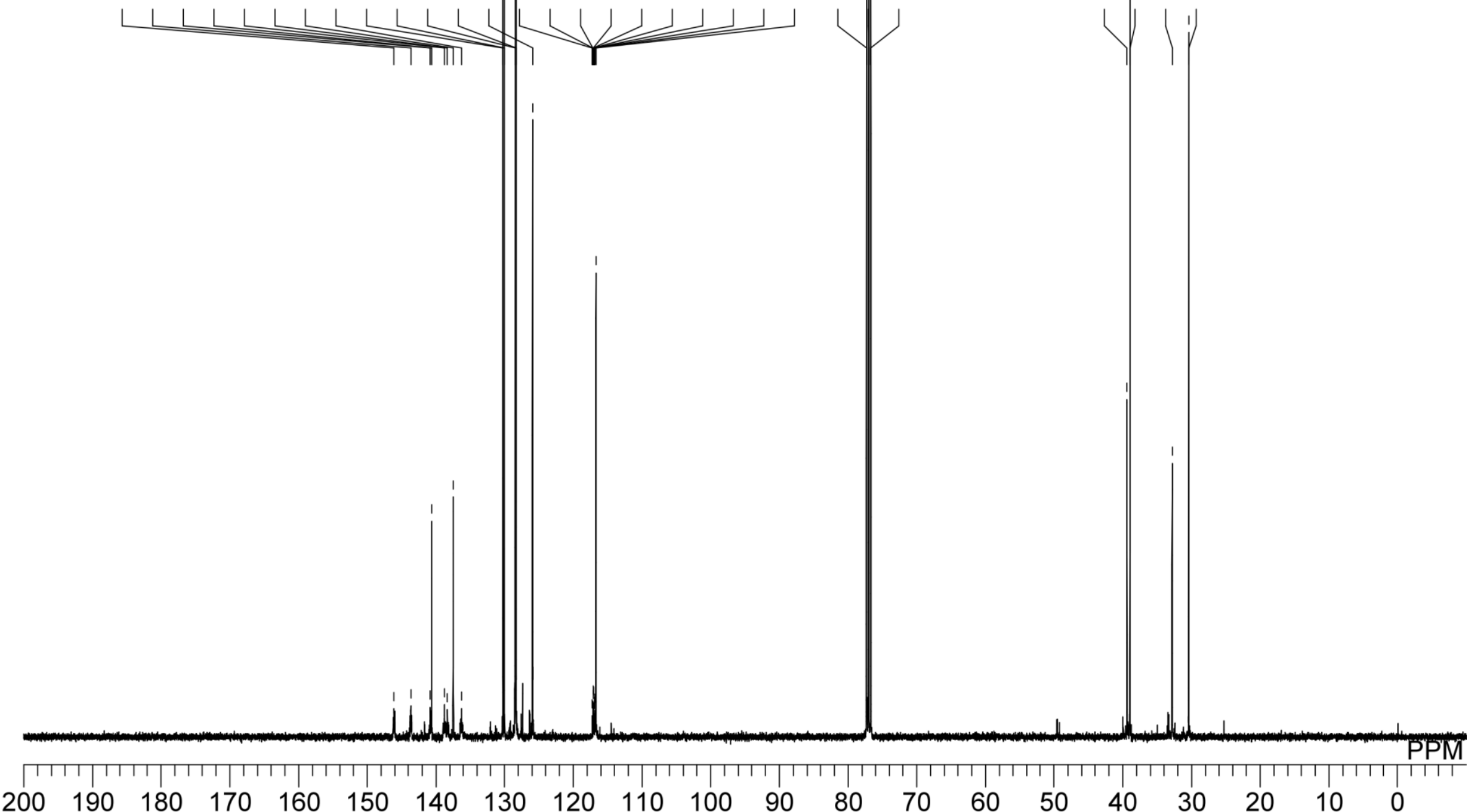


${ }^{19}$ F NMR of $\mathbf{4 a b}$

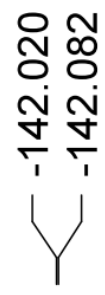

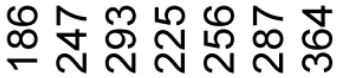

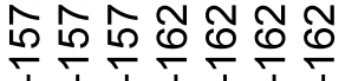
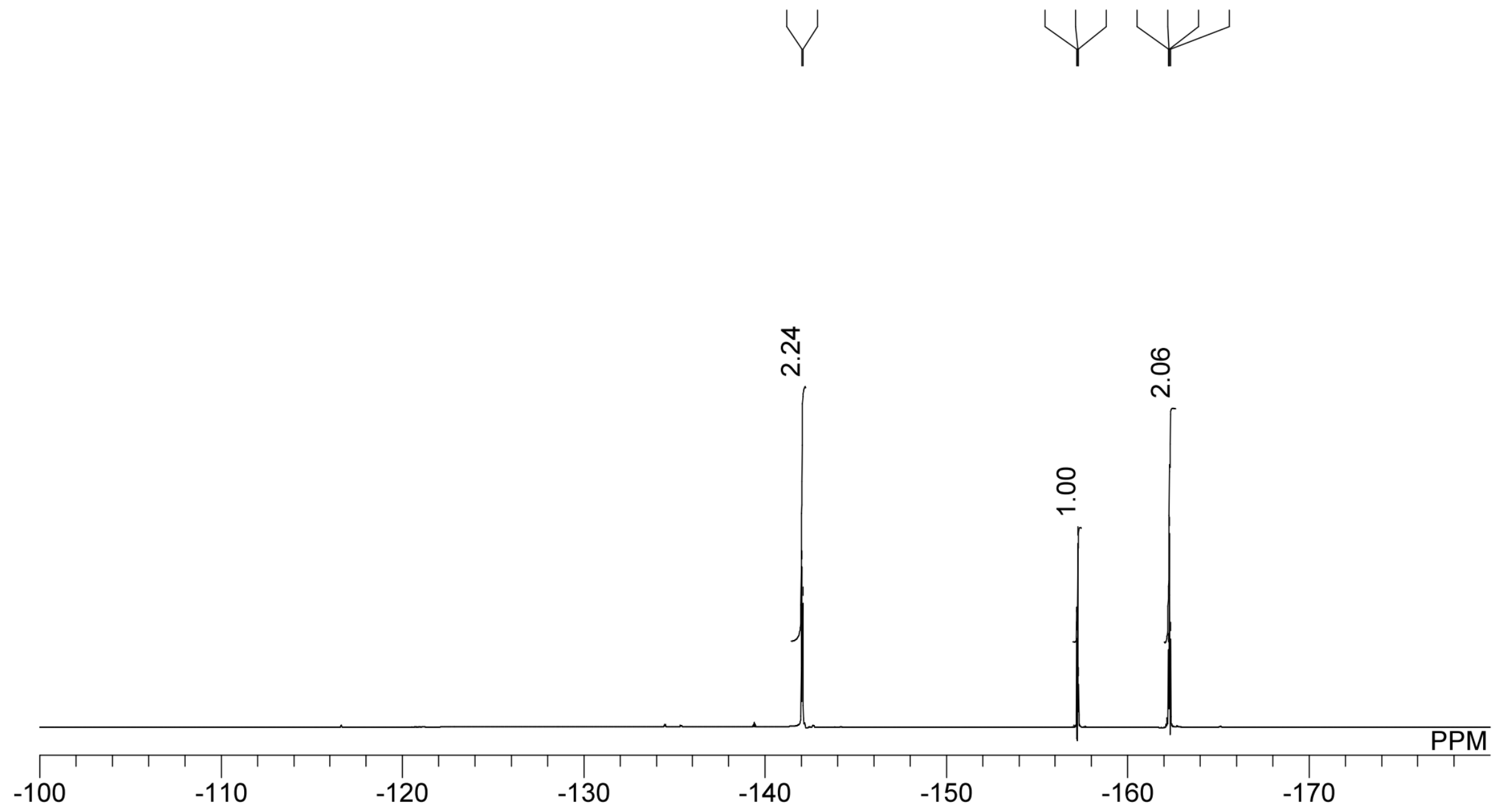


\section{${ }^{1} \mathrm{H}$ NMR of $\mathbf{4 a c}$}

ㅅㅇㅇㅇㅠ

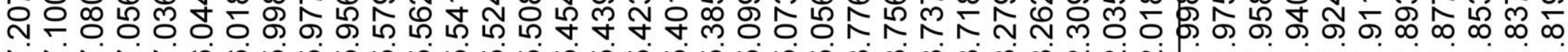
N N N

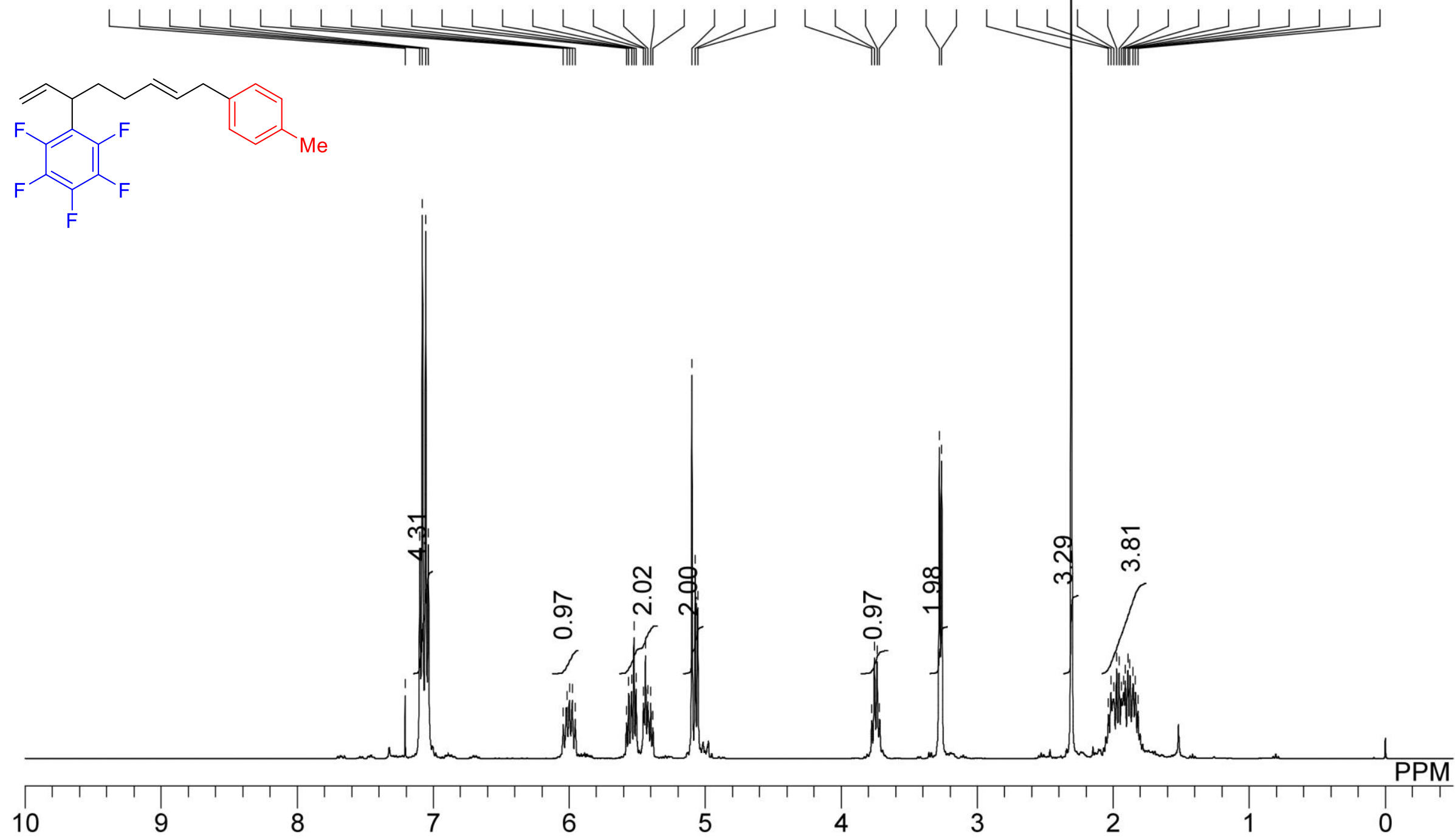


${ }^{13} \mathrm{C}\left\{{ }^{1} \mathrm{H}\right\}$ NMR of $\mathbf{4 a c}$

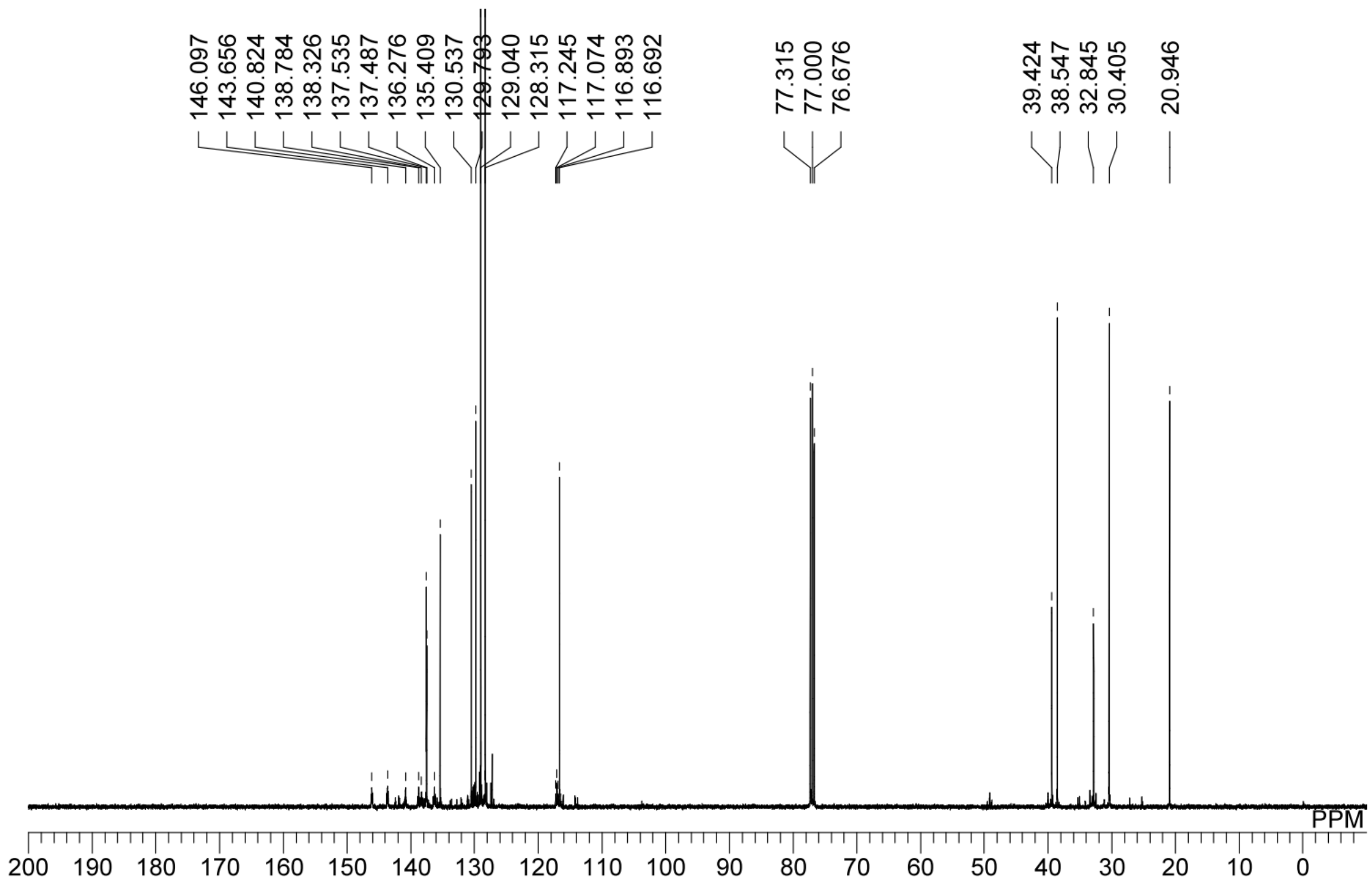


${ }^{19} \mathrm{~F}$ NMR of $\mathbf{4 a c}$

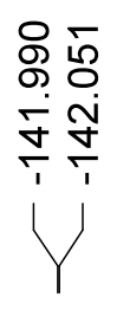

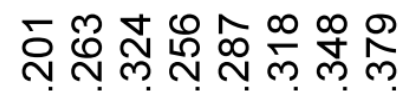

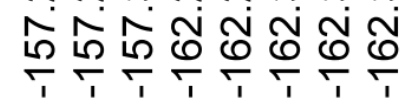

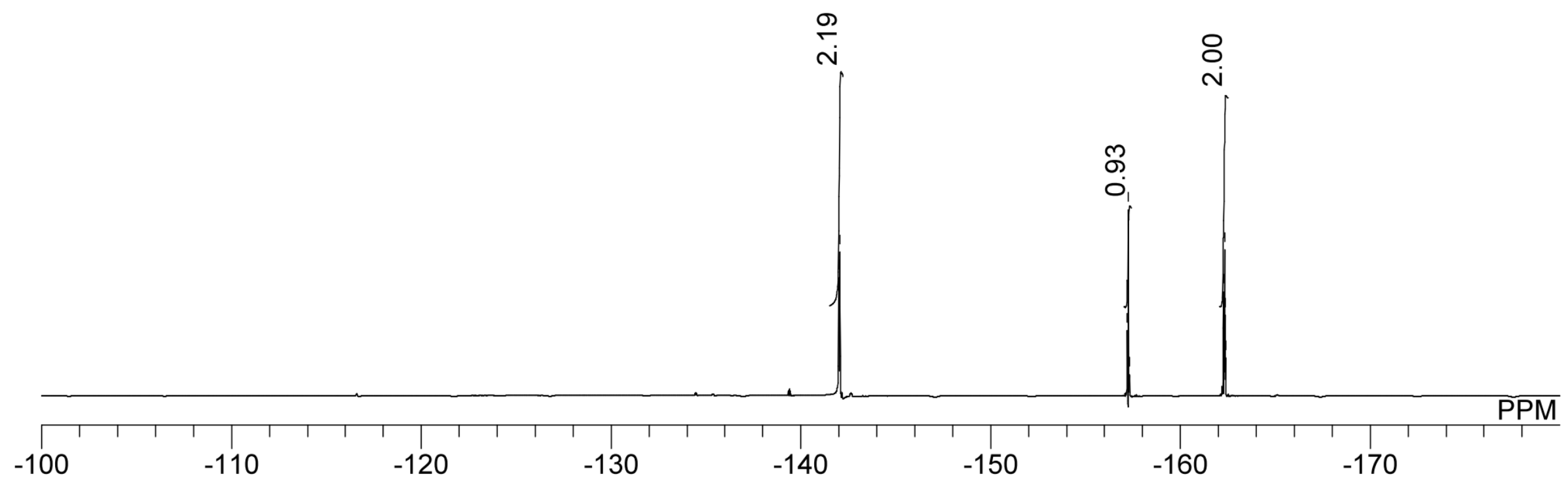




\section{${ }^{1} \mathrm{H}$ NMR of $\mathbf{4 a d}$}

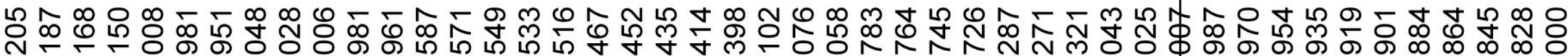
N N N
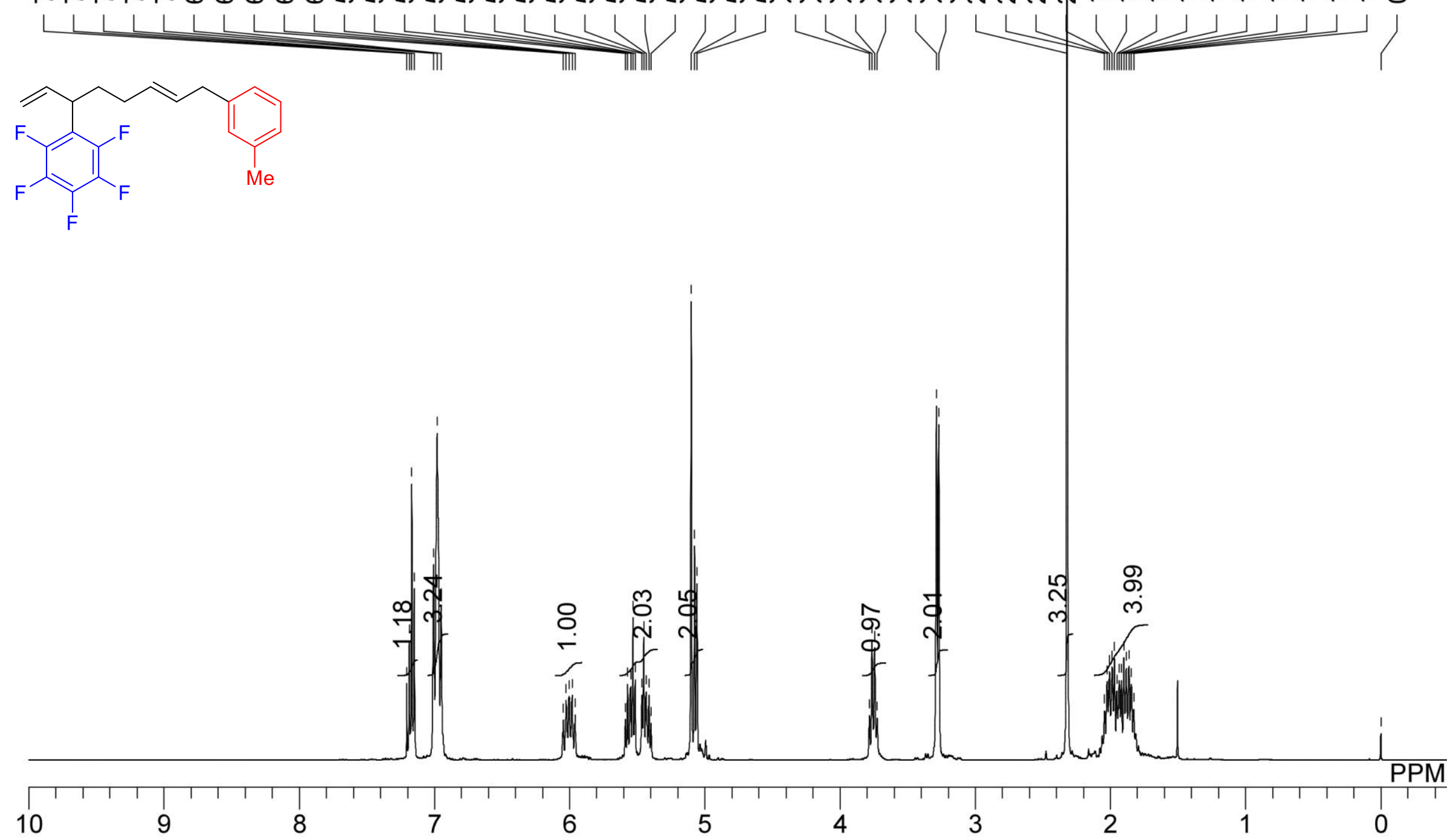
${ }^{13} \mathrm{C}\left\{{ }^{1} \mathrm{H}\right\}$ NMR of $\mathbf{4 a d}$

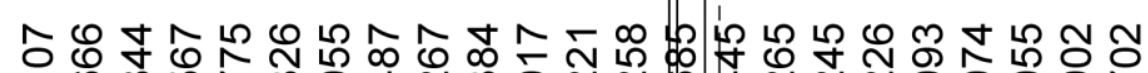
은

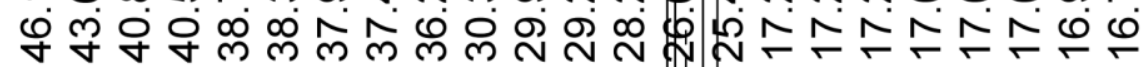

సั่ NヘN

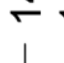
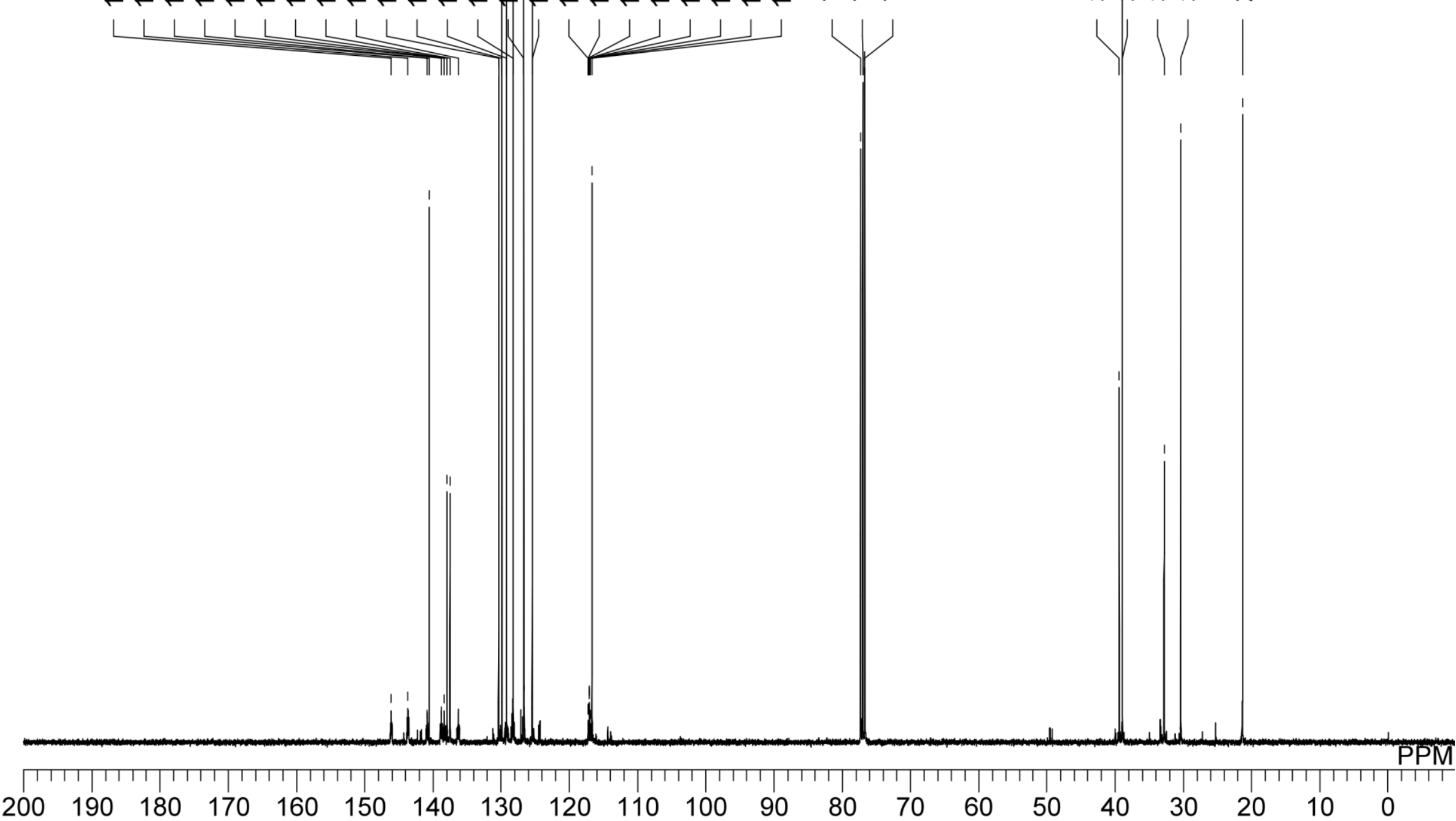

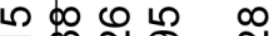

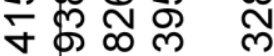

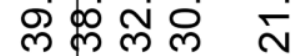


${ }^{19} \mathrm{~F}$ NMR of $\mathbf{4 a d}$
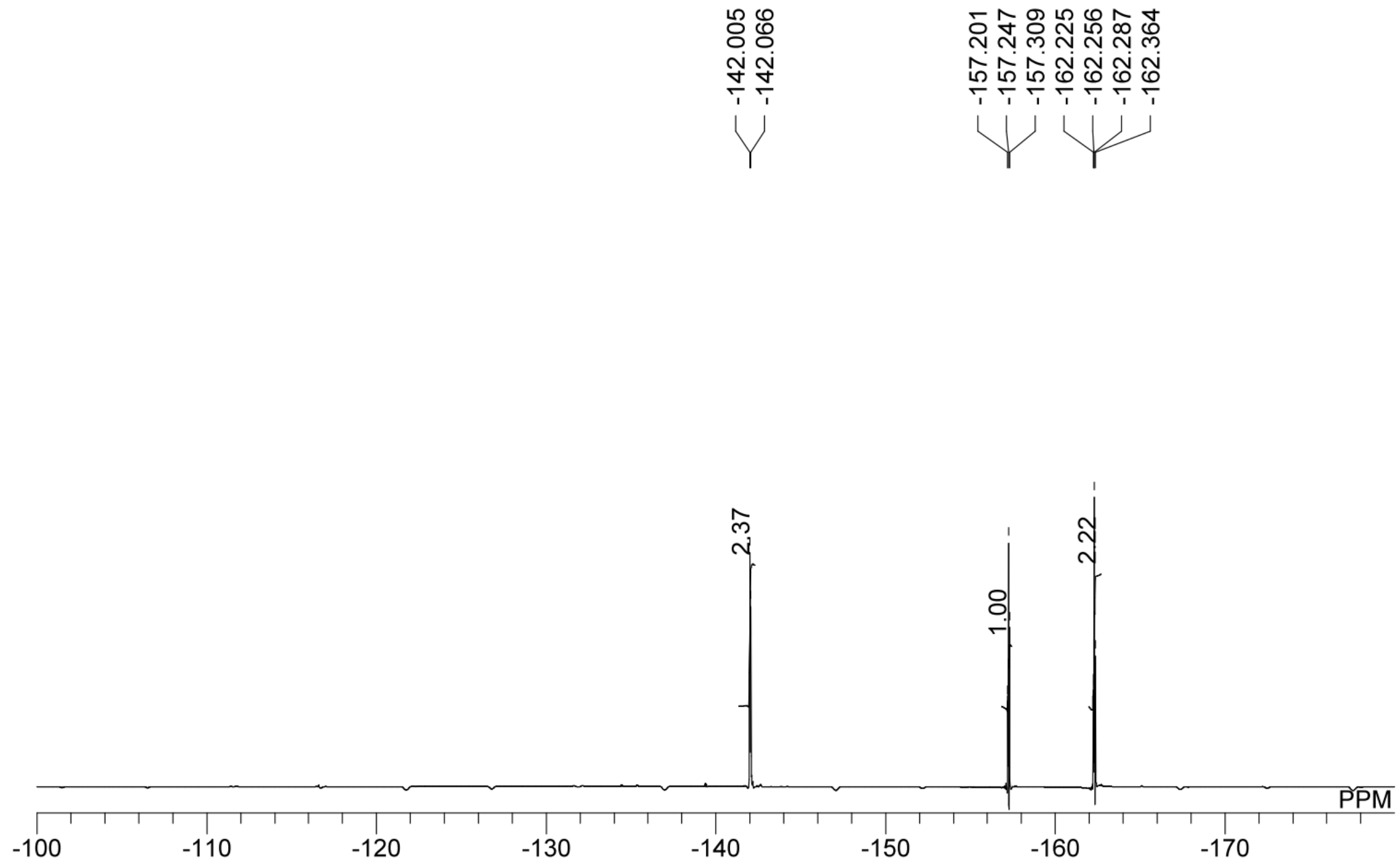
${ }^{1} \mathrm{H}$ NMR of $4 \mathbf{a e}$

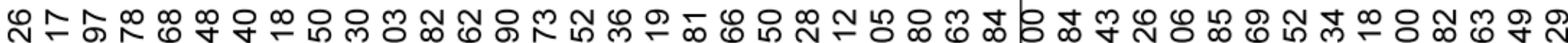

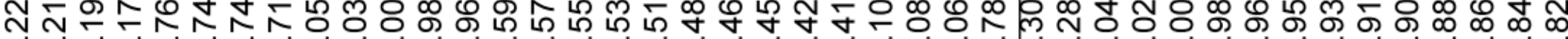

N N N
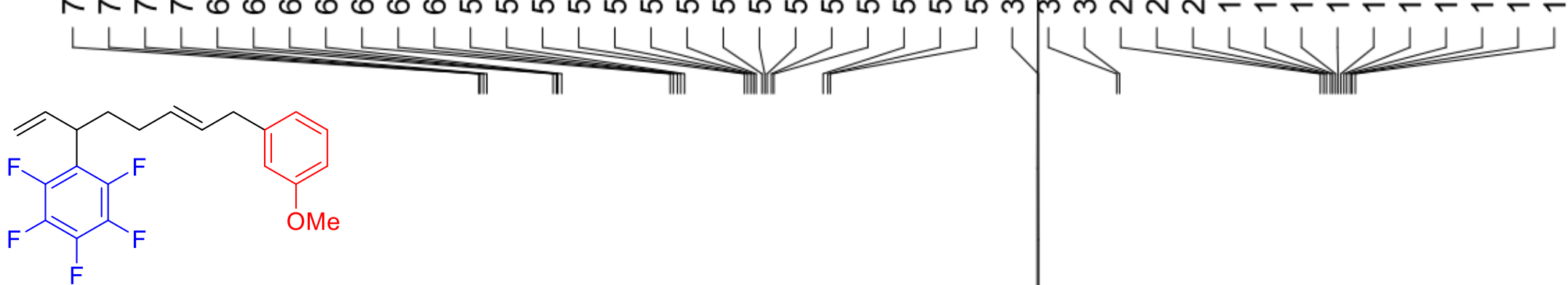

웅

\section{(1)}

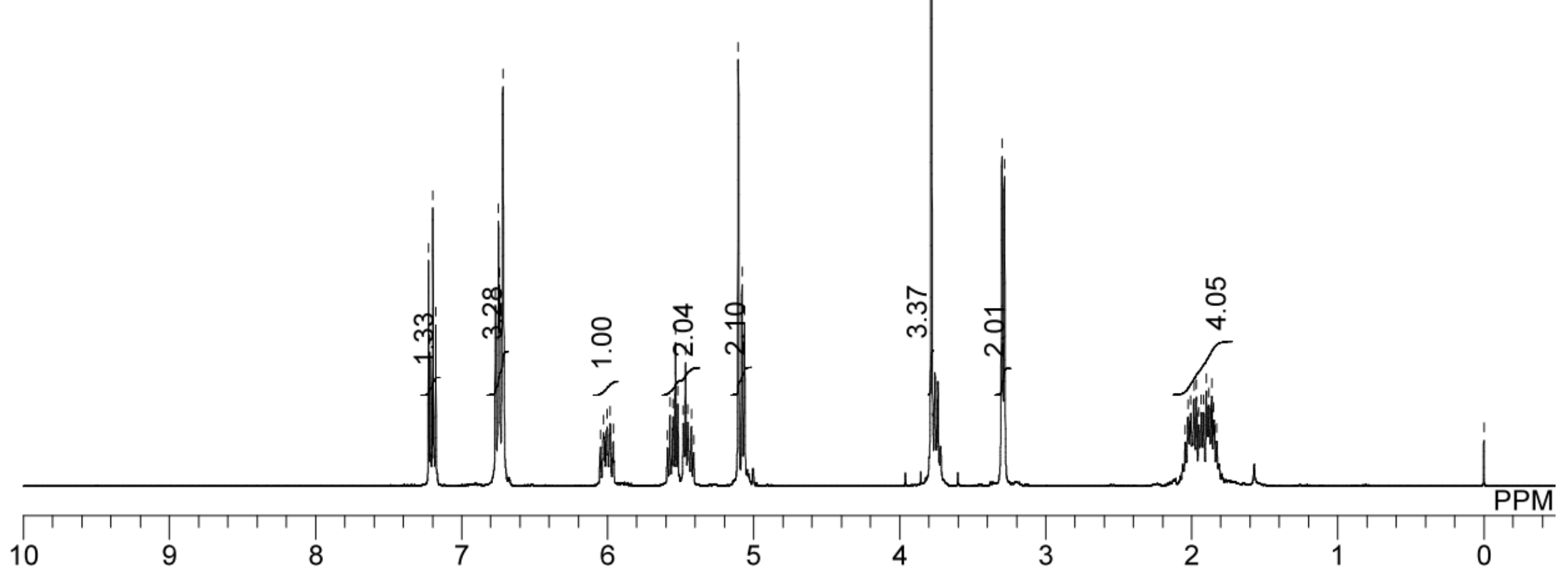




\section{${ }^{13} \mathrm{C}\left\{{ }^{1} \mathrm{H}\right\}$ NMR of 4ae}

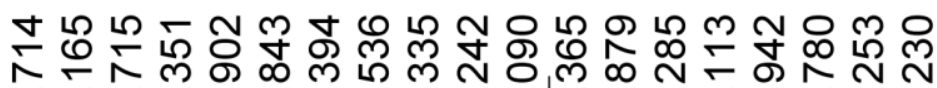

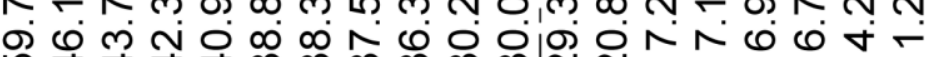

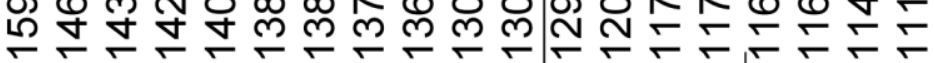

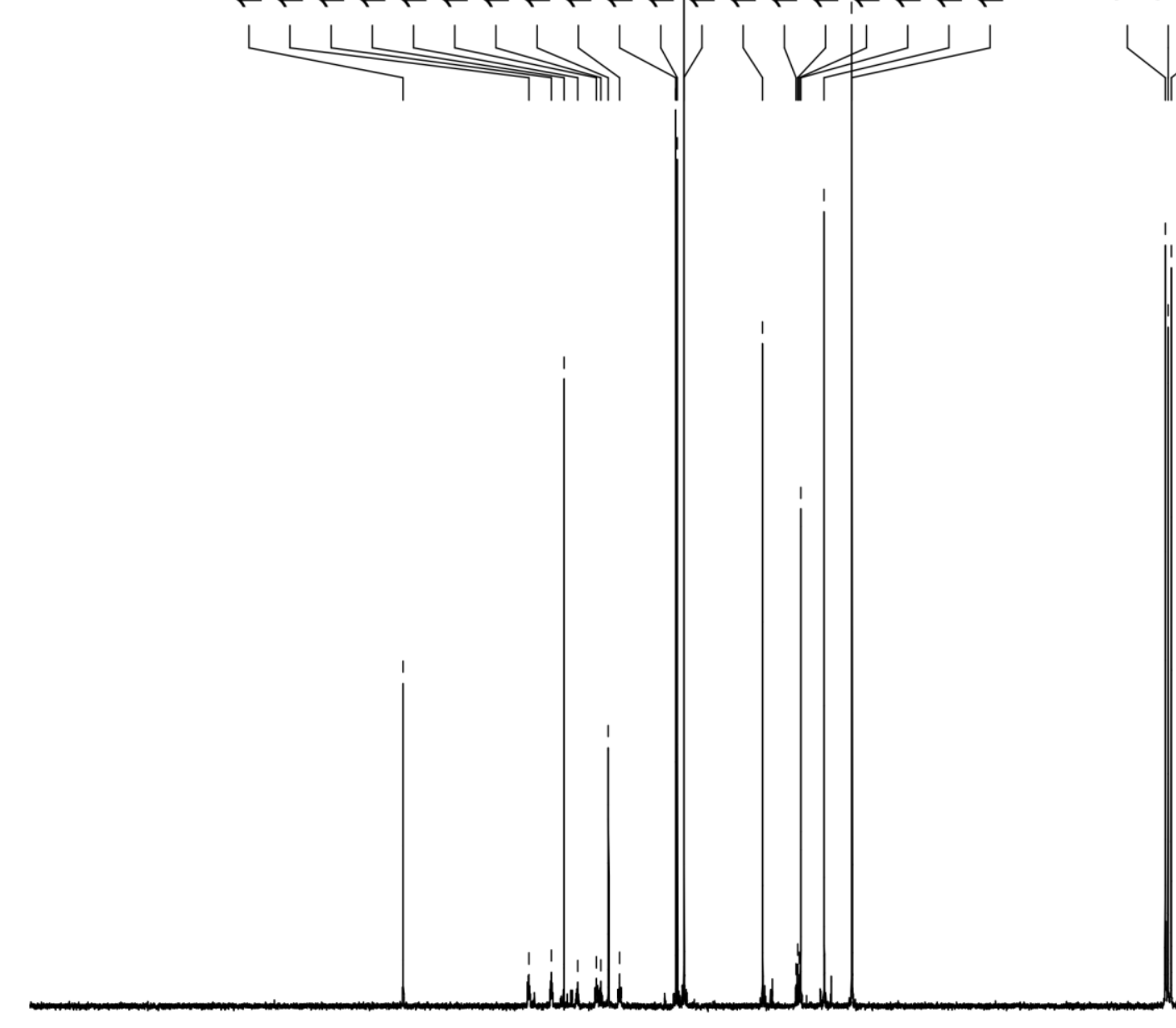

ก m동 ヘペ

ㄱ. 융ำ

L ग्लें స్లి
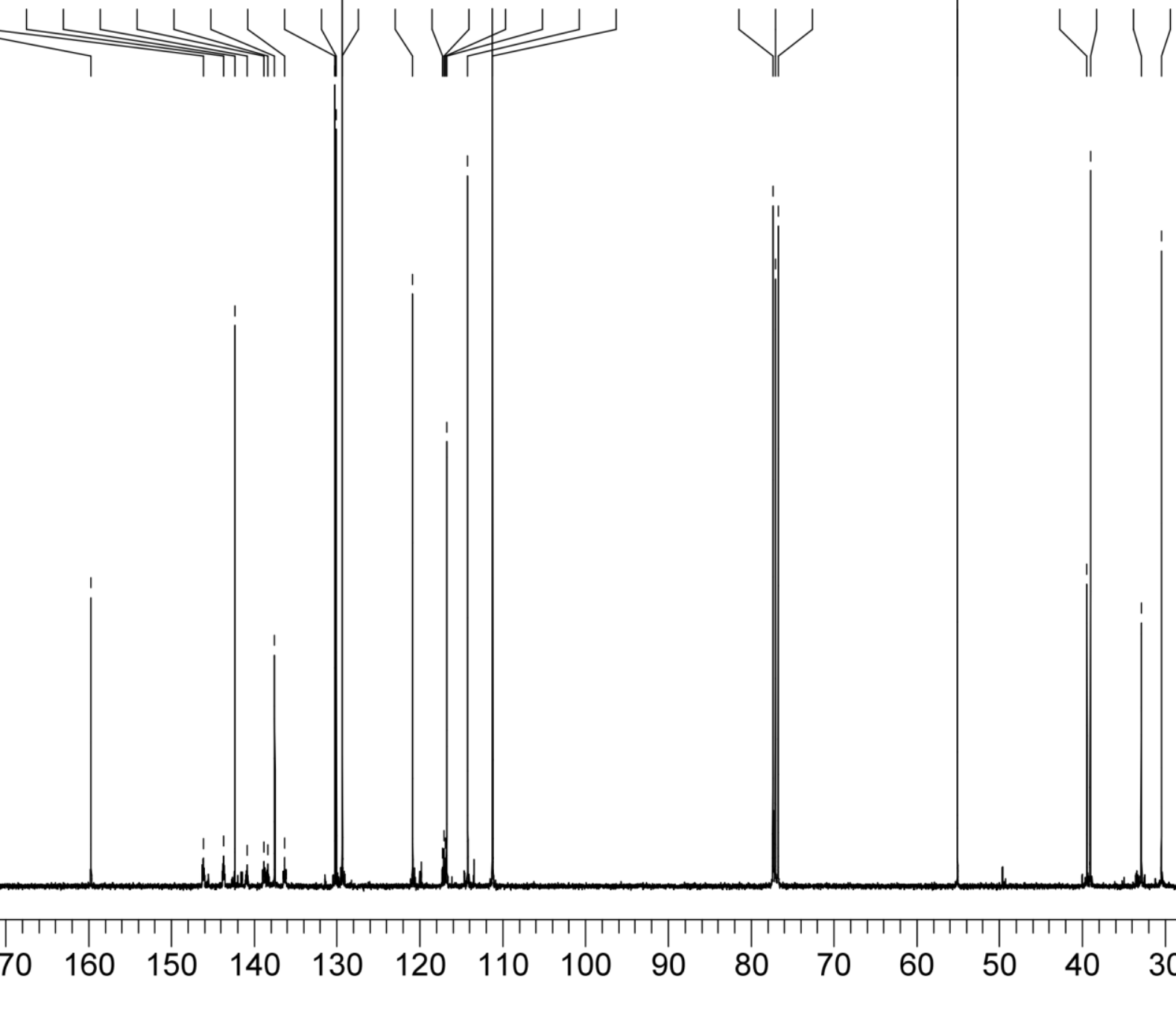
${ }^{19}$ F NMR of $4 a e$
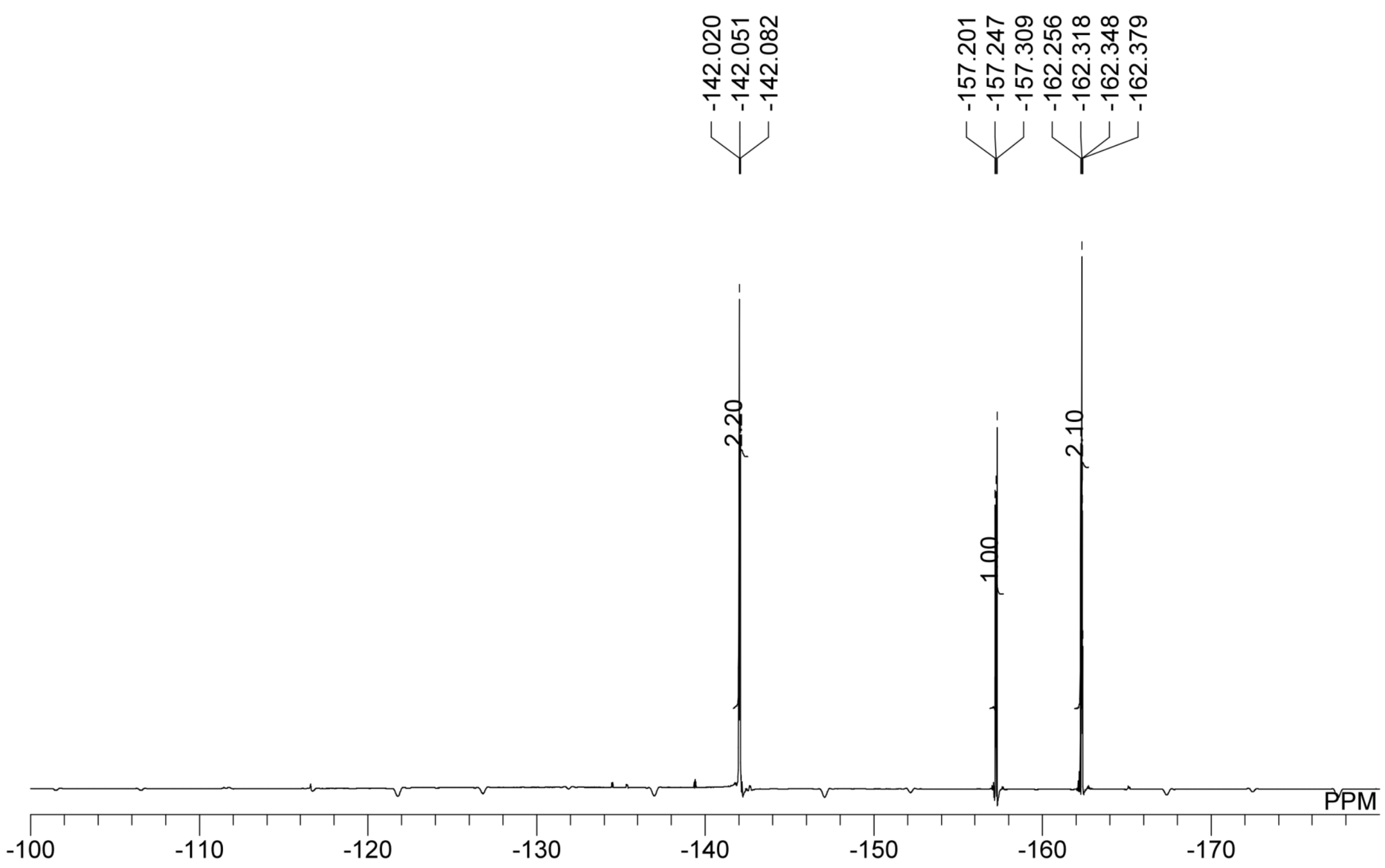


\section{${ }^{1} \mathrm{H}$ NMR of $\mathbf{4 a f}$}

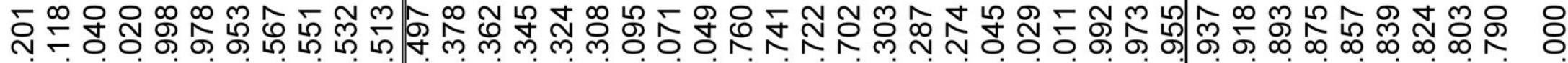
ヘ
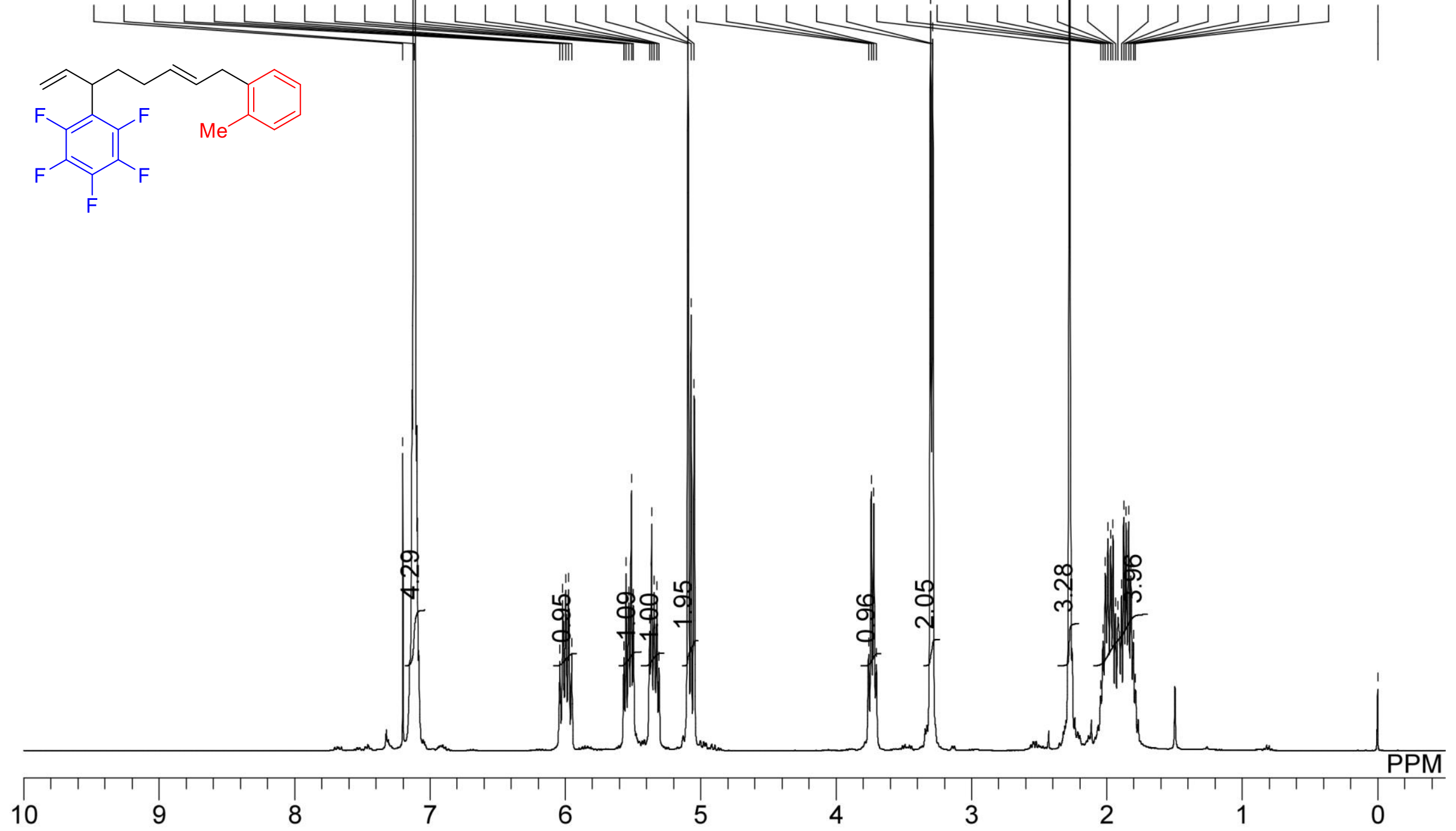
${ }^{13} \mathrm{C}\left\{{ }^{1} \mathrm{H}\right\}$ NMR of 4 af

슨 守 守

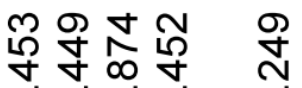

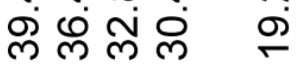
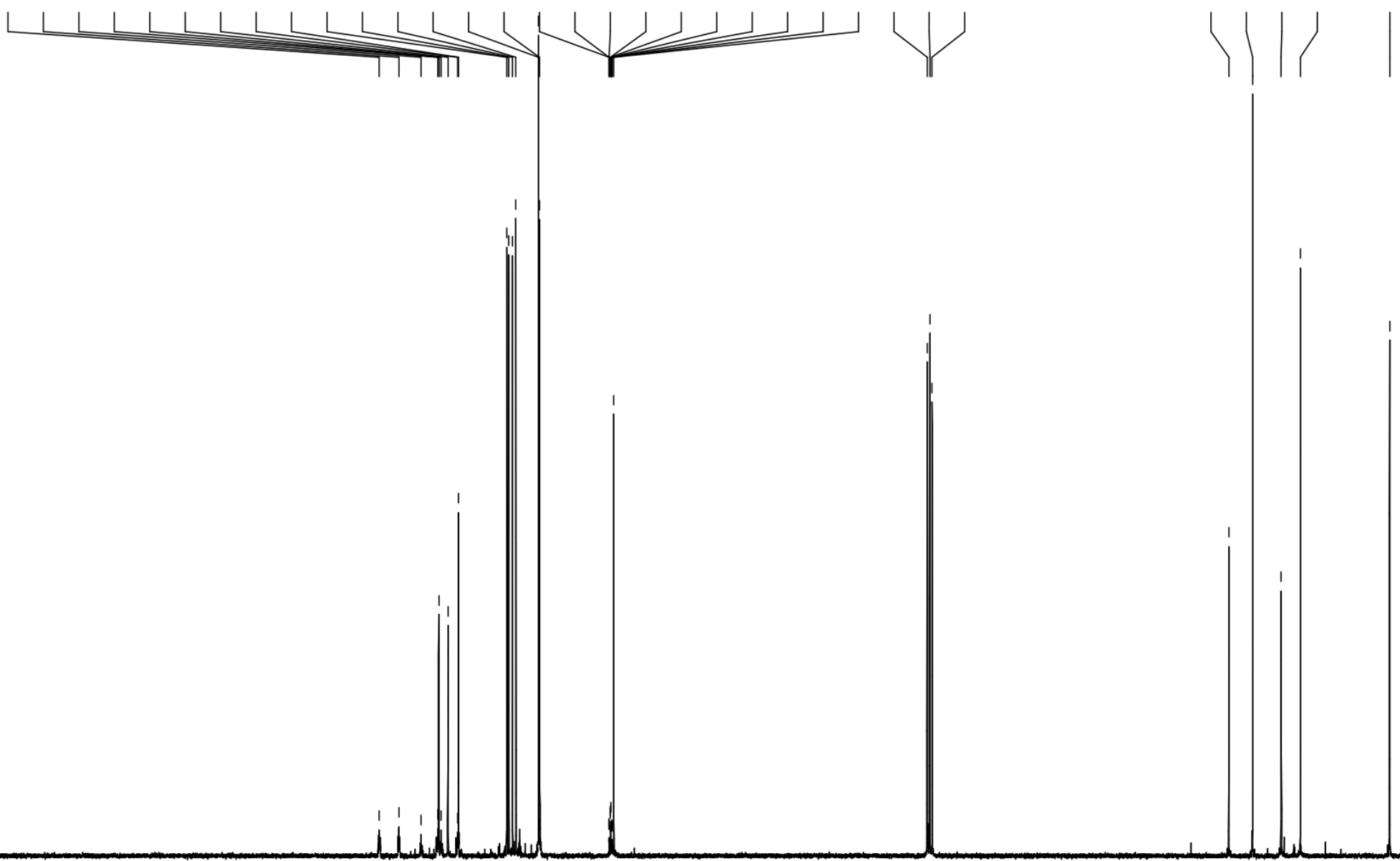
${ }^{19}$ F NMR of 4 af
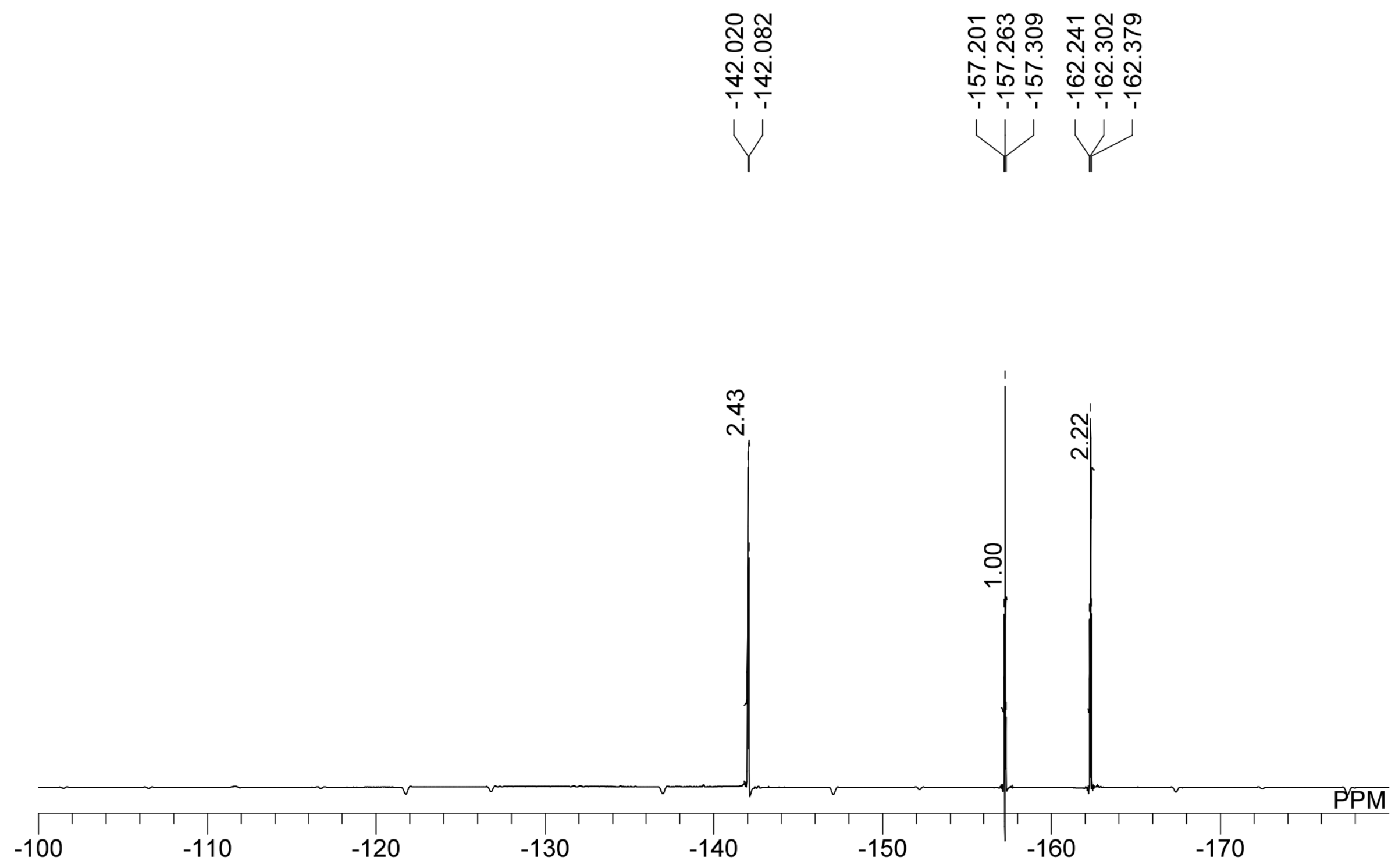
${ }^{1} \mathrm{H}$ NMR of $\mathbf{4 a g}$

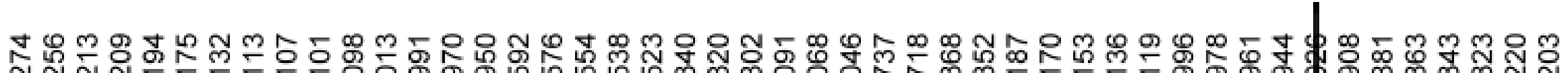

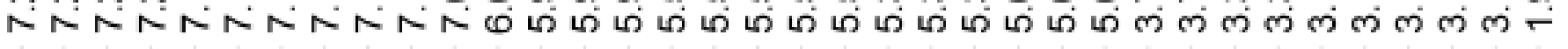
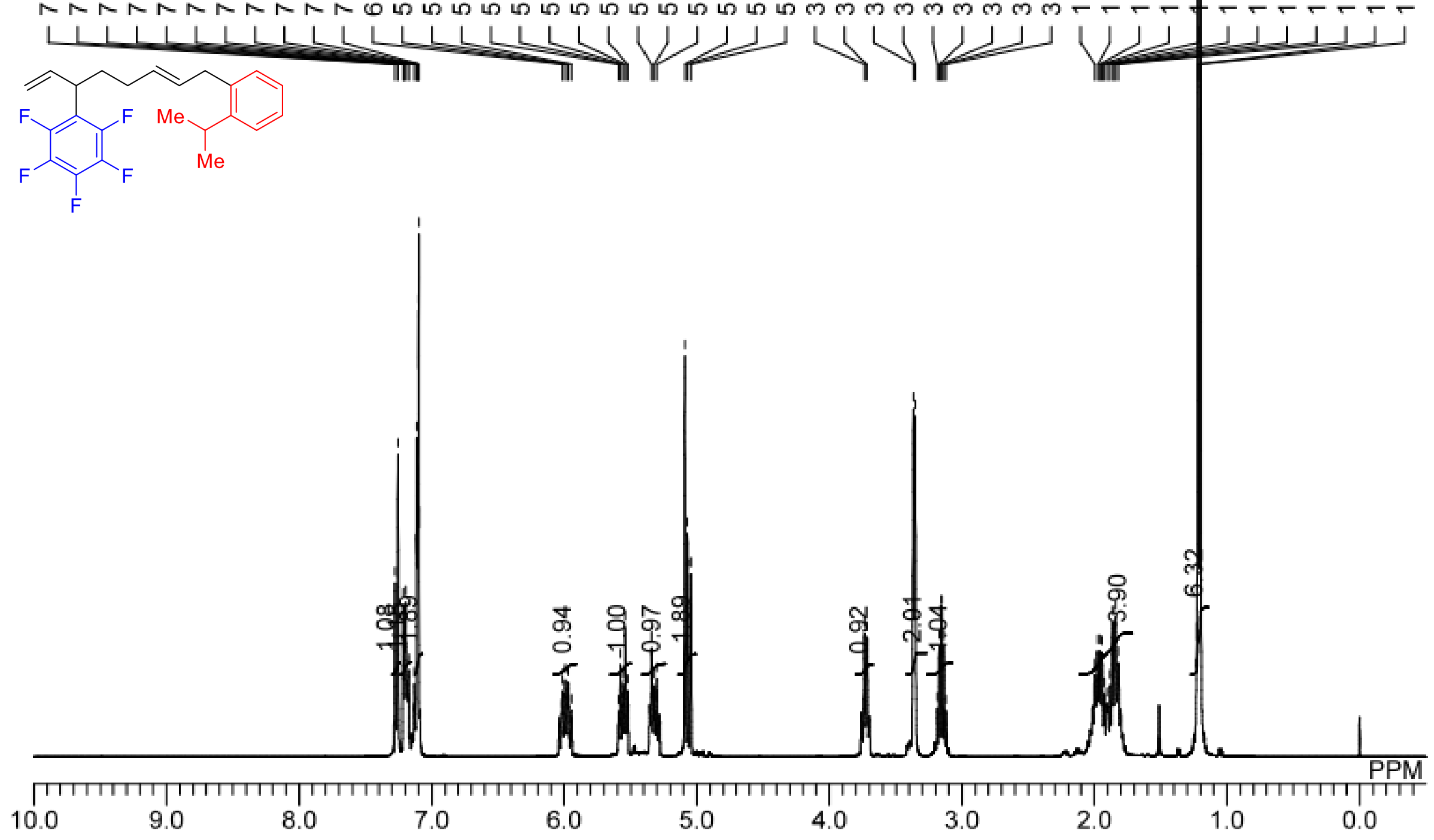


\section{${ }^{13} \mathrm{C}\left\{{ }^{1} \mathrm{H}\right\}$ NMR of $\mathbf{4 a g}$}

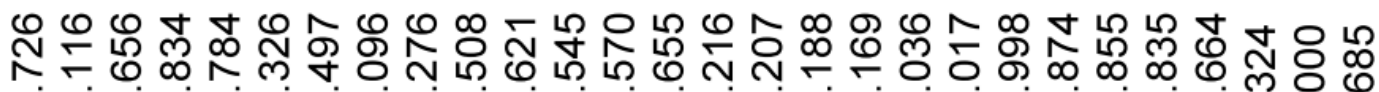

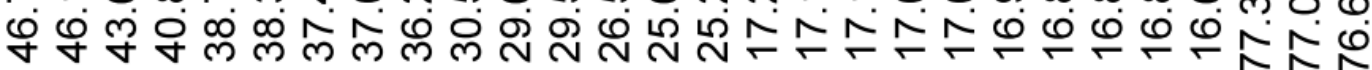

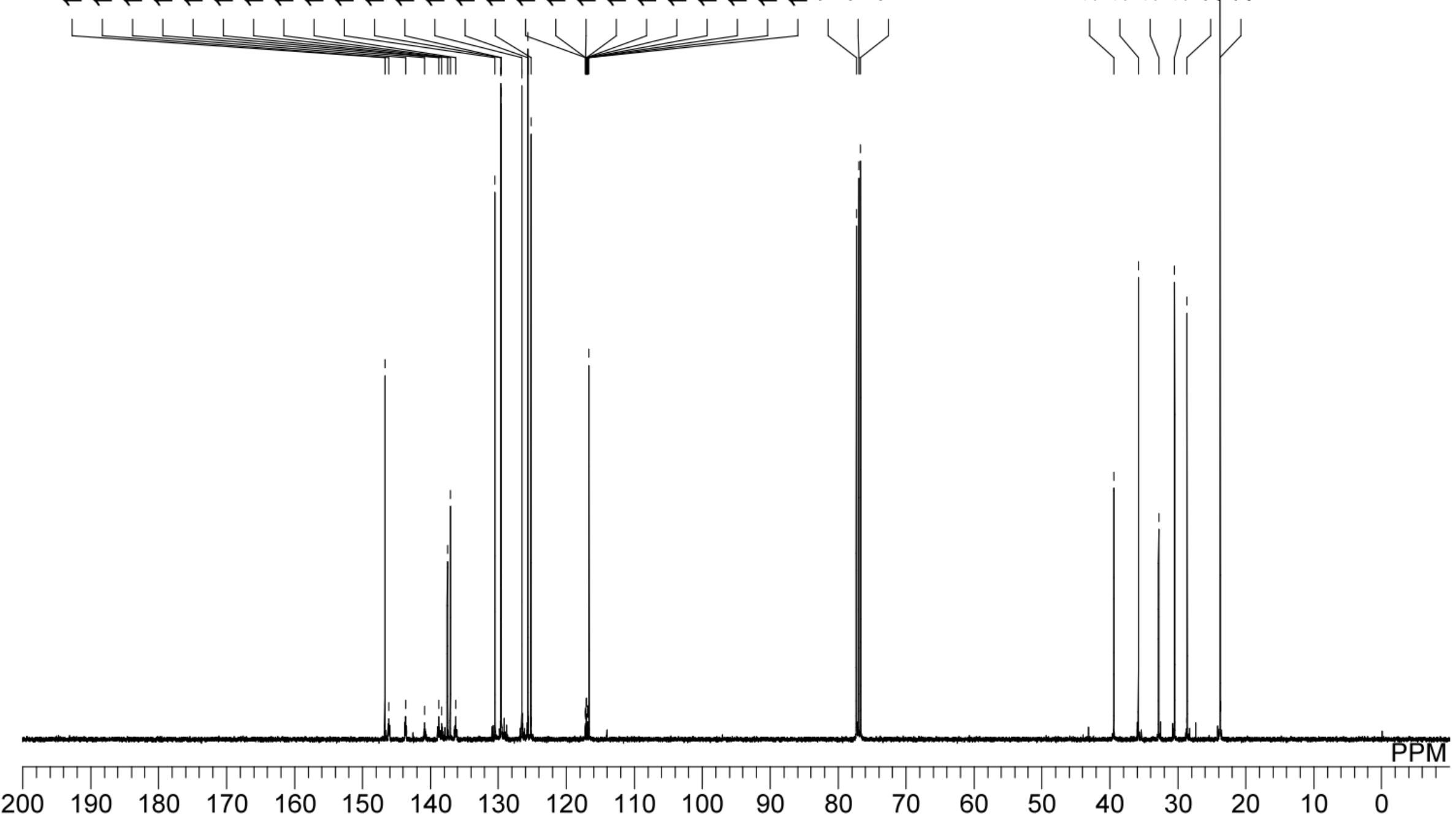


${ }^{19}$ F NMR of $\mathbf{4 a g}$

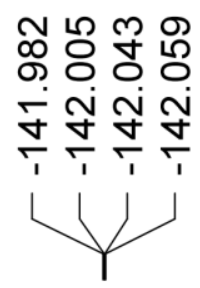



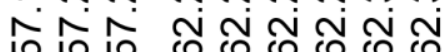

눈
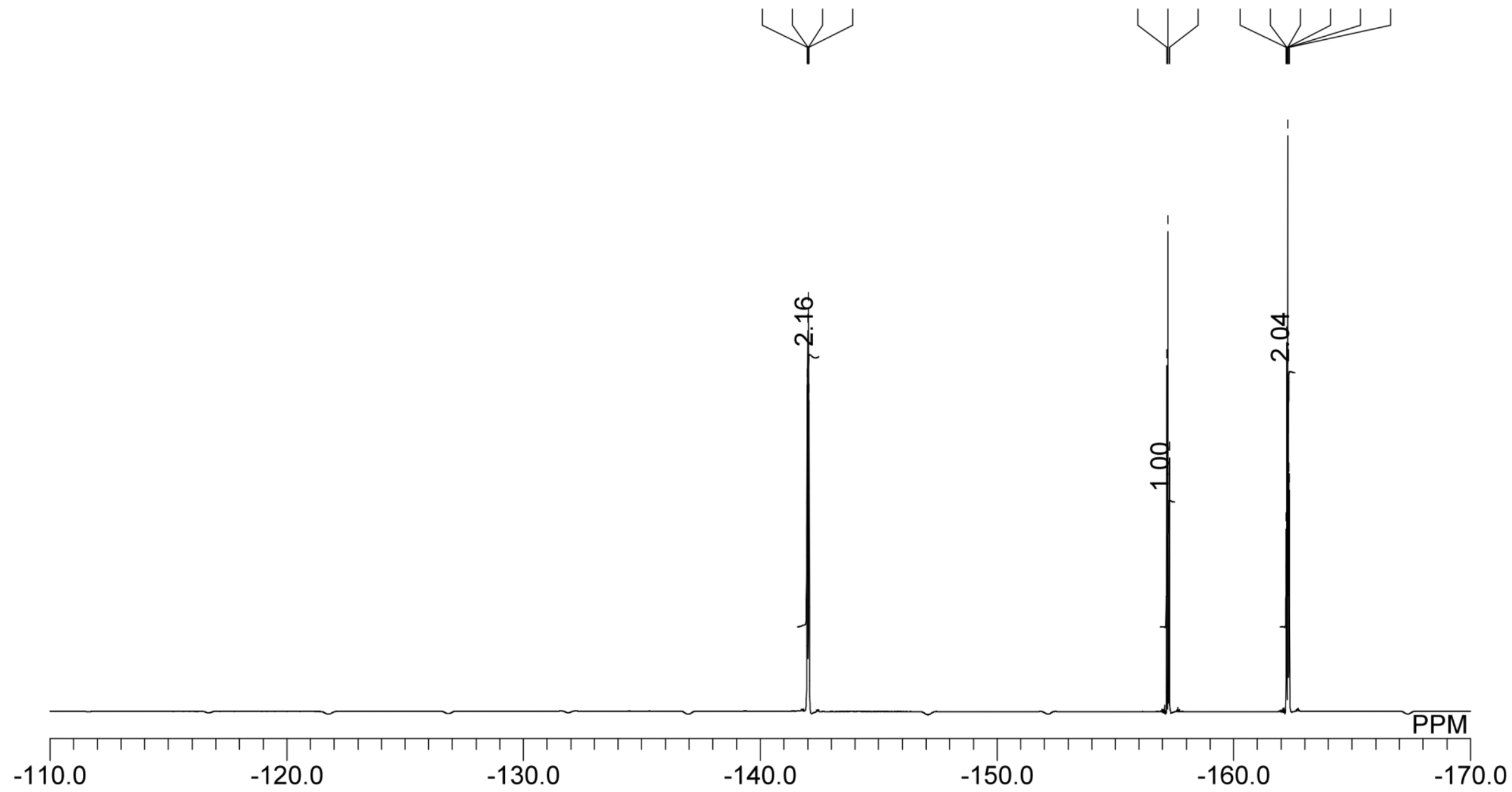


\section{${ }^{1} \mathrm{H}$ NMR of 4ah}

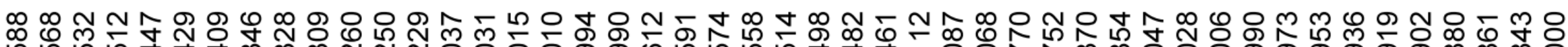

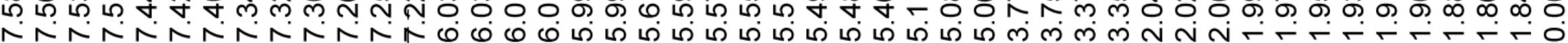

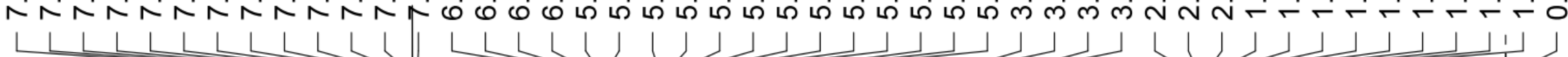

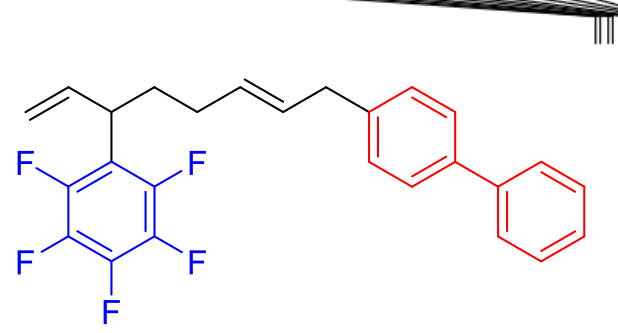

\|\|\|\|\|\|
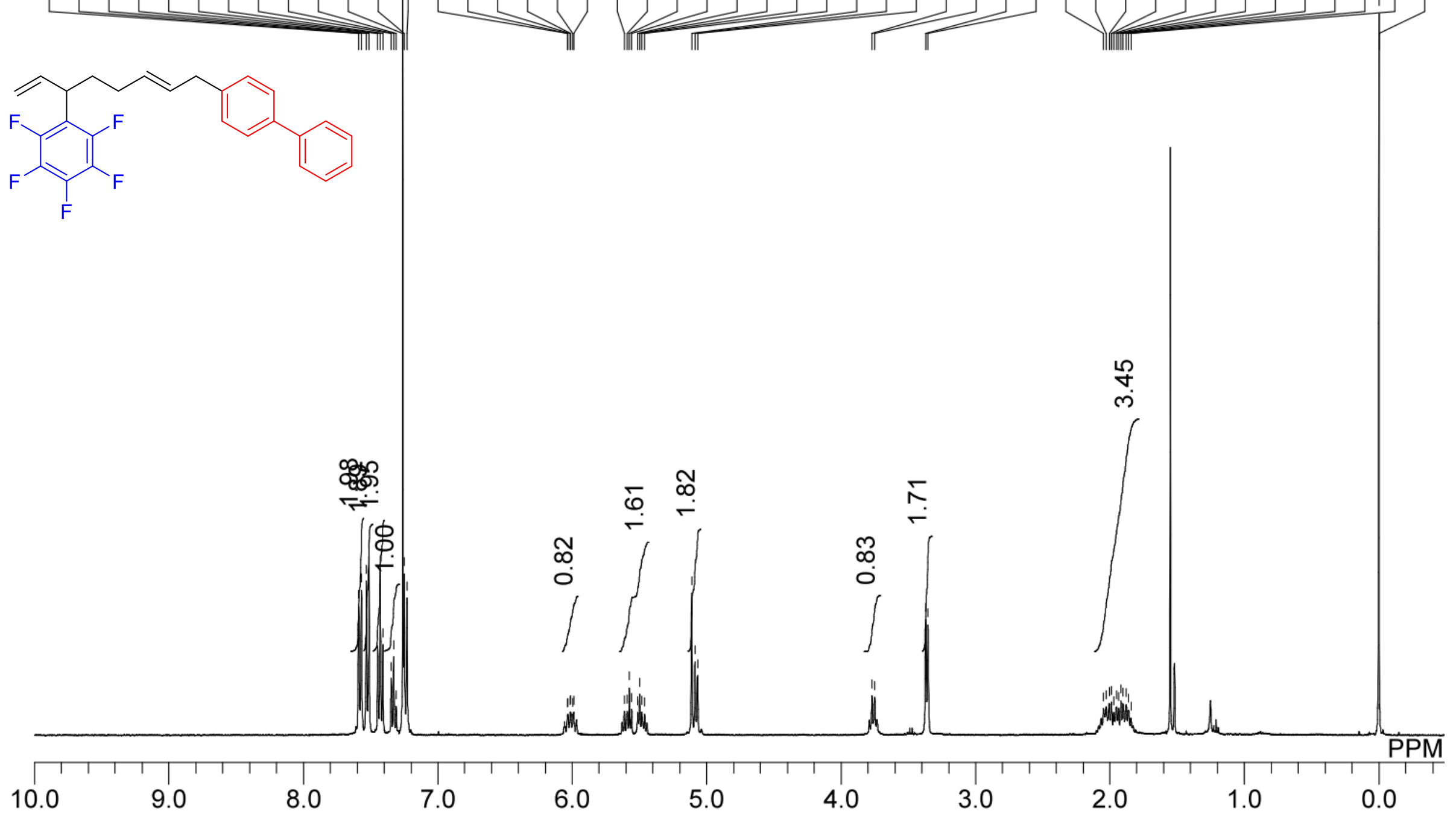
${ }^{13} \mathrm{C}\left\{{ }^{1} \mathrm{H}\right\}$ NMR of $\mathbf{4 a h}$

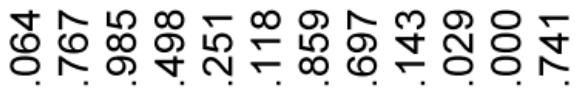

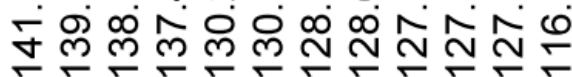

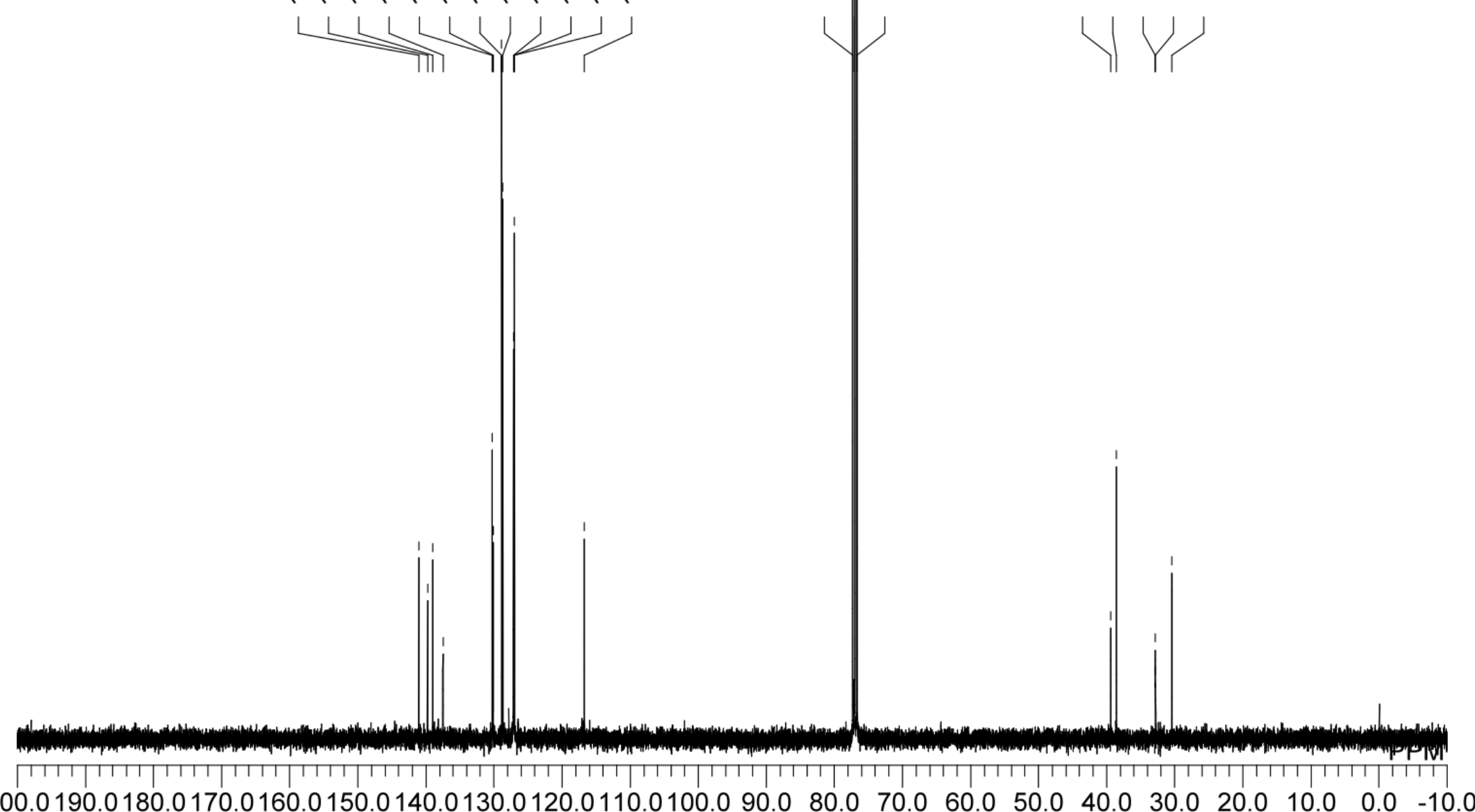

m

人⿻上丨

mָ

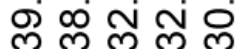


${ }^{19} \mathrm{~F}$ NMR of $\mathbf{4 a h}$
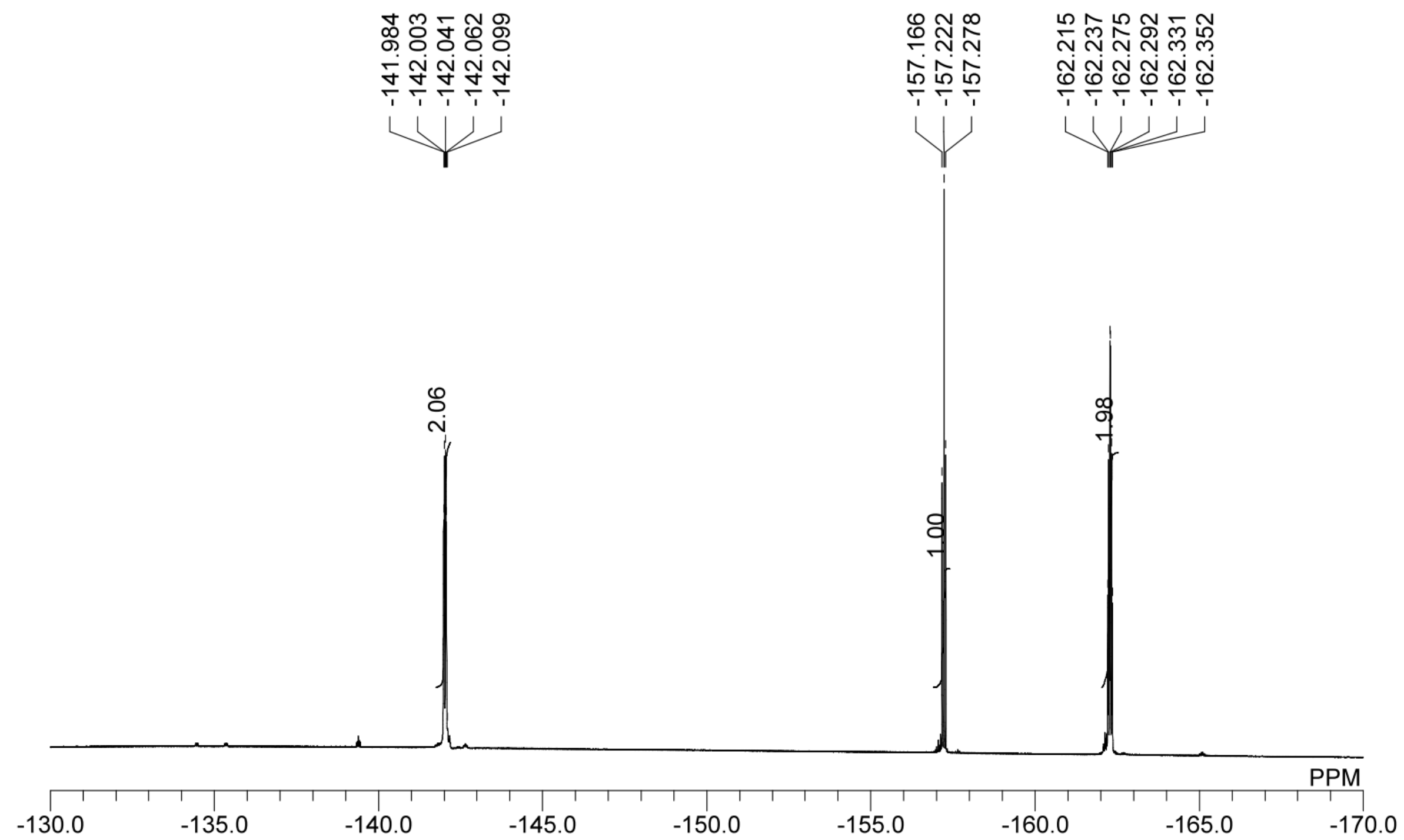


\section{${ }^{1} \mathrm{H}$ NMR of $\mathbf{4 a i}$}

广

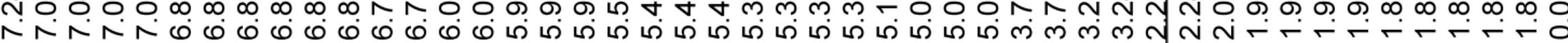
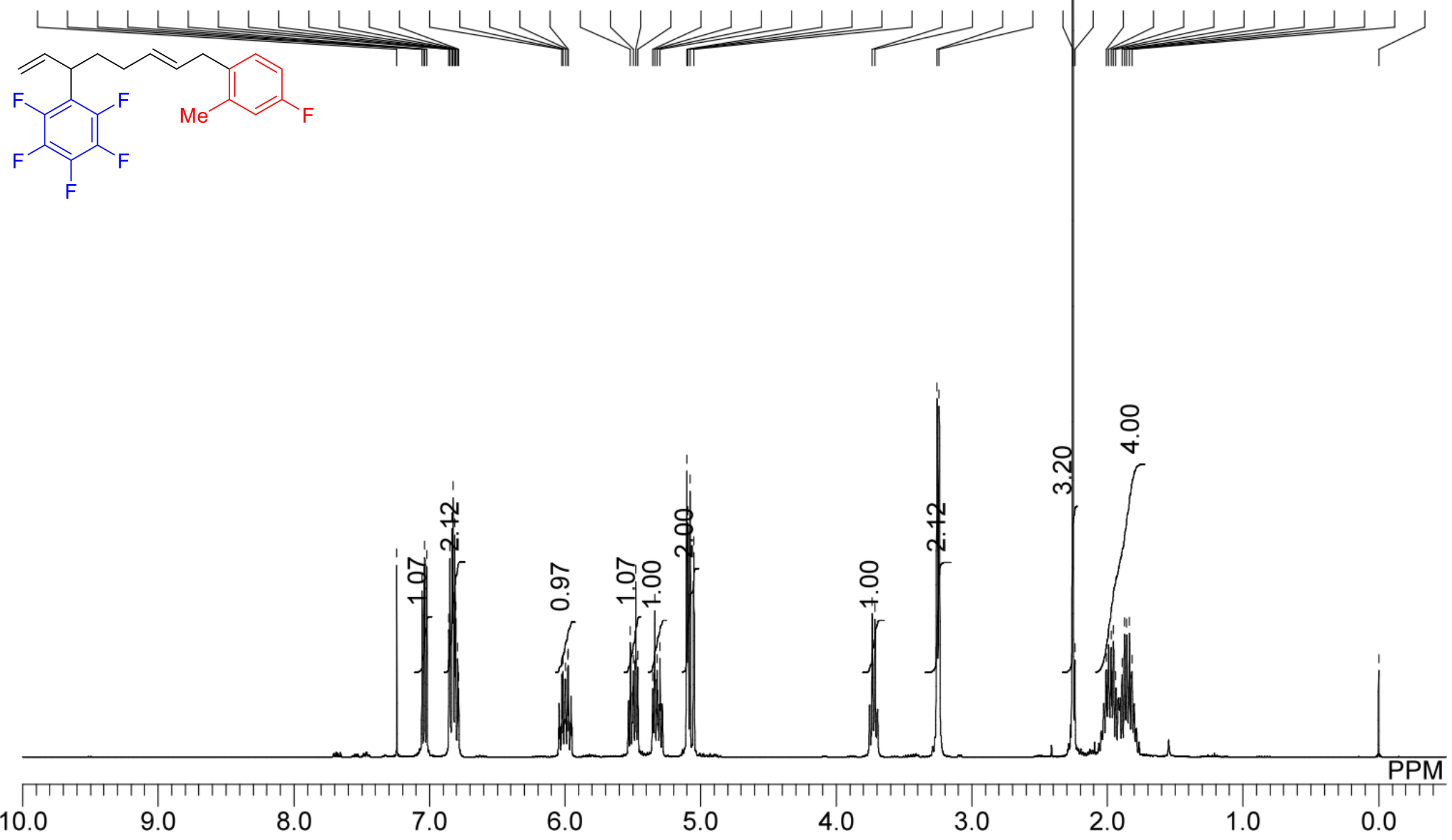
${ }^{13} \mathrm{C}\left\{{ }^{1} \mathrm{H}\right\}$ NMR of 4 ai

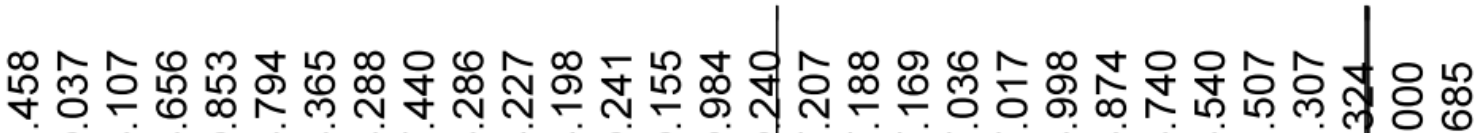
ஸ்

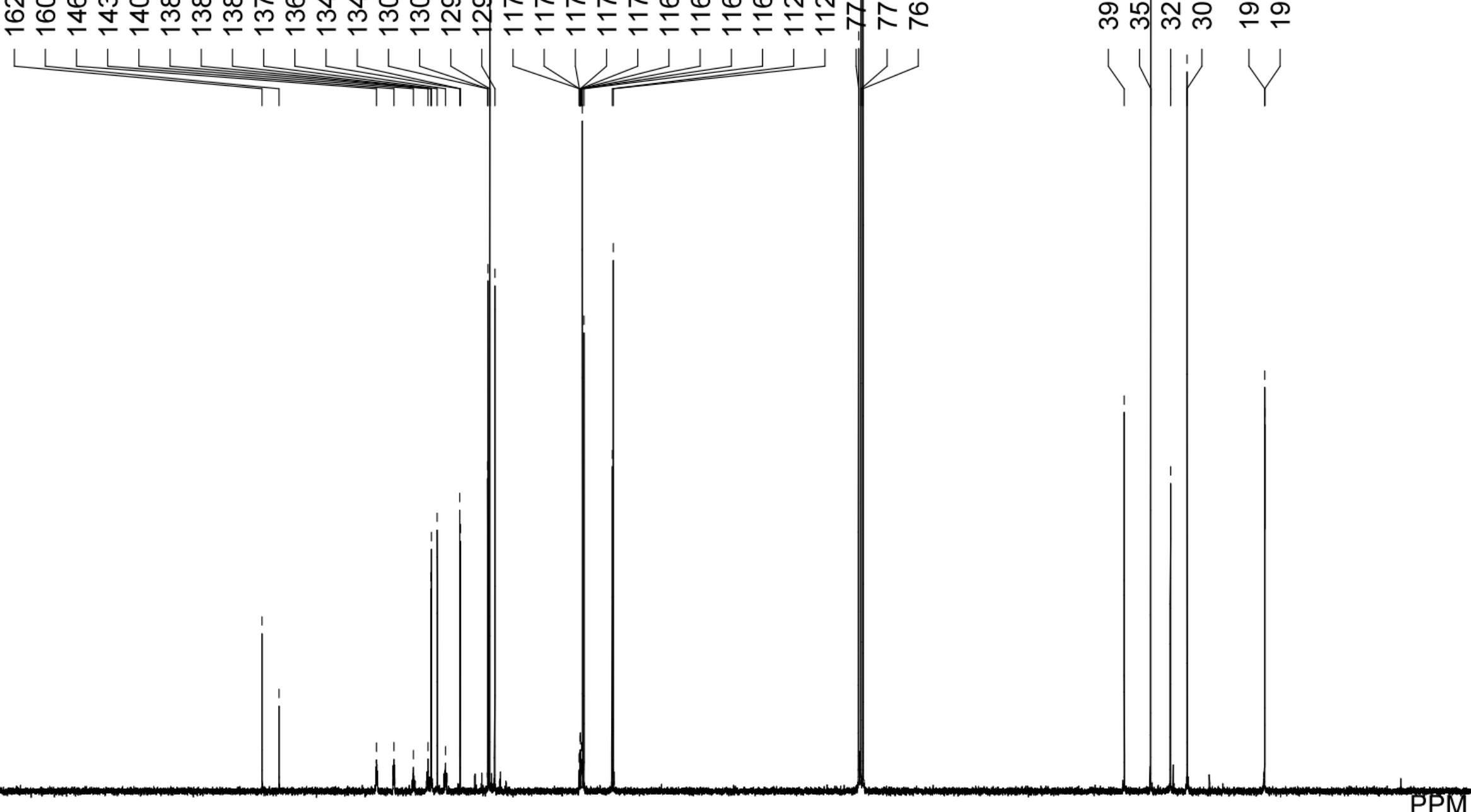


${ }^{19}$ F NMR of $4 a i$
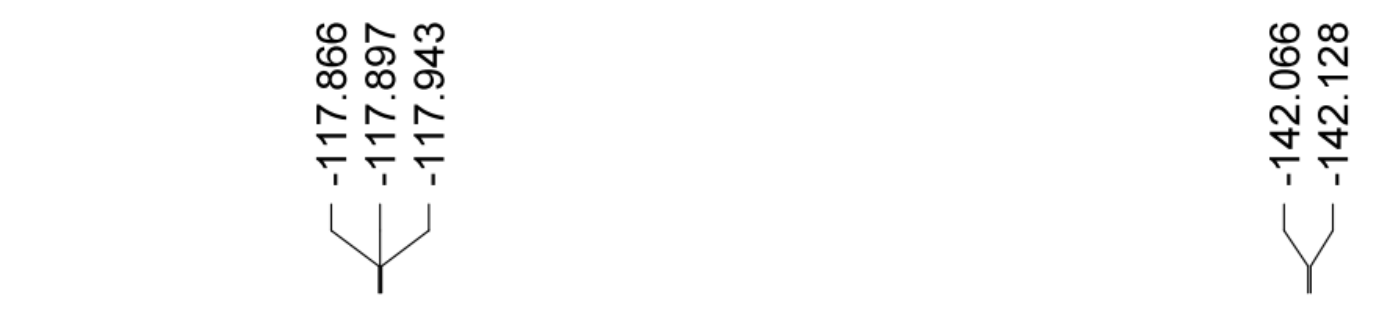

온 ํำ กิก กิชู่

$8 \stackrel{2}{\circ}$

\&

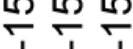

$\div \div$

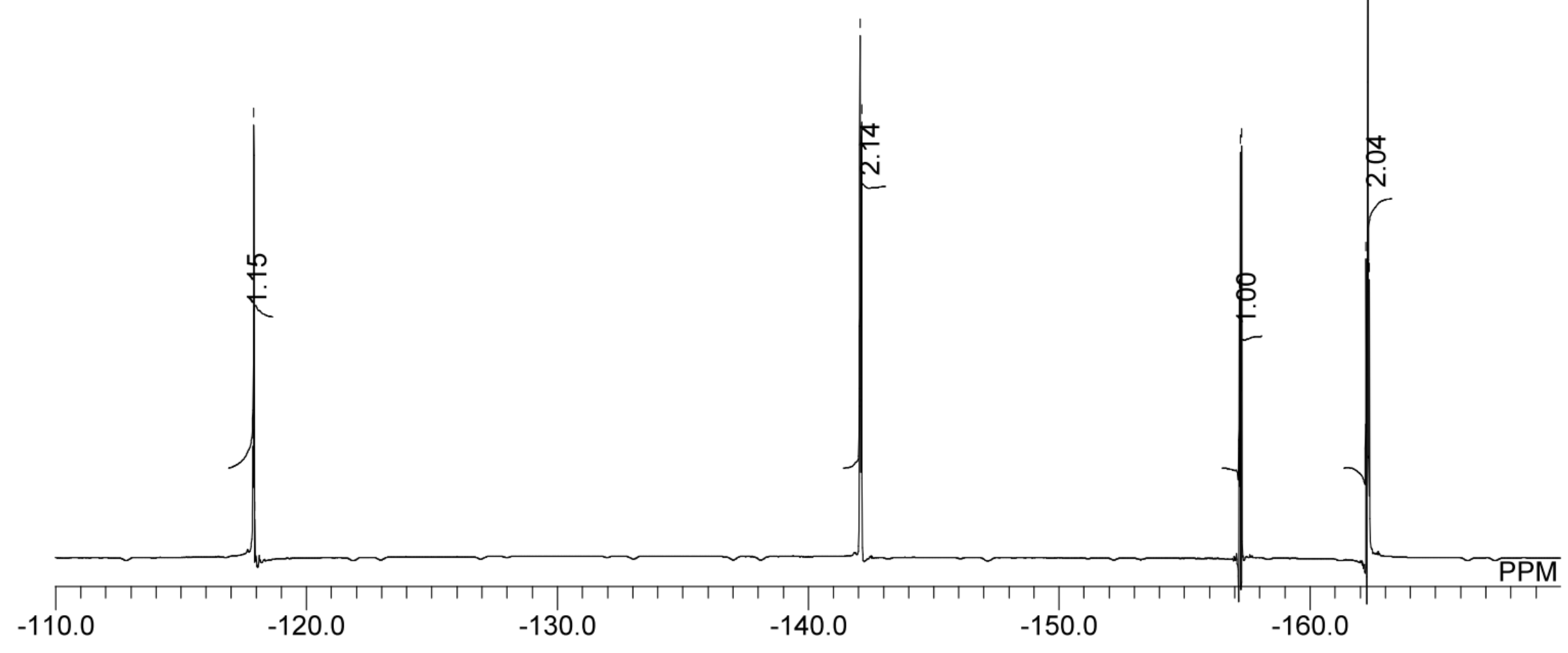




\section{${ }^{1} \mathrm{H}$ NMR of 4aj}

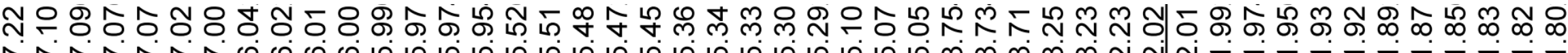

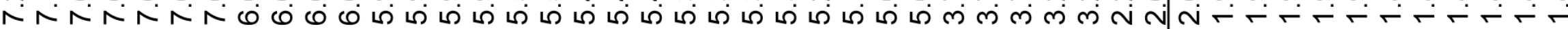
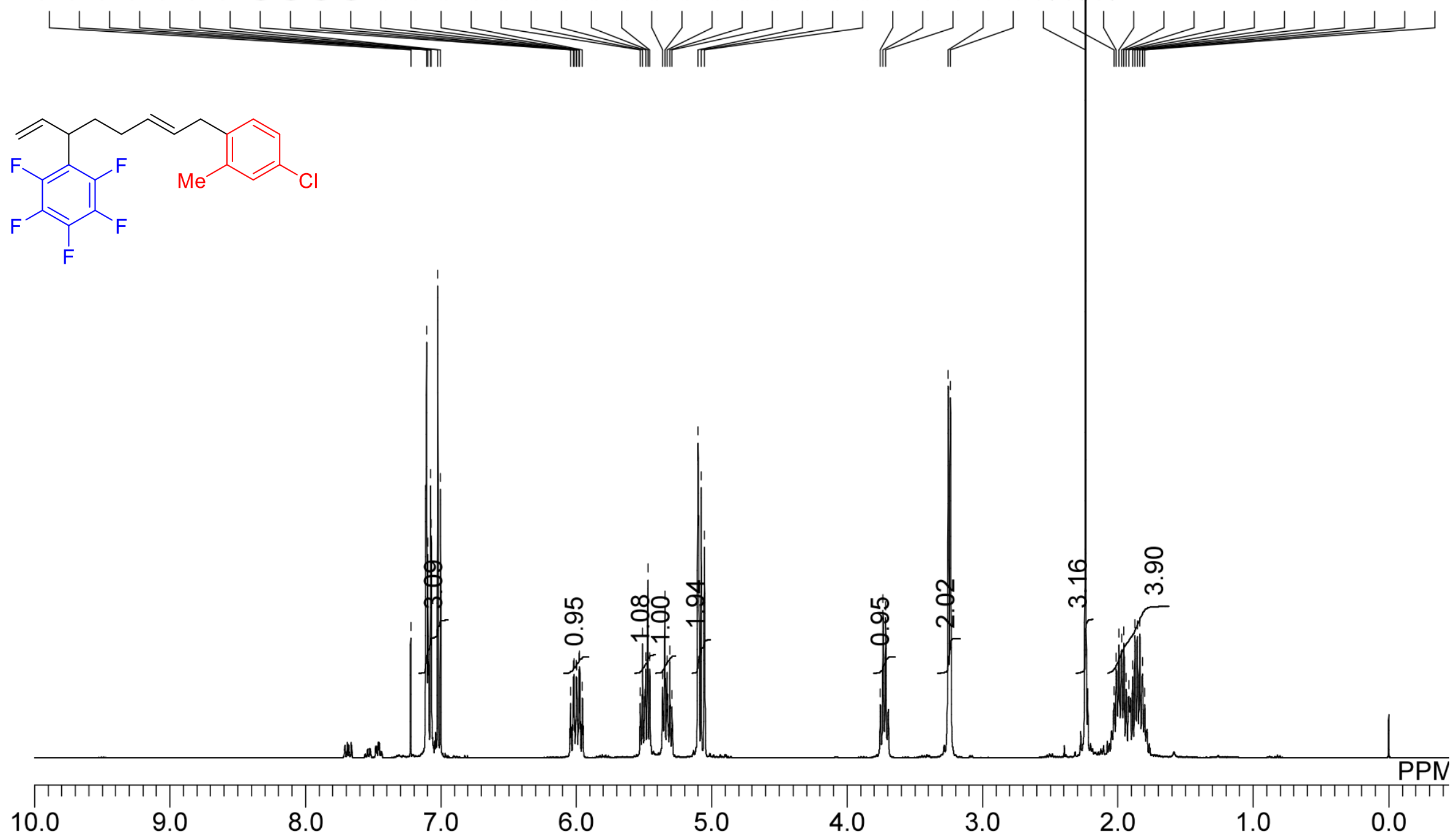
${ }^{13} \mathrm{C}\left\{{ }^{1} \mathrm{H}\right\}$ NMR of $\mathbf{4 a j}$

œ

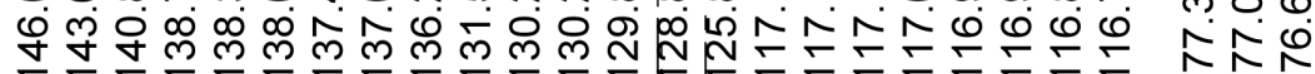

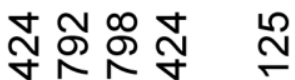

लेंल्लिंग्ले
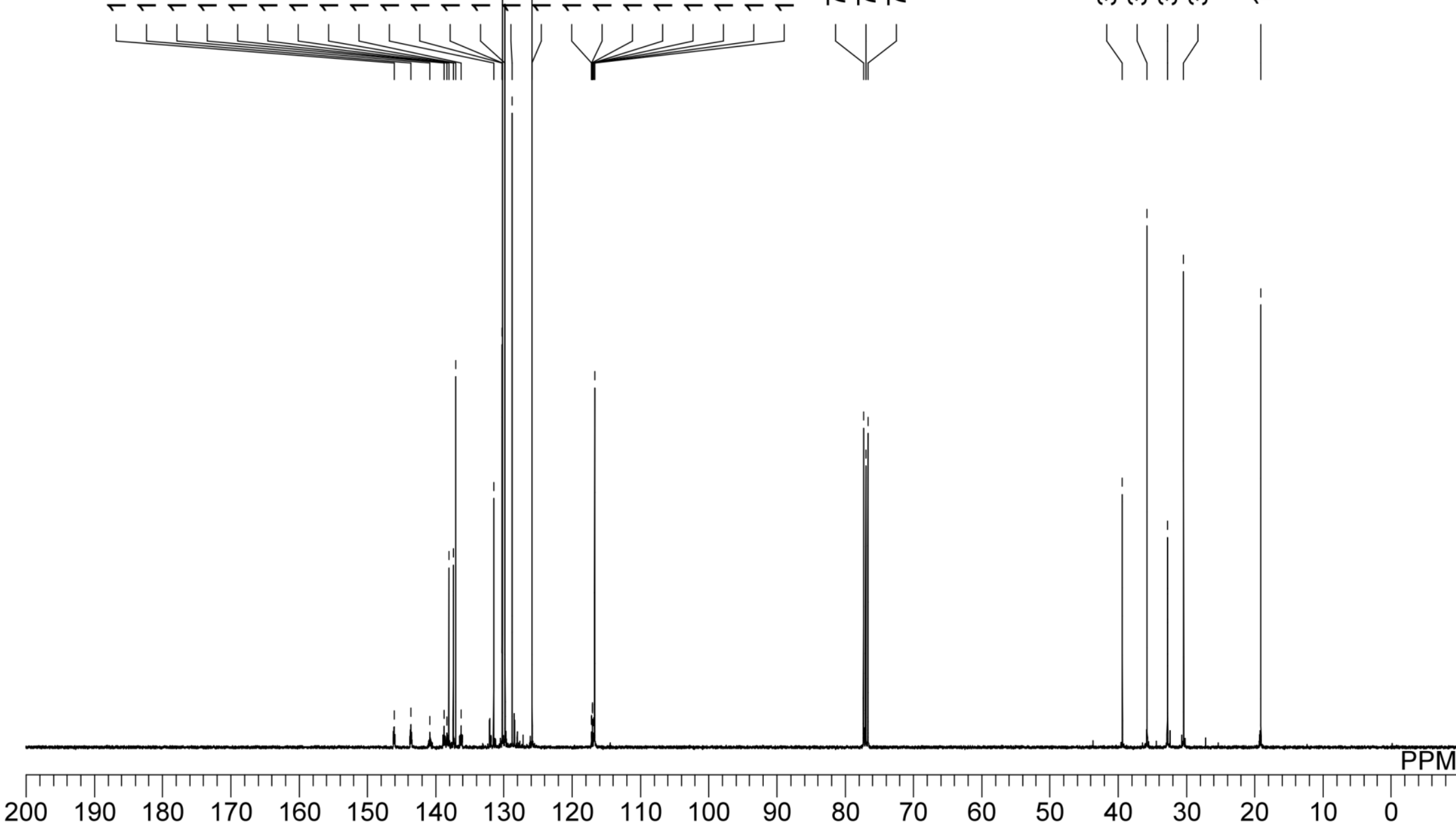
${ }^{19}$ F NMR of $\mathbf{4 a j}$

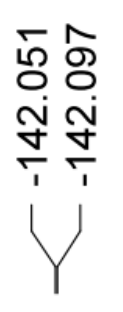

읃ำ ำ 워ำ

กิก กิ ชู่ ชู่

단 $\frac{0}{1} \frac{0}{1}$
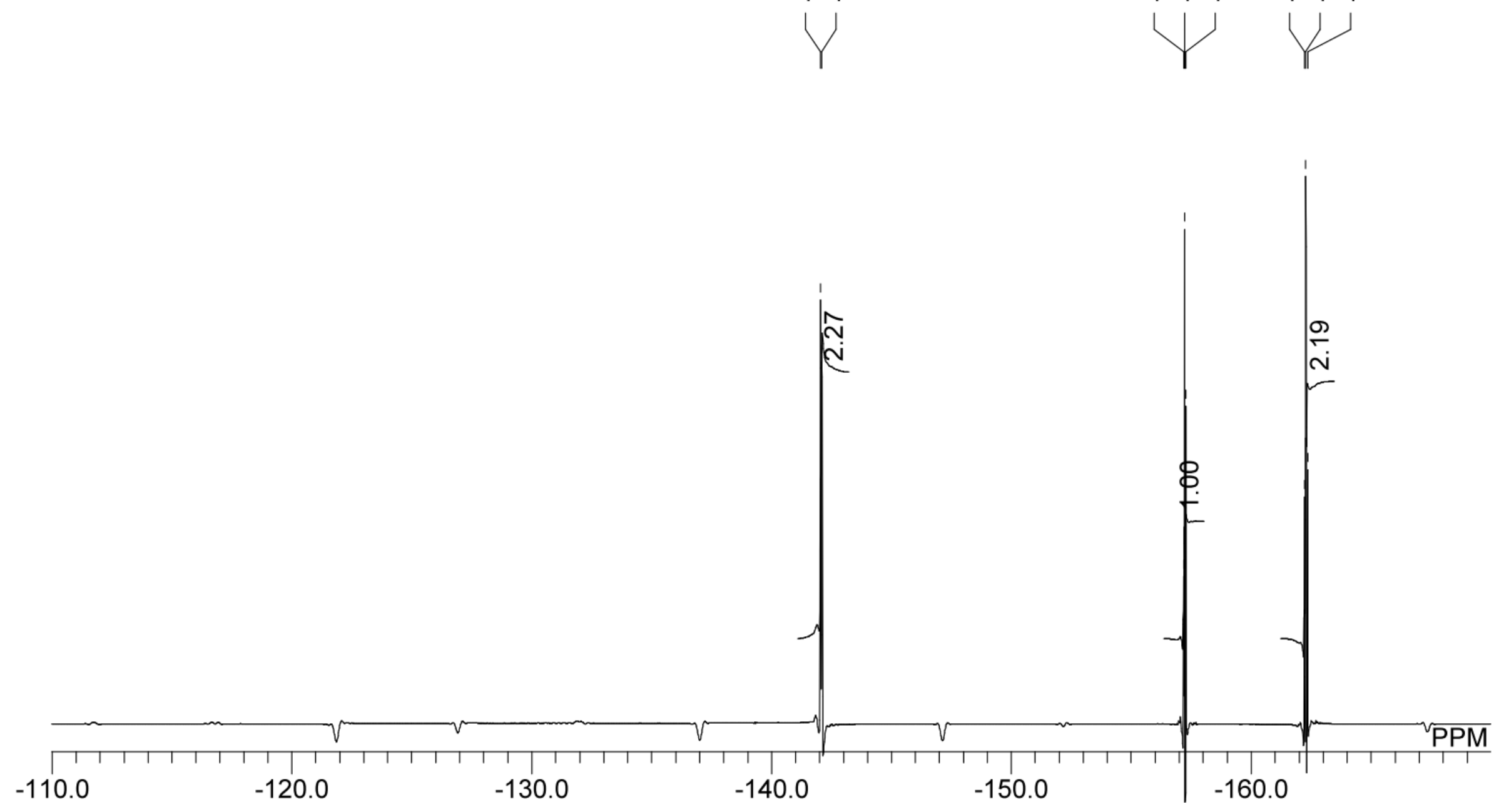


\section{${ }^{1} \mathrm{H}$ NMR of 4ak}

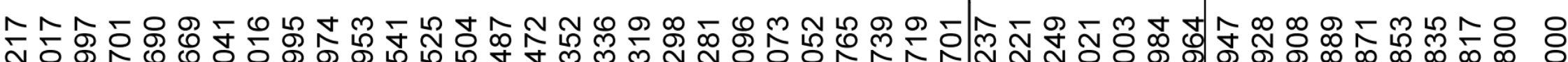
ก
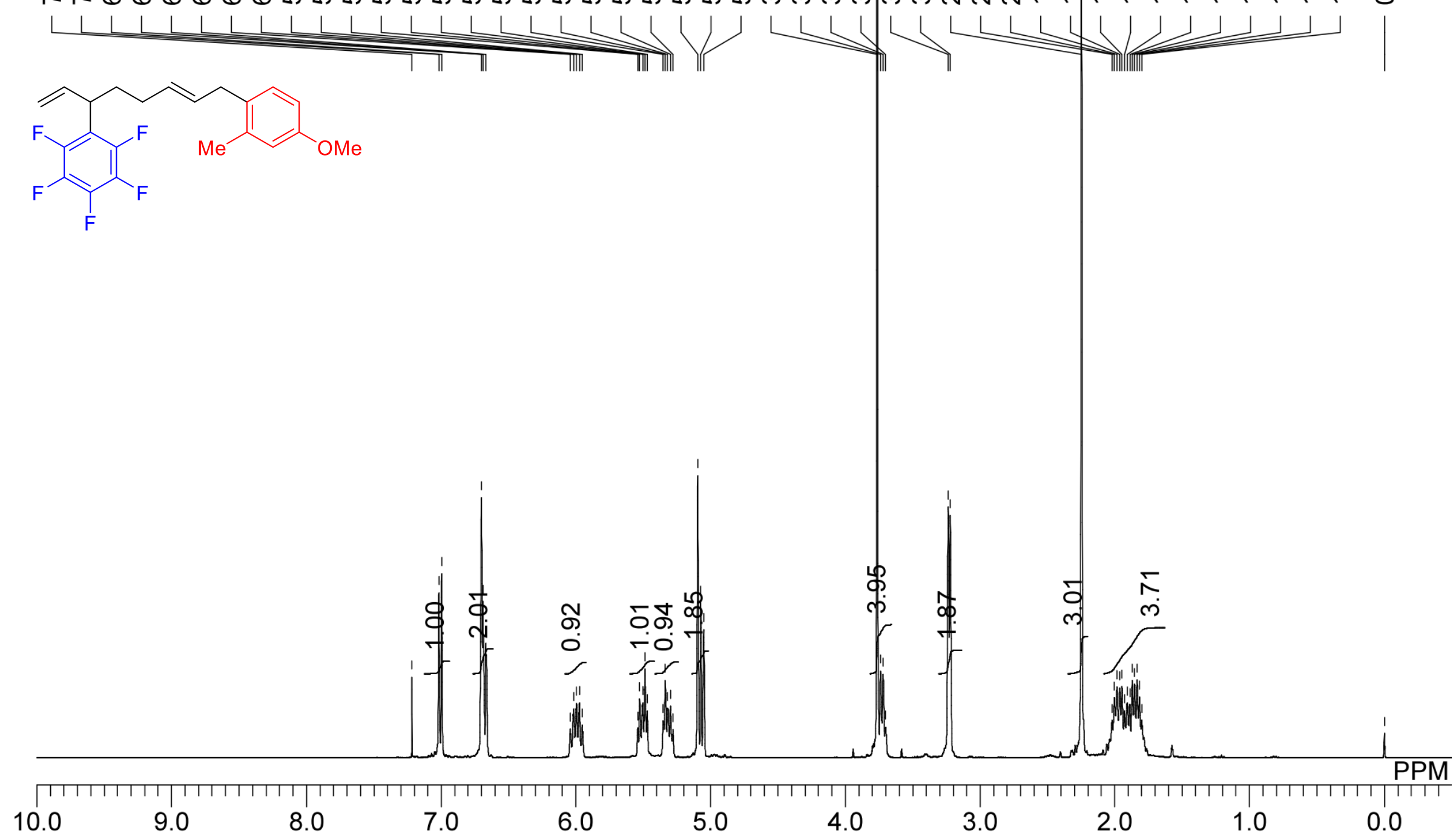


\section{${ }^{13} \mathrm{C}\left\{{ }^{1} \mathrm{H}\right\}$ NMR of $\mathbf{4 a k}$}

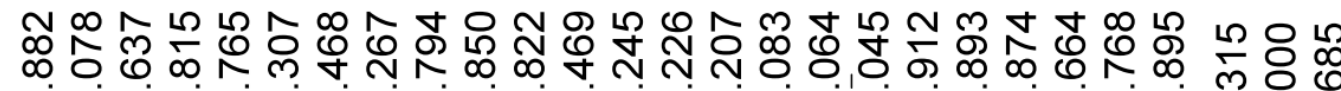

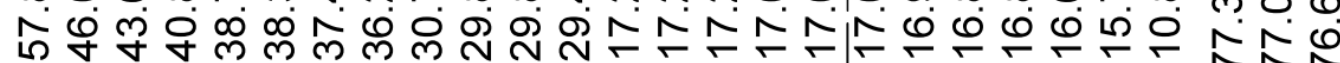

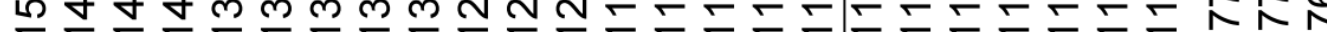
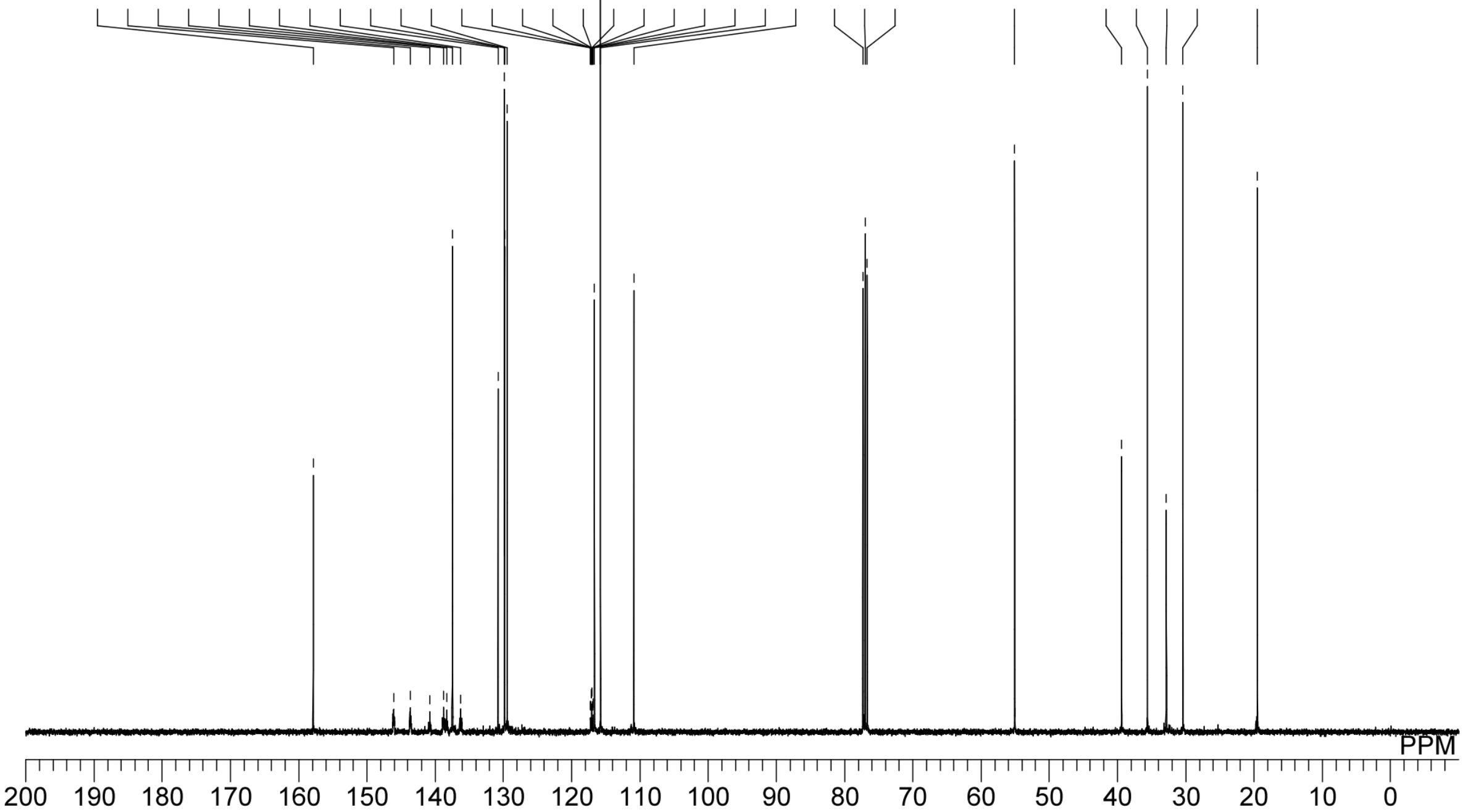
${ }^{19}$ F NMR of $\mathbf{4 a k}$
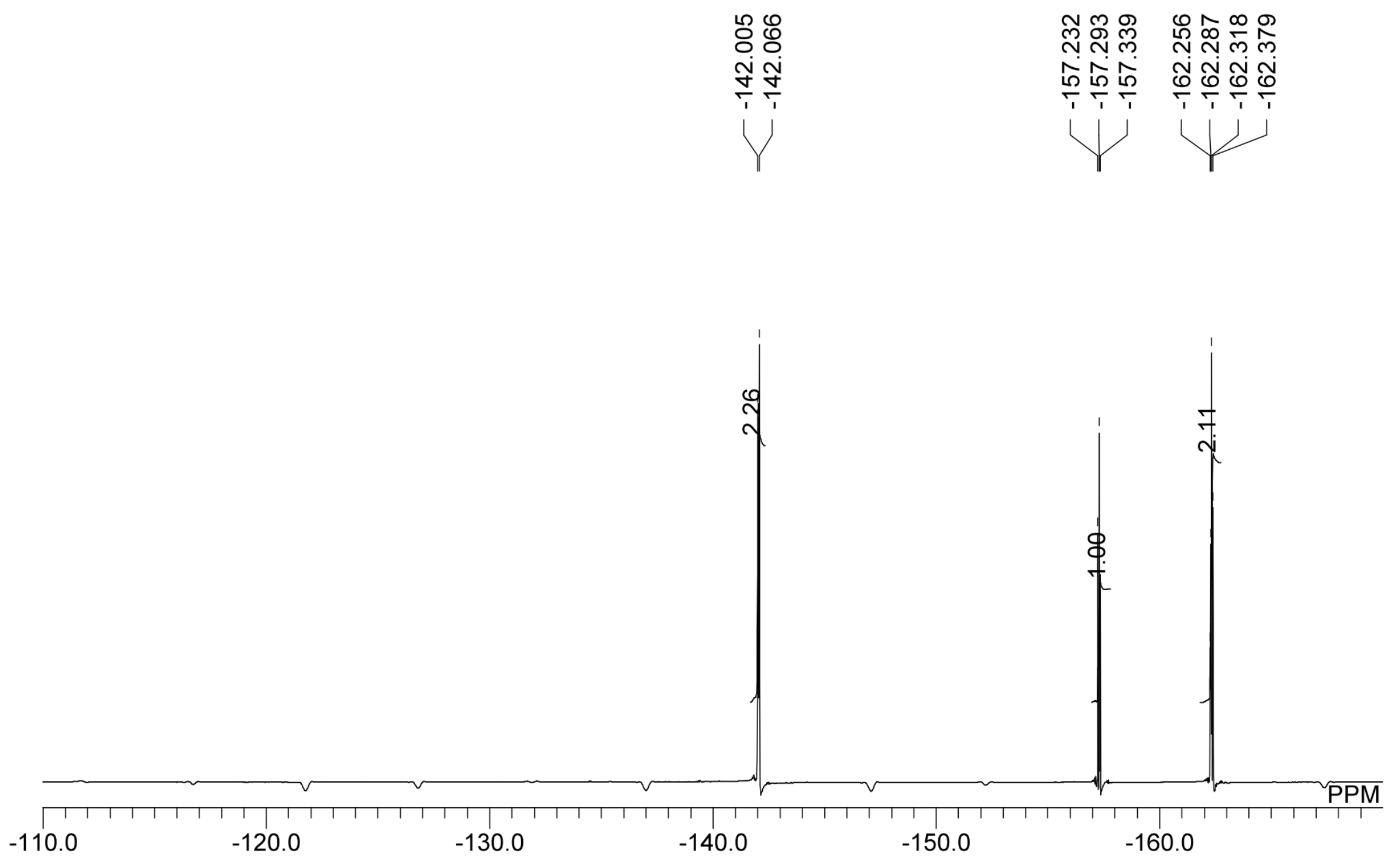


\section{${ }^{1} \mathrm{H}$ NMR of $4 \mathbf{a l}$}

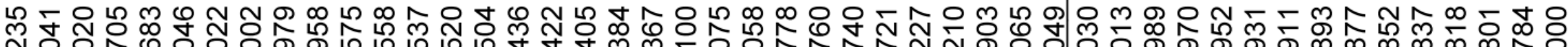
ヘ

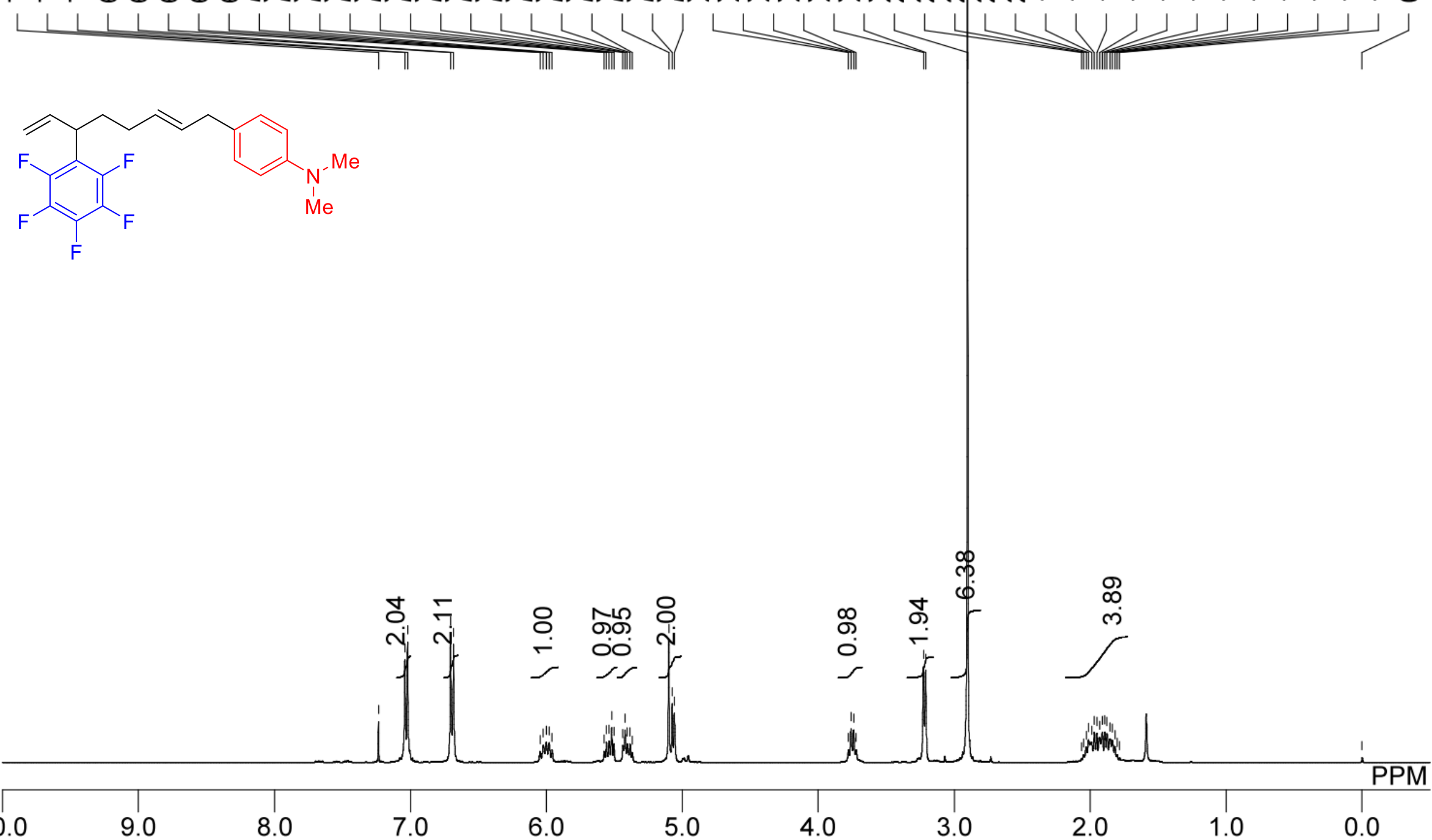


${ }^{13} \mathrm{C}\left\{{ }^{1} \mathrm{H}\right\}$ NMR of $\mathbf{4 a l}$

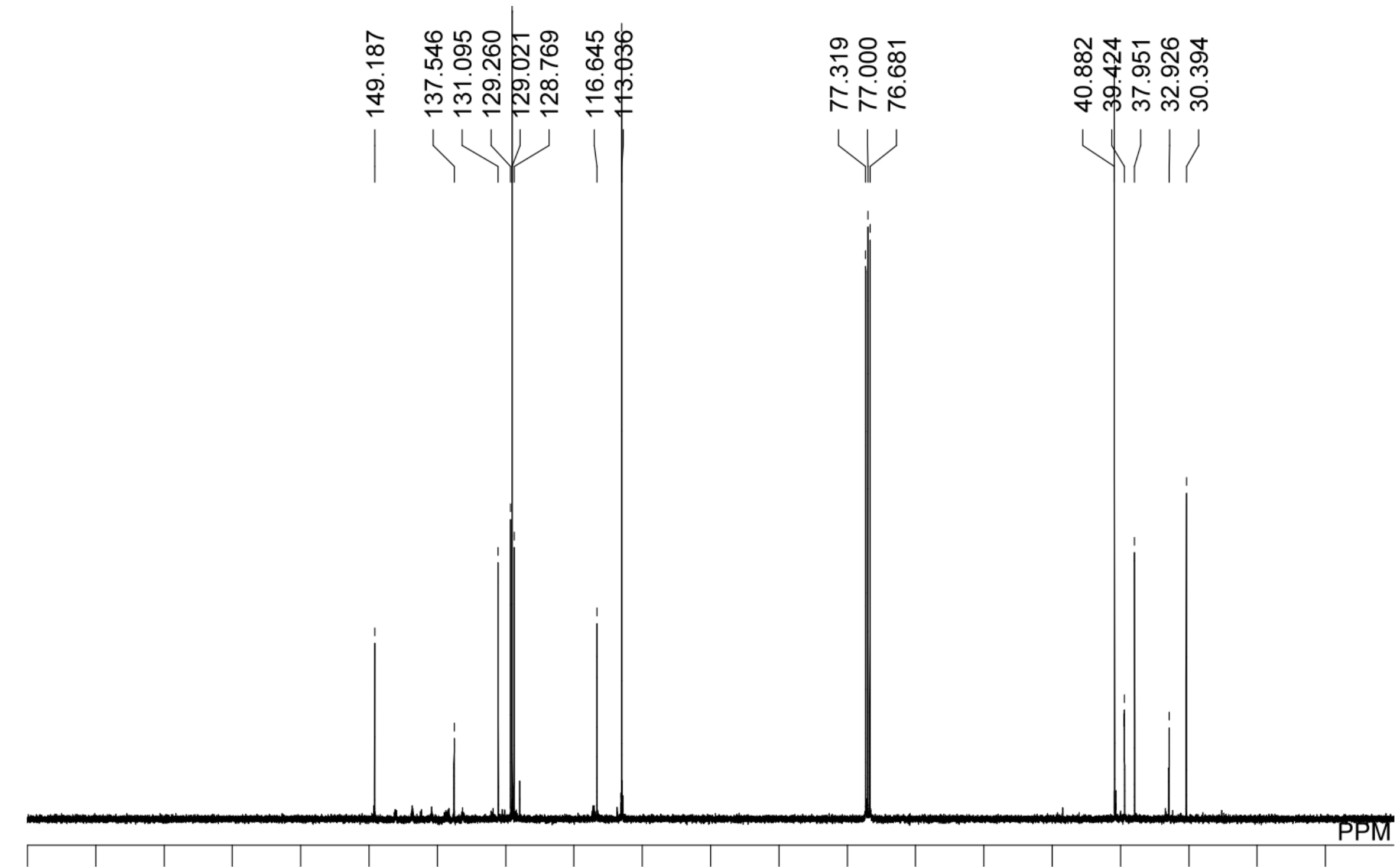

$200.0190 .0180 .0170 .0160 .0150 .0140 .0130 .0120 .0110 .0100 .0 \quad 90.0 \quad 80.0 \quad 70.0 \quad 60.0 \quad 50.0 \quad 40.0 \quad 30.0 \quad 20.0 \quad 10.0$ 
${ }^{19} \mathrm{~F}$ NMR of $\mathbf{4 a} \boldsymbol{l}$

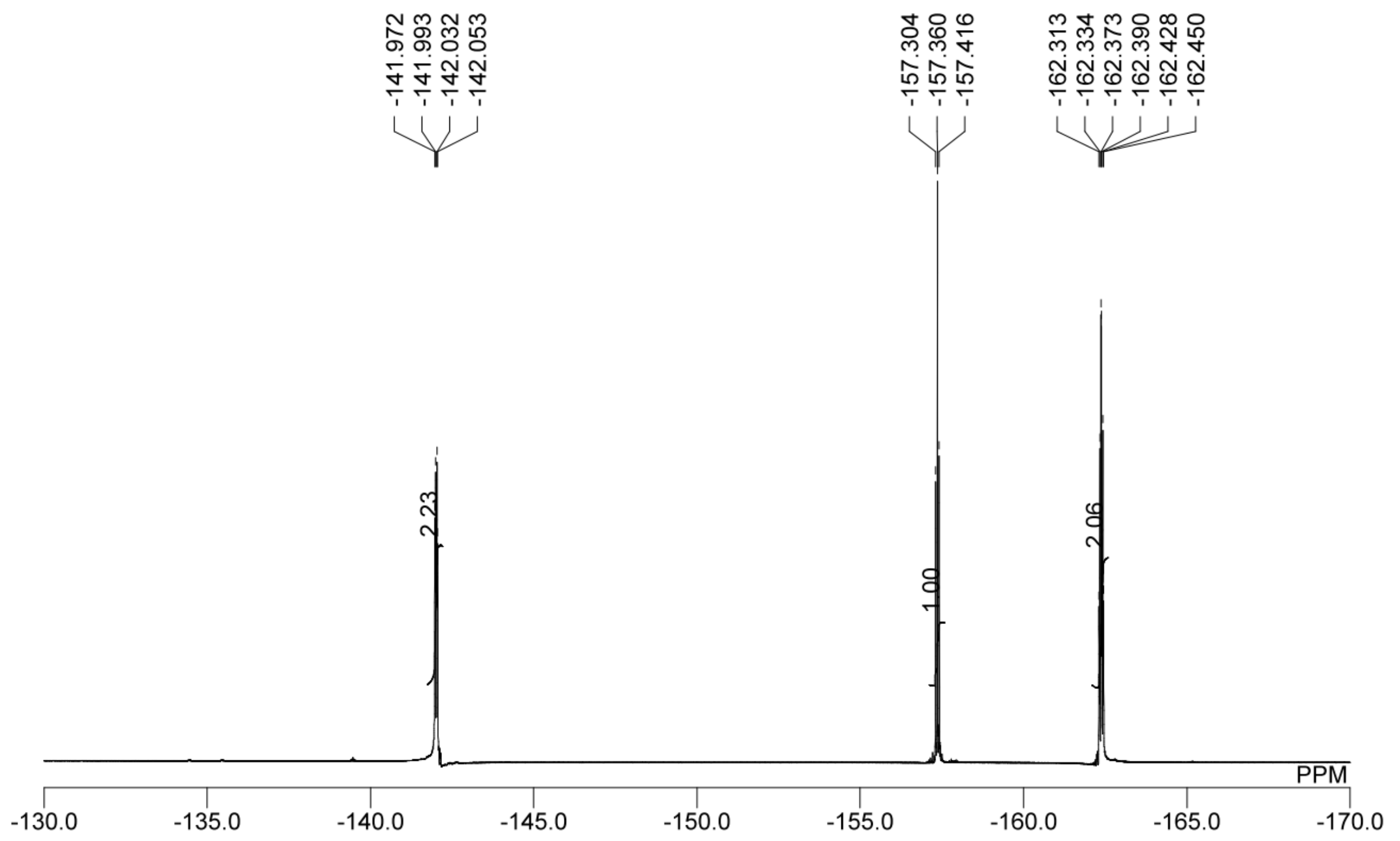




\section{${ }^{1} \mathrm{H}$ NMR of $4 \mathbf{a m}$}

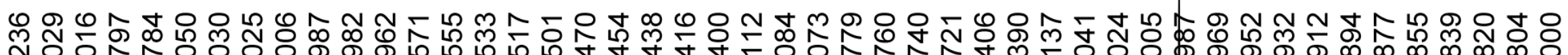
N
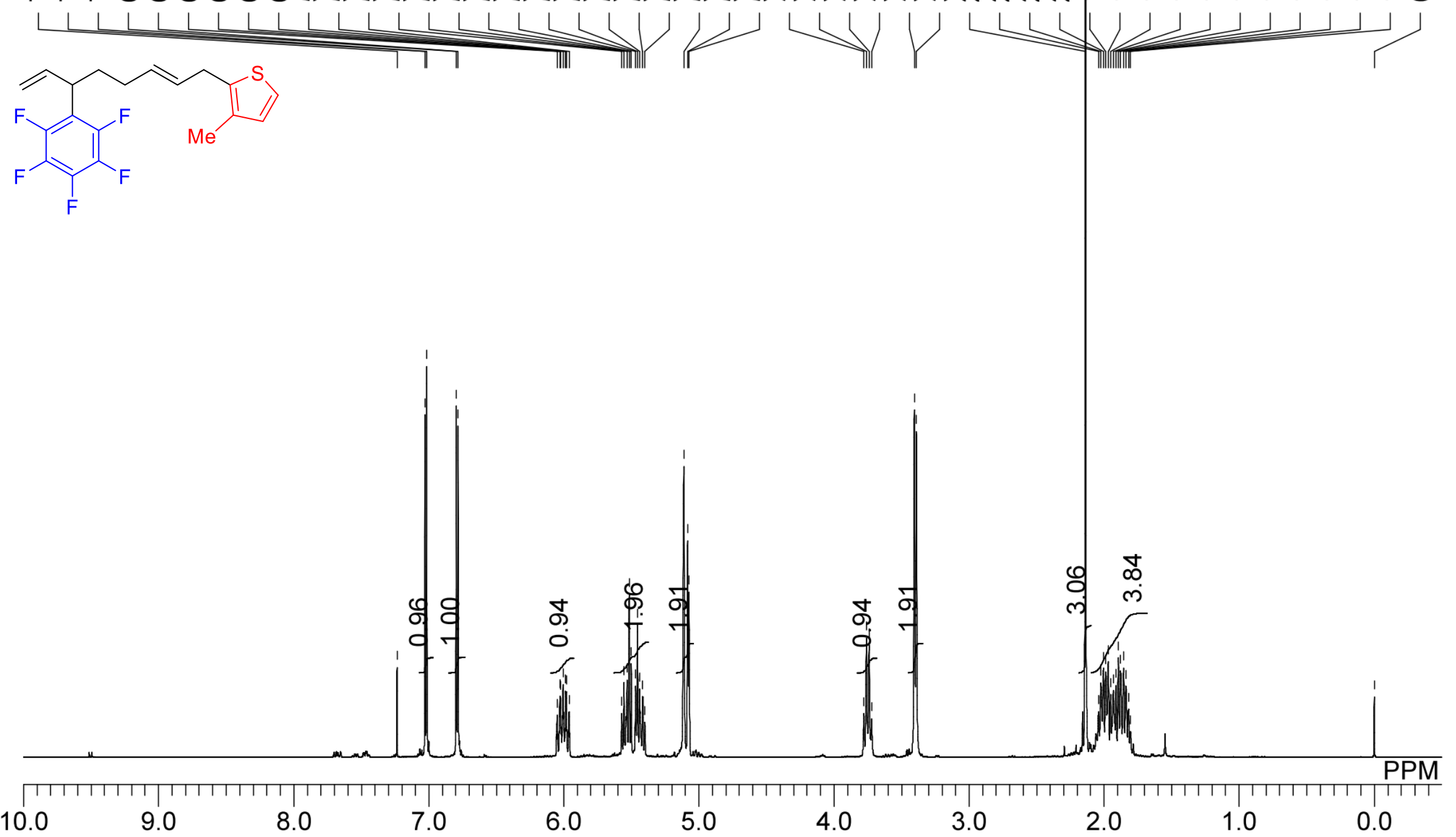


\section{${ }^{13} \mathrm{C}\left\{{ }^{1} \mathrm{H}\right\}$ NMR of $\mathbf{4 a m}$}

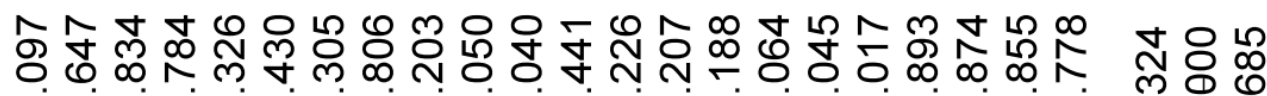

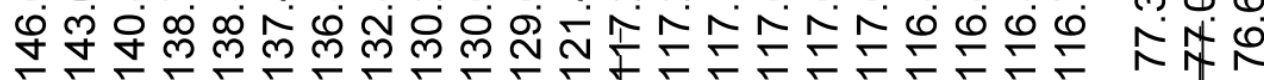

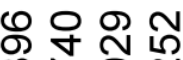

ก $\mathrm{N}$

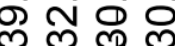

$\underset{+\infty}{\infty}$
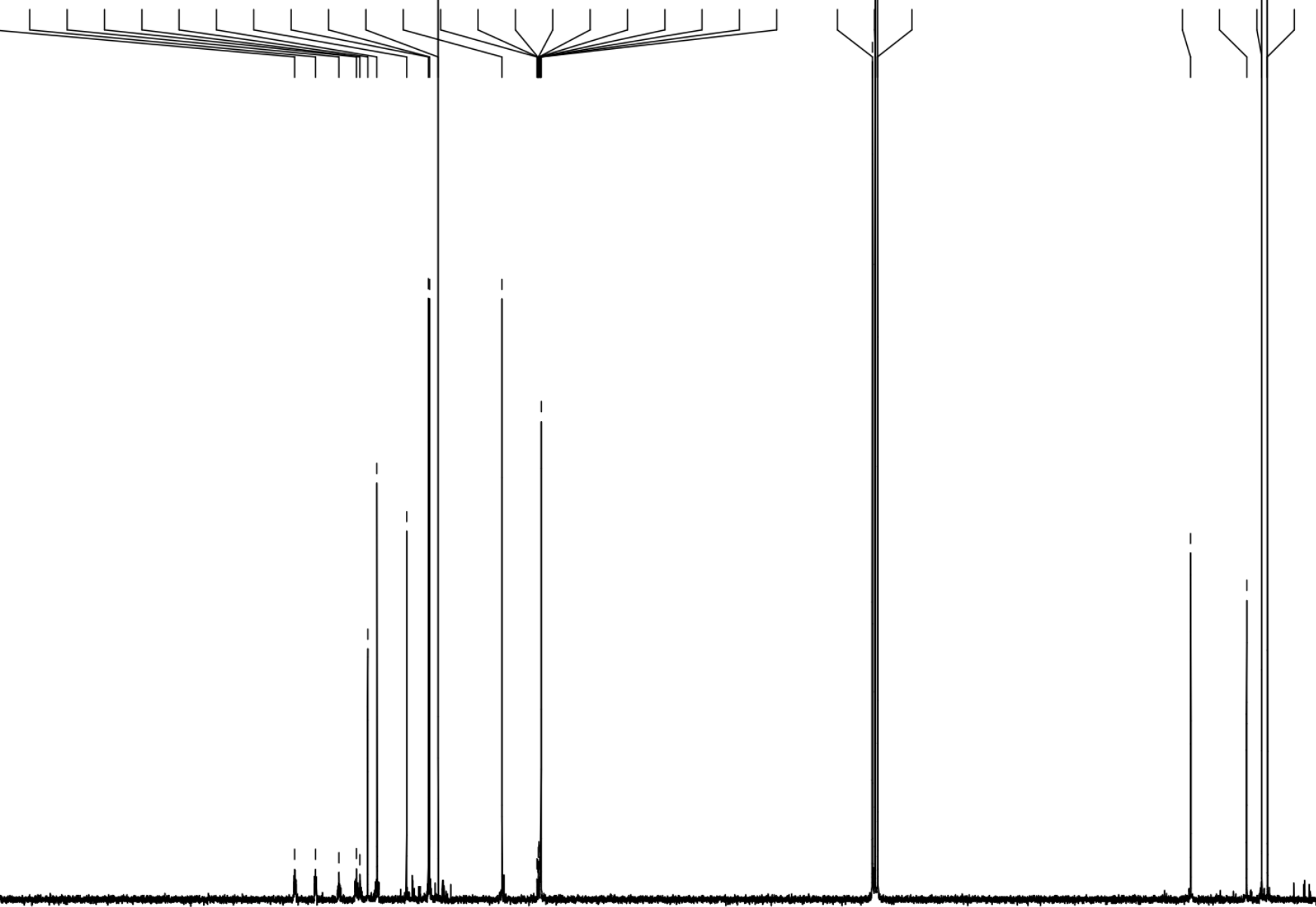

PPM

|

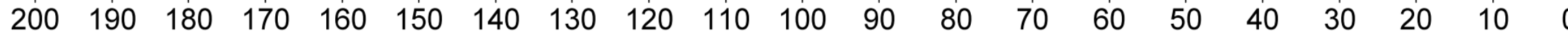


${ }^{19} \mathrm{~F}$ NMR of 4 am

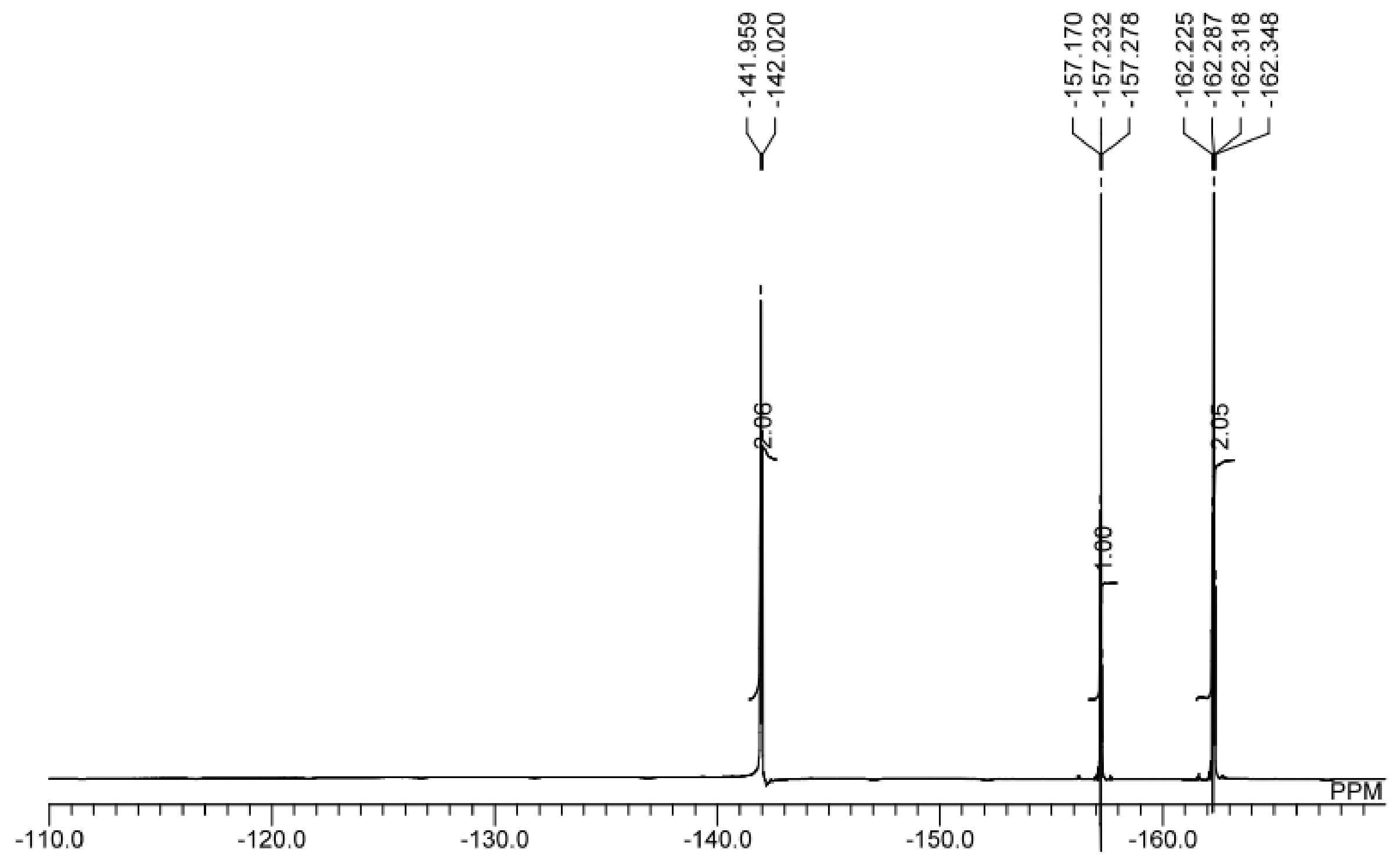


${ }^{1} \mathrm{H}$ NMR of 4an

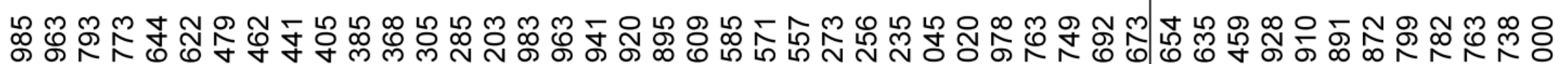

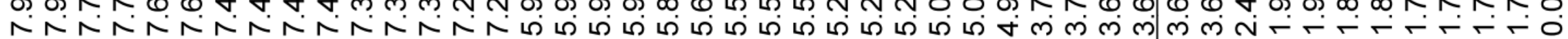
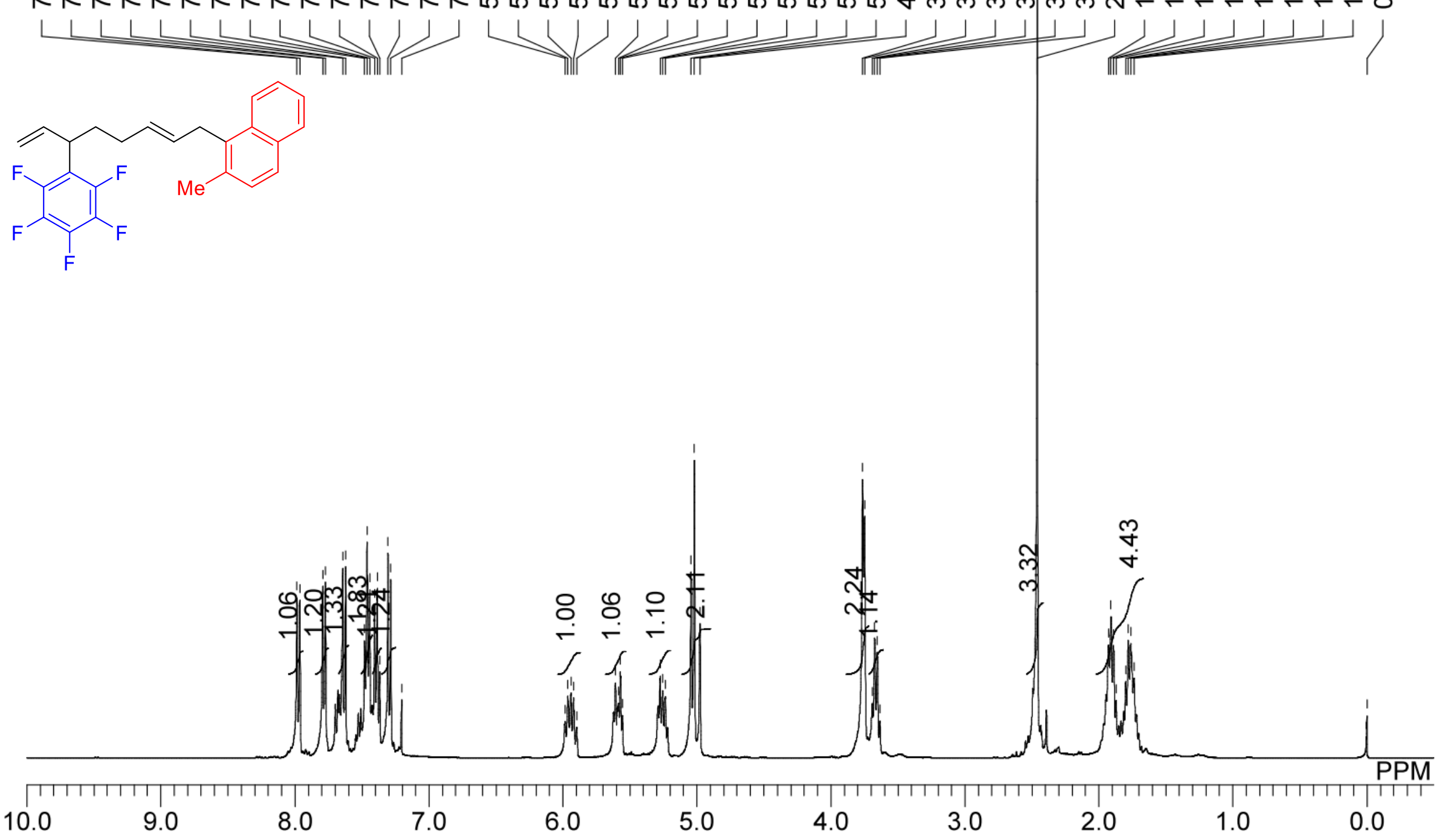


\section{${ }^{13} \mathrm{C}\left\{{ }^{1} \mathrm{H}\right\}$ NMR of 4an}

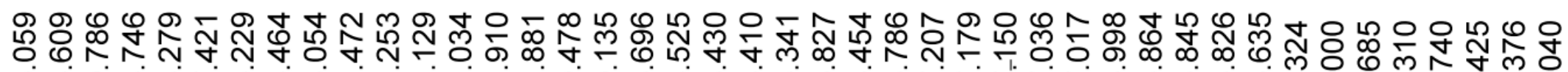

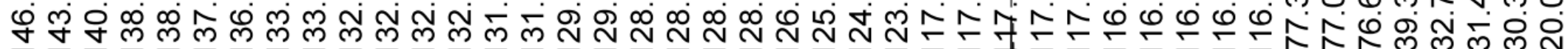

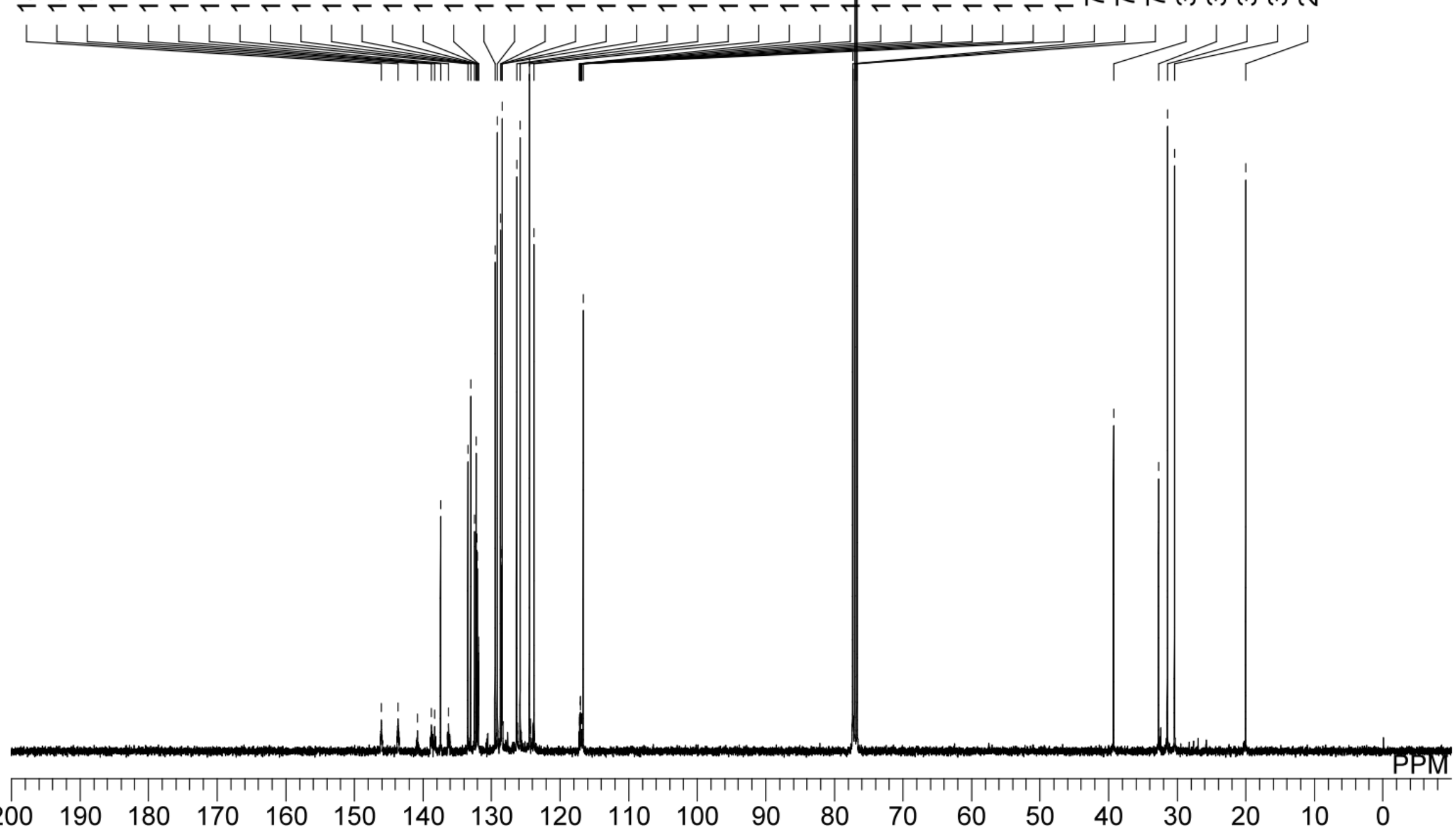


${ }^{19} \mathrm{~F}$ NMR of $4 \mathbf{a n}$

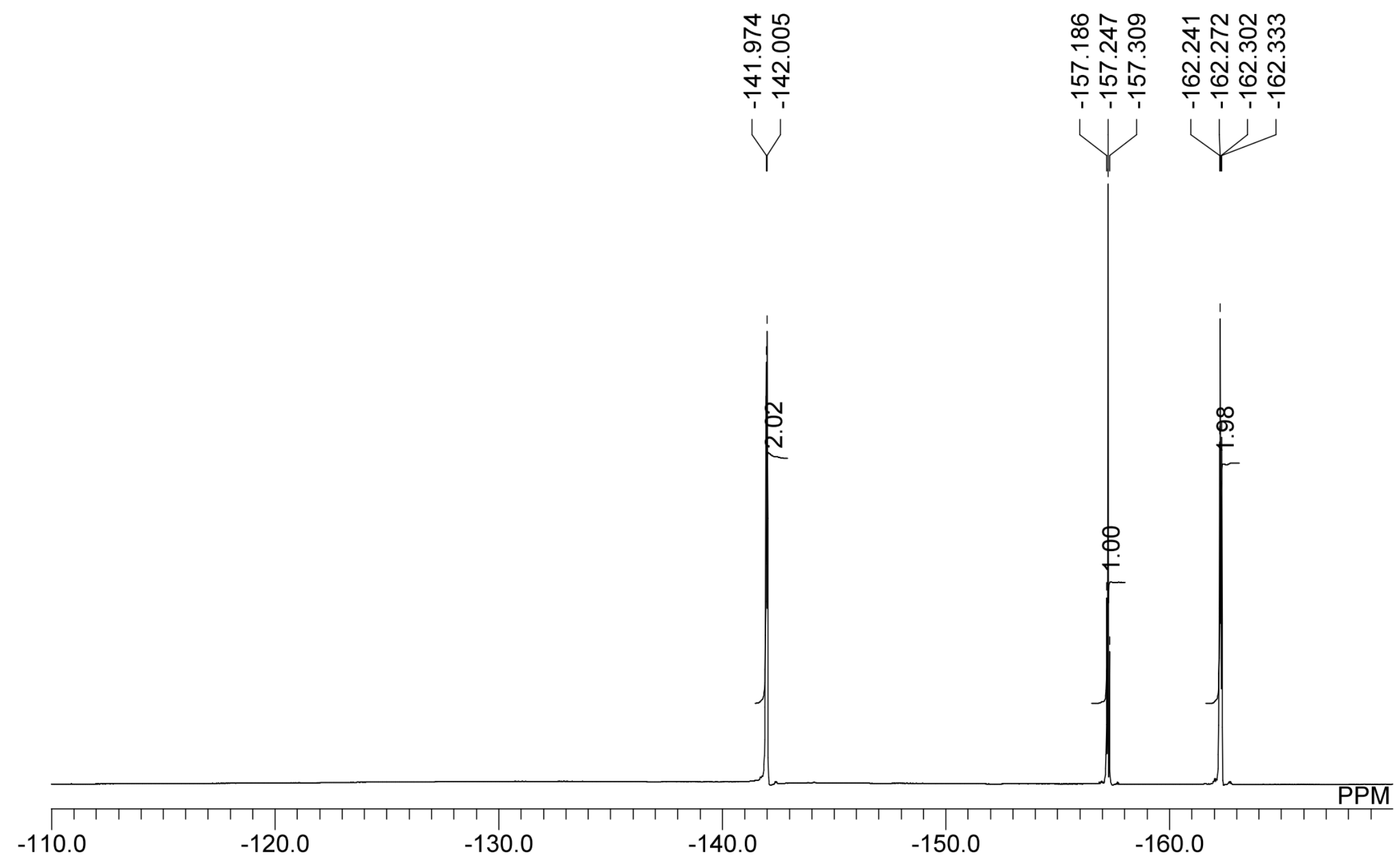




\section{${ }^{1} \mathrm{H}$ NMR of 4 ao}

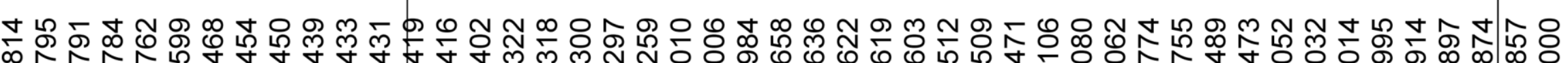

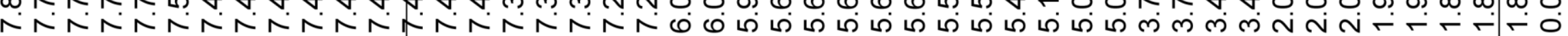
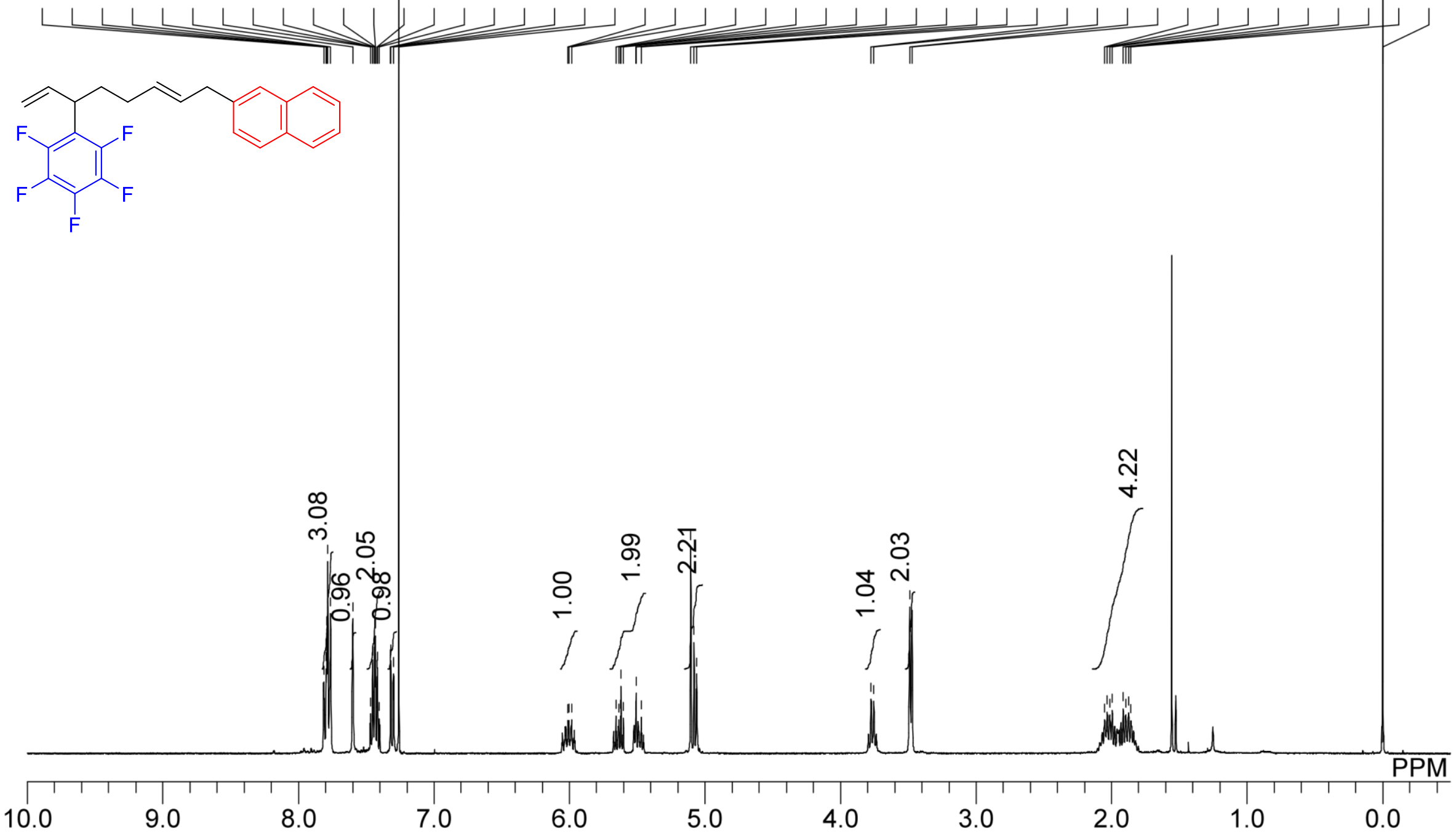
${ }^{13} \mathrm{C}\left\{{ }^{1} \mathrm{H}\right\}$ NMR of $4 \mathbf{a o}$

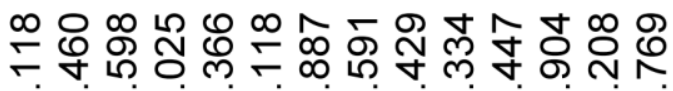

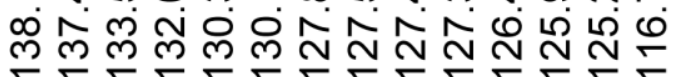
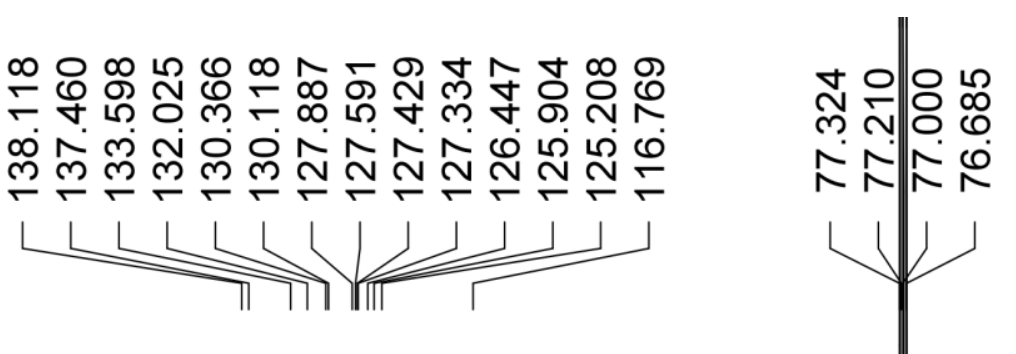

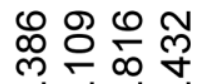

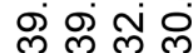
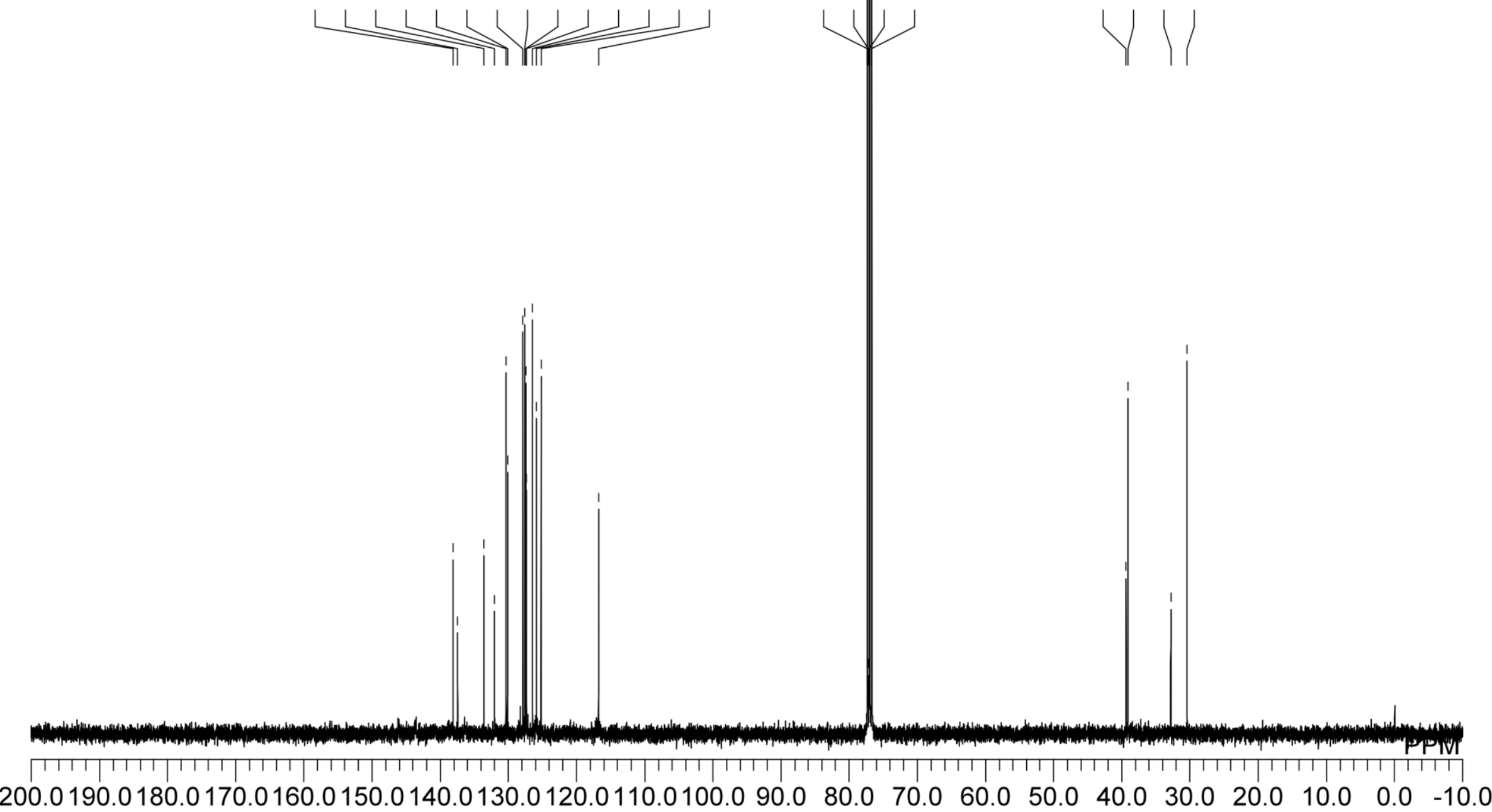
${ }^{19} \mathrm{~F}$ NMR of $4 \mathbf{a o}$

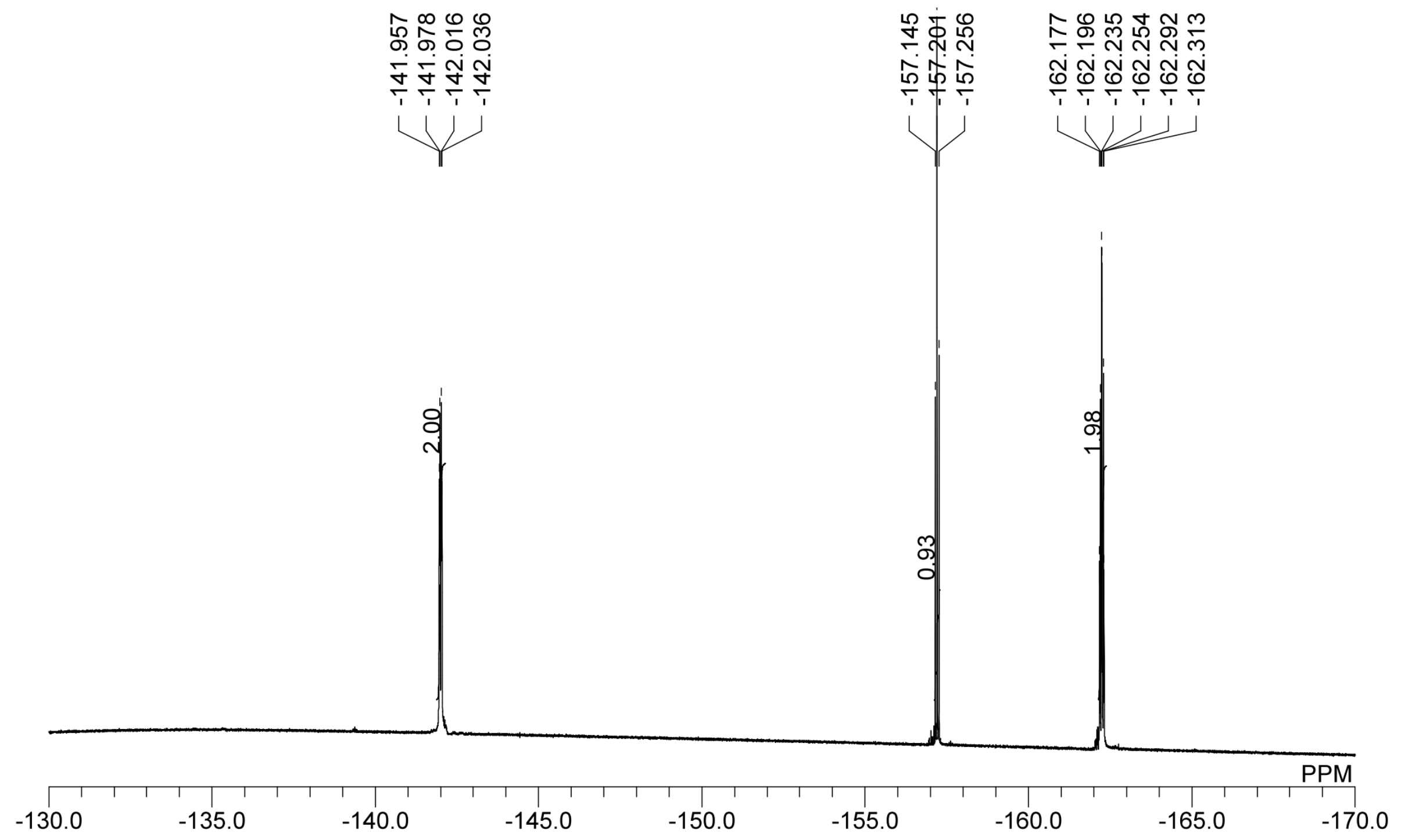


${ }^{1} \mathrm{H}$ NMR of mixture of $\mathbf{4 b a}$ and its regioisomer ( $\left.p: m=94: 6\right)$

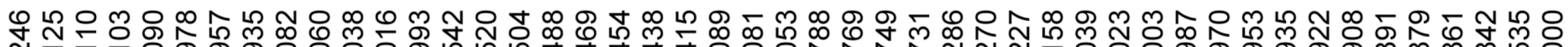
느능 N
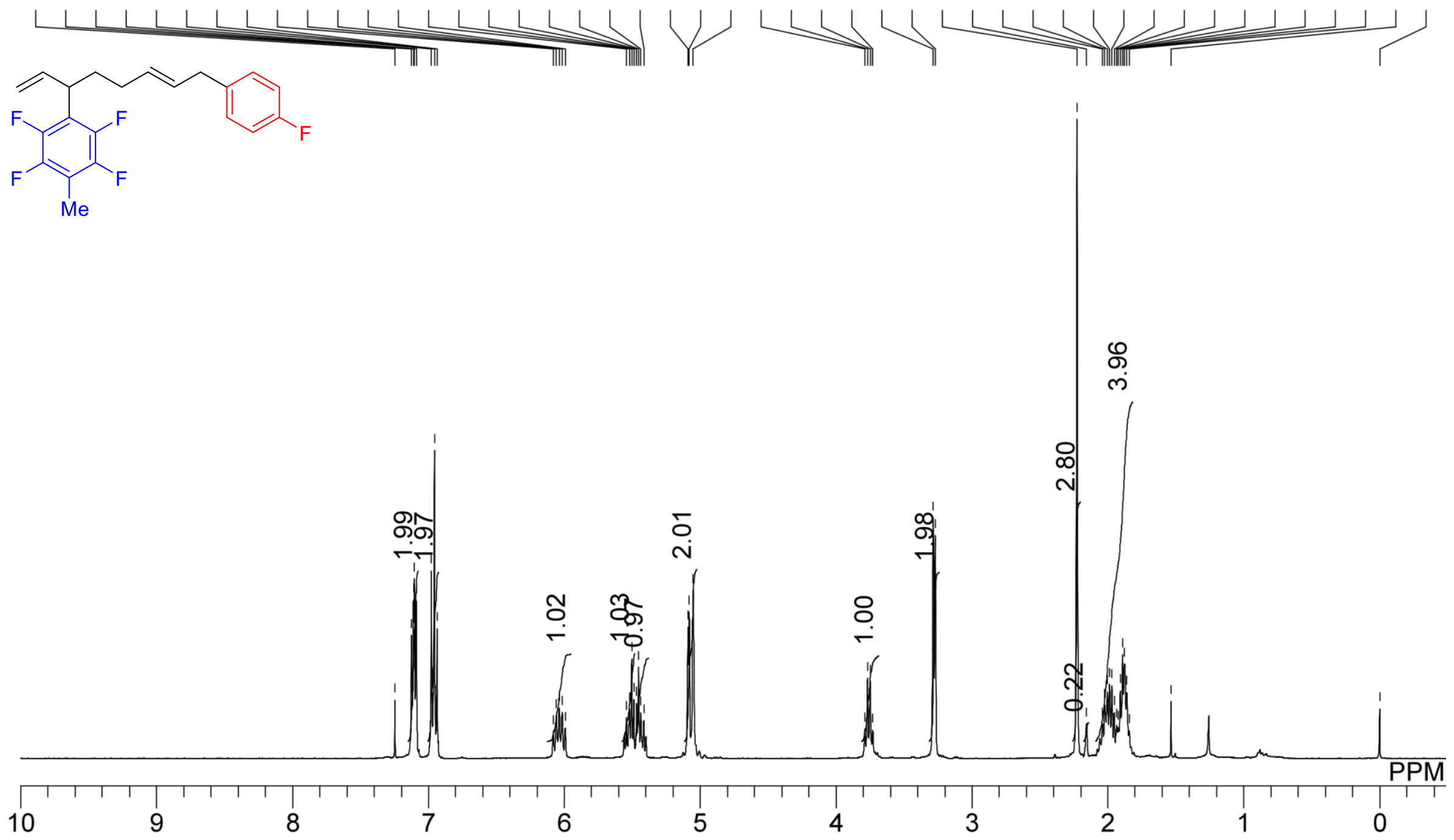
${ }^{13} \mathrm{C}\left\{{ }^{1} \mathrm{H}\right\}$ NMR of mixture of $\mathbf{4 b a}$ and its regioisomer ( $p: m=94: 6$ )

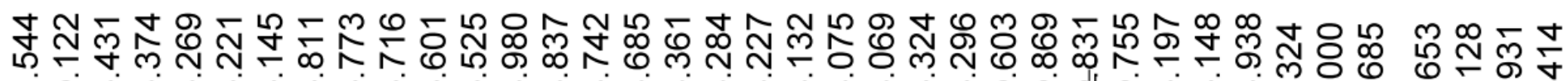
ชิ

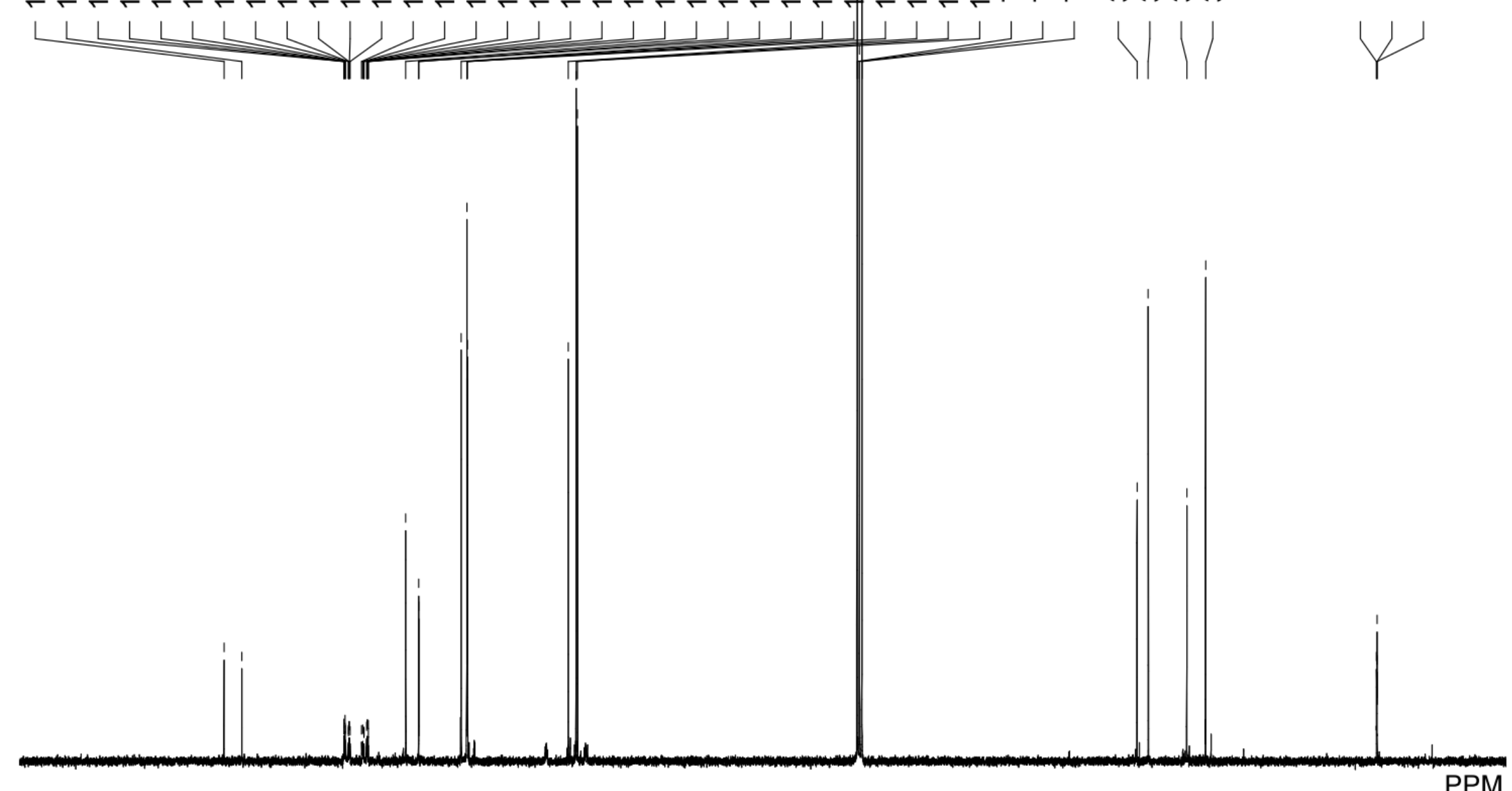


${ }^{13} \mathrm{C}\left\{{ }^{19} \mathrm{~F}\right\}$ NMR of mixture of $\mathbf{4 b a}$ and its regioisomer $(p: m=94: 6)$
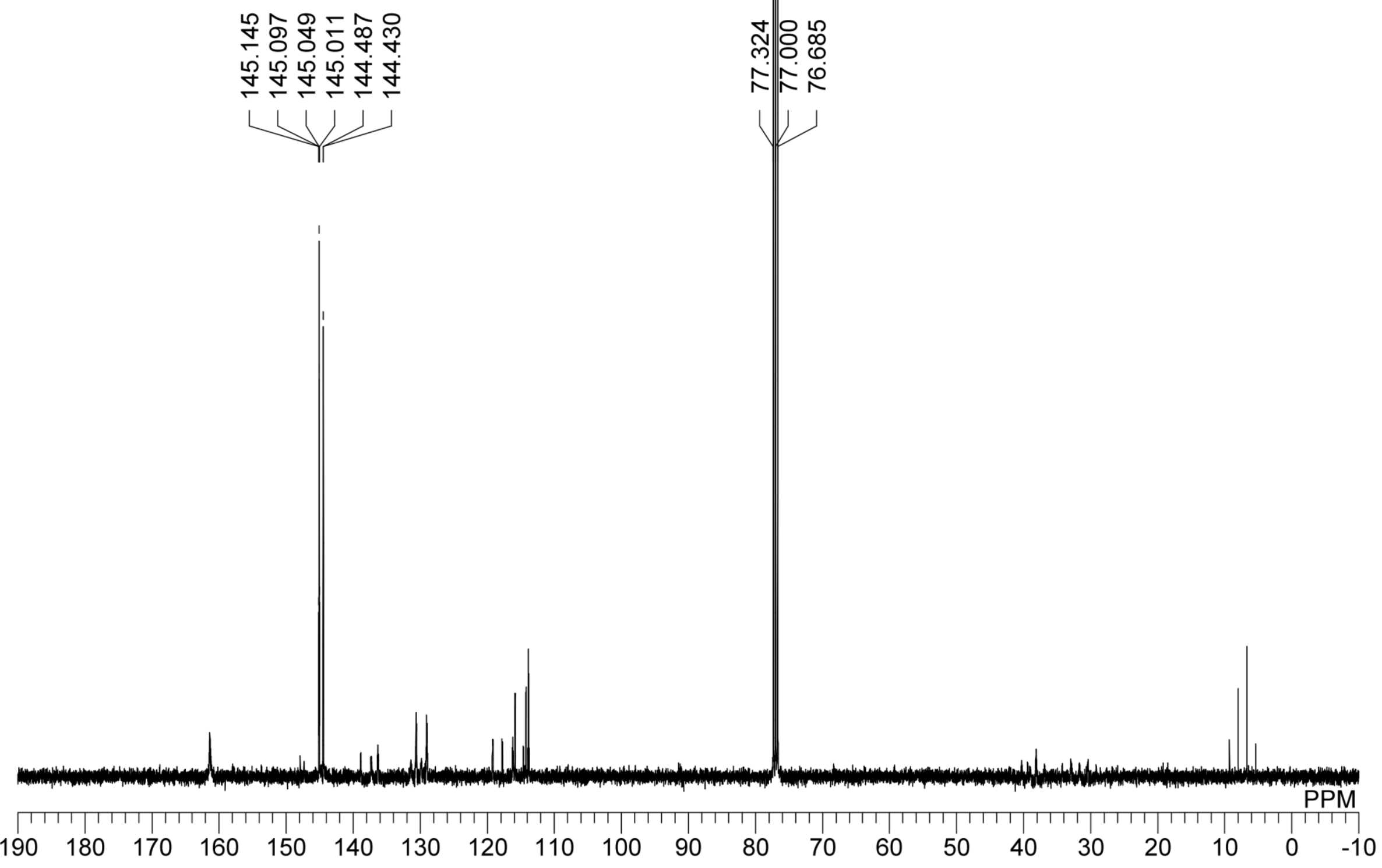
${ }^{19} \mathrm{~F}$ NMR of mixture of $\mathbf{4 b a}$ and its regioisomer $(p: m=94: 6)$
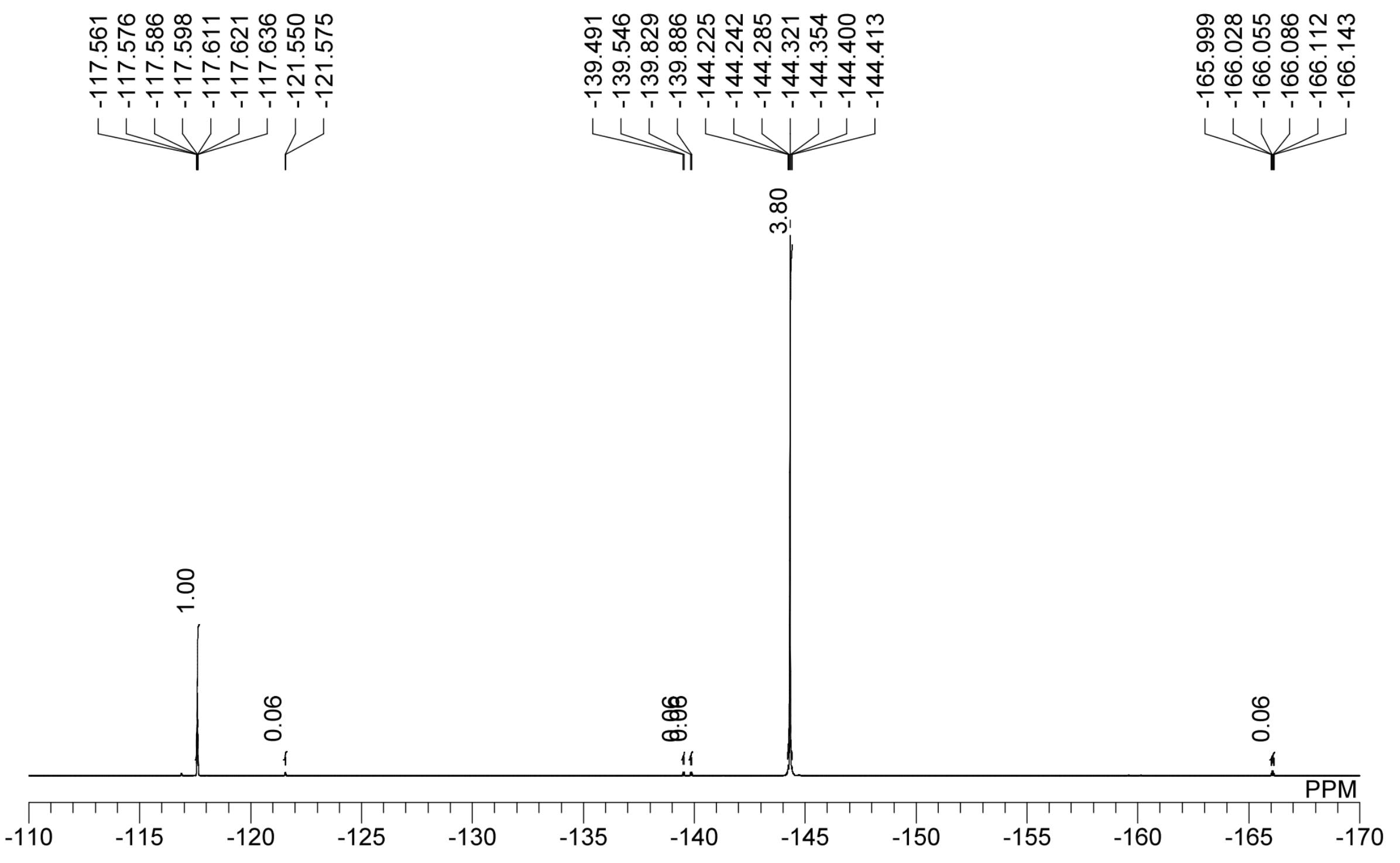


\section{${ }^{1} \mathrm{H}$ NMR of $4 \mathbf{c a}$}

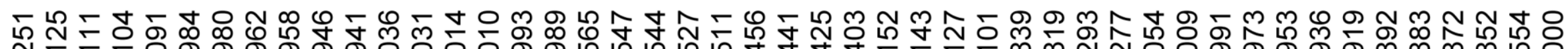
NTír

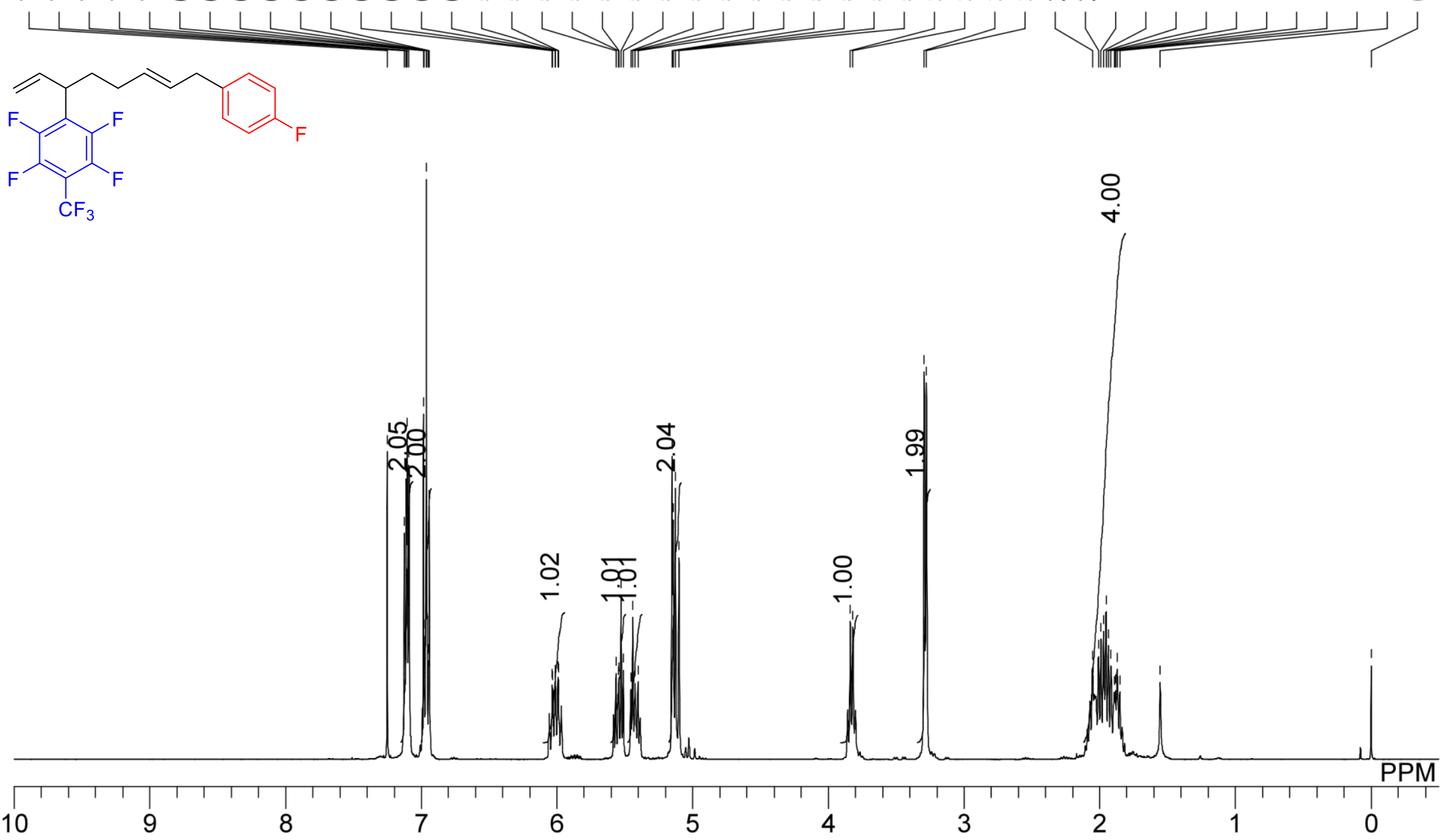


${ }^{13} \mathrm{C}\left\{{ }^{1} \mathrm{H}\right\}$ NMR of $\mathbf{4 c a}$

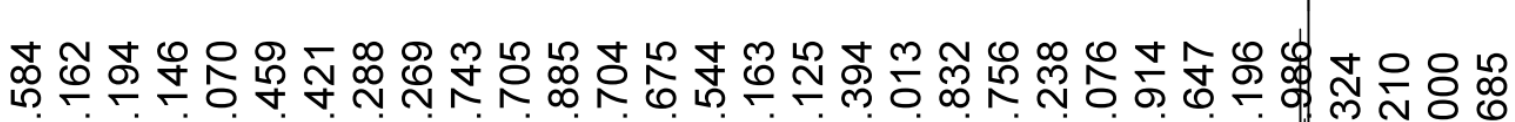
ชิ

은 응

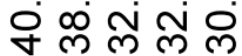
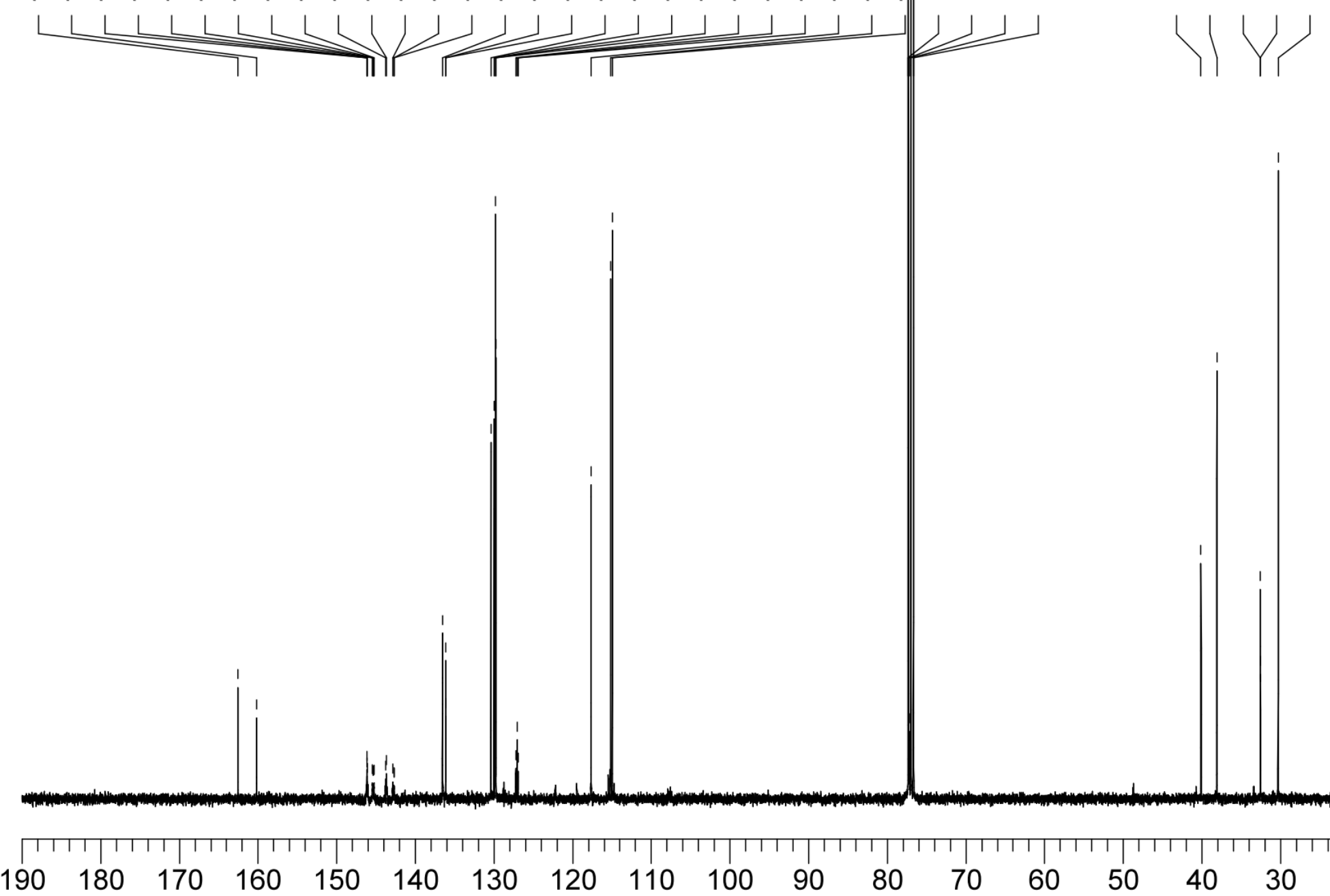

90

80

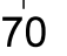

60

50

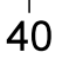

30

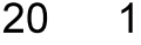

0 
${ }^{13} \mathrm{C}\left\{{ }^{19} \mathrm{~F}\right\}$ NMR of $\mathbf{4 c a}$

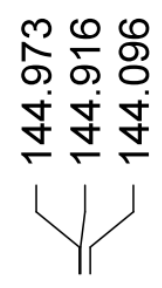

능요

令余
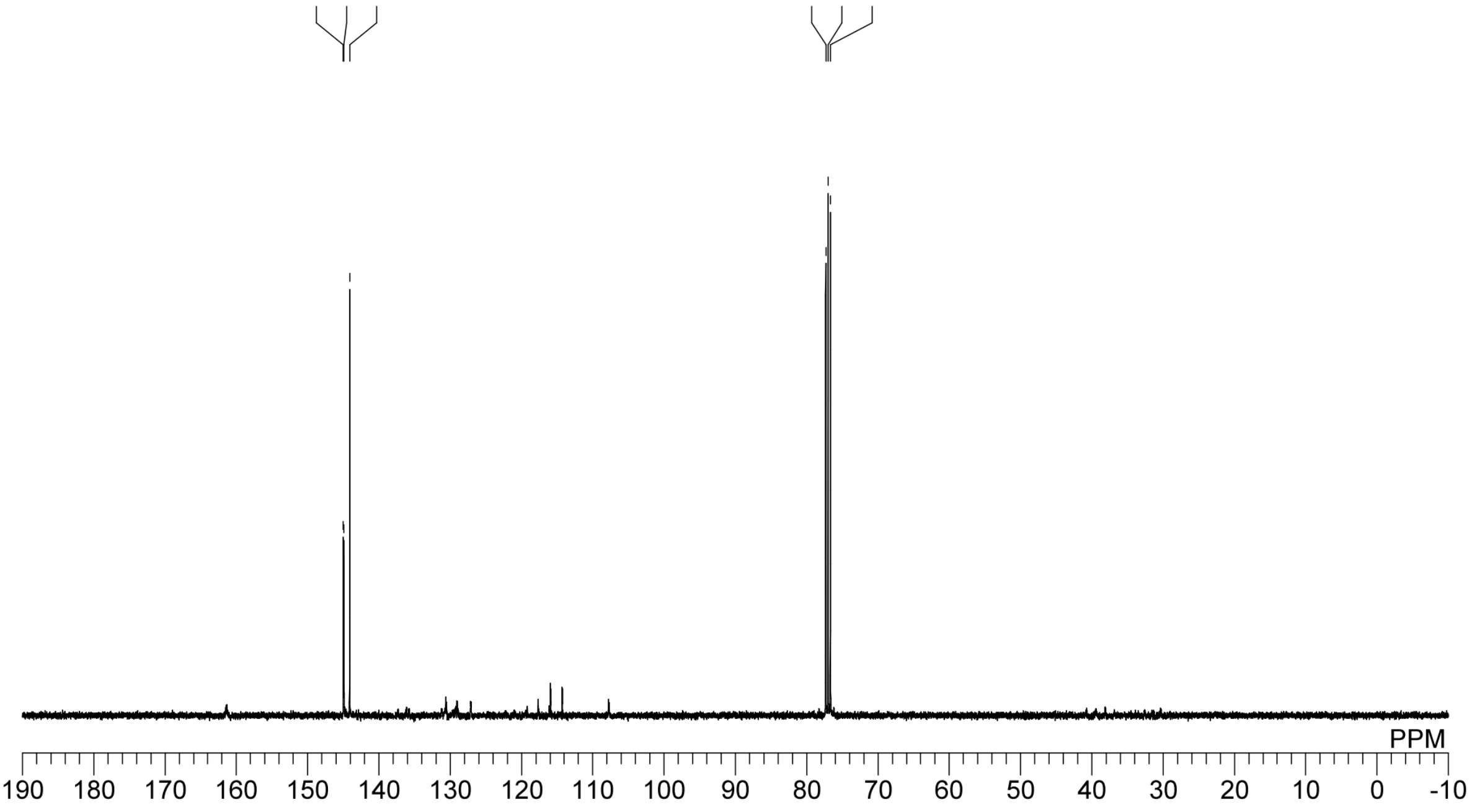
${ }^{19} \mathrm{~F}$ NMR of $\mathbf{4} \mathbf{c a}$
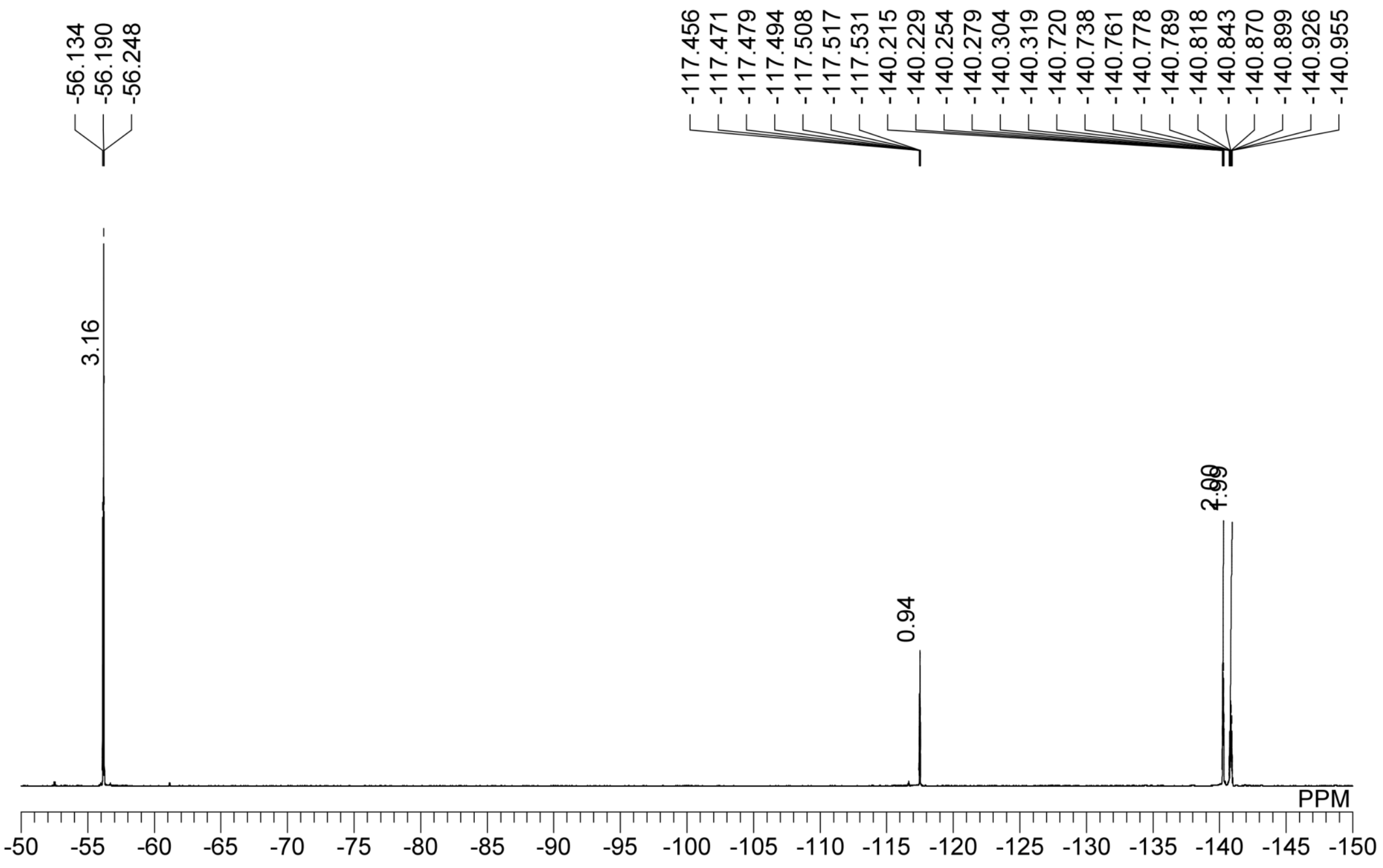
${ }^{1} \mathrm{H}$ NMR of mixture of 4da and its regioisomers ( $\left.p: m: o=61: 35: 4\right)$

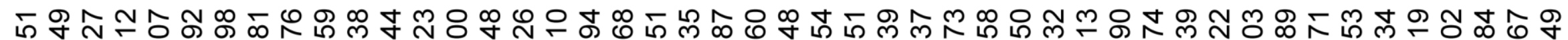

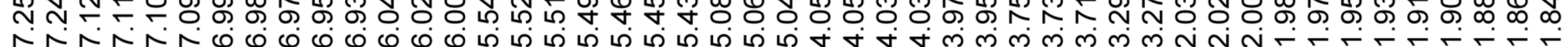
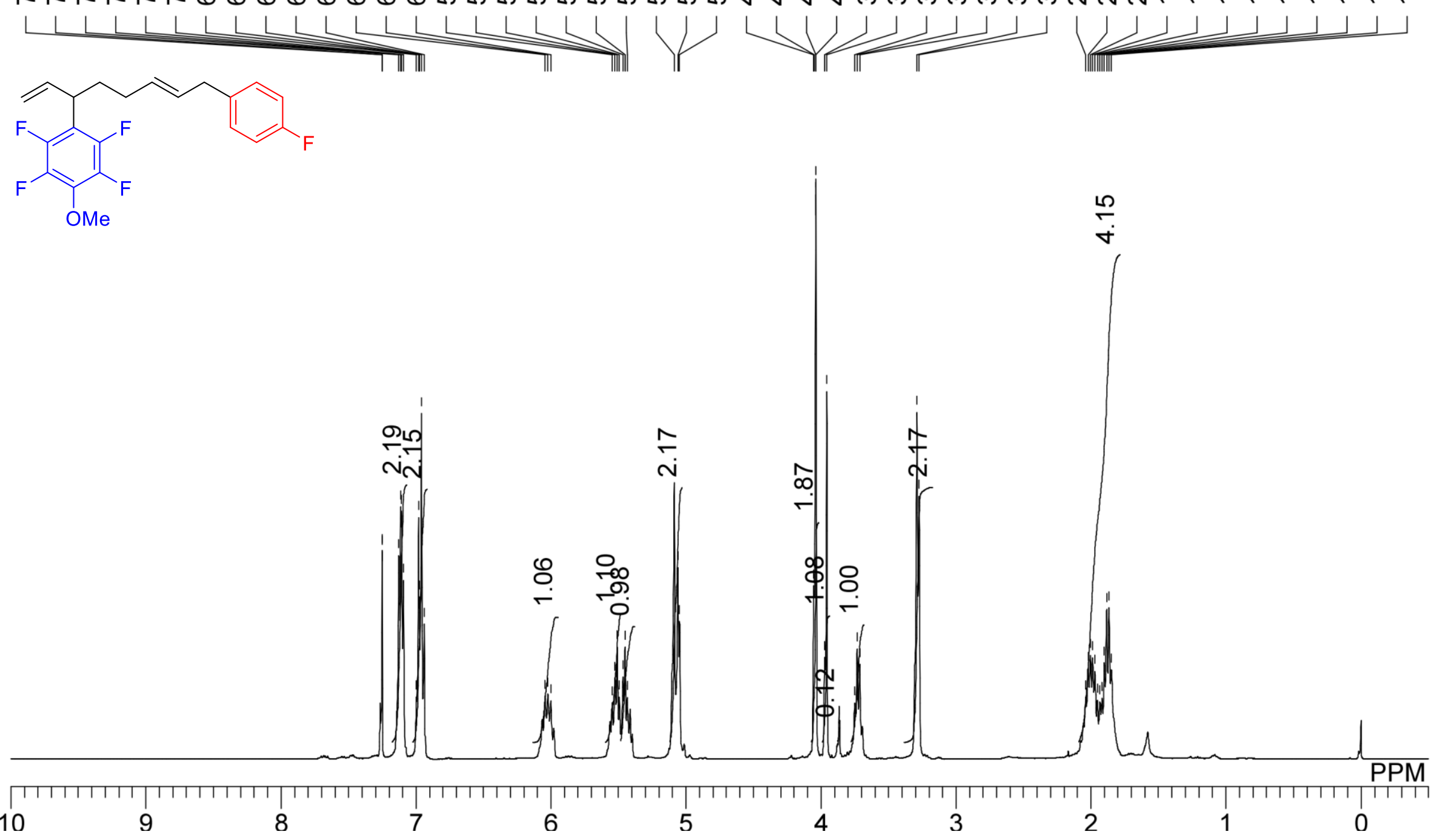
${ }^{13} \mathrm{C}\left\{{ }^{1} \mathrm{H}\right\}$ NMR of mixture of $\mathbf{4 d a}$ and its regioisomers ( $\left.p: m: o=61: 35: 4\right)$

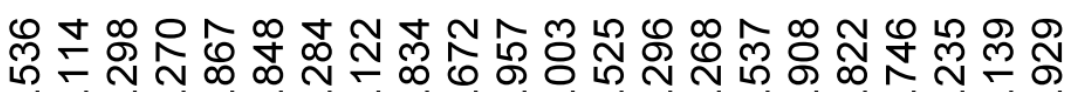

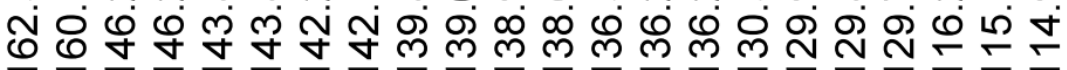

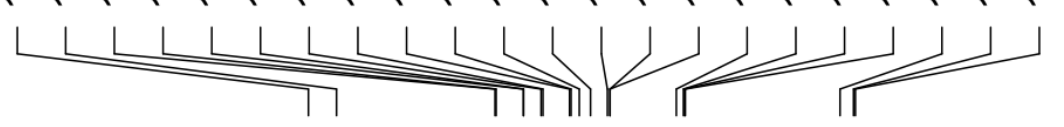

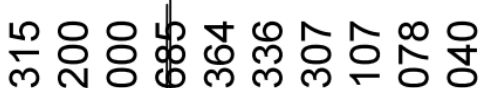

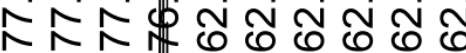

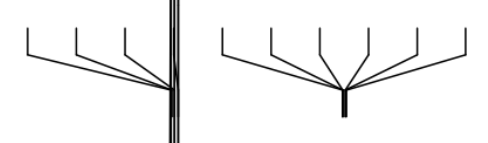

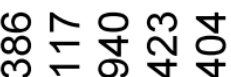

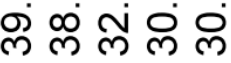

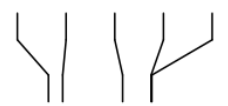

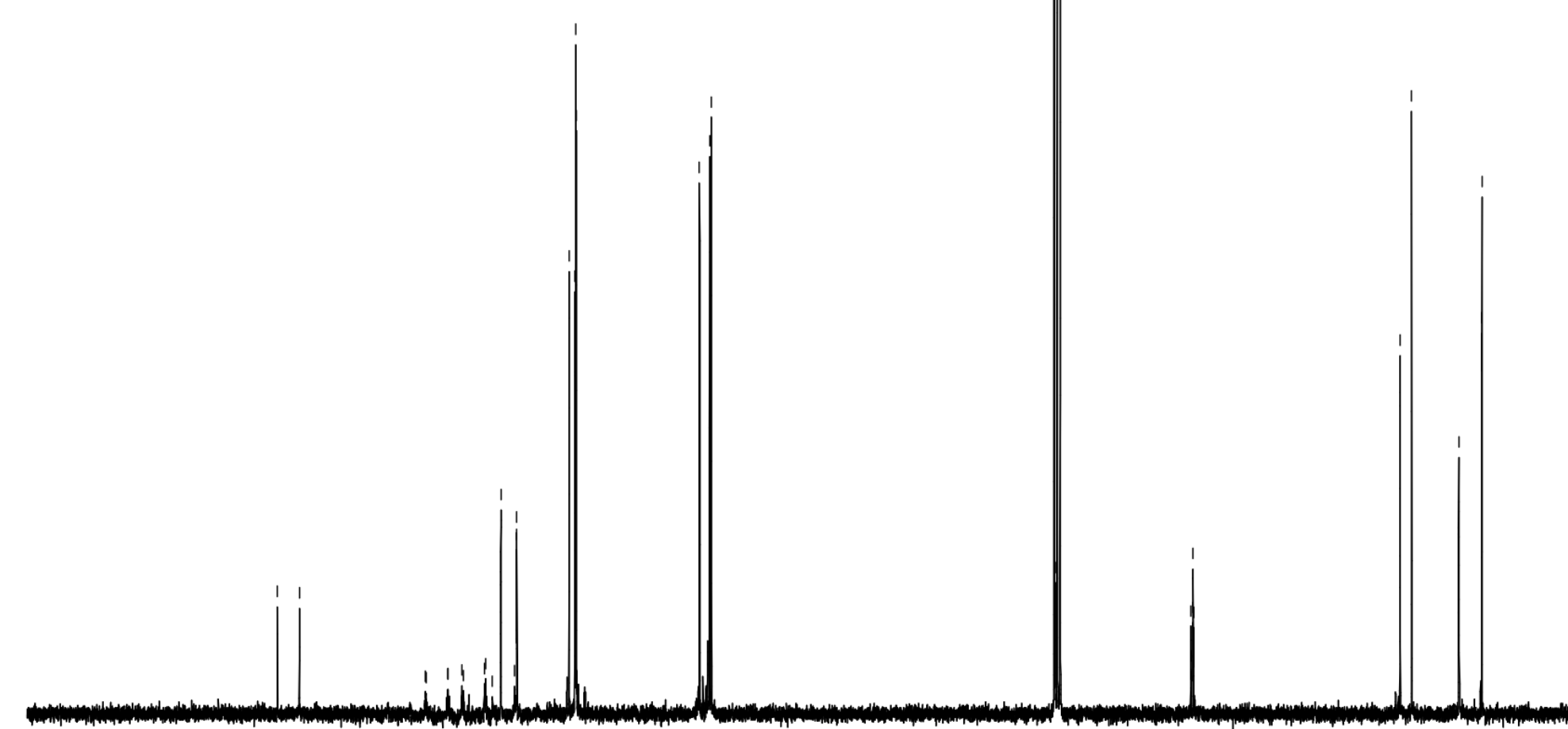


${ }^{13} \mathrm{C}\left\{{ }^{19} \mathrm{~F}\right\} \mathrm{NMR}$ of mixture of $\mathbf{4 d a}$ and its regioisomers $(p: m: o=61: 35: 4)$

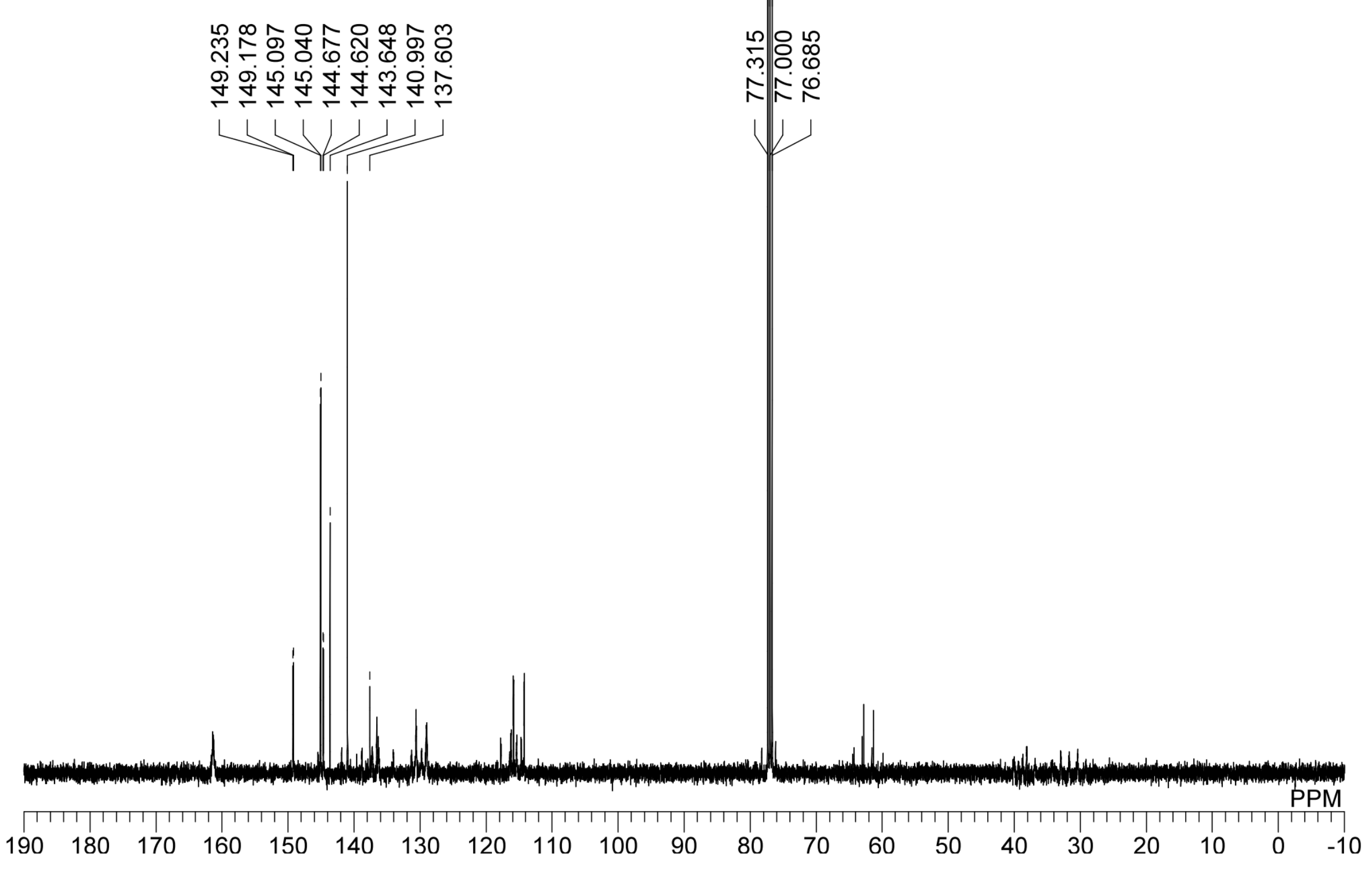


${ }^{19} \mathrm{~F}$ NMR of mixture of $\mathbf{4 d a}$ and its regioisomers ( $\left.p: m: o=61: 35: 4\right)$
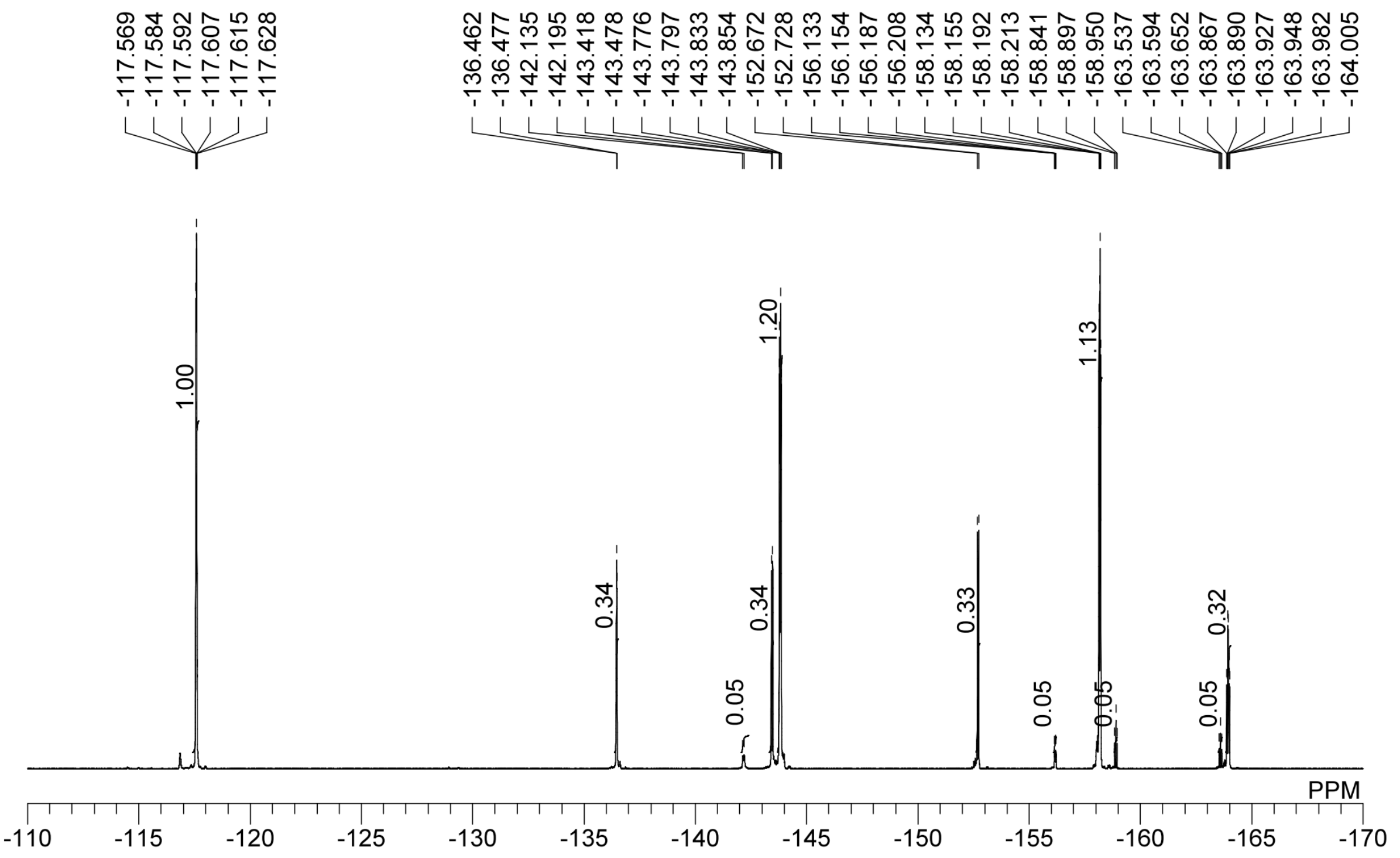
${ }^{1} \mathrm{H}$ NMR of 4ea

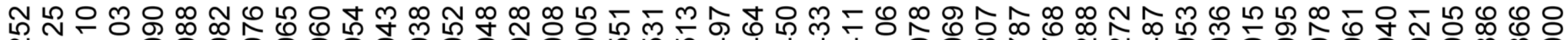

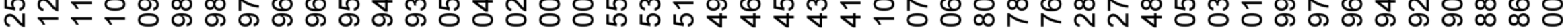
N N N

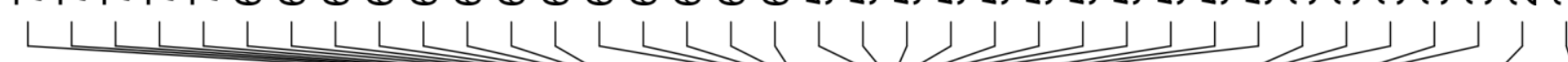

Tा\|ा
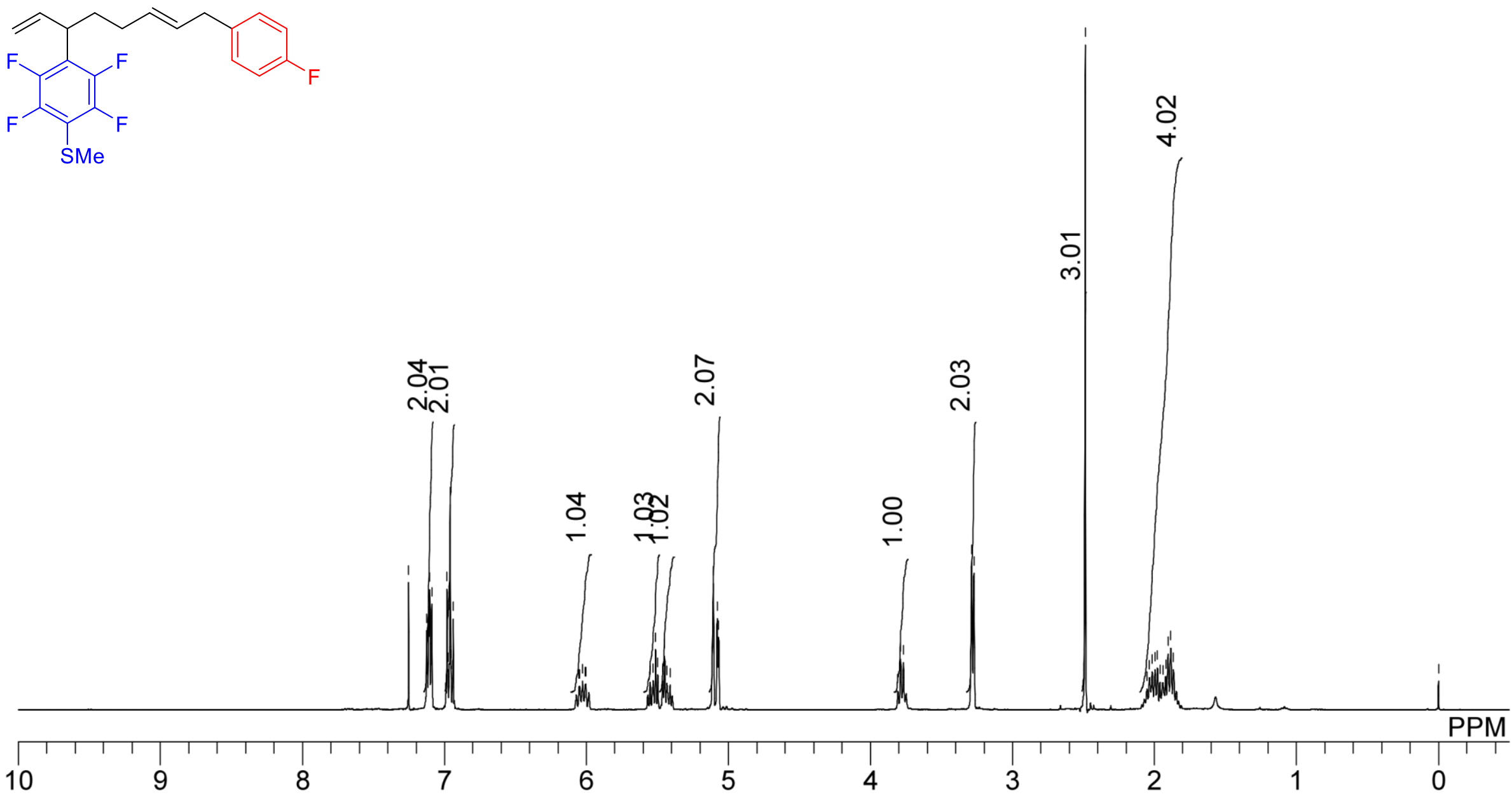


\section{${ }^{13} \mathrm{C}\left\{{ }^{1} \mathrm{H}\right\}$ NMR of 4ea}

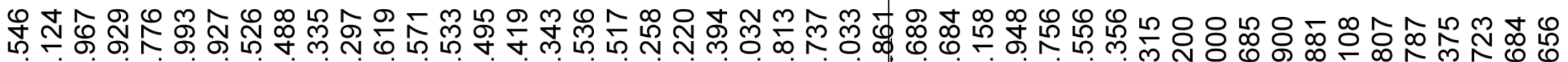

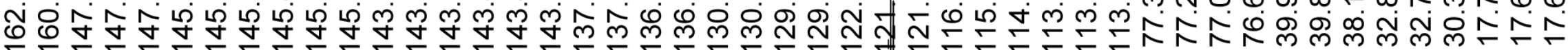
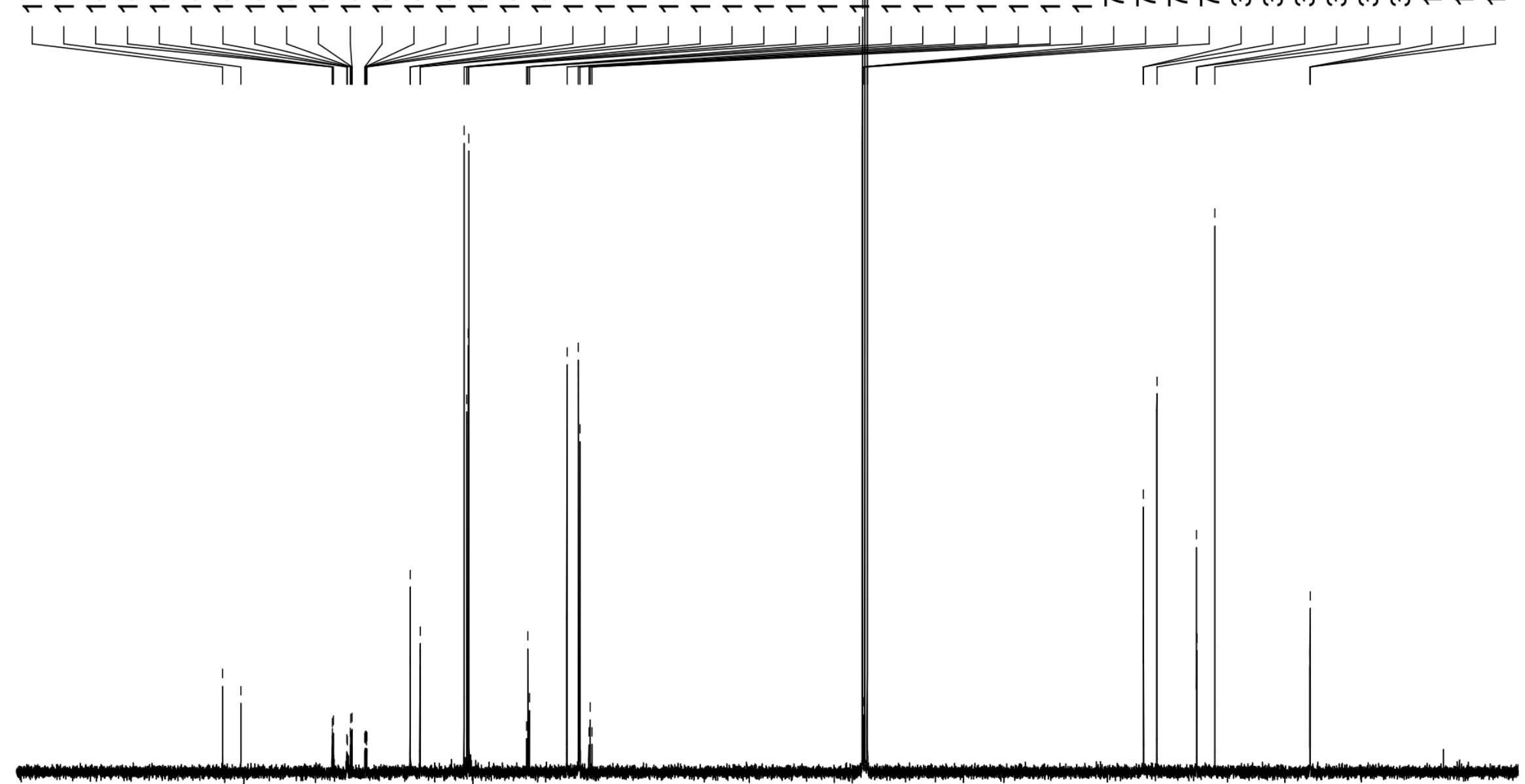
${ }^{13} \mathrm{C}\left\{{ }^{19} \mathrm{~F}\right\}$ NMR of $\mathbf{4 e a}$

$$
\text { 倍 }
$$

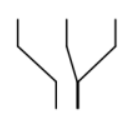

능융

Nヘ்

NN

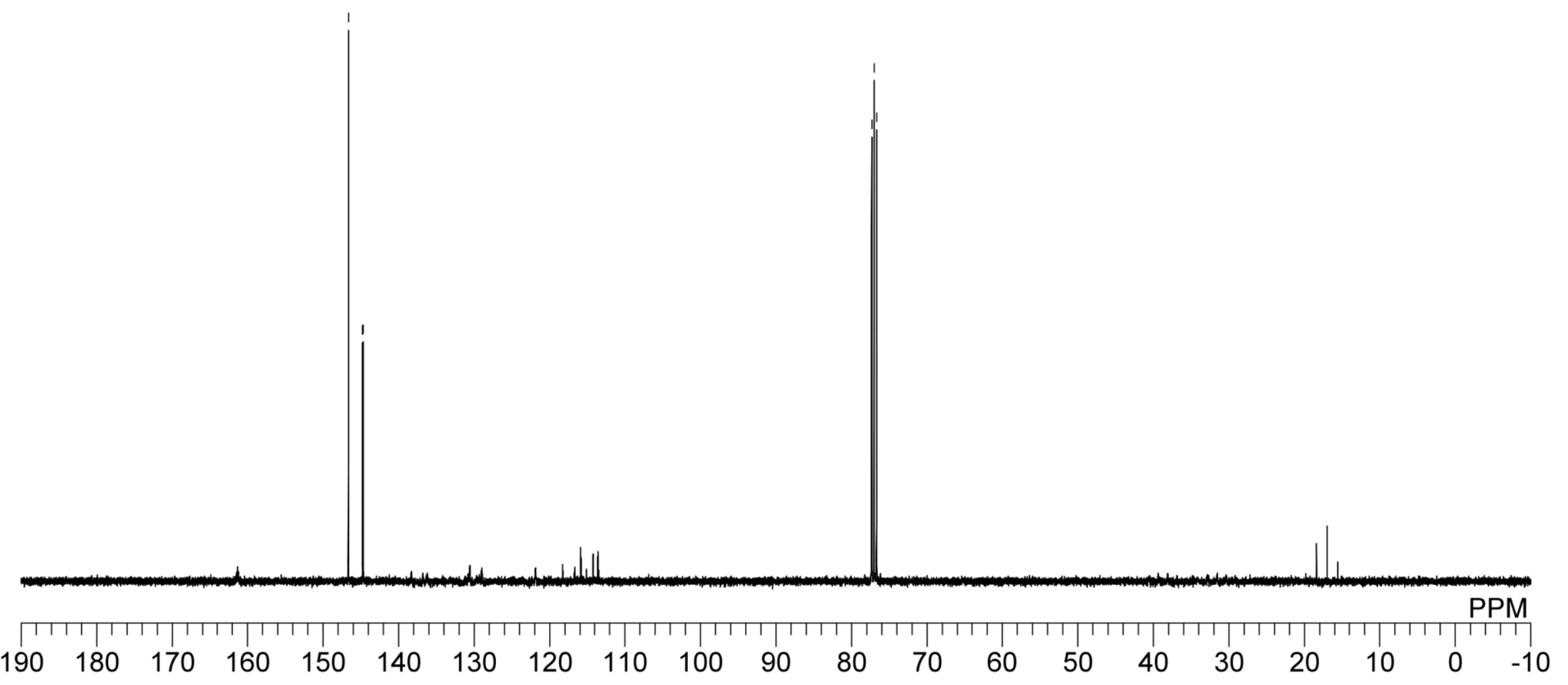


${ }^{19}$ F NMR of 4ea
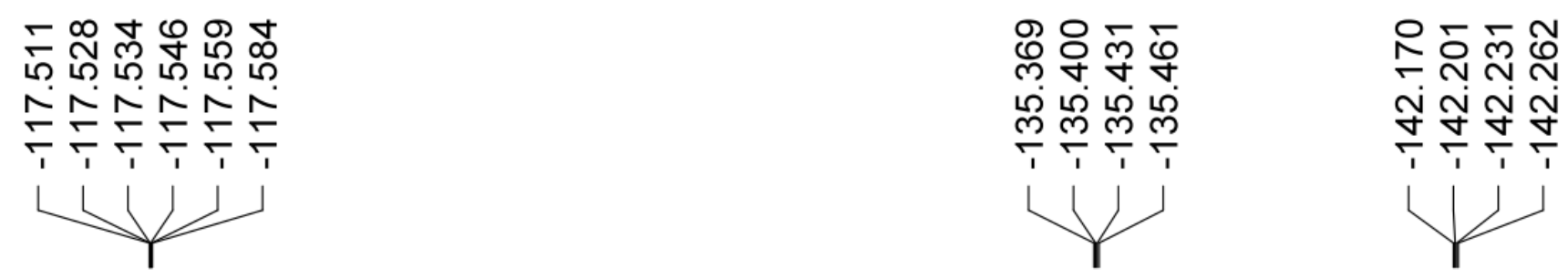

오.

응

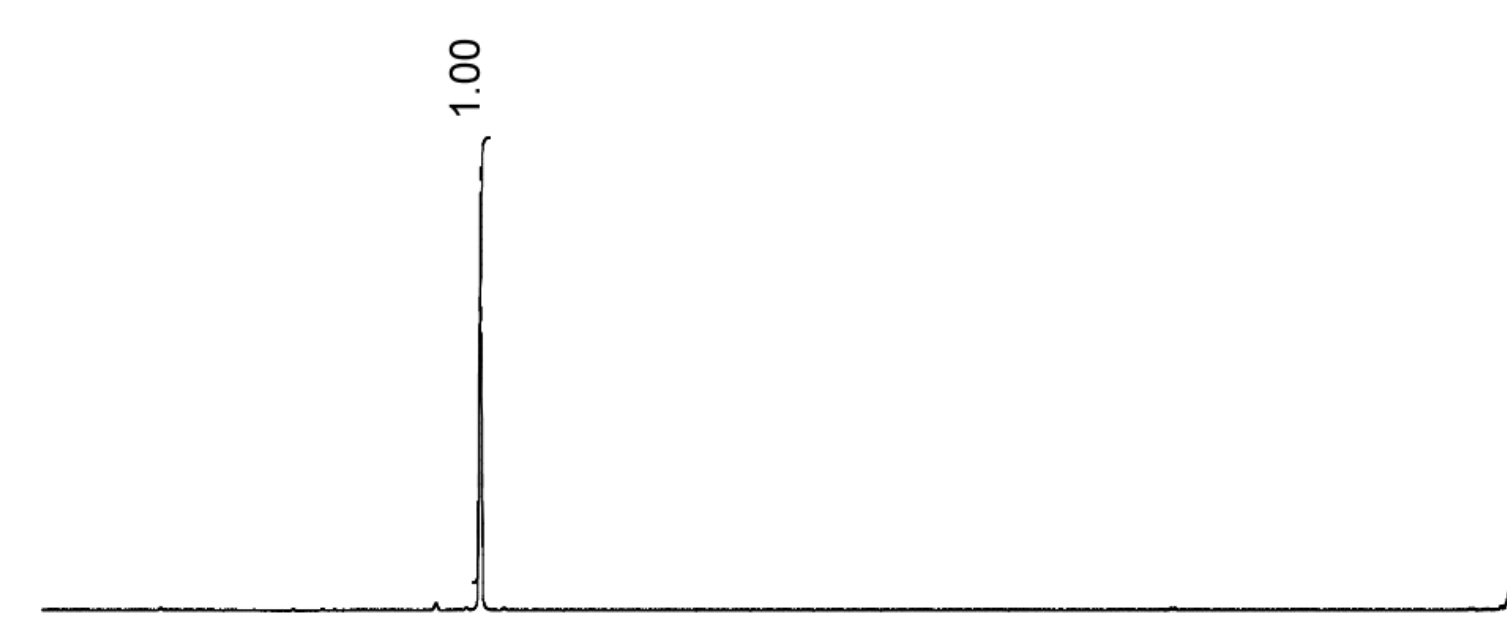

PPM

$\begin{array}{lllllllllllllllllllll} & 10 & -112 & -114 & -116 & -118 & -120 & -122 & -124 & -126 & -128 & -130 & -132 & -134 & -136 & -138 & -140 & -142 & -144 & -146 & -148\end{array}$ 
${ }^{1} \mathrm{H}$ NMR of mixture of $\mathbf{4 f a}$ and its regioisomers ( $p: m: o=86: 6: 8$ )

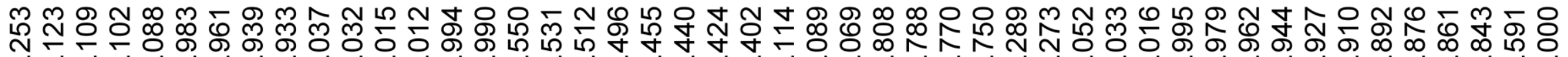
N N N N
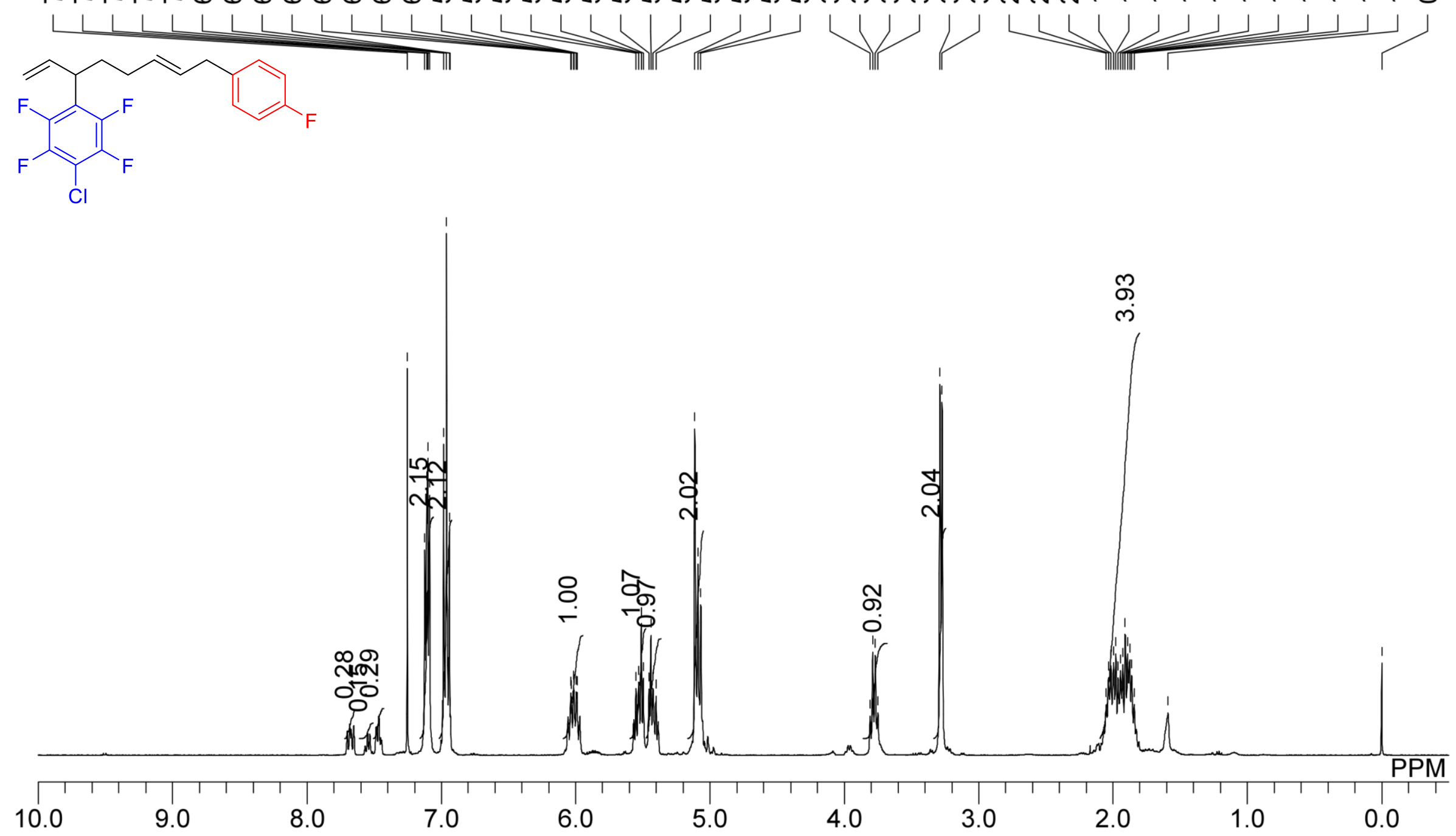
${ }^{13} \mathrm{C}\left\{{ }^{1} \mathrm{H}\right\}$ NMR of mixture of $\mathbf{4 f a}$ and its regioisomers ( $\left.p: m: o=86: 6: 8\right)$

只 ஸீ

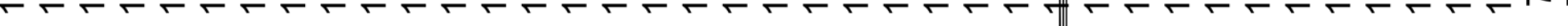
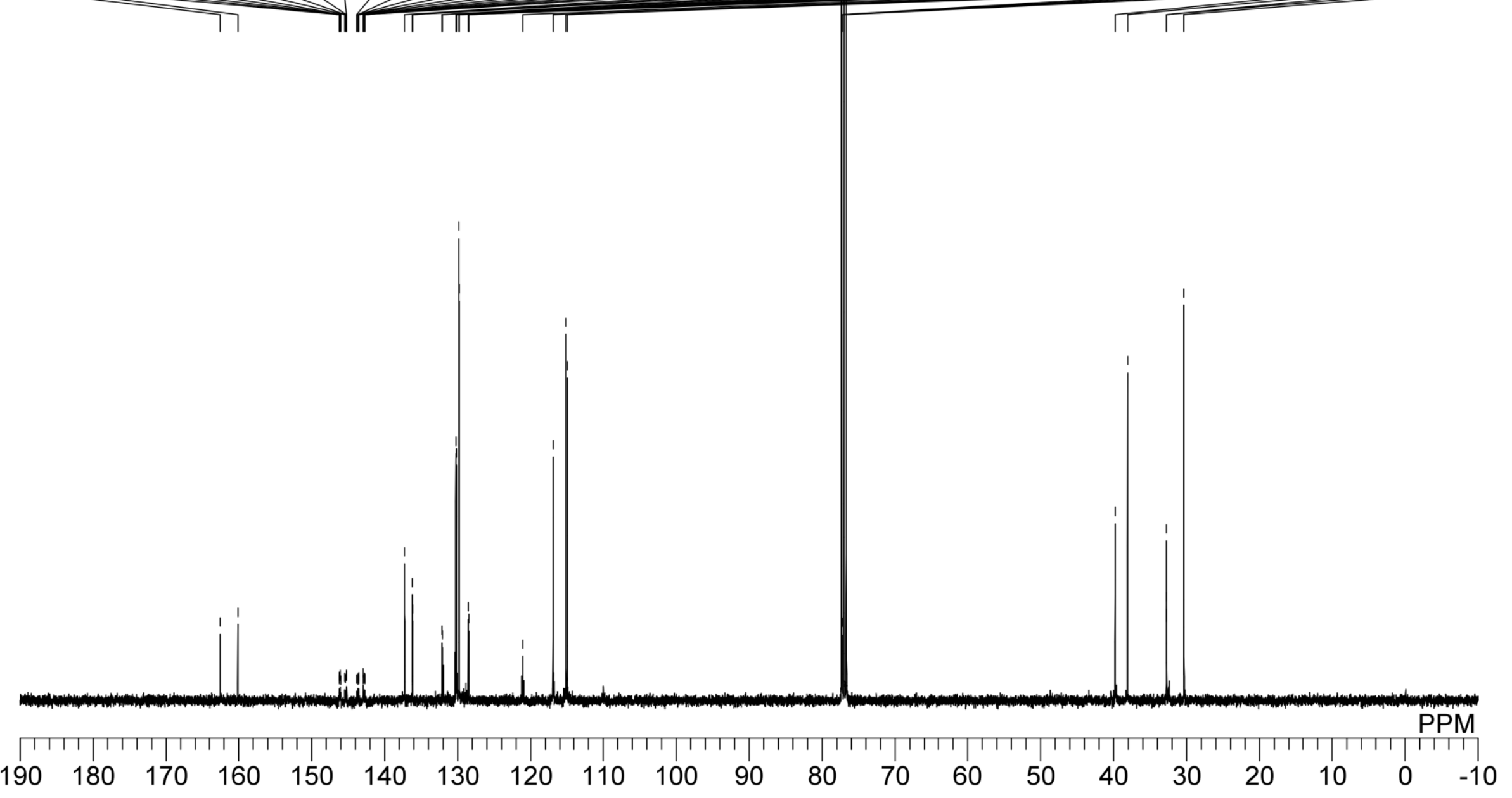
${ }^{13} \mathrm{C}\left\{{ }^{19} \mathrm{~F}\right\}$ NMR of mixture of $\mathbf{4 f a}$ and its regioisomers ( $\left.p: m: o=86: 6: 8\right)$
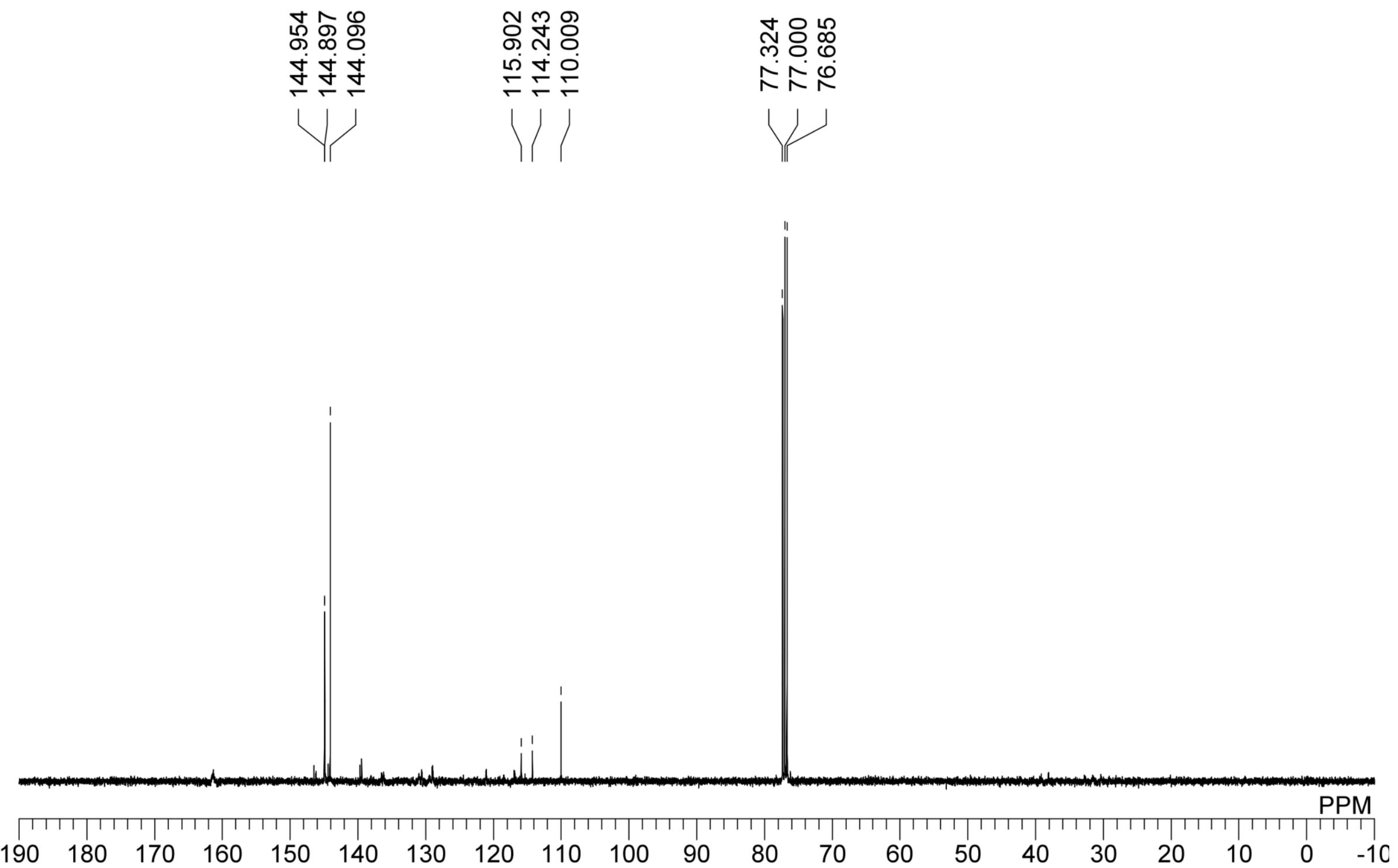
${ }^{19} \mathrm{~F}$ NMR of mixture of $\mathbf{4 f a}$ and its regioisomers ( $\left.p: m: o=86: 6: 8\right)$

గ

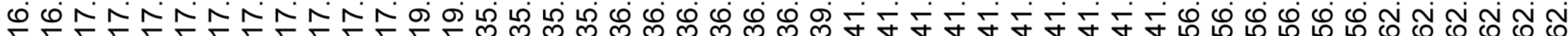

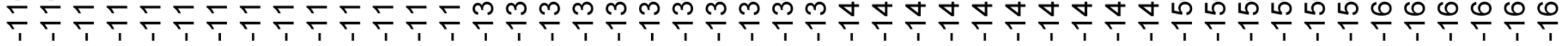
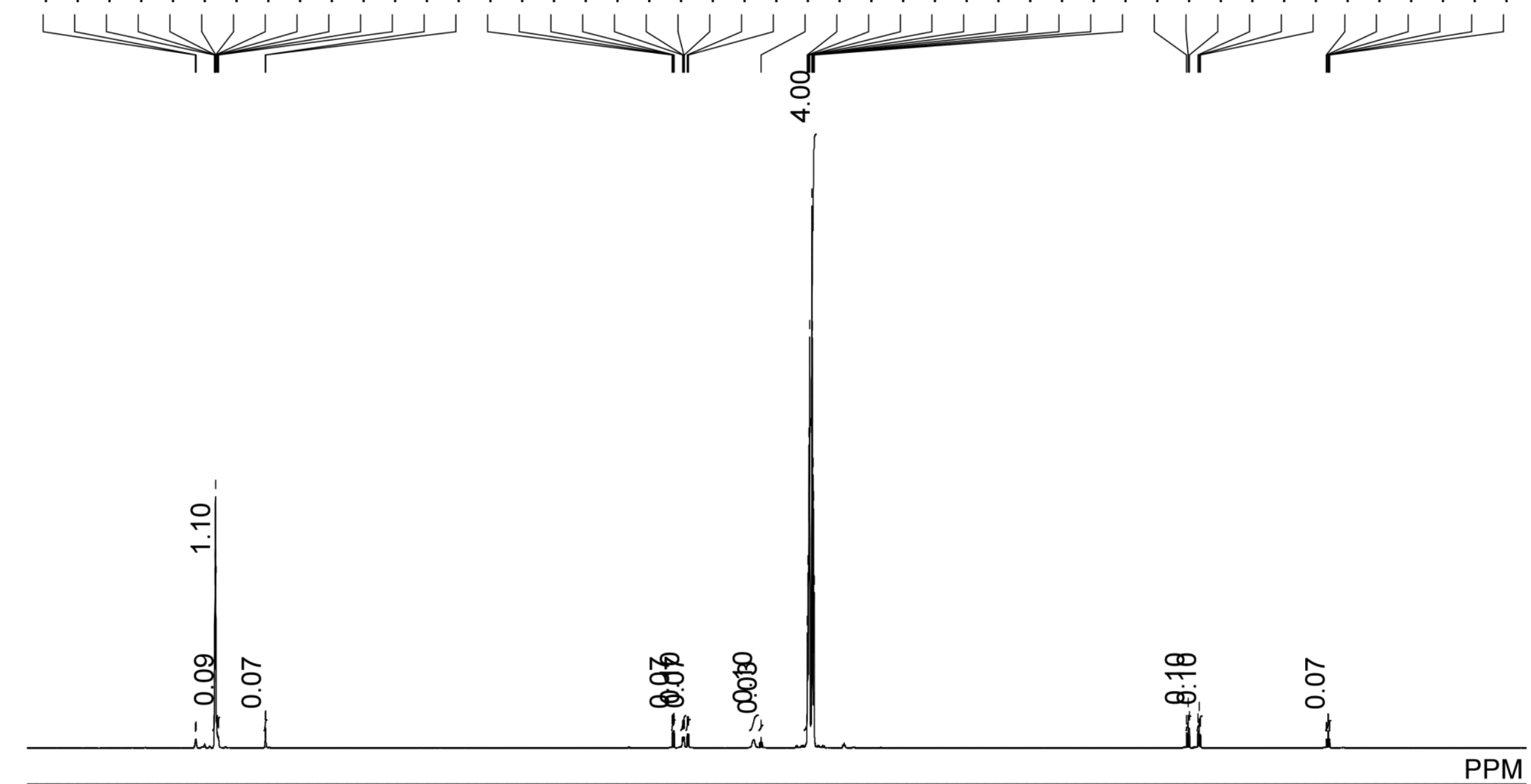


\section{${ }^{1} \mathrm{H}$ NMR of 4 ga}

б

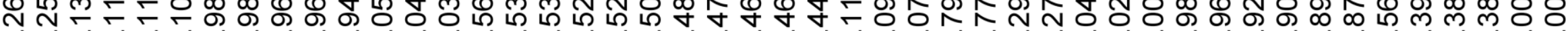
NNNNN

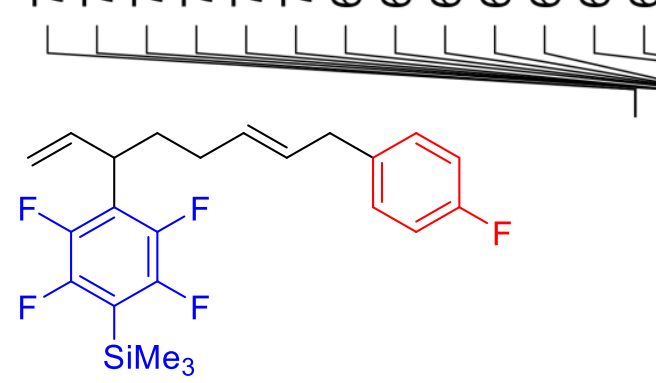

|II||
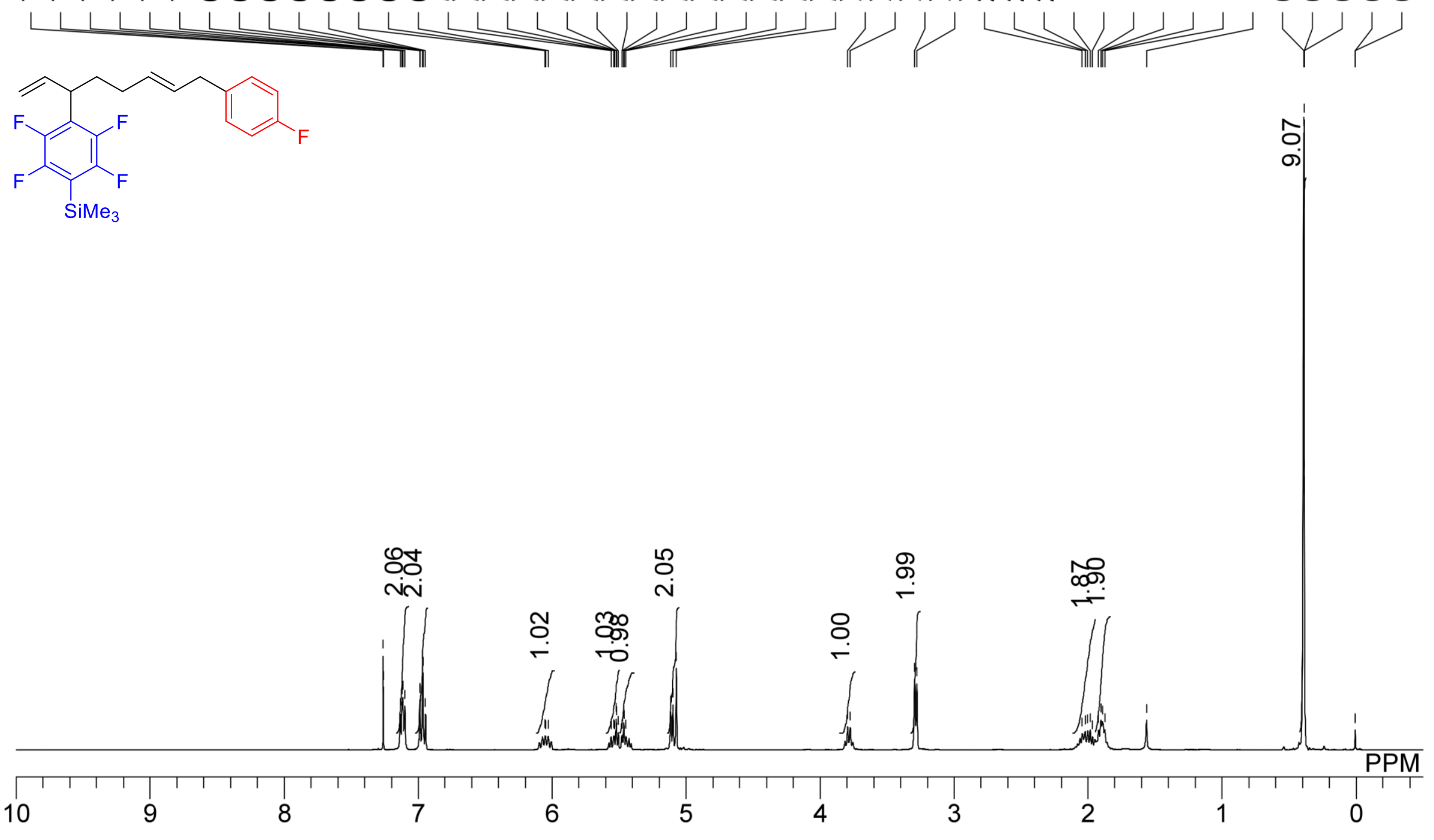


\section{${ }^{13} \mathrm{C}\left\{{ }^{1} \mathrm{H}\right\}$ NMR of 4 ga}

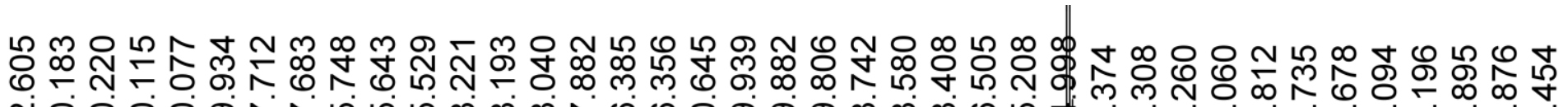
ช่
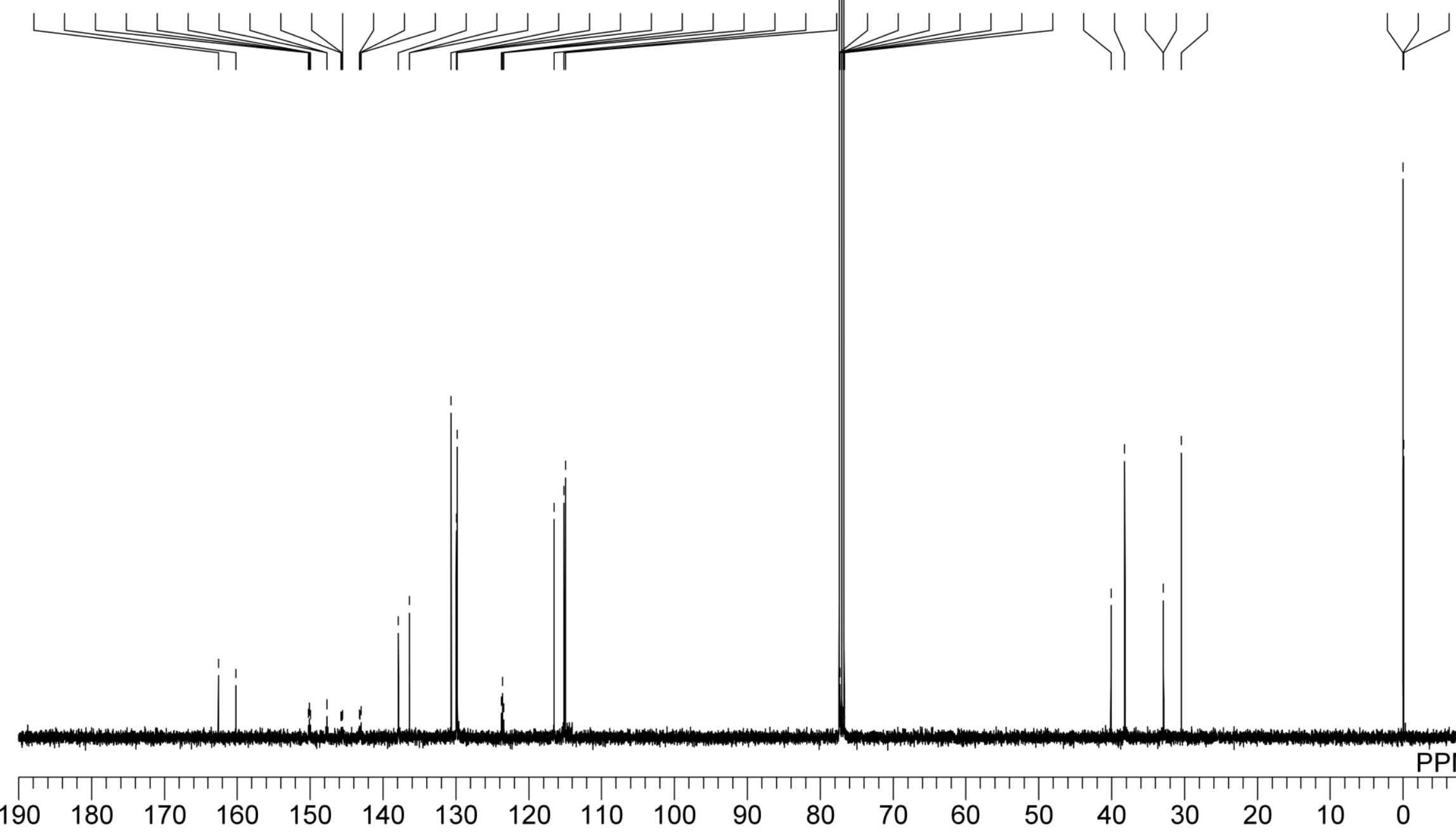
${ }^{13} \mathrm{C}\left\{{ }^{19} \mathrm{~F}\right\}$ NMR of 4 ga

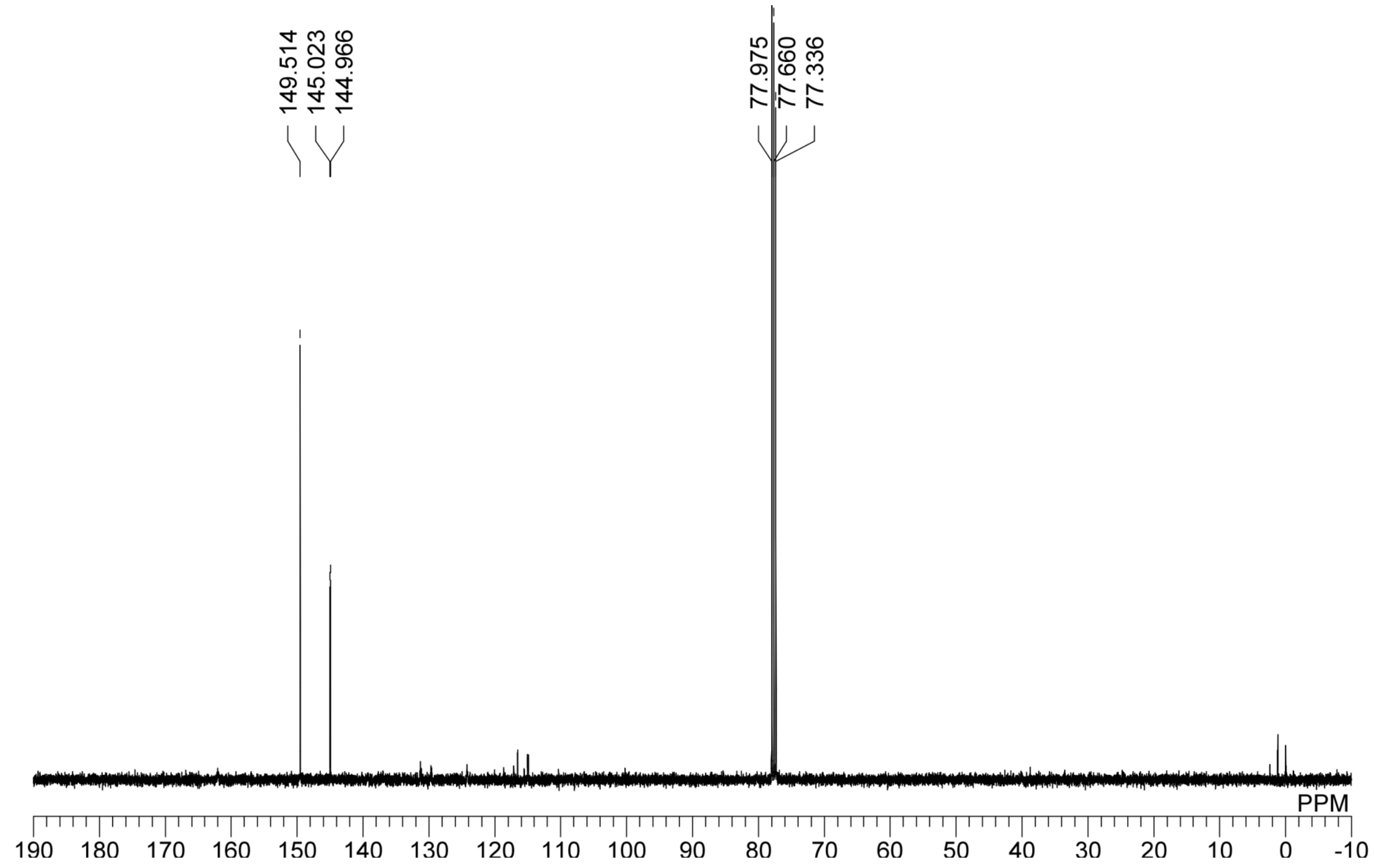


${ }^{19}$ F NMR of 4ga
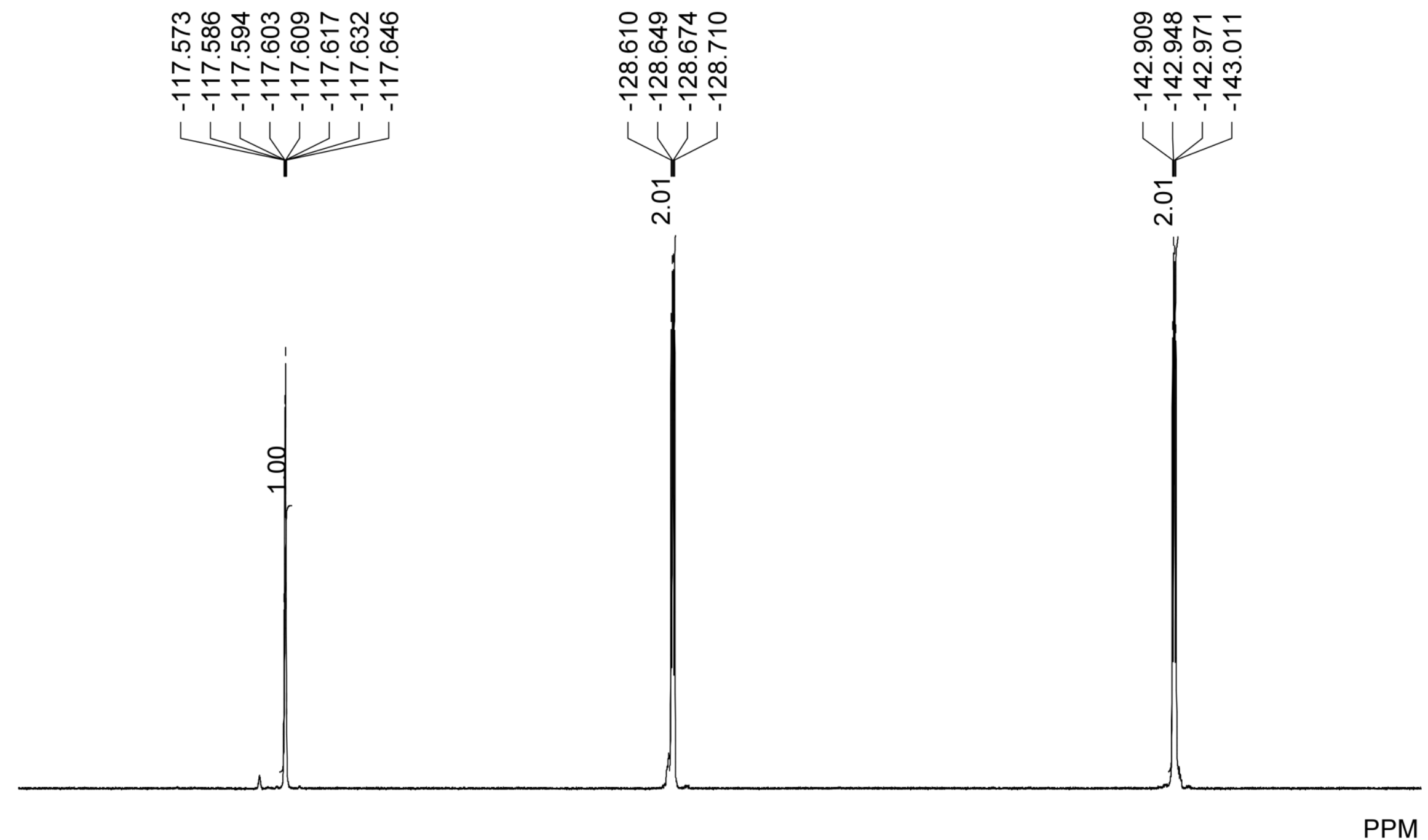

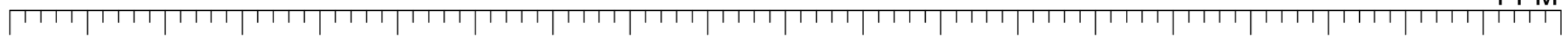
$\begin{array}{lllllllllllllllllllll}-110 & -112 & -114 & -116 & -118 & -120 & -122 & -124 & -126 & -128 & -130 & -132 & -134 & -136 & -138 & -140 & -142 & -144 & -146 & -148 & -150\end{array}$ 
${ }^{1} \mathrm{H}$ NMR of mixture of 4 ha and its regioisomers ( $\left.p: m: o=96: 2: 2\right)$

N

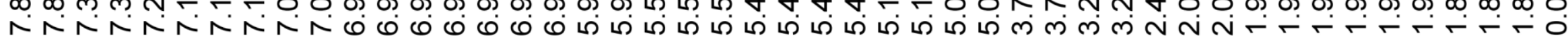

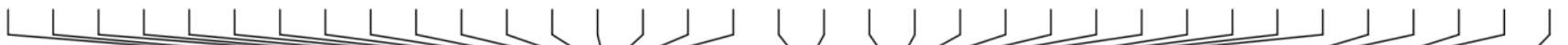

n| 117
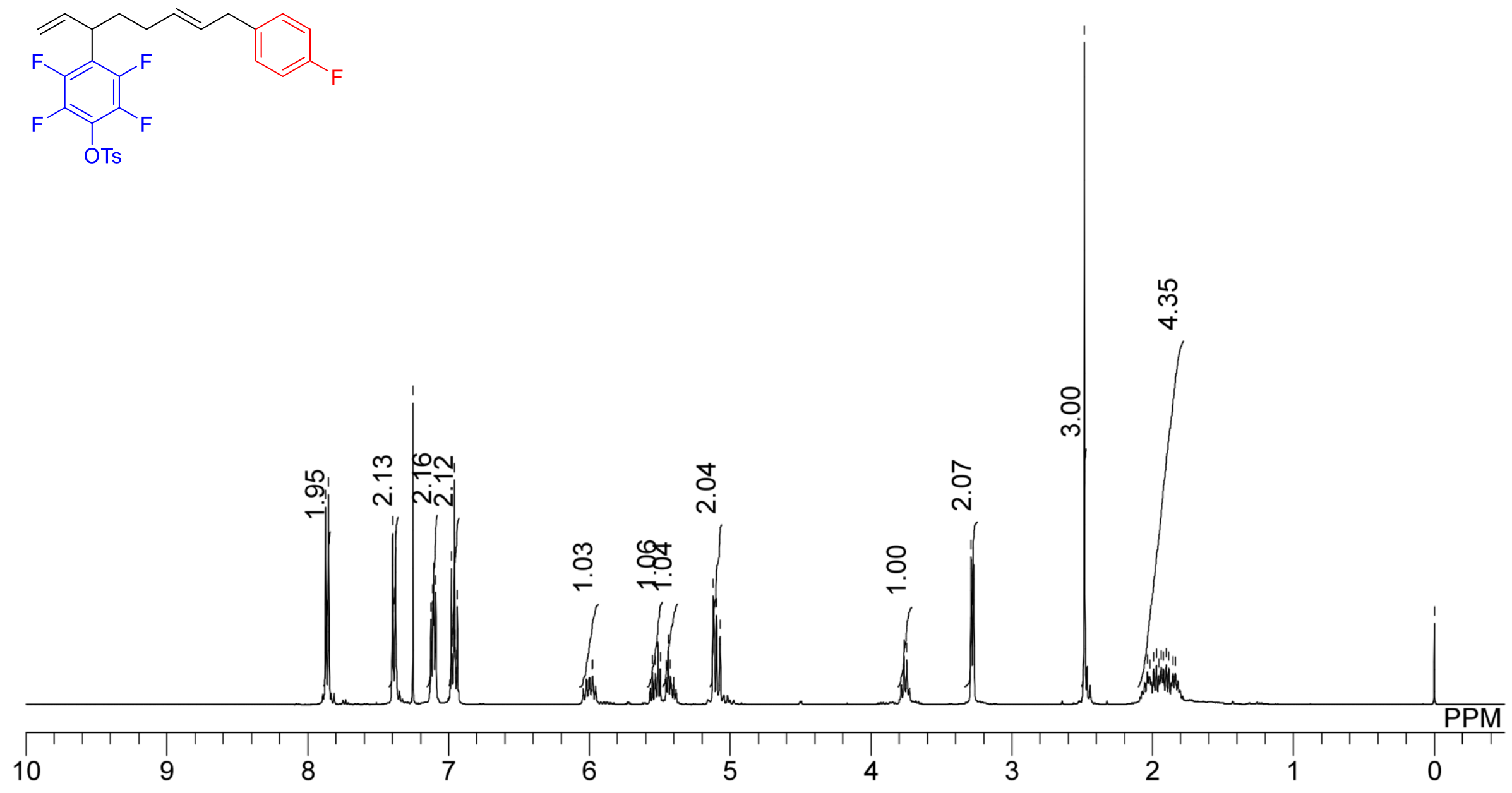
${ }^{13} \mathrm{C}\left\{{ }^{1} \mathrm{H}\right\}$ NMR of mixture of 4 ha and its regioisomers ( $p: m: o=96: 2: 2$ )

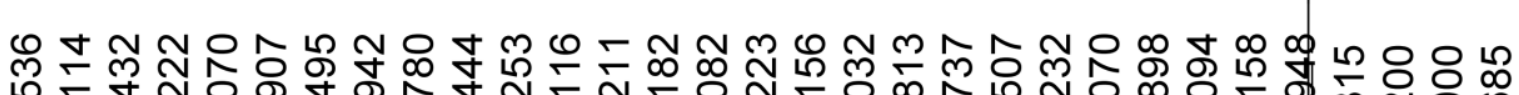
负 ஸ゚
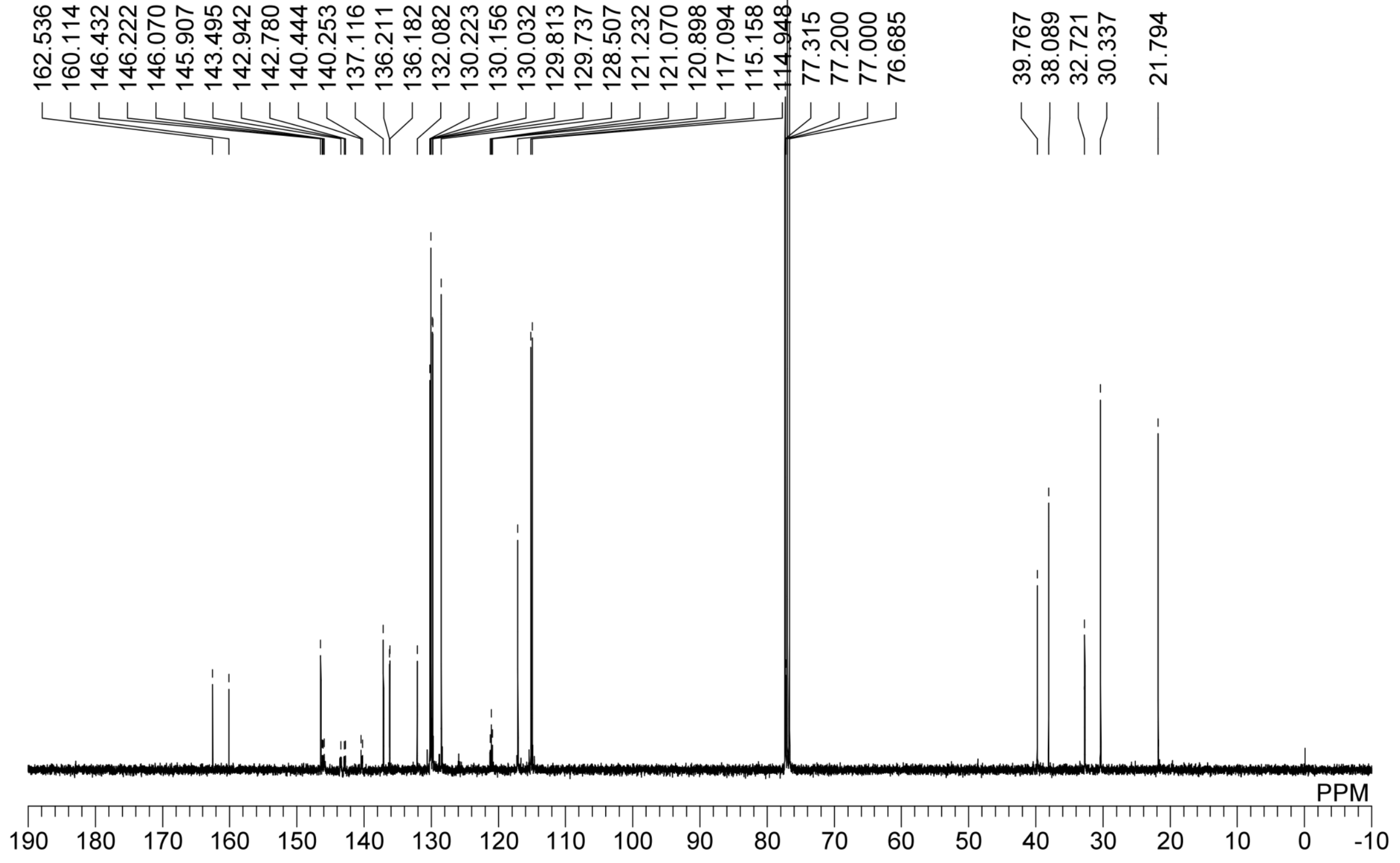
${ }^{13} \mathrm{C}\left\{{ }^{19} \mathrm{~F}\right\}$ NMR of mixture of 4 ha and its regioisomers ( $p: m: o=96: 2: 2$ )

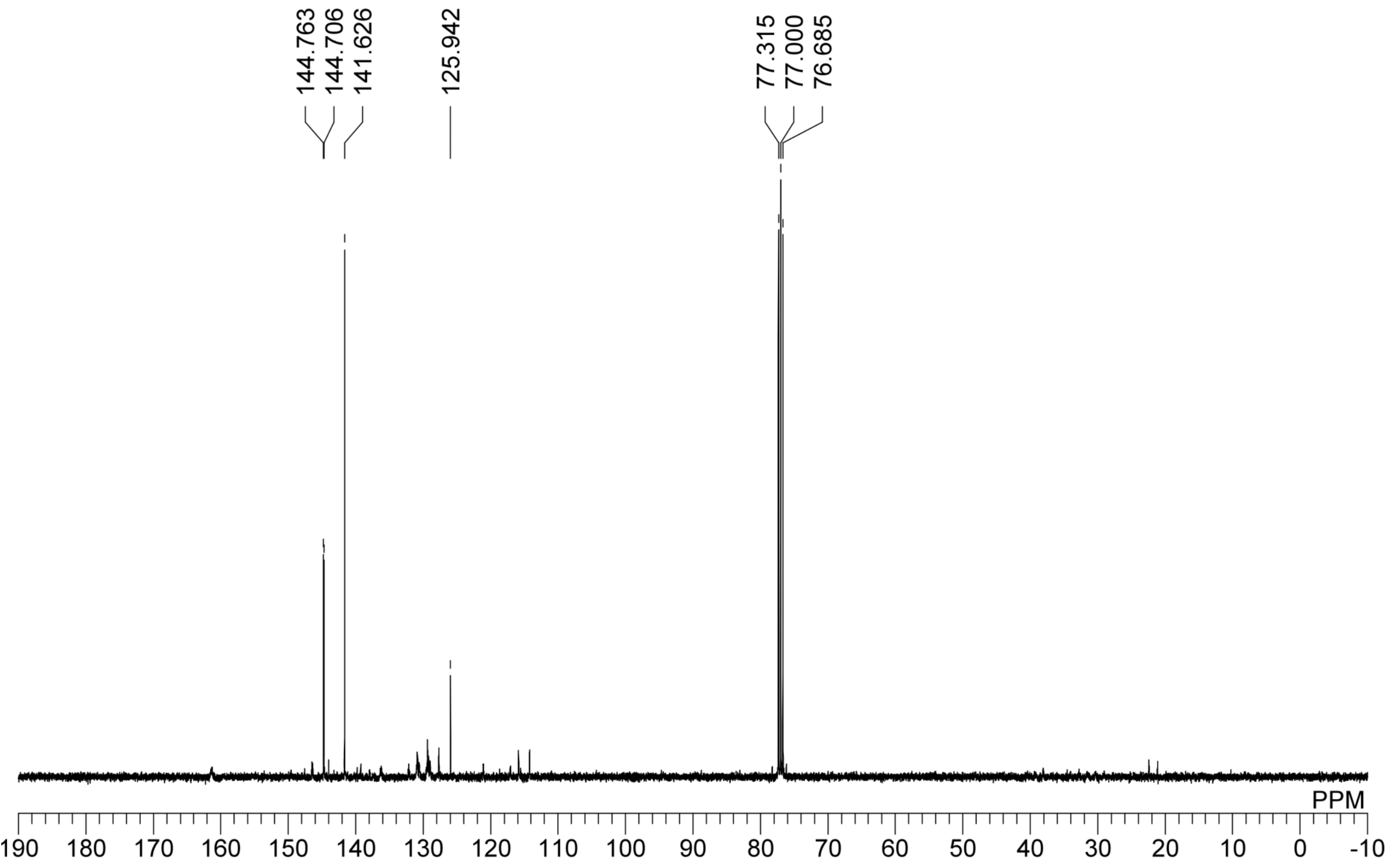


${ }^{19} \mathrm{~F}$ NMR of mixture of 4 ha and its regioisomers ( $\left.p: m: o=96: 2: 2\right)$

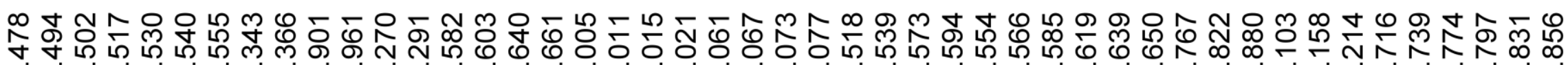

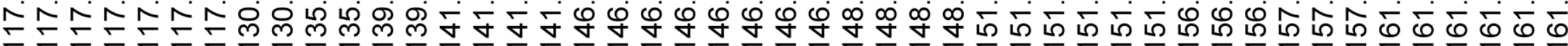

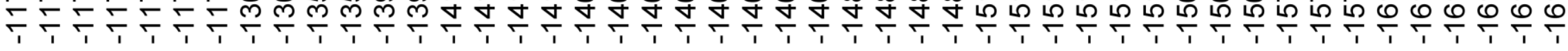
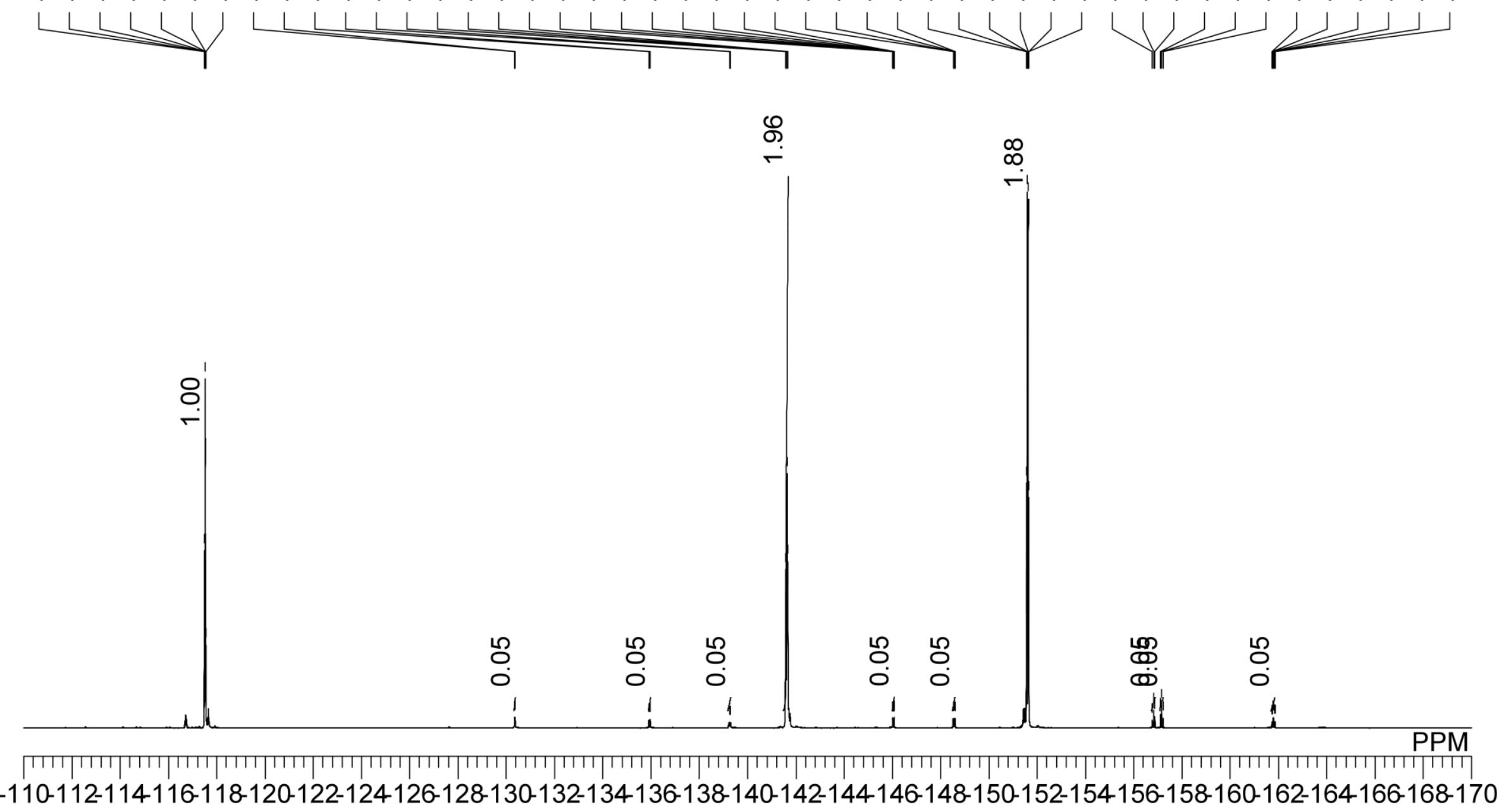


\section{${ }^{1} \mathrm{H}$ NMR of 4ia}

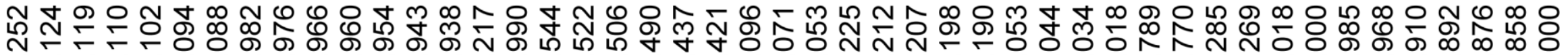

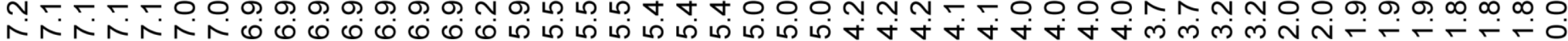
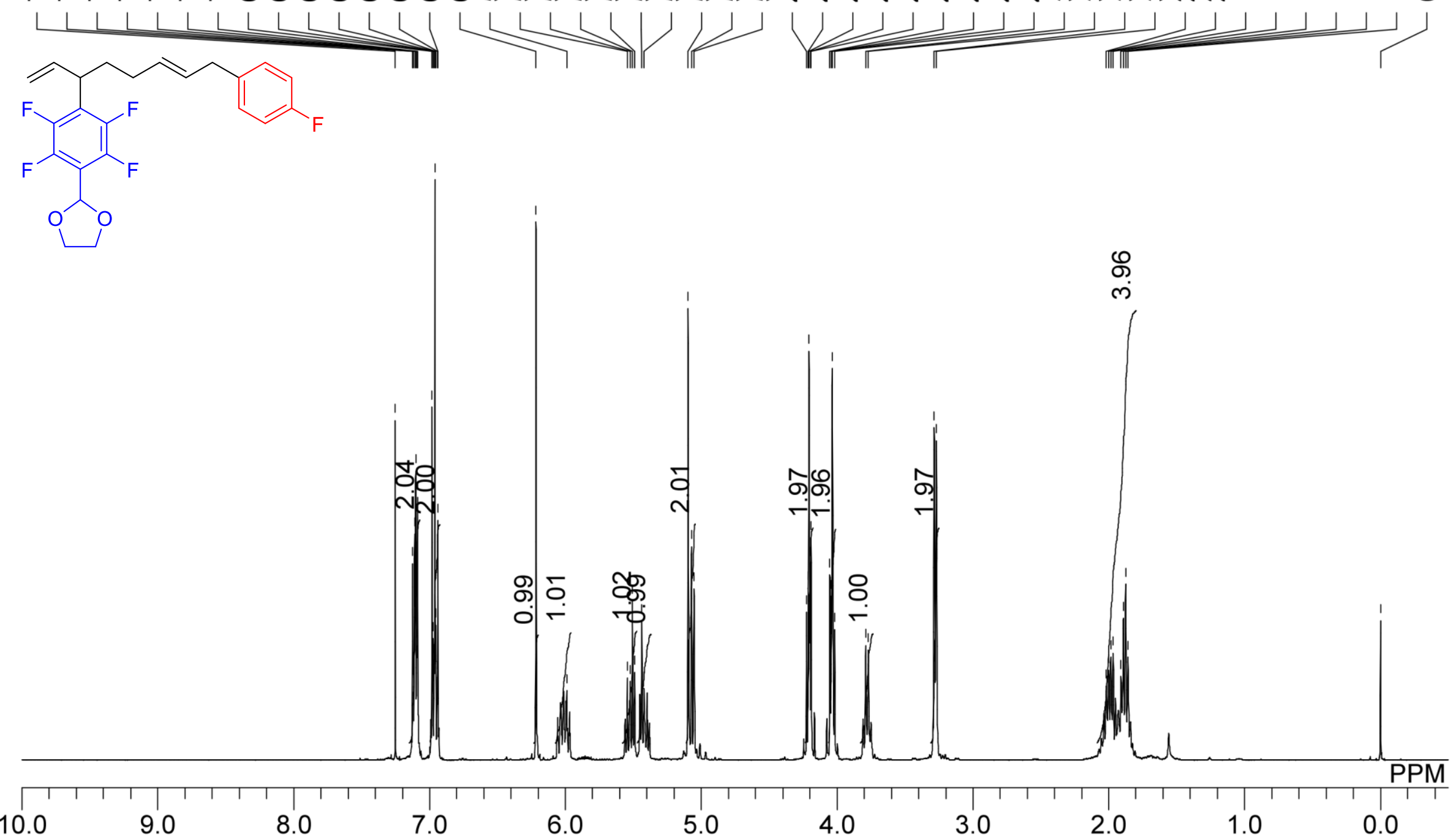


\section{${ }^{13} \mathrm{C}\left\{{ }^{1} \mathrm{H}\right\}$ NMR of 4 ia}

W ช TFF F F F F F F F F
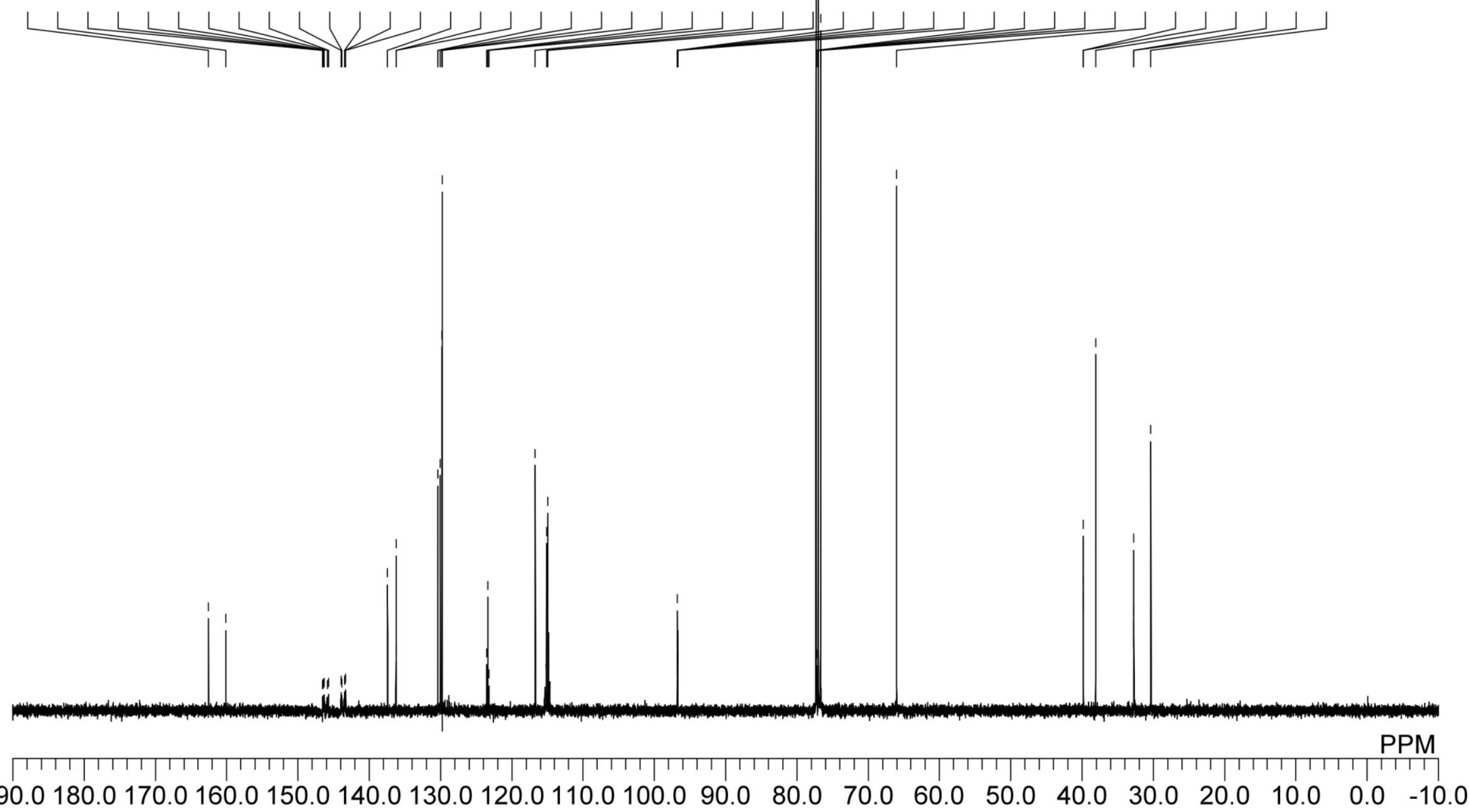
${ }^{13} \mathrm{C}\left\{{ }^{19} \mathrm{~F}\right\}$ NMR of 4ia
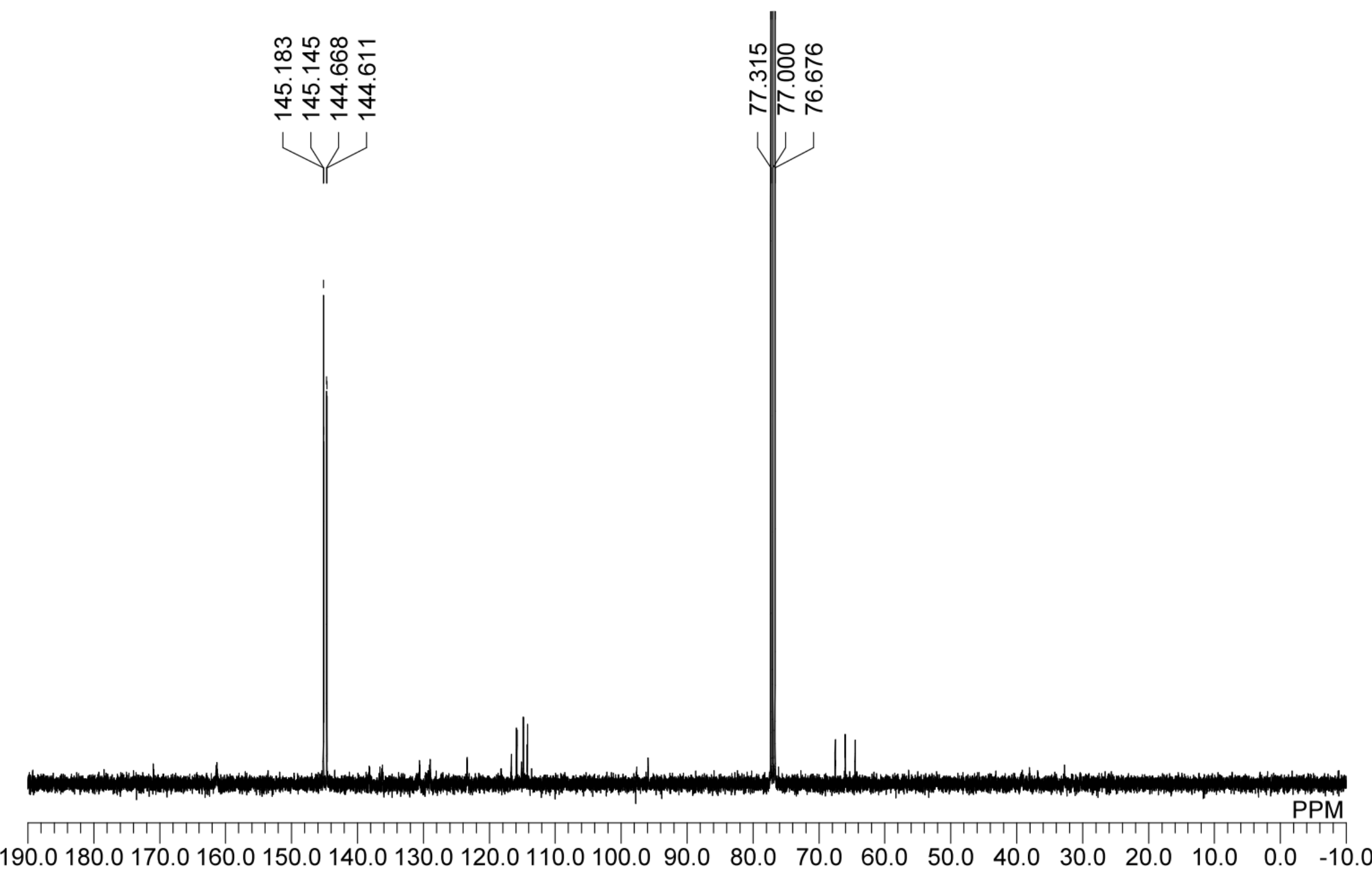
${ }^{19}$ F NMR of 4ia

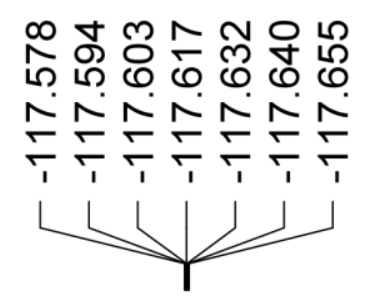

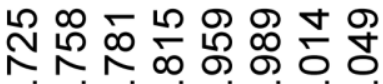

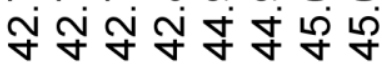
$\overbrace{1}^{1} \overbrace{1}^{1} \frac{1}{1}$
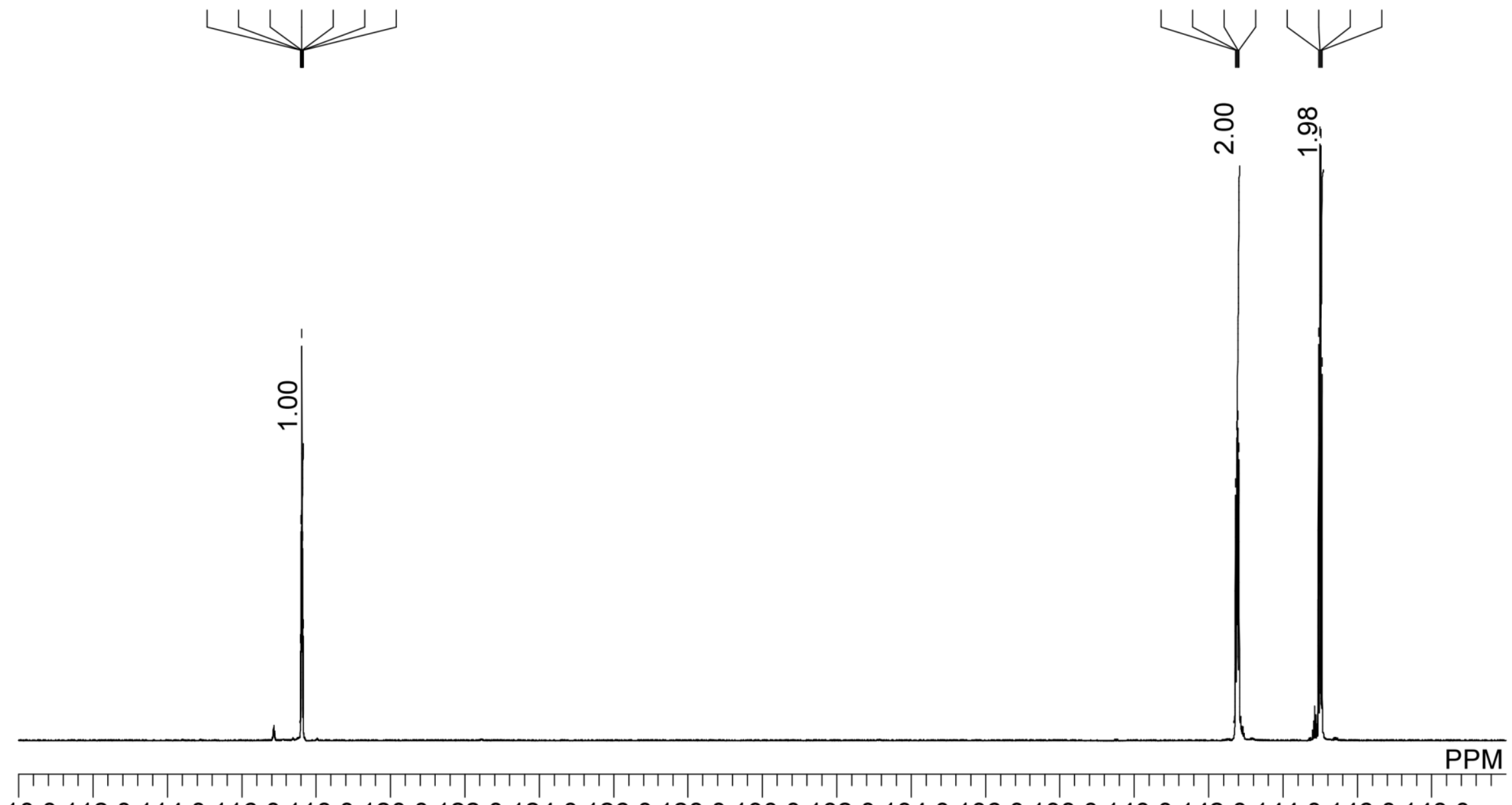

$-110.0-112.0-114.0-116.0-118.0-120.0-122.0-124.0-126.0-128.0-130.0-132.0-134.0-136.0-138.0-140.0-142.0-144.0-146.0-148.0$ 
${ }^{1} \mathrm{H}$ NMR of mixture of $\mathbf{4 j a}$ and its regioisomers ( $\left.p: m: o=64: 32: 4\right)$

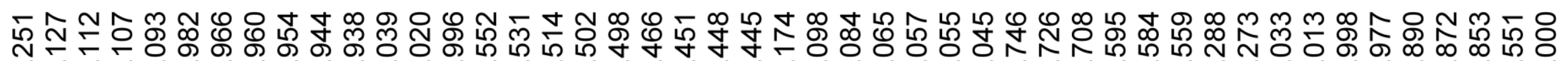

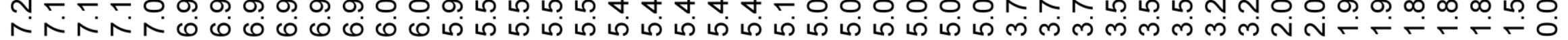
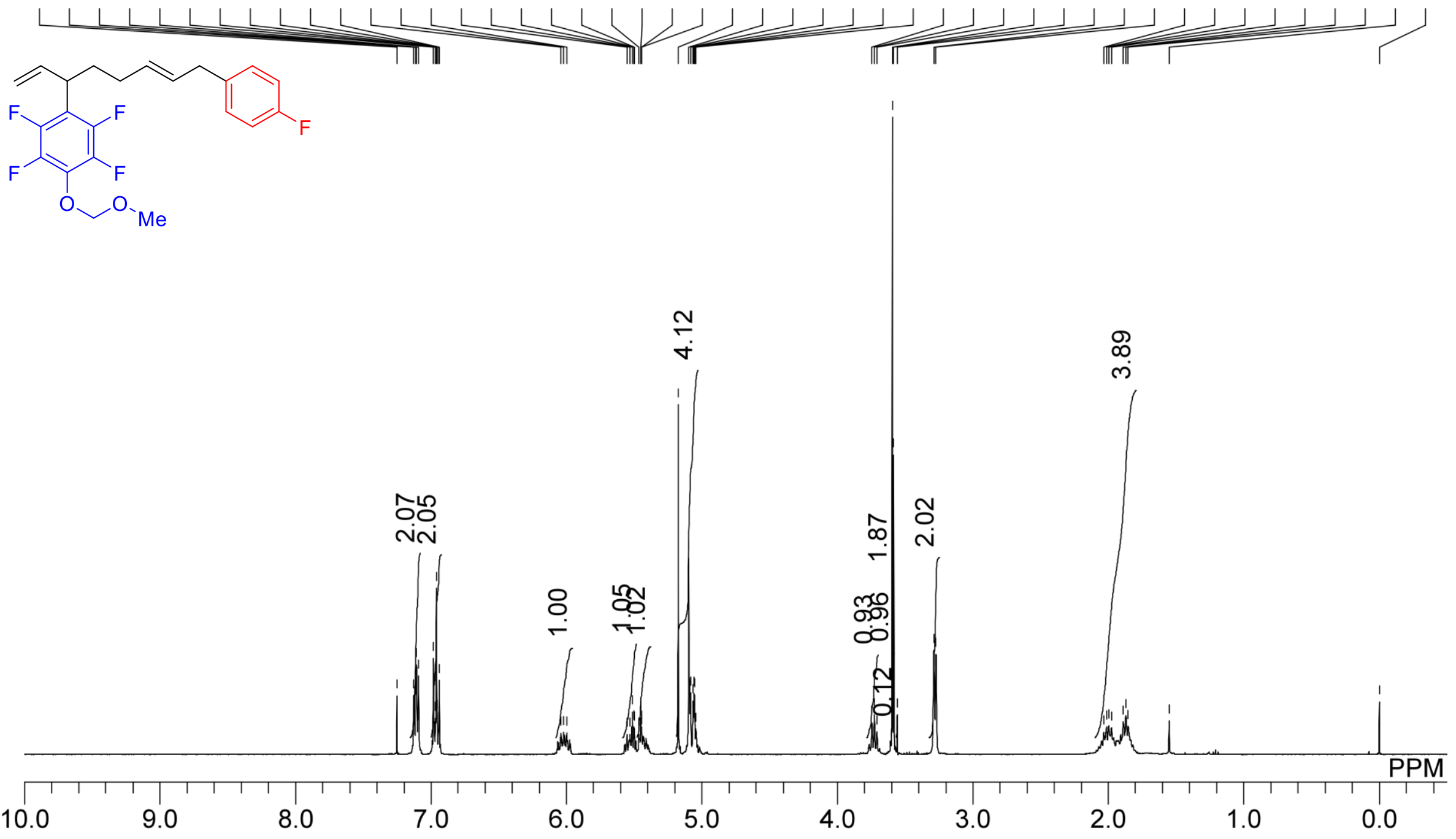
${ }^{13} \mathrm{C}\left\{{ }^{1} \mathrm{H}\right\}$ NMR of mixture of $\mathbf{4 j a}$ and its regioisomers ( $\left.p: m: o=64: 32: 4\right)$

ம ஸ்
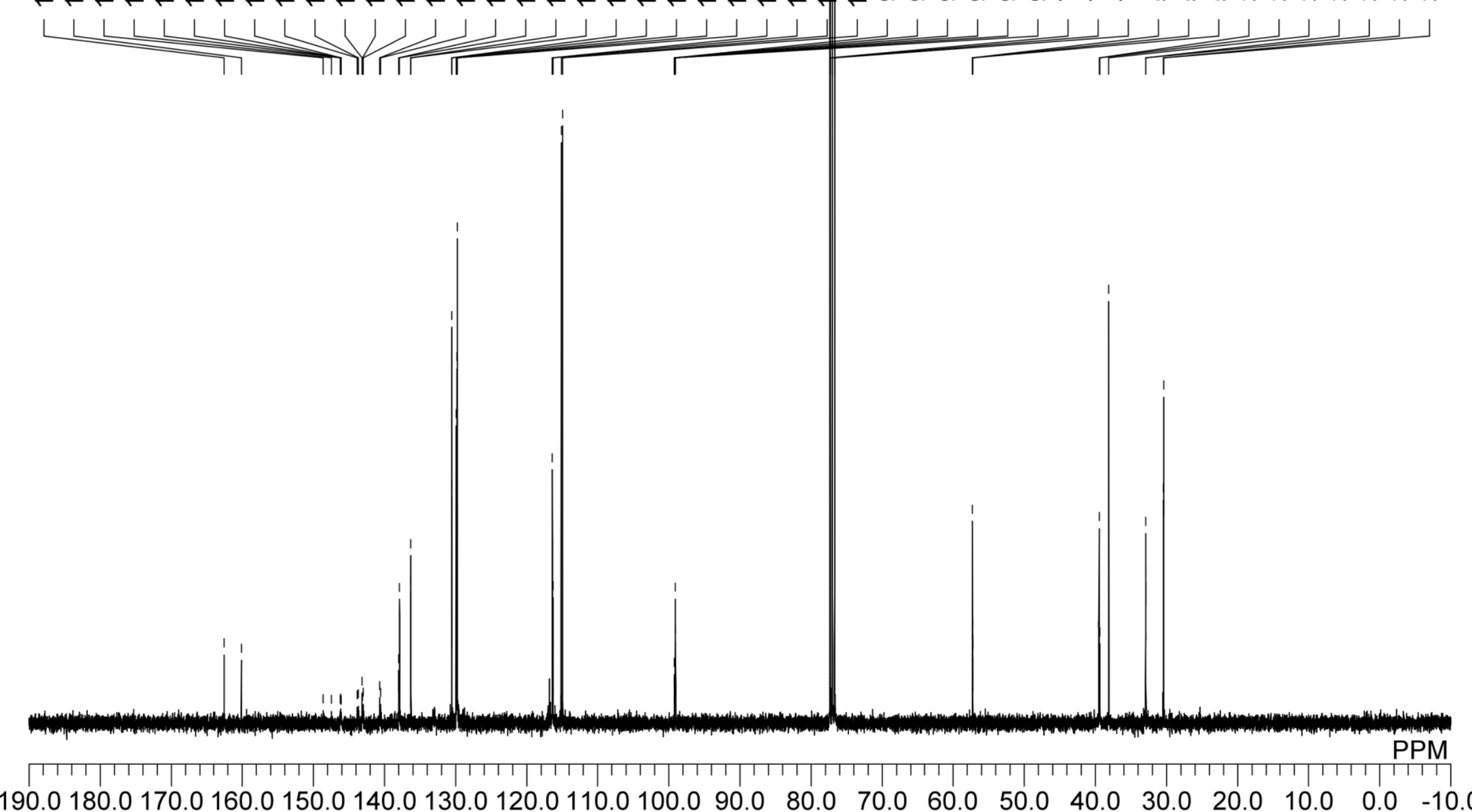
${ }^{13} \mathrm{C}\left\{{ }^{19} \mathrm{~F}\right\}$ NMR of mixture of $4 \mathbf{j a}$ and its regioisomers $(p: m: o=64: 32: 4)$
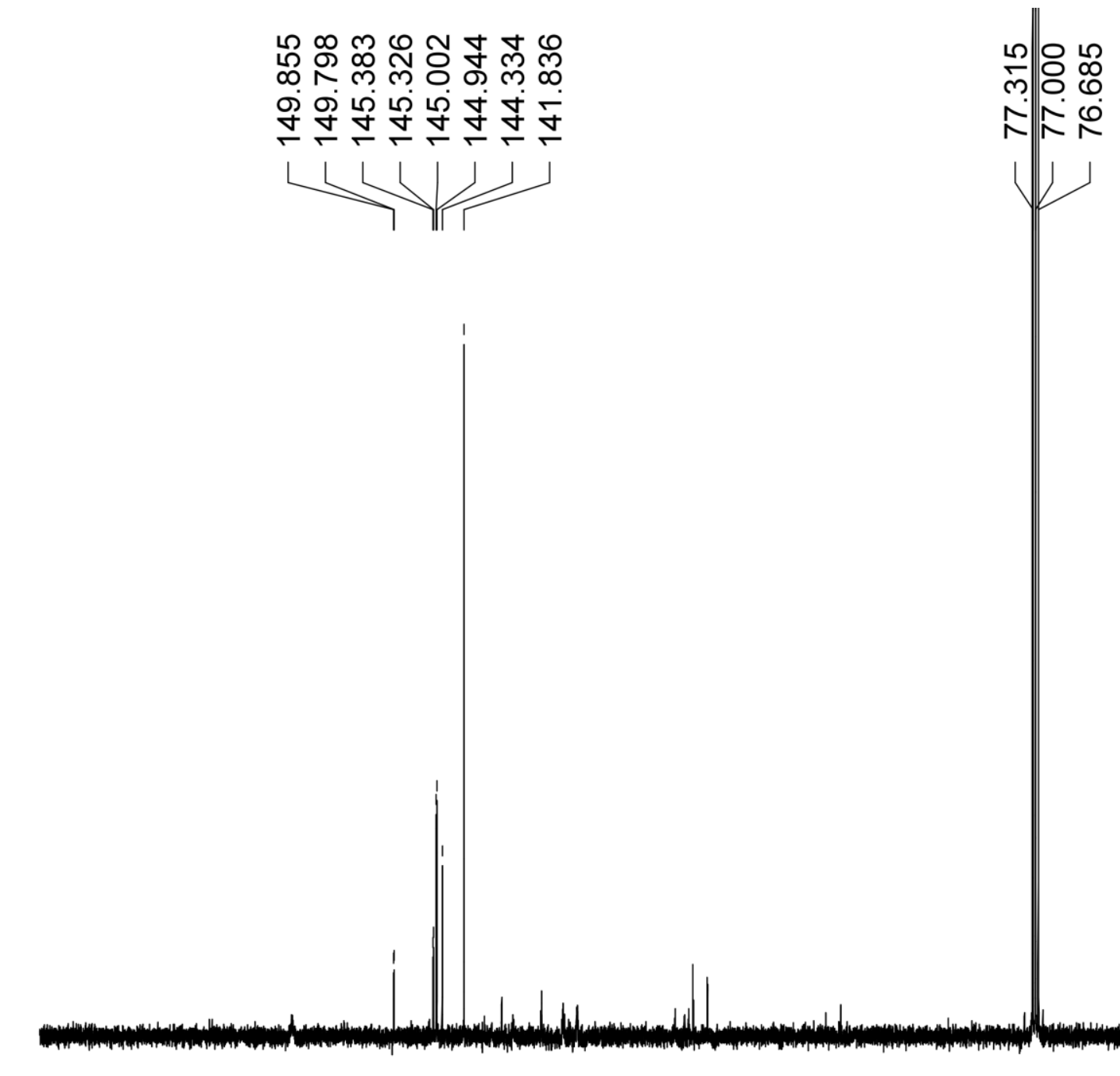

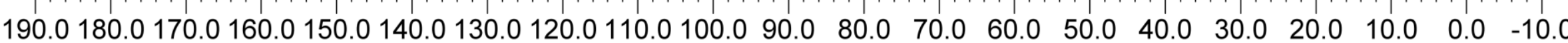


${ }^{19} \mathrm{~F}$ NMR of mixture of $\mathbf{4 j a}$ and its regioisomers ( $\left.p: m: o=64: 32: 4\right)$

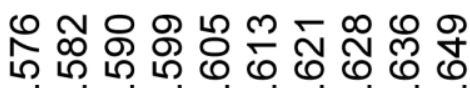

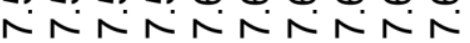

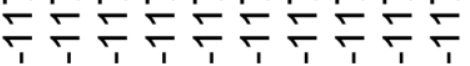

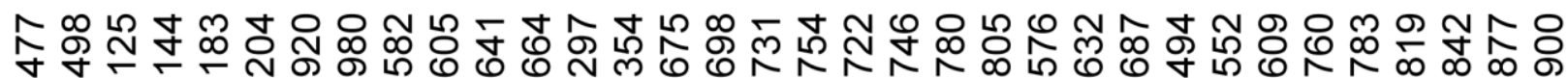
峁
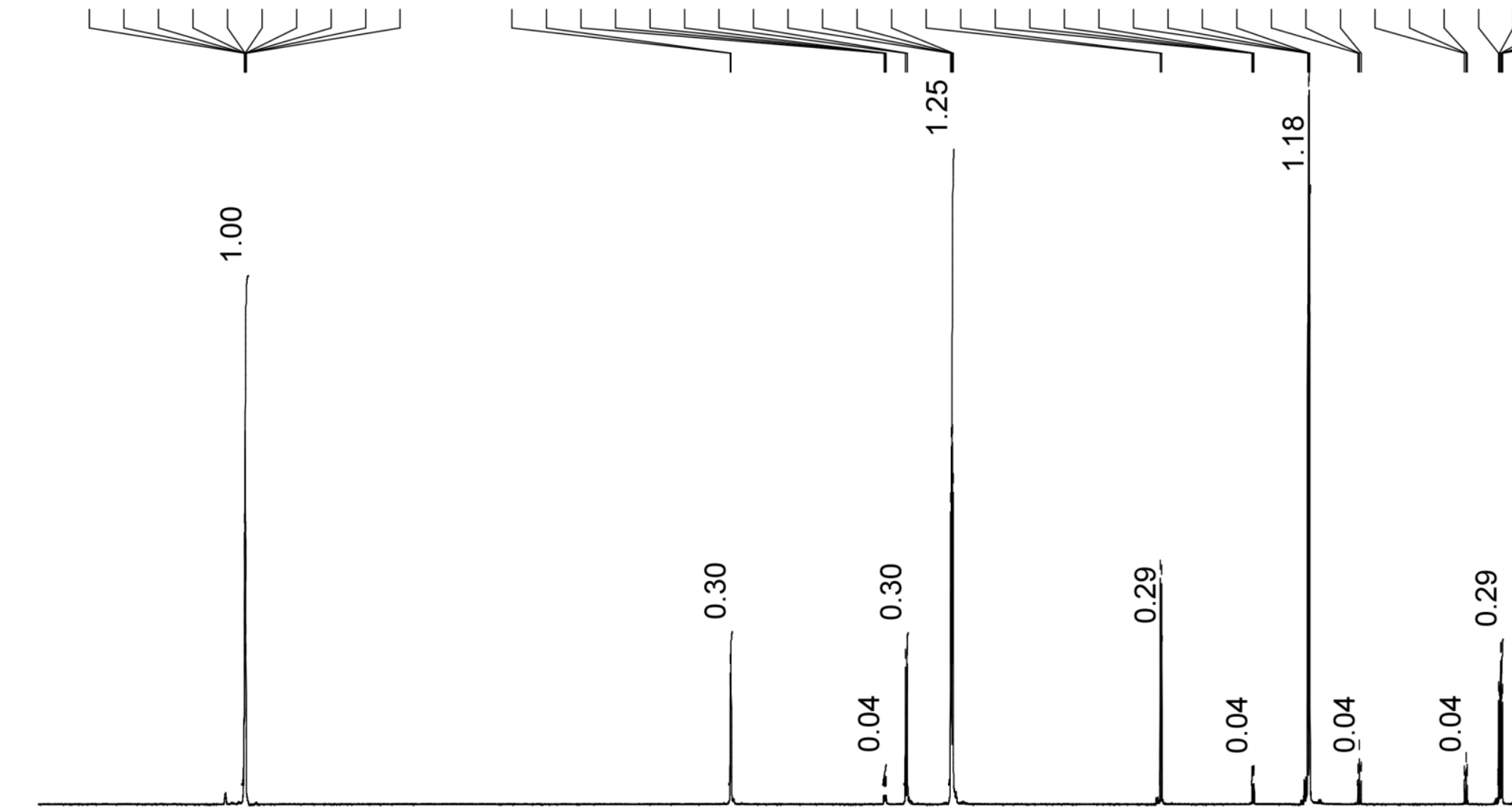

PPM

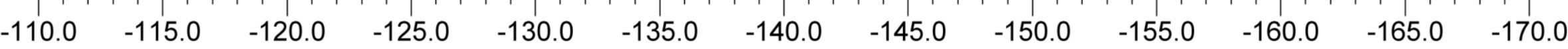


${ }^{1} \mathrm{H}$ NMR of mixture of $\mathbf{4 k a}$ and its regioisomer ( $p: m$ or $\left.o=96: 4\right)$

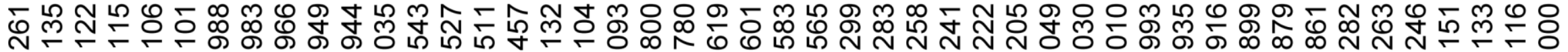

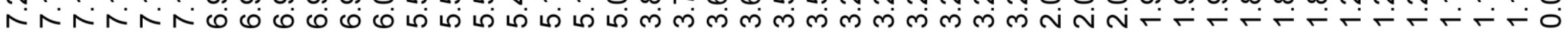

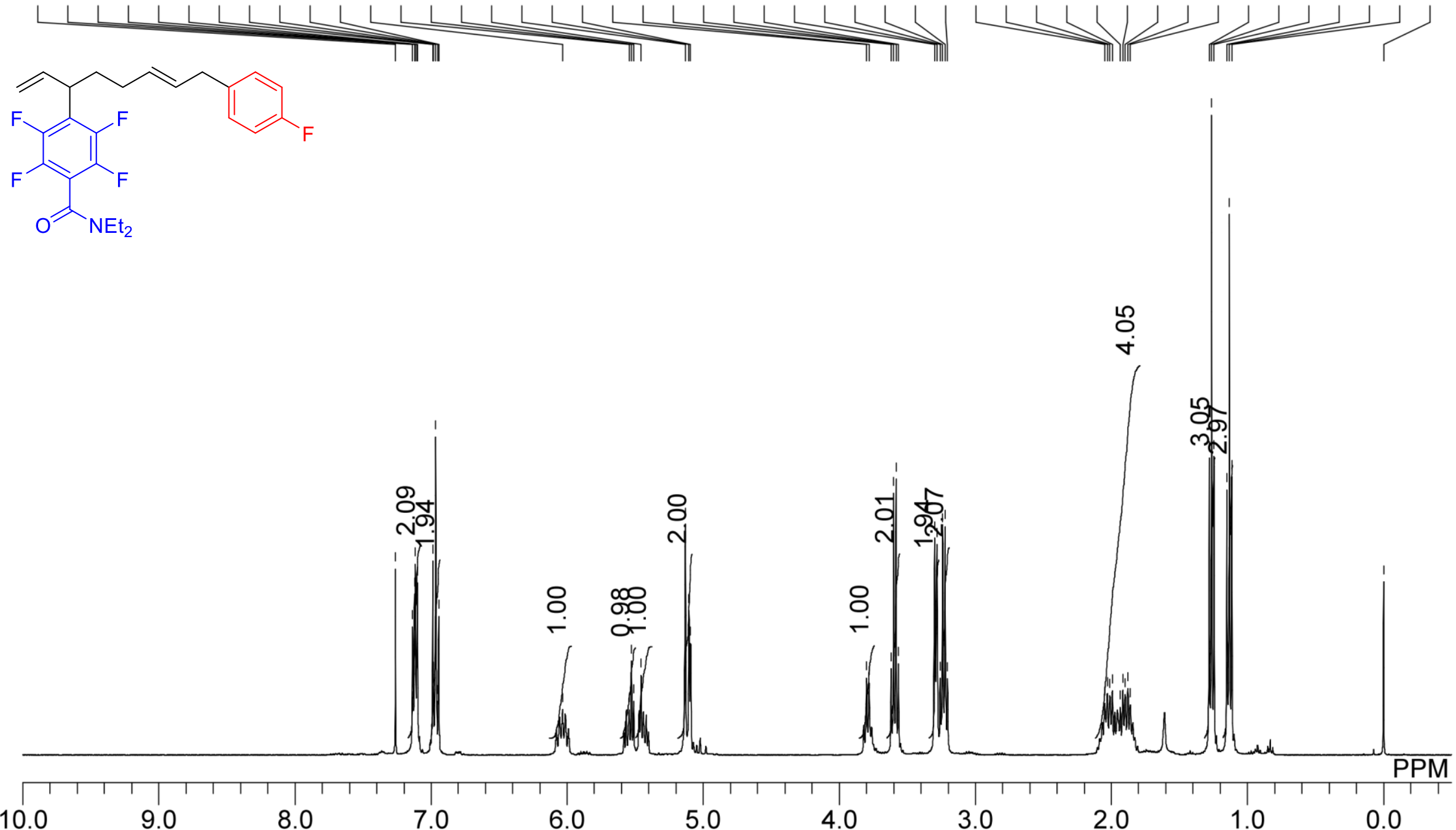


${ }^{13} \mathrm{C}\left\{{ }^{1} \mathrm{H}\right\}$ NMR of mixture of $\mathbf{4 k a}$ and its regioisomer ( $p: m$ or $o=96: 4$ )

只 ஸู

윰

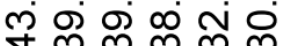

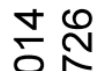
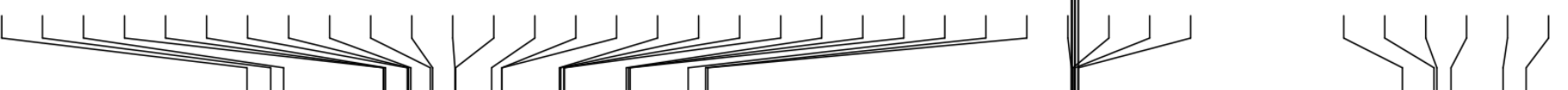

$\underset{2}{\sim}$
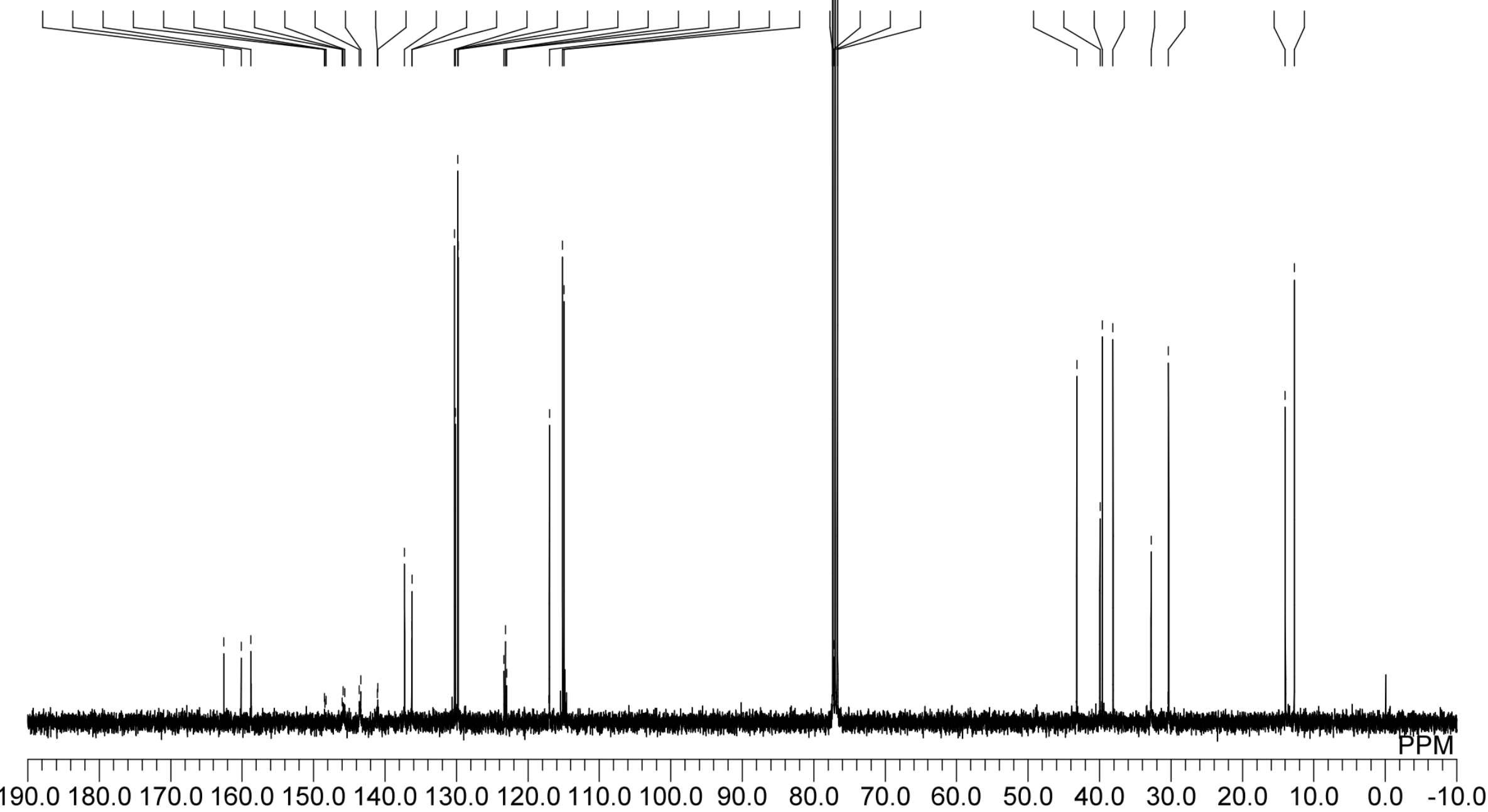
${ }^{13} \mathrm{C}\left\{{ }^{19} \mathrm{~F}\right\}$ NMR of mixture of $\mathbf{4 k a}$ and its regioisomer ( $p: m$ or $\left.o=96: 4\right)$
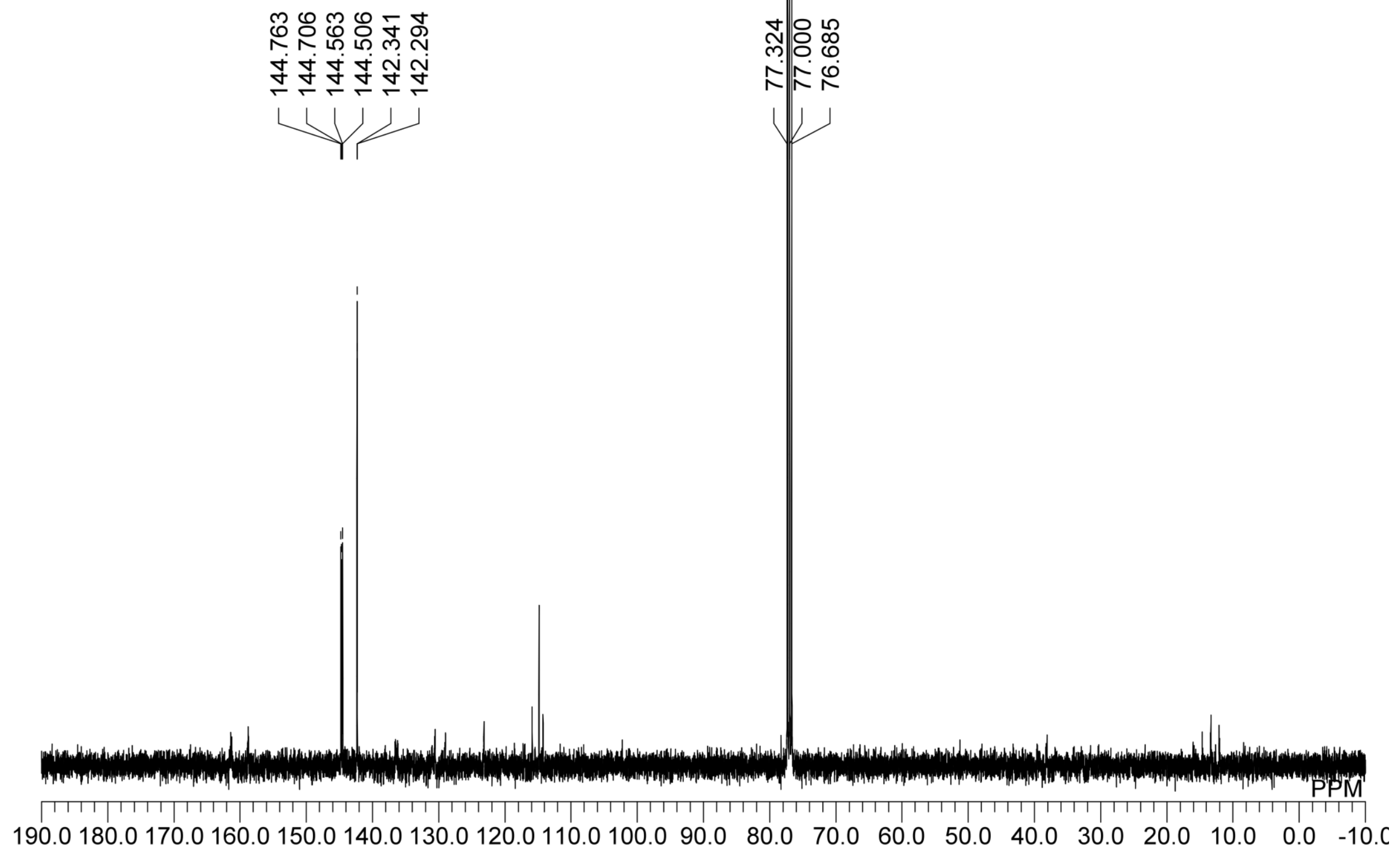
${ }^{19} \mathrm{~F}$ NMR of mixture of $\mathbf{4 k a}$ and its regioisomer ( $p: m$ or $o=96: 4$ )

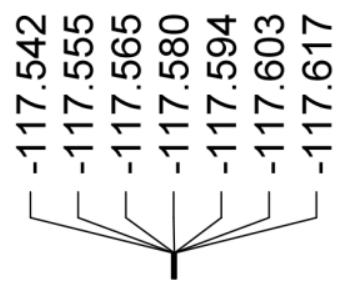

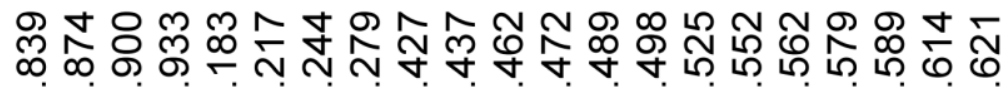

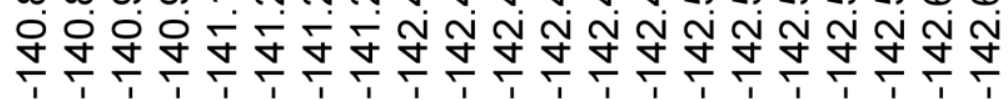

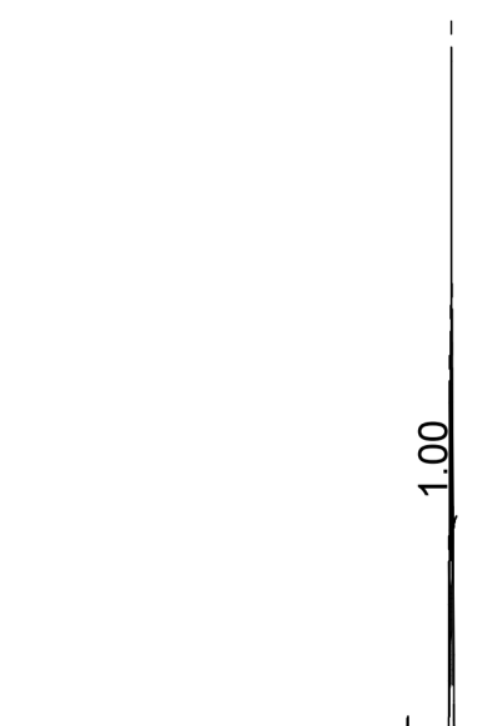


${ }^{1} \mathrm{H}$ NMR of $\mathbf{5 k a}$

œ 요

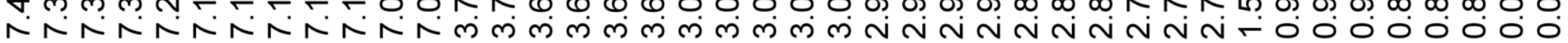
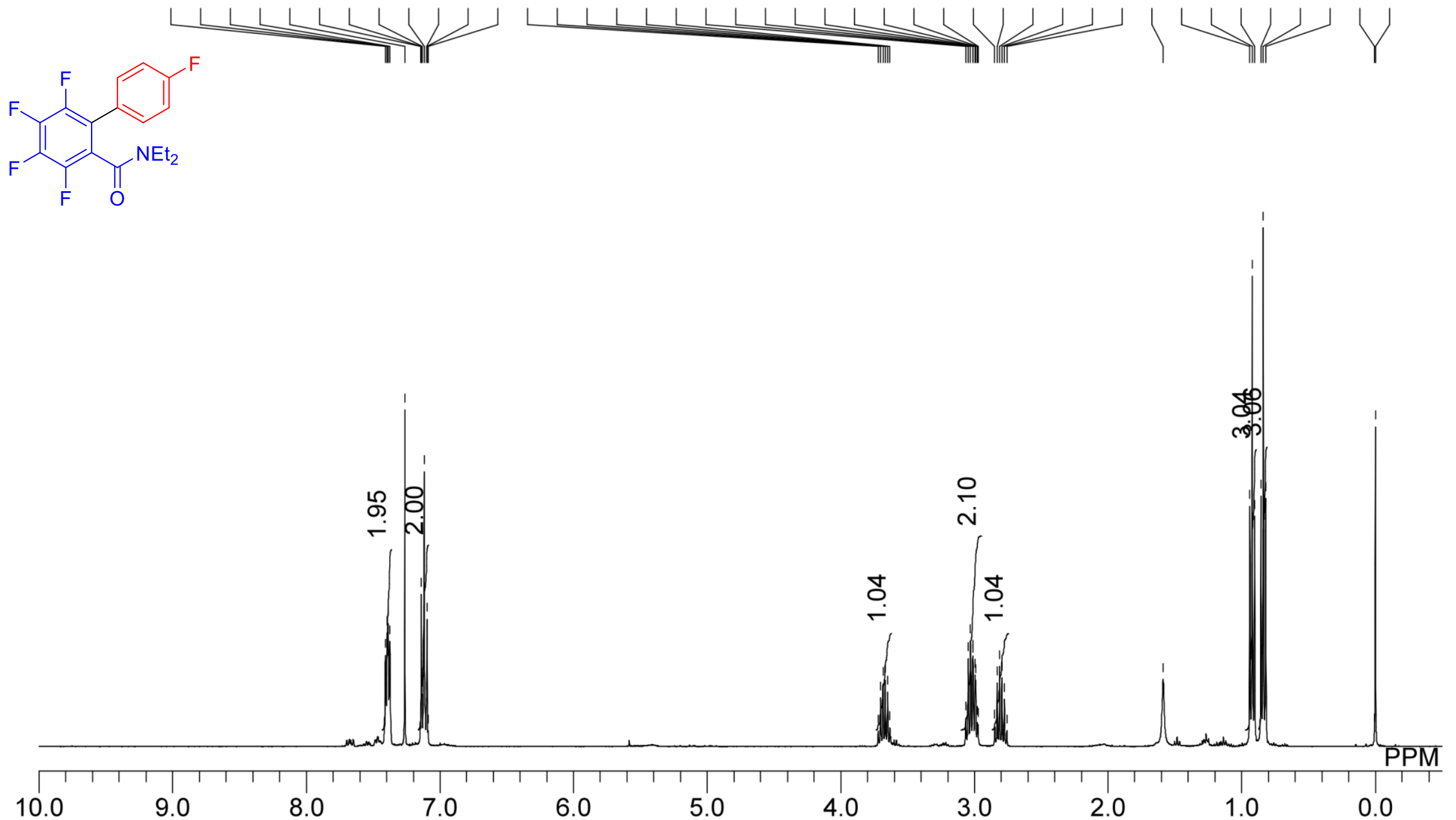
${ }^{13} \mathrm{C}\left\{{ }^{1} \mathrm{H}\right\}$ NMR of 5 ka

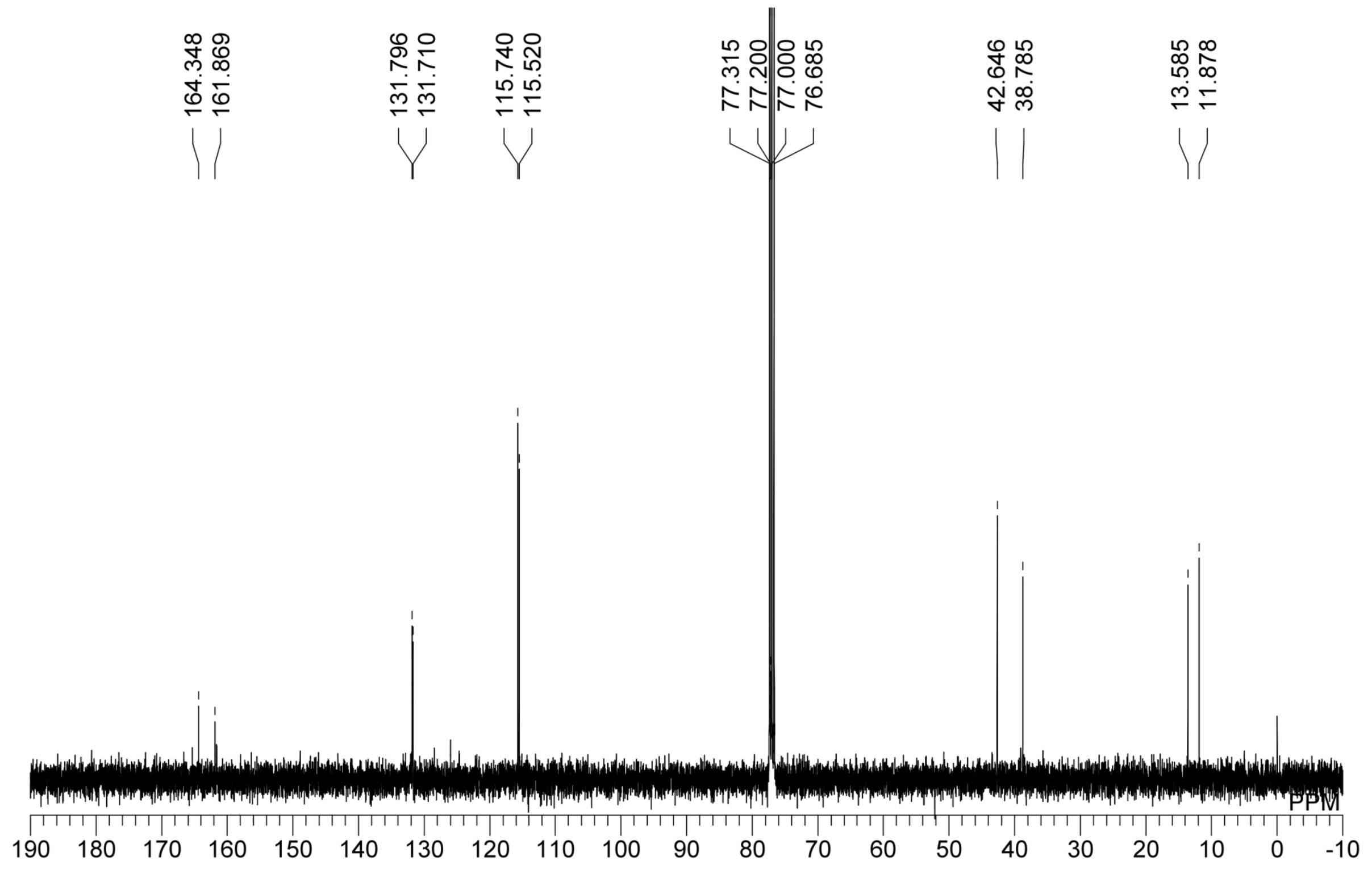


${ }^{19} \mathrm{~F}$ NMR of $\mathbf{5 k a}$

요

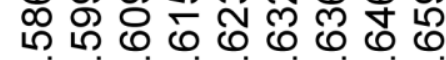

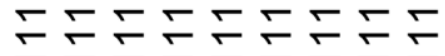

$\frac{1}{1} \frac{1}{1} \frac{1}{1} \frac{1}{1} \frac{1}{1}$

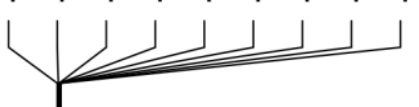

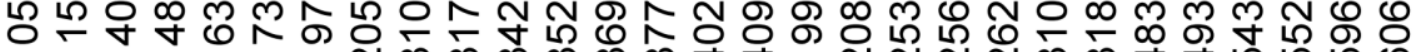

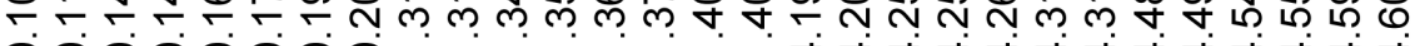

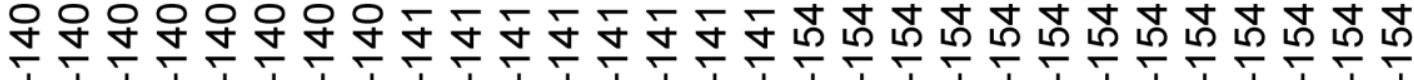
11

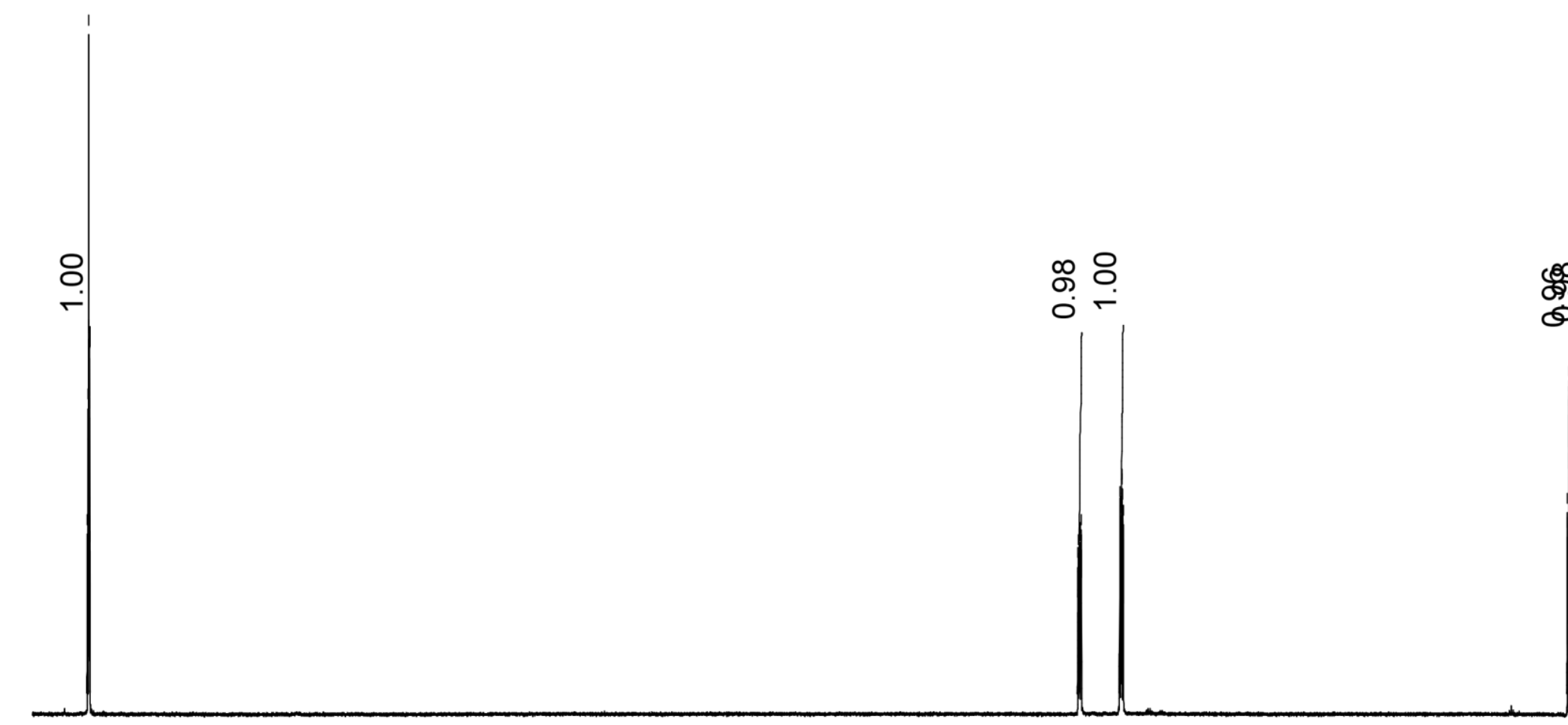

PPM

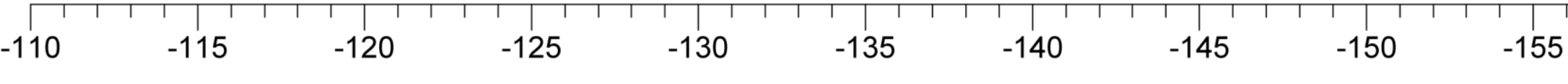




\section{${ }^{1} \mathrm{H}$ NMR of $\mathbf{4 k p}$}

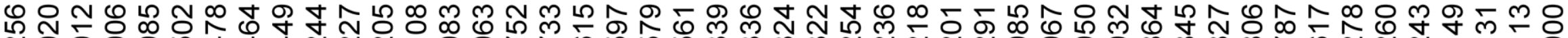
N
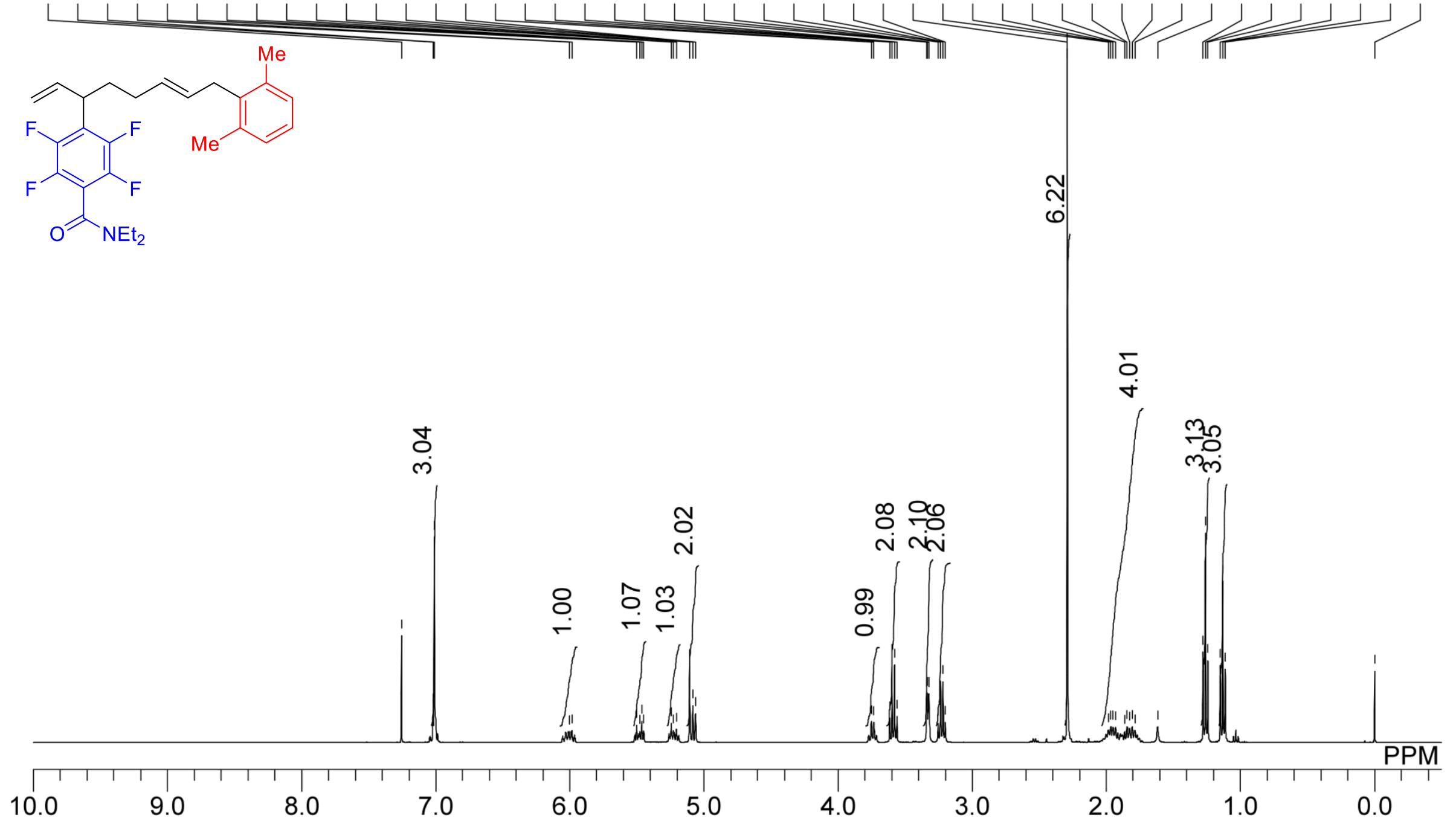


\section{${ }^{13} \mathrm{C}\left\{{ }^{1} \mathrm{H}\right\}$ NMR of $\mathbf{4 k p}$}

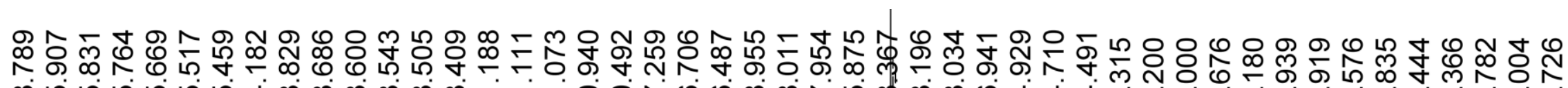

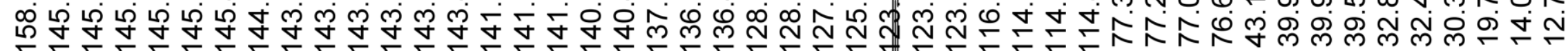
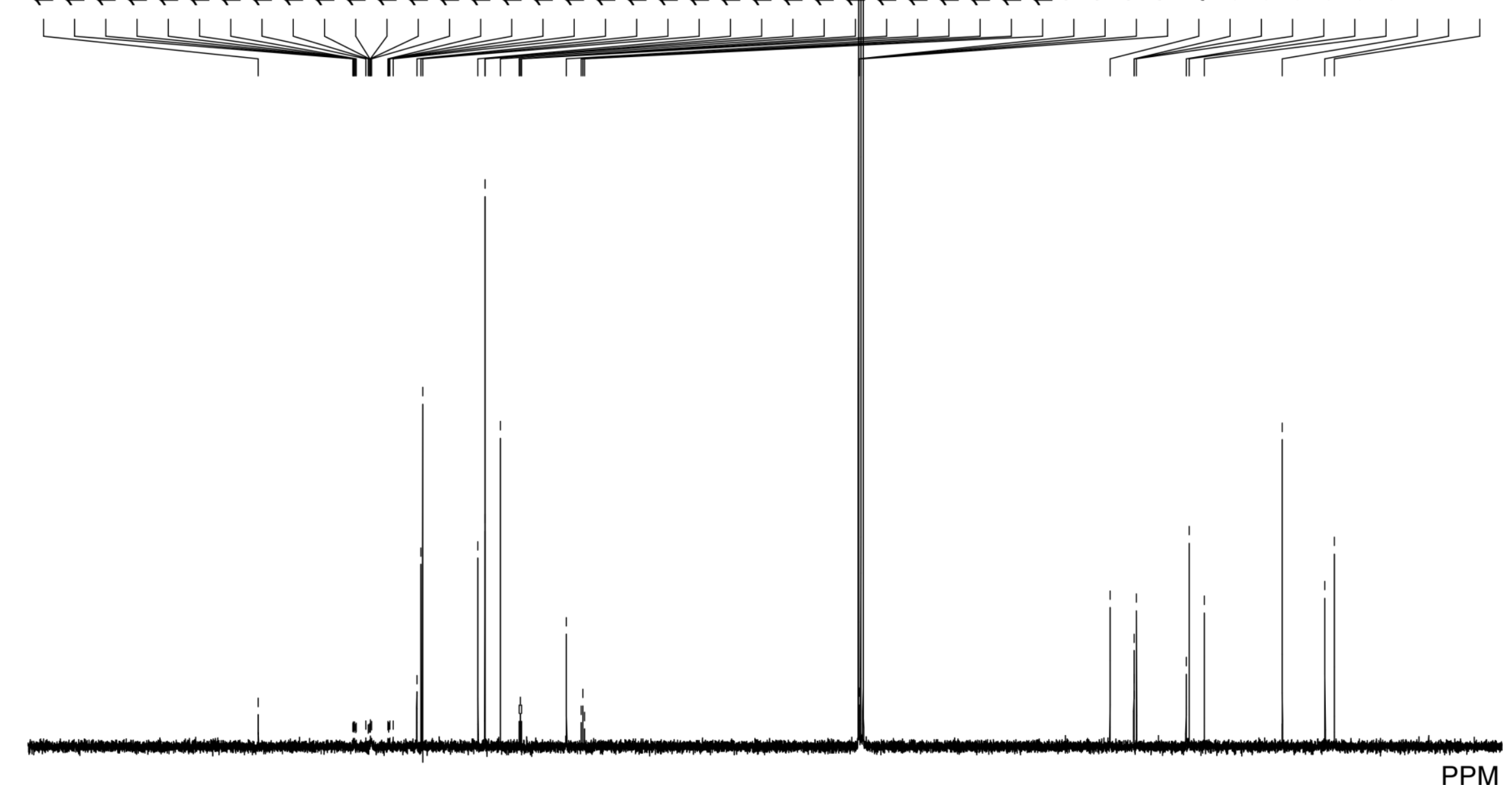

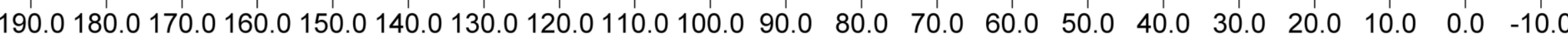


${ }^{13} \mathrm{C}\left\{{ }^{19} \mathrm{~F}\right\}$ NMR of $\mathbf{4 k p}$

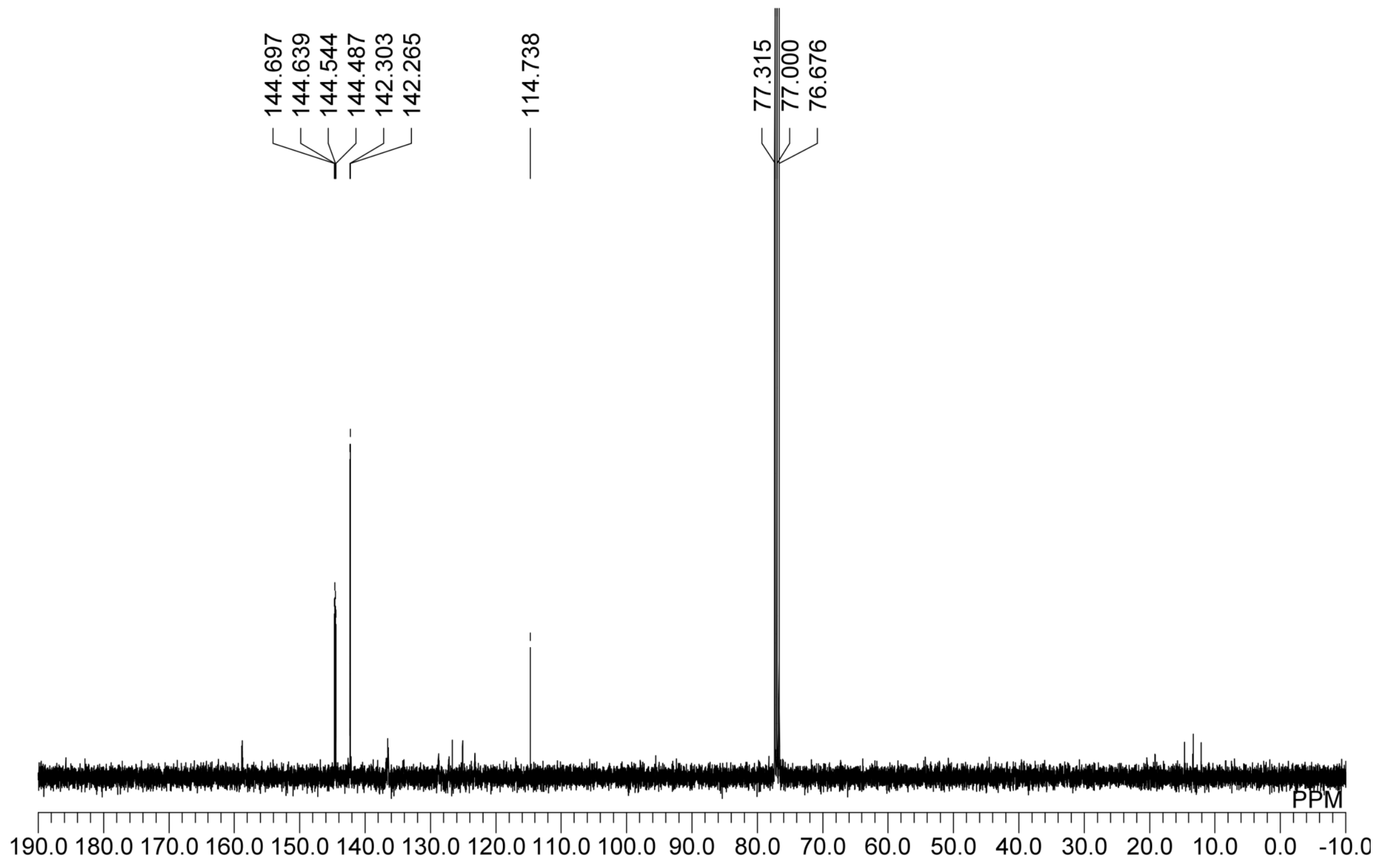


${ }^{19}$ F NMR of $\mathbf{4 k p}$

낭

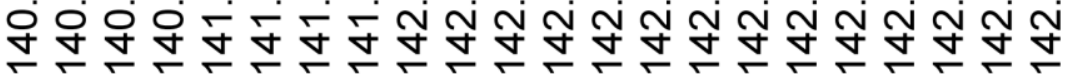
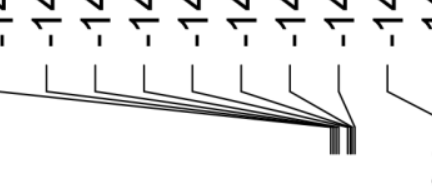

깅

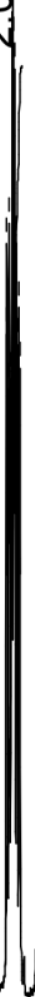

$\begin{array}{lllllllllllllll}-130.0 & -132.0 & -134.0 & -136.0 & -138.0 & -140.0 & -142.0 & -144.0 & -146.0 & -148.0 & -150.0 & -152.0 & -154.0 & -156.0 & -158.0\end{array}$ 
${ }^{1} \mathrm{H}$ NMR of mixture of $4 \mathbf{l a}$ and its regioisomer $(p: m=90: 10)$

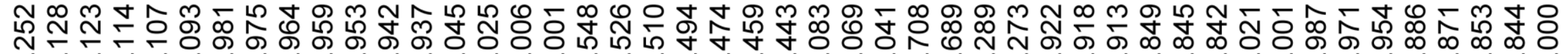
N
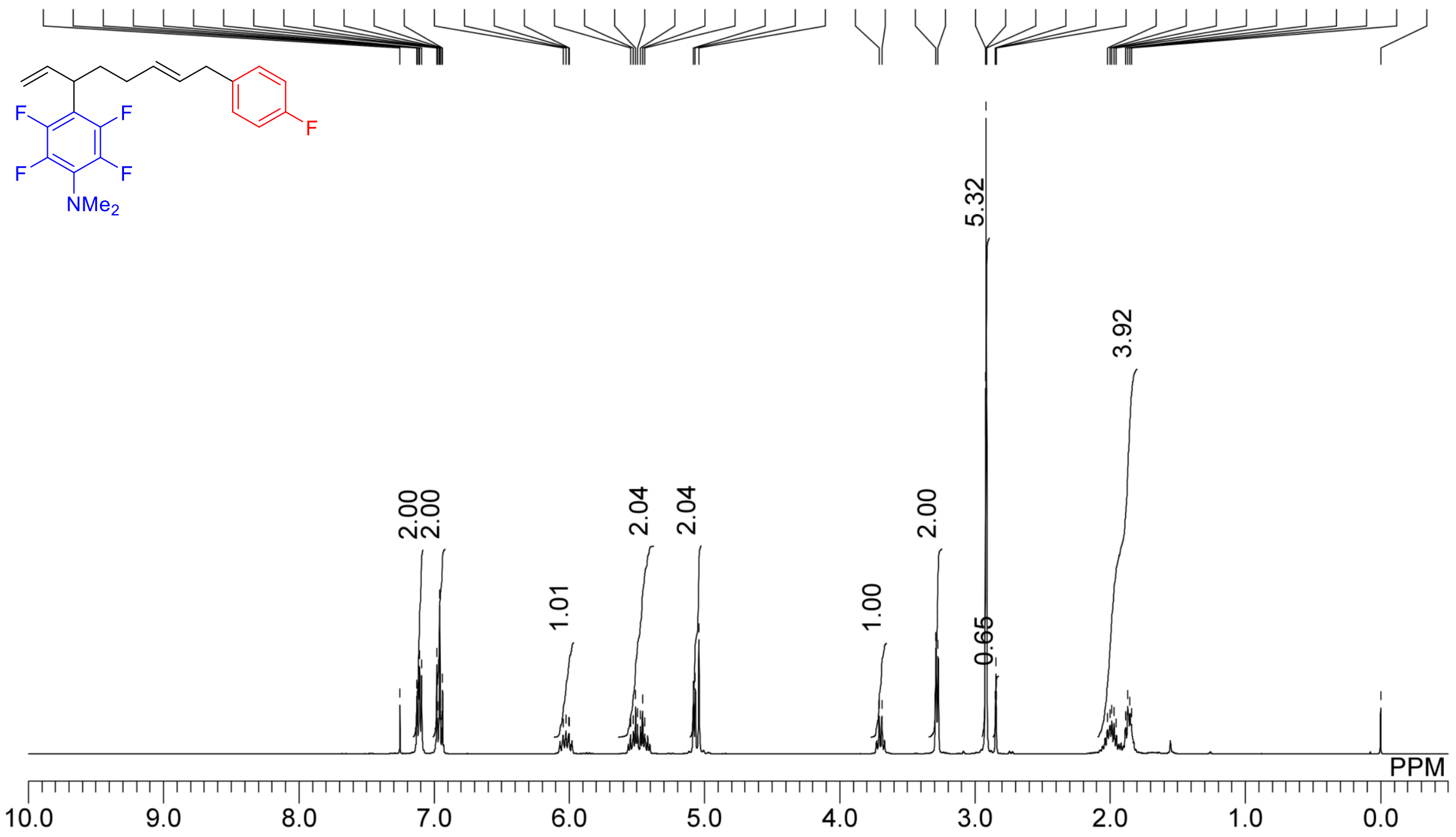
${ }^{13} \mathrm{C}\left\{{ }^{1} \mathrm{H}\right\}$ NMR of mixture of $\mathbf{4 l a}$ and its regioisomer $(p: m=90: 10)$

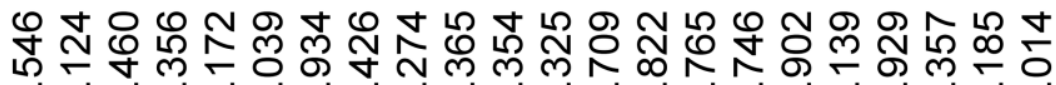
đீ

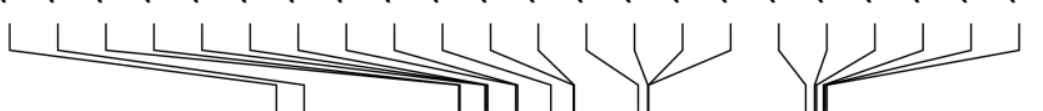

๓ NヘN

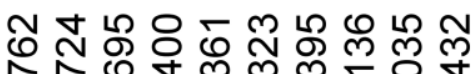

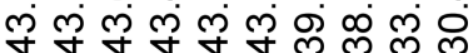

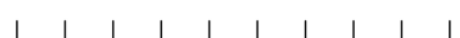

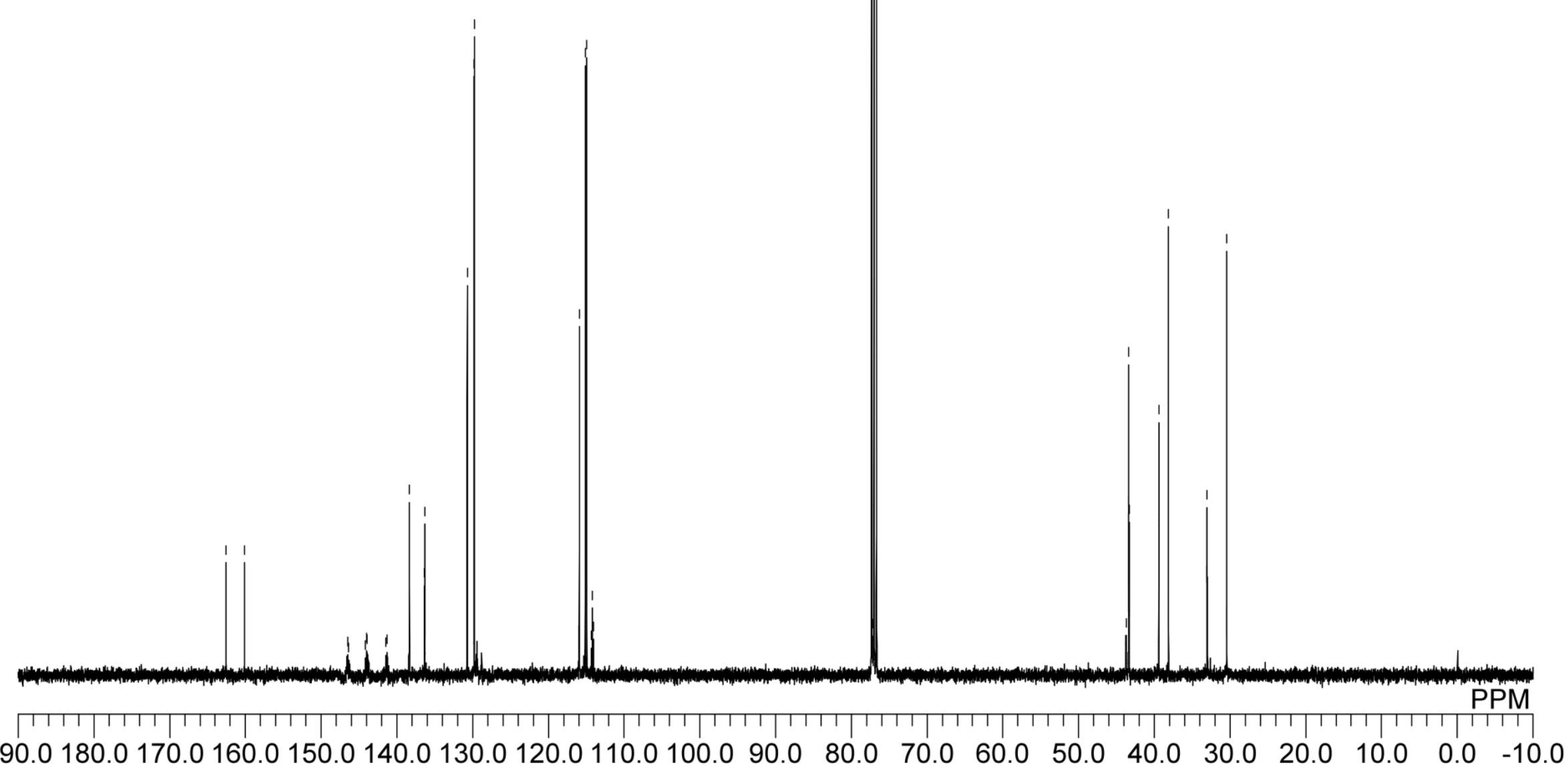


${ }^{13} \mathrm{C}\left\{{ }^{19} \mathrm{~F}\right\}$ NMR of mixture of $\mathbf{4 l a}$ and its regioisomer ( $p: m=90: 10$ )

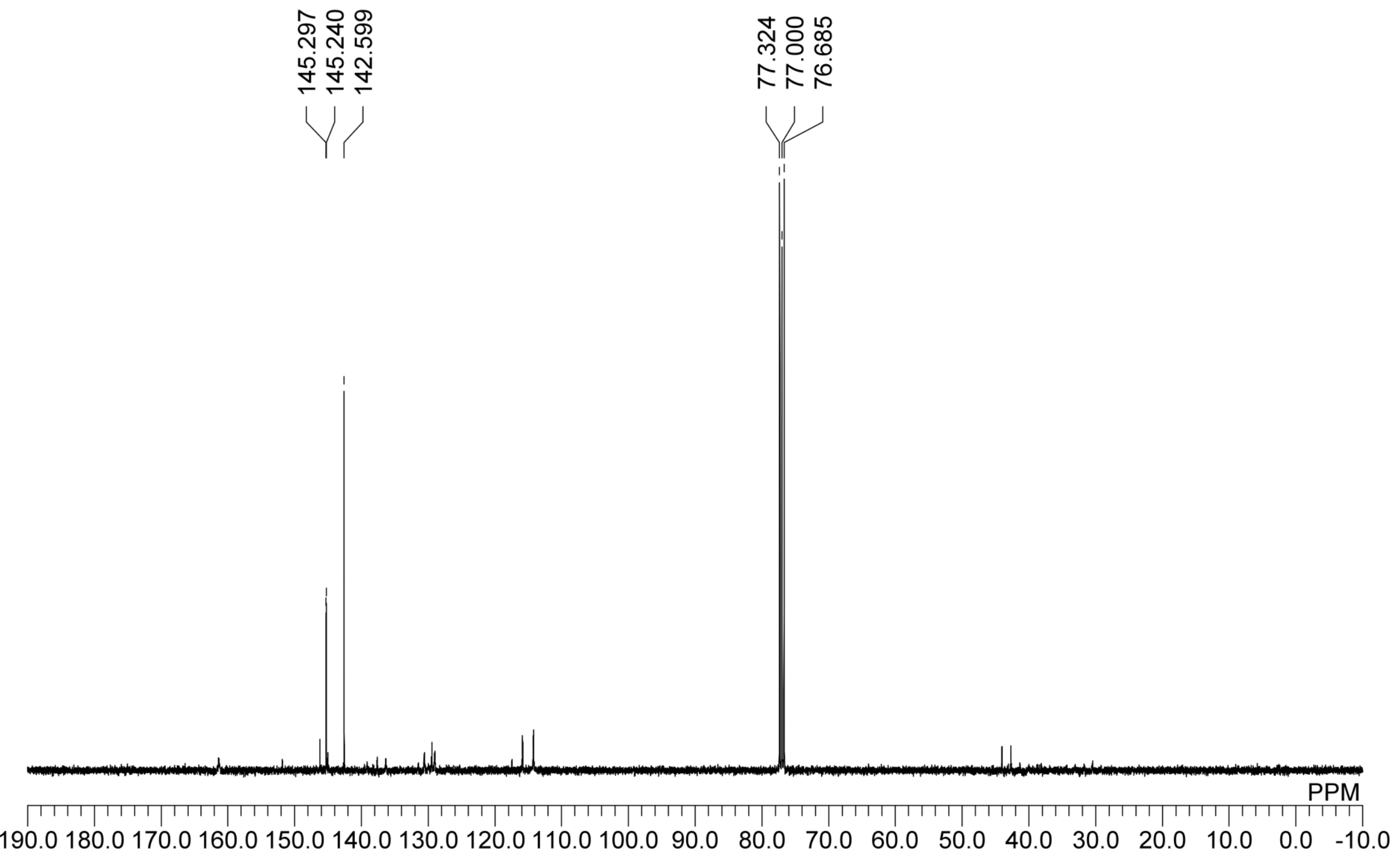


${ }^{19} \mathrm{~F}$ NMR of mixture of $\mathbf{4 l a}$ and its regioisomer $(p: m=90: 10)$

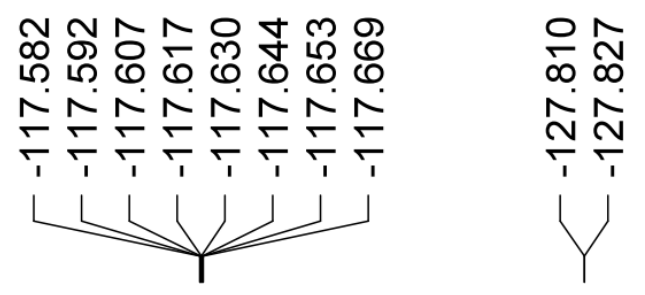

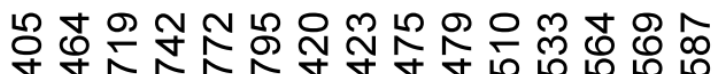

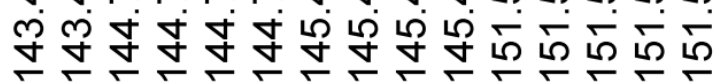

オ⿻

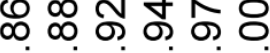

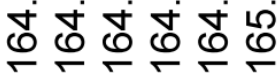
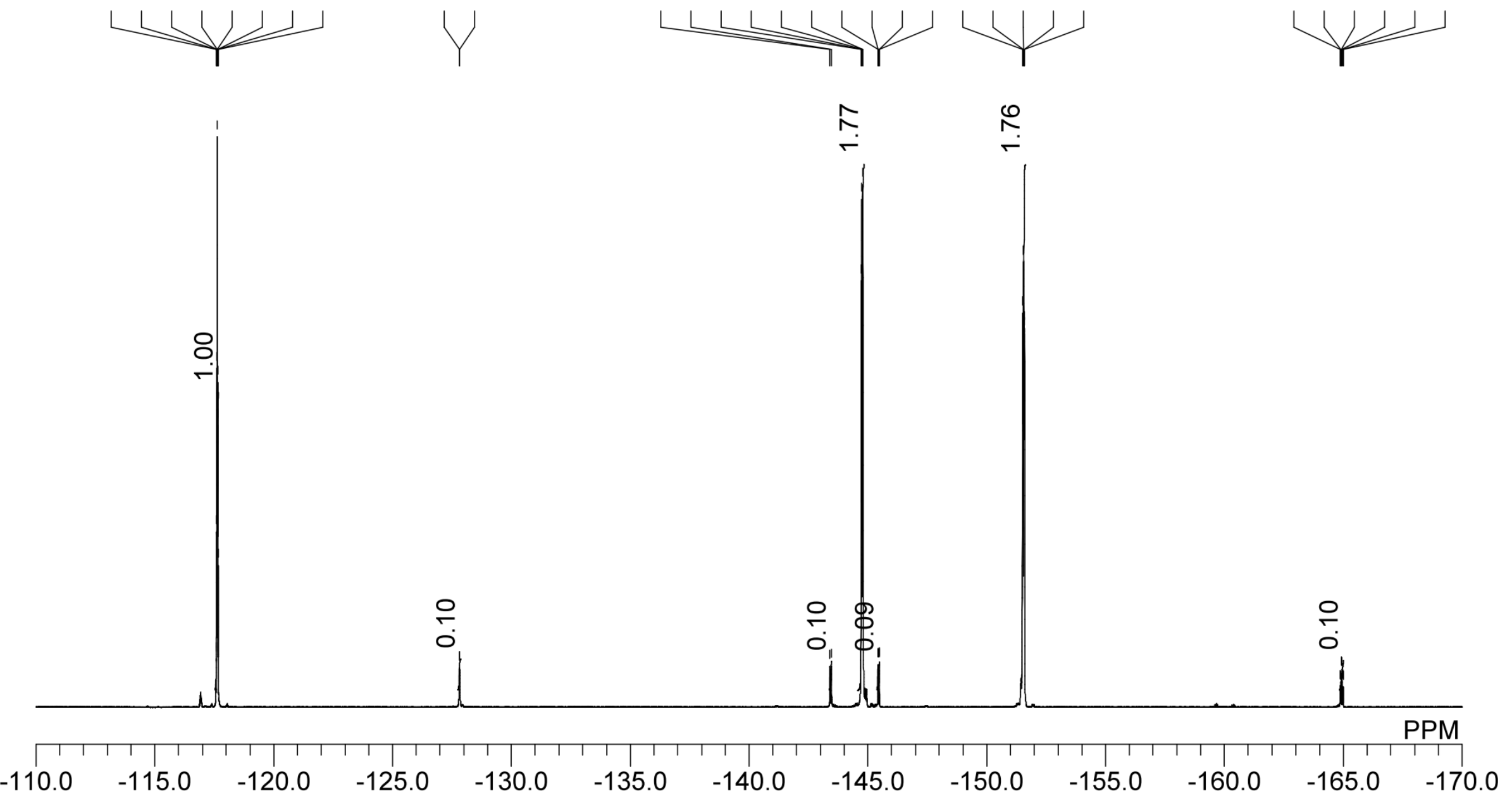


\section{${ }^{1} \mathrm{H}$ NMR of 4 ma}

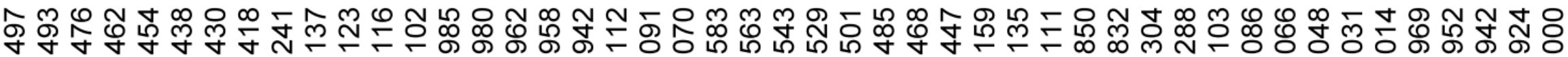

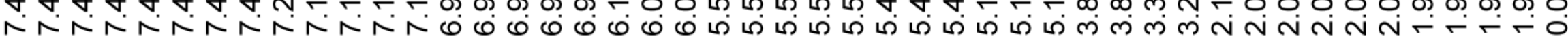
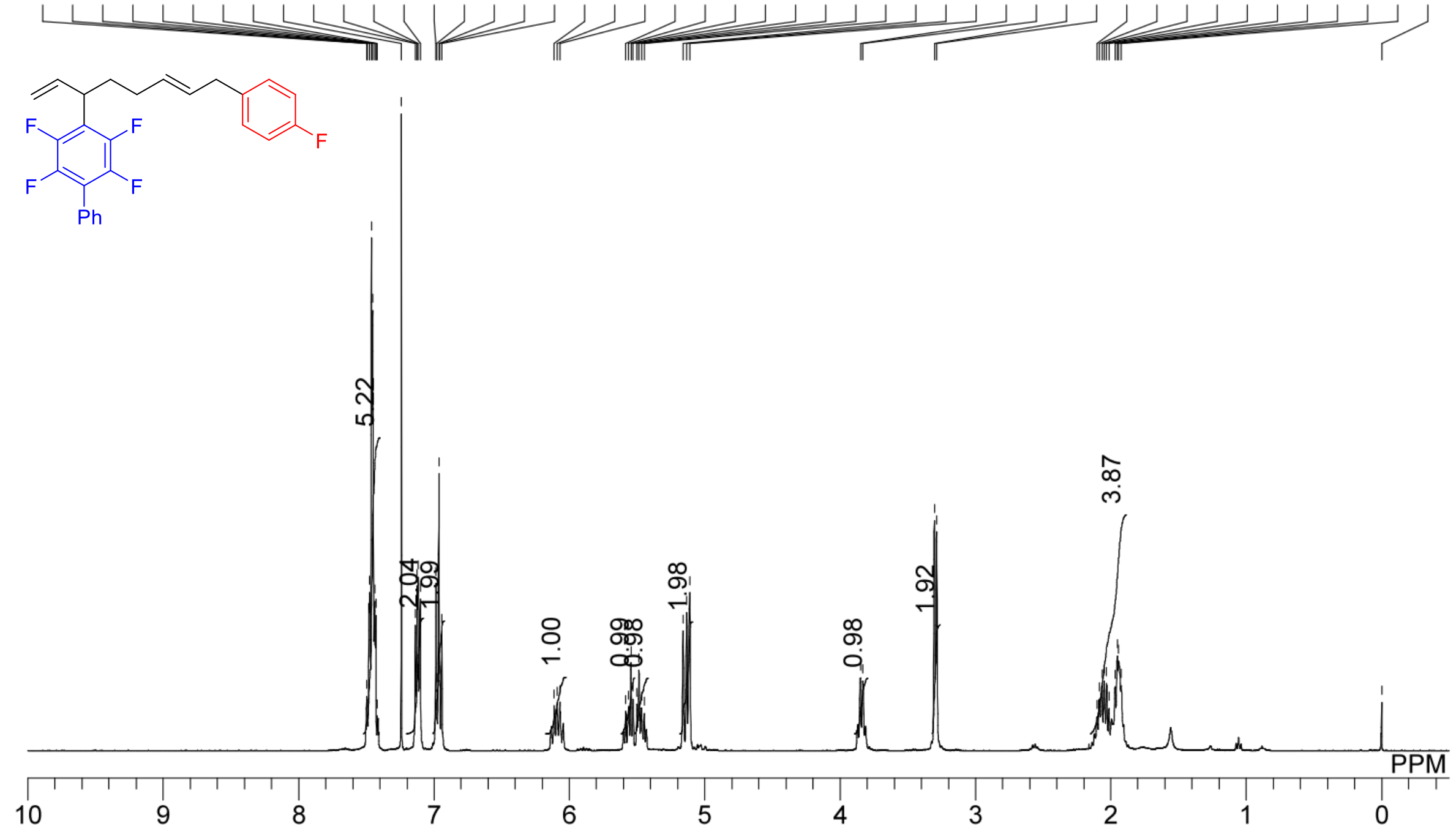


\section{${ }^{13} \mathrm{C}\left\{{ }^{1} \mathrm{H}\right\}$ NMR of $\mathbf{4 m a}$}

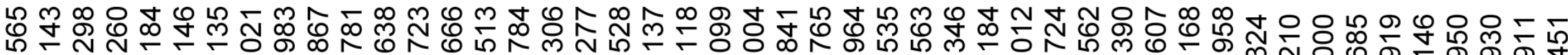
ஸ்

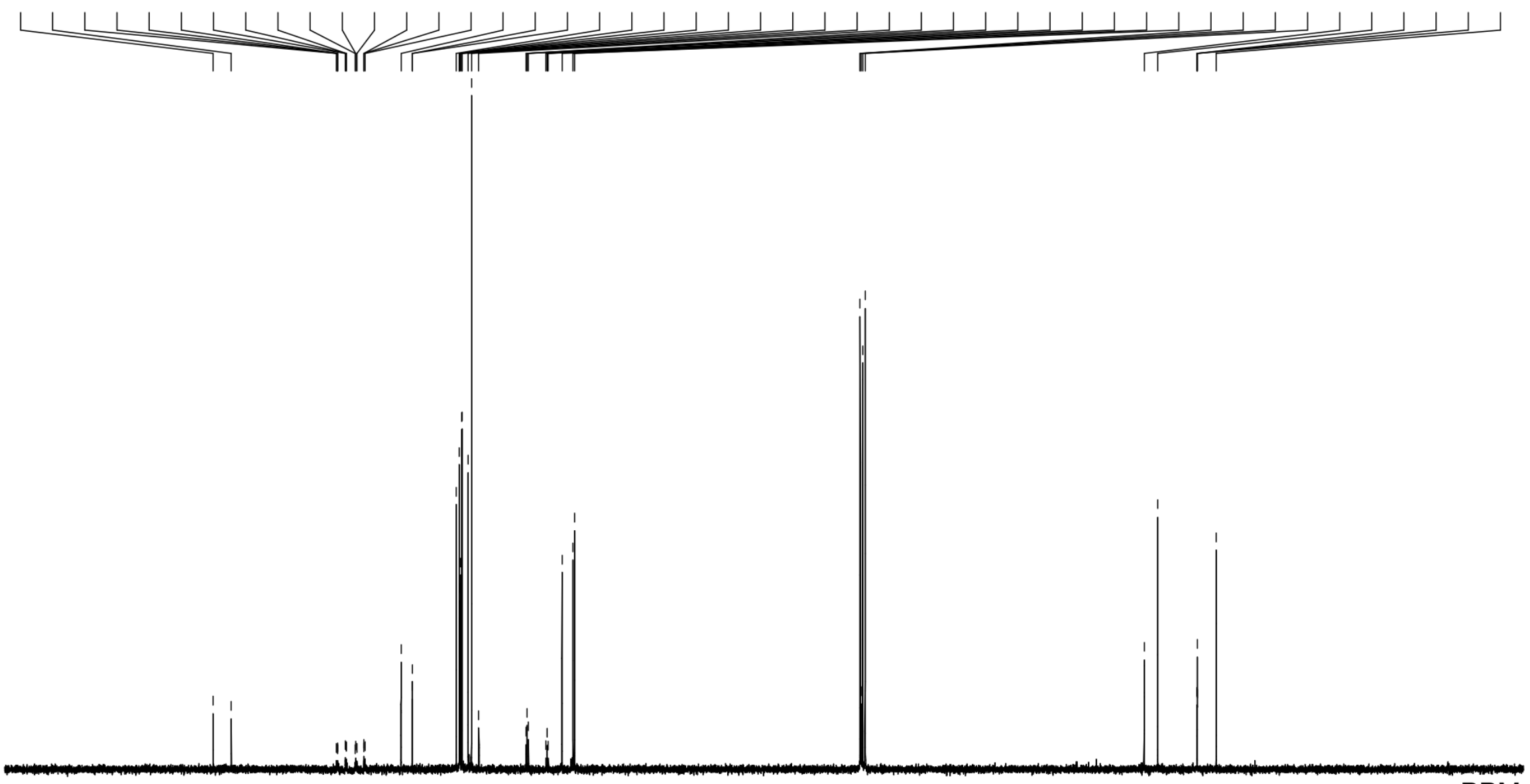

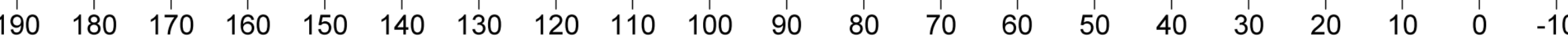


${ }^{13} \mathrm{C}\left\{{ }^{19} \mathrm{~F}\right\}$ NMR of $\mathbf{4 m a}$

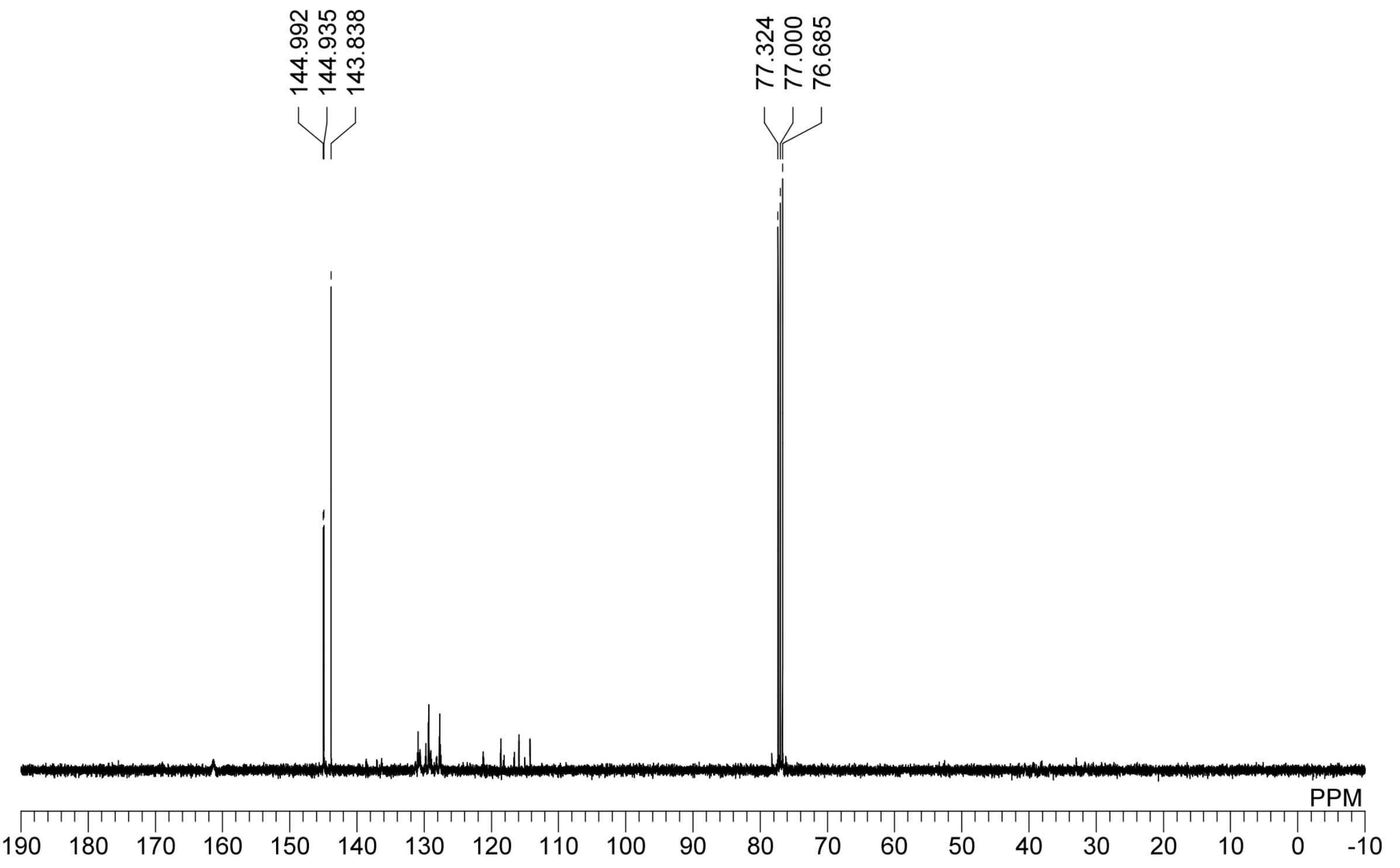


${ }^{19} \mathrm{~F}$ NMR of $\mathbf{4 m a}$

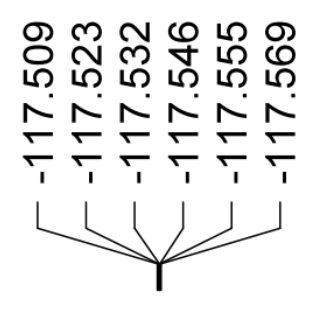

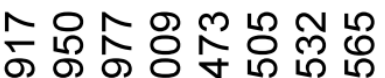

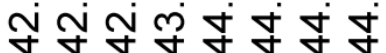
$\div \div \frac{1}{1} \div \frac{1}{1}$
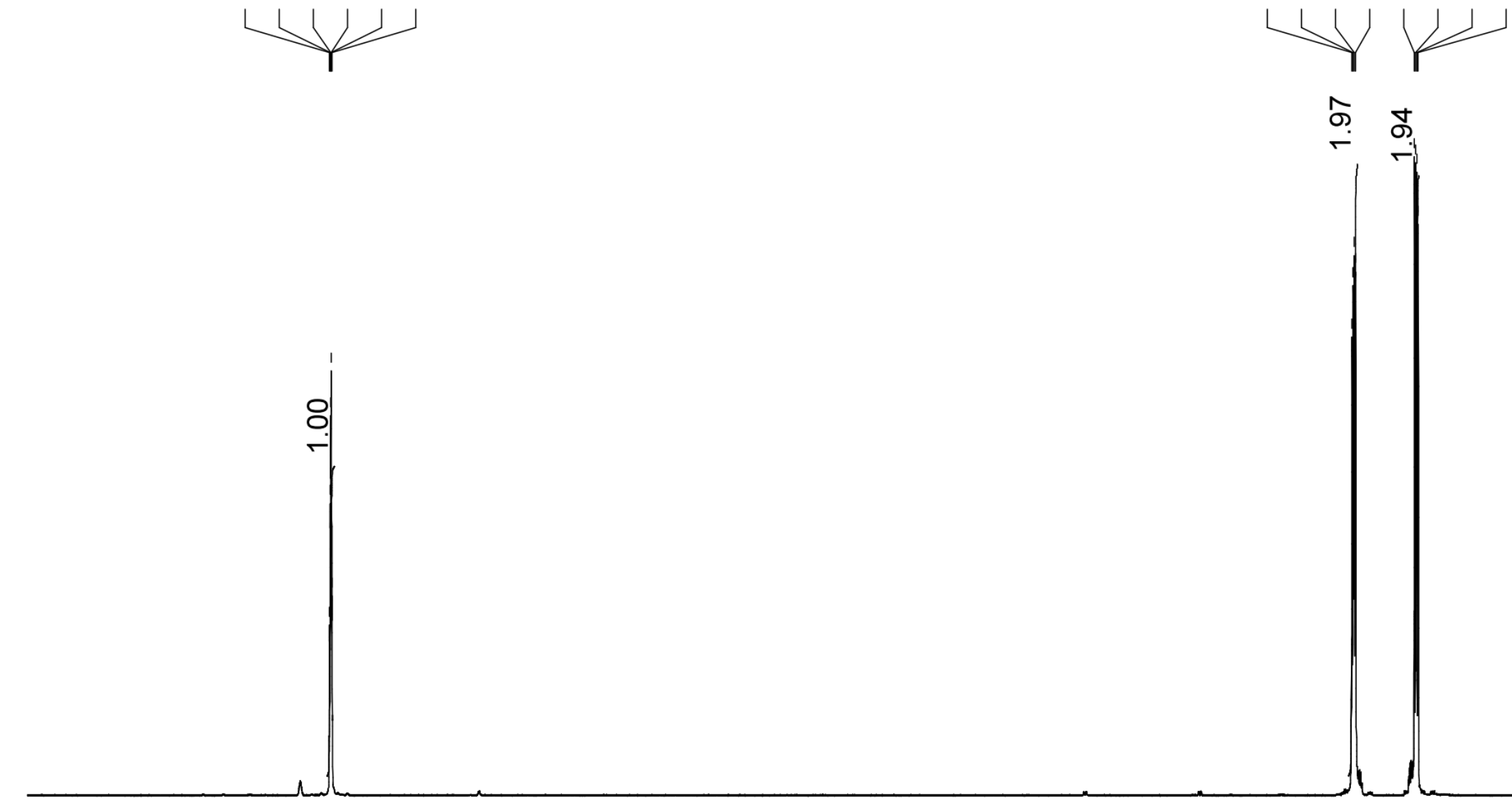

PPM

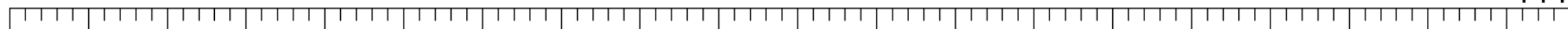
$\begin{array}{lllllllllllllllllllll}-110 & -112 & -114 & -116 & -118 & -120 & -122 & -124 & -126 & -128 & -130 & -132 & -134 & -136 & -138 & -140 & -142 & -144 & -146 & -148 & -150\end{array}$ 


\section{${ }^{1} \mathrm{H}$ NMR of 4na}

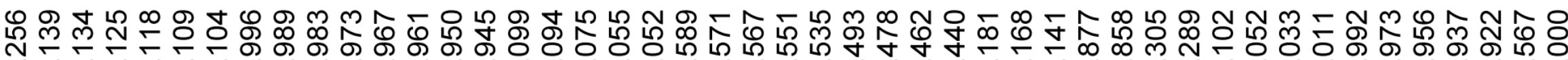
N N
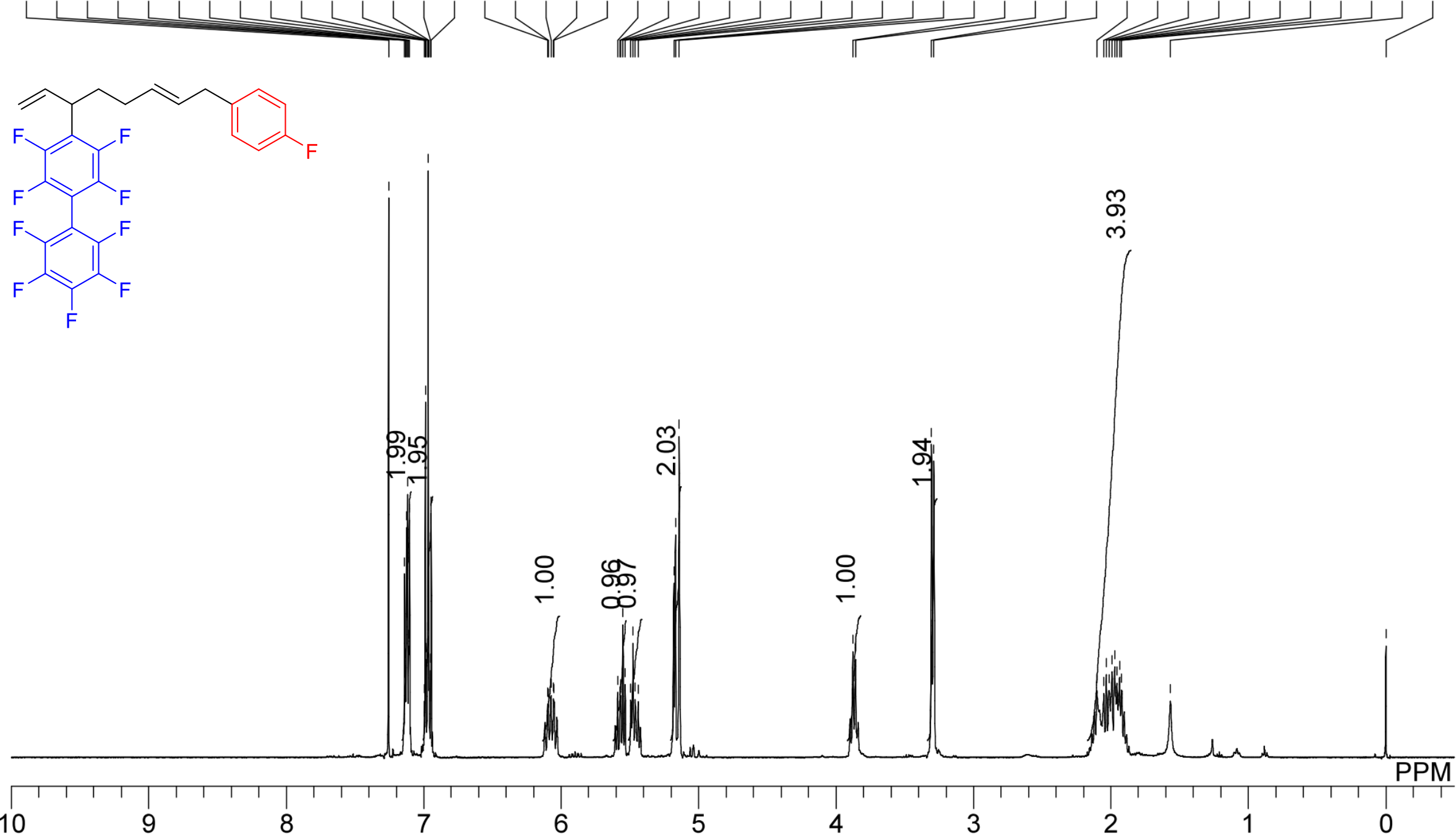
${ }^{13} \mathrm{C}\left\{{ }^{1} \mathrm{H}\right\}$ NMR of 4na

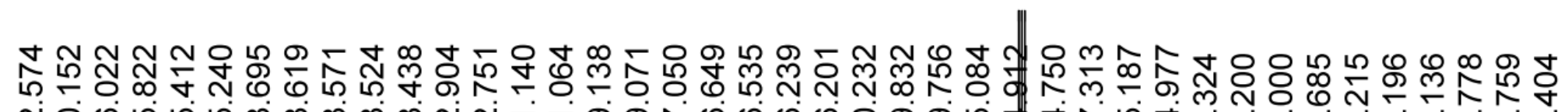
ชิ

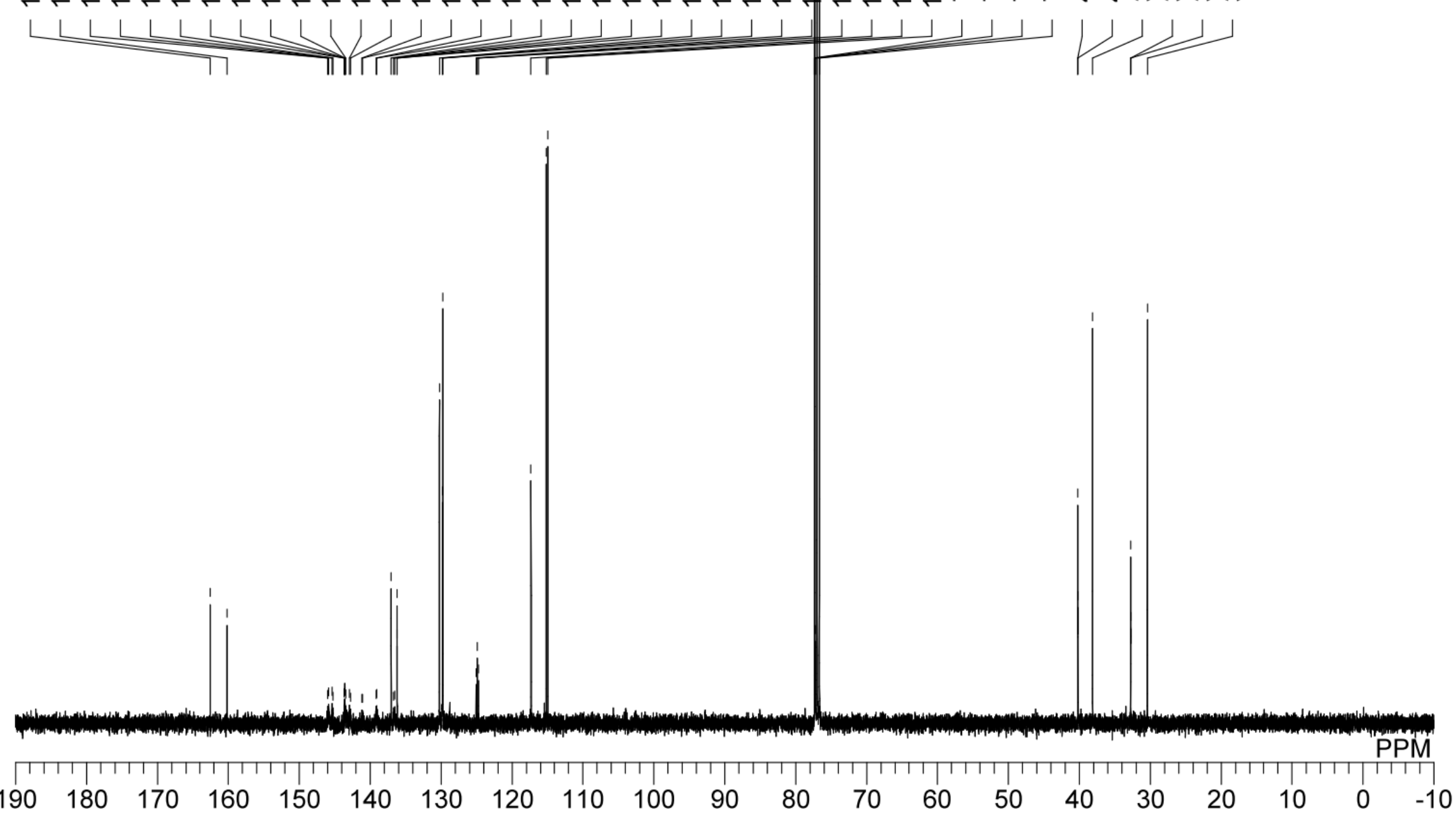


${ }^{13} \mathrm{C}\left\{{ }^{19} \mathrm{~F}\right\}$ NMR of 4 na

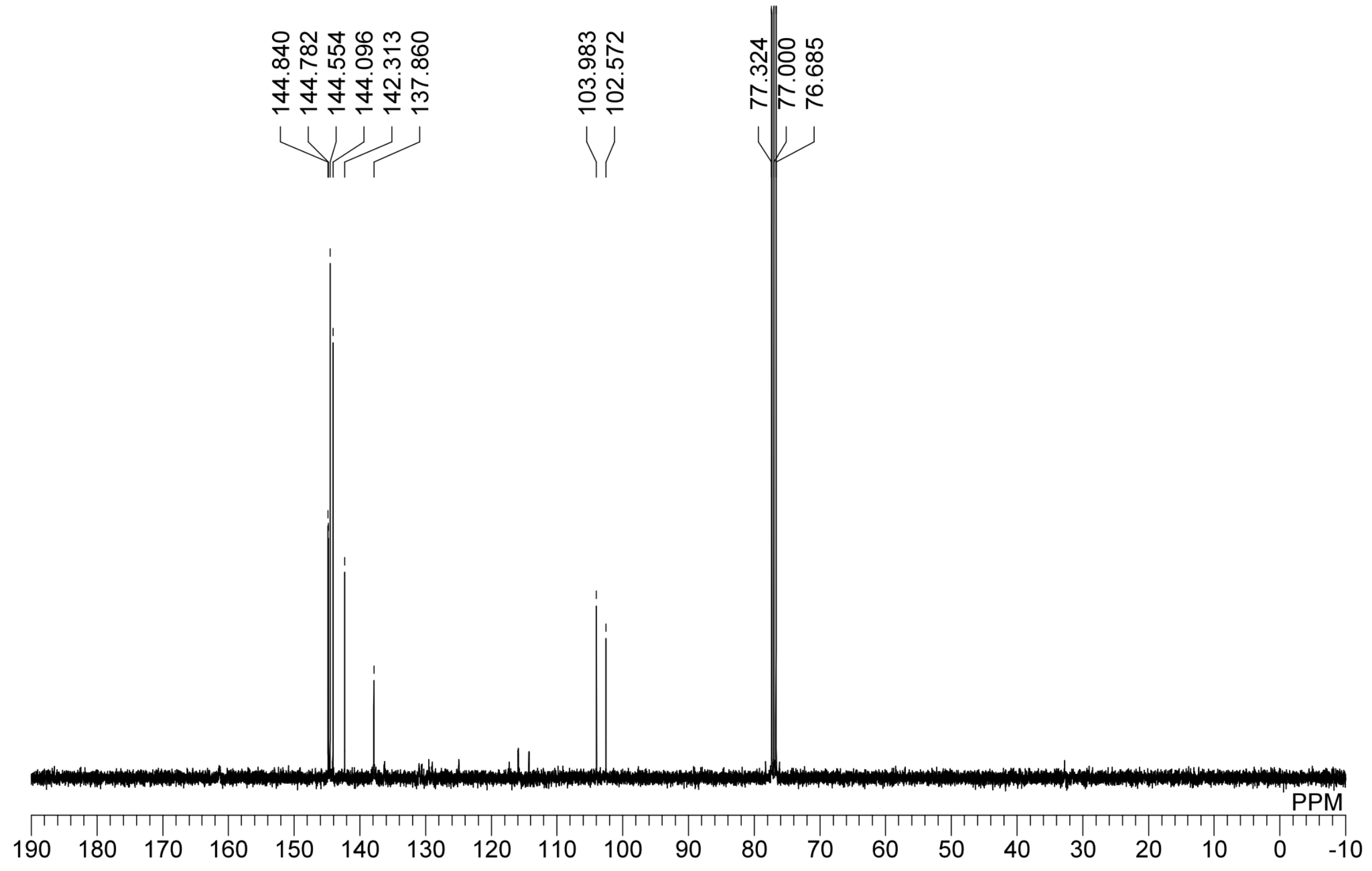




\section{${ }^{19}$ F NMR of 4na}

ᄃ슈

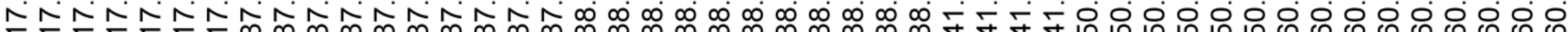

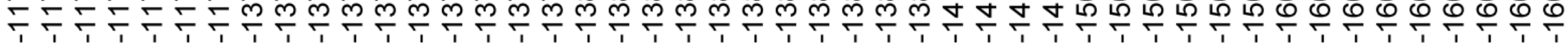
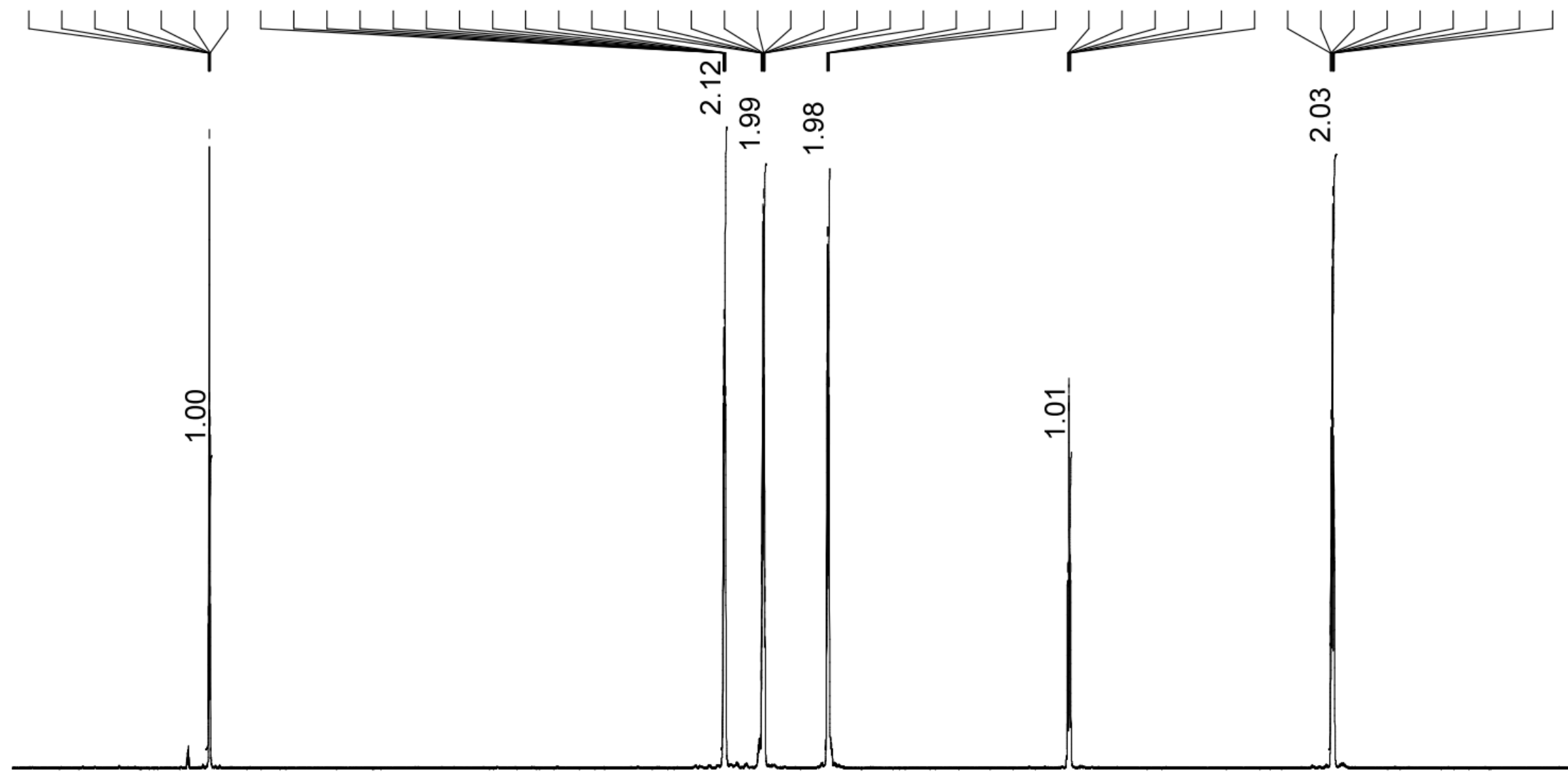

m.

$\infty$ 


\section{${ }^{1} \mathrm{H}$ NMR of $4 \mathbf{o a}$}

次

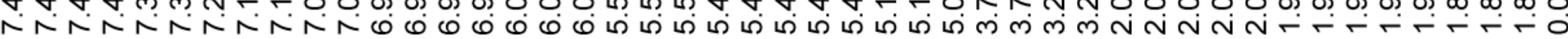

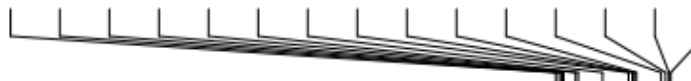
ता1
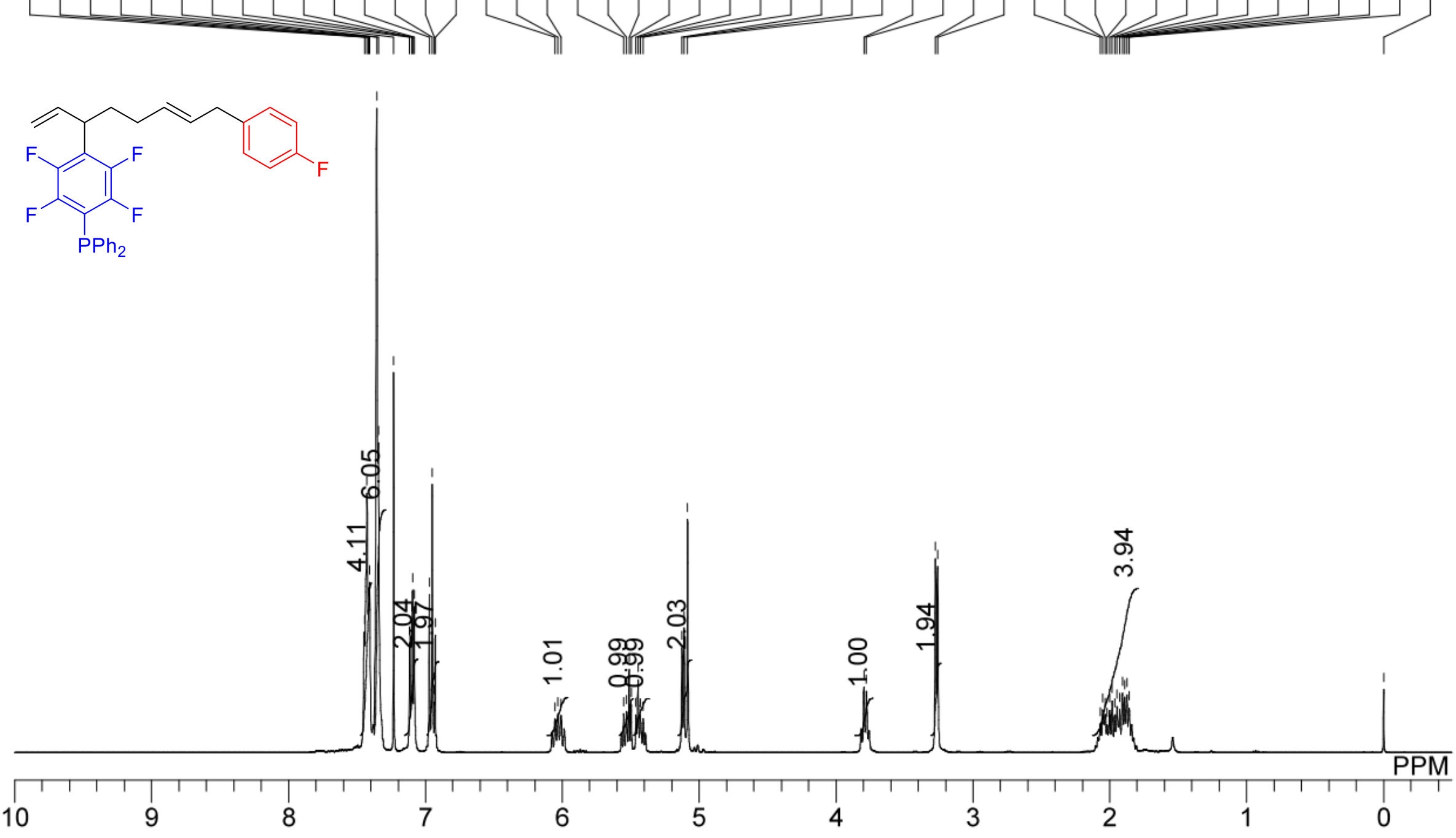


\section{${ }^{13} \mathrm{C}\left\{{ }^{1} \mathrm{H}\right\}$ NMR of $4 \mathbf{o a}$}

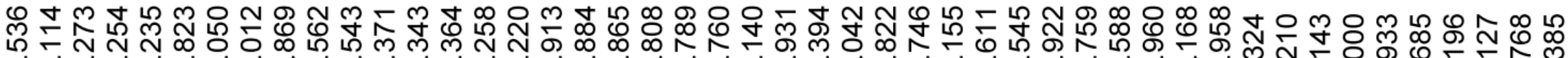

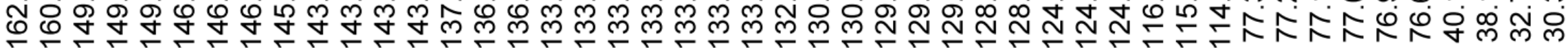
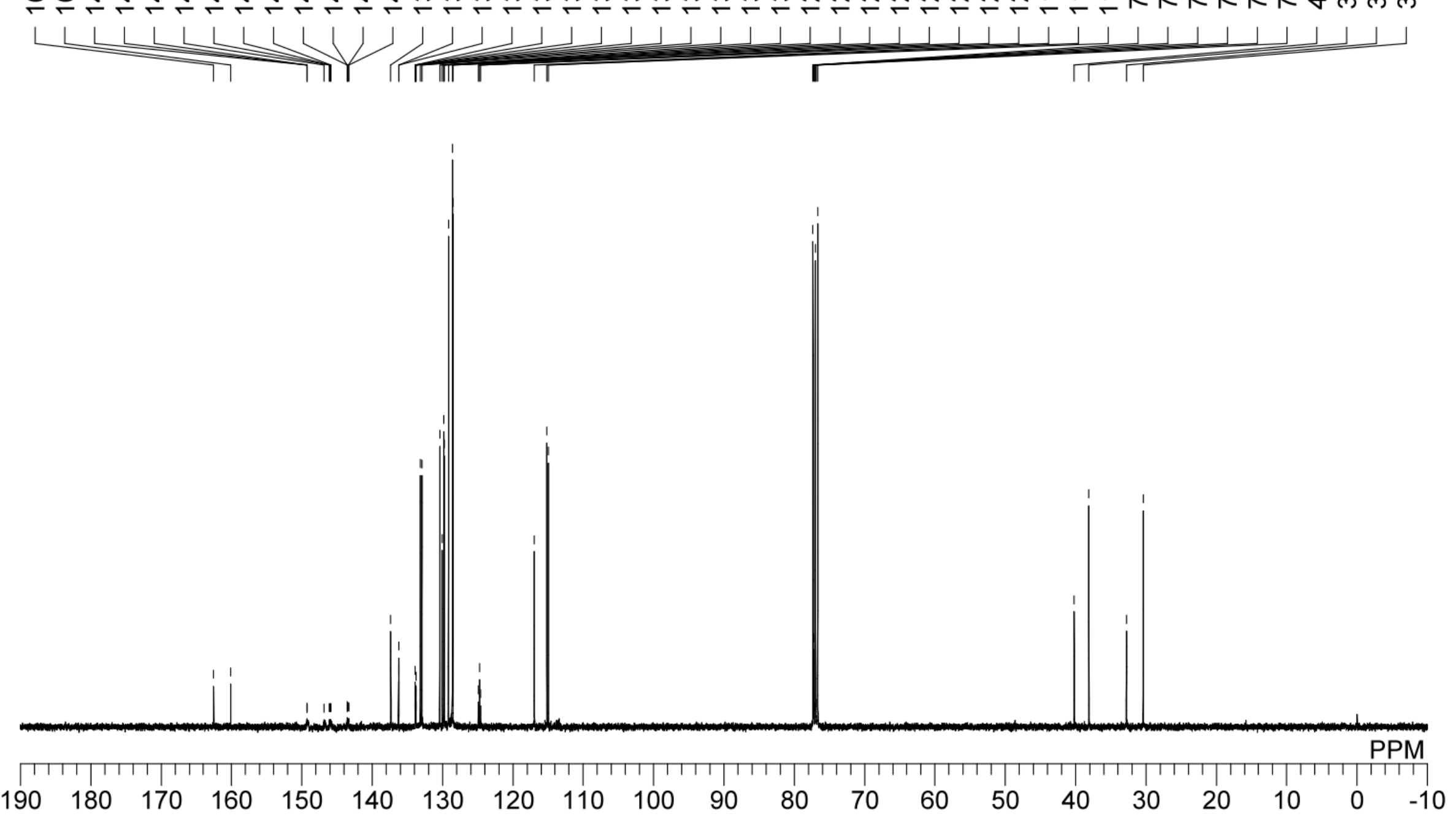
${ }^{13} \mathrm{C}\left\{{ }^{19} \mathrm{~F}\right\}$ NMR of $\mathbf{4 0 a}$

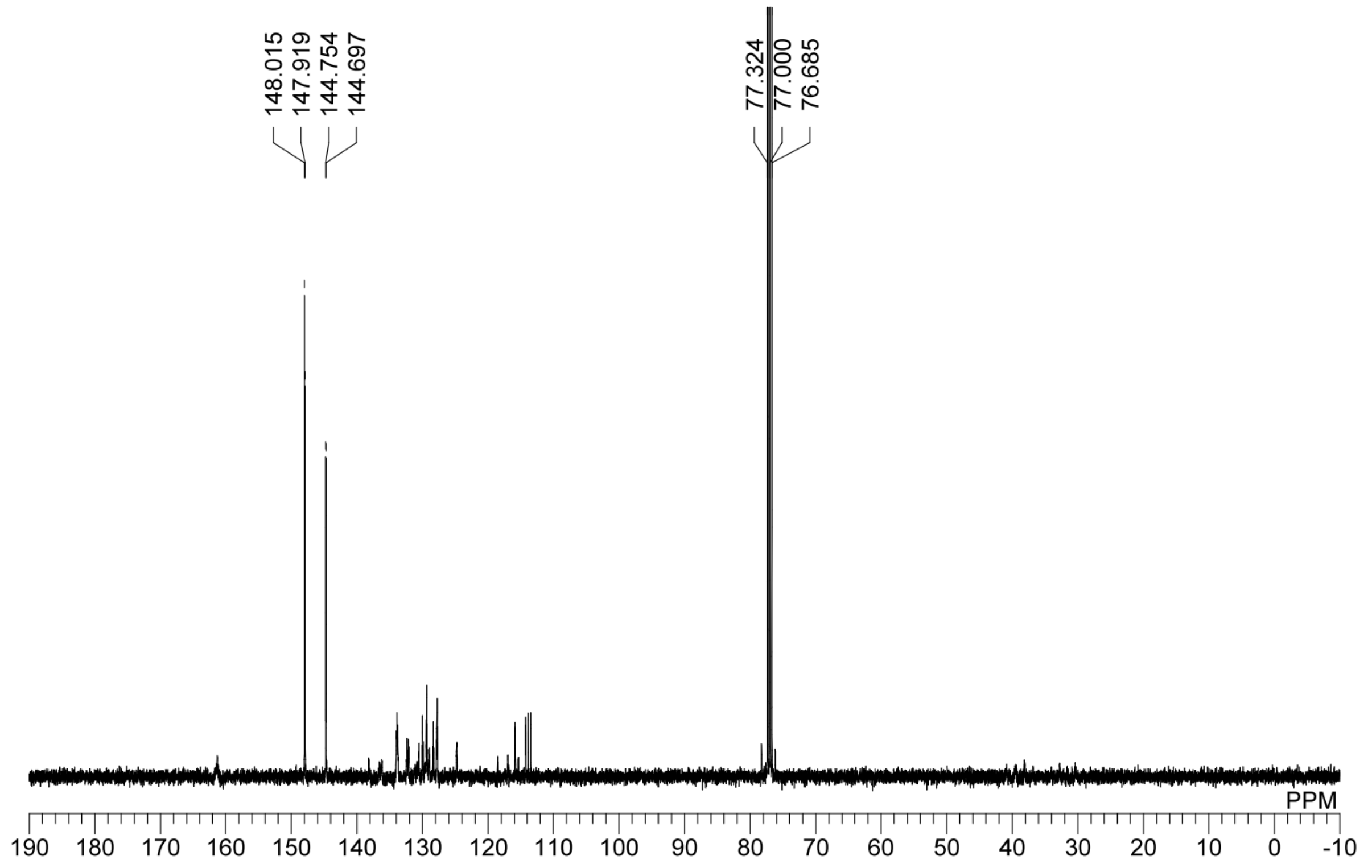


${ }^{19}$ F NMR of $\mathbf{4 o a}$
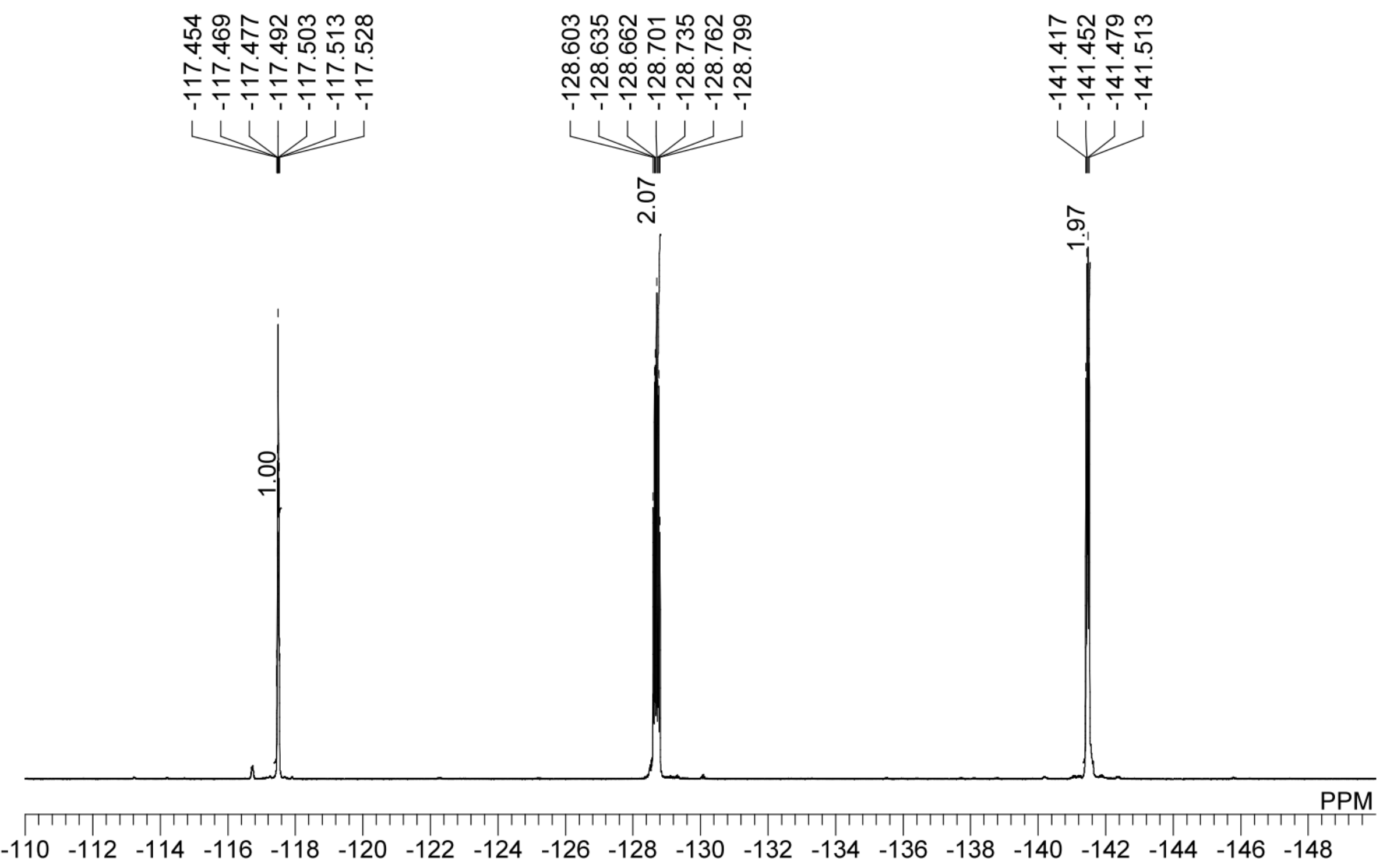
${ }^{41} \mathrm{P}$ NMR of $4 \mathbf{o a}$
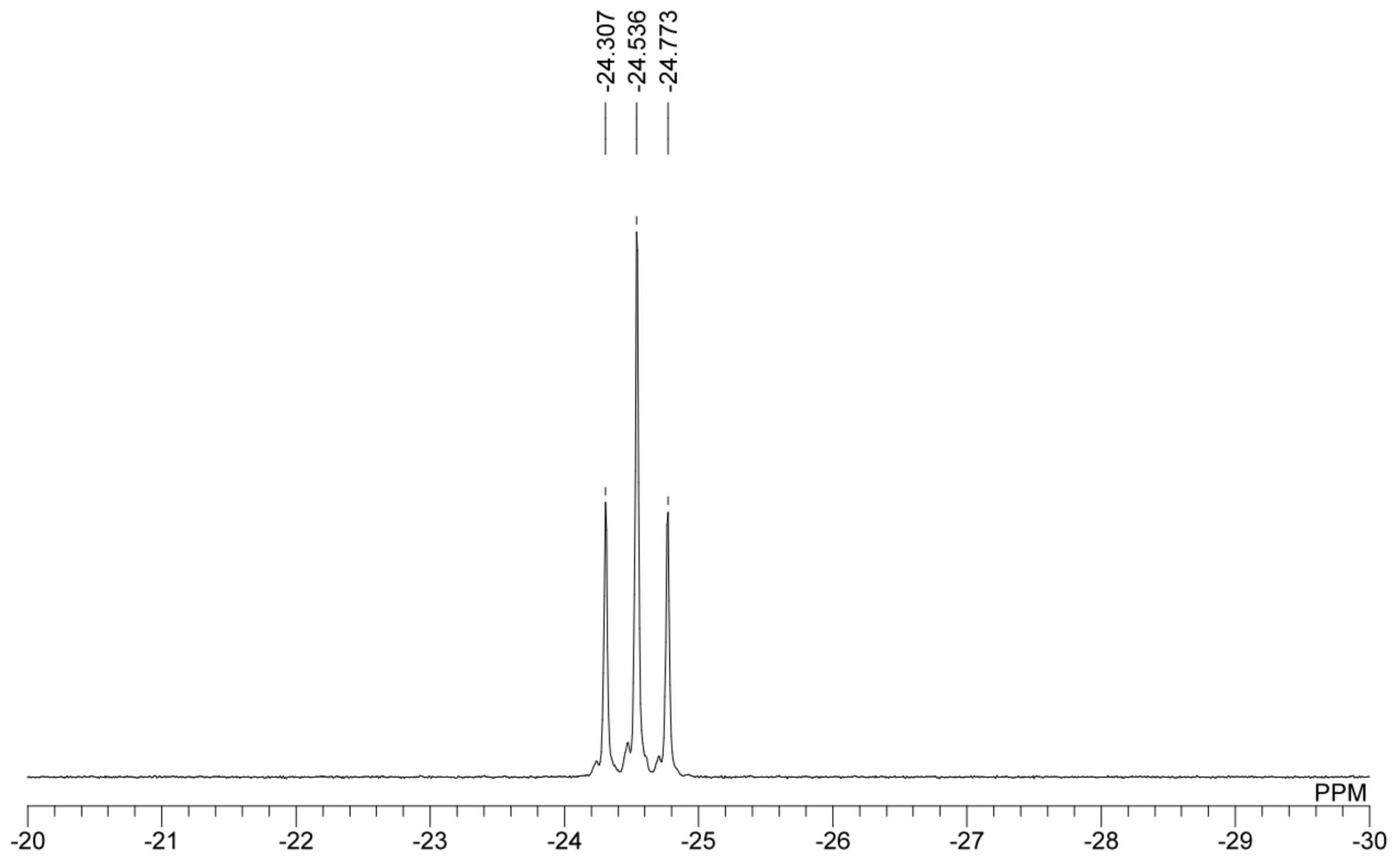


\section{${ }^{1} \mathrm{H}$ NMR of 4 pa}

숭

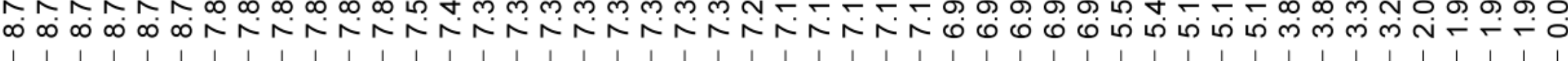
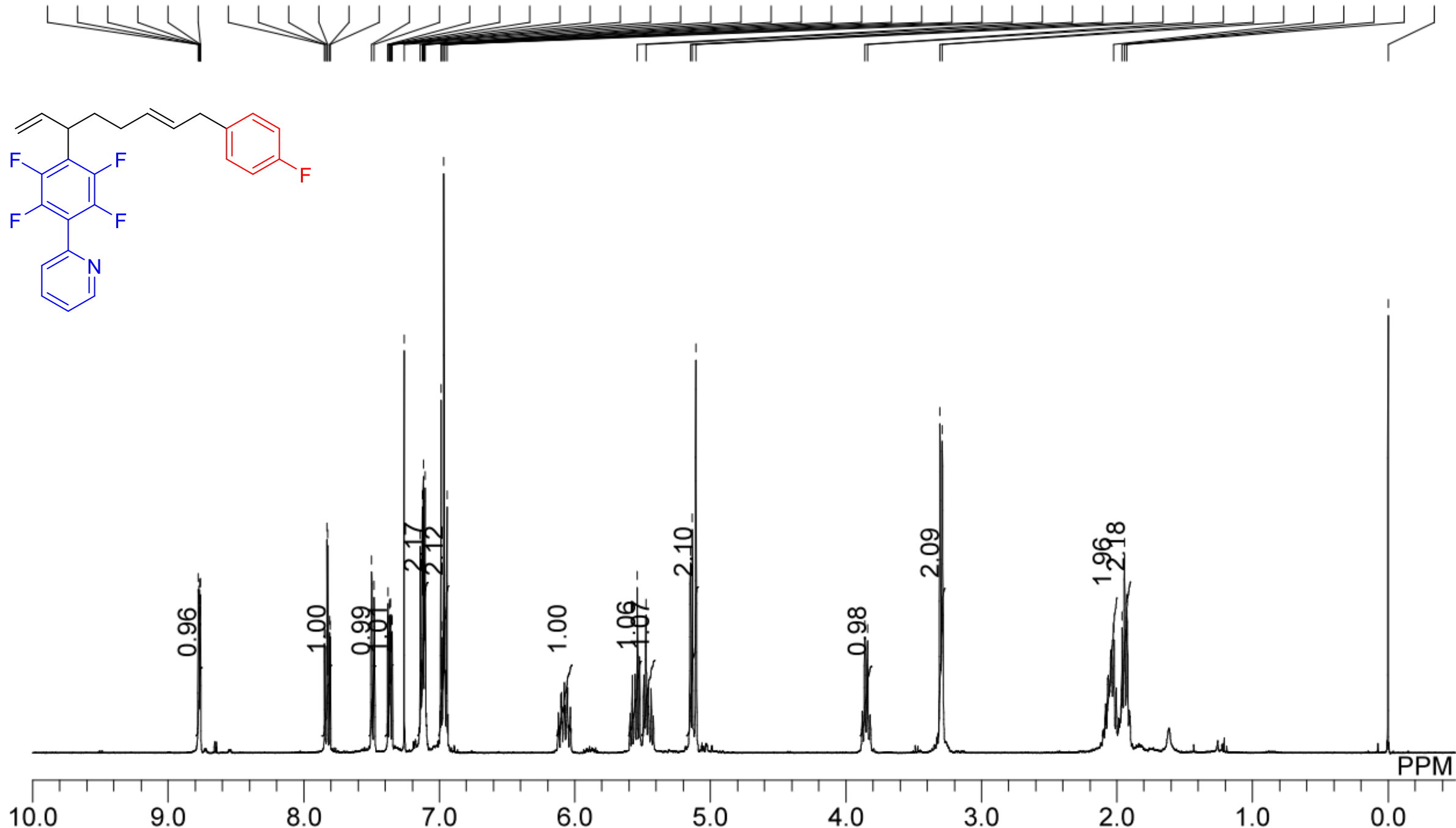
${ }^{13} \mathrm{C}\left\{{ }^{1} \mathrm{H}\right\}$ NMR of 4pa

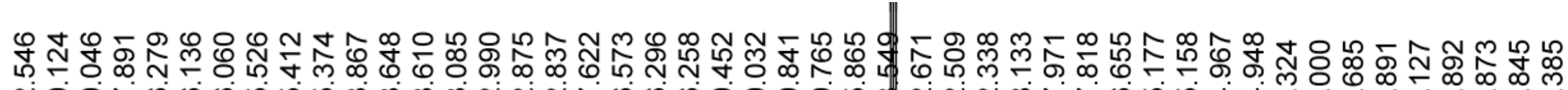
ஸ่
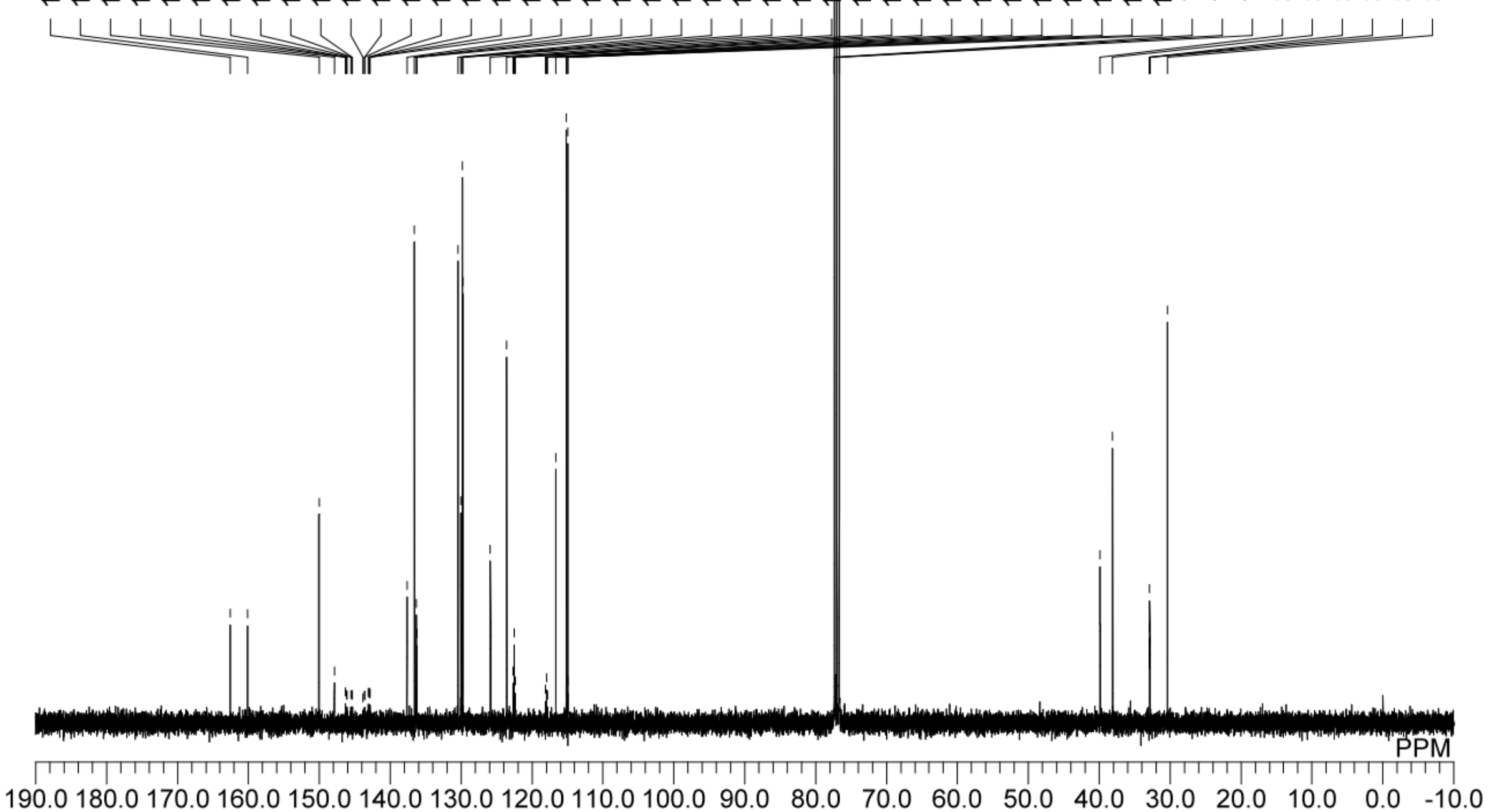
${ }^{13} \mathrm{C}\left\{{ }^{19} \mathrm{~F}\right\}$ NMR of $\mathbf{4 p a}$

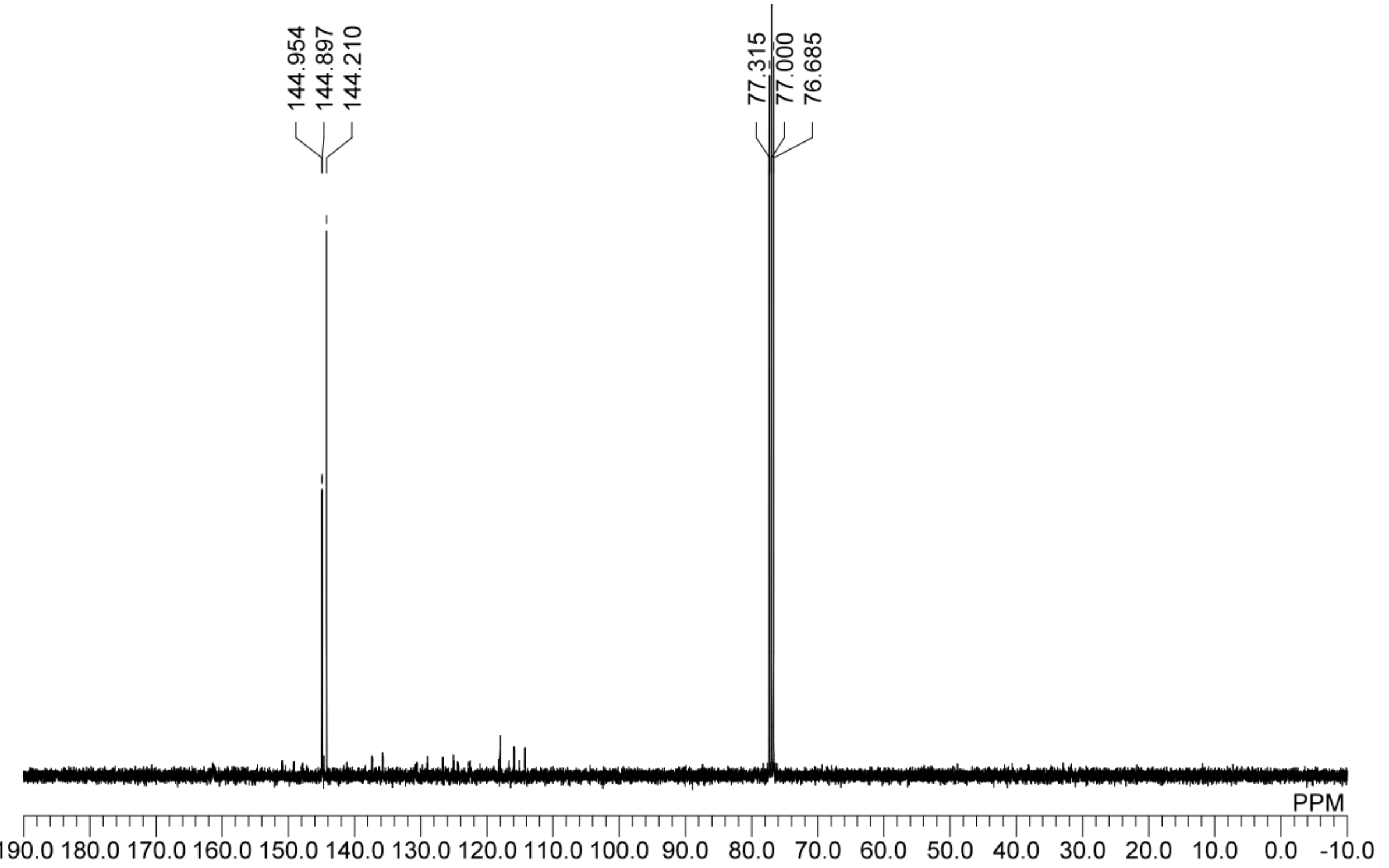


${ }^{19}$ F NMR of 4pa

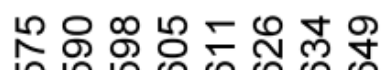

ก

슫슫슫듣드듣

๑ 귬

约

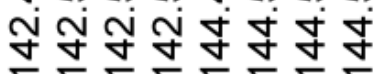
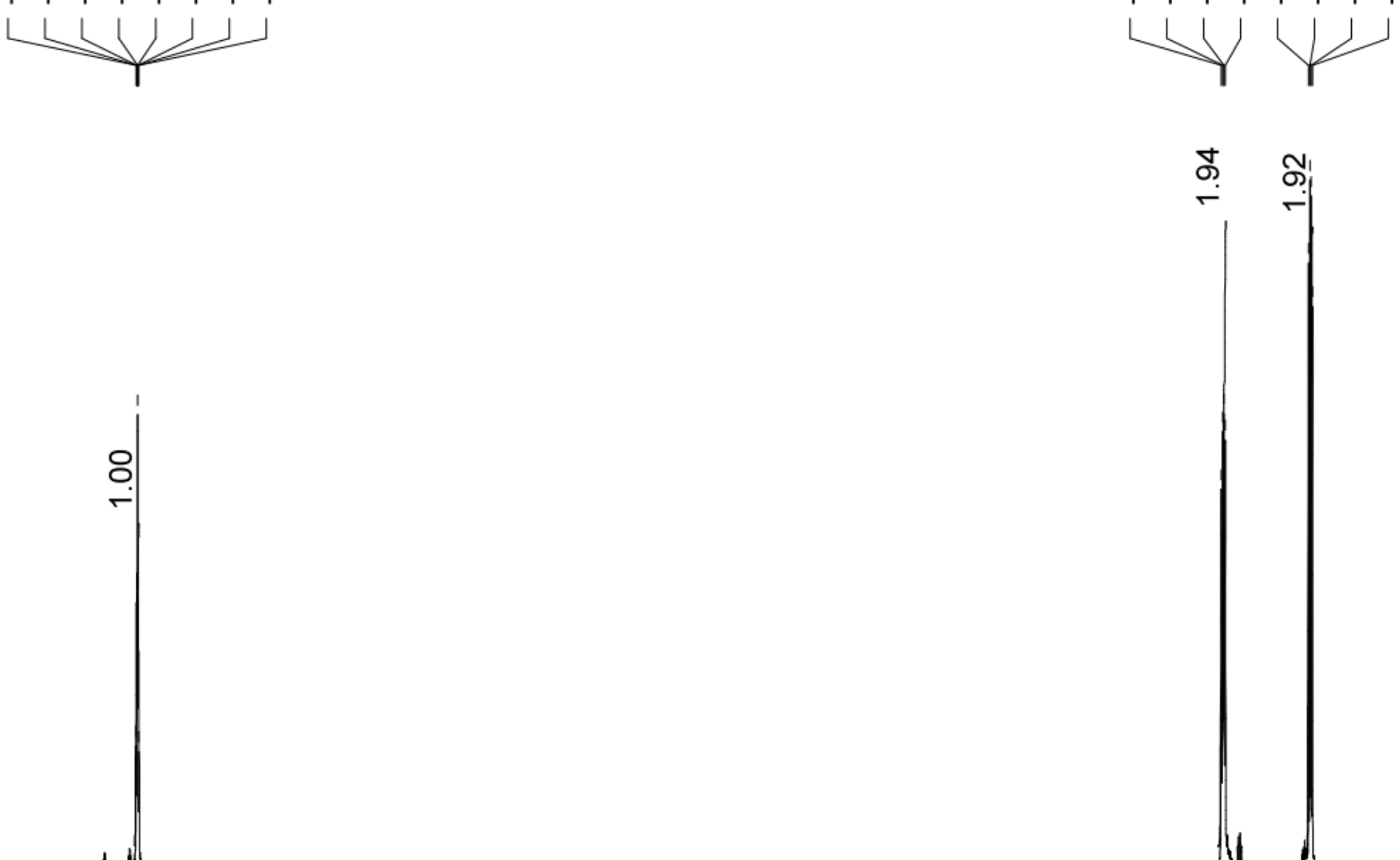

PPM

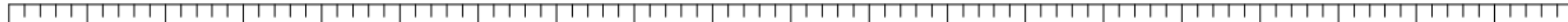

$-110.0-112.0-114.0-116.0-118.0-120.0-122.0-124.0-126.0-128.0-130.0-132.0-134.0-136.0-138.0-140.0-142.0-144.0-146.0-148.0-150.0$ 


\section{${ }^{1} \mathrm{H}$ NMR of 5pa}

내튜

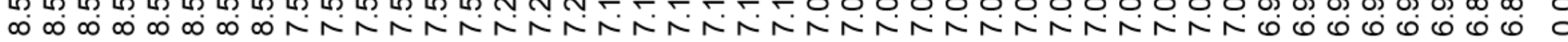

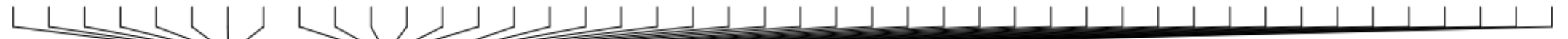

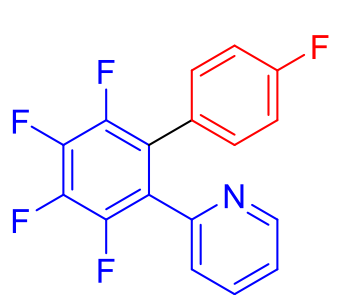

TTII

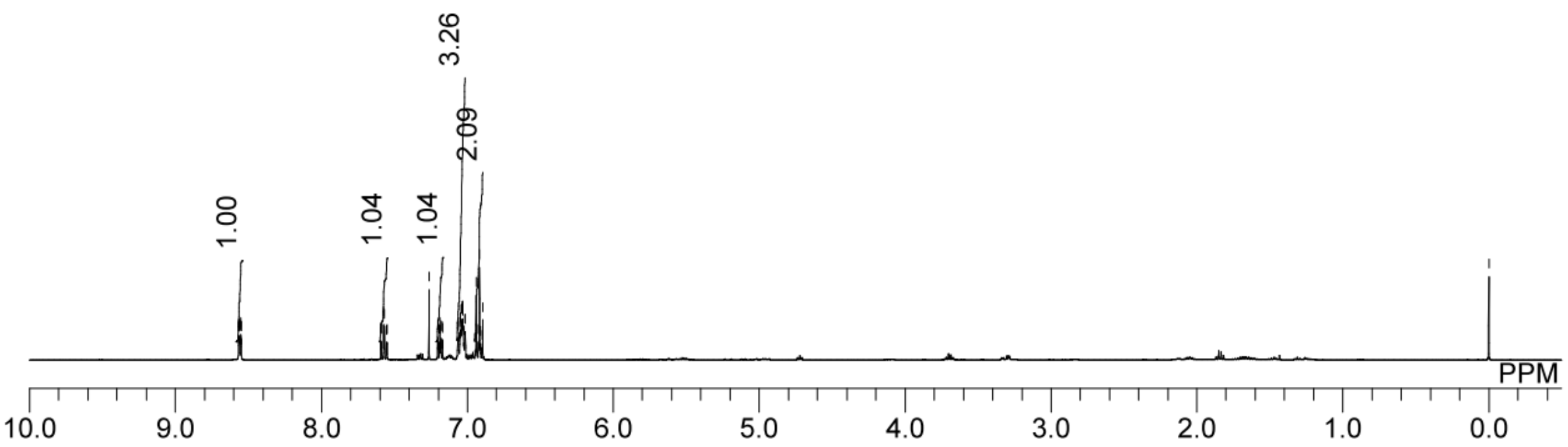


${ }^{13} \mathrm{C}\left\{{ }^{1} \mathrm{H}\right\}$ NMR of $\mathbf{5 p a}$

ம

ஸூ
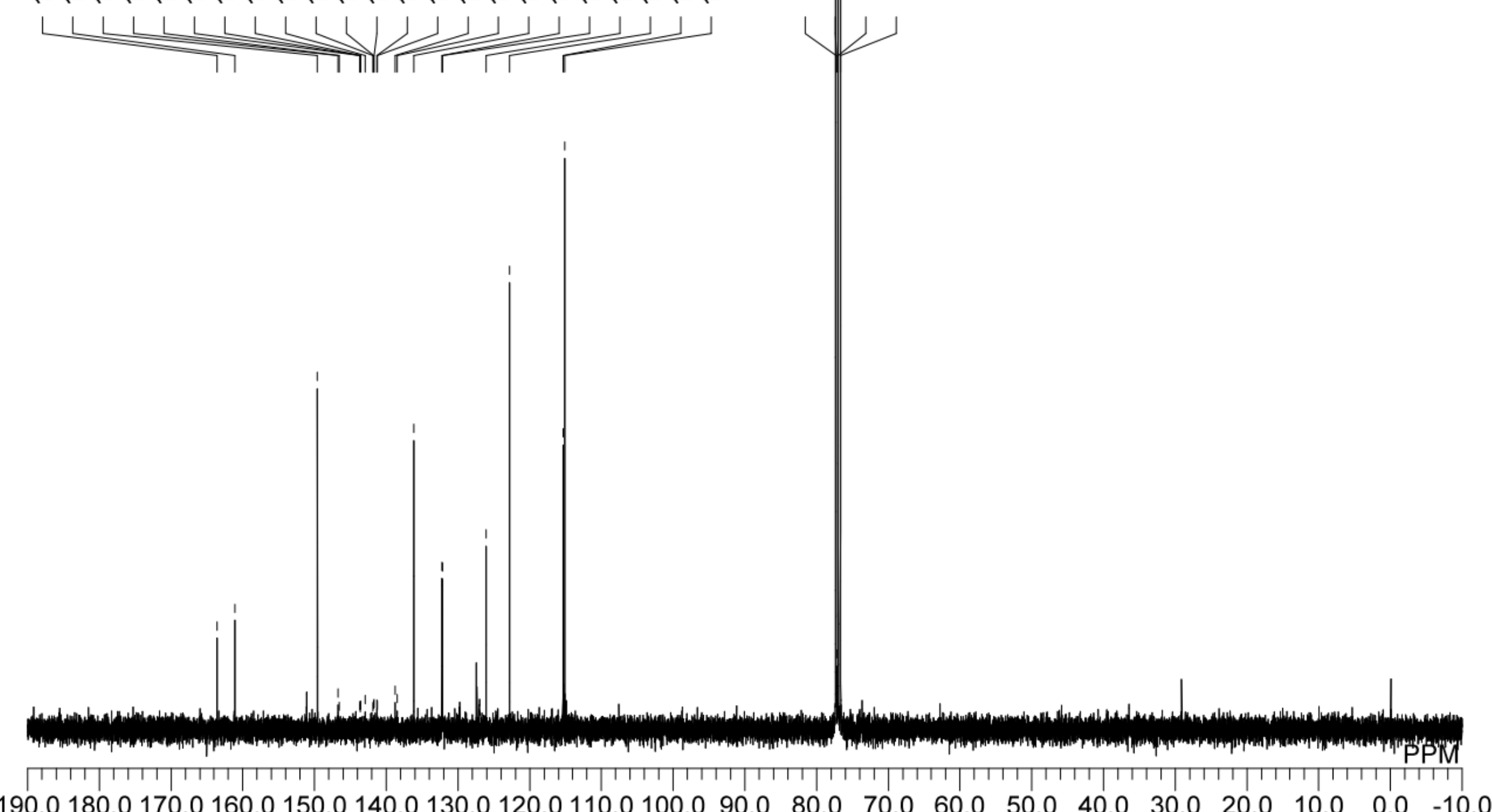
${ }^{13} \mathrm{C}\left\{{ }^{19} \mathrm{~F}\right\}$ NMR of $5 \mathbf{p a}$

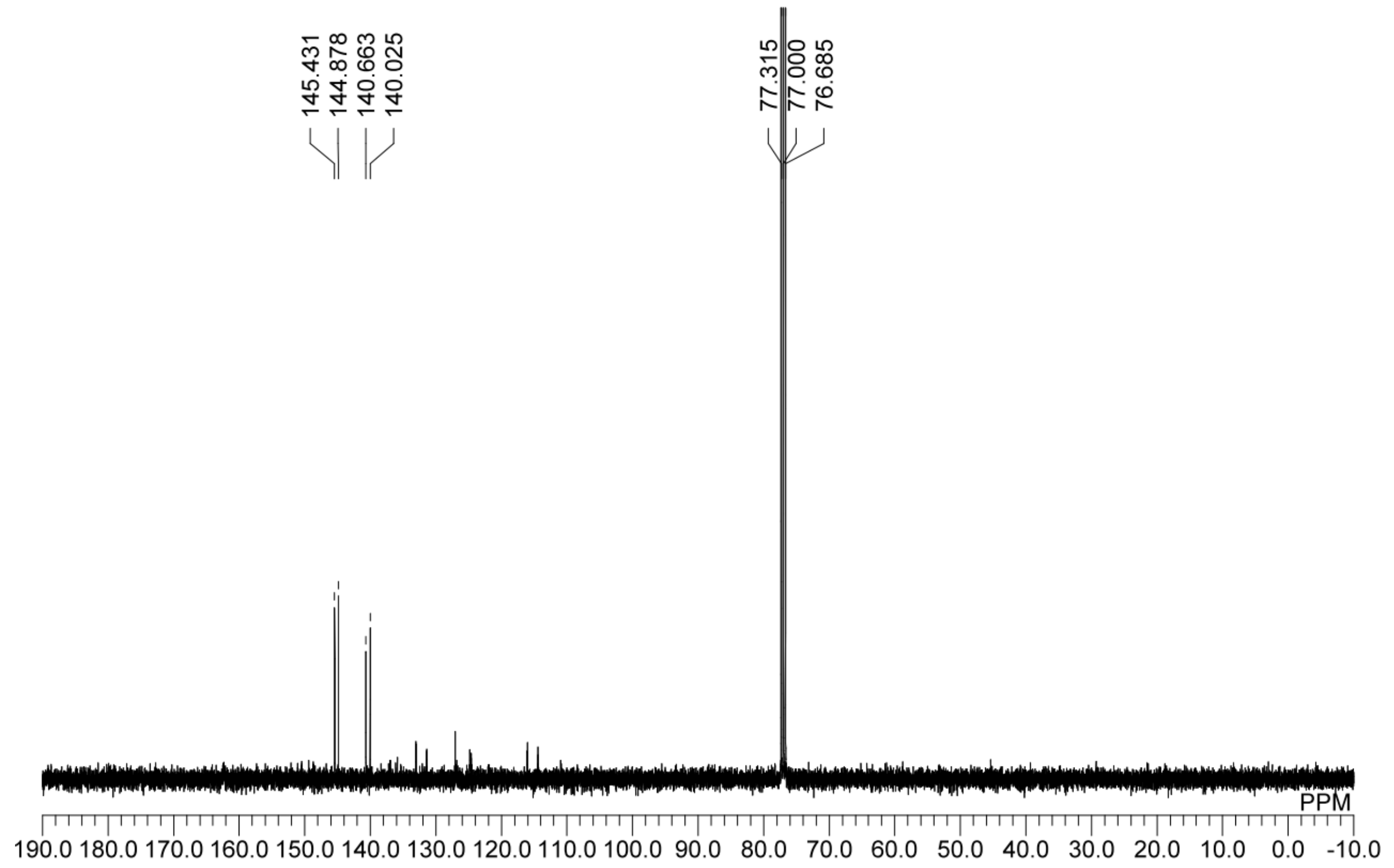


${ }^{19}$ F NMR of 5pa

เి

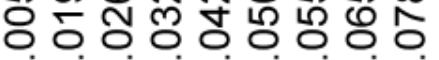
m m m m m m m m

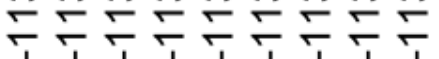
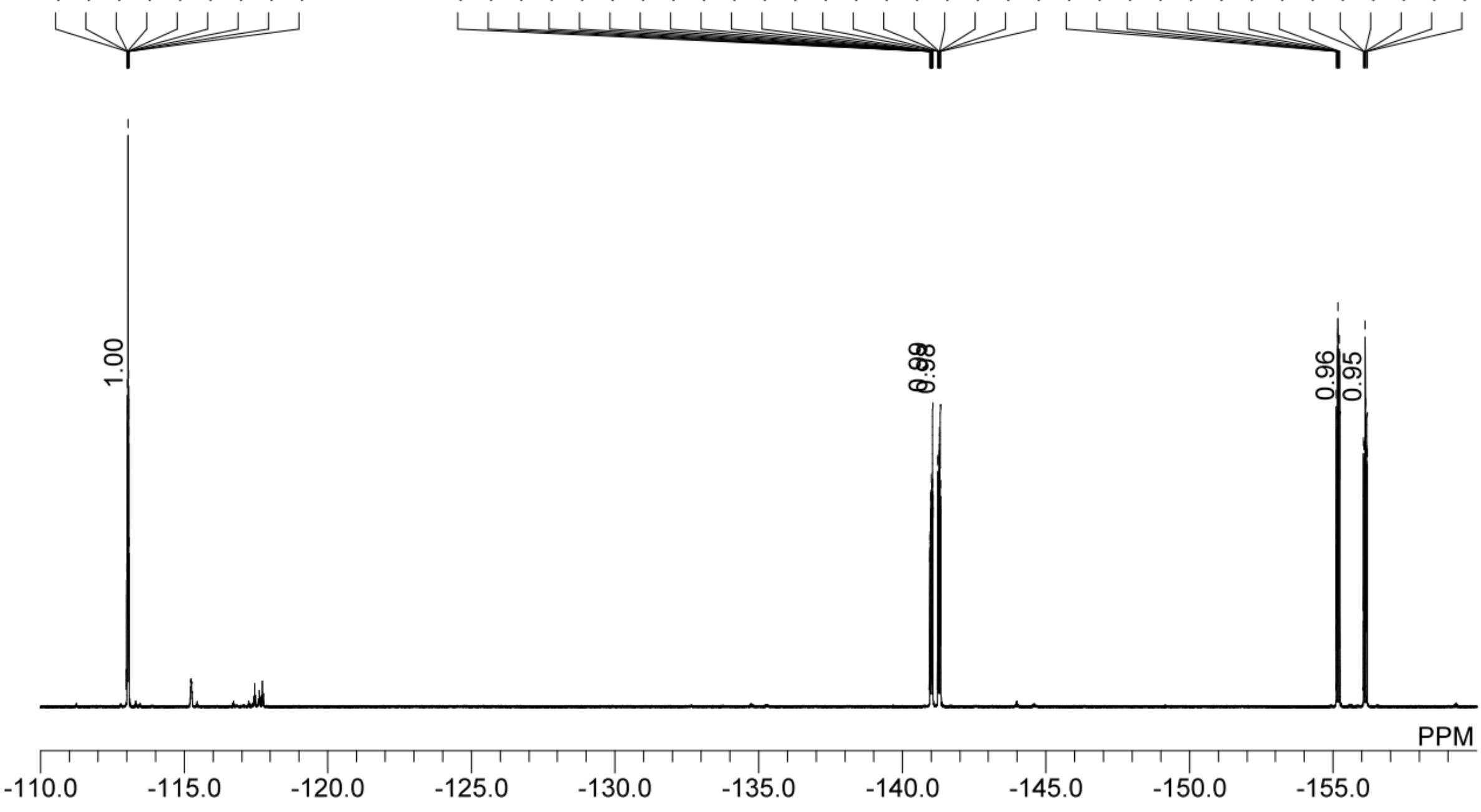

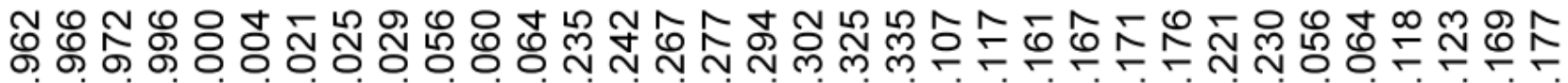

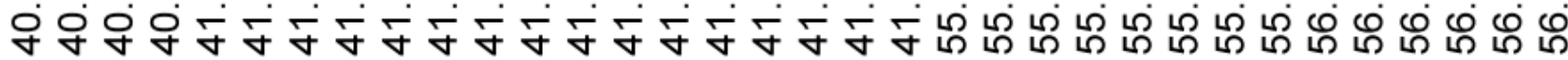

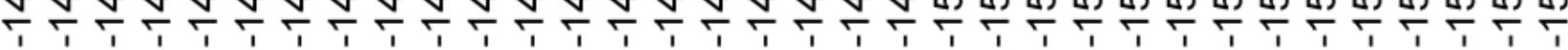


${ }^{1} \mathrm{H}$ NMR of mixture of $\mathbf{4 q a}$ and its regioisomer ( $p: m$ or $o=98: 2$ )

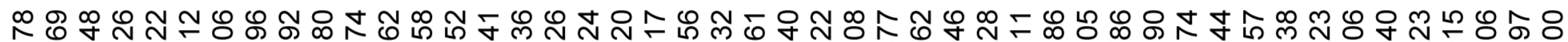

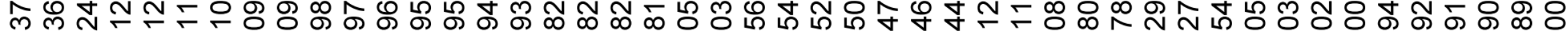
N
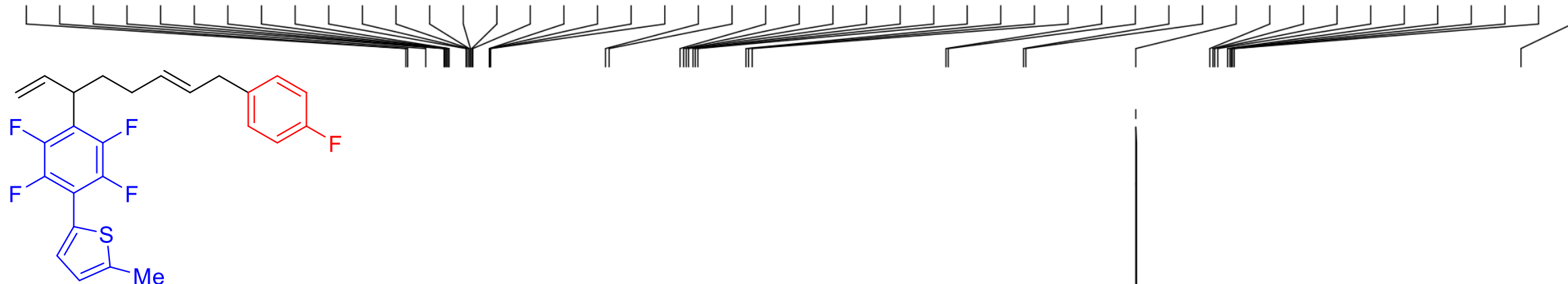

T111

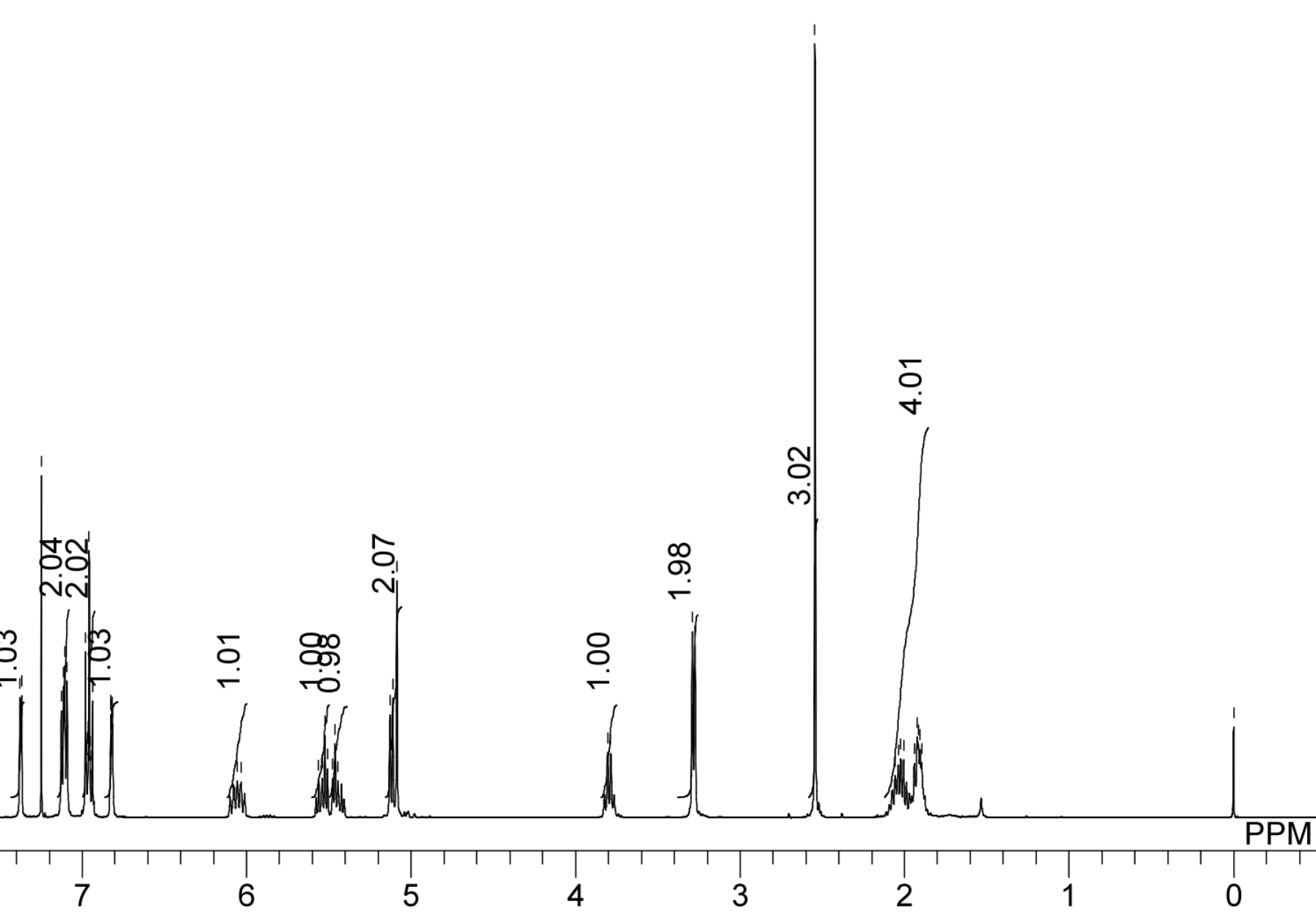


${ }^{13} \mathrm{C}\left\{{ }^{1} \mathrm{H}\right\}$ NMR of mixture of $\mathbf{4 q a}$ and its regioisomer ( $p: m$ or $o=98: 2$ )

년 @゚
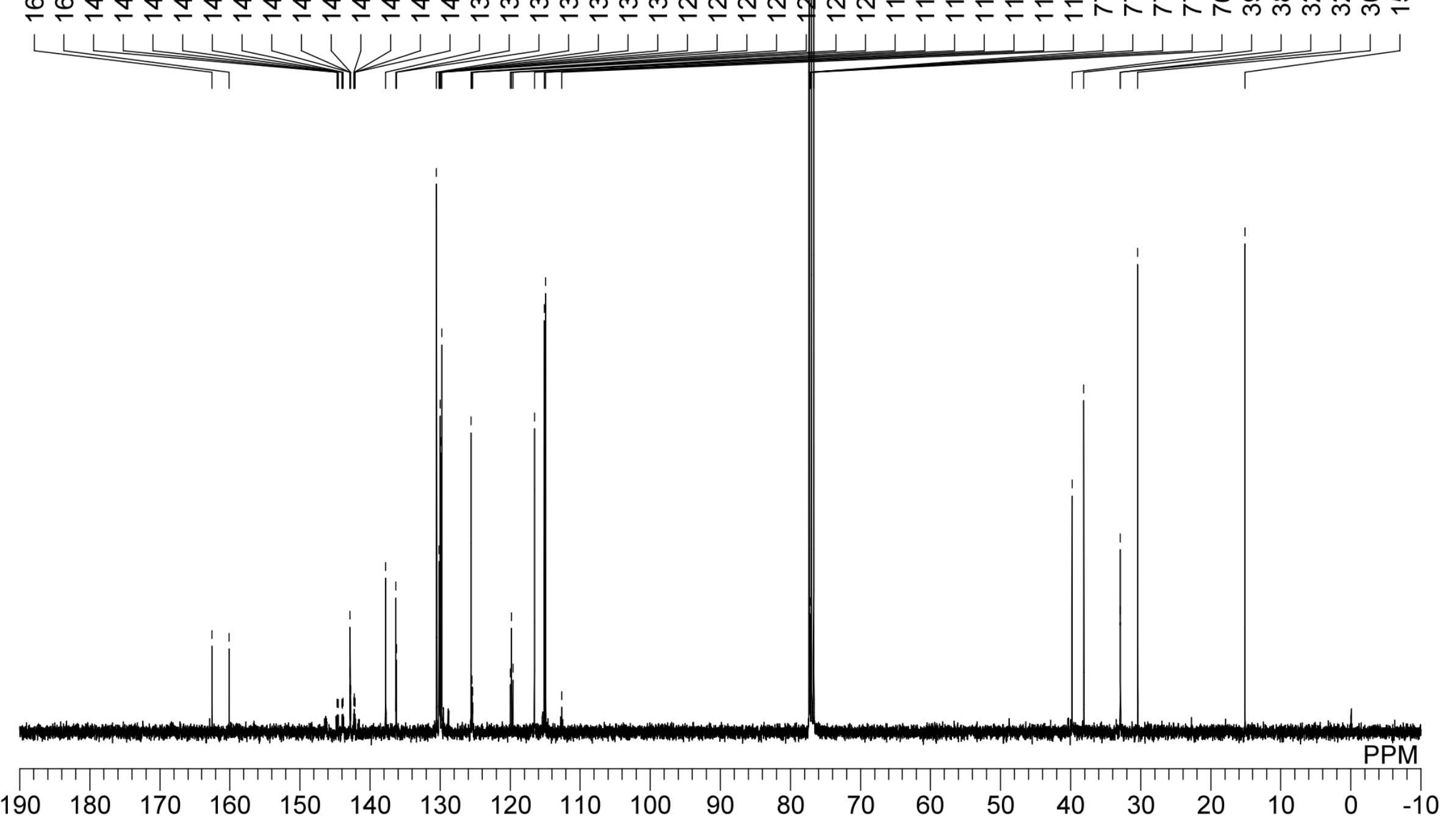
${ }^{13} \mathrm{C}\left\{{ }^{19} \mathrm{~F}\right\}$ NMR of mixture of $\mathbf{4 q a}$ and its regioisomer ( $p: m$ or $o=98: 2$ )
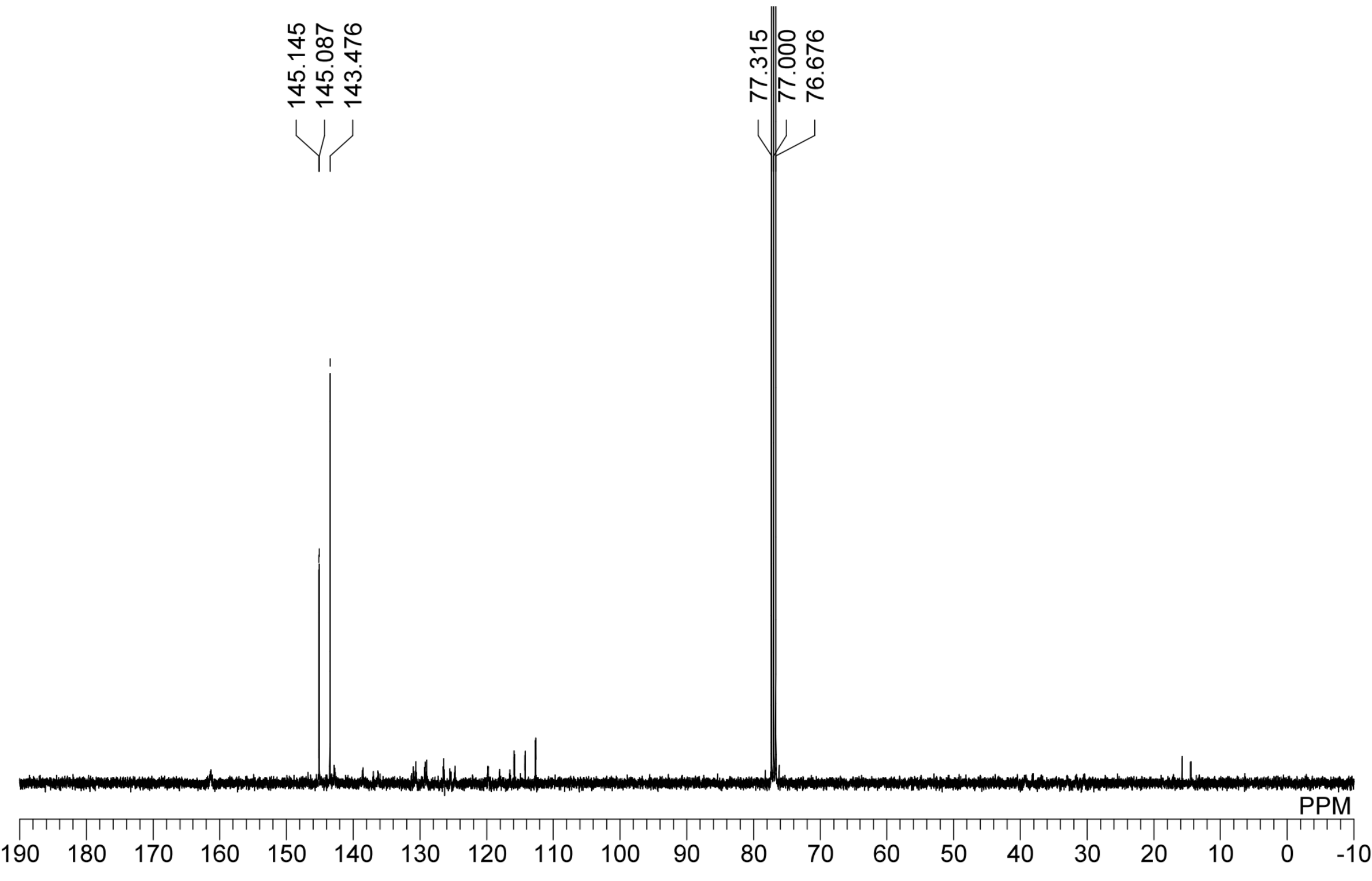
${ }^{19} \mathrm{~F}$ NMR of mixture of $\mathbf{4 q a}$ and its regioisomer ( $p: m$ or $o=98: 2$ )

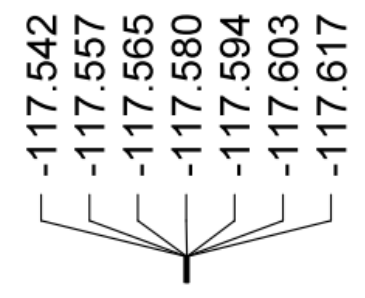

잉

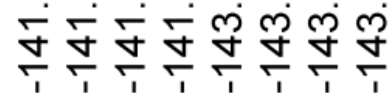
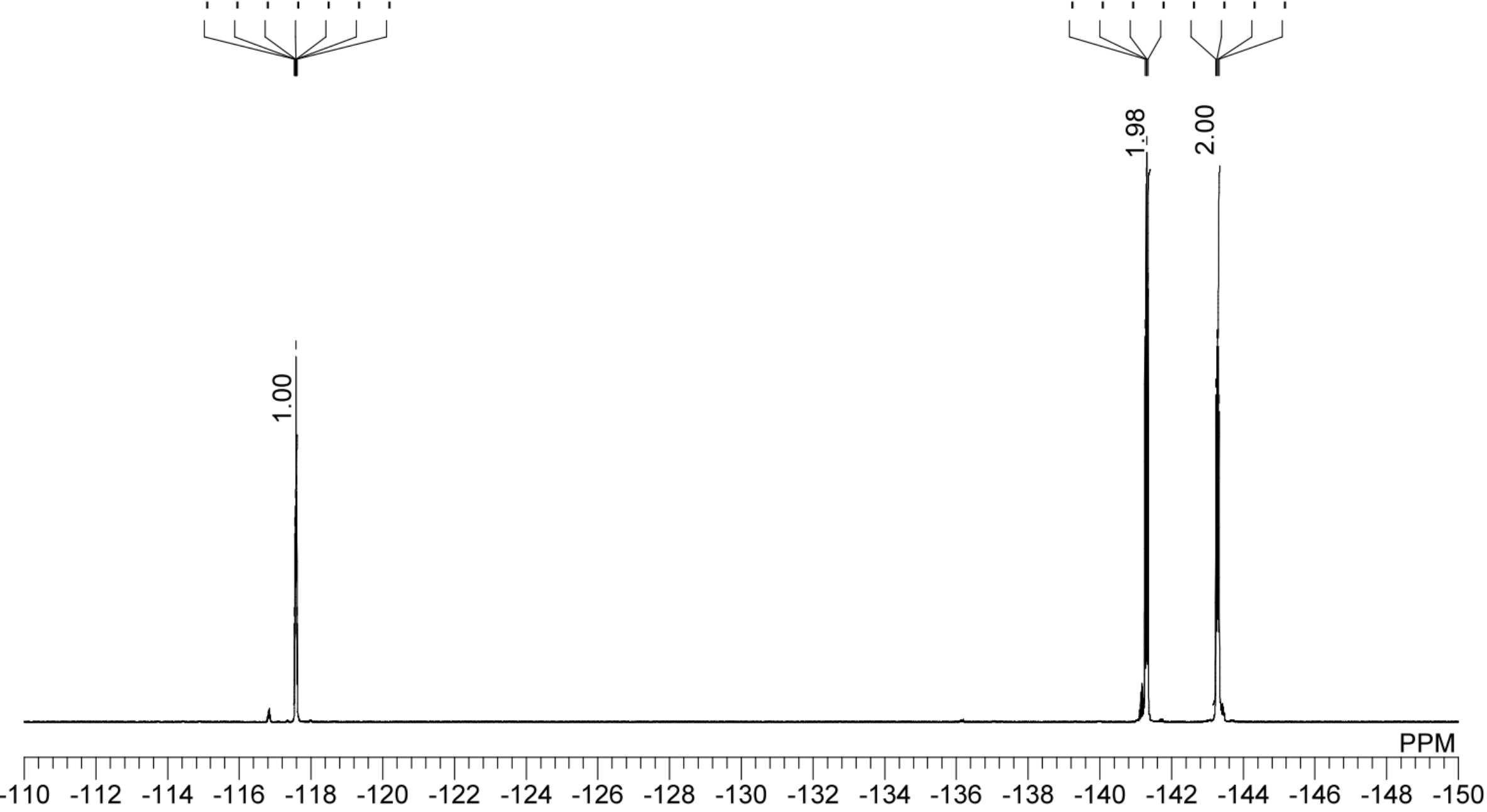


\section{${ }^{1} \mathrm{H}$ NMR of 4ra}

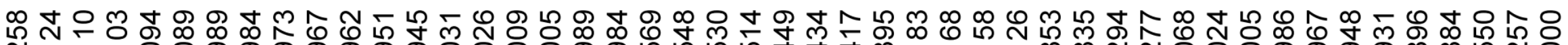

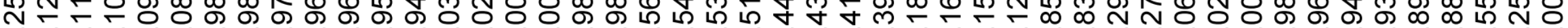
N N N
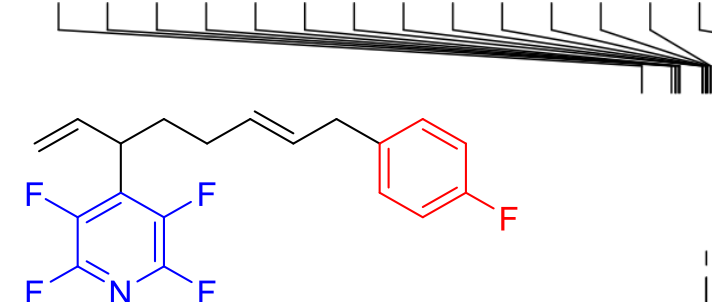

1III
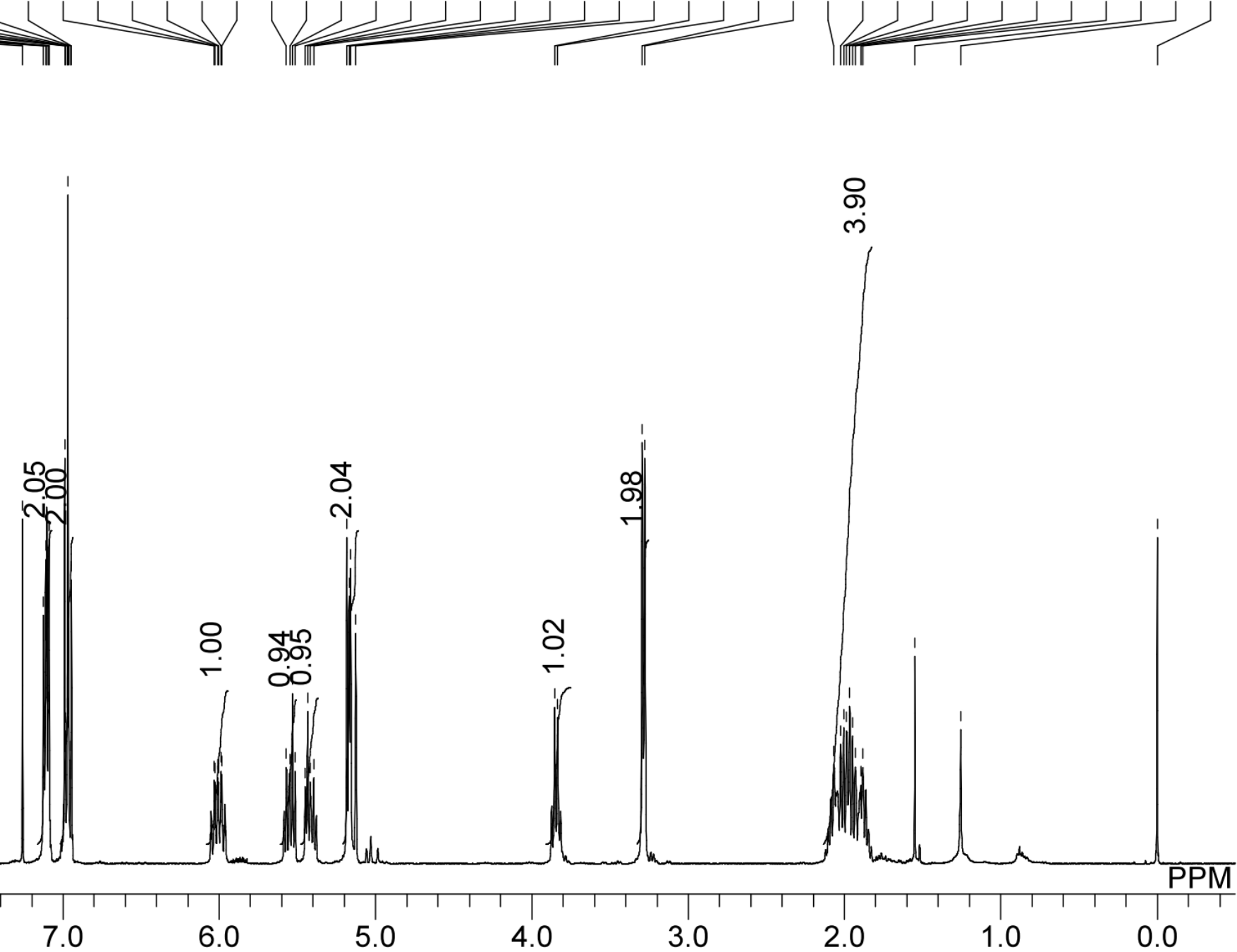
${ }^{13} \mathrm{C}\left\{{ }^{1} \mathrm{H}\right\}$ NMR of 4 ra

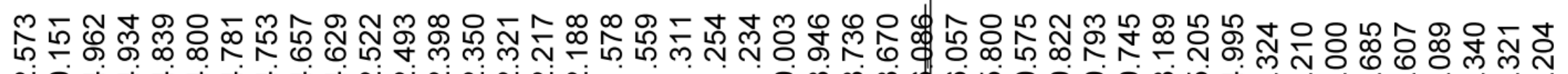
ஸூ
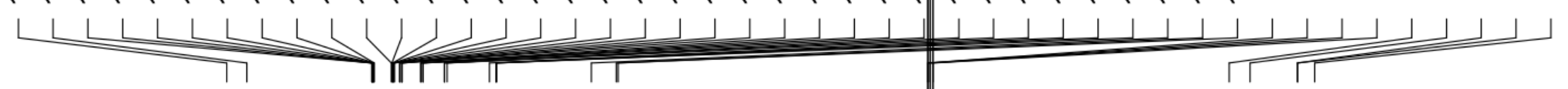

\section{I."}

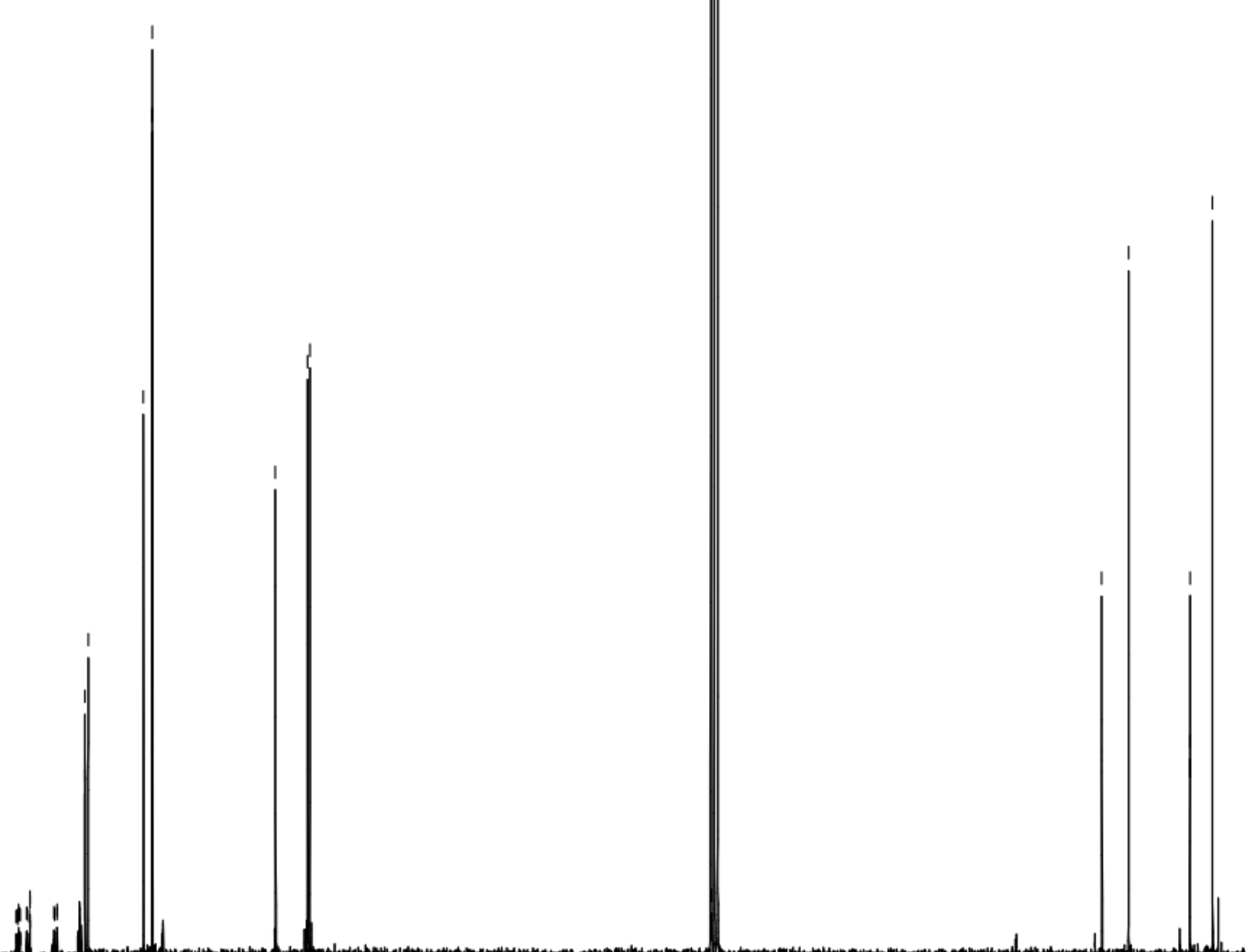


${ }^{13} \mathrm{C}\left\{{ }^{19} \mathrm{~F}\right\}$ NMR of 4 ra

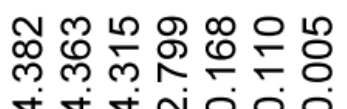

导㠻守守守守守
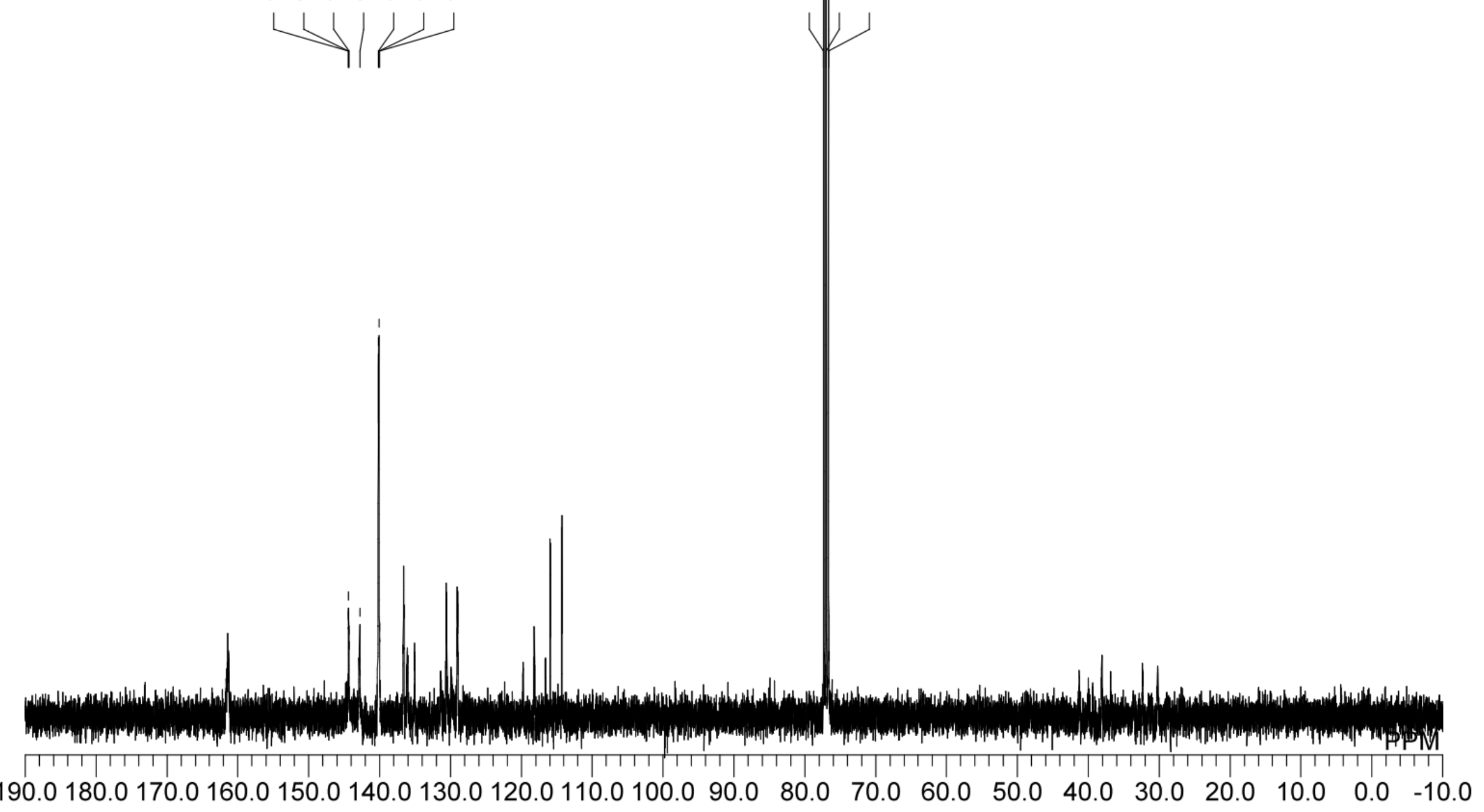
${ }^{19} \mathrm{~F}$ NMR of 4 ra

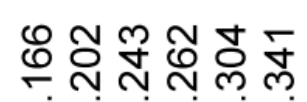

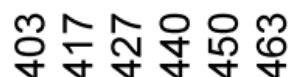

চ চரচ চ

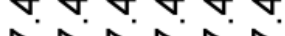

슫ㅊㄷㄷㄷㄷㄷ

గొ

ตं

m
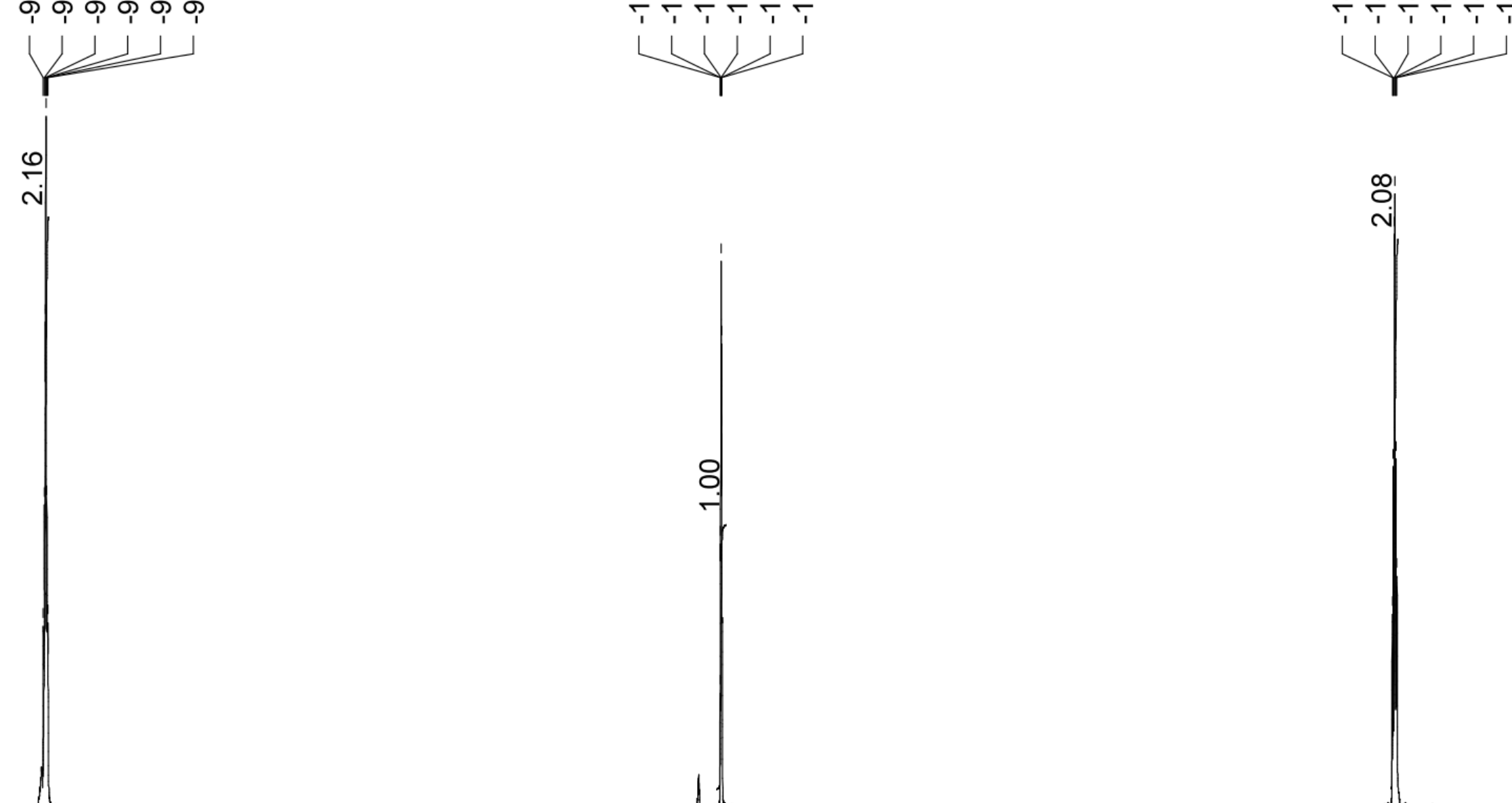

PPM

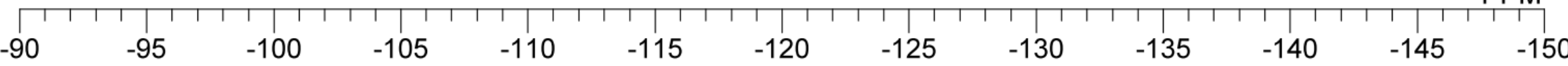


${ }^{1} \mathrm{H}$ NMR of mixture of 4sa and its regioisomer (7-:8- = 94:6)

和

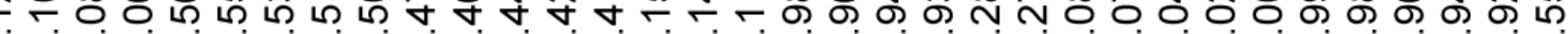
N

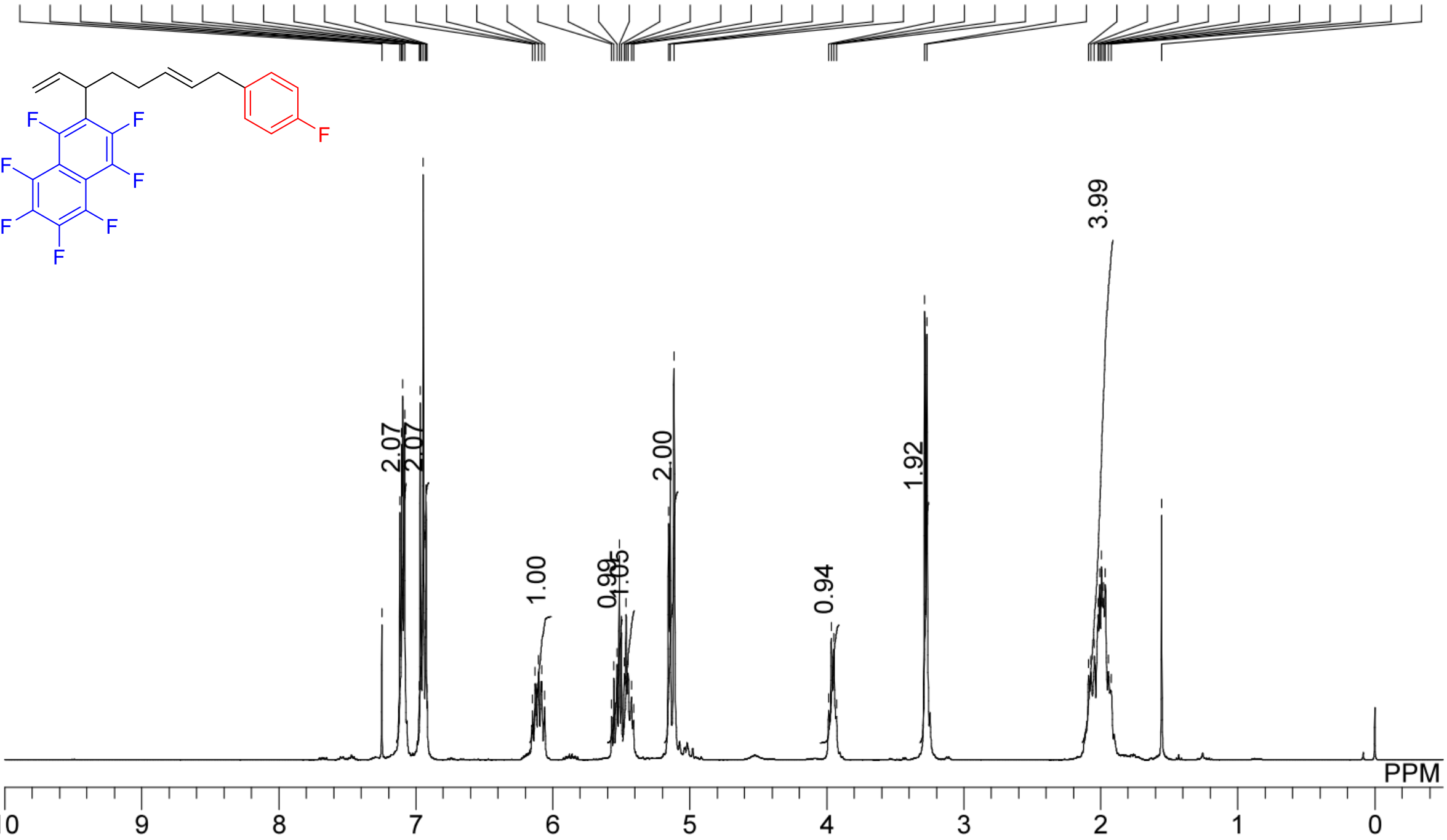


${ }^{13} \mathrm{C}\left\{{ }^{1} \mathrm{H}\right\}$ NMR of mixture of 4 sa and its regioisomer (7-:8- = 94:6)

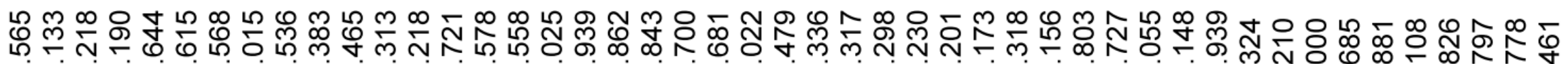

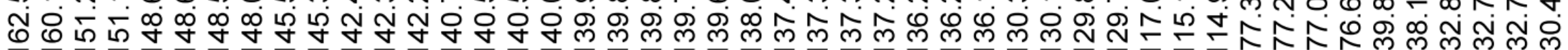
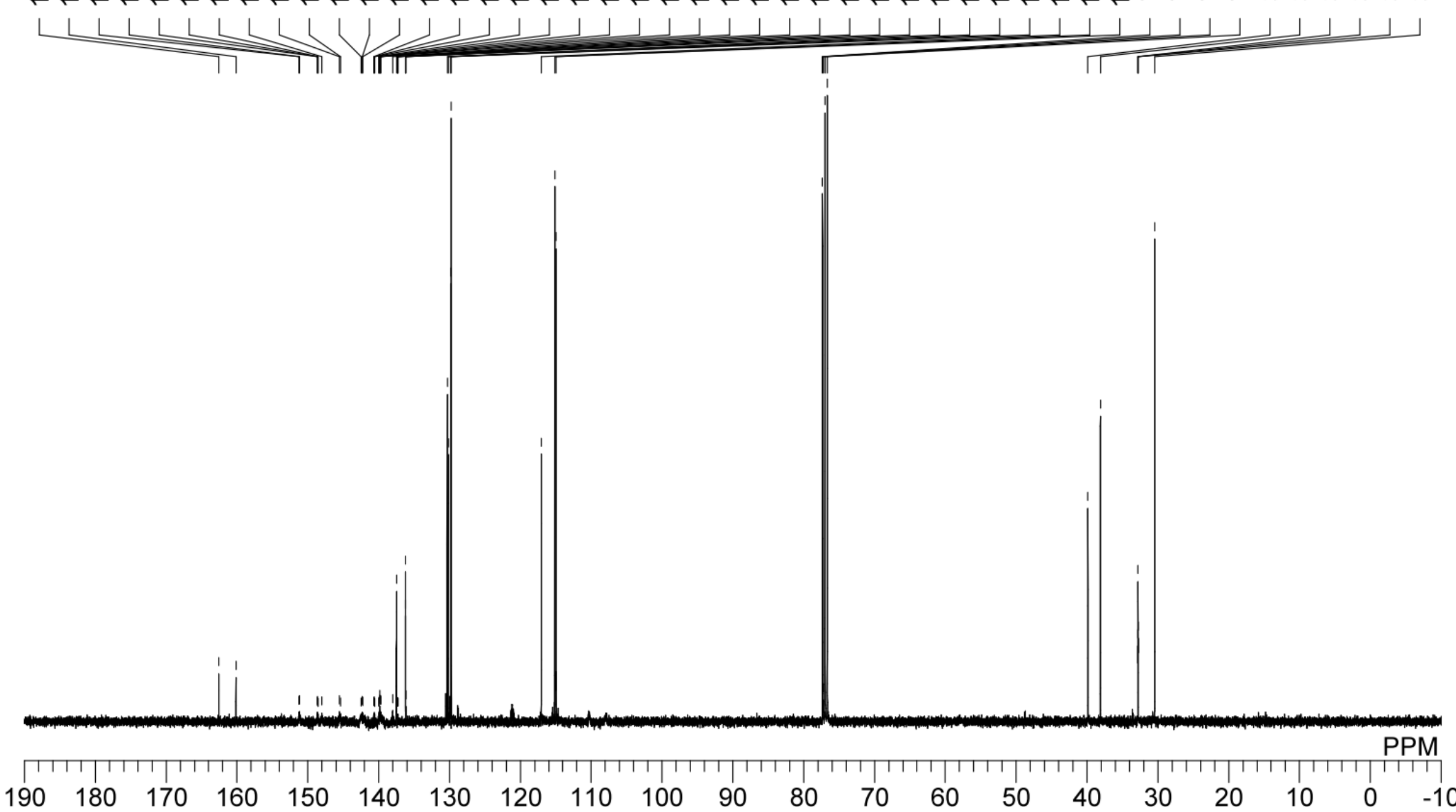
${ }^{13} \mathrm{C}\left\{{ }^{19} \mathrm{~F}\right\}$ NMR of mixture of $4 \mathbf{s a}$ and its regioisomer (7-:8- = 94:6)

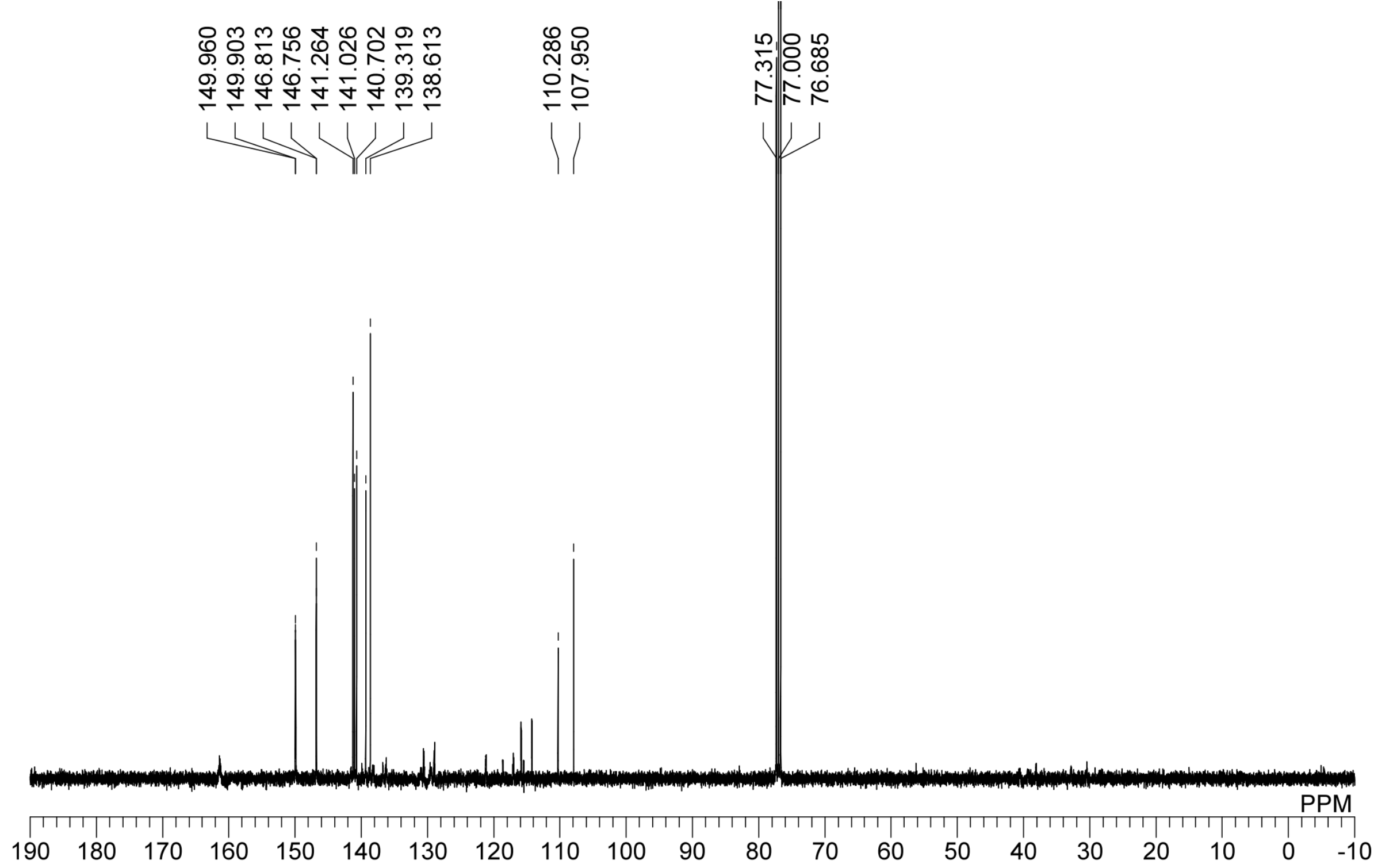


${ }^{19} \mathrm{~F}$ NMR of mixture of 4 sa and its regioisomer (7-:8- =94:6)

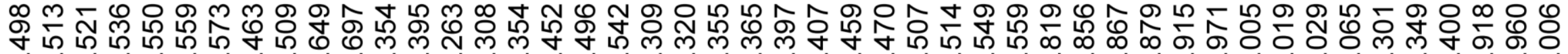

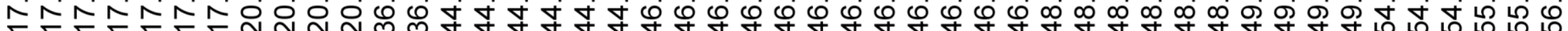

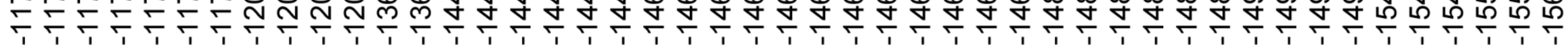

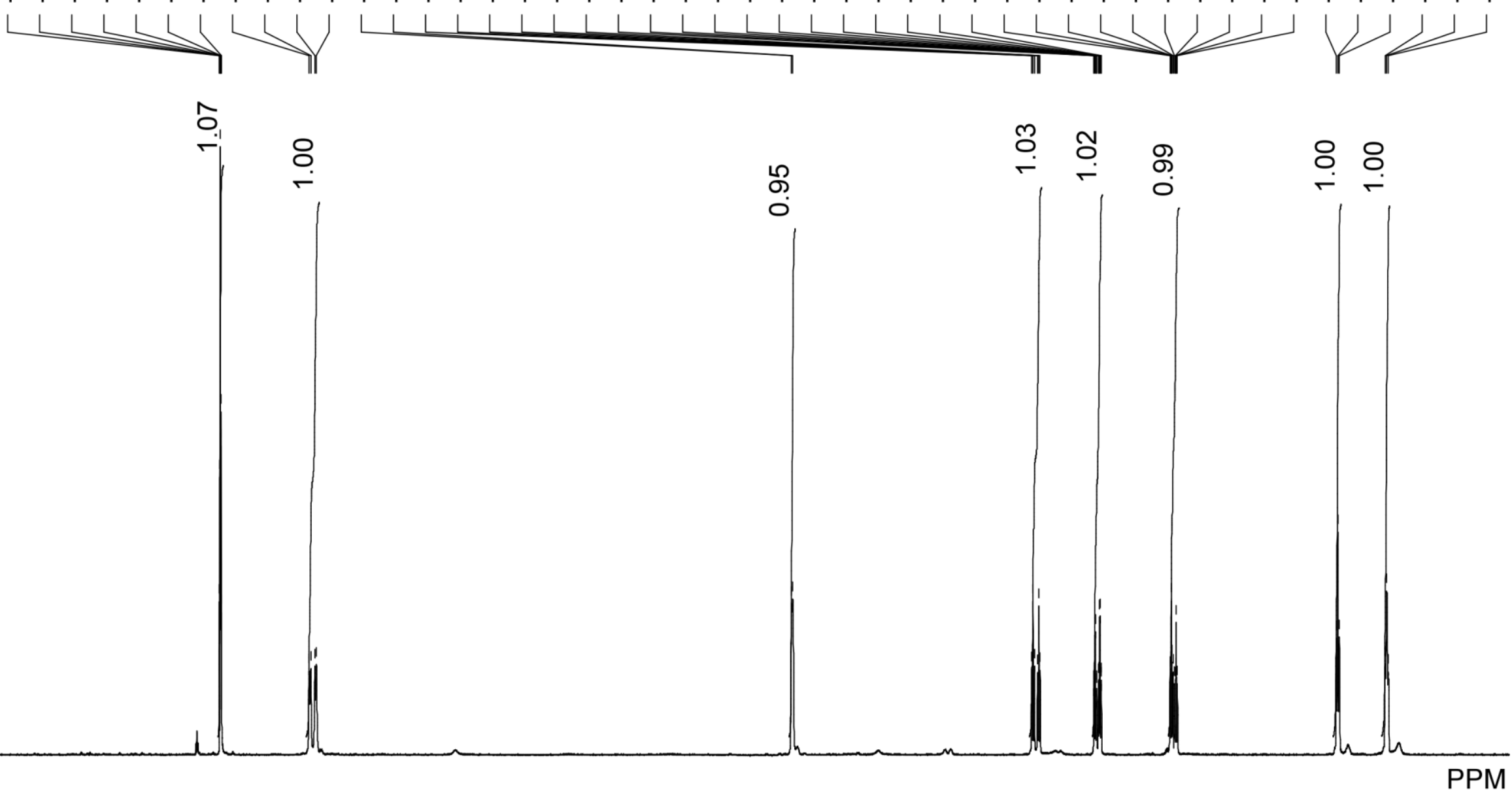

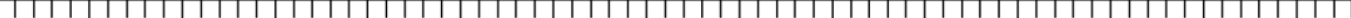
$-110-112-114-116-118-120-122-124-126-128-130-132-134-136-138-140-142-144-146-148-150-152-154-156-158$ 
${ }^{1} \mathrm{H}$ NMR of mixture of 4 ta and its regioisomer (3-:4- = 67:33)

넌

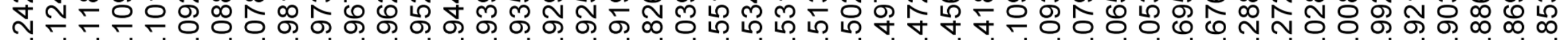

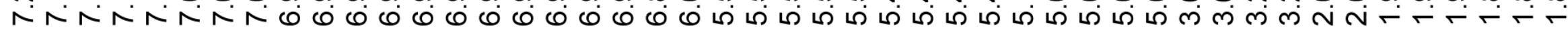

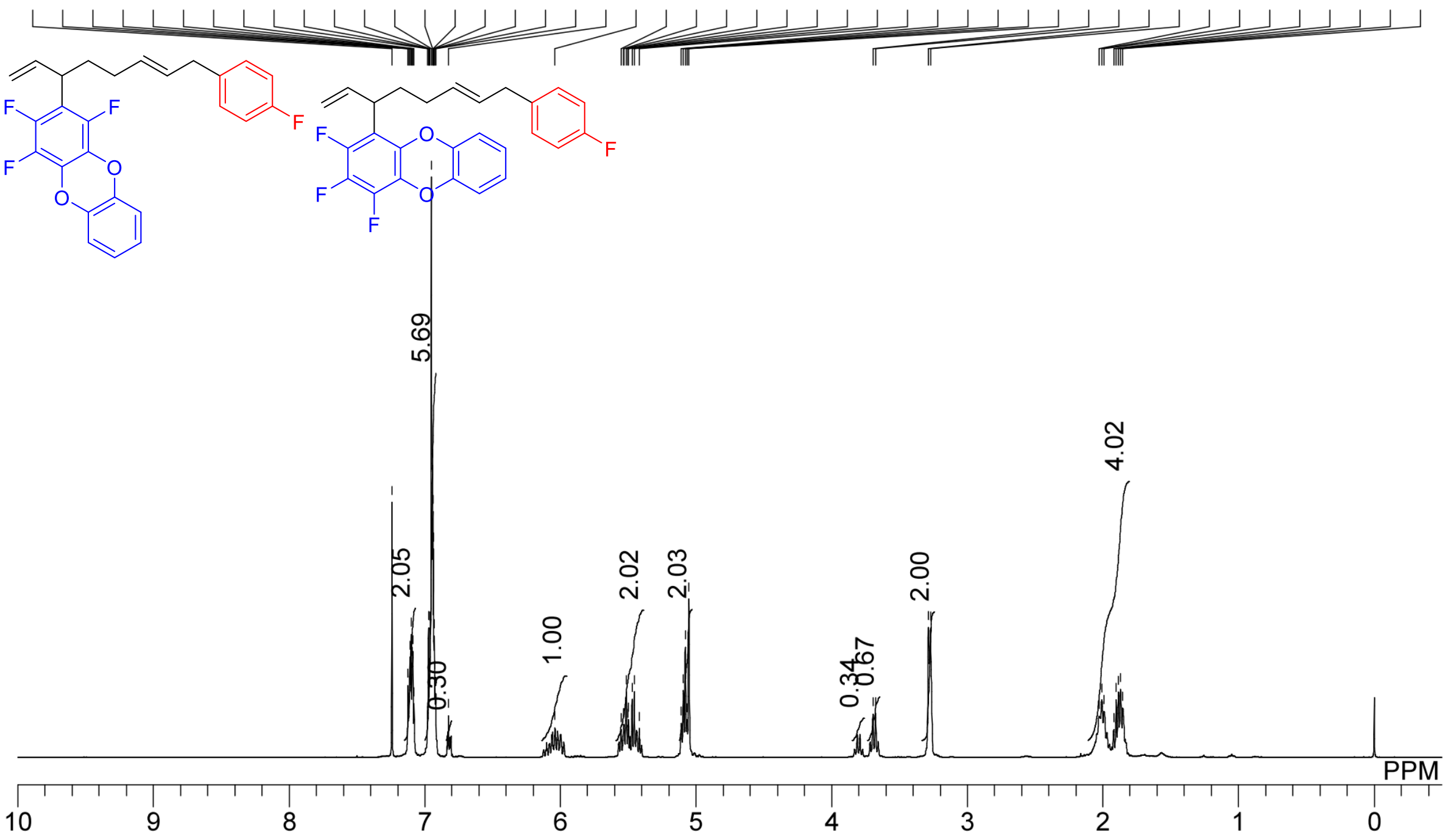


${ }^{13} \mathrm{C}\left\{{ }^{1} \mathrm{H}\right\}$ NMR of mixture of 4 ta and its regioisomer $(3-: 4-=67: 33)$

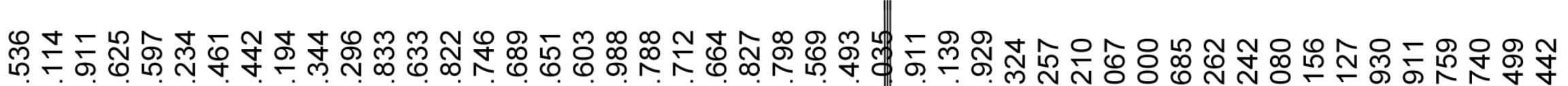
@守
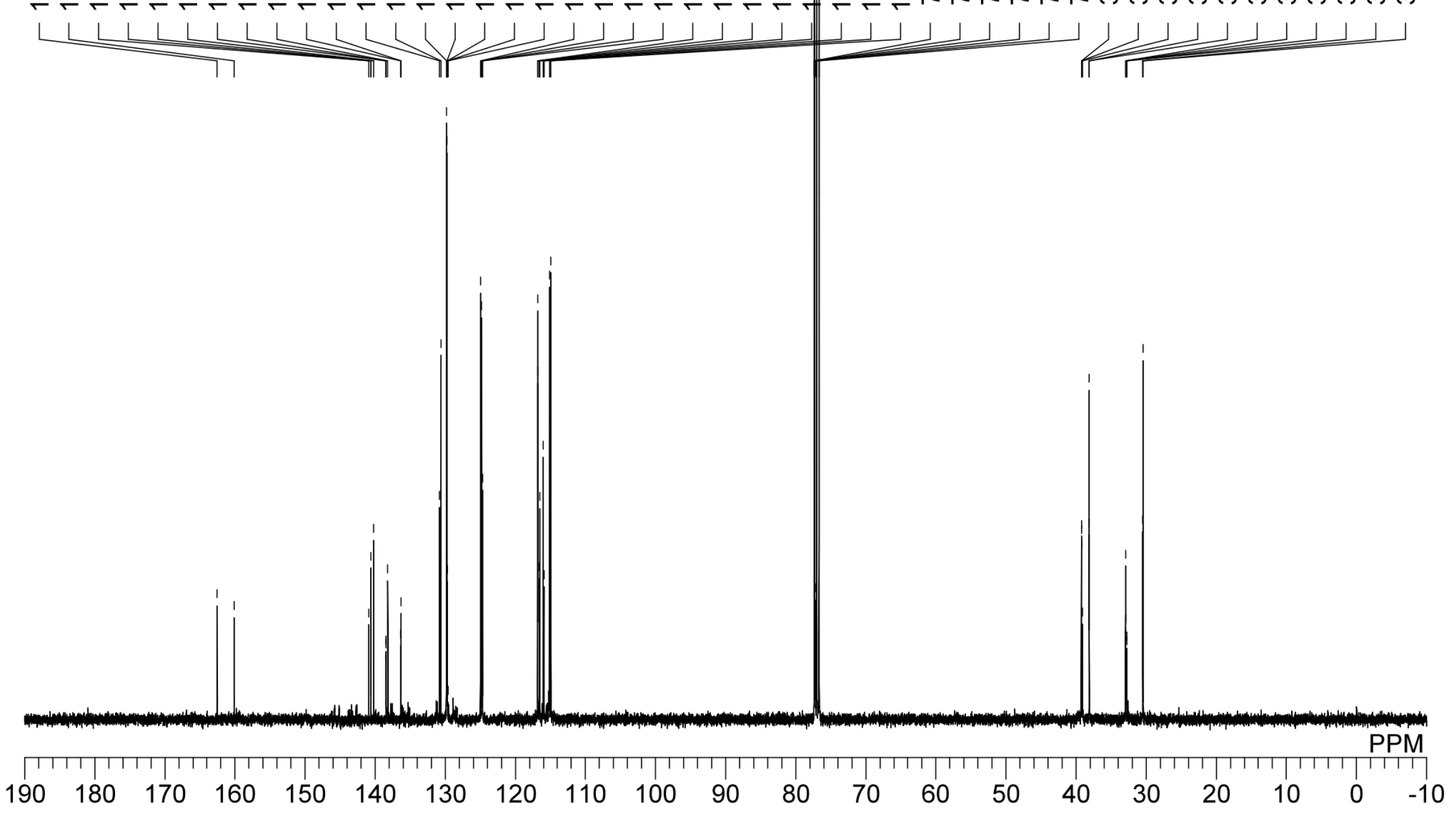
${ }^{13} \mathrm{C}\left\{{ }^{19} \mathrm{~F}\right\}$ NMR of mixture of 4 ta and its regioisomer $(3-: 4-=67: 33)$
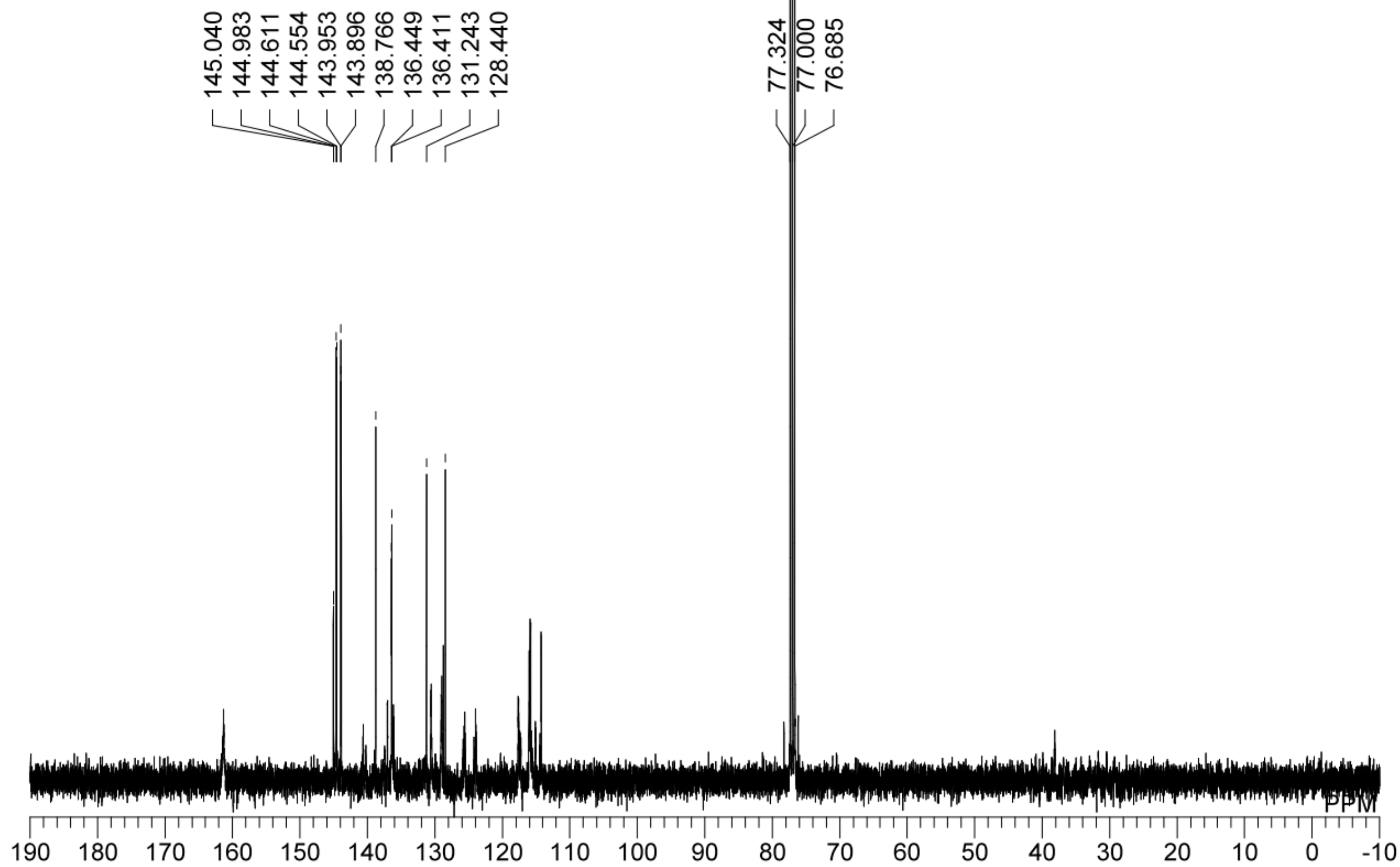
${ }^{19}$ F NMR of mixture of 4ta and its regioisomer (3-:4- = 67:33)

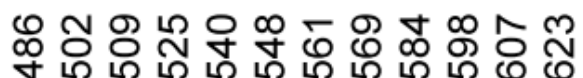

Mํ.

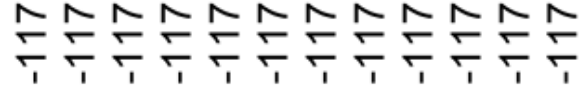

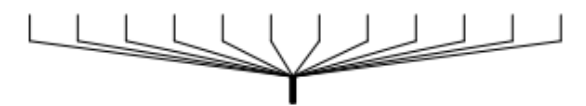

8

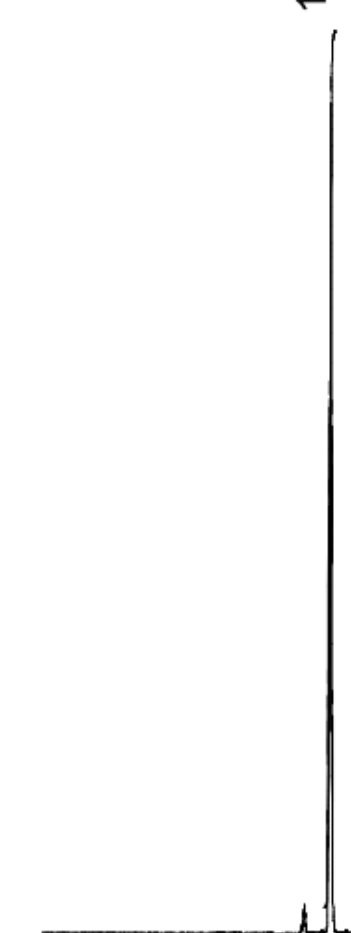

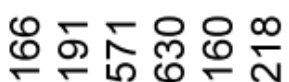

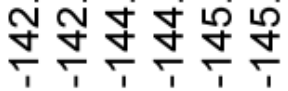
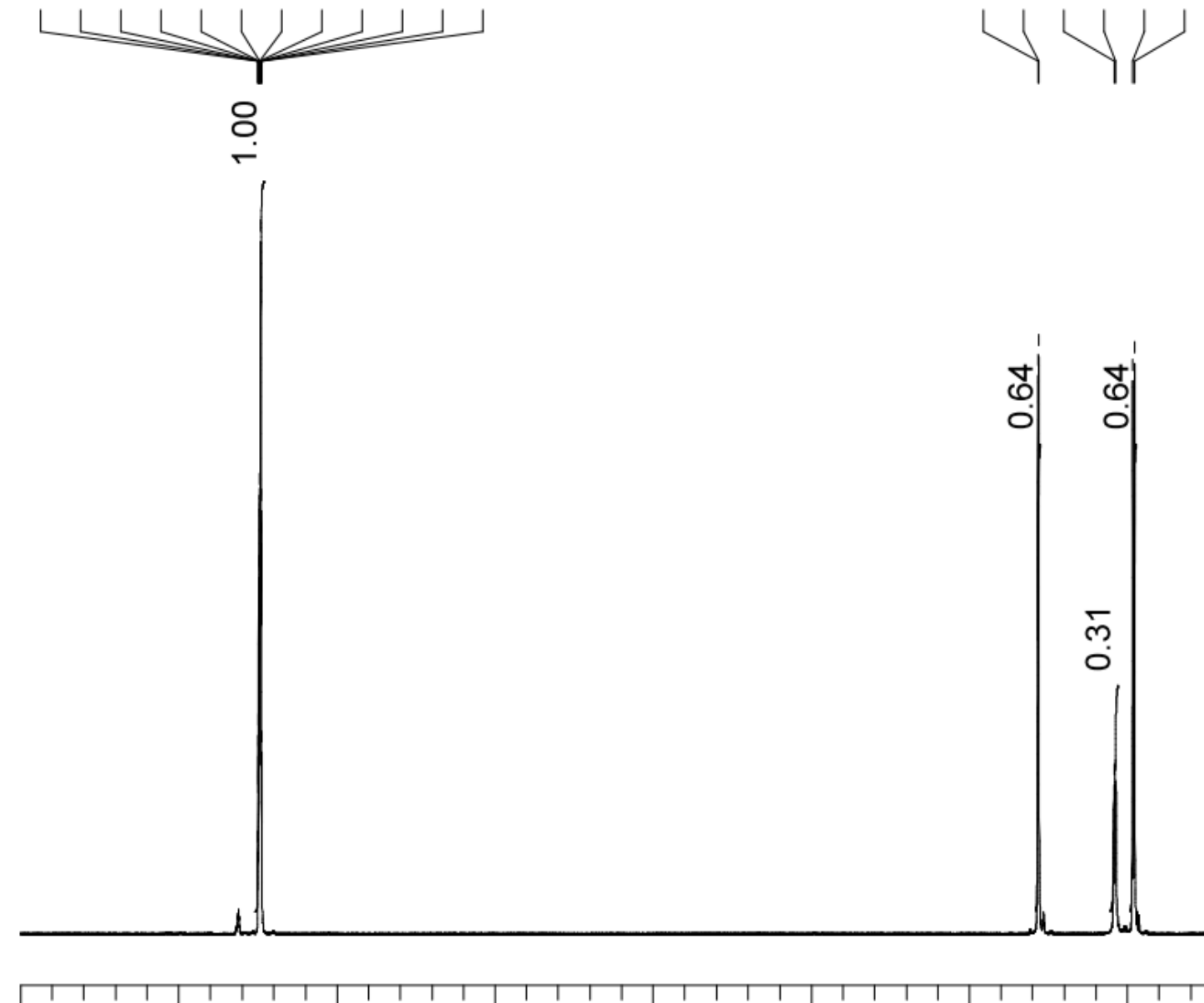

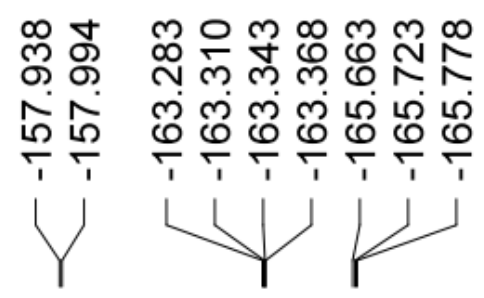
กิ

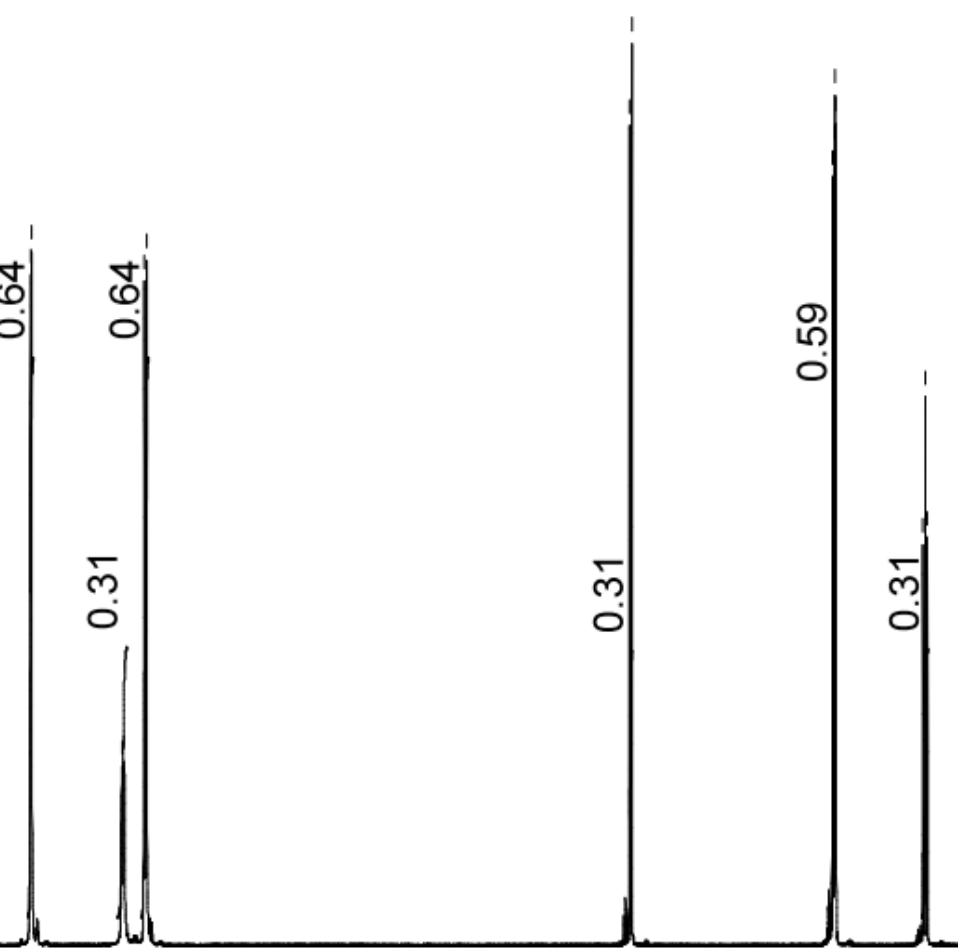

\section{PPM}

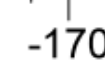


${ }^{1} \mathrm{H}$ NMR of mixture of 4ua its regioisomers $(p: m+o=87: 13)$

훈

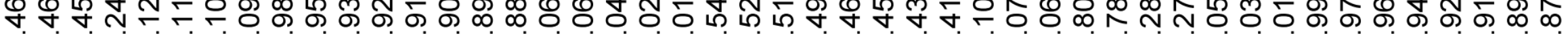
N
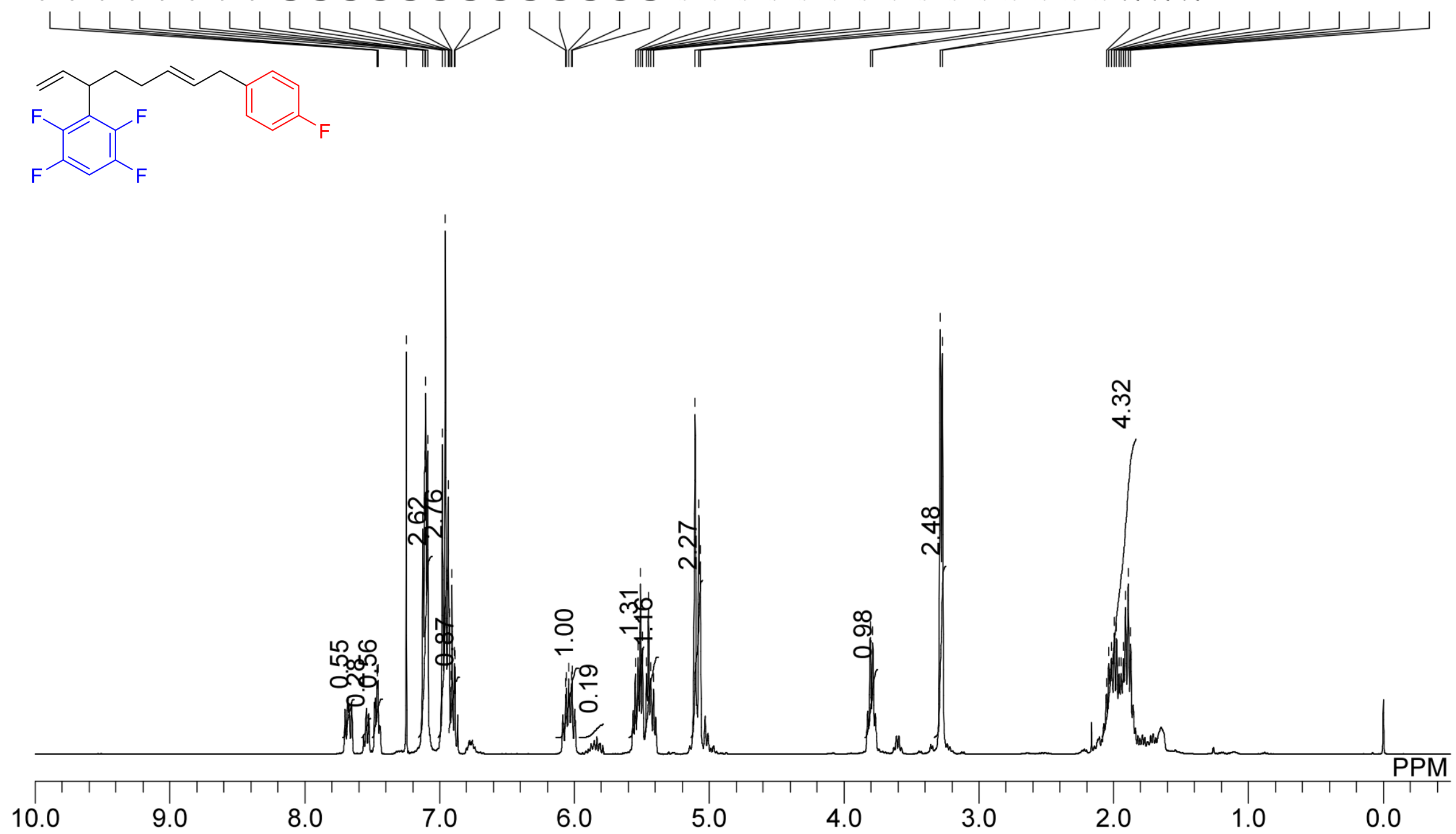
${ }^{13} \mathrm{C}\left\{{ }^{1} \mathrm{H}\right\}$ NMR of mixture of 4ua its regioisomers ( $p: m+o=87: 13$ )

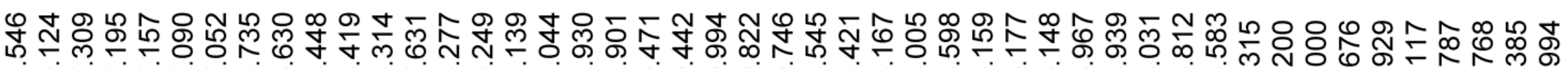

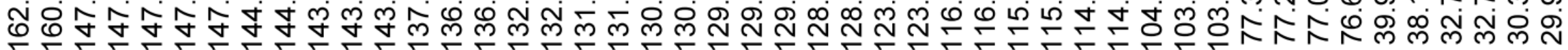
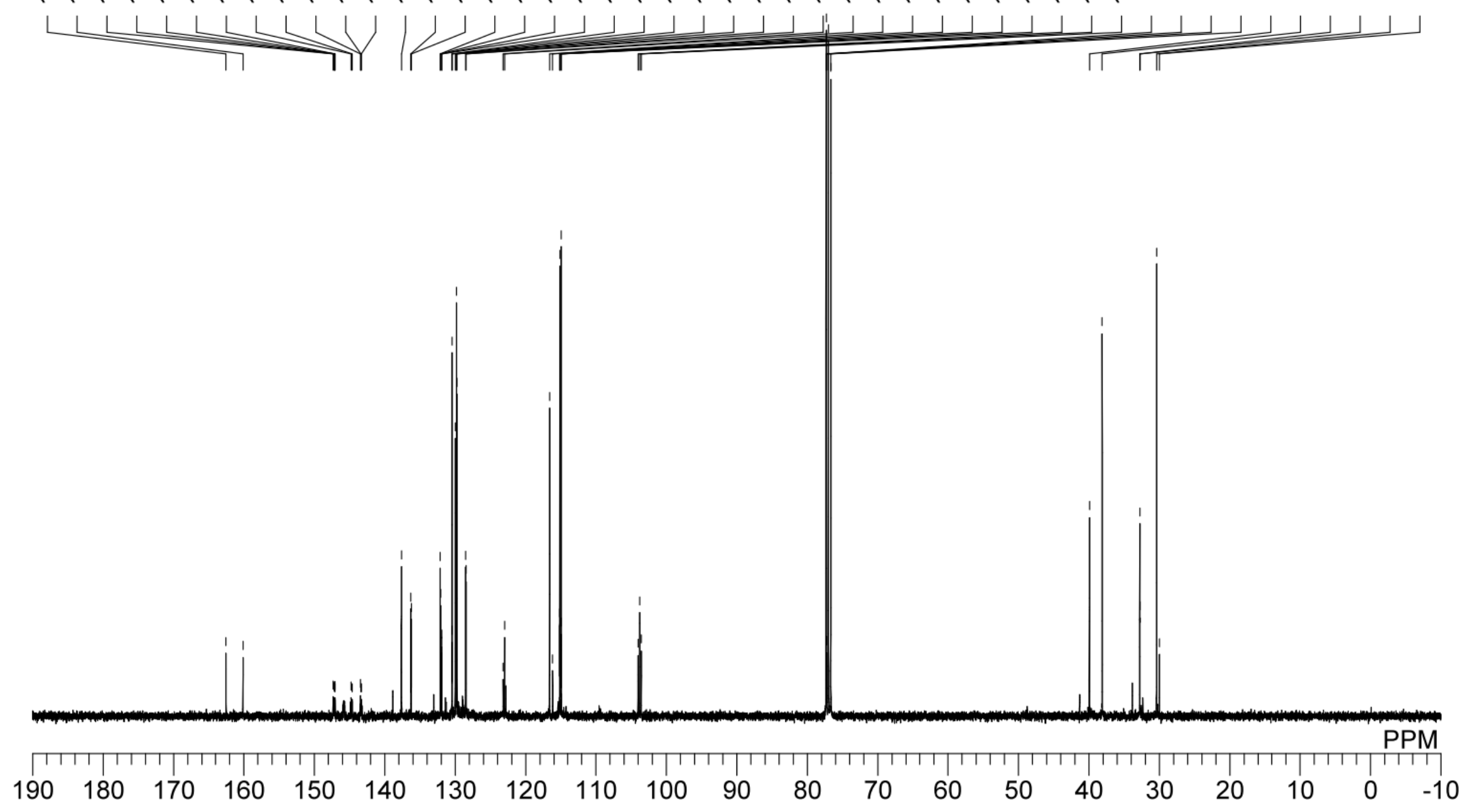
${ }^{13} \mathrm{C}\left\{{ }^{19} \mathrm{~F}\right\}$ NMR of mixture of 4 ua its regioisomers $(p: m+o=87: 13)$
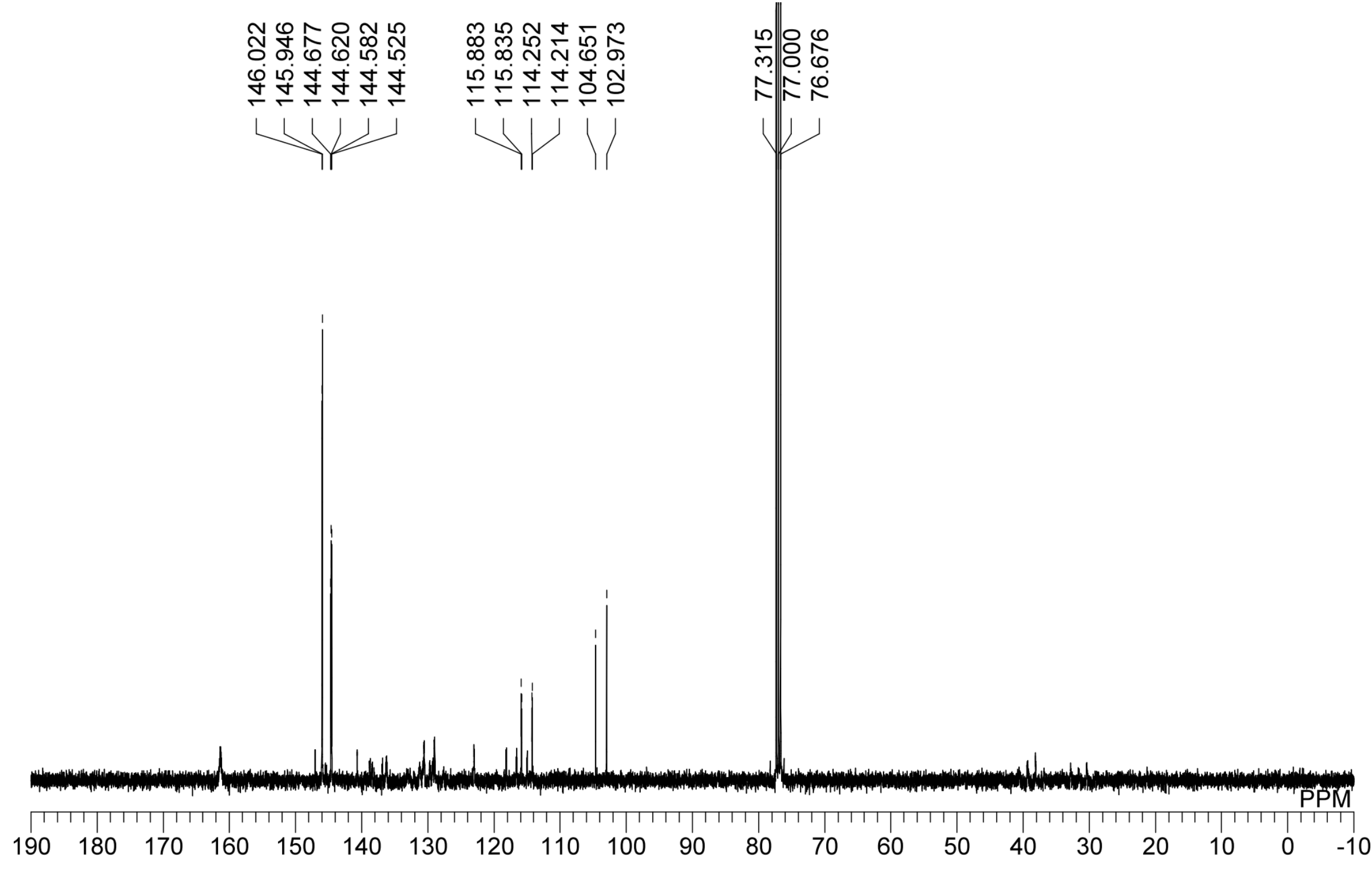
${ }^{19} \mathrm{~F}$ NMR of mixture of 4 ua its regioisomers $(p: m+o=87: 13)$

菖

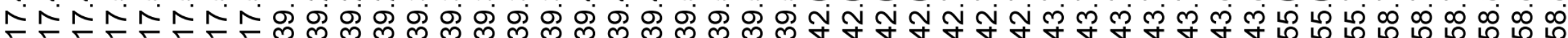

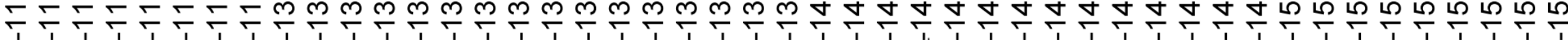
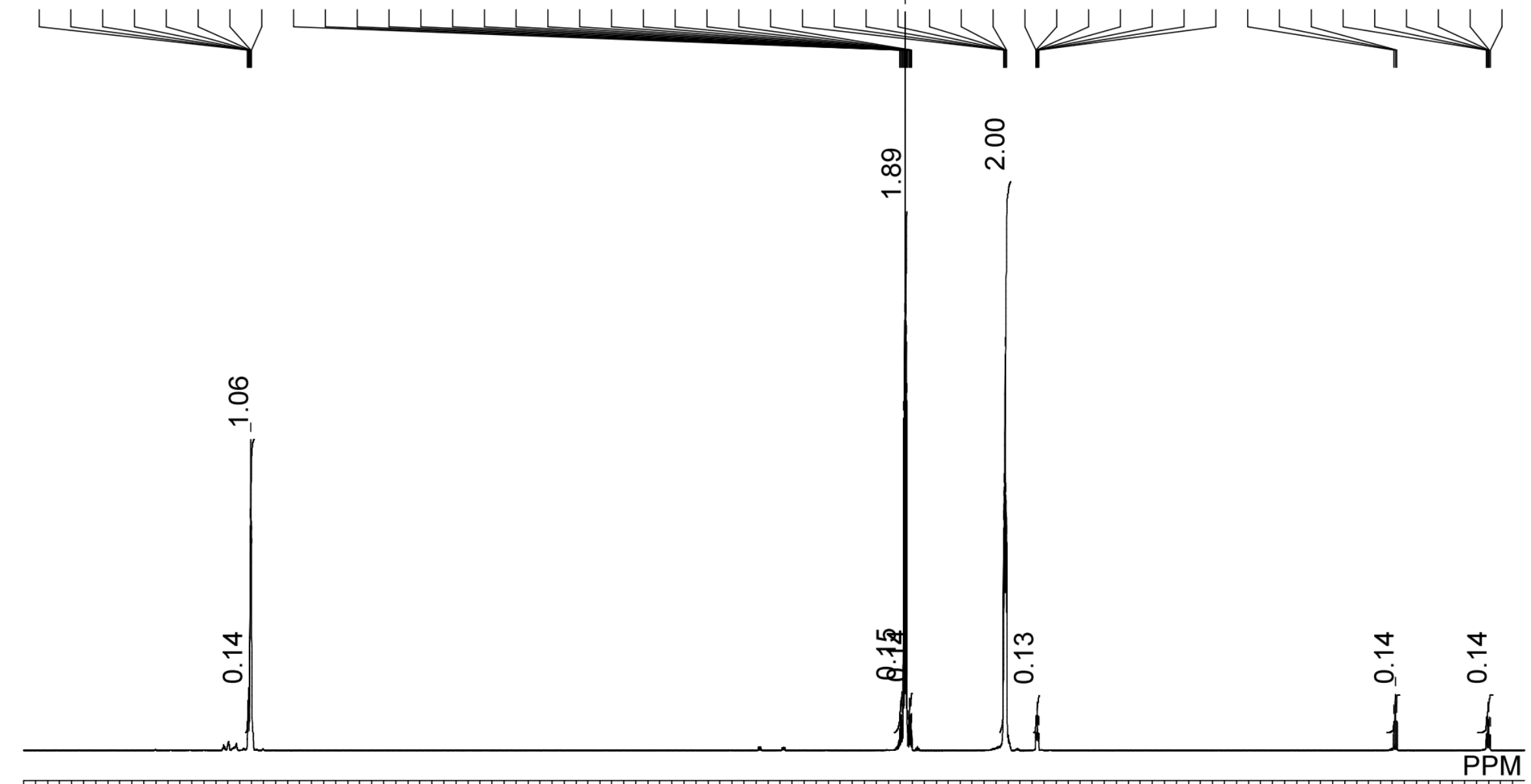

(13) $-110-112-114-116-118-120-122-124-126-128-130-132-134-136-138-140-142-144-146-148-150-152-154-156-158$ 


\section{${ }^{1} \mathrm{H}$ NMR of $\mathbf{6 a}$}

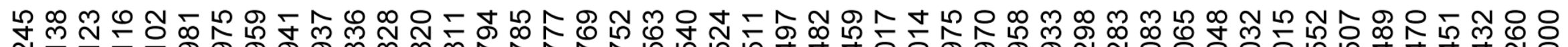

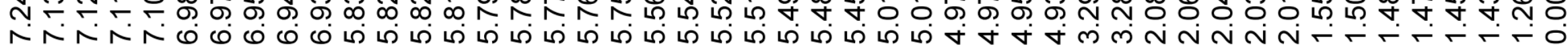
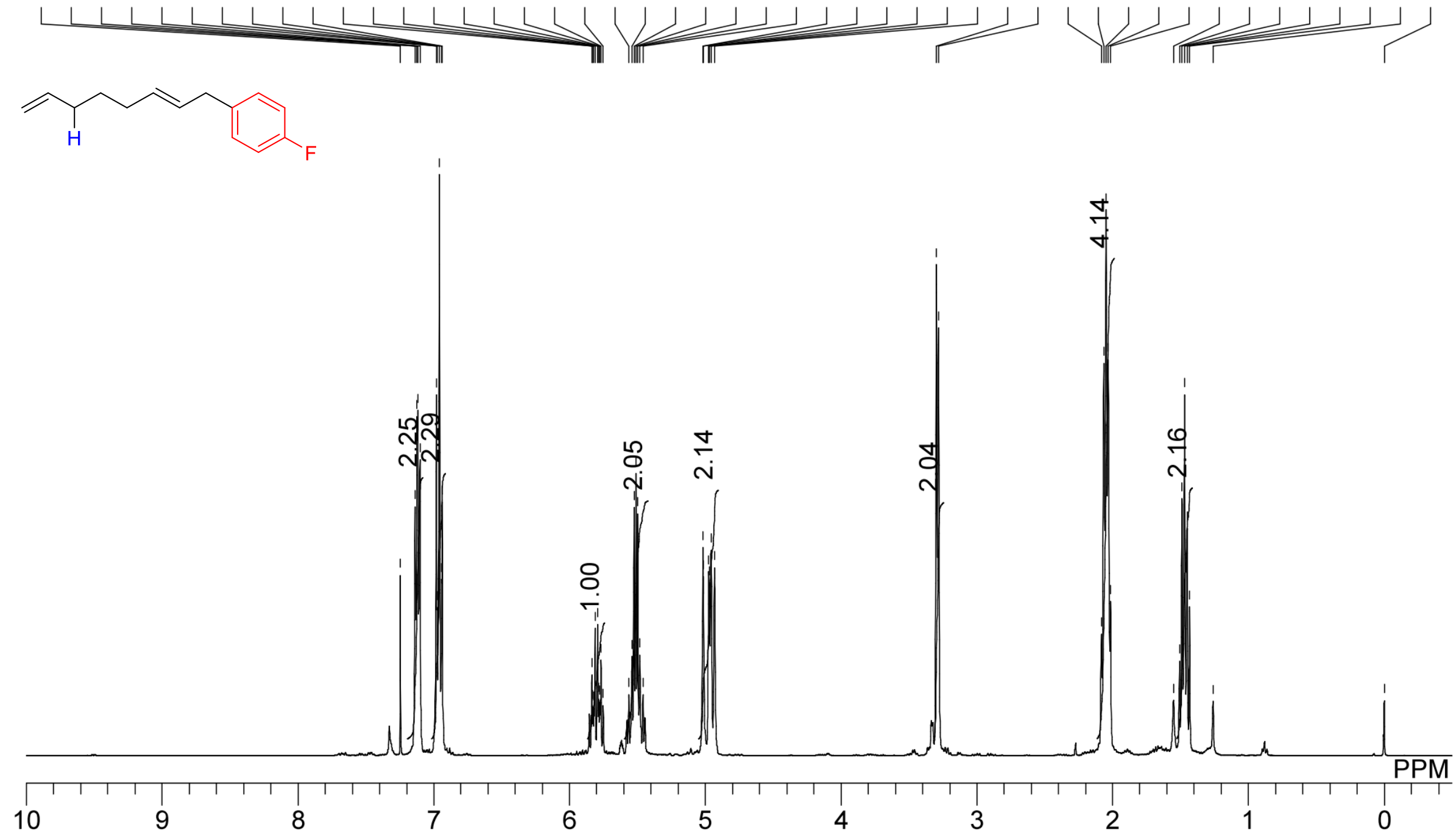
${ }^{13} \mathrm{C}\left\{{ }^{1} \mathrm{H}\right\}$ NMR of $\mathbf{6 a}$

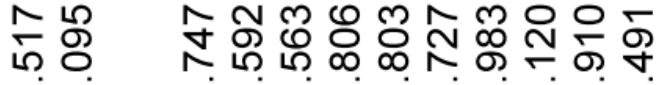

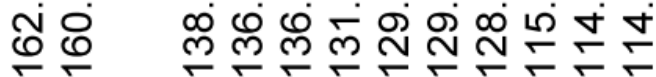
స్ㅇㅇㅛ
ヘド

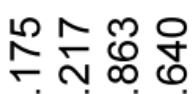

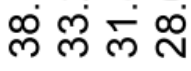
11
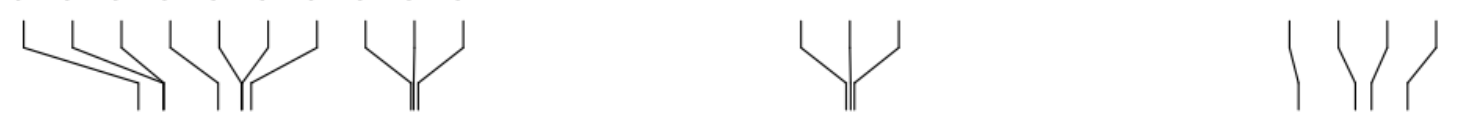

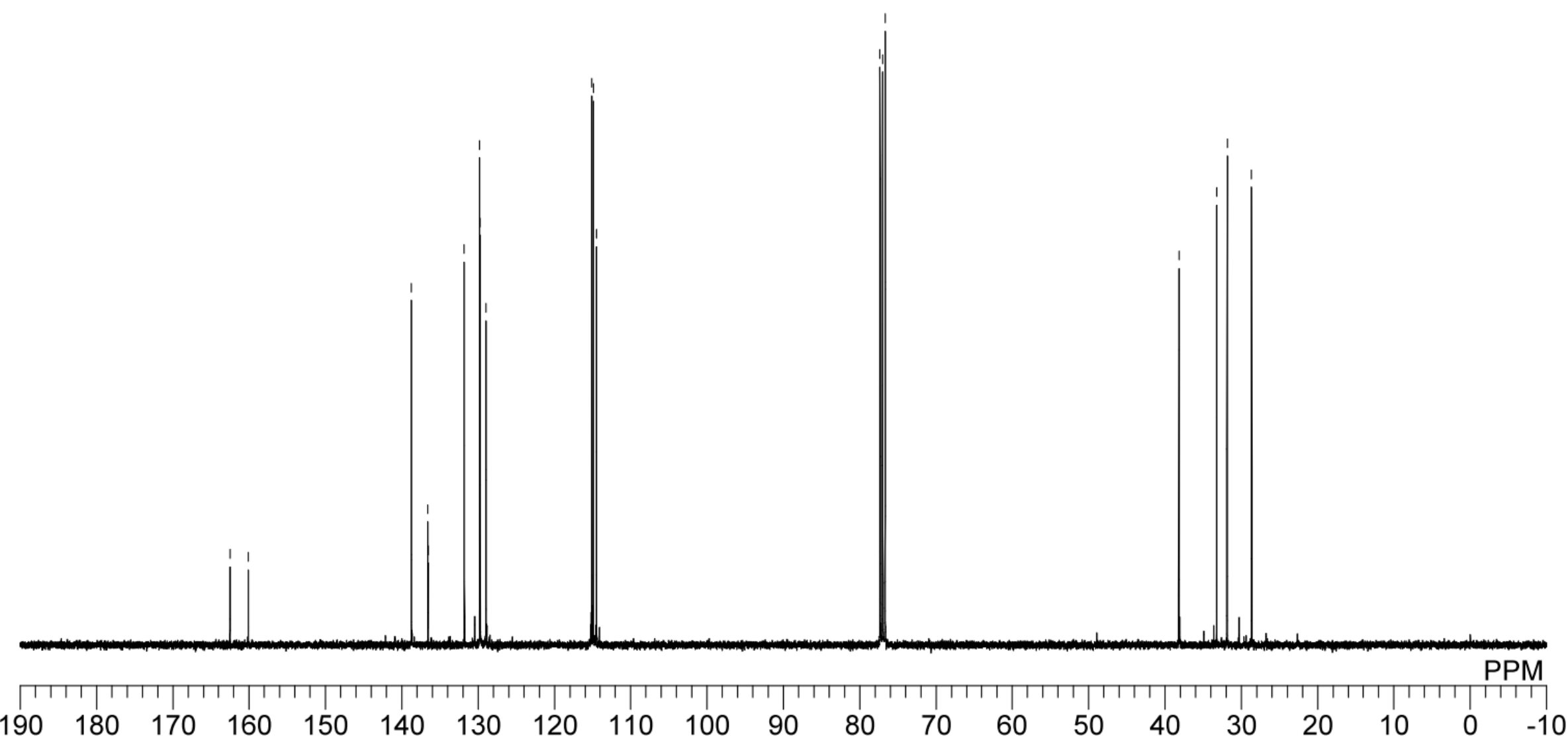


${ }^{19}$ F NMR of $6 \mathbf{a}$
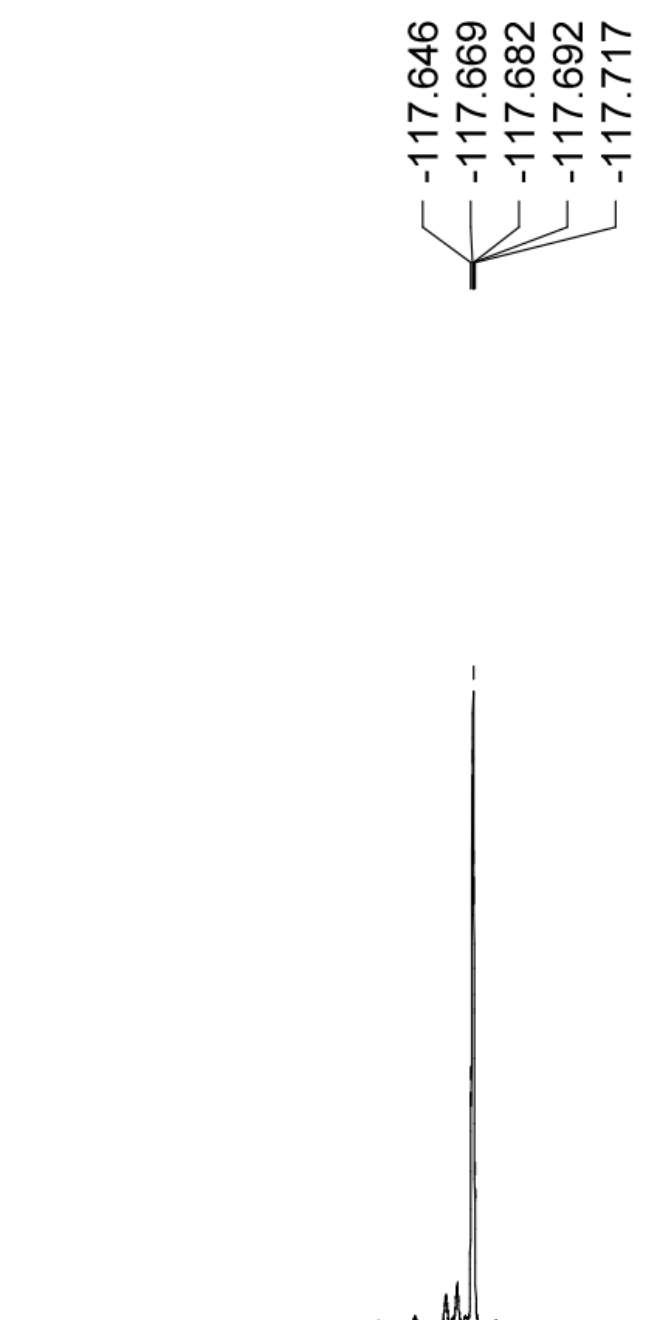

PPM

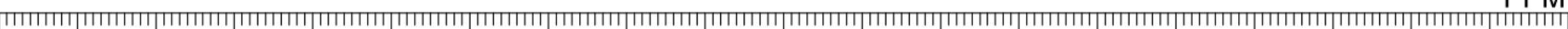
$\begin{array}{lllllllllllllllllllllll}-110 & -112 & -114 & -116 & -118 & -120 & -122 & -124 & -126 & -128 & -130 & -132 & -134 & -136 & -138 & -140 & -142 & -144 & -146 & -148 & -150\end{array}$ 